ORNL-6958

(Edition 19 of ORNL-5 198)

Center for Transportation Analysis

Energy Division

\title{
TRANSPORTATION ENERGY DATA BOOK: EDITION 19
}

\author{
Stacy C. Davis \\ Oak Ridge National Laboratory
}

September 1999

Prepared for

Office of Transportation Technologies

U.S. Department of Energy

Prepared by

OAK RIDGE NATIONAL LABORATORY

Oak Ridge, Tennessee 3783 1-6073

managed by

LOCKHEED MARTIN ENERGY RESEARCH CORP.

for the

U.S. DEPARTMENT OF ENERGY

under Contract No. DE-AC05-96OR22464 
This report has been reproduced directly from the best available copy.

Available to DOE and DOE contractors from the Office of Scientific and Technical Information, P.O. Box 62, Oak Ridge, TN 37831; prices available from (423) 576-8401.

Available to the public from the National Technical Information Service, U.S. Department of Commerce, 5285 Port Royal Rd., Springfield, VA 22161.

This report was prepared as an account of work sponsored by an agency of the United States Government. Neither the United States Government nor any agency thereof, nor any of their employees, makes any warranty, express or implied, or assumes any legal liability or responsibility of the accuracy, completeness, or usefulness of any information, apparatus, product, or process disclosed, or represents that its use would not infringe privately owned rights. Reference herein to any specific commercial product, process, or service by, trade name, trademark, manufacturer, or otherwise, does not necessarily constitute or imply its endorsement, recommendation, or favoring by the United States Government or any agency thereof. The views and opinions of authors expressed herein do not necessarily state or reflect those of the United States Government or any agency thereof. 
Users of the Transportation Energy Data Book are encouraged to comment on errors, omissions, emphases, and organization of this report to one of the persons listed below. Requests for additional complementary copies of this report, additional data, or information on an existing table should be referred to Ms. Stacy Davis, Oak Ridge National Laboratory.

Stacy C. Davis

Oak Ridge National Laboratory

P. 0. Box 2008

Building 3 156, MS-6073

Oak Ridge, Tennessee 3783 1-6073

Telephone*:

(423) 574-5957

FAX*:

(423) 574-3851

E-mail: DAVISSC@ornl.gov

Web Site Location: http://www-cta.ornl.gov

Philip D. Patterson

Office of Transportation Technologies

Department of Energy, EE-30

Forrestal Building, Room 5F-034

1000 Independence Avenue, S. W.

Washington, D.C. 20585

Telephone: (202) 586-9121

FAX: $\quad$ (202) 586-1637

E-mail: $\quad$ PHILIP.PATTERSON@hq.doe.gov

Web Site Location: http://www.ott.doe.gov

analytic page: http://www.ott.doe.gov/fact.html

This and previous editions of the Transportation Energy Data Book can be found on the web at: http://www-cta.ornl.gov/data/tedb.htm

* The Oak Ridge area code (423) will change to area code 865 as of February 2000. 


\section{TABLE OF CONTENTS}

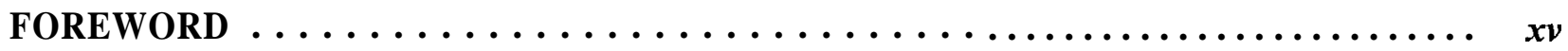

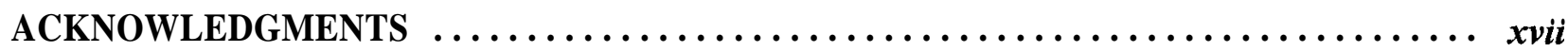

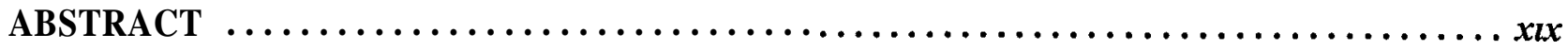

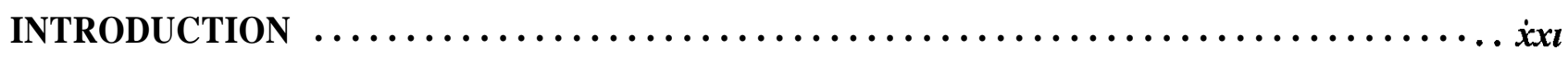

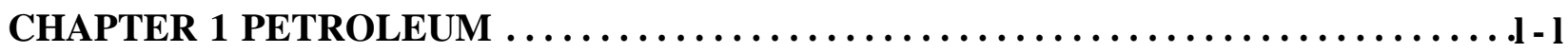

Figure $1.1 \quad$ USGS World Oil Resource Estimates, 1993 ......................... 1-2

Table $1.1 \quad$ Summary of Recent World Oil Assessments .................... 1-2

Table $1.2 \quad$ World Crude Oil Production, $1960-97 \ldots \ldots \ldots \ldots \ldots \ldots \ldots \ldots \ldots \ldots$ 1-3

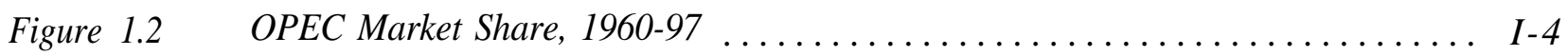

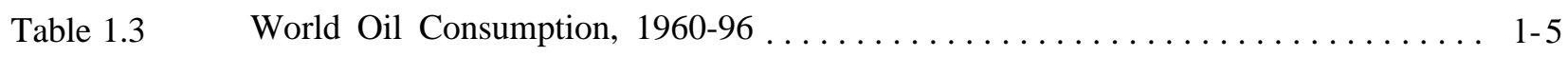

Table 1.4 Petroleum Stocks in OECD Countries, End of Year 1973-97 ............. 1-6

Figure I. 3 Share of OECD Petroleum Stocks to OECD Petroleum Consumption, 1973-96 _ . . l-7

Figure $1.4 \quad$ Share of Petroleum Stocks to Petroleum Consumption by Country, $1996 \ldots \ldots \ldots$. . 1-8

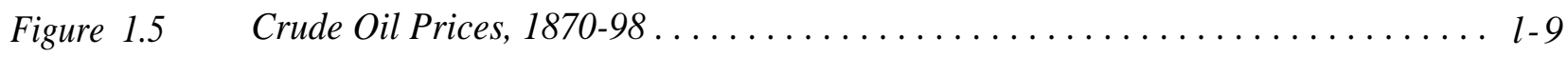

Table 1.5 U.S. Petroleum Net Imports by World Region of Origin, 1960-97 . ......... 1-10

Table 1.6 Summary of 1996 Military Expenditures for Defending Oil Supplies from the Middle East .................................. $1-11$

Figure $1.6 \quad$ Refinery Gross Output by World Region, $1998 \ldots \ldots \ldots \ldots \ldots \ldots \ldots \ldots \ldots$. 12

Table 1.7 U.S. Refinery Input of Crude Oil and Petroleum Products, 1987-97 . . . . . . . . 1-13

Table 1.8 Refinery Yield of Petroleum Products from a Barrel of Crude Oil, 1978-97 .... . 1-14

Table 1.9 United States Petroleum Production and Consumption, 1973-98 ........... 1-15

Figure I. 7. United States Petroleum Production and Consumption, $1973-98 \ldots \ldots \ldots \ldots$. .l 6

Figure I. 8. Import Share of U.S. Petroleum Consumption, 1973-98 .............. l-l 7 


\section{TABLE OF CONTENTS (Continued)}

Table $1.10 \quad$ Consumption by Petroleum by End-Use Sector, $1973-98 \ldots \ldots \ldots \ldots \ldots \ldots$ 1- 18

Table 1.11 Transportation of Petroleum and Petroleum Products in the U.S.

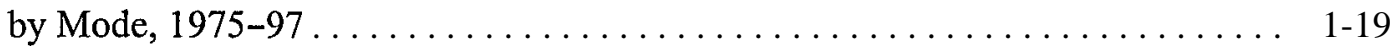

CHAPTER 2 ENERGY $\ldots \ldots \ldots \ldots \ldots \ldots \ldots \ldots \ldots \ldots \ldots \ldots \ldots \ldots \ldots \ldots \ldots \ldots \ldots \ldots$

Table 2.1 World Production of Primary Energy by Selected Country Groups, 1988-97 . . . . 2-2

Table 2.2 World Consumption of Primary Energy by Selected Country Groups, 1988-97 . . . 2-3

Table $2.3 \quad$ U. S. Consumption of Total Energy by End-Use Sector, 1970-98 . . . . . . . . . 2-4

Table 2.4 Distribution of Energy Consumption by Source, 1973, 1980, and $1998 \ldots \ldots \ldots$ 2-5

Table 2.5 Domestic Consumption of Transportation Energy by Mode and

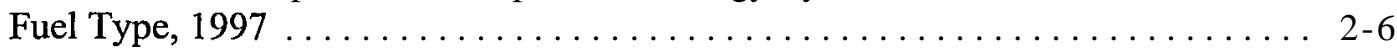

Table $2.6 \quad$ Transportation Energy Use by Mode, $1996-97 \ldots \ldots \ldots \ldots \ldots \ldots \ldots \ldots \ldots$

Table 2.7 Transportation Energy Consumption by Mode, $1970-97 \ldots \ldots \ldots \ldots \ldots \ldots$

Table $2.8 \quad$ Highway Usage of Gasoline and Special Fuels, $1973-97 \ldots \ldots \ldots \ldots \ldots \ldots \ldots$. . . . .

Figure $2.1 \quad$ Motor Gasoline Quantities by Type, 1981 and 1997................ 2-10

Table $2.9 \quad$ Alternative Vehicle Fuel Consumption, $1992-99 \ldots \ldots \ldots \ldots \ldots \ldots \ldots \ldots \ldots$ 2-1 1

Table $2.10 \quad$ U.S. Production and Imports of MTBE and Fuel Ethanol, 1978-98 ......... 2-12

Table $2.11 \quad$ Passenger Travel and Energy Use in the United States, $1997 \ldots \ldots \ldots \ldots \ldots$. . . 13

Table $2.12 \quad$ Energy Intensities of Passenger Modes, $1970-97 \ldots \ldots \ldots \ldots \ldots \ldots \ldots \ldots$. . . . . . . .

Figure 2.2 Energy Intensity for Transit in the U.S., 1997................. 2-15

Table 2.13 Intercity Freight Movement and Energy Use in the United States, $1997 \ldots \ldots$. $2-16$

Table $2.14 \quad$ Energy Intensities of Freight Modes, $1970-97 \ldots \ldots \ldots \ldots \ldots \ldots \ldots \ldots \ldots$

CHAPTER 3 GREENHOUSE GAS EMISSIONS $\ldots \ldots \ldots \ldots \ldots \ldots \ldots \ldots \ldots \ldots \ldots \ldots$

Table 3.1 International Man-Made Emissions of All Greenhouse Gases, 1990-96 . . . . . . 3-2

Table 3.2 International Man-Made Emissions of Carbon Dioxide, 1990-96 . . . . . . . . 3-3 


\section{TABLE OF CONTENTS (Continued)}

Table 3.3 International Man-Made Emissions of Carbon Dioxide by

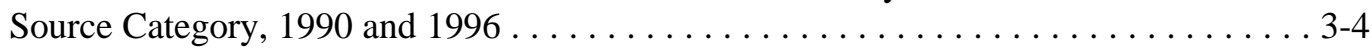

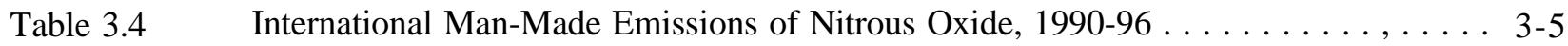

Table $3.5 \quad$ Estimated U.S. Emissions of Greenhouse Gases, 1990-97 . . . . . . . . . . . . . 3-6

Table $3.6 \quad$ U.S. Carbon Dioxide Emissions from Fossil Energy Consumption by

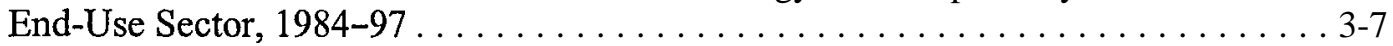

Table 3.7 U.S. Carbon Dioxide Emissions from Energy Use in the Transportation

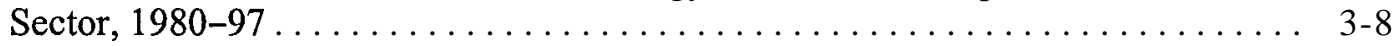

Table $3.8 \quad$ Numerical Estimates of Global Warming Potentials Compared With Carbon Dioxide . . . . . . . . . . . . . . . . . . . . . . . . . 3-9

Table 3.9 Changes in Per-Mile, Fuel-Cycle Energy Use and Greenhouse Gas Emissions for Passenger Cars using Near-Term Technologies . . . . . . . . . . . . . 3-12

Table 3.10 Changes in Per-Mile, Fuel-Cycle Energy Use and Greenhouse Gas Emissions for Passenger Cars using Long-Term Technologies . . . . . . . . . . . . . . . 3-1 3

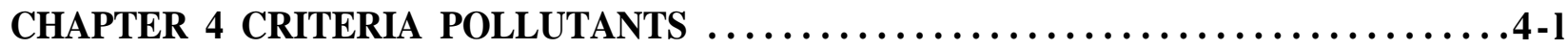

Table $4.1 \quad$ Total National Emissions of the Criteria Air Pollutants by Sector, $1997 \ldots \ldots$. . . 4-2

Table 4.2 Total National Emissions of Carbon Monoxide, 1970-97 . . . . . . . . . . . . . . . 4 4-3

Table 4.3 Emissions of Carbon Monoxide from Highway Vehicles, 1970-97 . . . . . . . . . . . 4-4

Table $4.4 \quad$ Total National Emissions of Nitrogen Oxides, $1970-97 \ldots \ldots \ldots \ldots \ldots \ldots \ldots \ldots$

Table $4.5 \quad$ Emissions of Nitrogen Oxides from Highway Vehicles, 1970-97 . . . . . . . . . . 4-6

Table 4.6 Total National Emissions of Volatile Organic Compounds, 1970-97. . . . . . . . . 4 4-7

Table 4.7 Emissions of Volatile Organic Compounds from Highway Vehicles, 1970-97 . . . . 4-8

Table $4.8 \quad$ Total National Emissions of Particulate Matter (PM-1 0), 1970-97 . . . . . . . . . 4-9

Table 4.9 Emissions of Particulate Matter (PM-IO) from Highway Vehicles, 1970-97 . . . . 4-10

Table $4.10 \quad$ Total National Emissions of Particulate Matter (PM-2.5), 1990-97 . . . . . . . . . 4-1 1

Table 4.11 Emissions of Particulate Matter (PM-2.5) from Highway Vehicles, 1990-97 . . . . 4-12

Table $4.12 \quad$ National Lead Emission Estimates, $1970-97 \ldots \ldots \ldots \ldots \ldots \ldots \ldots \ldots \ldots \ldots$. . . . 3

Table $4.13 \quad$ State-level Emissions for Criteria Pollutants, $1997 \ldots \ldots \ldots \ldots \ldots \ldots \ldots \ldots \ldots$. . . . . . . . . . 


\section{TABLE OF CONTENTS (Continued)}

Table 4.14 Changes in Per-Mile, Fuel-Cycle Energy Use and Criteria Pollutant Emissions for Passenger Cars using Near-Term Technologies . . . . . . . . . . . . . . 4-1 7

Table 4.15 Changes in Per-Mile, Fuel-Cycle Energy Use and Criteria Pollutant Emissions for Passenger Cars using Long-Term Technologies . . . . . . . . . . . . . . . . . 4-1 9

Table 4.16 Federal Exhaust Emission Certification Standards for Gasoline- and Diesel-Powered Light Vehicles . . . . . . . . . . . . . . . . . . . . . 4 4-28

Table 4.17 Federal Exhaust Emission Certification Standards for Gasoline- and Diesel Powered Light Trucks (Category LDT1) . . . . . . . . . . . . . . . . 4-29

Table 4.18 Federal Exhaust Emission Certification Standards for Gasoline- and Diesel Powered Light Trucks (Category LDT2) . . . . . . . . . . . . . . . . . 4-30

Table 4.19 Federal Exhaust Emission Certification Standards for Gasoline- and Diesel Powered Light Trucks (Category LDT3) . . . . . . . . . . . . . . . 4-3 1

Table 4.20 Federal Exhaust Emission Certification Standards for Gasoline- and Diesel Powered Light Trucks (Category LDT4) . . . . . . . . . . . . . . 4-32

Table 4.2 1 Federal Exhaust Emission Certification Standards for Gasoline- and Diesel Powered Light Heavy Trucks . . . . . . . . . . . . . . . . . . . . 4-33

Table 4.22 Federal Exhaust Emission Certification Standards for Gasoline- and Diesel Powered Heavy Heavy Trucks . . . . . . . . . . . . . . . . . . . . . . . 4-34

Table 4.23 California Passenger Cars and Light Trucks Emission Certification Standards. . . . . . . . . . . . . . . . . . . . . . . . . . . . . . . . . 4 4-35

Table 4.24 California Vehicle Emission Reduction for Passenger Cars and Light Trucks ................................ 4-36

Table 4.25 California Air Resources Board Requirements for Meeting Emission Standards. . . 4-37

CHAPTER 5 TRANSPORTATION AND THE ECONOMY $\ldots \ldots \ldots \ldots \ldots \ldots \ldots \ldots \ldots$.

Table 5.1 Gasoline Prices for Selected Countries, 1978-98 . . . . . . . . . . . . . . . . 5 5-2

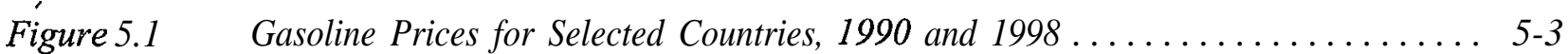

Table $5.2 \quad$ Diesel Fuel Prices for Selected Countries, $1978-98 \ldots \ldots \ldots \ldots \ldots \ldots \ldots \ldots \ldots$. . . . .

Figure $5.2 \quad$ Diesel Fuel Prices for Selected Countries, 1990 and $1998 \ldots \ldots \ldots \ldots \ldots \ldots \ldots$

Table 5.3 Prices for a Barrel of Crude Oil and a Gallon of Gasoline, 1978-98 ........ 5-6

Table $5.4 \quad$ Retail Prices for Motor Fuel, $1978-98 \ldots \ldots \ldots \ldots \ldots \ldots \ldots \ldots \ldots \ldots \ldots$

Table $5.5 \quad$ Prices for Selected Transportation Fuels, $1978-98 \ldots \ldots \ldots \ldots \ldots \ldots \ldots \ldots$ 5-8 


\section{TABLE OF CONTENTS (Continued)}

Table 5.6

State Taxes on Motor Fuels, 1999 $5-9$

Table 5.7

State Tax Exemptions for Gasohol, November 1998

$5-11$

Table 5.8

Federal Excise Taxes on Motor Fuels

5-1 1

Table 5.9 States With Ethanol Tax Incentives

Table 5.10

Average Price of a New Car, 1970-97

Table 5.11

Automobile Operating Cost per Mile, 1975-98.

$5-14$

Table 5.12

Fixed Automobile Operating Costs per Year, 1975-98

$5-15$

Table 5.13

Economic Indicators, 1970-98

$5-16$

Table 5.14

Consumer Price Indices, 1970-98

5-1 6

Table 5.15

Motor Vehicle Manufacturing Employment Statistics, 1972-97

$5-17$

Table 5.16

Employees of Motor Vehicle and Related Industries, 1990 and 1995

$5-18$

Table 5.17

Employment in Transportation and Related Industries, 1960-97

5-1 9

CHAPTER 6 HIGHWAY VEHICLES AND CHARACTERISTICS

Table 6.1

Automobile Registrations for Selected Countries, 1950-96

$6-2$

Table 6.2

Truck and Bus Registrations for Selected Countries, 1950-96 6-3

Table 6.3

Table 6.4

Table 6.5

Table 6.6

Table 6.7

Table 6.8

Table 6.9

Table 6.10
Automobiles and Trucks in Use, 1970-97

Vehicle Stock and New Sales in United States, 1997 Calendar Year $6-6$

Highway Vehicle Miles Traveled by Vehicle Type, 1970-97 $6-7$

Automobiles in Operation and Vehicle Travel by Age, 1970 and 1997 $6-8$

Trucks in Operation and Vehicle Travel by Age, 1970 and 1997 $6-9$ Average Age of Automobiles and Trucks in Use, 1970-97 6-10 Scrappage and Survival Rates for Automobiles 1970, 1980 and 1990 Model Years . . . . . . . . . . . . . . . . . . . . . 6- 11 Scrappage and Survival Rates for Trucks $6-12$ 


\section{TABLE OF CONTENTS (Continued)}

CHAPTER 7 LIGHT VEHICLES AND CHARACTERISTICS $\ldots \ldots \ldots \ldots \ldots \ldots \ldots \ldots \ldots$

Table $7.1 \quad$ Summary Statistics for Passenger Cars, $1970-97 \ldots \ldots \ldots \ldots \ldots \ldots \ldots \ldots \ldots \ldots$. . . . . . .

Table $7.2 \quad$ New Retail Automobile Sales in the United States, 1970-97 . . . . . . . . . . . . . 7-3

Table 7.3 Period Sales, Market Shares, and Sales-Weighted Fuel Economies of New Domestic and Import Automobiles, Selected Sales Periods 1976-98 . . . . . . . . . . . 7-4

Table 7.4 Sales-Weighted Engine Size of New Domestic and Import Automobiles

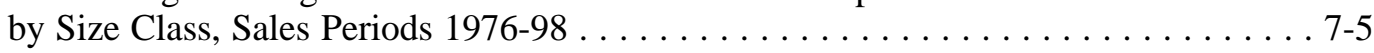

Table 7.5 Sales-Weighted Curb Weight of New Domestic and Import Automobiles by Size Class, Sales Periods 1976-98 . . . . . . . . . . . . . . . . . . . . . . . 7 7-6

Table 7.6 Sales-Weighted Interior Space of New Domestic and Import Automobiles by Size Class, Sales Periods 1976-98 . . . . . . . . . . . . . . . . . . . . . 7-7

Figure 7. I Engine Size, Curb Weight, and Interior Space of New Domestic and

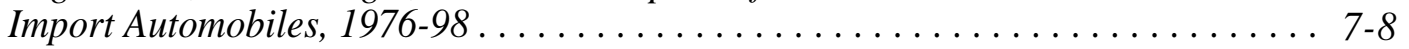

Table 7.7 Average Material Consumption for a Domestic Automobile,

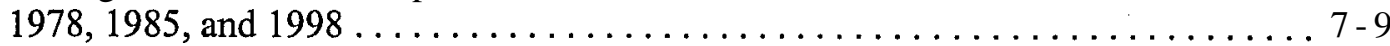

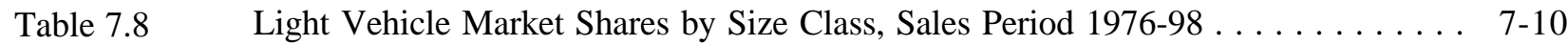

Table 7.9 Sales-Weighted Wheelbase of New Automobiles and Light Trucks, Sales Period $1976-98 \ldots \ldots \ldots \ldots \ldots \ldots \ldots \ldots \ldots \ldots \ldots \ldots \ldots \ldots \ldots \ldots$. 11

Table $7.10 \quad$ Summary Statistics for Two-Axle, Four-Tire Trucks, 1970-97 . . . . . . . . . . . 7-12

Table 7.11 New Retail Sales of Trucks 10,000 pounds GVW and Less in the

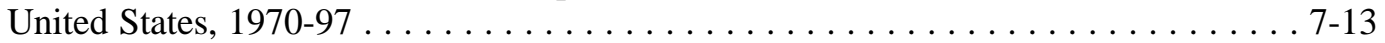

Table 7.12 Period Sales, Market Shares, and Sales-Weighted Fuel Economies of New Domestic and Import Light Trucks, Selected Sales Period 1976-98 . . . . . . . . . . 7-14

Table 7.13 Sales-Weighted Engine Size of New Domestic and Import Light Trucks by Size Class Sales Periods 1976-98 ....................... 7-15

Table $7.14 \quad$ New Light Vehicle Dealerships and Sales, 1970-97 . . . . . . . . . . . . . . . . . 7-16

Table 7.15 Corporate Average Fuel Economy (CAFE) Standards versus Sales-Weighted Fuel Economy Estimates for Automobiles and Light Trucks, 1978-98 . . . . . . . . . 7-17

Table 7.16 Corporate Average Fuel Economy (CAFE) Fines Collected, 1983-97 . . . . . . . . 7-18

Table $7.17 \quad$ Tax Receipts from the Sale of Gas Guzzlers, $1980-97 \ldots \ldots \ldots \ldots \ldots \ldots \ldots \ldots$. . . . . . . . .

Table $7.18 \quad$ The Gas Guzzler Tax on New Cars $\ldots \ldots \ldots \ldots \ldots \ldots \ldots \ldots \ldots \ldots \ldots \ldots$. $\ldots \ldots$ 


\section{TABLE OF CONTENTS (Continued)}

Table $7.19 \quad$ Vehicle Specifications for Tested Vehicles $\ldots \ldots \ldots \ldots \ldots \ldots \ldots \ldots \ldots \ldots$. 1

Table $7.20 \quad$ Fuel Economy by Speed, 1973, 1984 and $1997 \ldots \ldots \ldots \ldots \ldots \ldots \ldots \ldots \ldots$

Figure $7.2 \quad$ Fuel Economy by Speed, 1973, 1984 and 1997................. 7-23

Table $7.21 \quad$ Steady Speed Fuel Economy for Tested Vehicles $\ldots \ldots \ldots \ldots \ldots \ldots \ldots \ldots \ldots$. 7-24 $^{2}$

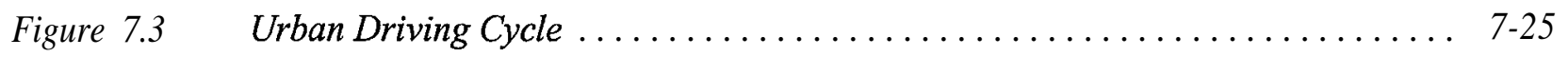

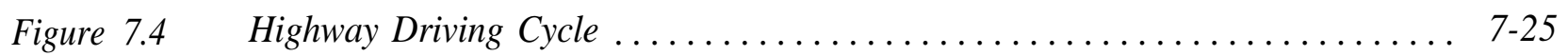

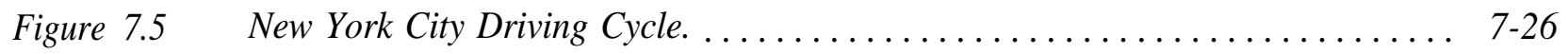

Figure 7.6 Representative Number Five Driving Cycle .................. 7-26

Figure $7.7 \quad$ US06 Driving Cycle $\ldots \ldots \ldots \ldots \ldots \ldots \ldots \ldots \ldots \ldots \ldots \ldots \ldots \ldots \ldots \ldots$

CHAPTER 8 HEAVY VEHICLES AND CHARACTERISTICS $\ldots \ldots \ldots \ldots \ldots \ldots \ldots \ldots . . .8$.

Table $8.1 \quad$ Summary Statistics for Other Single-Unit and Combination Trucks,

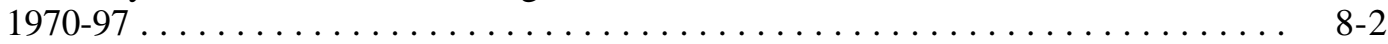

Table $8.2 \quad$ New Retail Truck Sales by Gross Vehicle Weight, $1970-97 \ldots \ldots \ldots \ldots \ldots$. . . . 8-3

Table $8.3 \quad$ New Retail Sales of Light Trucks by Gross Vehicle Weight, 1970-97 . . . . . . 8 8-4

Table $8.4 \quad$ Truck Statistics by Gross Vehicle Weight Class, $1992 \ldots \ldots \ldots \ldots \ldots \ldots \ldots \ldots$. . . . . . . . .

Table $8.5 \quad$ Percentage of Trucks by Size Class, 1977, 1982, 1987, and $1992 \ldots \ldots \ldots \ldots$

Table $8.6 \quad$ Truck Fuel Economy by Size Class, 1977, 1982, 1987, and $1992 \ldots \ldots \ldots \ldots$.

Table $8.7 \quad$ Percentage of Trucks by Fleet Size and Primary Refueling Facility, $1992 \ldots \ldots$. . . 8 8-7

Table $8.8 \quad$ Truck Fuel Economy by Fuel Type and Size Class, $1992 \ldots \ldots \ldots \ldots \ldots \ldots$. . . . . 8

Table $8.9 \quad$ Truck Statistics by Size, $1992 \ldots \ldots \ldots \ldots \ldots \ldots \ldots \ldots \ldots \ldots \ldots \ldots \ldots \ldots$

Table 8.10 Percentage of Trucks by Major Use and Primary Refueling Facility, $1992 \ldots \ldots$. . 8-10

Table $8.11 \quad$ Percentage of Trucks by Size Ranked by Major Use, $1992 \ldots \ldots \ldots \ldots \ldots \ldots$. 8 -1 1

Table 8.12 1993 Commodity Flow Survey: Shipment Characteristics by Mode of Transportation ....................... 8-13

Table $8.13 \quad$ Value, Tons, and Ton-Miles of Commodity Shipments, $1993 \ldots \ldots \ldots \ldots$. . . . 8-14

Table $8.14 \quad$ Summary Statistics on Buses by Type, $1970-97 \ldots \ldots \ldots \ldots \ldots \ldots \ldots$.1 5 


\section{TABLE OF CONTENTS (Continued)}

CHAPTER 9 ALTERNATIVE FUEL VEHICLES AND CHARACTERISTICS . . . . . . . 9-1

Table 9.1 Estimates of Alternative Fuel Vehicles in Use, 1992-99 . . . . . . . . . . . . . . . 9-3

Table 9.2 Estimates of Light Alternative Fuel Vehicles, 1995, 1997, and 1999 ......... 9-4

Table 9.3 Estimates of Heavy Alternative Fuel Vehicles, 1995, 1997, and $1999 \ldots \ldots \ldots \ldots$. $9-5$

Table $9.4 \quad$ Alternative Fuel Vehicles Available by Manufacturer, Model Year $1999 \ldots . . .$. 9-6

Table $9.5 \quad$ Number of Alternative Refuel Sites by State and Fuel Type, $1998 \ldots \ldots \ldots \ldots \ldots$. $9-7$

Table $9.6 \quad$ List of Clean Cities as of $9 / 1 / 99$ by Designation. . . . . . . . . . . . . . $9-8$

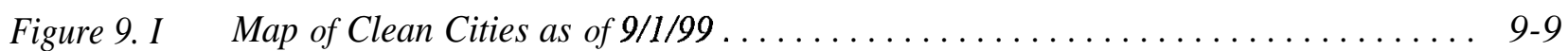

Table 9.7 U.S. Advanced Battery Consortium Research Agreements, Phase II . . . . . . . . 9 9-10

Table $9.8 \quad$ U.S. Advanced Battery Consortium Goals for Electric Vehicle Batteries ....... 9-1 1

CHAPTER 10 FLEET VEHICLES AND CHARACTERISTICS $\ldots \ldots \ldots \ldots \ldots \ldots \ldots \ldots$. 10

Figure $10.1 \quad$ Fleet Vehicles in Service as of January I, I998 ................ IO-2

Table $10.1 \quad$ Top Ten States with Fleets of Ten Vehicles or More, $1999 \ldots \ldots \ldots \ldots \ldots \ldots$. . . . . .

Table 10.2 Fleet Vehicle Composition by Vehicle Type, $1991 \ldots \ldots \ldots \ldots \ldots \ldots \ldots \ldots$ 10 $\ldots \ldots$

Table 10.3 Average Length of Time Fleet Vehicles are Kept Before Sold to Others, 1991 . . . 10-4

Table 10.4 Average Annual and Daily Vehicle-Miles of Travel for Fleet Vehicles, 1991 . . . . 10-4

Figure $10.2 \quad$ Worldwide Federal Inventory, $1992-97 \ldots \ldots \ldots \ldots \ldots \ldots \ldots \ldots \ldots$

Figure 10.3 Average Miles per Federal Vehicle by Vehicle Type, $1997 \ldots \ldots \ldots \ldots \ldots \ldots$........ $10-5$

Table 10.5 Federal Government Vehicles by Agency, Fiscal Year $1997 \ldots \ldots \ldots \ldots \ldots \ldots$. . . . . .

Table 10.6 Federal Fleet Vehicle Acquisitions by Fuel Type, FY $1997 \ldots \ldots \ldots \ldots \ldots \ldots$. . . . . .

Table 10.7 Fuel Consumed by Federal Government Fleets, FY $1997 \ldots \ldots \ldots \ldots \ldots \ldots$. $\ldots$. .

Table 10.8 Energy Policy Act Purchase Requirements of Light Alternative

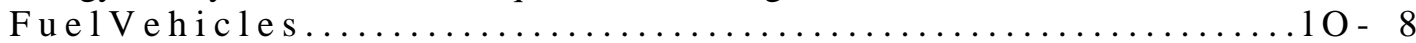

CHAPTER 11 HOUSEHOLD VEHICLES AND CHARACTERISTICS ............. 11-I

Table $11.1 \quad$ Population and Vehicle Profile, $1950-97 \ldots \ldots \ldots \ldots \ldots \ldots \ldots \ldots \ldots \ldots \ldots$

Table 11.2 Average Annual Expenditures of Households by Income, $1997 \ldots \ldots \ldots \ldots \ldots$. . . . . 11 


\section{TABLE OF CONTENTS (Continued)}

Table 11.3 Average Number of Vehicles and Vehicle Travel per Household, 1991

and 1994 RTECS . . . . . . . . . . . . . . . . . . . . . . . . . . . . 11-4

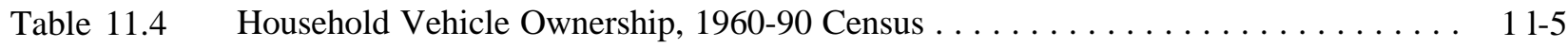

Table 11.5 Demographic Statistics, 1969, 1977, 1983, 1990, and 1995 NPTS . . . . . . . 1 1-6

Table 11.6 Average Annual Vehicle-Miles, Vehicle Trips and Trip Length per Household 1969, 1977, 1983, 1990, and 1995 NPTS . . . . . . . . . . . . . . . . 11-7

Table 11.7 Average Annual Person-Miles Traveled (PMT), Person Trips and Trip Length per Household by Selected Trip Purposes, 1983, 1990, and 1995 NPTS . . . . . . . . 11 1-8

Figure 11.7 Average Vehicle Occupancy by Vehicle Type, 1995 NPTS . . . . . . . . . . . . . 11-9

Figure 11.2 Average Vehicle Occupancy by Trip Purpose, 1977, 1983, 1990, and 1995 NPTS . . . . . . . . . . . . . . . . . . . . . . . . . . . . II 10

Table $11.8 \quad$ Vehicle-Miles by Trip Purpose, 1995 NPTS $\ldots \ldots \ldots \ldots \ldots \ldots \ldots \ldots \ldots \ldots \ldots \quad 1111$

Table 11.9 Average Annual Miles per Vehicle by Household Vehicle Ownership,

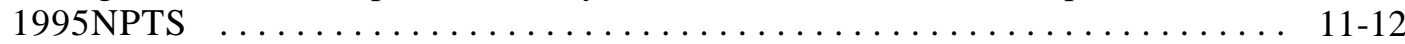

Table 11.10 Average Age of Vehicles by Household Vehicle Ownership, 1995 NPTS . . . . . . 11-12

Table 11.11 Average Annual Miles per Household Vehicle by Vehicle Age . . . . . . . . . 11 1-13

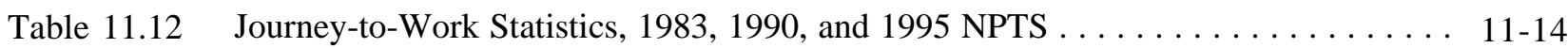

Table 11.13 Means of Transportation to Work, 1980 and 1990 Census . . . . . . . . . . . 11-15

Table 11.14 National and Metropolitan Area Comparisons of Journey-to-Work Statistics,

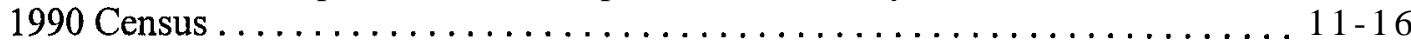

Figure $11.3 \quad$ Long-Distance Trips by Destination, $1995 \ldots \ldots \ldots \ldots \ldots \ldots \ldots \ldots \ldots \ldots$ II-17

Table 11.15 Long-Distance Trips by Mode and Purpose, $1995 \ldots \ldots \ldots \ldots \ldots \ldots \ldots \ldots \ldots$

Figure 11.4 Long-Distance Household Trips by Mode and Trip Distance, 1995. . . . . . . . . 11-19

Figure 11.5 Shares of Long-Distance Person Trips by Mode and Household Income, 1995 . . . . I I-20

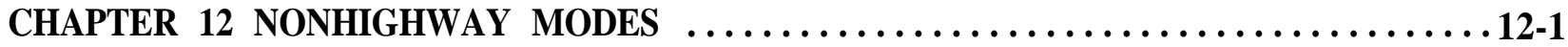

Table 12.1 Summary Statistics for U.S. Domestic and International Certificated

Route Air Carriers (Combined Totals), 1970-97 . . . . . . . . . . . . . . . . 12-2

Table 12.2 Summary Statistics for General Aviation, $1970-97 \ldots \ldots \ldots \ldots \ldots \ldots \ldots \ldots \ldots$ 12-3

Table 12.3 Tonnage Statistics for Domestic and International Waterborne

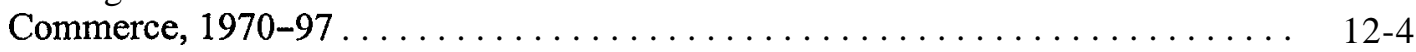




\section{TABLE OF CONTENTS (Continued)}

Table $12.4 \quad$ Summary Statistics for Domestic Waterborne Commerce, 1970-97 . . . . . . . . . 12-5

Table $12.5 \quad$ Breakdown of Domestic Marine Cargo by Commodity

Class 1997 . . . . . . . . . . . . . . . . . . . . . . . . . . 12-6

Table 12.6 Class I Railroad Freight Systems in the United States

Ranked by Revenue Ton-Miles, $1997 \ldots \ldots \ldots \ldots \ldots \ldots \ldots \ldots$. . . . . . . . . 12-7

Table $12.7 \quad$ Summary Statistics for Class I Freight Railroads, $1970-97 \ldots \ldots \ldots \ldots \ldots \ldots \ldots \ldots$. . . . $12-8$

Table 12.8 Railroad Revenue Carloads by Commodity Group,

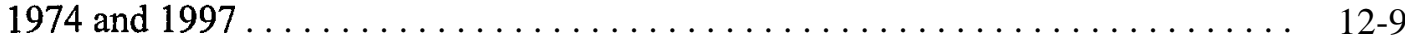

Table $12.9 \quad$ Intermodal Rail Traffic, $1965-97 \ldots \ldots \ldots \ldots \ldots \ldots \ldots \ldots \ldots \ldots \ldots \ldots$

Table 12.10 Summary Statistics for the National Railroad Passenger

Corporation (Amtrak), 1971-97 . . . . . . . . . . . . . . . . . . . . . . . . . . . 12-11

Table 12.11 Summary Statistics for Rail Transit Operations, $1970-97 \ldots \ldots \ldots \ldots \ldots$. . . . . . 12-12

APPENDIX A. SOURCES $\ldots \ldots \ldots \ldots \ldots \ldots \ldots \ldots \ldots \ldots \ldots \ldots \ldots \ldots \ldots \ldots \ldots \ldots \ldots$, A-I

APPENDIX B. CONVERSIONS $\ldots \ldots \ldots \ldots \ldots \ldots \ldots \ldots \ldots \ldots \ldots \ldots \ldots \ldots \ldots \ldots$, B -I

APPENDIX C. CENSUS DIVISIONS AND REGIONS $\ldots \ldots \ldots \ldots \ldots \ldots \ldots \ldots \ldots \ldots$ C-I

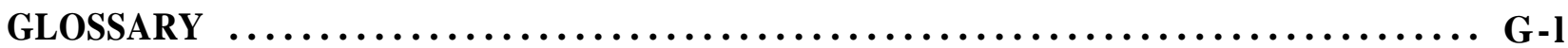

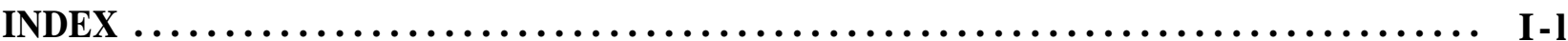




\section{FOREWORD}

This edition continues to emphasize oil and its importance to the U.S. transportation system. The increase in oil prices that has taken place in the first half of 1999 is partly due to the reduction in production of oil by OPEC countries and four other countries that joined forces with OPEC. The prices that U.S. drivers pay for gasoline are still influenced by members of a cartel. Chapter 1 shows that world oil production grew by 5.3 million barrels per day in the three years between 1994 and 1997, whereas it took 21 years for it to grow that much prior to 1994. Even though projections are not contained in this document, most projections show large increases in future oil production and use.

Chapter 2 contains data on transportation energy use. One of the most dramatic changes we have witnessed over the past three decades is the reduction in the amount of energy it takes to move a person a mile in an automobile or in an airplane. On the other hand, rail and bus transit has become less efficient over that same period.

Chapter 3 deals with greenhouse gases. The full fuel-cycle greenhouse gases from many types of alternative fuel/engine combinations are provided.

Chapter 4 provides data on criteria emissions and the importance of transportation emissions is made clear.

Chapter 5 highlights the role of transportation in the U.S. economy. The taxes that the U.S. places on gasoline and diesel fuel are much lower than those in many developed countries.

Chapter 6 provides data on highway vehicles. Highway vehicle-miles-of-travel continues to grow at around 3\% a year in spite of past projections that should be much lower by now. The average age of both cars and trucks on the road is quite a bit higher than it was 25 years ago.

Chapter 7 provides more detailed data on light vehicles (cars and light trucks). Light trucks continue to increase their share of new vehicle sales and of the vehicle stock. Both types of vehicles have not improved in fuel economy over the last 13 years.

Heavy vehicle data is presented in Chapter 8. Alternative fuel vehicle data makes up Chapter 9. Chapter 10 deals with fleet vehicles and their characteristics. 
In Chapter 11, household vehicle data is presented. Since 1950, the number of vehicles per capita grew from 0.28 to 0.75 , the vehicle miles traveled per capita grew from 3.0 to 9.6 thousand, and the number of licensed drivers nearly tripled.

Data for the nonhighway modes are presented in Chapter 12. Appendix A provides the data sources and Appendix B gives conversion factors.

Take advantage of the websites listed throughout this document to find more detailed information and to update data where possible. Please send any comments and/or suggestions to me or to the author, Stacy Davis.

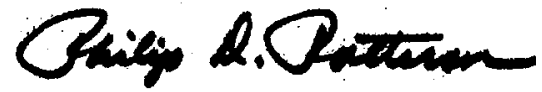




\section{ACKNOWLEDGMENTS}

I would like to express my gratitude to the many individuals who assisted in the preparation of this document. First, I would like to thank Phil Patterson and the staff of the Office of Transportation Technologies (OTT) for their continued support of the Transportation Energy Data Book project. I would also like to thank Patricia Hu of Oak Ridge National Laboratory (ORNL) for her dedicated leadership of this project. This document benefits from the criticism and careful review of Phil Patterson, OTT, and John Maples, Trancon, Inc. I would also like to thank Robert Gibson, University of Tennessee, for compiling new emission standard tables; John Maples, Trancon, for the methodology for Class $2 b$ truck sales; Jamie Payne, ORNL, who designed the new cover; Sherry Campbell Gambrell, ORNL, who prepared the title index; and Bob Boundy, Q Systems, who assisted in a variety of ways. Finally, this book would not have been possible without the dedication of Debbie Bain, ORNL, who masterfully prepared the manuscript. 


\begin{abstract}
The Transportation Energy Data Book: Edition 19 is a statistical compendium prepared and published by Oak Ridge National Laboratory (ORNL) under contract with the Office of Transportation Technologies in the Department of Energy (DOE). Designed for use as a desk-top reference, the data book represents an assembly and display of statistics and information that characterize transportation activity, and presents data on other factors that influence transportation energy use. The purpose of this document is to present relevant statistical data in the form of tables and graphs. The latest editions of the Data Book are available to a larger audience via the Internet (http://www-cta.ornl.gov/data/tedb.htm).

This edition of the Data Book has 12 chapters which focus on various aspects of the transportation industry. Chapter 1 focuses on petroleum; Chapter 2 - energy; Chapter 3 greenhouse gas emissions; Chapter 4 - criteria pollutant emissions; Chapter 5 - transportation and the economy; Chapter 6-highway vehicles; Chapter 7 - light vehicles; Chapter 8-heavy vehicles; Chapter 9 - alternative fuel vehicles; Chapter 10 - fleet vehicles; Chapter 11 - household vehicles; and Chapter 12- nonhighway modes. The sources used represent the latest available data. There are also three appendices which include detailed source information for some tables, measures of conversion, and the definition of Census divisions and regions. A glossary of terms and a title index are also included for the readers convenience.
\end{abstract}




\section{INTRODUCTION}

In January 1976, the Transportation Energy Conservation (TEC) Division of the Energy Research and Development Administration contracted with Oak Ridge National Laboratory (ORNL) to prepare a Transportation Energy Conservation Data Book to be used by TEC staff in their evaluation of current and proposed conservation strategies. The major purposes of the data book were to draw together, under one cover, transportation data from diverse sources, to resolve data conflicts and inconsistencies, and to produce a comprehensive document. The first edition of the TEC Data Book was published in October 1976. With the passage of the Department of Energy (DOE) Organization Act, the work being conducted by the former Transportation Energy Conservation Division fell under the purview of the DOE's Office of Transportation Programs (now the Office of Transportation Technologies). DOE, through the Office of Transportation Technologies, has supported the compilation of Editions 3 through 19.

Policymakers and analysts need to be well-informed about activity in the transportation sector. The organization and scope of the data book reflect the need for different kinds of information. For this reason, Edition 19 updates much of the same type of data that is found in previous editions.

In any attempt to compile a comprehensive set of statistics on transportation activity, numerous instances of inadequacies and inaccuracies in the basic data are encountered. Where such problems occur, estimates are developed by ORNL. To minimize the misuse of these statistics, an appendix (Appendix A) is included to document the estimation procedures. The attempt is to provide sufficient information for the conscientious user to evaluate the estimates and to form their own opinions as to their utility. Clearly, the accuracy of the estimates cannot exceed the accuracy of the primary data, an accuracy which in most instances is unknown. In cases where data accuracy is known or substantial errors are strongly suspected in the data, the reader is alerted. In all cases it should be recognized that the estimates are not precise.

The majority of the statistics contained in the data book are taken directly from published sources, although these data may be reformatted for presentation by ORNL. Consequently, neither ORNL nor DOE endorses the validity of these data. 


\section{Chapter 1 \\ Petroleum}

Summary Statistics from Tables/Figures in this Chapter

Source

Figure 1.1 World oil proved resources, 1993 (most recent survey)

billion barrels

Proved resources (USGS)

1,092

Ultimate resources (USGS)

1,574

Table 1.2 World Oil Production, 1997

U.S. Oil Production (million barrels per day)

U.S. Share

$9.7 \%$

Table 1.3 World Oil Consumption, 1996

U.S. Oil Consumption (million barrels per day)

18.3

U.S. Share

$25.6 \%$

Figure 1.6 Refinery yield, 1997

OECD North America

Gasoline

$31.1 \%$

$41.7 \%$

Diesel fuel

$28.1 \%$

$22.3 \%$

Residual fuel

$11.8 \%$

$5.5 \%$

Kerosene

$8.9 \%$

$9.3 \%$

Other

$20.2 \%$

$21.2 \%$

Table 1.8 U.S. transportation oil use as a percent of U.S. oil production, $1998 \quad 196 \%$

Table 1.8 Net imports as a percentage of U.S. oil consumption, $1998 \quad 51 \%$

Table 1.9 Transportation share of oil consumption, 1998

$66 \%$ 
No one knows the exact amount of oil which is in the Earth. The U.S. Geological Survey (USGS) produces estimates of the ultimate world oil resources periodically - the latest done in 1993. Because of the evolving understanding of world recoverable resources, the USGS assessments are valid for the perceptions at that point in time. The surveys that produced the data shown in Table 1.1 were conducted using consistent methodologies and the same core group ofgeologists. The differences among the surveys are largely due to better understanding of world recoverable resources and technological change. As understanding of the subject grows, so may the estimates of world oil resources. A new study will be available in the year 2000.

Figure 1.1. USGS World Oil Resource Estimates, 1993

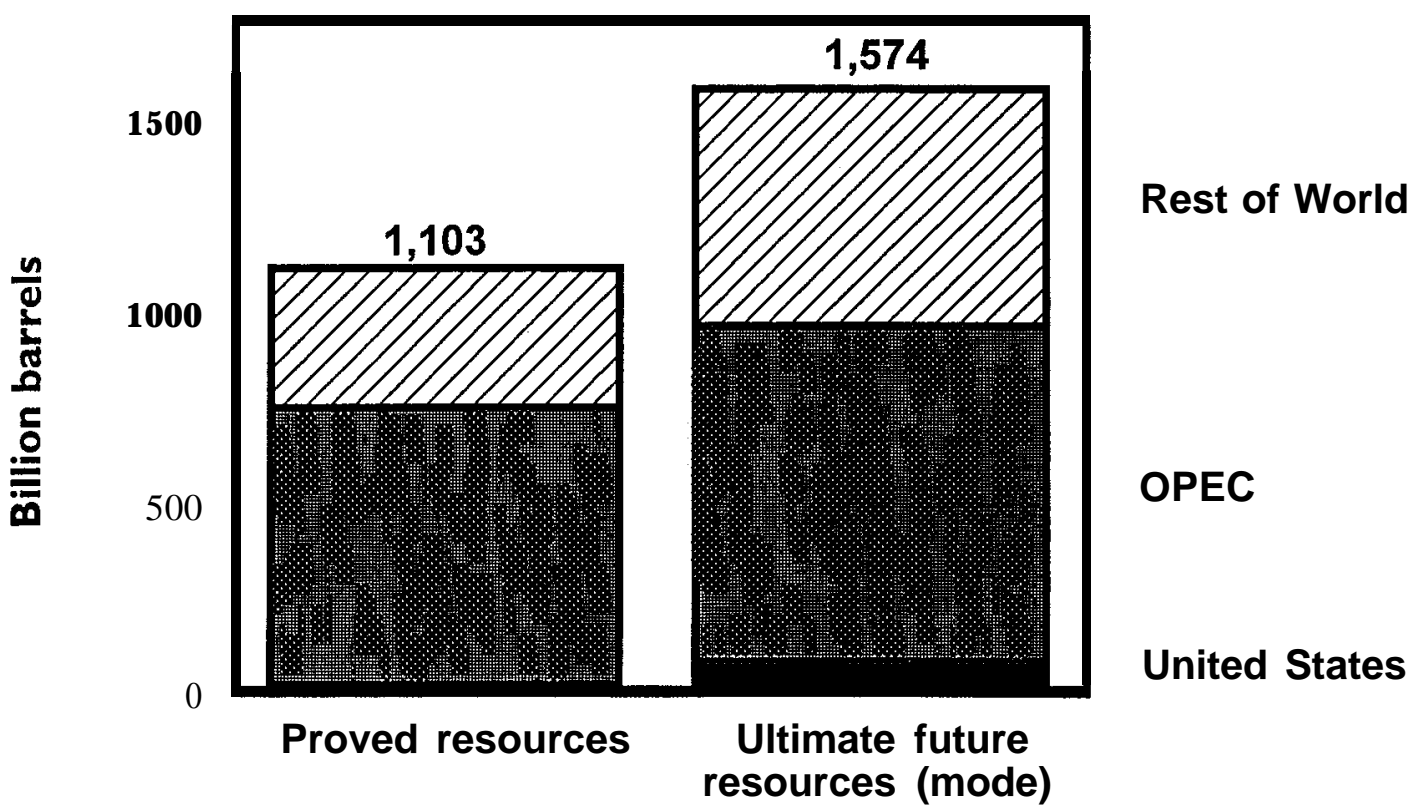

Source:

U.S. Geological Survey, U.S. Geological Survey Fact Sheet, FS-145-97, 1997. Also see: Masters C. D., E. D. Attanasi and D. H. Root, World Petroleum Assessment and Analysis, U.S. Geological Survey, National Center, Reston, VA, 1994, Table 1. (Additional resources: http://energy.er.usgs.gov)

Table 1.1

Summary of Recent World Oil Assessments (billion barrels of oil)

\begin{tabular}{lccrc}
\hline & \multicolumn{3}{c}{ Effective date of assessment } \\
\cline { 2 - 5 } & $1 / 1 / 81$ & $1 / 1 / 83$ & $1 / 1 / 90$ & $1 / 1 / 93$ \\
\hline 1. Cumulative production & 445 & 524 & 629 & 699 \\
2. Identified (discovered) resources & 724 & 795 & 1,053 & 1,103 \\
3. Undiscovered conventional resources (mode) & 550 & 425 & 489 & 471 \\
4. Future resources (mode) (categories 2+3) & 1,274 & 1,220 & 1,542 & 1,574 \\
5. Total resources (categories 1+2+3) & 1,719 & 1,744 & 2,171 & 2,273 \\
\hline
\end{tabular}

\section{Source:}

U.S. Geological Survey, U.S. Geological Survey Fact Sheet, FS-145-97, 1997.

(Additional resources: http://energy.er.usgs.gov) 
Table 1.2

'World Crude Oil Production, 1960-97'

(million barrels per day)

\begin{tabular}{|c|c|c|c|c|c|c|}
\hline Year & $\begin{array}{l}\text { United } \\
\text { States }\end{array}$ & $\begin{array}{l}\text { U.S. } \\
\text { Share }\end{array}$ & $\begin{array}{l}\text { Total } \\
\text { OPEC }^{b}\end{array}$ & $\begin{array}{c}\text { Total } \\
\text { Non-OPEC }\end{array}$ & $\begin{array}{c}\text { Persian Gulf } \\
\text { nations } \mathbf{s}^{\mathbf{c}}\end{array}$ & World \\
\hline 1960 & 7.04 & $33.5 \%$ & 8.70 & 12.29 & 5.27 & 20.99 \\
\hline 1965 & 7.80 & $25.7 \%$ & 14.35 & 15.98 & 8.37 & 30.33 \\
\hline 1970 & 9.64 & $21.0 \%$ & 23.30 & 22.59 & 13.39 & 45.89 \\
\hline 1971 & 9.46 & $19.5 \%$ & 25.21 & 23.31 & 15.77 & 48.52 \\
\hline 1972 & 9.44 & $18.5 \%$ & 26.89 & 24.25 & 17.54 & 51.14 \\
\hline 1973 & 9.21 & $16.5 \%$ & 30.63 & 25.05 & 20.67 & 55.68 \\
\hline 1974 & 8.77 & $15.7 \%$ & 30.35 & 25.37 & 21.28 & 55.72 \\
\hline 1975 & 8.37 & $15.8 \%$ & 26.77 & 26.06 & 18.93 & 52.83 \\
\hline 1976 & 8.13 & $14.2 \%$ & 30.33 & 27.01 & 21.51 & 57.34 \\
\hline 1977 & 8.24 & $13.8 \%$ & 30.89 & 28.82 & 21.73 & 59.71 \\
\hline 1978 & 8.71 & $14.5 \%$ & 29.46 & 30.70 & 20.61 & 60.16 \\
\hline 1979 & 8.55 & $13.6 \%$ & 30.58 & 32.09 & 21.07 & 62.67 \\
\hline 1980 & 8.60 & $14.4 \%$ & 26.61 & 32.99 & 17.96 & 59.60 \\
\hline 1981 & 8.57 & $15.3 \%$ & 22.48 & 33.60 & 15.25 & 56.08 \\
\hline 1982 & 8.65 & $16.2 \%$ & 18.78 & 34.70 & 12.16 & 53.48 \\
\hline 1983 & 8.69 & $16.3 \%$ & 17.50 & 35.76 & 11.08 & 53.26 \\
\hline 1984 & 8.88 & $16.3 \%$ & 17.44 & 37.05 & 10.78 & 54.49 \\
\hline 1985 & 8.97 & $16.6 \%$ & 16.18 & 37.80 & 9.63 & 53.98 \\
\hline 1986 & 8.68 & $15.4 \%$ & 18.28 & 37.95 & 11.70 & 56.23 \\
\hline 1987 & 8.35 & $14.7 \%$ & 18.52 & 38.15 & 12.10 & 56.67 \\
\hline 1988 & 8.14 & $13.9 \%$ & 20.32 & 38.42 & 13.46 & 58.74 \\
\hline 1989 & 7.61 & $12.7 \%$ & 22.07 & 37.79 & 14.84 & 59.86 \\
\hline 1990 & 7.36 & $12.2 \%$ & 23.20 & 37.37 & 15.28 & 60.57 \\
\hline 1991 & 7.42 & $12.3 \%$ & 23.27 & 36.94 & 14.74 & 60.21 \\
\hline 1992 & 7.17 & $11.9 \%$ & 24.40 & 35.82 & 15.97 & 60.22 \\
\hline 1993 & 6.85 & $11.4 \%$ & 25.12 & 35.13 & 16.71 & 60.25 \\
\hline 1994 & 6.66 & $10.9 \%$ & 25.51 & 35.49 & 16.96 & 61.00 \\
\hline 1995 & 6.56 & $10.5 \%$ & 26.09 & 36.36 & 17.30 & 62.45 \\
\hline 1996 & 6.46 & $10.1 \%$ & 26.77 & 37.23 & 17.37 & 63.97 \\
\hline 1997 & 6.41 & $9.7 \%$ & 28.36 & 37.91 & 18.50 & 66.27 \\
\hline \multicolumn{7}{|c|}{ Average annual percentage change } \\
\hline $1960-97$ & $-0.3 \%$ & & $3.2 \%$ & $3.1 \%$ & $3.5 \%$ & $3.2 \%$ \\
\hline $1970-97$ & $-1.5 \%$ & & $0.7 \%$ & $1.9 \%$ & $1.2 \%$ & $1.4 \%$ \\
\hline $1987-97$ & $-2.6 \%$ & & $4.4 \%$ & $-0.1 \%$ & $4.3 \%$ & $1.6 \%$ \\
\hline
\end{tabular}

Source:

U.S. Department of Energy, Energy Information Administration, Annual Energy Review 1997, Washington, DC, July 1998, p. 273.

"Includes lease condensate. Excludes natural gas plant liquids.

borganization of Petroleum Exporting Countries. See Glossary for membership.

"See Glossary for Persian Gulf nations. 
Figure 1.2. OPEC Market Share, 1960-97

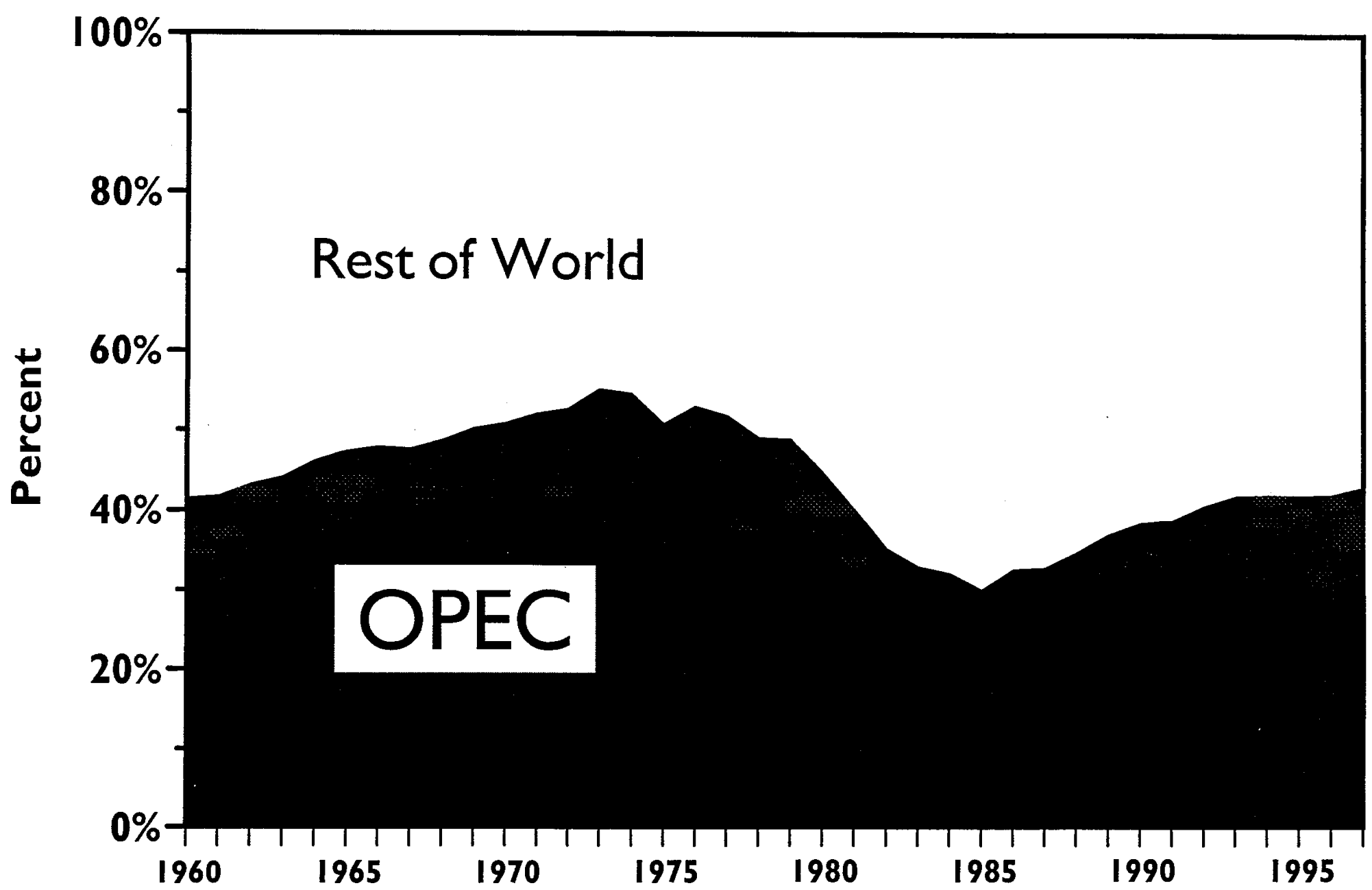

U.S. Department of Energy, Energy Information Administration, Annual Energy Review 1997, Washington, DC, July 1998 , p. 273. 
These data are the latest available; oil consumption data generally lags behindproduction data (previous table) by one year.

Table 1.3

World Oil Consumption, 1960-96 (million barrels per day)

\begin{tabular}{|c|c|c|c|c|c|}
\hline Year & $\begin{array}{l}\text { United } \\
\text { States }\end{array}$ & $\begin{array}{l}\text { U.S. } \\
\text { Share }\end{array}$ & Total OECD" & $\begin{array}{c}\text { Total } \\
\text { Non-OECD }\end{array}$ & World \\
\hline 1960 & 9.80 & $45.9 \%$ & 15.78 & 5.56 & 21.34 \\
\hline 1965 & 11.51 & $37.0 \%$ & 22.81 & 8.33 & 31.14 \\
\hline 1970 & 14.70 & $31.4 \%$ & 34.49 & 12.32 & 46.81 \\
\hline 1971 & 15.21 & $30.8 \%$ & 36.07 & 13.35 & 49.42 \\
\hline 1972 & 16.37 & $30.8 \%$ & 38.74 & 14.35 & 53.09 \\
\hline 1973 & 17.31 & $30.2 \%$ & 41.53 & 15.71 & 57.24 \\
\hline 1974 & 16.65 & $29.4 \%$ & 40.12 & 16.56 & 56.68 \\
\hline 1975 & 16.32 & $29.0 \%$ & 38.82 & 17.38 & 56.20 \\
\hline 1976 & 17.46 & $29.3 \%$ & 41.39 & 18.28 & 59.67 \\
\hline 1977 & 18.43 & $29.8 \%$ & 42.43 & 19.40 & 61.83 \\
\hline 1978 & 18.85 & $29.4 \%$ & 43.62 & 20.54 & 64.16 \\
\hline 1979 & 18.51 & $28.4 \%$ & 44.01 & 21.21 & 65.22 \\
\hline 1980 & 17.06 & $27.0 \%$ & 41.41 & 21.66 & 63.07 \\
\hline 1981 & 16.06 & $26.4 \%$ & 39.14 & 21.76 & 60.90 \\
\hline 1982 & 15.30 & $25.7 \%$ & 37.45 & 22.05 & 59.50 \\
\hline 1983 & 15.23 & $25.9 \%$ & 36.59 & 22.15 & 58.74 \\
\hline 1984 & 15.73 & $26.3 \%$ & 37.43 & 22.41 & 59.84 \\
\hline 1985 & 15.73 & $26.2 \%$ & 37.23 & 22.87 & 60.10 \\
\hline 1986 & 16.28 & $26.4 \%$ & 38.28 & 23.48 & 61.76 \\
\hline 1987 & 16.67 & $26.5 \%$ & 38.96 & 24.04 & 63.00 \\
\hline 1988 & 17.28 & $26.7 \%$ & 40.24 & 24.58 & 64.82 \\
\hline 1989 & 17.33 & $26.3 \%$ & 40.88 & 25.04 & 65.92 \\
\hline 1990 & 16.99 & $25.7 \%$ & 40.92 & 25.07 & 65.99 \\
\hline 1991 & 16.71 & $25.1 \%$ & 41.40 & 25.18 & 66.58 \\
\hline 1992 & 17.03 & $25.5 \%$ & 42.41 & 24.33 & 66.74 \\
\hline 1993 & 17.24 & $25.7 \%$ & 43.05 & 23.99 & 67.04 \\
\hline 1994 & 17.72 & $25.9 \%$ & 44.20 & 24.11 & 68.31 \\
\hline 1995 & 17.72 & $25.3 \%$ & 45.07 & 24.86 & 69.93 \\
\hline 1996 & 18.31 & $\begin{array}{l}25.6 \% \\
\text { Avera\& }\end{array}$ & $\begin{array}{l}\quad 46.15 \\
\text { annualpercentage }\end{array}$ & $\begin{array}{l}25.37 \\
\text { change }\end{array}$ & 71.52 \\
\hline 1960-96 & $1.8 \%$ & & $3.0 \%$ & $4.3 \%$ & $3.4 \%$ \\
\hline 1970-96 & $0.8 \%$ & & $1.1 \%$ & $2.8 \%$ & $1.6 \%$ \\
\hline 1986-96 & $1.2 \%$ & & $1.9 \%$ & $0.8 \%$ & $1.5 \%$ \\
\hline
\end{tabular}

Source:

U.S. Department of Energy, Energy Information Administration, Annual Energy Review 1997, Washington, DC, July 1998, p. 283.

a Organization for Economic Cooperation and Development. See Glossary for membership. 
The United States has increased its petroleum stocks by 55\% from 1973 to 1984; there has been no significant change in the stocks since 1984. Petroleum demand, however, has increased $87 \%$ in that same time period (see Table 1.3). The Strategic Petroleum Reserve accountedfor 36\% of total U.S. stocks at the end of 1997.

Table 1.4

Petroleum Stocks in OECD Countries, End of Year 1973-97” (million barrels)

\begin{tabular}{|c|c|c|c|c|c|c|c|c|c|c|c|c|}
\hline Year & France & Germany $^{b}$ & Italy & $\begin{array}{c}\text { United } \\
\text { Kingdom }\end{array}$ & $\begin{array}{c}\text { Other } \\
\text { OECD } \\
\text { Europe }\end{array}$ & $\begin{array}{l}\text { OECD } \\
\text { Europe }\end{array}$ & Canada & Japan & $\begin{array}{c}\text { U.S. Strategic } \\
\text { Petroleum } \\
\text { Reserve }\end{array}$ & $\begin{array}{l}\text { United } \\
\text { States } \\
\text { total } \\
\end{array}$ & $\begin{array}{c}\text { Other } \\
\text { OECD }^{d}\end{array}$ & $\mathrm{OECD}^{\circ}$ \\
\hline 1973 & 201 & 181 & 152 & 156 & 380 & 1,070 & 140 & 303 & e & 1,008 & 67 & 2,588 \\
\hline 1980 & 243 & 319 & 170 & 168 & 564 & & 164 & 495 & 108 & & 72 & \\
\hline 1981 & 214 & 297 & 167 & 143 & 516 & 1,337 & 161 & 482 & 230 & 1,484 & 67 & 3,531 \\
\hline 1982 & 193 & 272 & 179 & 125 & 489 & 1,258 & 136 & 484 & 294 & 1,430 & 68 & 3,376 \\
\hline 1983 & 153 & 249 & 149 & 118 & 473 & 1,142 & 121 & 470 & 379 & 1,454 & 68 & 3,255 \\
\hline 1985 & 139 & 233 & 157 & 123 & 440 & 1,092 & 113 & 494 & 493 & 1,519 & 66 & 3,362 \\
\hline 1986 & 127 & 252 & 155 & 124 & 475 & & 111 & 509 & 512 & & 72 & \\
\hline 1987 & 127 & 259 & 169 & 121 & 454 & 1,130 & 126 & 540 & 541 & 1,607 & 71 & 3,474 \\
\hline 1988 & 140 & 266 & 155 & 112 & 445 & 1,118 & 116 & 538 & 560 & 1,597 & 71 & 3,440 \\
\hline 1989 & 138 & 271 & 164 & 118 & 442 & 1,133 & 114 & 577 & 580 & 1,581 & 71 & 3,476 \\
\hline 1990 & 140 & 265 & 172 & 112 & 474 & 1,163 & 121 & 590 & 586 & 1,621 & 73 & 3,568 \\
\hline 1992 & 153 & 310 & 174 & 113 & 476 & $1,2 \overline{19}$ & 107 & 603 & 575 & 1,592 & 67 & 3,588 \\
\hline 1994 & 158 & 312 & 164 & 115 & 490 & 1,240 & 119 & 645 & 592 & 1,653 & 69 & 3,726 \\
\hline 1995 & 159 & 301 & 162 & 107 & 499 & 1,228 & 109 & 630 & 592 & 1,563 & 71 & 3,601 \\
\hline 1996 & 158 & 300 & 152 & 108 & 538 & 1,256 & 103 & 651 & 566 & 1,507 & 74 & 3,591 \\
\hline 1997 & 164 & 298 & 147 & 105 & 542 & 1,256 & 115 & 685 & 563 & 1,560 & 74 & 3,689 \\
\hline \multicolumn{13}{|c|}{ Average annualpercentage change } \\
\hline $1973-97$ & $-0.8 \%$ & $2.1 \%$ & $-0.1 \%$ & $-1.6 \%$ & $1.5 \%$ & $0.7 \%$ & $-0.8 \%$ & $3.5 \%$ & $e$ & $1.8 \%$ & $0.4 \%$ & $1.5 \%$ \\
\hline 1987-97 & $2.6 \%$ & $1.4 \%$ & $-1.4 \%$ & $-1.4 \%$ & $1.8 \%$ & $1.1 \%$ & $-0.9 \%$ & $2.4 \%$ & $0.4 \%$ & $-0.3 \%$ & $0.4 \%$ & $0.6 \%$ \\
\hline
\end{tabular}

Source:

Country stocks-U.S.Department of Energy, Energy Information Administration, International Petroleum Statistics Report, Washington, DC, January1999.

U.S.Strategic Petroleum Reserve - U.S. Department of Energy, Energy Information Administration, Annual Energy Review, 1997 , Washington, DC, July 1998 , p. 147.

${ }^{a}$ Includes crude oil (including strategic reserves), lease condensate, natural gas plant liquids, unfinished oils, and finished petroleum products. Oil stocks include all non-military stocks held by importers, refiners, Governments, major non-importing final consumers and by foreign entities in certain facilities. See Stocks in Glossary for details.

$\mathrm{b}$ Through 1990, the data for Germany are for the former West Germany only. Beginning in 1991, the data for Germany are for the unified Germany, i.e., the former East Germany and West Germany.

${ }^{c}$ Organization for Economic Cooperation and Development (OECD). See Glossary for membership.

d Australia, New Zealand, and United States Territories. Data for Mexico, which joined the OECD on May 18, 1994, are not available.

e Data are not available. The Energy Policy and Conservation Act, effective February 1976, authorized the establishment of the U.S. Strategic Petroleum Reserve. 
The countri vhich make up the Organization for Economic Cooperation and Development (OECD) had combined stocks which to $2 d 21 \%$ of the

petroleum c umption in 1996; this is up from 17\% in 1973. This includes stocks which are privately-controlled as well as governmer. wned holding

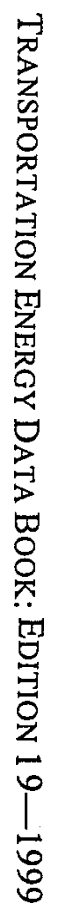

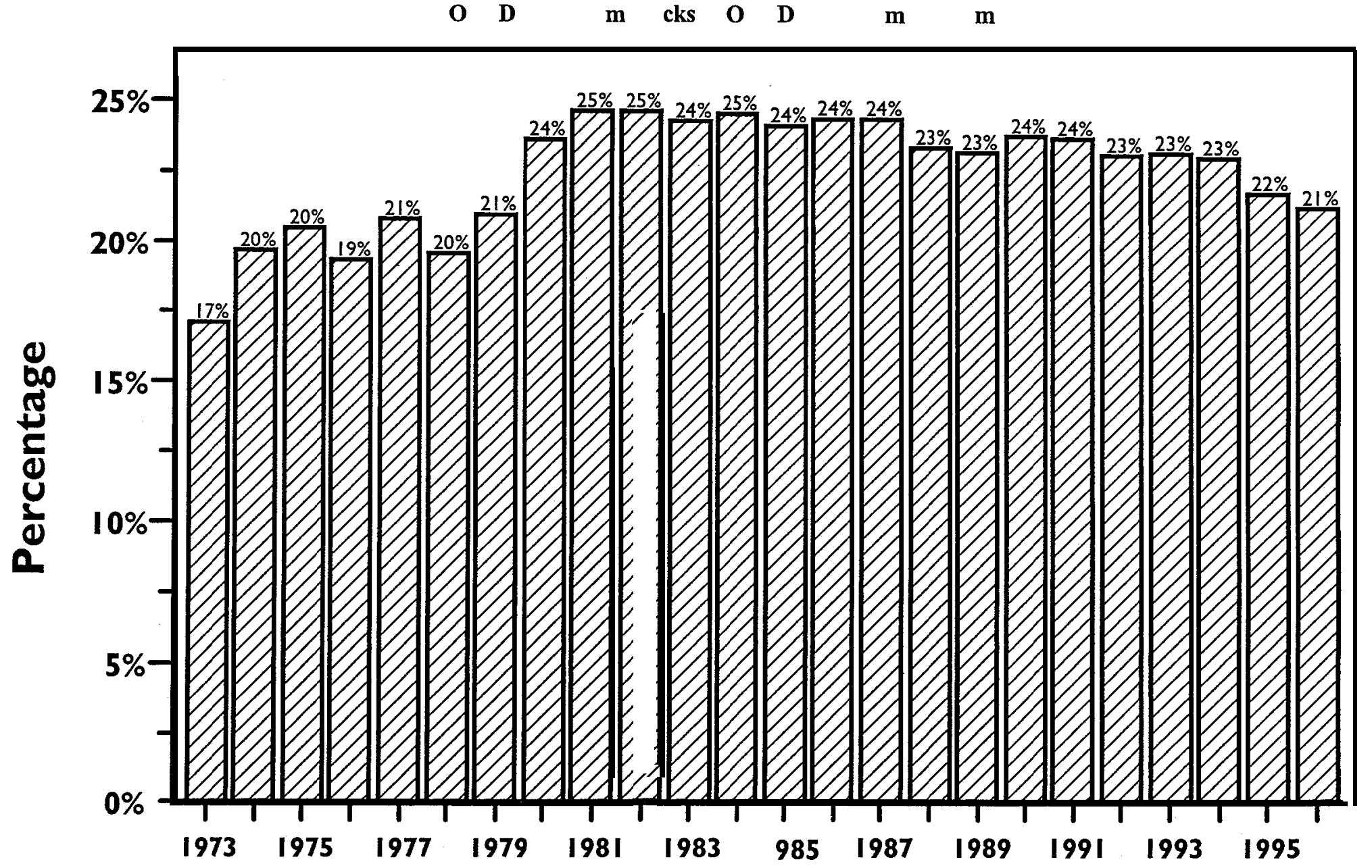

Source: See Tables 1.3 and 1.4 . 
The United States had petroleum stocks of almost one quarter of U.S. petroleum consumption in 1996, which was slightly above the average for $O E C D$ countries. Germany and Japan held higher reserves relative to their consumption of petroleum.

Figure 1.4. Share of Petroleum Stocks to Petroleum Consumption by Country, 1996

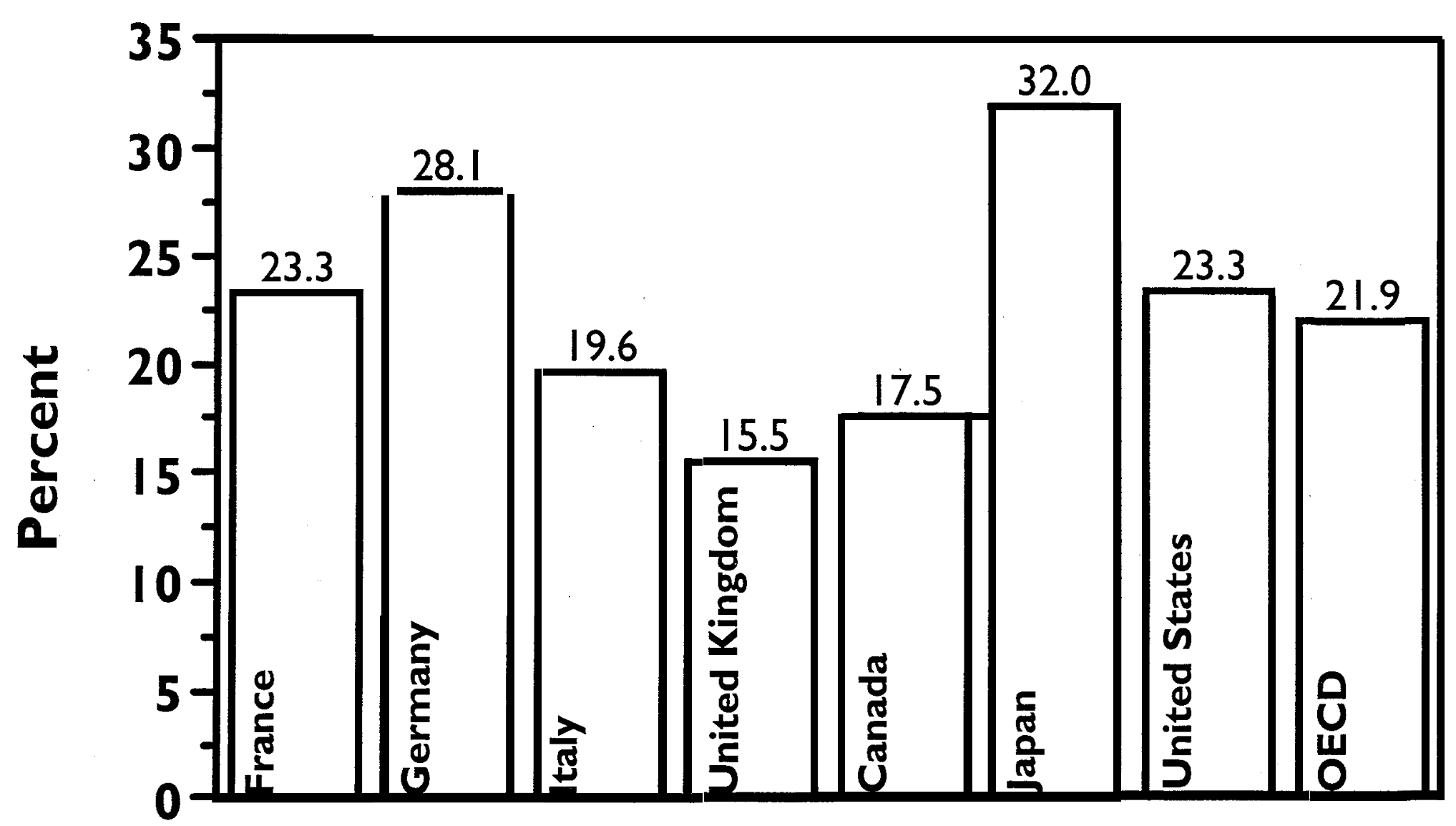

Source: Table 1.4 and U.S. Department of Energy, Energy Information Administration, Annual Energy Review, 1997, Washington, DC, July 1998 , p. 307. 
Figure 1.5. Crude Oil Prices, 1870-98

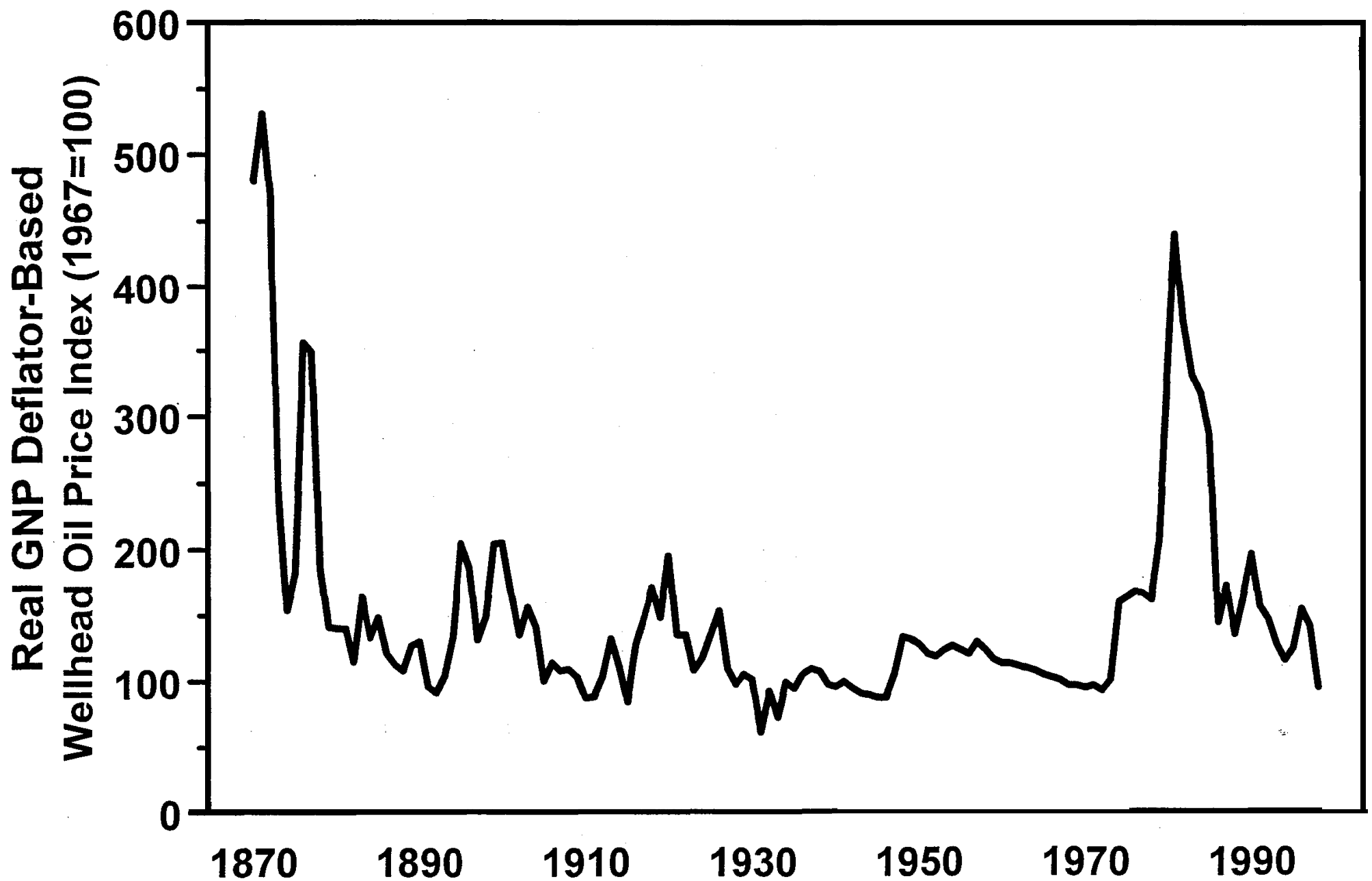

Source:

Santini, Danilo J., "An Assessment of Oil Supply and Its Implications for Future Prices," Nonrenewable Resources, Vol. 7, No. 2, 1998, pp. 101-121, and 1994-98 data update. 
Table 1.5

U.S. Petroleum Net Imports by World Region of Origin, 1960-97 (thousand barrels per day)

\begin{tabular}{|c|c|c|c|c|c|c|}
\hline Year & $\begin{array}{c}\text { Total } \\
\text { OPEC" }\end{array}$ & $\begin{array}{c}\text { Total } \\
\text { Non-OPEC }\end{array}$ & $\begin{array}{l}\text { Persian Gulf } \\
\text { nations }{ }^{b}\end{array}$ & $\begin{array}{l}\text { Total net } \\
\text { imports }\end{array}$ & $\begin{array}{l}\text { OPEC share of } \\
\text { net imports }\end{array}$ & $\begin{array}{l}\text { OPEC share of } \\
\text { consumption" }\end{array}$ \\
\hline 1960 & 1,311 & 302 & $\mathrm{~d}$ & 1,613 & $81.3 \%$ & $13.4 \%$ \\
\hline 1965 & 1,475 & 806 & d & 2,281 & $64.7 \%$ & $12.8 \%$ \\
\hline 1970 & 1,343 & 1,817 & d & 3,161 & $42.5 \%$ & $9.1 \%$ \\
\hline 1971 & 1,671 & 2,030 & d & 3,701 & $45.2 \%$ & $11.0 \%$ \\
\hline 1972 & 2,061 & 2,458 & d & 4,519 & $45.6 \%$ & $12.6 \%$ \\
\hline 1973 & 2,991 & 3,034 & d & 6,025 & $49.6 \%$ & $17.3 \%$ \\
\hline 1974 & 3,277 & 2,615 & d & 5,892 & $55.6 \%$ & $19.7 \%$ \\
\hline 1975 & 3,599 & 2,248 & d & 5,846 & $61.6 \%$ & $22.1 \%$ \\
\hline 1976 & 5,063 & 2,027 & d & 7,090 & $71.4 \%$ & $29.0 \%$ \\
\hline 1977 & 6,190 & 2,375 & d & 8,565 & $72.3 \%$ & $33.6 \%$ \\
\hline 1978 & 5,747 & 2,255 & d & 8,002 & $71.8 \%$ & $30.5 \%$ \\
\hline 1979 & 5,633 & 2,352 & d & 7,985 & $70.5 \%$ & $30.4 \%$ \\
\hline 1980 & 4,293 & 2,071 & d & 6,365 & $67.5 \%$ & $25.2 \%$ \\
\hline 1981 & 3,315 & 2,086 & 1,215 & 5,401 & $61.4 \%$ & $20.6 \%$ \\
\hline 1982 & 2,136 & 2,163 & 692 & 4,298 & $49.7 \%$ & $14.0 \%$ \\
\hline 1983 & 1,843 & 2,469 & 439 & 4,312 & $42.7 \%$ & $12.1 \%$ \\
\hline 1984 & 2,037 & 2,679 & 502 & 4,715 & $43.2 \%$ & $13.0 \%$ \\
\hline 1985 & 1,821 & 2,465 & 309 & 4,286 & $42.5 \%$ & $11.6 \%$ \\
\hline 1986 & 2,828 & 2,611 & 909 & 5,439 & $52.0 \%$ & $17.4 \%$ \\
\hline 1987 & 3,055 & 2,859 & 1,074 & 5,914 & $51.7 \%$ & $18.3 \%$ \\
\hline 1988 & 3,513 & 3,074 & 1,529 & 6,587 & $53.3 \%$ & $20.3 \%$ \\
\hline 1989 & 4,124 & 3,078 & 1,858 & 7,202 & $57.3 \%$ & $23.8 \%$ \\
\hline 1990 & 4,285 & 2,876 & 1,962 & 7,161 & $59.8 \%$ & $25.2 \%$ \\
\hline 1991 & 4,065 & 2,561 & 1,833 & 6,626 & $61.3 \%$ & $24.3 \%$ \\
\hline 1992 & 4,071 & 2,867 & 1,773 & 6,938 & $58.7 \%$ & $23.9 \%$ \\
\hline 1993 & 4,253 & 3,365 & 1,774 & 7,618 & $55.8 \%$ & $24.7 \%$ \\
\hline 1994 & 4,233 & 3,822 & 1,723 & 8,054 & $52.6 \%$ & $23.9 \%$ \\
\hline 1995 & 3,980 & 3,906 & 1,563 & 7,886 & $50.5 \%$ & $22.5 \%$ \\
\hline 1996 & 4,193 & 4,305 & 1,596 & 8,498 & $49.3 \%$ & $22.9 \%$ \\
\hline 1997 & 4,461 & 4,443 & 1,731 & 8,904 & $50.1 \%$ & $24.0 \%$ \\
\hline & \multicolumn{6}{|c|}{ Average annual percentage change } \\
\hline 1960-97 & $3.4 \%$ & $7.5 \%$ & d & $4.7 \%$ & & \\
\hline 1970-97 & $4.5 \%$ & $3.4 \%$ & d & $3.9 \%$ & & \\
\hline $1987-97$ & $3.9 \%$ & $4.5 \%$ & $4.9 \%$ & $4.2 \%$ & & \\
\hline
\end{tabular}

Source:

U.S. Department of Energy, Energy Information Administration, Annual Energy Review 1997, Washington, DC, July 1998, p. 149.

a Organization of Petroleum Exporting Countries. See Glossary for membership.

${ }^{\mathrm{b}}$ See Glossary for Persian Gulf nations.

c See Table 1.8 for U.S. petroleum consumption.

${ }^{d}$ Data are not available. 
Estimates of 1996 military expenditures for defending oil supplies in the Middle East rangefrom $\$ 6$ to $\$ 60$ billion per year. This wide range in estimates reflects the difficulty in assigning a precise figure to the military cost of defending the U.S. interests in the Middle East. The two main reasons for the difficulty are 1) the Department of Defense does not divide the budget into regional defense sectors and 2) it is difficult to determine how much of the cost is attributable to defending Persian Gulf oil.

Table 1.6

Summary of 1996 Military Expenditures for Defending Oil Supplies from the Middle East

\begin{tabular}{lccc}
\hline \multicolumn{1}{c}{ Source } & $\begin{array}{c}\text { Original estimates } \\
\text { (billion dollars) }\end{array}$ & $\begin{array}{c}\text { Year of } \\
\text { original estimate }\end{array}$ & $\begin{array}{c}\text { 1996 estimate } \\
\text { (constant 1996 } \\
\text { billion dollars) }\end{array}$ \\
\hline General Accounting Office [1] & $\$ 33$ & 1990 & $\$ 28^{\prime \prime}$ \\
Congressional Research Service [2] & $\$ 6.4$ & 1990 & $\$ 6^{\prime \prime}$ \\
Greene and Leiby [3] & $\$ 14.3$ & 1990 & $\$ 12^{\prime \prime}$ \\
Ravenal [4] & $\$ 50$ & 1992 & $\$ 60^{\mathrm{b}}$ \\
Kaufinann and Steinbruner [5] & $\$ 64.5$ & 1990 & $\$ 55^{\mathrm{b}}$ \\
Delucchi and Murphy" [6] & $\$ 20-40$ & 1996 & $\$ 20-40^{\mathrm{b}}$ \\
\hline
\end{tabular}

Average estimate is $\$ 32$ billion, with a standard deviation of $\$ 22$ billion.

[1] U.S. General Accounting Offices, Southwest Asia: Cost of Protecting U.S. Interests, GAO/NSIAD-9 1-250, Washington, DC, August 1991.

[2] Congressional Research Service, The External Costs of Oil Used in Transportation, prepared for the U.S. Alternative Fuels Council, Washington, DC, June 1992.

[3] Greene, D.L., and P. Leiby, The Social Costs to the U.S. of Monopolization of the World Oil Market, 1972- 199 1, ORNL-6744, Oak Ridge National Laboratory, Oak Ridge, TN, March 1993.

[4] Ravenal, E.C., Designing Defense for a New World Order: The Military Budget in 1992 and Beyond, Cato Institute, Washington, DC, 1991.

[5] Kaufmann, W.W., and J.D. Steinbruner, Decisions for Defense: Prospects for a New Order, The Brookings Institution, Washington, DC, 1991.

[6] Delucchi, M.A., and J. Murphy, U.S. Military Expenditures to Protect the Use of Persian-Gulf Oil for Motor Vehicles, UCD-ITS-RR-96-3 (15), University of California, Davis, California, April 1996.

Source:

Hu, P.S., "Estimates of 1996 U.S. Military Expenditures on Defending Oil Supplies from the Middle East: A Literature Review,” Oak Ridge National Laboratory, Oak Ridge, TN, March 1996.

aEstimated based on a 3\% annual inflation rate and a decrease of 30\% in the total Defense budget from 1990 to 1996.

'Provided by the author(s); thus, assumptions used for the projection are different from those used in the other estimates.

"Annual cost to defend all U.S. interests in the Persian Gulf. 
Figure 1.6. Refinery Gross Output by World Region, 1998

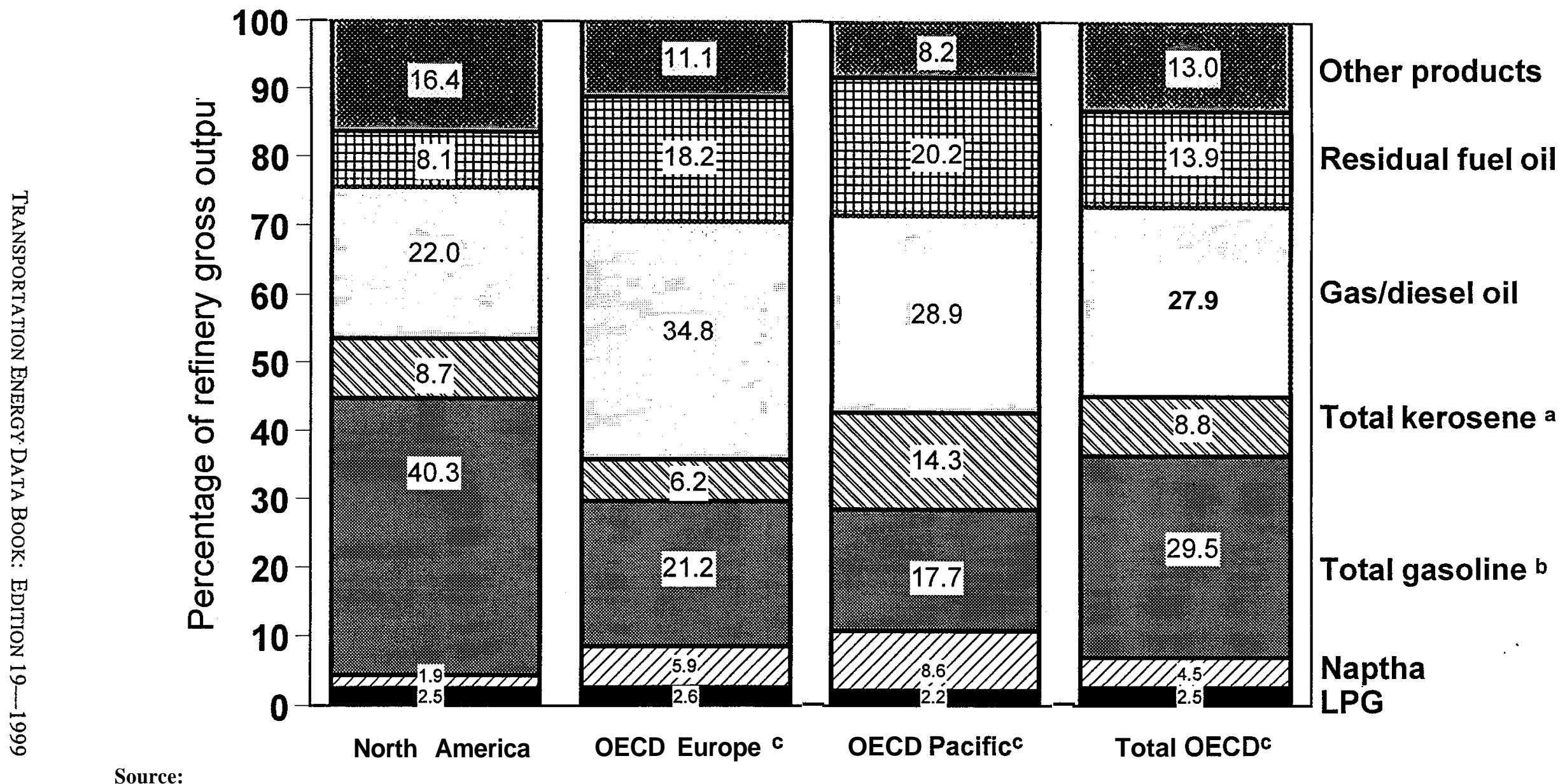

International Energy Agency, Monthly Oil Survey, January 1999, Paris, France, Table 7.

\footnotetext{
a Includes jet kerosene and other kerosene.

${ }^{\mathbf{b}}$ Includes motor gasoline, jet gasoline, and aviation gasoline.

c Organization for Economic Cooperation and Development. See Glossary for membership.
} 
Oxygenate refinery input increased significantly in 1995, most certainly due to the Clean Air Act Amendments of 1990 which mandated the sale of

reformulated gasoline in certain areas beginning in January 1995.

Table 1.7

U.S. Refinery Input of Crude Oil and Petroleum Products, 1987-97

(thousand barrels)

\begin{tabular}{|c|c|c|c|c|c|c|c|c|c|}
\hline \multirow[b]{2}{*}{ Year } & \multirow[b]{2}{*}{ Crude oil } & \multirow[b]{2}{*}{$\begin{array}{c}\text { Natural gas } \\
\text { liquids }\end{array}$} & \multicolumn{4}{|c|}{ Oxygenates } & \multirow[b]{2}{*}{$\begin{array}{c}\text { Other } \\
\text { hydrocarbons" }\end{array}$} & \multirow[b]{2}{*}{ Other liquids } & \multirow[b]{2}{*}{$\begin{array}{c}\text { Total input to } \\
\text { refineries }\end{array}$} \\
\hline & & & $\begin{array}{c}\text { Fuel } \\
\text { ethanol }\end{array}$ & Methanol & MTBE" & $\begin{array}{c}\text { Other } \\
\text { oxygenates }^{\mathrm{b}}\end{array}$ & & & \\
\hline 1987 & $4,691,783$ & 280,889 & $d$ & d & d & $\mathrm{d}$ & 23,304 & 220,296 & $5,105,392$ \\
\hline \multirow[t]{2}{*}{1988} & $4,848,175$ & 304,566 & d & d & d & d & 19,515 & 203,794 & $5,258,386$ \\
\hline & $4,891,381$ & 182,109 & & & & & 21,757 & 202,040 & $5,297,287$ \\
\hline 1990 & $4,894,379$ & 170,589 & $d$ & d & $\mathrm{d}$ & d & 28,642 & 231,466 & $5,325,076$ \\
\hline 1991 & $4,855,016$ & 172,306 & d & d & d & d & 31,574 & 248,691 & $5,307,587$ \\
\hline 1992 & $4,908,603$ & 171,701 & d & d & d & d & 47,918 & 224,758 & $5,352,980$ \\
\hline 1993 & $4,968,641$ & 179,213 & 3,351 & 782 & 49,393 & 1,084 & 15,543 & 264,531 & $5,482,538$ \\
\hline 1994 & $5,061,111$ & 169,868 & 3,620 & 242 & 52,937 & 1,676 & 14,130 & 179,678 & $5,483,262$ \\
\hline 1995 & $5,100,317$ & 172,026 & 9,055 & 246 & 79,396 & 3,876 & 14,668 & 175,743 & $5,555,327$ \\
\hline 1996 & $5,195,265$ & 164,552 & 11,156 & 126 & 79,407 & 3,444 & 20,587 & 193,695 & $5,668,232$ \\
\hline 1997 & $5,351,466$ & 151,769 & 11,803 & 496 & 86,240 & 3,750 & 22,976 & 178,292 & $5,806,792$ \\
\hline 1987-97 & $1.3 \%$ & $-6.0 \%$ & e & e & ge annuc & $\underset{\mathbf{e}}{\text { entage }}$ cha & $-0.1 \%$ & $-2.1 \%$ & $1.3 \%$ \\
\hline 1993-97 & $1.9 \%$ & $-4.1 \%$ & $37.0 \%$ & $-10.8 \%$ & $15.0 \%$ & $36.4 \%$ & $10.3 \%$ & $-9.4 \%$ & $1.4 \%$ \\
\hline
\end{tabular}

Source:
U.S. Department of Energy, Energy Information Administration, Petroleum Supply Annual, 1997, Vol. 1, June 1998, Table 16, p. 49, and annual.

(Additional resources: http://www.eia.doe.gov)

"Methyl tertiary butyl ether (MTBE).

'Includes ethyl tertiary butyl ether (ETBE), tertiary amyl methyl ether (TAME), tertiary butyl alcohol (TBA), and other aliphatic alcohols and ethers intended for motor gasoline blending.

'For 1987-92, includes other hydrocarbons/hydrogen/oxygenates. For 1993-on, includes other hydrocarbons/hydrogen.

'Reported in "Other hydrocarbons" category in this year.

'Data are not available. 
When crude oil and other hydrocarbons are processed into products that are, on average, less dense than the input, a processing volume gain occurs. Due to this gain, the product yield from a barrel of crude oil is more than 100\%. The processing volume gain has been growing over the years.

Table 1.8

Refinery Yield of Petroleum Products from a Barrel of Crude Oil, 1978-97 (percentage)

\begin{tabular}{|c|c|c|c|c|c|c|}
\hline Year & $\begin{array}{l}\text { Motor } \\
\text { gasoline }\end{array}$ & $\begin{array}{c}\text { Distillate } \\
\text { fuel oil }\end{array}$ & Jet fuel & $\begin{array}{c}\text { Liquified } \\
\text { petroleum gas }\end{array}$ & Other" & Total $^{\mathrm{b}}$ \\
\hline 1978 & 44.1 & 21.4 & 6.6 & 2.3 & 29.6 & 104.0 \\
\hline 1979 & 43.0 & 21.5 & 6.9 & 2.3 & 30.3 & 104.0 \\
\hline 1980 & 44.5 & 19.7 & 7.4 & 2.4 & 30.0 & 104.0 \\
\hline 1981 & 44.8 & 20.5 & 7.6 & 2.4 & 28.7 & 104.0 \\
\hline 1982 & 46.4 & 21.5 & 8.1 & 2.2 & 26.2 & 104.4 \\
\hline 1983 & 47.6 & 20.5 & 8.5 & 2.7 & 24.8 & 104.1 \\
\hline 1984 & 46.7 & 21.5 & 9.1 & 2.9 & 24.2 & 104.4 \\
\hline 1985 & 45.6 & 21.6 & 9.6 & 3.1 & 24.6 & 104.5 \\
\hline 1986 & 45.7 & 21.2 & 9.8 & 3.2 & 24.8 & 104.7 \\
\hline 1987 & 46.4 & 20.5 & 10.0 & 3.4 & 24.5 & 104.8 \\
\hline 1988 & 46.0 & 20.8 & 10.0 & 3.6 & 24.4 & 104.8 \\
\hline 1989 & 45.7 & 20.8 & 10.1 & 4.0 & 24.2 & 104.8 \\
\hline 1990 & 45.6 & 20.9 & 10.7 & 3.6 & 24.1 & 104.9 \\
\hline 1991 & 45.7 & 21.3 & 10.3 & 3.8 & 24.1 & 105.2 \\
\hline 1992 & 46.0 & 21.2 & 9.9 & 4.3 & 24.0 & 105.4 \\
\hline 1993 & 46.1 & 21.9 & 10.0 & 4.1 & 23.3 & 105.4 \\
\hline 1994 & 45.5 & 22.3 & 10.1 & 4.2 & 23.2 & 105.3 \\
\hline 1995 & 46.4 & 21.8 & 9.7 & 4.5 & 22.9 & 105.3 \\
\hline 1996 & 45.7 & 22.7 & 10.4 & 4.5 & 22.4 & 105.7 \\
\hline 1997 & 45.7 & 22.5 & 10.3 & 4.6 & 22.5 & 105.6 \\
\hline
\end{tabular}

\section{Source:}

Department of Energy, Energy Information Administration, Petroleum Supply Annual 1997, Vol. 1, June 1998, Table 19, p. 54, and annual. (Additional resources: http://www.eia.doe.gov)

\footnotetext{
a Includes aviation gasoline, kerosene, naphtha and other oils for petrochemical feedstock use, special naphthas, lubricants, waxes, petroleum coke, asphalt and road oil, still gas, and miscellaneous products. b Products sum greater than $100 \%$ due to processing gain. The processing gain for years 1978 to 1980 is assumed to be 4\% Transportation ENERgy DATa Book: Edition 19-1999
} 
Table 1.9

United States Petroleum Production and Consumption, 1973-98

(million barrels per day)

\begin{tabular}{|c|c|c|c|c|c|c|c|c|c|c|c|}
\hline \multirow[b]{2}{*}{ Year } & \multirow{2}{*}{$\begin{array}{l}\text { Domestic } \\
\text { crude oil } \\
\text { production }\end{array}$} & \multicolumn{3}{|c|}{ Net imports } & \multicolumn{2}{|c|}{ Exports } & \multirow{2}{*}{$\begin{array}{c}\text { U.S. } \\
\text { petroleum } \\
\text { consumption }\end{array}$} & \multirow{2}{*}{$\begin{array}{l}\text { World } \\
\text { petroleum } \\
\text { consumption }\end{array}$} & \multirow{2}{*}{$\begin{array}{l}\text { Net imports as } \\
\text { a percentage } \\
\text { of U.S. } \\
\text { petroleum } \\
\text { consumption }\end{array}$} & \multirow{2}{*}{$\begin{array}{l}\text { U.S. petroleum } \\
\text { consumption as a } \\
\text { percentage } \\
\text { of world } \\
\text { consumption }\end{array}$} & \multirow{2}{*}{$\begin{array}{c}\text { Transportation } \\
\text { petroleum use } \\
\text { as a percentage } \\
\text { of domestic } \\
\text { production }^{\mathrm{b}}\end{array}$} \\
\hline & & $\begin{array}{c}\text { Crude } \\
\text { oil } \\
\end{array}$ & $\begin{array}{c}\text { Petroleum } \\
\text { products }\end{array}$ & Total & Crude oil & $\begin{array}{c}\text { Petroleum } \\
\text { products }\end{array}$ & & & & & \\
\hline 1973 & 9.21 & 3.24 & 2.78 & 6.03 & 0.00 & 0.23 & 17.31 & 56.39 & $34.8 \%$ & $30.7 \%$ & $76.7 \%$ \\
\hline 1974 & 8.77 & 3.47 & 2.42 & 5.89 & 0.00 & 0.22 & 16.65 & 55.91 & $35.4 \%$ & $29.8 \%$ & $78.3 \%$ \\
\hline 1975 & 8.37 & 4.10 & 1.75 & 5.85 & 0.00 & 0.20 & 16.32 & 55.48 & $35.8 \%$ & $29.4 \%$ & $82.8 \%$ \\
\hline 1976 & 8.13 & 5.28 & 1.81 & 7.09 & 0.00 & 0.22 & 17.46 & 58.74 & $40.6 \%$ & $29.7 \%$ & $89.5 \%$ \\
\hline 1977 & 8.25 & 6.57 & 2.00 & 8.57 & 0.05 & 0.19 & 18.43 & 61.63 & $46.5 \%$ & $29.9 \%$ & $91.7 \%$ \\
\hline 1978 & 8.71 & 6.20 & 1.80 & 8.00 & 0.16 & 0.20 & 18.85 & 63.30 & $42.5 \%$ & $29.8 \%$ & $91.7 \%$ \\
\hline 1979 & 8.55 & 6.28 & 1.70 & 7.99 & 0.24 & 0.24 & 18.51 & 65.17 & $43.1 \%$ & $28.4 \%$ & $92.0 \%$ \\
\hline 1980 & 8.60 & 4.98 & 1.39 & 6.37 & 0.29 & 0.26 & 17.06 & 63.07 & $37.3 \%$ & $27.0 \%$ & $87.9 \%$ \\
\hline 1981 & 8.57 & 4.17 & 1.23 & 5.40 & 0.23 & 0.37 & 16.06 & 60.87 & $33.6 \%$ & $26.4 \%$ & $86.9 \%$ \\
\hline 1982 & 8.65 & 3.25 & 1.05 & 4.30 & 0.24 & 0.58 & 15.30 & 59.50 & $28.1 \%$ & $25.7 \%$ & $84.9 \%$ \\
\hline 1983 & 8.69 & 3.17 & 1.15 & 4.31 & 0.16 & 0.58 & 15.23 & 58.74 & $28.3 \%$ & $25.9 \%$ & $85.3 \%$ \\
\hline 1984 & 8.88 & 3.25 & 1.47 & 4.72 & 0.18 & 0.54 & 15.73 & 59.84 & $30.0 \%$ & $26.3 \%$ & $86.0 \%$ \\
\hline 1985 & 8.97 & 3.00 & 1.29 & 4.29 & 0.20 & 0.58 & 15.73 & 60.10 & $27.3 \%$ & $26.2 \%$ & $86.6 \%$ \\
\hline 1986 & 8.68 & 4.02 & 1.41 & 5.44 & 0.15 & 0.63 & 16.28 & 61.76 & $33.4 \%$ & $26.4 \%$ & $93.1 \%$ \\
\hline 1988 & 8.14 & 4.95 & 1.63 & 6.59 & 0.16 & 0.66 & 17.28 & 64.82 & $38.1 \%$ & $26.7 \%$ & $104.1 \%$ \\
\hline 1989 & 7.61 & 5.70 & 1.50 & 7.20 & 0.14 & 0.72 & 17.33 & 65.92 & $41.6 \%$ & $26.3 \%$ & $112.1 \%$ \\
\hline 1990 & 7.36 & 4.79 & 1.38 & 7.16 & 0.11 & 0.75 & 16.99 & 65.98 & $42.2 \%$ & $25.8 \%$ & $114.5 \%$ \\
\hline 1991 & 7.42 & 5.67 & 0.96 & 6.63 & 0.12 & 0.89 & 16.71 & 66.57 & $39.6 \%$ & $25.1 \%$ & $110.6 \%$ \\
\hline 1992 & 7.17 & 5.99 & 0.94 & 6.94 & 0.09 & 0.86 & 17.03 & 66.74 & $40.7 \%$ & $25.5 \%$ & $114.5 \%$ \\
\hline 1993 & 6.85 & 6.69 & 0.93 & 7.62 & 0.10 & 0.90 & 17.24 & 66.99 & $44.2 \%$ & $25.7 \%$ & $118.7 \%$ \\
\hline 1994 & 6.66 & 6.96 & 1.09 & 8.05 & 0.10 & 0.84 & 17.72 & 68.30 & $45.5 \%$ & $25.9 \%$ & $124.7 \%$ \\
\hline 1995 & 6.56 & 7.14 & 0.75 & 7.89 & 0.10 & 0.86 & 17.73 & 69.89 & $44.5 \%$ & $25.4 \%$ & $127.6 \%$ \\
\hline 1996 & 6.47 & 7.40 & 1.10 & 8.50 & 0.11 & 0.87 & 18.31 & 71.32 & $46.4 \%$ & $25.7 \%$ & $130.8 \%$ \\
\hline 1997 & 6.45 & 8.12 & 1.04 & 9.16 & 0.11 & 0.90 & 18.62 & 73.01 & $49.2 \%$ & $25.5 \%$ & $132.3 \%$ \\
\hline 1998 & 6.24 & 8.44 & 1.01 & 9.45 & 0.11 & 0.82 & 18.68 & & $50.6 \%$ & & $137.0 \%$ \\
\hline \multicolumn{12}{|c|}{ Average annual percentage change } \\
\hline 1973-98 & $-1.5 \%$ & $3.9 \%$ & $-4.0 \%$ & $1.8 \%$ & - & $5.2 \%$ & $0.3 \%$ & $1.0 \%{ }^{\mathrm{d}}$ & & & \\
\hline 1988-98 & $-2.6 \%$ & $5.5 \%$ & $-4.7 \%$ & $3.7 \%$ & $-3.7 \%$ & $2.2 \%$ & $0.8 \%$ & $1.2 \%{ }^{\mathrm{d}}$ & & & \\
\hline
\end{tabular}

Source:

U.S. Department of Energy, Energy Information Administration, Monthly Energy Review, March 1999, Washington, DC, $1999, \mathrm{pp} .42-47$.

World petroleum consumption - U.S. Department of Energy, Energy Information Administration, International Energy Annual 1997, April 1999, Table1.1. (Additional resources: http://www.eia.doe.gov)

${ }^{2}$ Best estimate for U.S. petroleum consumption is the amount of petroleum products supplied to the U.S. in a given year. This is not the sum of crude oil production and net imports due to processing gain and stock changes.

'Transportation petroleum use can be found on Table 1.10. This column has been revised to include domestic production of crude oil, natural gas plant liquids, and other hydrocarbons/hydrogen/oxygenates as shown in the Monthly Energy Review, Table 3.1 a.

c Data are not available.

${ }^{d}$ Average annual percentage change is to the latest possible year. 
Figure 1.7. United States Petroleum Production and Consumption, 1973-98

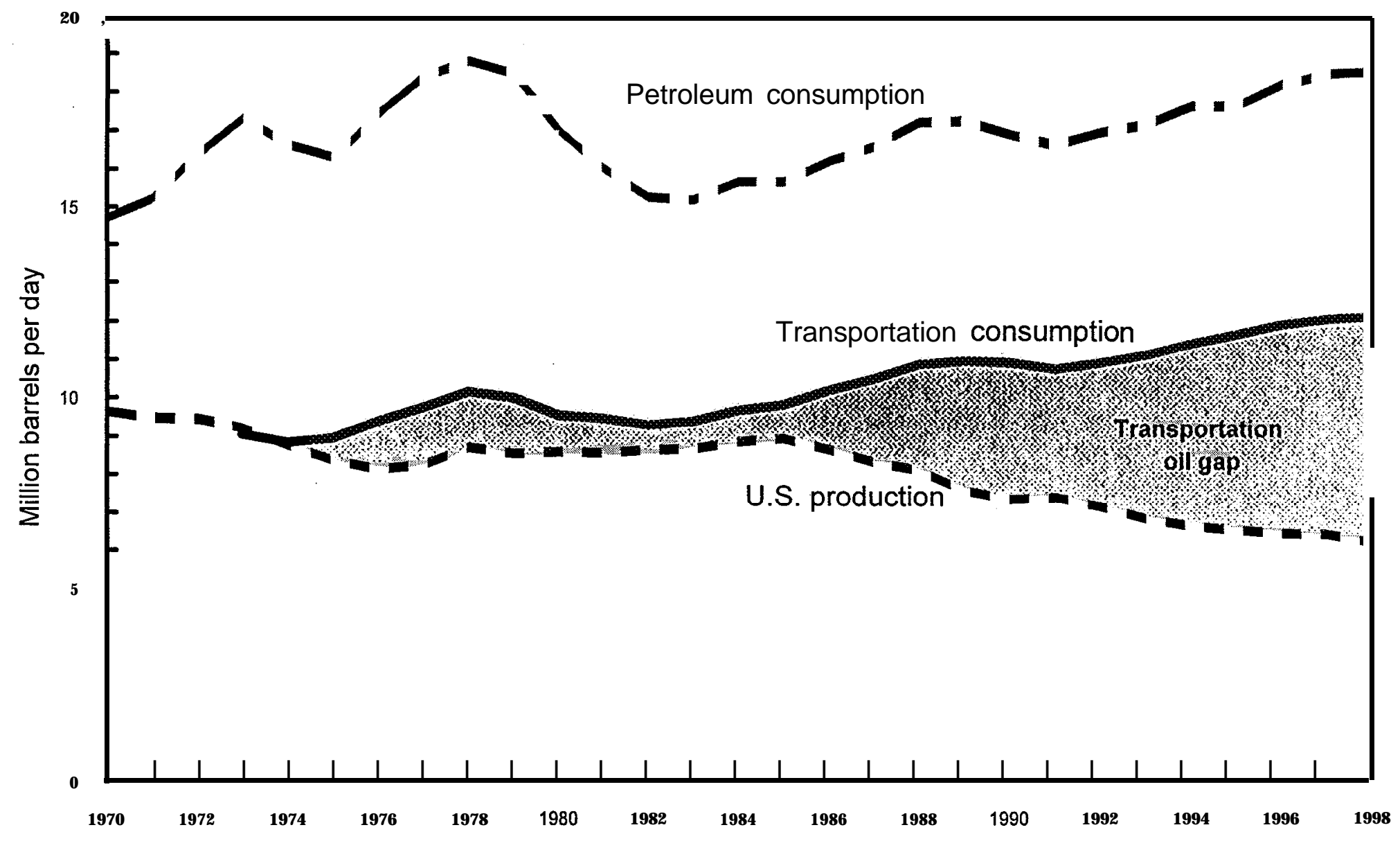

Source: See Tables 1.8 and 1.9. 
The share ofpetroleum imported to the U.S. can be calculated using total imports or net imports. Net imports, which is the preferred data, rose to $50 \%$ for the first time in 1998, while total imports reached 50\% for the first time in 1993. The reliance on imports from the Persian Gulf region has increased in 1998 to the highest point since 1990.

Figure 1.8. Import Share of U.S. Petroleum Consumption, 1973-98

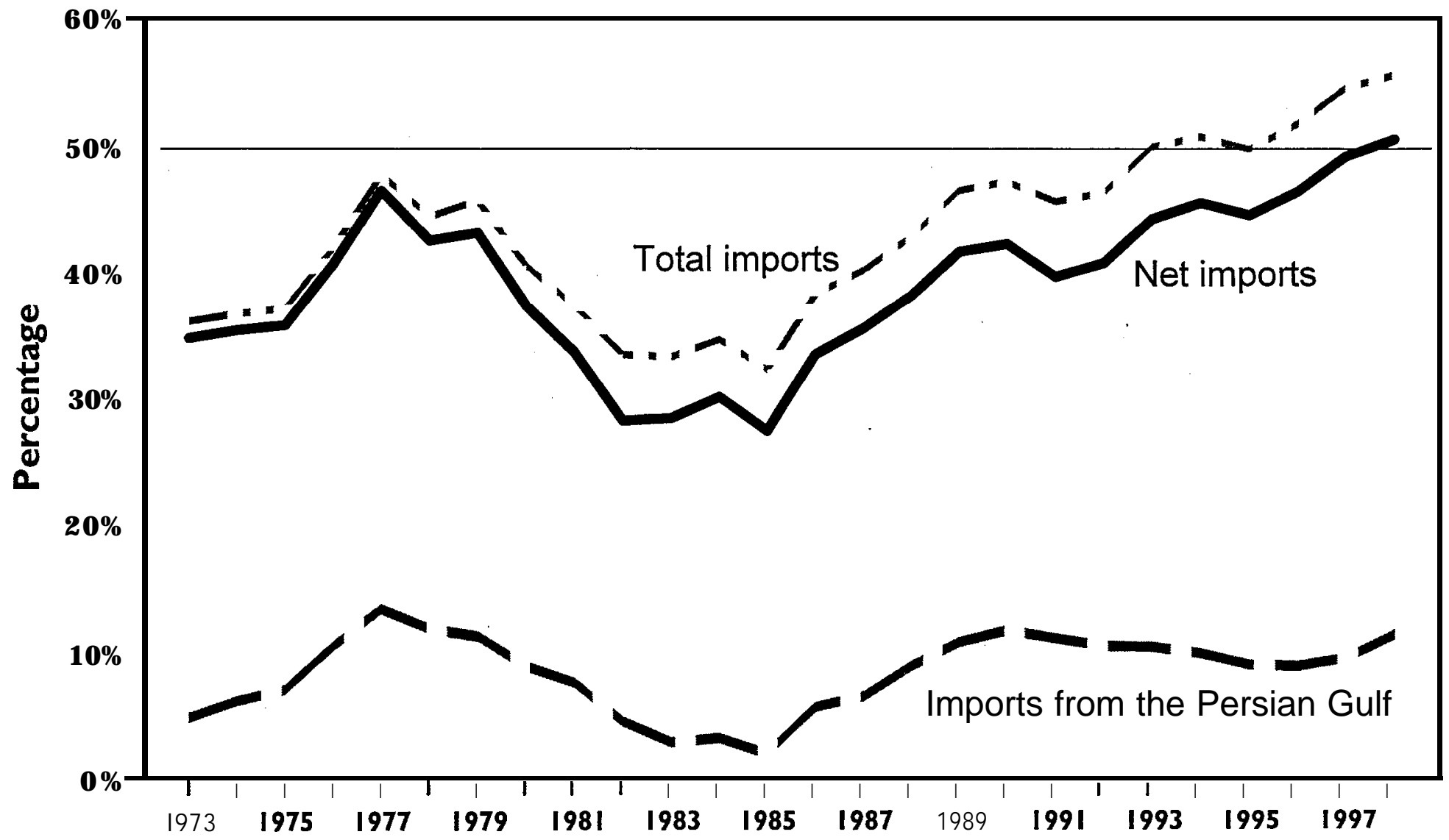

Source: Table 1.8 and the U.S. Department of Energy, Energy Information Administration, Monthly Energy Review, March 1999, Washington, DC, 1999 , p. 15. 
Table 1.10

Consumption of Petroleum by End-Use Sector, 1973-98 (quadrillion Btu)

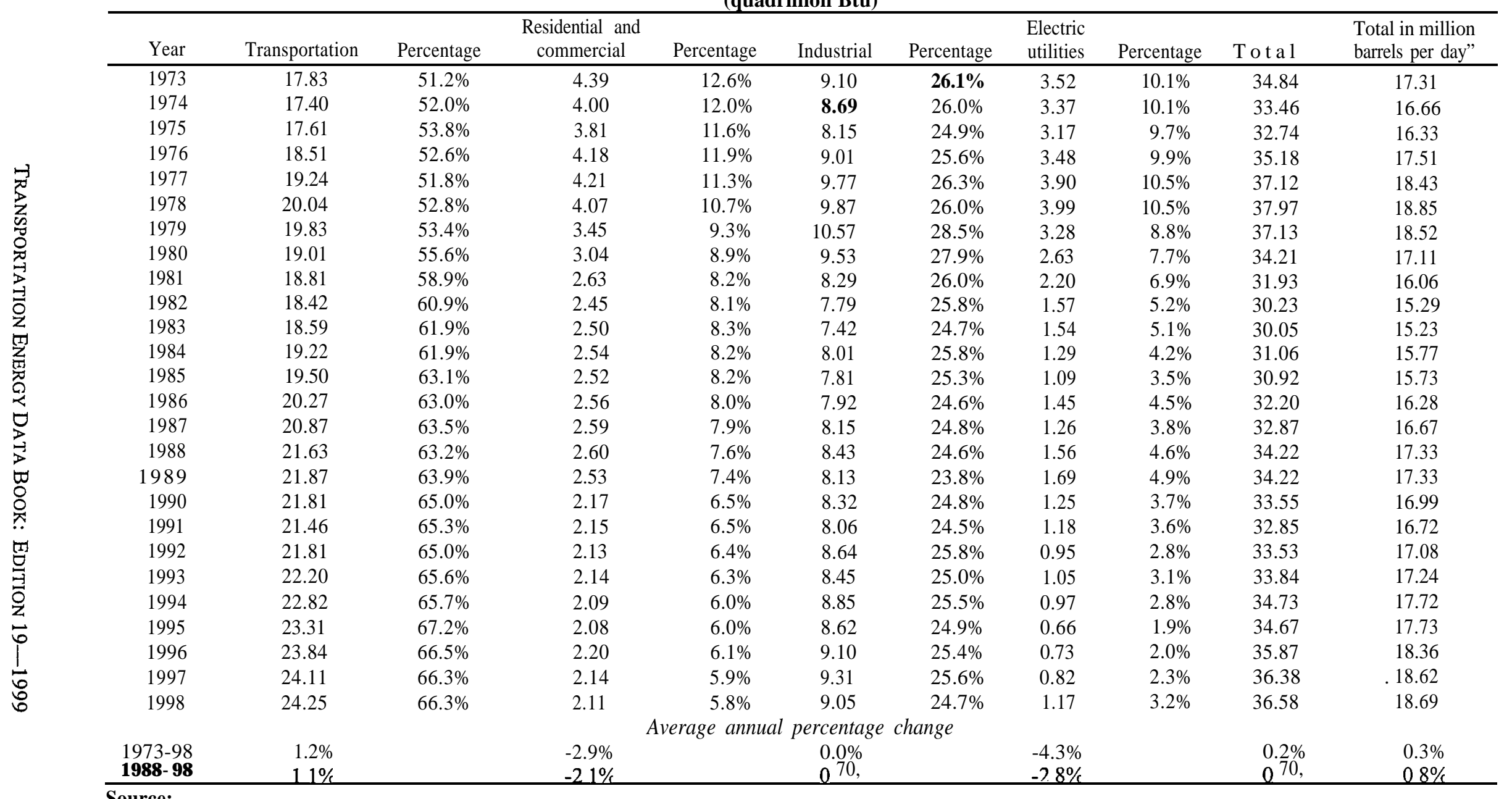

\section{Source:}

U.S. Department of Energy, Energy Information Administration, Monthly Energy Review, March 1999, pp. $27,29,3$ 1, 33. (Additional resources: http://www.eia.doe.gov)

a Calculated from Total column using Table A.3. Approximate Heat Content of Petroleum Products, Weighted Average, from the Monthly Energy Review, March 1997. 
Table 1.11

Transportation of Petroleum and Petroleum Products in the U.S. by Mode, 1975-97

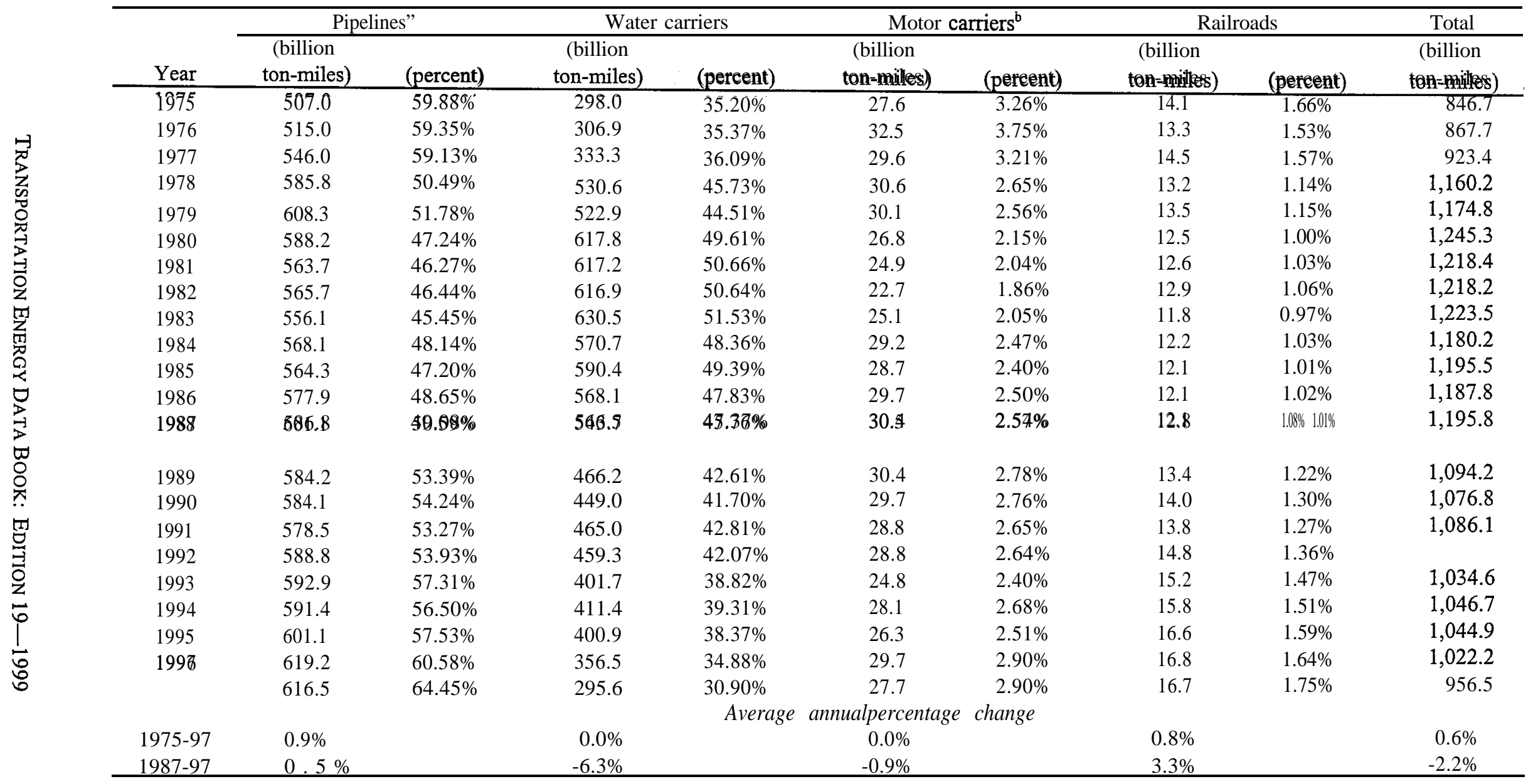

Source:

Association of Oil Pipelines, Shifts in Petroleum Transportation, Washington, DC, February 1999, Table 1.

a The amounts carried by pipeline are based on ton-miles of crude and petroleum products for Federally regulated pipelines (84 percent) plus au estimated breakdown of crude and petroleum products of the ton-miles for pipelines not Federally regulated (16 percent).

b The amounts carried by motor carriers are estimated. 


\section{Chapter 2 \\ Energy}

Summary Statistics from Tables in this Chapter

\begin{tabular}{|c|c|c|}
\hline Source & & \\
\hline Table 2.3 & Transportation share of U.S. energy consumption, 1998 & $27.7 \%$ \\
\hline Table 2.4 & Petroleum share of Transportation energy consumption, 1998 & $96.8 \%$ \\
\hline \multirow[t]{9}{*}{ Table 2.6} & Transportation energy use by mode, 1997 & (trillion Btu) \\
\hline & Automobiles & 8,743 \\
\hline & Light trucks & 6,189 \\
\hline & Heavy trucks & 4,069 \\
\hline & Buses & 184 \\
\hline & Air & 2,325 \\
\hline & Water & 1,309 \\
\hline & Pipeline & 987 \\
\hline & Rail & 579 \\
\hline \multirow[t]{7}{*}{ Table 2.9} & Alternative vehicle fuel consumption, 1998 (thousand gasolin & quivalent gallons) \\
\hline & Liquified petroleum gas & 245,058 \\
\hline & Compressed natural gas & 76,852 \\
\hline & Liquified natural gas & 6,338 \\
\hline & $M 85 / M 100$ & 3,318 \\
\hline & $E 85 / E 100$ & 1,674 \\
\hline & Electricity & 1,301 \\
\hline \multirow[t]{3}{*}{ Table 2.9} & \multicolumn{2}{|c|}{ Oxygenate consumption, 1998 (thousand gasoline equivalent gallons) } \\
\hline & $M T B E$ & $3,080,600$ \\
\hline & Ethanol in gasohol & 857,100 \\
\hline
\end{tabular}


Table 2.1

World Production of Primary Energy by Selected Country Groups, 1988-97 (quadrillion Btu)

\begin{tabular}{|c|c|c|c|c|c|c|c|c|c|c|}
\hline & 1988 & 1989 & 1990 & 1991 & 1992 & 1993 & 1994 & 1995 & 1996 & 1997" \\
\hline \multicolumn{11}{|c|}{ Petroleum } \\
\hline World total & 132.57 & 134.66 & 136.35 & 135.90 & 135.90 & 136.50 & 136.52 & 138.30 & 141.47 & 150.62 \\
\hline $\mathrm{OECD}^{\mathrm{b}}$ & 39.71 & 38.07 & 38.20 & 38.20 & 39.70 & 39.70 & 39.57 & 40.94 & 41.66 & 43.60 \\
\hline 'Non OECD & 92.86 & 96.58 & 98.15 & 98.15 & 96.81 & 96.81 & 96.95 & 97.36 & 99.81 & 107.20 \\
\hline \multicolumn{11}{|c|}{ Natural gas } \\
\hline World total & 71.91 & 74.47 & 76.10 & 76.72 & 76.94 & 78.35 & 79.1 & 80.27 & 84.06 & 84.26 \\
\hline $\mathrm{OECD}^{\mathrm{b}}$ & 30.12 & 30.84 & 31.63 & 32.22 & 33.05 & 34.1 & 35.52 & 36.08 & 38.05 & 38.18 \\
\hline Non OECD & 41.79 & 43.63 & 44.47 & 44.50 & 43.89 & 44.26 & 43.58 & 44.18 & 46.01 & 46.08 \\
\hline \multicolumn{11}{|c|}{ Coal } \\
\hline World total & 89.58 & 91.02 & 92.27 & 87.67 & 88.41 & 86.31 & 88.48 & 90.14 & 91.54 & 92.20 \\
\hline $\mathrm{OECD}^{\mathbf{b}}$ & 41.68 & 42.13 & 42.01 & 39.97 & 39.13 & 38.68 & 40.26 & 39.59 & 40.49 & 41.12 \\
\hline Non OECD & 47.9 & 48.90 & 50.26 & 47.7 & 49.28 & 47.63 & 48.22 & 50.55 & 51.05 & 51.08 \\
\hline \multicolumn{11}{|c|}{ Hydroelectric power } \\
\hline World total & 21.92 & 21.72 & 22.59 & 22.98 & 22.98 & 24.35 & 24.52 & 25.85 & 26.11 & 26.35 \\
\hline $\mathrm{OECD}^{\mathrm{b}}$ & 12.16 & 11.82 & 12.24 & 12.34 & 12.20 & 12.93 & 12.44 & 13.34 & 13.73 & 13.89 \\
\hline Non OECD & 9.76 & 9.90 & 10.35 & 10.64 & 10.78 & 11.42 & 12.08 & 12.51 & 12.38 & 12.47 \\
\hline \multicolumn{11}{|c|}{ Nuclear electric power } \\
\hline World total & 19.30 & 19.81 & 20.37 & 21.29 & 21.36 & 22.07 & 22.50 & 23.35 & 24.17 & 23.97 \\
\hline $\mathrm{OECD}^{\mathbf{b}}$ & 15.96 & 16.38 & 16.99 & 17.93 & 18.15 & 18.99 & 19.61 & 30.35 & 20.84 & 20.59 \\
\hline Non OECD & 3.34 & 3.44 & 3.38 & 3.36 & 3.21 & 3.08 & 2.89 & 3.01 & 3.33 & 3.37 \\
\hline \multicolumn{11}{|c|}{ Total energy' } \\
\hline World total & 335.93 & 342.34 & 351.22 & 348.13 & 349.96 & 351.42 & 356.79 & 364.99 & 374.89 & 381.34 \\
\hline $\mathrm{OECD}^{\mathrm{b}}$ & 140.09 & 139.70 & 144.40 & 145.02 & 145.77 & 147.84 & 152.40 & 154.64 & 160.02 & 160.98 \\
\hline Non OECD & 195.84 & 202.65 & 206.82 & 203.12 & 204.19 & 203.58 & 204.39 & 210.35 & 214.87 & 220.36 \\
\hline
\end{tabular}

Source:

U.S. Department of Energy, Energy Information Administration, International Energy Annual 1997, Washington, DC, April 1999, Table 2.9. (Additional resources: http://www.eia.doe.gov)

\footnotetext{
a Preliminary.

- Organization for Economic Cooperation and Development (OECD). See Glossary for membership.

c Geothermal, solar, and wind electric power are included in the total though not shown separately on this table.
} 
Table 2.2

World Consumption of Primary Energy by Selected Country Groups, 1988-97 (quadrillion Btu)

\begin{tabular}{|c|c|c|c|c|c|c|c|c|c|c|}
\hline & 1988 & 1989 & 1990 & 1991 & 1992 & 1993 & 1994 & 1995 & 1996 & $1997^{a}$ \\
\hline \multicolumn{11}{|c|}{ Petroleum } \\
\hline World total & 132.96 & 134.82 & 134.87 & 136.11 & 136.59 & 136.59 & 139.14 & 142.44 & 145.36 & 148.71 \\
\hline $\mathrm{OECD}^{\mathrm{b}}$ & 81.61 & 82.63 & 82.70 & 83.63 & 85.70 & 86.56 & 88.93 & 90.43 & 92.70 & 93.79 \\
\hline Non OECD & 51.35 & 52.19 & 52.17 & 52.48 & 50.89 & 50.03 & 50.21 & 52.01 & 52.66 & 54.92 \\
\hline \multicolumn{11}{|c|}{ Natural gas } \\
\hline World total & 71.24 & 74.31 & 75.10 & 76.21 & 76.33 & 78.47 & 78.31 & 80.20 & 84.13 & 83.86 \\
\hline $\mathrm{OECD}^{\mathrm{b}}$ & 35.54 & 36.23 & 36.58 & 37.88 & 38.70 & 40.28 & 41.31 & 43.40 & 45.83 & 45.88 \\
\hline Non OECD & 36.70 & 38.08 & 38.52 & 38.33 & 37.63 & 38.19 & 37.01 & 36.80 & 38.31 & 37.98 \\
\hline \multicolumn{11}{|c|}{ Coal } \\
\hline World total & 90.63 & 90.30 & 90.45 & 87.16 & 88.08 & 88.15 & 89.32 & 89.83 & 91.78 & 92.76 \\
\hline $\mathrm{OECD}^{\mathrm{b}}$ & 42.55 & 42.76 & 41.87 & 40.76 & 39.79 & 41.63 & 41.57 & 40.83 & 41.61 & 42.95 \\
\hline Non OECD & 48.08 & 47.54 & 48.58 & 46.40 & 48.30 & 46.52 & 47.75 & 49.00 & 50.18 & 49.81 \\
\hline \multicolumn{11}{|c|}{ Hydroelectric power } \\
\hline World total & 22.24 & 21.83 & 22.69 & 23.18 & 23.22 & 24.60 & 24.81 & 26.12 & 26.43 & 26.57 \\
\hline $\mathrm{OECD}^{\mathrm{b}}$ & 12.48 & 11.93 & 12.33 & 12.55 & 12.44 & 13.18 & 12.73 & 13.60 & 14.06 & 14.11 \\
\hline Non OECD & 9.76 & 9.90 & 10.35 & 10.64 & 10.78 & 11.42 & 12.08 & 12.51 & 12.38 & 12.47 \\
\hline \multicolumn{11}{|c|}{ Nuclear electric power } \\
\hline World total & 19.30 & 19.81 & 20.37 & 21.29 & 21.36 & 22.07 & 22.50 & 23.35 & 24.17 & 23.97 \\
\hline $\mathrm{OECD}^{\mathrm{b}}$ & 15.96 & 16.38 & 16.99 & 17.93 & 18.15 & 18.99 & 19.61 & 20.35 & 20.84 & 20.59 \\
\hline Non OECD & 3.34 & 3.44 & 3.38 & 3.36 & 3.21 & 3.08 & 2.89 & 3.01 & 3.33 & 3.37 \\
\hline \multicolumn{11}{|c|}{ Total energy $^{c}$} \\
\hline World total & 336.70 & 341.63 & 346.85 & 347.26 & 349.04 & 353.35 & 357.60 & 365.60 & 375.56 & 379.53 \\
\hline $\mathrm{OECD}^{\mathrm{b}}$ & 187.54 & 190.52 & 193.80 & 195.99 & 198.05 & 203.92 & 207.45 & 211.90 & 218.46 & 220.71 \\
\hline Non OECD & 149.16 & 151.11 & 153.05 & 151.28 & 150.99 & 149.44 & 150.16 & 153.59 & 157.10 & 158.82 \\
\hline
\end{tabular}

Source:

U.S. Department of Energy, Energy Information Administration, International Energy Annual 1997, Washington, DC, April 1999, Table 1.8. (Additional resources: http://www.eia.doe.gov)

a Preliminary

b Organization for Economic Cooperation and Development (OECD). See Glossary for membership.

c Geothermal, solar, and wind electric power are included in the total though not shown separately on this table. 
Total energy use in the U.S. remained over 90 quads in 1998, but declinedslightlyfrom 1997. The transportation sector commtiummes to accountfor more than $27 \%$ of total energy use.

Table 2.3

U. S. Consumption of Total Energy by End-Use Sector, 1970-98" (quadrillion Btu)

\begin{tabular}{|c|c|c|c|c|c|}
\hline Year & Transportation & $\begin{array}{l}\text { Percentage } \\
\text { transportation } \\
\text { of total }\end{array}$ & $\begin{array}{l}\text { Residential and } \\
\text { commercial }\end{array}$ & Industrial & Total \\
\hline 1970 & 16.07 & $24.2 \%$ & 21.71 & 28.65 & $\overline{66.43}$ \\
\hline 1971 & 16.70 & $24.6 \%$ & 22.59 & 28.59 & 67.88 \\
\hline 1972 & 17.70 & $24.8 \%$ & 23.69 & 29.88 & 71.27 \\
\hline 1973 & 18.61 & $25.1 \%$ & 24.14 & 31.53 & 74.28 \\
\hline 1974 & 18.12 & $25.0 \%$ & 23.73 & 30.69 & 72.54 \\
\hline 1975 & 18.24 & $25.9 \%$ & 23.90 & 28.40 & 70.54 \\
\hline 1976 & 19.10 & $25.7 \%$ & 25.02 & 30.24 & 74.36 \\
\hline 1977 & 19.82 & $26.0 \%$ & 25.39 & 31.08 & 76.29 \\
\hline 1978 & 20.61 & $26.4 \%$ & 26.08 & 31.39 & 78.09 \\
\hline 1979 & 20.47 & $25.9 \%$ & 25.81 & 32.62 & 78.90 \\
\hline 1980 & 19.70 & $25.9 \%$ & 25.66 & 30.61 & 75.96 \\
\hline 1981 & 19.51 & $26.4 \%$ & 25.24 & 29.24 & 73.99 \\
\hline 1982 & 19.07 & $26.9 \%$ & 25.63 & 26.15 & 70.85 \\
\hline 1983 & 19.13 & $27.1 \%$ & 25.63 & 25.76 & 70.52 \\
\hline 1984 & 19.80 & $26.7 \%$ & 26.47 & 27.87 & 74.14 \\
\hline 1985 & 20.07 & $27.1 \%$ & 26.70 & 27.21 & 73.98 \\
\hline 1986 & 20.81 & $28.0 \%$ & 26.85 & 26.63 & 74.30 \\
\hline 1987 & 21.45 & $27.9 \%$ & 27.62 & 27.83 & 76.89 \\
\hline 1988 & 22.31 & $27.8 \%$ & 28.93 & 28.99 & 80.22 \\
\hline 1989 & 22.56 & $27.7 \%$ & 29.40 & 29.35 & 81.33 \\
\hline 1990 & 22.54 & $27.7 \%$ & 28.79 & 29.94 & 81.27 \\
\hline 1991 & 22.12 & $27.3 \%$ & 29.42 & 29.57 & 81.12 \\
\hline 1992 & 22.46 & $27.3 \%$ & 29.11 & 30.58 & 82.15 \\
\hline 1993 & 22.88 & $27.3 \%$ & 30.24 & 30.75 & 83.87 \\
\hline 1994 & 23.57 & $27.5 \%$ & 30.44 & 31.59 & 85.60 \\
\hline 1995 & 24.07 & $27.6 \%$ & 31.27 & 31.86 & 87.21 \\
\hline 1996 & 24.62 & $27.3 \%$ & 32.65 & 32.77 & 90.04 \\
\hline 1997 & 24.93 & $27.5 \%$ & 32.80 & 32.90 & 90.63 \\
\hline 1998 & 25.03 & $27.7 \%$ & 32.91 & 32.45 & 90.42 \\
\hline \multicolumn{6}{|c|}{ Average annualpercentage change } \\
\hline $1970-98$ & $1.6 \%$ & & $1.5 \%$ & $0.4 \%$ & $1.1 \%$ \\
\hline $1988-98$ & $1.2 \%$ & & $1.3 \%$ & $1.1 \%$ & $1.2 \%$ \\
\hline
\end{tabular}

Source:

U.S. Department of Energy, Energy Information Administration, Monthly Energy Review, March 1999, Washington, DC, Table 2.2, p. 25. (Additional resources: http:Nwww.eia.doe.gov)

"Electrical energy losses have been distributed among the sectors. 
Due to the lack of consistent historical data, renewable energy sources are not includedfor sectors other than the electric utilities.

Additional detailed data about oxygenates and otherfuel types for the Transportation sector are found on Table 2.9.

Table 2.4

Distribution of Energy Consumption by Source, 1973, 1980, and 1998

(percentage)

\begin{tabular}{|c|c|c|c|c|c|c|c|c|c|c|c|c|}
\hline \multirow[b]{2}{*}{ Energy source } & \multicolumn{3}{|c|}{ Transportation } & \multicolumn{3}{|c|}{$\begin{array}{c}\text { Residential \& } \\
\text { Commercial }\end{array}$} & \multicolumn{3}{|c|}{ Industrial } & \multicolumn{3}{|c|}{ Electric utilities } \\
\hline & 1973 & 1980 & 1998 & 1973 & 1980 & 1998 & 1973 & 1980 & 1998 & 1973 & 1980 & 1998 \\
\hline Petroleum & 95.8 & 96.5 & 96.8 & 18.2 & 11.8 & 6.4 & 28.9 & 31.1 & 27.9 & 17.7 & 10.7 & 3.4 \\
\hline Naturalgas $^{\mathbf{a}}$ & 4.0 & 3.3 & 3.0 & 31.6 & 29.4 & 23.7 & 32.9 & 27.4 & 30.8 & 18.9 & 15.5 & 9.8 \\
\hline Coal & 0.0 & 0.0 & 0.0 & 1.1 & 0.6 & 0.4 & 12.8 & 10.3 & 7.1 & 43.6 & 49.5 & 55.2 \\
\hline Hydroelectric & 0.0 & 0.0 & 0.0 & 0.0 & 0.0 & 0.0 & 0.1 & 0.1 & 0.1 & 15.0 & 12.6 & 10.2 \\
\hline Nuclear & 0.0 & 0.0 & 0.0 & 0.0 & 0.0 & 0.0 & 0.0 & 0.0 & 0.0 & 4.6 & 11.2 & 21.1 \\
\hline Electricity ${ }^{b}$ & 0.2 & 0.2 & 0.2 & 49.2 & 58.2 & 69.5 & 25.2 & 31.1 & 34.1 & 0.0 & 0.0 & 0.0 \\
\hline Other" & 0.0 & 0.0 & 0.0 & 0.0 & 0.0 & 0.0 & 0.0 & 0.0 & 0.0 & 0.2 & 0.5 & 0.4 \\
\hline Total & 100.0 & 100.0 & 100.0 & 100.0 & 100.0 & 100.0 & 100.0 & 100.0 & 100.0 & 100.0 & 100.0 & 100.0 \\
\hline
\end{tabular}

\section{Source:}

U.S. Department of Energy, Energy Information Administration, Monthly Energy Review, March 1999, Washington, DC, pp. 27, $29,31,33$. (Additional resources: http://www.eia.doe.gov)

"Includes supplemental gaseous fuels. Transportation sector includes pipeline fuel and natural gas vehicle use.

b Includes electrical system energy losses.

c Energy generated from geothermal, wood, waste, wind, photovoltaic, and solar thermal energy sources. 
As data about alternativefuel use come available, an attempt is made to incorporate it into this table. Sometimes assumptions must be made in order to use the data. Please see Appendix A for detailed methodology of all energy data.

Table 2.5

Domestic Consumption of Transportation Energy by Mode and Fuel Type, 1997"

(trillion Btu)

\begin{tabular}{|c|c|c|c|c|c|c|c|c|}
\hline & Gasoline & Diesel fuel & $\begin{array}{l}\text { Liquified } \\
\text { petroleum } \\
\text { gas }\end{array}$ & Jet fuel & $\begin{array}{l}\text { Residual } \\
\text { fuel oil }\end{array}$ & $\begin{array}{c}\text { Natural } \\
\text { gas }\end{array}$ & Electricity & Methanol \\
\hline HIGHWAY & $15,228.3$ & $3,949.8$ & 27.5 & & & 4.2 & 0.9 & 0.0 \\
\hline$\overline{\text { Automobiles }}$ & $8,617.0^{b}$ & 126.0 & & & & 0.2 & & 0.0 \\
\hline Motorcycles & 25.2 & & & & & & & \\
\hline Buses & 32.2 & 147.0 & 0.5 & & & 3.4 & 0.9 & 0.0 \\
\hline Transit & 5.0 & 78.2 & 0.5 & & & 3.4 & 0.9 & 0.0 \\
\hline Intercity" & & 23.6 & & & & & & \\
\hline School" & 27.2 & 45.2 & & & & & & 0.0 \\
\hline Trucks & $6,553.9$ & $3,676.8$ & 27.0 & & & 0.6 & & 0.0 \\
\hline Light trucks ${ }^{d}$ & $5,949.1$ & 226.0 & 13.4 & & & 0.6 & & 0.0 \\
\hline Other trucks & 604.8 & $3,450.8$ & 13.6 & & & 0.0 & & 0.0 \\
\hline OFF-HIGHWAY & 160.7 & $570.1^{\mathbf{e}}$ & & & & & & \\
\hline Construction & 37.6 & $178.5^{\mathrm{e}}$ & & & & & & \\
\hline Agriculture & 123.1 & $391.6^{\mathbf{e}}$ & & & & & & \\
\hline NONHIGHWAY & 334.5 & 810.2 & & $2,290.0$ & 717.0 & 774.9 & 273.1 & \\
\hline Air & 34.8 & & & $2,290.0$ & & & & \\
\hline General aviation & 34.8 & & & 86.3 & & & & \\
\hline Domestic air carriers & & & & $1,857.3$ & & & & \\
\hline International air carriers' & & & & 346.4 & & & & \\
\hline Water & 299.7 & 292.3 & & & 717.0 & & & \\
\hline Freight & & 292.3 & & & 717.0 & & & \\
\hline Recreational & 299.7 & & & & & & & \\
\hline Pipeline & & & & & & 774.9 & 212.1 & \\
\hline Rail & & 517.9 & & & & & 61.0 & \\
\hline Freight (Class I) & & 499.7 & & & & & & \\
\hline Passenger & & 18.2 & & & & & 61.0 & \\
\hline Transit & & & & & & & 42.6 & \\
\hline Commuter & & 8.9 & & & & & 15.1 & \\
\hline Intercity" & & 9.3 & & & & & 3.3 & \\
\hline TOTAL & $15,723.5$ & $5,330.1$ & 27.5 & $2,290.0$ & 717.0 & 779.1 & 274.0 & 0.0 \\
\hline
\end{tabular}

Source:

See Appendix A for Table 2.5.

a Civilian consumption only. Totals may not include all possible uses of fuels for transportation (e.g., snowmobiles).

b Includes gasohol.

c Estimated using vehicle travel information.

d Two-axle, four-tire trucks.

e 1985 data.

f One half of fuel used by domestic carriers in international operation. 
The 1996 data have been revised to include the latest data available.

Table 2.6

Transportation Energy Use by Mode, 1996-97"

\begin{tabular}{|c|c|c|c|c|c|c|}
\hline & \multicolumn{2}{|c|}{ Trillion Btu } & \multicolumn{2}{|c|}{$\begin{array}{l}\text { Thousand barrels per day } \\
\text { crude oil equivalent }\end{array}$} & \multicolumn{2}{|c|}{ Percentage of total } \\
\hline & 1996 & 1997 & 1996 & 1997 & 1996 & 1997 \\
\hline HIGHWAY & $18,850.4$ & $19,210.7$ & $9,483.1$ & $9,664.4$ & $76.0 \%$ & $76.4 \%$ \\
\hline Automobiles & $8,662.3$ & $8,743.2$ & $4,357.8$ & $4,398.5$ & $34.9 \%$ & 34.8 \\
\hline Motorcycles & 24.8 & 25.2 & 12.5 & 12.7 & $0.1 \%$ & $0.1 \%$ \\
\hline Buses & 194.1 & 184.0 & 97.6 & 92.6 & $0.8 \%$ & $0.7 \%$ \\
\hline Transit & 89.3 & 89.3 & 44.9 & 44.9 & $0.4 \%$ & $0.4 \%$ \\
\hline Intercity & 22.6 & $82.2^{\prime}$ & 11.4 & $41.4^{\prime}$ & $0.1 \%$ & $0.3 \%$ \\
\hline School & 82.2 & $82.2^{\mathrm{c}}$ & 41.4 & $41.4^{\prime}$ & $0.3 \%$ & $0.3 \%$ \\
\hline Trucks & $9,969.2$ & $10,258.3$ & $\$ 015.2$ & $5,160.7$ & $40.2 \%$ & $40.8 \%$ \\
\hline Light trucks ${ }^{\mathbf{d}}$ & $5,936.3$ & $6,189.1$ & $2,986.4$ & $3,113.6$ & $23.9 \%$ & $24.6 \%$ \\
\hline Other trucks & $4,032.9$ & $4,069.2$ & $2,028.8$ & $2,047.1$ & $16.3 \%$ & $16.2 \%$ \\
\hline OFF-HIGHWAY & 720.4 & 730.8 & 362.4 & 367.6 & $2.9 \%$ & $2.9 \%$ \\
\hline Construction & 214.0 & 216.1 & 107.7 & 108.7 & $0.9 \%$ & $0.9 \%$ \\
\hline Agriculture & 506.4 & 514.7 & 254.8 & 258.9 & $2.0 \%$ & $2.0 \%$ \\
\hline NONHIGHWAY & $5,217.4$ & $5,199.7$ & $2,624.7$ & $2,615.8$ & $21.0 \%$ & $20.7 \%$ \\
\hline Air & $2,195.9$ & $2,324.8$ & $1,104.7$ & $1,169.5$ & $8.9 \%$ & $9.2 \%$ \\
\hline General aviation & 110.0 & 121.1 & 55.8 & 60.9 & $0.4 \%$ & $0.5 \%$ \\
\hline Domestic air carriers & $1,773.1$ & $1,857.3$ & 892.0 & 934.4 & $7.2 \%$ & $7.4 \%$ \\
\hline International air carriers & 311.8 & 346.4 & 156.9 & 174.3 & $1.3 \%$ & $1.4 \%$ \\
\hline Water & $1,460.2$ & $1,309.0$ & 734.6 & 658.5 & $5.9 \%$ & $5.2 \%$ \\
\hline Freight & $1,171.0$ & $1,009.3$ & 589.1 & 507.7 & $4.7 \%$ & $4.0 \%$ \\
\hline Recreational & 289.2 & 299.7 & 145.5 & 150.8 & $1.2 \%$ & $1.2 \%$ \\
\hline Pipeline & 983.5 & 987.0 & 494.8 & 496.5 & $4.0 \%$ & $3.9 \%$ \\
\hline Rail & 577.8 & 578.9 & 290.7 & 291.2 & $2.3 \%$ & $2.3 \%$ \\
\hline Freight & 499.4 & 499.7 & 251.2 & 251.4 & $2.0 \%$ & $2.0 \%$ \\
\hline Passenger & 78.4 & 79.2 & 39.4 & 39.8 & $0.3 \%$ & $0.3 \%$ \\
\hline Transit & 43.0 & 42.6 & 21.6 & 21.4 & $0.2 \%$ & $0.2 \%$ \\
\hline Commuter & 23.3 & 24.0 & 11.7 & 12.1 & $0.1 \%$ & 0.1 \\
\hline Intercity & $12.1^{\mathrm{c}}$ & $12.6 "$ & 6.1 & $6.3^{\prime}$ & $0.0 \%$ & $0.1 \%$ \\
\hline TOTAL & $24,788.2$ & $25,141.2$ & $12,470.2$ & $12,647.8$ & $100.0 \%$ & $100.0 \%$ \\
\hline
\end{tabular}

Source: See Appendix A for Table 2.5 (detailed breakdown).

"Civilian consumption only. Totals may not include all possible uses of fuels for transportation (e.g., snowmobiles).

bThousand barrels per day crude oil equivalents based average on the EIA weighted average of heat content of petroleum products used in transportation.

'Estimated using vehicle travel information.

'Two-axle, four-tire trucks. 
The Federal Highway Administration produced revised estimates of auto, light truck, and other truck historical fuel use in order to produce a consistent trend. Light trucks

include pickups, vans, and sport utility vehicles.

Table 2.7

Transportation Energy Consumption by Mode, 1970-97 (trillion Btu)

\begin{tabular}{|c|c|c|c|c|c|c|c|c|c|c|c|c|c|}
\hline Year & Autos & $\begin{array}{l}\text { Light } \\
\text { trucks }\end{array}$ & $\begin{array}{c}\text { Light } \\
\text { vehicles } \\
\text { subtotal }\end{array}$ & $\begin{array}{c}\text { Motor- } \\
\text { cycles }\end{array}$ & Buses" & $\begin{array}{l}\text { Other } \\
\text { trucks }\end{array}$ & $\begin{array}{l}\text { Highway } \\
\text { subtotal }\end{array}$ & Air & Water & Pipeline & Rail & $\begin{array}{c}\text { Nonhighway } \\
\text { subtotal }\end{array}$ & $\begin{array}{c}\text { Total } \\
\text { transportation }^{\mathrm{b}}\end{array}$ \\
\hline 1970 & 8,527 & 1,540 & 10,067 & 7 & 109 & 1,503 & 11,686 & 1,307 & 753 & 985 & 558 & 3,603 & 15,289 \\
\hline 1971 & 8,970 & 1,686 & 10,656 & 9 & 108 & 1,569 & 12,342 & 1,304 & 698 & 1,007 & 560 & 3,569 & 15,911 \\
\hline 1972 & 9,547 & 1,895 & 11,442 & 11 & 106 & 1,722 & 13,281 & 1,314 & 703 & 1,039 & 583 & 3,639 & 16,920 \\
\hline 1973 & 9,836 & 2,105 & 11,941 & 13 & 109 & 1,902 & 13,965 & 1,377 & 827 & 996 & 619 & 3,819 & 17,784 \\
\hline 1974 & 9,332 & 2,083 & 11,415 & 14 & 113 & 1,904 & 13,446 & 1,254 & 804 & 932 & 624 & 3,614 & 17,060 \\
\hline 1975 & 9,321 & 2,386 & 11,707 & 14 & 119 & 1,939 & 13,779 & 1,274 & 851 & 835 & 563 & 3,523 & 17,302 \\
\hline 1976 & 9,844 & 2,605 & 12,449 & 15 & 129 & 2,046 & 14,639 & 1,333 & 1,001 & 803 & 585 & 3,722 & 18,361 \\
\hline 1977 & 9,940 & 2,799 & 12,739 & 16 & 132 & 2,268 & 15,155 & 1,411 & 1,103 & 781 & 595 & 3,890 & 19,045 \\
\hline 1978 & 10,140 & 3,022 & 13,162 & 18 & 135 & 2,539 & 15,854 & 1,467 & 1,311 & 781 & 589 & 4,148 & 20,002 \\
\hline 1979 & 9,629 & 3,057 & 12,686 & 22 & 137 & 2,644 & 15,489 & 1,568 & 1,539 & 856 & 613 & 4,576 & 20,065 \\
\hline 1980 & 8,798 & 2,976 & 11,774 & 26 & 139 & 2,651 & 14,590 & 1,528 & 1,677 & 889 & 596 & 4,690 & 19,280 \\
\hline 1981 & 8,695 & 2,964 & 11,659 & 27 & 143 & 2,706 & 14,535 & 1,455 & 1,562 & 899 & 565 & 4,481 & 19,016 \\
\hline 1982 & 8,695 & 2,839 & 11,534 & 25 & 146 & 2,707 & 14,412 & 1,468 & 1,290 & 853 & 488 & 4,099 & 18,511 \\
\hline 1983 & 8,814 & 2,995 & 11,809 & 22 & 145 & 2,757 & 14,733 & 1,505 & 1,187 & 738 & 482 & 3,912 & 18,645 \\
\hline 1984 & 8,857 & 3,202 & 12,059 & 22 & 154 & 2,846 & 15,081 & 1,633 & 1,251 & 780 & 523 & 4,187 & 19,268 \\
\hline 1985 & 8,954 & 3,422 & 12,376 & 23 & 161 & 2,842 & 15,402 & 1,678 & 1,311 & 758 & 487 & 4,234 & 19,636 \\
\hline 1986 & 9,162 & 3,636 & 12,798 & 23 & 154 & 2,903 & 15,878 & 1,823 & 1,295 & 738 & 423 & 4,279 & 20,157 \\
\hline 1987 & 9,179 & 3,827 & 13,006 & 24 & 157 & 2,990 & 16,177 & 1,894 & 1,326 & 775 & 485 & 4,480 & 20,657 \\
\hline 1988 & 9,180 & 4,096 & 13,276 & 25 & 159 & 3,117 & 16,577 & 1,978 & 1,338 & 878 & 498 & 4,692 & 21,269 \\
\hline 1989 & 9,251 & 4,173 & 13,424 & 26 & 163 & 3,196 & 16,809 & 1,981 & 1,376 & 895 & 501 & 4,753 & 21,562 \\
\hline 1990 & 8,707 & 4,467 & 13,174 & 24 & 163 & 3,329 & 16,690 & 2,059 & 1,487 & 928 & 492 & 4,966 & 21,656 \\
\hline 1991 & 8,048 & 4,793 & 12,841 & 23 & 174 & 3,396 & 16,434 & 1,926 & 1,567 & 864 & 463 & 4,820 & 21,254 \\
\hline 1992 & 8,188 & 5,134 & 13,322 & 24 & 182 & 3,460 & 16,988 & 1,971 & 1,641 & 849 & 476 & 4,937 & 21,925 \\
\hline 1993 & 8,389 & 5,375 & 13,764 & 25 & 192 & 3,567 & 17,548 & 1,996 & 1,473 & 889 & 513 & 4,871 & 22,419 \\
\hline 1994 & 8,494 & 5,530 & 14,024 & 26 & 202 & 3,772 & 18,024 & 2,056 & 1,414 & 955 & 546 & 4,971 & 22,995 \\
\hline 1995 & 8,519 & 5,717 & 14,236 & 25 & 179 & 3,950 & 18,390 & 2,117 & 1,522 & 971 & 565 & 5,175 & 23,565 \\
\hline 1996 & 8,622 & 5,936 & 14,558 & 25 & 194 & 4,033 & 18,850 & 2,196 & 1,460 & 984 & 578 & 5,218 & 24,068 \\
\hline 1997 & 8,743 & 6,189 & 14,932 & 25 & 184 & 4,069 & 19,211 & 2,325 & 1,309 & 987 & 579 & 5,200 & 24,411 \\
\hline \multicolumn{14}{|c|}{ Average annual percentage change } \\
\hline $1970-97$ & $0.1 \%$ & $5.3 \%$ & $1.5 \%$ & $4.8 \%$ & $2.0 \%$ & $3.8 \%$ & $1.9 \%$ & $2.2 \%$ & $2.1 \%$ & $0.0 \%$ & $0.1 \%$ & $1.4 \%$ & $1.7 \%$ \\
\hline $1987-97$ & $-0.5 \%$ & $4.9 \%$ & $1.4 \%$ & $0.4 \%$ & $1.6 \%$ & $3.1 \%$ & $1.7 \%$ & $2.1 \%$ & $-0.1 \%$ & $2.4 \%$ & $1.8 \%$ & $1.5 \%$ & $1.7 \%$ \\
\hline
\end{tabular}

Source:

See Appendix A for Table 2.7.

a Beginning in 1992 data became available on alternative fuel use by transit buses.

b Total transportation figures do not include military and off-highway energy use and may not include all possible uses of fuel for transportation (e.g. snowmobiles). 
The Federal Highway Administration cautions that data from 1993-on may not be directly comparable to earlier years. Some states have improved reporting procedures in recent years, and the estimation procedures were revised in 1994. Prior to the Energy Policy Act of 1992, gasohol was defined as a blend of gasoline and at least 10\%, by volume, alcohol. Effective January 1,1993, three types of gasohol were defined: 10\%gasohol-containing at least 10\%alcohol;7.7\% gasohol-containing 7.7\% alcohol but less than 10\%; and $5.7 \%$ gasohol-containing at least $5.7 \%$ alcohol but less than $7.7 \%$. See Table 2.9 for details on oxygenate usage.

Table 2.8

Highway Usage of Gasoline and Special Fuels, 1973-97 (million gallons)

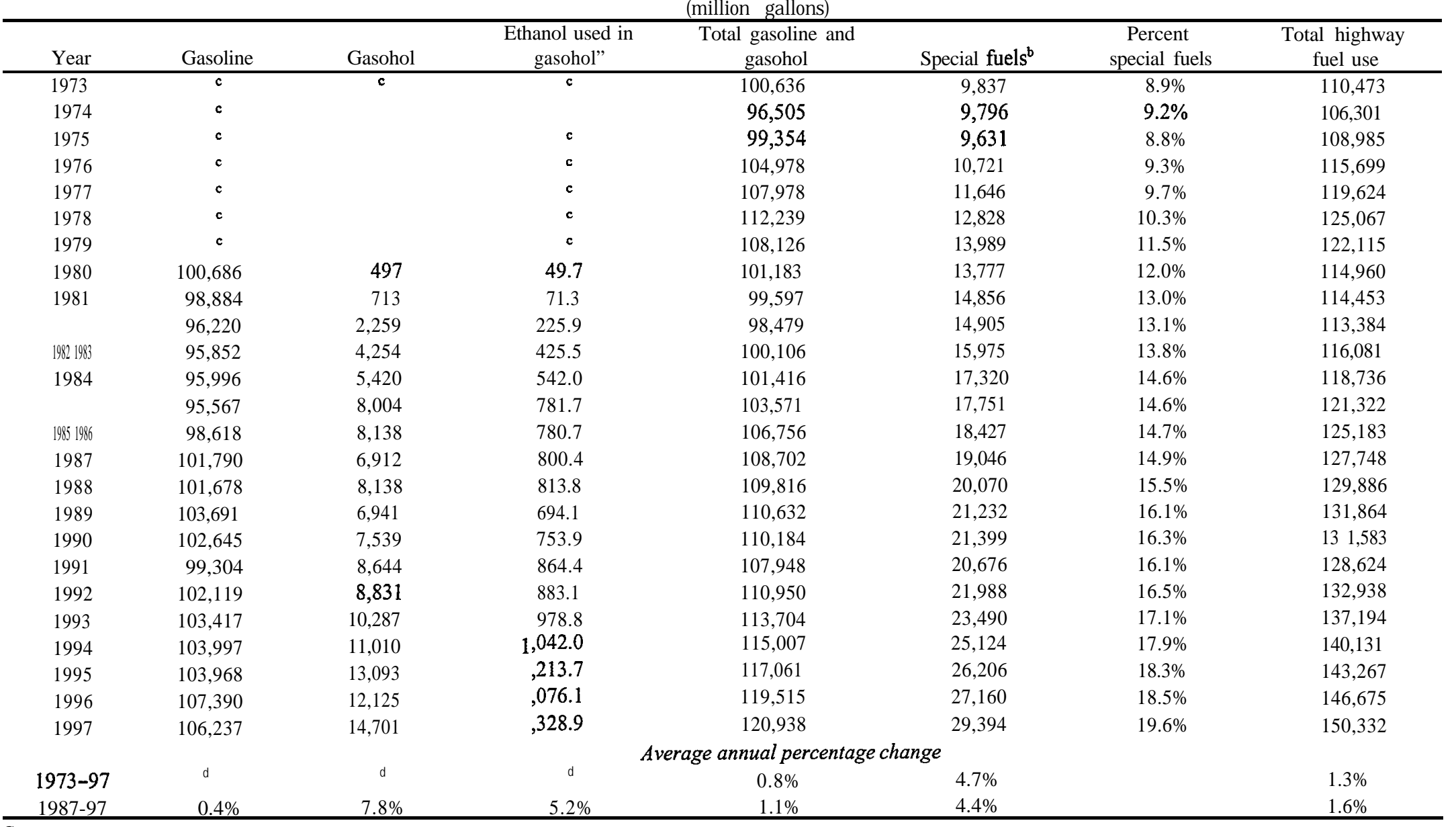

\section{Source:}

U.S. Department of Transportation, Federal Highway Administration, Highway Statistics 1997, Washington, DC, 1998, Tables MF-21 and MF-33E, pp. I-3, I-6, and annual. (Additional resources: http://www.fhwa.dot.gov)

\footnotetext{
Estimated for $1980-92$ as $10 \%$ of gasohol consumption.

b Special fuels consist primarily of diesel fuel, with small quantities of liquified petroleum gas.

c Data for gasoline and gasohol. cannot be separated in this year.

d Data are not available.
} 
Figure 2.1. Motor Gasoline Quantities by Type, 1981 and 1997

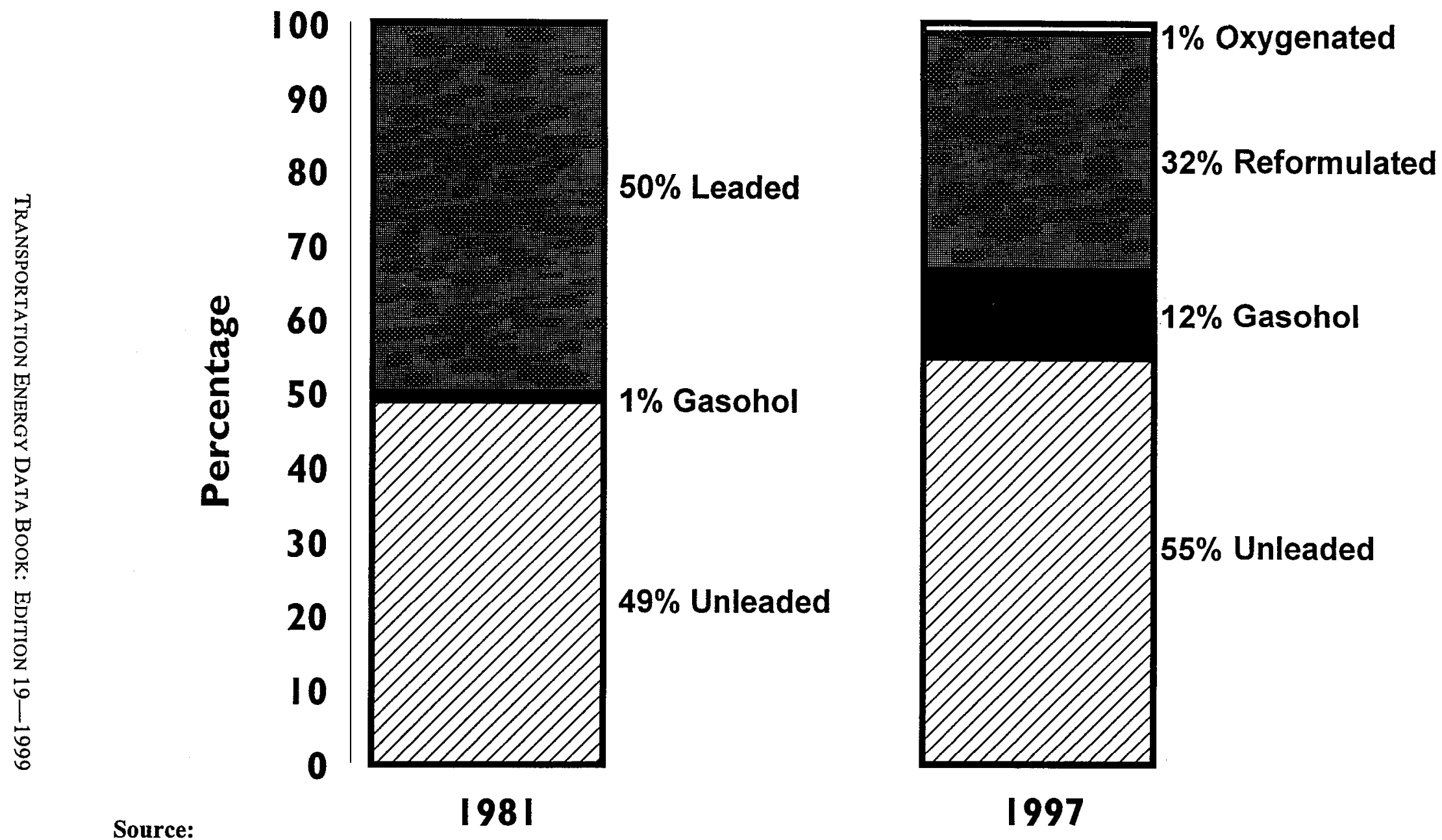

U.S. Department of Energy, Energy Information Administration, Petroleum Supply Annual 1997, Washington, DC, Tables 17 and 20.

U.S. Department of Energy, Energy Information Administration, The Motor Gasoline Industry: Past, Present and Future, Washington, DC, Table 5.

U.S. Department of Transportation, Federal Highway Administration, Highway Statistics 1997, Washington, DC, Tables MF-21 and MF-33E, and annual. 
Table 2.9

Alternative Vehicle Fuel Consumption, 1992-99

(thousand gasoline equivalent gallons)

\begin{tabular}{|c|c|c|c|c|c|c|c|c|c|}
\hline Alternative fuel & 1992 & 1993 & 1994 & 1995 & 1996 & 1997 & 1998 & $1999 ”$ & $\begin{array}{c}1999 \\
\text { Percentage }\end{array}$ \\
\hline Liqnified petroleum gas & 208,142 & 264,655 & 248,467 & 232,701 & 239,158 & 238,356 & 245,058 & 250,322 & $5.9 \%$ \\
\hline Compressed natural gas & 16,823 & 21,603 & 24,160 & 35,162 & 46,923 & 64,295 & 76,852 & 87,389 & $2.0 \%$ \\
\hline Liqnified natural gas & 585 & 1,901 & 2,345 & 2,759 & 3,247 & 3,714 & 6,338 & 6,888 & $0.2 \%$ \\
\hline $\mathrm{M}^{8} 5^{\mathrm{b}}$ & 1,069 & 1,593 & 2,340 & 2,023 & 1,775 & 1,554 & 1,395 & 1,301 & $0.0 \%$ \\
\hline M100 & 2,547 & 3,166 & 3,190 & 2,150 & 347 & 347 & 1,923 & 1,923 & $0.0 \%$ \\
\hline $\mathrm{E} 85^{\mathrm{b}}$ & 21 & 48 & 80 & 190 & 694 & 1,280 & 1,615 & 2,243 & $0.1 \%$ \\
\hline $\mathrm{E} 95^{\mathrm{b}}$ & 85 & 80 & 140 & 995 & 2,699 & 1,136 & 59 & 59 & $0.0 \%$ \\
\hline Electricity & 359 & 288 & 430 & 663 & 773 & 1,010 & 1,301 & 1,414 & $0.0 \%$ \\
\hline Subtotal & 229,631 & 293,334 & 281,152 & 216,643 & 295,616 & 311,692 & 334,541 & 351,539 & $8.2 \%$ \\
\hline \multicolumn{10}{|l|}{ Oxygenates } \\
\hline $\mathrm{MTBE}^{\mathrm{c}}$ & 1,$175 ; 000$ & $2,069,200$ & $2,018,800$ & $2,691,200$ & $2,749,700$ & $3,104,200$ & $3,080,600$ & $3,087,100$ & $72.3 \%$ \\
\hline Ethanol in gasohol & 701,000 & 760,000 & 845,900 & 910,700 & 660,200 & 830,700 & 857,100 & $831,400.0$ & $19.5 \%$ \\
\hline Total & $2,105,631$ & $3,122,534$ & $3,145,852$ & $3,878,543$ & $3,705,516$ & $4,246,592$ & $4,272,241$ & $4,270,039$ & $100.0 \%$ \\
\hline
\end{tabular}

Source:

U.S. Department of Energy, Energy Information Administration, Alternatives to Traditional Transportation Fuels, 1997, Washington, DC, 1998, web site http://www.eia.doe.gov/cneaf/solar.renewables/alt_trans_fue197/atf.html. (Additional resources: http://www.eia.doe.gov)

${ }^{a}$ Based on plans or projections.

'Consumption includes gasoline portion of the mixture.

'Methyl Tertiary Bntyl Ether. This category includes a very small amount of other ethers, primarily Tertiary Amyl Methyl Ether (TAME) and Ethyl Tertiary Bntyl Ether (ETBE). 
Table 2.10

U.S. Production and Imports of MTBE" and Fuel Ethanol, 1978-98 (million gallons)

\begin{tabular}{|c|c|c|c|c|}
\hline \multirow[b]{2}{*}{ Year } & \multicolumn{2}{|c|}{ Production } & \multicolumn{2}{|c|}{ Imports } \\
\hline & Fuel ethanol & MTBE & Fuel ethanol & MTBE" \\
\hline 1978 & 20 & $\mathrm{~b}$ & $\mathrm{~b}$ & $\mathrm{~b}$ \\
\hline 1979 & 40 & b & $\mathrm{b}$ & b \\
\hline 1980 & 80 & $\mathrm{~b}$ & b & $\mathrm{b}$ \\
\hline 1981 & 85 & 122 & $\mathrm{~b}$ & $\mathrm{~b}$ \\
\hline 1982 & 234 & 132 & b & $\mathrm{b}$ \\
\hline 1983 & 443 & 134 & $\mathrm{~b}$ & $\mathrm{~b}$ \\
\hline 1984 & 567 & 235 & b & $\mathrm{b}$ \\
\hline 1985 & 793 & 302 & b & $\mathrm{b}$ \\
\hline 1986 & 798 & 359 & $\mathrm{~b}$ & b \\
\hline 1987 & 825 & $\mathrm{~b}$ & b & $\mathrm{b}$ \\
\hline 1988 & 800 & b & $\mathrm{b}$ & $\mathrm{b}$ \\
\hline 1989 & 750 & $\mathrm{~b}$ & $\mathrm{~b}$ & $\mathrm{~b}$ \\
\hline 1990 & 756 & $\mathrm{~b}$ & $\mathrm{~b}$ & $\mathrm{~b}$ \\
\hline 1991 & 875 & b & $\mathrm{b}$ & $\mathrm{b}$ \\
\hline 1992 & 1,080 & 1,542 & $\mathrm{~b}$ & $\mathrm{~b}$ \\
\hline 1993 & 1,156 & 2,081 & b & $\mathrm{b}$ \\
\hline 1994 & 1,280 & 2,205 & 10 & 306 \\
\hline 1995 & 1,355 & 2,506 & 12 & 595 \\
\hline 1996 & 974 & 2,846 & 16 & 692 \\
\hline 1997 & 1,274 & 3,011 & 13 & 733 \\
\hline \multirow[t]{2}{*}{1998} & 1,387 & 3,151 & 4 & 918 \\
\hline & \multicolumn{4}{|c|}{ Average annual percentage change } \\
\hline 1978-98 & $23.6 \%$ & b & b & $\mathrm{b}$ \\
\hline $1988-98$ & $5.7 \%$ & b & b & b \\
\hline
\end{tabular}

Source:

Production - 1992-98 Ethanol and MTBE: U.S. Department of Energy, Energy Information Administration, Petroleum Supply Monthly, Washington, DC, January 1999, Table D 1. 1978-90 Ethanol: Information Resources, Inc., Washington, DC, 1991. 198 1-86 MTBE: EA-Mueller,Inc., Baltimore, MD, 1992.

Imports - U.S. Department of Energy, Energy Information Administration, Petroleum Supply Annual, 1997, Volume 1, Washington, DC, 1999, Table 20, and annual.

"Methyl tertiary-butyl ether.

bData are not available. 
Great care should be taken when comparing modal energy intensity data among modes. Because of the inherent differences between the

transportation modes in the nature of services, routes available, and many additional factors, it is not possible to obtain truly comparable

national energy intensities among modes.

Table 2.11

Passenger Travel and Energy Use in the United States, 1997

\begin{tabular}{|c|c|c|c|c|c|c|c|}
\hline & \multirow[b]{2}{*}{$\begin{array}{c}\text { Number of vehicles } \\
\text { (thousands) }\end{array}$} & \multirow{2}{*}{$\begin{array}{l}\text { Vehicle- } \\
\text { miles } \\
\text { (millions) }\end{array}$} & \multirow{2}{*}{$\begin{array}{l}\text { Passenger- } \\
\text { miles } \\
\text { (millions) }\end{array}$} & \multirow[b]{2}{*}{$\begin{array}{c}\text { Load factor } \\
\text { (persons/vehicle) }\end{array}$} & \multicolumn{2}{|c|}{ Energy intensities } & \multirow[b]{2}{*}{$\begin{array}{l}\text { Energy use } \\
\text { (trillion Btu) }\end{array}$} \\
\hline & & & & & $\begin{array}{c}\text { (Btu per } \\
\text { vehicle-mile) }\end{array}$ & $\begin{array}{c}\text { (Btu per } \\
\text { passenger-mile) }\end{array}$ & \\
\hline Automobiles & $129,748.7$ & $1,501,820$ & $2,402,912$ & 1.6 & 5,822 & 3,639 & $8,743.2$ \\
\hline Personal trucks & $52,993.5$ & 598,589 & 957,743 & 1.6 & 6,781 & 4,238 & $4,059.1$ \\
\hline Motorcycles & $3,826.4$ & 10,076 & 12,091 & 1.2 & 2,501 & 2,084 & 25.2 \\
\hline Buses & 658.5 & 7,983 & 132,857 & 16.6 & 23,024 & 1,383 & 183.8 \\
\hline Transit & 69.5 & 2,307 & 20,357 & 8.8 & 38,101 & 4,318 & 87.9 \\
\hline Intercity & 20.9 & 1,276 & 29,600 & 23.2 & $18,495^{\mathrm{a}}$ & 797 & 23.6 \\
\hline School & 568.1 & 4,400 & 82,900 & 18.8 & 16432 & $872 ”$ & $72.3 ”$ \\
\hline Air & $\mathbf{b}$ & 8,365 & 64,989 & 7.8 & 233,365 & 30,037 & $1,952.1$ \\
\hline Certificated route & $\mathrm{b}$ & 4,997 & 452,489 & 90.6 & 366,420 & 4,047 & $1,831.0$ \\
\hline General aviation & 192.4 & $3,368^{\prime}$ & 12,500 & 3.7 & 35,956 & 9,688 & 121.1 \\
\hline Recreational boats & $12,313.0$ & $b$ & b & b & b & $b$ & 299.7 \\
\hline Rail & 18.3 & 1,137 & 26,299 & 23.1 & 69,745 & 3,015 & 79.3 \\
\hline Intercity $^{d}$ & $1.9^{\prime}$ & $287^{\prime}$ & $5,166^{\mathrm{g}}$ & 18.0 & 44,251 & 2,458 & $12.7 "$ \\
\hline Transit $^{\mathrm{h}}$ & 11.5 & 599 & 13,095 & 21.9 & 71,119 & 3,253 & 42.6 \\
\hline Commuter & 4.9 & 251 & 8,038 & 32.0 & 95,618 & 2,986 & 24.0 \\
\hline
\end{tabular}

See Appendix A for Table 2.11

'Estimated using vehicle travel data.

bata are not available.

"Nautical miles.

'Amtrak only.

'Sum of passenger train cars and locomotiveunits.

passenger train car-miles.

Revenue passenger miles.

hLight and heavy rail. 
Great care should be taken when comparing modal energy intensity data among modes. Because of the inherent differences between the transportation modes

in the nature of services, routes available, and many additionalfactors, it is notpossible to obtain truly comparable national energy intensities among modes.

Table 2.12

Energy Intensities of Passenger Modes, 1970-97

\begin{tabular}{|c|c|c|c|c|c|c|c|c|c|c|c|}
\hline \multirow[b]{3}{*}{ Year } & & & \multirow{3}{*}{$\begin{array}{l}\text { Light } \\
\text { truck }^{\mathbf{2}} \\
\text { (Btu per } \\
\text { vehicle- } \\
\text { mile) }\end{array}$} & \multicolumn{4}{|c|}{ Buses } & \multicolumn{2}{|c|}{ Air } & \multicolumn{2}{|c|}{ Rail } \\
\hline & \multicolumn{2}{|c|}{ Automobiles } & & \multicolumn{2}{|c|}{ Transit $^{\mathrm{b}}$} & \multirow{2}{*}{$\begin{array}{l}\text { Intercity } \\
\text { (Btu per } \\
\text { passenger- } \\
\text { mile) }\end{array}$} & \multirow{2}{*}{$\begin{array}{l}\text { School } \\
\text { (Btu per } \\
\text { vehicle- } \\
\text { mile) }\end{array}$} & \multirow{2}{*}{$\begin{array}{c}\text { Certificated } \\
\text { air carriers } \\
\text { (Btu per } \\
\text { passenger-mile) }\end{array}$} & \multirow{2}{*}{$\begin{array}{c}\text { General } \\
\text { aviation } \\
\text { (Btu per } \\
\text { passenger-mile) }\end{array}$} & \multirow{2}{*}{$\begin{array}{c}\text { Intercity } \\
\text { Amtrak } \\
\text { (Btu per } \\
\text { passenger-mile) }\end{array}$} & \multirow{2}{*}{$\begin{array}{c}\text { Rail } \\
\text { transit } \\
\text { (Btu per } \\
\text { passenger-mile) }\end{array}$} \\
\hline & $\begin{array}{l}\text { (Btu per } \\
\text { vehicle- } \\
\text { mile) }\end{array}$ & $\begin{array}{l}\text { (Btu per } \\
\text { passenger- } \\
\text { mile) }\end{array}$ & & $\begin{array}{c}\text { (Btu per } \\
\text { vehicle- } \\
\text { mile) }\end{array}$ & $\begin{array}{c}\text { (Btu per } \\
\text { passenger- } \\
\text { mile) }\end{array}$ & & & & & & \\
\hline 1970 & 9,301 & 4,896 & 12,492 & 31,796 & 2,472 & 1,051 & 17,857 & 10,351 & 10,374 & $\bar{c}$ & 2,453 \\
\hline 1975 & 9,015 & 4,745 & 11,890 & 33,748 & 2,814 & 976 & 17,040 & 7,883 & 10,658 & 3,677 & 2,962 \\
\hline 1976 & 9,130 & 4,805 & 11,535 & 34,598 & 2,896 & 996 & 17,051 & 7,481 & 10,769 & 3,397 & 2,971 \\
\hline 1977 & 8,961 & 4,716 & 11,171 & 35,120 & 2,889 & 961 & 16,983 & 7,174 & 11,695 & 3,568 & 2,691 \\
\hline 1978 & 8,844 & 4,655 & 10,815 & 36,603 & 2,883 & 953 & 17,018 & 6,333 & 11,305 & 3,683 & 2,210 \\
\hline 1979 & 8,647 & 4,551 & 10,473 & 36,597 & 2,795 & 963 & 16,980 & 5,858 & 10,787 & 3,472 & 2,794 \\
\hline 1980 & 7,915 & 4,166 & 10,230 & 36,553 & 2,813 & 1,069 & 16,379 & 5,837 & 11,497 & 3,176 & 3,008 \\
\hline 1981 & 7,672 & 4,038 & 10,001 & 37,745 & 3,027 & 1,155 & 16,385 & 5,743 & 11,123 & 2,957 & 2,946 \\
\hline 1982 & 7,485 & 3,939 & 9,275 & 38,766 & 3,237 & 1,149 & 16,296 & 5,147 & 13,015 & 3,156 & 3,069 \\
\hline 1983 & 7,376 & 4,098 & 9,141 & 37,962 & 3,177 & 1,174 & 16,236 & 5,107 & 11,331 & 2,957 & 3,212 \\
\hline 1984 & 7,218 & 4,010 & 8,945 & 37,507 & 3,204 & 1,247 & 14,912 & 5,031 & 11,454 & 3,027 & 3,732 \\
\hline 1985 & 7,182 & 3,990 & 8,754 & 38,862 & 2,421 & 1,324 & 16,531 & 5,679 & 11,707 & 2,800 & 3,461 \\
\hline 1986 & 7,213 & 4,007 & 8,578 & 39,869 & 3,512 & 869 & 15,622 & 5,447 & 11,935 & 2,574 & 3,531 \\
\hline 1987 & 6,975 & 3,875 & 8,376 & 38,557 & 3,542 & 939 & 15,615 & 4,753 & 11,496 & 2,537 & 3,534 \\
\hline 1988 & 6,700 & 3,722 & 8,155 & 39,121 & 3,415 & 965 & 15,585 & 4,814 & 11,794 & 2,462 & 3,585 \\
\hline 1989 & 6,602 & 3,668 & 7,779 & 36,583 & 3,711 & 963 & 15,575 & 4,796 & 10,229 & 2,731 & 3,397 \\
\hline 1990 & 6,183 & 3,864 & 7,774 & 36,647 & 3,735 & 944 & 16,368 & 4,811 & 10,146 & 2,609 & 3,453 \\
\hline 1991 & 5,925 & 3,703 & 7,381 & 36,939 & 3,811 & 978 & 16,419 & 4,560 & 9,869 & 2,503 & 3,710 \\
\hline 1992 & 5,970 & 3,731 & 7,263 & 40,472 & 4,303 & 978 & 16,386 & 4,482 & 9,785 & 2,610 & 3,575 \\
\hline 1993 & 6,103 & 3,814 & 7,208 & 39,005 & 4,257 & 972 & 19,093 & 4,304 & 9,653 & 2,646 & 3,687 \\
\hline 1994 & 6,041 & 3,775 & 7,232 & 40,102 & 4,604 & 876 & 20,591 & 4,455 & 9,163 & 2,351 & 3,828 \\
\hline 1995 & 5,923 & 3,702 & 7,237 & 40,175 & 4,650 & 816 & 13,680 & 4,236 & 10,152 & 2,314 & 3,818 \\
\hline 1996 & 5,874 & 3,671 & 7,247 & 39,307 & 4,512 & 816 & 13,680 & 4,081 & 10,481 & 2,389 & 3,444 \\
\hline 1997 & 5,822 & 3,639 & 6,981 & 38,101 & 4,318 & 872 & 16,432 & 4,047 & 9,688 & 2,458 & 3,253 \\
\hline \multicolumn{12}{|c|}{ Average annual percentage change } \\
\hline 1970-97 & $-1.7 \%$ & $-1.1 \%$ & $-2.1 \%$ & $0.7 \%$ & $2.1 \%$ & $-0.7 \%$ & $-0.3 \%$ & $-3.4 \%$ & $-0.3 \%$ & $-1.5 \%^{\mathrm{d}}$ & $1.1 \%$ \\
\hline $1987-97$ & $-1.8 \%$ & $-0.6 \%$ & $-1.8 \%$ & $-0.1 \%$ & $2.0 \%$ & $-0.7 \%$ & $-0.5 \%$ & $-1.6 \%$ & $-1.7 \%$ & $-0.3 \%$ & $-0.8 \%$ \\
\hline
\end{tabular}

Source:

See Appendix A for Table 2.12 .

"All two-axle, four-tire trucks.

'Series not continuous between 1983 and 1984 because of a change in data source by the American Public Transit Association (APTA).

'Data are not available.

'Average annual percentage change is from earliest year possible. 
Figure 2.2. Energy Intensity for Transit in the U.S., 1997

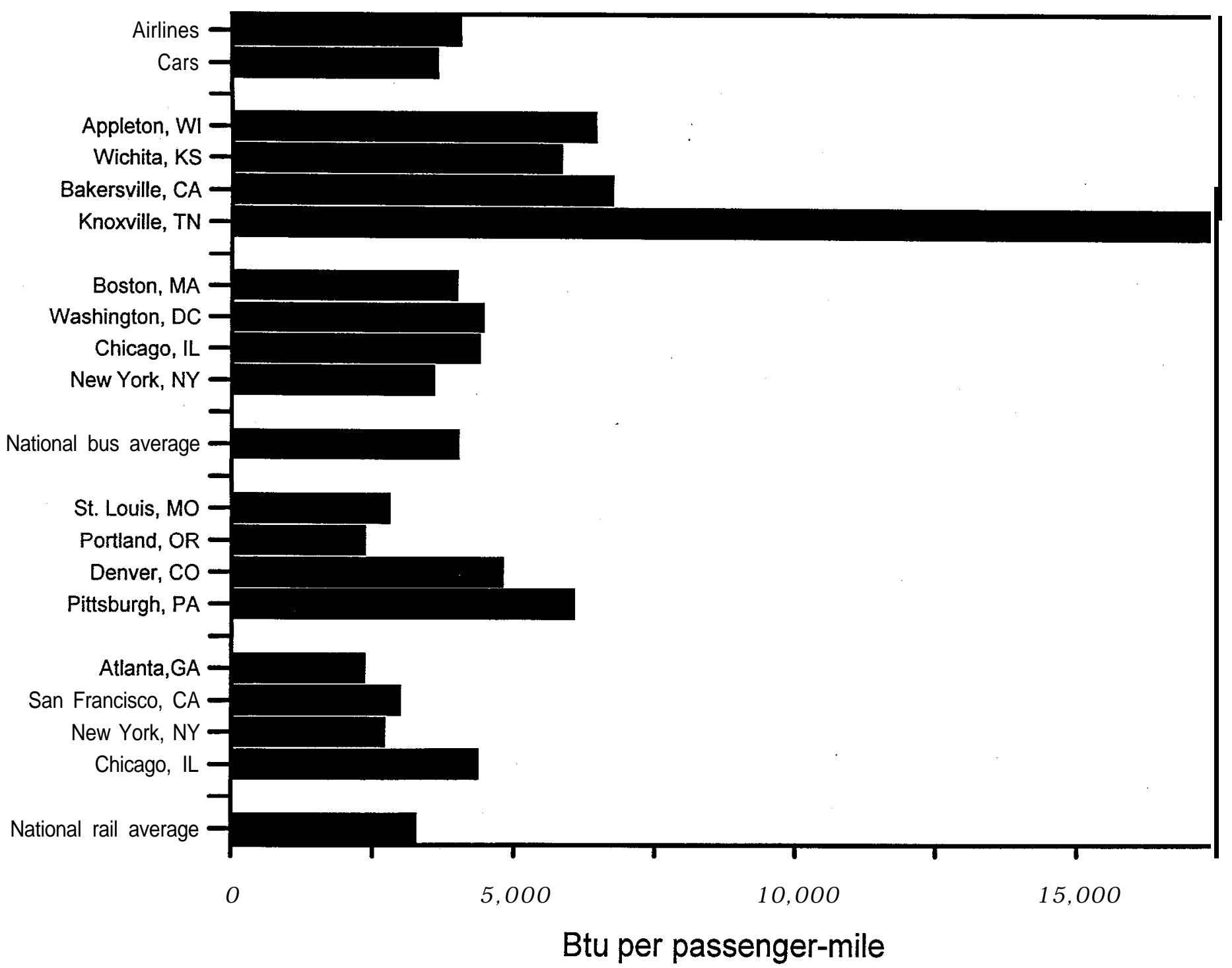

Source:

U.S. Department of Transportation, Federal Transit Administration, 1997 National Transit Database, Washington, DC. (Additional resources: http://www.fta.dot.gov/ntl) 
Great care should be taken when comparing modal energy intensity data among modes. Because of the inherent differences between the transportation modes

in the nature of services, routes available, and many additionalfactors, it is notpossible to obtain truly comparable national energy intensities among modes.

Table 2.13

Intercity Freight Movement and Energy Use in the United States, 1997

\begin{tabular}{|c|c|c|c|c|c|c|c|}
\hline & $\begin{array}{l}\text { Number of } \\
\text { vehicles } \\
\text { (thousands) }\end{array}$ & $\begin{array}{l}\text { Vehicle-miles } \\
\text { (millions) }\end{array}$ & $\begin{array}{l}\text { Ton-miles } \\
\text { (millions) }\end{array}$ & $\begin{array}{l}\text { Tons shipped } \\
\text { (millions) }\end{array}$ & $\begin{array}{l}\text { Average length } \\
\text { of haul (miles) }\end{array}$ & $\begin{array}{l}\text { Energy intensity } \\
\text { (Btu/ton-mile) }\end{array}$ & $\begin{array}{l}\text { Energy use } \\
\text { (trillion Btu) }\end{array}$ \\
\hline Truck" & 1,911 & 118,952 & $1,051,000$ & 3,745 & $629^{b}$ & 2,635 & $2,763.5$ \\
\hline Waterborne commerce' & 41 & d & 707,410 & 1,106 & 640 & 415 & 293.2 \\
\hline Coastwise & d & d & 349,843 & 263 & 1,330 & d & d \\
\hline Lakewise & d & d & 62,166 & 122 & 507 & d & d \\
\hline Internal and local & d & d & 295,401 & 720 & 410 & d & d \\
\hline Pipeline & d & d & d & 1,644 & d & d & 973.4 \\
\hline Natural gas & d & d & d & 490 & d & d & 815.0 \\
\hline Crude oil and products & d & d & 628,000 & 1,154 & d & 252 & 158.4 \\
\hline Class I railroads' & 568 & 31,660 & $1,348,926$ & 1,585 & 851 & 370 & 499.7 \\
\hline
\end{tabular}

Source:

See Appendix A for Table 2.13.

"The definition of intercity truck was "tightened" to exclude smaller trucks. See Appendix A for details.

${ }^{\text {b } 629 ~ m i l e s ~ i s ~ f o r ~ g e n e r a l ~ f r e i g h t ~(l e s s ~ t h a n ~ t r u c k l o a d) . ~ B a s e d ~ o n ~ d a t a ~ f r o m ~ t h e ~ E n o ~ T r a n s p o r t a t i o n ~ F o u n d a t i o n, ~ t h e ~ a v e r a g e ~ l e n g t h ~ o f ~}$ haul for specialized freight (truckload) was 289 miles.

"Includes commerce by foreign and domestic carriers in the U.S.

Data are not available.

'Railroad measures are: number vehicles $=$ number freight cars, vehicle-miles $=$ car-miles, ton-miles $=$ revenue ton-miles. 
Great care should be taken when comparing modal energy intensity data among modes. Because of the inherent differences between the transportation modes in the nature of services, routes available, and many additional factors, it is not possible to obtain truly comparable national energy intensities among modes.

Table 2.14

Energy Intensities of Freight Modes, 1970-97

\begin{tabular}{|c|c|c|c|c|}
\hline \multirow[b]{2}{*}{ Year } & \multirow{2}{*}{$\begin{array}{l}\text { Heavy single-unit and } \\
\text { combination trucks } \\
\text { (Btu per vehicle-mile) }\end{array}$} & \multicolumn{2}{|c|}{ Class I freight railroad } & \multirow{2}{*}{$\begin{array}{c}\text { Domestic waterborne } \\
\text { commerce } \\
\text { (Btu per ton-mile) }\end{array}$} \\
\hline & & (Btu per freight car-mile) & (Btu per ton-mile) & \\
\hline 1970 & 24,154 & 17,668 & 691 & 545 \\
\hline 1971 & 23,694 & 18,814 & 717 & 506 \\
\hline 1972 & 23,871 & 18,292 & 714 & 522 \\
\hline 1973 & 23,977 & 18,468 & 677 & 576 \\
\hline 1974 & 23,983 & 18,852 & 681 & 483 \\
\hline 1975 & 23,836 & 18,741 & 687 & 549 \\
\hline 1976 & 23,773 & 18,938 & 680 & 468 \\
\hline 1977 & 23,873 & 19,225 & 669 & 458 \\
\hline 1978 & 24,013 & 18,930 & 641 & 383 \\
\hline 1979 & 24,260 & 19,187 & 618 & 457 \\
\hline 1980 & 24,431 & 18,742 & 597 & 358 \\
\hline 1981 & 24,892 & 18,628 & 572 & 360 \\
\hline 1982 & 24,296 & 18,403 & 553 & 310 \\
\hline 1983 & 23,740 & 17,863 & 525 & 319 \\
\hline 1984 & 23,363 & 17,797 & 510 & 346 \\
\hline 1985 & 23,015 & 17,500 & 497 & 446 \\
\hline 1986 & 22,917 & 17,265 & 486 & 463 \\
\hline 1987 & 22,391 & 16,791 & 456 & 402 \\
\hline 1988 & 22,586 & 16,758 & 443 & 361 \\
\hline 1989 & 22,391 & 16,896 & 437 & 403 \\
\hline 1990 & 22,765 & 16,618 & 420 & 388 \\
\hline 1991 & 22,710 & 15,834 & 391 & 386 \\
\hline 1992 & 22,559 & 16,044 & 393 & 398 \\
\hline 1993 & 22,308 & 16,055 & 389 & 389 \\
\hline 1994 & 22,159 & 16,338 & 388 & 369 \\
\hline 1995 & 22,172 & 15,993 & 372 & 374 \\
\hline 1996 & 21,964 & 15,747 & 368 & 412 \\
\hline 1997 & 21,266 & 15,783 & 370 & 415 \\
\hline \multicolumn{5}{|c|}{ Average annualpercentage change } \\
\hline $1970-97$ & $-0.5 \%$ & $-0.4 \%$ & $-2.3 \%$ & $-1.0 \%$ \\
\hline $1987-97$ & $-0.5 \%$ & $-0.6 \%$ & $-2.1 \%$ & $0.3 \%$ \\
\hline
\end{tabular}

Source:

See Appendix A for Table 2.14 . 


\section{Chapter 3 Greenhouse Gas Emissions}

Summary Statistics from Tables in this Chapter

\begin{tabular}{|c|c|c|c|}
\hline \multicolumn{4}{|l|}{ Source } \\
\hline \multirow[t]{6}{*}{ Table 3.1} & Greenhous gasemissions (million metric tonnes) & 1990 & 1996 \\
\hline & France & 557 & 562 \\
\hline & Germany & 1,209 & 1,091 \\
\hline & United Kingdom & 757 & 717 \\
\hline & Japan & 1,222 & $1,352 ”$ \\
\hline & United States & 5,999 & 6,557 \\
\hline \multirow[t]{4}{*}{ Table 3.6} & \multicolumn{3}{|c|}{$\begin{array}{l}\text { Transportation share of U.S. carbon dioxide emissions from fossil fuel } \\
\text { consumption }\end{array}$} \\
\hline & 1984 & & $30.5 \%$ \\
\hline & 1990 & & $32.1 \%$ \\
\hline & 1997 & & $31.2 \%$ \\
\hline \multirow[t]{10}{*}{ Table 3.7} & \multicolumn{3}{|c|}{ Carbon dioxide emissions from U.S. transportation energy use, 1997} \\
\hline & Motor gasoline & & $61.0 \%$ \\
\hline & Liquified petroleum gas & & $0.1 \%$ \\
\hline & Jet fuel & & $13.4 \%$ \\
\hline & Distillate fuel & & $19.4 \%$ \\
\hline & Residual fuel & & $3.4 \%$ \\
\hline & Lubricants & & $0.4 \%$ \\
\hline & Aviation gas & & $0.1 \%$ \\
\hline & Natural gas & & $2.2 \%$ \\
\hline & Electricity & & $0.1 \%$ \\
\hline
\end{tabular}

${ }^{a} 1995$ data are the latest available. 
Table 3.1

International Man-Made Emissions of All Greenhouse Gases, 1990-96" (CO, equivalent)

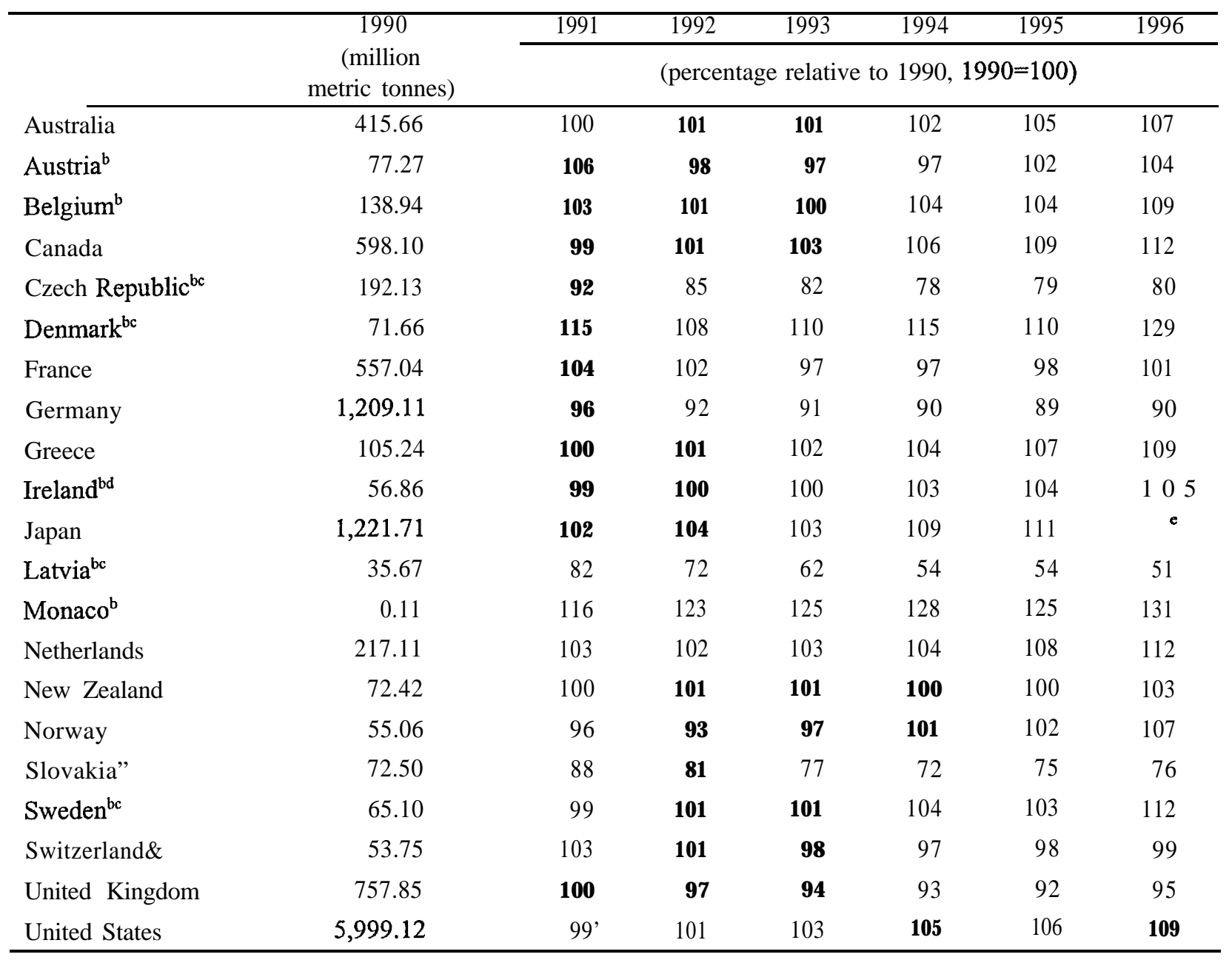

Source:

United Nations Framework Convention on Climate Change, FCCC/CP/1998/INF.9, October 1998.

(Additional resources: http://www.unfccc.de)

${ }^{a}$ Excluding land-use change and forestry.

${ }^{\mathbf{b}}$ As estimates for HFCs, PFCs and $\mathrm{SF}_{6}$ were not provided, or not provided for all years, estimates given here are for $\mathrm{CO}, \mathrm{CH}_{4}$ and $\mathrm{N}_{2} \mathrm{O}$ only.

${ }^{\mathbf{c}}$ As estimates for 1990-1995 were not provided in the inventory submission, data from the second national communication are used in this table.

dAs estimates for 1990-1994 were not provided in the inventory submission, data from the second national communication are used in this table.

'Data are not available. 
Table 3.2

International Man-Made Emissions of Carbon Dioxide, 1990-96”

\begin{tabular}{|c|c|c|c|c|c|c|c|}
\hline & \multirow{2}{*}{$\begin{array}{c}1990 \\
\text { (million } \\
\text { metric tonnes) }\end{array}$} & 1991 & 1992 & 1993 & 1994 & 1995 & 1996 \\
\hline & & \multicolumn{6}{|c|}{ (percentage relative to $1990,1990=100$ ) } \\
\hline Australia & 275.34 & 101 & 102 & 103 & 104 & 108 & 112 \\
\hline Austria & 62.10 & 108 & 98 & 96 & 97 & 102 & 105 \\
\hline Belgium & 116.09 & 103 & 102 & 100 & 104 & 105 & 111 \\
\hline Canada & 460.90 & 98 & 101 & 101 & 103 & 107 & 110 \\
\hline Czech Republic ${ }^{\mathrm{b}}$ & 165.49 & 93 & 85 & 81 & 77 & 78 & 80 \\
\hline Denmark $^{\mathbf{b}}$ & 52.28 & 120 & 110 & 114 & 121 & 114 & 140 \\
\hline France & 390.71 & 107 & 105 & 99 & 99 & 101 & 104 \\
\hline Germany & $1,014.16$ & 96 & 91 & 91 & 89 & 88 & 90 \\
\hline Greece & 85.35 & 100 & 102 & 102 & 104 & 106 & 108 \\
\hline Ireland" & 30.72 & 103 & 105 & 104 & 108 & 111 & 113 \\
\hline Japan & $1,124.53$ & 102 & 103 & 102 & 108 & 109 & 110 \\
\hline Latvia $^{b}$ & 24.77 & 78 & 66 & 58 & 48 & 49 & 45 \\
\hline Monaco & 0.11 & 116 & 123 & 125 & 128 & 125 & 131 \\
\hline Netherlands & 161.36 & 103 & 102 & 104 & 104 & 110 & 115 \\
\hline New Zealand & 25.24 & 102 & 110 & 107 & 107 & 107 & 115 \\
\hline Norway & 35.46 & 95 & 97 & 101 & 107 & 108 & 116 \\
\hline Slovakia $^{b}$ & 60.03 & 88 & 81 & 77 & 72 & 76 & 77 \\
\hline Sweden $^{b}$ & 55.45 & 100 & 101 & 101 & 106 & 105 & 114 \\
\hline Switzerland $^{\mathrm{b}}$ & 45.07 & 104 & 101 & 98 & 96 & 98 & 100 \\
\hline United Kingdom & 583.17 & 101 & 98 & 95 & 95 & 93 & 97 \\
\hline United States & $4,943.30$ & 99 & 100 & 103 & 104 & 105 & 109 \\
\hline
\end{tabular}

Source:

United Nations Framework Convention on Climate Change, FCCC/CP/1998/INF.9, October 1998.

(Additional resources: http://www.unfccc.de)

"Excluding land-use change and forestry.

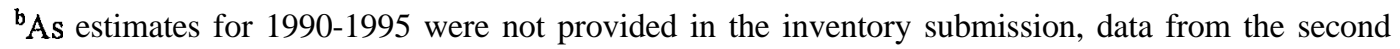
national communication are used in this table.

'As estimates for 1990-1994 were not provided in the inventory submission, data from the second national communication are used in this table. 
Table 3.3

International Man-Made Emissions of Carbon Dioxide by Source Category, 1990 and 1996"

\begin{tabular}{|c|c|c|c|c|c|c|c|c|c|c|c|c|c|c|}
\hline & \multicolumn{4}{|c|}{ Energy (excl transport) } & \multicolumn{4}{|c|}{ Transport } & \multicolumn{4}{|c|}{ Industrial processes } & \multicolumn{2}{|c|}{ Total } \\
\hline & \multicolumn{2}{|c|}{1990} & \multicolumn{2}{|c|}{1996} & \multicolumn{2}{|c|}{1990} & \multicolumn{2}{|c|}{1996} & \multicolumn{2}{|c|}{1990} & \multicolumn{2}{|c|}{1996} & \multirow{2}{*}{$\frac{\text { '1990 }}{\text { (MMT) }}$} & 1996 \\
\hline & (MMT) & $\%$ & (MMT) & $\%$ & (MMT) & $\%$ & (MMT) & $\%$ & (MMT) & $\%$ & (MMT) & $\%$ & & (MMT) \\
\hline Australia & 209 & 75.9 & 234 & 75.9 & 60 & 21.6 & 67 & 21.8 & 7 & 2.4 & 7 & 2.3 & 275 & 308 \\
\hline Austria & 35 & 56.2 & 38 & 58.2 & 14 & 22.4 & 16 & 24.2 & 13 & 20.5 & 11 & 16.8 & 62 & 65 \\
\hline Belgium & 86 & 74.0 & 94 & 72.9 & 20 & 17.2 & 22 & 17.4 & 9 & 7.9 & 11 & 8.8 & 116 & 129 \\
\hline Canada & 275 & 59.6 & 300 & 59.1 & 147 & 31.9 & 167 & 32.8 & 32 & 6.9 & 39 & 7.7 & 461 & 509 \\
\hline Czech Republic ${ }^{\mathrm{b}}$ & 152 & 91.9 & 120 & 90.3 & 8 & 4.8 & 10 & 7.5 & 5 & 3.3 & 2 & 1.9 & 165 & 133 \\
\hline Denmark $^{b}$ & 41 & 77.8 & 60 & 82.0 & 10 & 20.0 & 12 & 16.0 & 1 & 1.9 & 1 & 1.9 & 52 & 73 \\
\hline France & 241 & 61.8 & 248 & 61.1 & 123 & 31.5 & 135 & 33.3 & 21 & 5.4 & 17 & 4.3 & 391 & 407 \\
\hline Germany & 824 & 81.3 & 712 & 78.2 & 162 & 16.0 & 173 & 19.0 & 28 & 2.7 & 25 & 2.7 & 1,014 & 910 \\
\hline Greece & 62 & 72.7 & 66 & 72.1 & 15 & 17.8 & 17 & 18.8 & 8 & 9.1 & 8 & 8.8 & 85 & 92 \\
\hline Ireland $^{b}$ & 24 & 78.6 & 26 & 76.1 & 5 & 15.9 & 7 & 18.8 & 2 & 5.3 & 2 & 5.0 & 31 & 35 \\
\hline Japan & 846 & 75.2 & 904 & 73.2 & 207 & 18.4 & 249 & 20.1 & 59 & 5.2 & 61 & 4.9 & 1,125 & 1,235 \\
\hline Latvia $^{b}$ & 18 & 74.2 & 9 & 83.7 & 6 & 23.5 & 2 & 14.6 & 563 & 2.3 & 185 & 1.7 & 25 & 11 \\
\hline Monaco & 67 & 61.6 & 91 & 64.4 & 39 & 36.5 & 47 & 33.1 & - & & - & - & 108 & 141 \\
\hline Netherlands & 129 & 80.2 & 149 & 80.3 & 29 & 17.7 & 33 & 18.1 & 2 & 1.2 & 2 & 0.9 & 161 & 185 \\
\hline New Zealand & 14 & 56.3 & 15 & 52.7 & 9 & 34.2 & 11 & 37.8 & 2 & 9.5 & 3 & 9.5 & 25 & 29 \\
\hline Norway & 15 & 41.5 & 18 & 44.4 & 14 & 39.2 & 16 & 37.8 & 7 & 18.9 & 7 & 17.4 & 35 & 41 \\
\hline Slovakia $^{\mathrm{b}}$ & 51 & 85.6 & 39 & 84.5 & 5 & 8.6 & 4 & 9.0 & 3 & 5.7 & 3 & 6.5 & 60 & 46 \\
\hline Sweden $^{b}$ & 33 & 59.0 & 40 & 62.9 & 19 & 33.6 & 20 & 30.9 & 4 & 6.8 & 4 & 5.9 & 55 & 63 \\
\hline Switzerland ${ }^{\mathrm{b}}$ & 26 & 57.1 & 26 & 58.8 & 15 & 32.5 & 15 & 33.2 & 3 & 7.5 & 2 & 4.9 & 45 & 45 \\
\hline United Kingdom & 453 & 77.6 & 429 & 76.2 & 116 & 19.9 & 122 & 21.6 & 14 & 2.4 & 12 & 2.1 & 583 & 563 \\
\hline United States & 3,390 & 68.6 & 3,699 & 68.6 & 1,499 & 30.3 & 1,631 & 30.2 & 55 & 1.1 & 63 & 1.2 & 4,943 & 5,394 \\
\hline
\end{tabular}

Source:

United Nations Framework Convention on Climate Change, FCC/CP/1998/INF.9, October 1998.

(Additional resources: http://www.unfccc.de)

${ }^{a}$ Excluding land-use change and forestry.

${ }^{\mathbf{b}}$ As estimates for 1990 were not provided in the inventory submission, data from the second national communication are used in this table. 
Table 3.4

International Man-Made Emissions of Nitrous Oxide, 1990-96

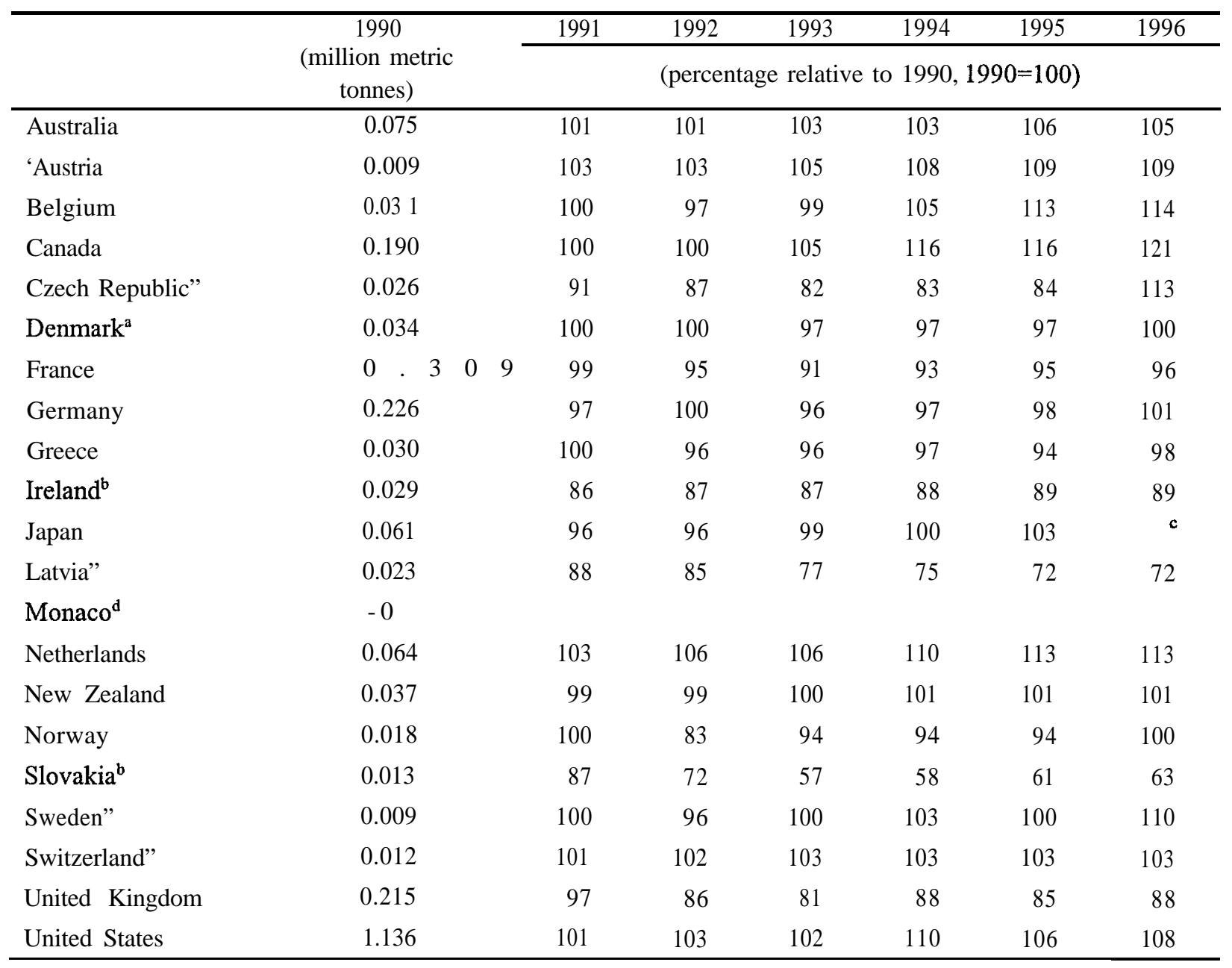

Source:

United Nations Framework Convention on Climate Change, FCCC/CP/1998/INF.9, October 1998.

(Additional resources: http://www.unfccc.de)

aAs estimates for 1990-1995 were not provided in the inventory submission, data from the second national communication are used in this table.

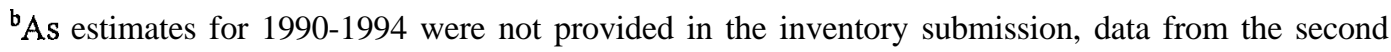
national communication are used in this table.

"Data are not available.

dThe trend in emissions is not shown here as estimates reported were approximately zero from 1990 to 1996. 
Table 3.5

Estimated U.S. Emissions of Greenhouse Gases, 1990-97

\begin{tabular}{|c|c|c|c|c|c|c|c|c|c|}
\hline Greenhouse gas & Unit of measure ${ }^{a}$ & 1990 & 1991 & 1992 & 1993 & 1994 & 1995 & 1996 & 1997 \\
\hline \multirow[t]{2}{*}{ Carbon dioxide } & million metric tons of gas & $4,971.7$ & $4,916.3$ & $4,988.8$ & $5,109.8$ & $5,183.9$ & $5,236.4$ & $5,422.3$ & $5,503.0$ \\
\hline & million metric tons of carbon & $1,356.0$ & $1,341.0$ & $1,361.0$ & $1,394.0$ & $1,414.0$ & $1,428.0$ & $1,479.0$ & $1,501.0$ \\
\hline \multirow[t]{2}{*}{ Methane } & million metric tons of gas & 30.2 & 30.4 & 30.4 & 29.7 & 29.9 & 30.0 & 29.1 & 29.1 \\
\hline & million metric tons of carbon $(\mathrm{gwp})^{\mathrm{b}}$ & 173.0 & 174.0 & 174.0 & 170.0 & 171.0 & 172.0 & 167.0 & 167.0 \\
\hline \multirow[t]{2}{*}{ Nitrous oxide } & million metric tons of gas & 1.0 & 1.0 & 1.0 & 1.0 & 1.1 & 1.0 & 1.0 & 1.0 \\
\hline & million metric tons of carbon $(\mathrm{gwp})^{\mathrm{b}}$ & 82.0 & 83.0 & 85.0 & 86.0 & 91.0 & 88.0 & 86.0 & 85.0 \\
\hline Carbon monoxide & million metric tons of gas & 87.6 & 89.3 & 86.3 & 86.4 & 90.4 & 81.4 & 80.6 & c \\
\hline Nitrogen oxide & million metric tons of gas & 21.6 & 21.5 & 21.9 & 22.2 & 22.5 & 21.7 & 21.3 & c \\
\hline Nonmethane VOCs ${ }^{\mathrm{d}}$ & million metric tons of gas & 19.0 & 19.1 & 18.8 & 19.0 & 19.5 & 18.7 & 17.3 & c \\
\hline CFC-11,12,113 ${ }^{\mathrm{d}}$ & million metric tons of gas & 0.2 & 0.2 & 0.2 & 0.1 & 0.1 & 0.1 & 0.1 & c \\
\hline $\mathrm{HCFC}-22^{\mathrm{d}}$ & million metric tons of gas & 0.1 & 0.1 & 0.1 & 0.1 & 0.1 & 0.1 & 0.1 & 0.1 \\
\hline HCFC-23 and PFCs ${ }^{d}$ & million metric tons of carbon $(\mathrm{gwp})^{\mathrm{b}}$ & 22.0 & 22.0 & 23.0 & 23.0 & 26.0 & 31.0 & 35.0 & 38.0 \\
\hline
\end{tabular}

Source:

U.S. Department of Energy, Energy Information Administration, Emissions of Greenhouse Gases in the United States, 1997, Washington, DC, October 1998, p. ix, x. (Additional resources: http://www.eia.doe.gov)

Criteria pollutants (CO, NO,, VOC) - U.S. Environmental Protection Agency, National Air Pollutant Emission Trends, 1900-1996, 1997, pp. A-6, A-1 1, A-18. (Additional resources: http://www.epa.gov/oar/oaqps)

${ }^{\mathrm{a}}$ Gases that contain carbon can be measured either in terms of the full molecular weight of the gas or just in terms of their carbon content. See Appendix B, Table B.5 for details.

${ }^{\mathrm{b}}$ Based on global warming potential.

'Data are not available.

${ }^{\mathrm{d}} \mathrm{VOC}=$ volatile organic compounds. $\mathrm{CFC}=$ chlorofluorocarbons. $\mathrm{HCFC}=$ hydrochlorofluorocarbons. $\mathrm{HFC}=$ hydrofluorocarbons. $\mathrm{PFC}=$ perfluorocarbons. 
Gases which contain carbon can be measured in terms of the $\mathbf{f} \mathbf{u l} \mathbf{I}$ molecular weight of the gas or just in terms of their carbon content. This

table presents carbon content. The ratio of the weight of carbon to carbon dioxide is 0.2727 .

Table 3.6

U.S. Carbon Dioxide Emissions from Fossil Energy Consumption

by End-Use Sector, 1984-97"

(million metric tons of carbon)

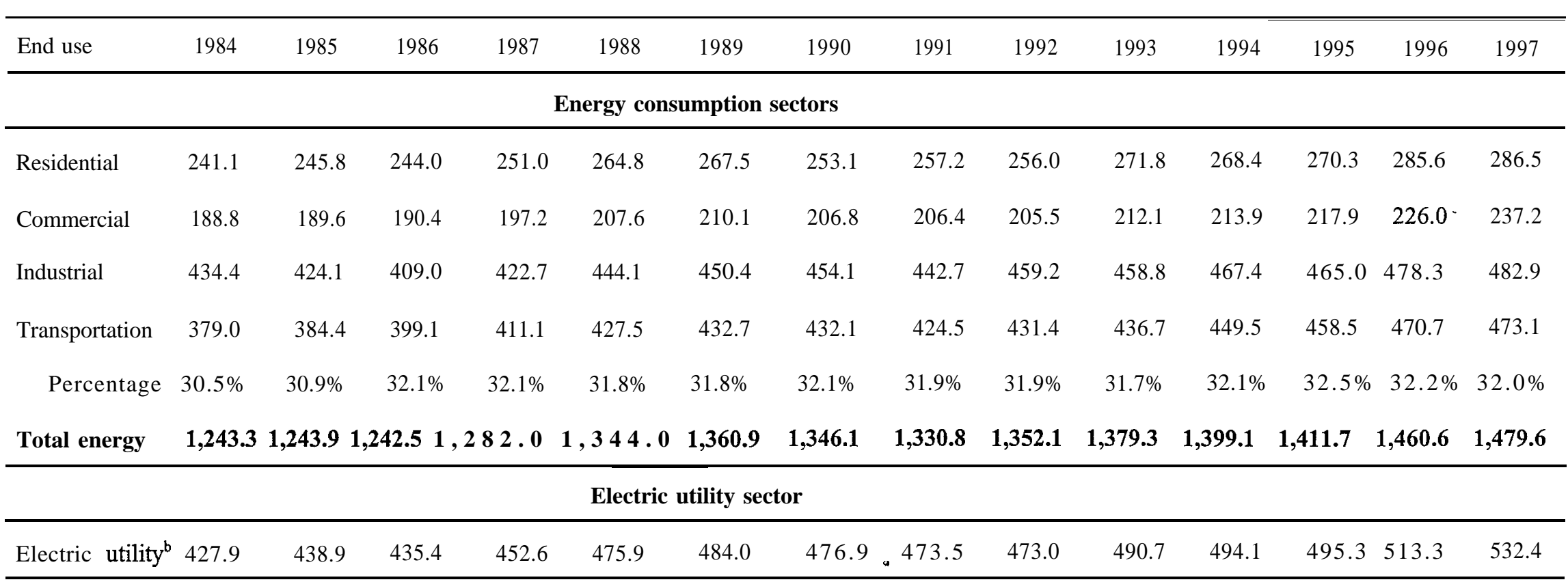

\section{Source:}

U.S. Department of Energy, Energy Information Administration, Emissions of Greenhouse Gases in the United States, 1997, Washington, DC, October 1998, p. 21, and annual. (Additional resources: http://www.eia.doe.gov)

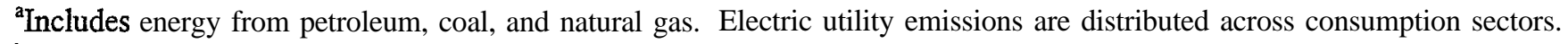

${ }^{b}$ Does not include estimates of carbon dioxide emissions from the use of flue gas desulfurization. 
Table 3.7

U.S. Carbon Dioxide Emissions from Energy Use in the Transportation Sector, 1980-97 (million metric tons of carbon)

\begin{tabular}{|c|c|c|c|c|c|c|c|c|c|c|}
\hline \multirow[b]{2}{*}{ Fuel } & \multicolumn{2}{|c|}{1980} & \multicolumn{2}{|c|}{1985} & \multicolumn{2}{|c|}{1990} & \multicolumn{2}{|c|}{1995} & \multicolumn{2}{|c|}{1997} \\
\hline & Emissions & Percentage & Emissions & Percentage & Emissions & Percentage & Emissions & Percentage & Emissions & Percentage \\
\hline & \multicolumn{10}{|c|}{ Petroleum } \\
\hline $\begin{array}{l}\text { Motor } \\
\text { gasoline }\end{array}$ & 238.1 & $62.9 \%$ & 245.1 & $63.8 \%$ & 260.9 & $60.4 \%$ & 279.9 & $61.1 \%$ & 288.3 & $61.0 \%$ \\
\hline LPG” & 0.3 & $0.1 \%$ & 0.5 & $0.1 \%$ & 0.4 & $0.1 \%$ & 0.3 & $0.1 \%$ & 0.3 & $0.1 \%$ \\
\hline Jet fuel & 42.0 & $11.1 \%$ & 48.0 & $12.5 \%$ & 60.1 & $13.9 \%$ & 60.0 & $13.1 \%$ & 63.3 & $13.4 \%$ \\
\hline Distillate fuel & 55.3 & $14.6 \%$ & 63.3 & $16.5 \%$ & 75.7 & $17.5 \%$ & 85.1 & $18.6 \%$ & 91.6 & $19.4 \%$ \\
\hline Residual fuel & 30.0 & $7.9 \%$ & 16.7 & $4.3 \%$ & 21.9 & $5.1 \%$ & 19.7 & $4.3 \%$ & 15.9 & $3.4 \%$ \\
\hline Lubricants & 1.8 & $0.5 \%$ & 1.6 & $0.4 \%$ & 1.8 & $0.4 \%$ & 1.7 & $0.4 \%$ & 1.7 & $0.4 \%$ \\
\hline Aviation gas & 1.2 & $0.3 \%$ & 0.9 & $0.2 \%$ & 0.8 & $0.2 \%$ & 0.7 & $0.2 \%$ & 0.7 & $0.1 \%$ \\
\hline \multirow[t]{2}{*}{ Total } & 368.7 & $97.4 \%$ & 376.1 & $97.8 \%$ & 421.5 & $97.5 \%$ & 447.4 & $97.6 \%$ & 461.9 & $97.8 \%$ \\
\hline & \multicolumn{10}{|c|}{ Other energy } \\
\hline Natural gas & 9.4 & $2.5 \%$ & 7.5 & $2.0 \%$ & 9.8 & $2.3 \%$ & 10.4 & $2.3 \%$ & 10.5 & $2.2 \%$ \\
\hline Electricity & 0.3 & $0.1 \%$ & 0.7 & $0.2 \%$ & 0.7 & $0.2 \%$ & 0.6 & $0.1 \%$ & 0.7 & $0.1 \%$ \\
\hline Total & 378.4 & $100.0 \%$ & 384.4 & $100.0 \%$ & 432.1 & $100.0 \%$ & 458.5 & $100.0 \%$ & 473.1 & $100.0 \%$ \\
\hline
\end{tabular}

Source:

U.S. Department of Energy, Energy Information Administration, Emissions of Greenhouse Gases in the United States, 1997, Washington, DC, October 1998, p. 23, and annual. (Additional resources: http://www.eia.doe.gov) 
Global Warming Potentials (GWP) were developed to allow comparison of each greenhouse gas' ability to trap heat in the atmosphere relative to carbon dioxide. Extensive research has been performed and it has been discovered that the effects of various gases on global warming are too complex to be precisely summarized by a single number. Further understanding of the subject also causes frequent changes to estimates. Despite that, the scientific community has developed approximations, which are shown below. Most analysts use the 100-year time horizon.

Table 3.8

Numerical Estimates of Global Warming Potentials Compared With Carbon Dioxide (kilogram of gas per kilogram of carbon dioxide)

\begin{tabular}{lcccc}
\hline & & \multicolumn{2}{c}{ Direct effect for time horizons of } \\
\cline { 2 - 4 } \multicolumn{1}{c}{ Gas } & Lifetime & & & \\
& (years) & 20 years & 100 years & 500 years \\
\hline Carbon Dioxide & Variable & 1 & 1 & 1 \\
Methane & $12 \pm 3$ & 56 & 21 & 7 \\
Nitrous Oxide & 120 & 280 & 310 & 170 \\
HFCs, PFCs, and other gases & & & & \\
HFC-23 & 264 & 9,200 & 12,100 & 9,900 \\
HFC- 125 & 33 & 4,800 & 3,200 & 11 \\
HFC-134a & 15 & 3,300 & 1,300 & 420 \\
HFC-152a & 2 & 460 & 140 & 42 \\
HFC-227ea & 37 & 4,300 & 2,900 & 950 \\
Perfluoromethane & 50,000 & 4,400 & 6,500 & 10,000 \\
Perfluoroethane & 10,000 & 6,200 & 9,200 & 14,000 \\
Sulfur hexafluoride & 3,200 & 16,300 & 23,900 & 34,900 \\
\hline
\end{tabular}

Source:

U.S. Department of Energy, Energy Information Administration, Emissions of Greenhouse Gases in the United States 1997, Washington, DC, October 1998, p. 8. Original source: Intergovernmental Panel on Climate Change. (Additional resources: http://www.eia.doe.gov, http://www.ipcc.ch)

Note:

The typical uncertainty for global warming potentials is estimated by the Intergovernmental Panel on Climate Change at \pm 35 percent. 


\section{The Greenhouse Gases, Regulated Emissions, and Energy Use in Transportation (GREET) Model}

The energy in greenhouse gas estimates of the most recent version (Version 1.4) of the GREET model are displayed in the next. two tables. The model estimates the full fuel-cycle emissions and energy use associated with various transportation fuels and advanced transportation technologies for light-duty vehicles. It calculates fuel-cycle emissions of three GREENHOUSE GASES (carbon dioxide, methane, and nitrous oxide) and five criteria pollutants (volatile organic compounds, carbon monoxide, nitrogen oxides, sulfur oxides, and particulate matter measuring 10 microns or less). See Chapter 4 for the criteria pollutant data from GREET. The model also calculates the total fuel-cycle energy consumption, fossil fuel consumption, and petroleum consumption using various transportation fuels. The fuel cycles that are included in the GREET model are:

- petroleum to conventional gasoline, reformulated gasoline, conventional diesel, reformulated diesel, liquefied petroleum gas, and electricity via residual oil;

- natural gas to compressed natural gas, liquefied natural gas, liquefied petroleum gas, methanol, FischerTropsch diesel, dimethyl ether, hydrogen, and electricity;

- coal to electricity;

- uranium to electricity;

- renewable energy (hydropower, solar energy, and wind) to electricity;

- corn, woody biomass, and herbaceous biomass to ethanol;

- $\quad$ soybeans to biodiesel; and

- landfill gases to methanol.

Near-term technologies are ones which may be applied to 2000 model-year cars and Long-term technologies are ones which may be applied to 2010 model-year cars.

For additional information about the GREET model, contact:

Michael Q. Wang

Argonne National Laboratory

9700 South Cass Avenuk, ES/362

Argonne, IL 60439-48 15

phone: 630-252-2819

fax: 630-252-3443

email: michaelwang@qmgate.anl.gov 


\section{Acronyms Used on Tables 3.9 and 3.10}

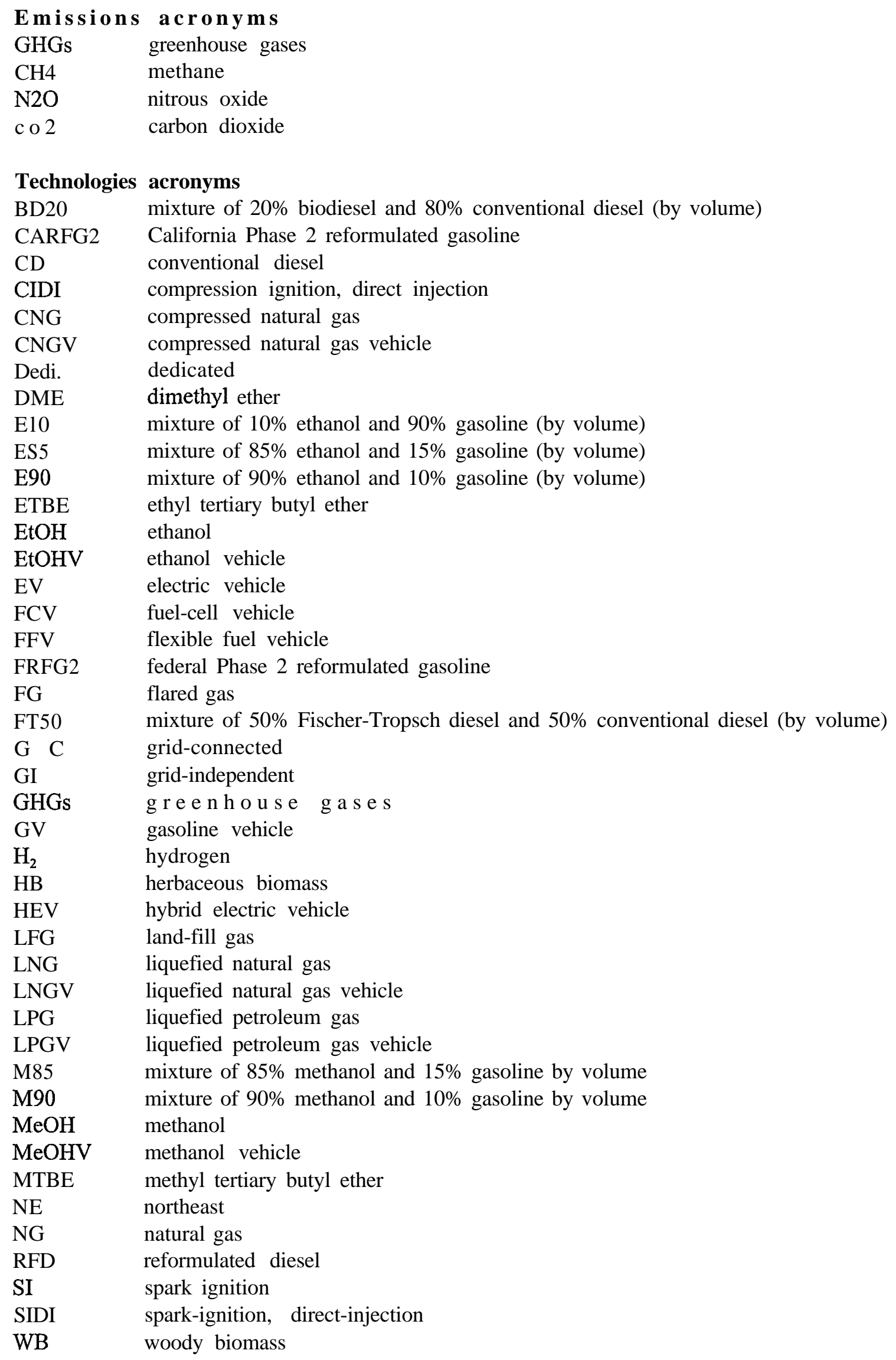




\section{Table 3.9}

\section{Near-Term Technology}

Changes in Per-Mile, Fuel-Cycle Energy Use and Greenhouse Gas Emissions for Passenger Cars (Percentage relative to conventional gasoline vehicles fueled with conventional gasoline)

\begin{tabular}{|c|c|c|c|c|c|c|c|c|c|}
\hline & $\begin{array}{c}\text { GV: } \\
\text { FRFG2, } \\
\text { MTBE }\end{array}$ & $\begin{array}{c}\text { GV: } \\
\text { CARFG2, } \\
\text { ETBE }\end{array}$ & $\begin{array}{c}\text { GV: } \\
\text { CARFG2, } \\
\text { EtOH }\end{array}$ & $\begin{array}{l}\text { CIDI: } \\
\text { CD }\end{array}$ & $\begin{array}{l}\text { Bi-fuel } \\
\text { CNGV }\end{array}$ & $\begin{array}{l}\text { Dedi. } \\
\text { CNGV }\end{array}$ & $\begin{array}{l}\text { Dedi. } \\
\text { LPGV: } \\
\text { crude }\end{array}$ & $\begin{array}{c}\text { Dedi. } \\
\text { LPGV: NG }\end{array}$ & $\begin{array}{c}\text { M85 FFV: } \\
\text { NG }\end{array}$ \\
\hline Total energy & $0.0 \%$ & $0.8 \%$ & $0.4 \%$ & $-29.7 \%$ & $6.7 \%$ & $3.3 \%$ & $-8.6 \%$ & $-9.6 \%$ & $14.5 \%$ \\
\hline Fossil fuels & $0.0 \%$ & $-3.1 \%$ & $-2.6 \%$ & $-29.6 \%$ & $5.7 \%$ & $2.3 \%$ & $-8.6 \%$ & $-9.3 \%$ & $15.0 \%$ \\
\hline Petroleum & $-11.0 \%$ & $-10.1 \%$ & $-2.5 \%$ & $-26.7 \%$ & $-99.4 \%$ & $-99.4 \%$ & $-3.4 \%$ & $-98.2 \%$ & $-72.6 \%$ \\
\hline $\mathrm{CH} 4$ & $18.2 \%$ & $18.8 \%$ & $-2.4 \%$ & $-38.8 \%$ & $211.0 \%$ & $205.1 \%$ & $-5.7 \%$ & $2.8 \%$ & $1.0 \%$ \\
\hline $\mathrm{CO} 2$ & $1.2 \%$ & $-0.9 \%$ & $-5.1 \%$ & $-25.8 \%$ & $-13.9 \%$ & $-16.7 \%$ & $-12.6 \%$ & $-14.3 \%$ & $-4.7 \%$ \\
\hline GHGs & $1.6 \%$ & $0.7 \%$ & $-4.4 \%$ & $-26.5 \%$ & $-8.2 \%$ & $-10.7 \%$ & $-12.2 \%$ & $-13.6 \%$ & $-4.4 \%$ \\
\hline
\end{tabular}

\begin{tabular}{|c|c|c|c|c|c|c|c|c|c|}
\hline & $\begin{array}{c}\text { E10 GV: } \\
\text { corn } \\
\end{array}$ & $\begin{array}{c}\text { E85 FFV: } \\
\text { corn } \\
\end{array}$ & $\begin{array}{c}\text { E85 FFV: } \\
\text { WB }\end{array}$ & $\begin{array}{c}\text { E85 FFV: } \\
\text { HB }\end{array}$ & $\begin{array}{c}\text { EV: } \\
\text { US mix }\end{array}$ & $\begin{array}{c}\text { EV: } \\
\text { NE mix }\end{array}$ & $\begin{array}{c}\text { EV: } \\
\text { CA mix }\end{array}$ & $\begin{array}{l}\text { GC SIDI } \\
\text { HEV: } \\
\text { ETBE, } \\
\text { CA mix }\end{array}$ & $\begin{array}{l}\text { GC SIDI } \\
\text { HEV: } \\
\text { EtOH, } \\
\text { CA mix }\end{array}$ \\
\hline Total energy & $2.1 \%$ & $17.9 \%$ & $90.4 \%$ & $79.1 \%$ & $-14.7 \%$ & $-14.0 \%$ & $-16.9 \%$ & $-35.9 \%$ & $-36.0 \%$ \\
\hline Fossil fuels & $-3.4 \%$ & $-41.8 \%$ & $-81.8 \%$ & $-72.7 \%$ & $-34.7 \%$ & $-46.0 \%$ & $-68.6 \%$ & $-52.9 \%$ & $-52.7 \%$ \\
\hline Petroleum & $-6.3 \%$ & $-73.8 \%$ & $-71.2 \%$ & $-73.2 \%$ & $-98.4 \%$ & $-96.9 \%$ & $-99.5 \%$ & $-64.9 \%$ & $-62.0 \%$ \\
\hline $\mathrm{CH} 4$ & $-1.5 \%$ & $-14.3 \%$ & $-62.9 \%$ & $-48.9 \%$ & $-15.5 \%$ & $-23.5 \%$ & $-49.2 \%$ & $-34.3 \%$ & $-42.5 \%$ \\
\hline $\mathrm{N} 2 \mathrm{O}$ & $45.4 \%$ & $500.3 \%$ & $187.1 \%$ & $607.8 \%$ & $-89.9 \%$ & $-90.2 \%$ & $-92.9 \%$ & $-4.9 \%$ & $-16.6 \%$ \\
\hline $\mathrm{CO} 2$ & $-2.9 \%$ & $-36.4 \%$ & $-113.1 \%$ & $-85.5 \%$ & $-24.3 \%$ & $-42.8 \%$ & $-70.7 \%$ & $-50.4 \%$ & $-51.9 \%$ \\
\hline GHGs & $-2.0 \%$ & $-26.3 \%$ & $-106.4 \%$ & $-72.2 \%$ & $-25.3 \%$ & $-43.1 \%$ & $-70.5 \%$ & $-49.1 \%$ & $-51.1 \%$ \\
\hline
\end{tabular}

\section{Source:}

Wang, Michael Q., GREET Model Results, Argonne National Laboratory, Argonne, IL, September 1998.

\section{Note:}

See page preceding table for acronym definitions. 
Table 3.10

Long-Term Technology

Changes in Per-Mile, Fuel-Cycle Energy Use and Greenhouse Gas Emissions for Passenger Cars

(Percentage relative to conventional gasoline vehicles fueled with conventional gasoline)

\begin{tabular}{|c|c|c|c|c|c|c|c|c|c|c|c|}
\hline Long term & $\begin{array}{l}\text { Dedi. } \\
\text { CNGV }\end{array}$ & $\begin{array}{c}\text { Dedi. } \\
\text { LNGV }\end{array}$ & $\begin{array}{l}\text { Dedi. } \\
\text { LPGV: } \\
\text { crude }\end{array}$ & $\begin{array}{c}\text { Dedi. } \\
\text { LPGV: } \\
\text { NG }\end{array}$ & $\begin{array}{c}\text { Dedi. } \\
\text { MeOHV: } \\
\text { M90, NG }\end{array}$ & $\begin{array}{c}\text { Dedi. } \\
\text { MeOHV: } \\
\text { M90, FG }\end{array}$ & $\begin{array}{c}\text { Dedi. } \\
\text { MeOHV: } \\
\text { M90, LFG }\end{array}$ & $\begin{array}{c}\text { Dedi. } \\
\text { EtOHV: } \\
\text { E90, corn }\end{array}$ & $\begin{array}{c}\text { Dedi. } \\
\text { EtOHV: } \\
\text { E90, WB } \\
\end{array}$ & $\begin{array}{c}\text { Dedi. } \\
\text { EtOHV: } \\
\text { E90, HB }\end{array}$ & $\begin{array}{c}\text { SIDI: } \\
\text { FRFG2, } \\
\text { MTBE }\end{array}$ \\
\hline Total energy & $-8.6 \%$ & $-5.7 \%$ & $-16.8 \%$ & $-17.7 \%$ & $8.8 \%$ & $13.8 \%$ & $-1.8 \%$ & $10.0 \%$ & $57.7 \%$ & $44.4 \%$ & $-20.0 \%$ \\
\hline Fossil fuels & $-9.5 \%$ & $-5.1 \%$ & $-16.9 \%$ & $-17.4 \%$ & $9.4 \%$ & $-45.4 \%$ & $-61.0 \%$ & $-50.1 \%$ & $-82.2 \%$ & $-78.2 \%$ & $-20.0 \%$ \\
\hline Petroleum & $-99.4 \%$ & $-96.0 \%$ & $-1.3 \%$ & $-98.2 \%$ & $-78.1 \%$ & $-78.1 \%$ & $-78.7 \%$ & $-81.5 \%$ & $-79.3 \%$ & $-81.1 \%$ & $-20.0 \%$ \\
\hline $\mathrm{CH} 4$ & $84.4 \%$ & $84.2 \%$ & $-28.9 \%$ & $-22.3 \%$ & $-22.2 \%$ & $-27.0 \%$ & $-409.0 \%$ & $-19.3 \%$ & $-60.9 \%$ & $-54.3 \%$ & $-18.0 \%$ \\
\hline $\mathrm{N} 2 \mathrm{O}$ & $-48.5 \%$ & $-47.7 \%$ & $-2.2 \%$ & $-2.2 \%$ & $-0.4 \%$ & $-2.7 \%$ & $0.4 \%$ & $307.9 \%$ & $91.3 \%$ & $337.6 \%$ & $-0.9 \%$ \\
\hline c o 2 & $-26.9 \%$ & $-27.0 \%$ & $-21.3 \%$ & $-22.9 \%$ & $-11.6 \%$ & $-77.3 \%$ & $-146.8 \%$ & $-49.4 \%$ & $-107.1 \%$ & $-88.7 \%$ & $-20.0 \%$ \\
\hline GHGs & $-23.8 \%$ & $-23.9 \%$ & $-21.2 \%$ & $-22.5 \%$ & $-11.7 \%$ & $-74.3 \%$ & $-152.2 \%$ & $-39.9 \%$ & $-101.1 \%$ & $-77.5 \%$ & $-19.6 \%$ \\
\hline
\end{tabular}

\begin{tabular}{|c|c|c|c|c|c|c|c|c|c|c|c|}
\hline & $\begin{array}{l}\text { SIDI: } \\
\text { FRFG2, } \\
\text { ETBE }\end{array}$ & $\begin{array}{l}\text { SIDI: } \\
\text { FRFG2 } \\
\text { EtOH }\end{array}$ & $\begin{array}{c}\text { SIDI: } \\
\text { C A R F G } 2, \\
\text { ETBE }\end{array}$ & $\begin{array}{l}\text { SIDI: } \\
\text { CARFG2 } \\
\text { EtOH }\end{array}$ & $\begin{array}{l}\text { Dedi. } \\
\text { 2, MeOH } \\
\text { SIDI: NG }\end{array}$ & $\begin{array}{c}\text { Dedi. } \\
\text { MeOH } \\
\text { SIDI: } \\
\text { M90, FG }\end{array}$ & $\begin{array}{c}\text { Dedi. } \\
\text { MeOH } \\
\text { SIDI: } \\
\text { M90, LFG }\end{array}$ & $\begin{array}{c}\text { Dedi } \\
\text { EtOH } \\
\text { SIDI: E90, } \\
\text { corn }\end{array}$ & $\begin{array}{c}\text { Dedi. } \\
\text { EtOH } \\
\text { SIDI: E90, } \\
\text { WB }\end{array}$ & $\begin{array}{c}\text { Dedi. } \\
\text { EtOH } \\
\text { SIDI: E90, } \\
\text { HB }\end{array}$ & $\begin{array}{l}\text { GI SIDI } \\
\text { HEV: } \\
\text { FRFG2, } \\
\text { MTBE }\end{array}$ \\
\hline Total energy & $-20.0 \%$ & $-20.0 \%$ & $-20.0 \%$ & $-20.0 \%$ & $-6.7 \%$ & $-2.3 \%$ & $-16.1 \%$ & $-3.2 \%$ & $38.7 \%$ & $27.1 \%$ & $-47.4 \%$ \\
\hline Fossil fuels & $-20.0 \%$ & $-20.0 \%$ & $-19.0 \%$ & $-19.3 \%$ & $-6.2 \%$ & $-68.4 \%$ & $-68.1 \%$ & $-56.1 \%$ & $-84.4 \%$ & $-80.8 \%$ & $-47.4 \%$ \\
\hline Petroleum & $-20.0 \%$ & $-20.0 \%$ & $-17.2 \%$ & $-19.2 \%$ & $-82.1 \%$ & $-82.1 \%$ & $-82.6 \%$ & $-83.7 \%$ & $-81.8 \%$ & $-83.4 \%$ & $-47.4 \%$ \\
\hline $\mathrm{CH} 4$ & $-18.0 \%$ & $-17.6 \%$ & $-18.0 \%$ & $-17.6 \%$ & $-35.3 \%$ & $-39.5 \%$ & $-375.7 \%$ & $-26.8 \%$ & $-63.5 \%$ & $-57.6 \%$ & $-42.6 \%$ \\
\hline $\mathrm{N} 2 \mathrm{O}$ & $-7.2 \%$ & $-4.7 \%$ & $-7.2 \%$ & $-4.7 \%$ & $-1.5 \%$ & $-3.5 \%$ & $-0.7 \%$ & $268.1 \%$ & $77.5 \%$ & $294.2 \%$ & $-2.1 \%$ \\
\hline c o 2 & $-20.0 \%$ & $-19.7 \%$ & $-20.0 \%$ & $-19.8 \%$ & $-24.7 \%$ & $-82.5 \%$ & $-143.7 \%$ & $-55.3 \%$ & $-106.2 \%$ & $-90.0 \%$ & $-47.4 \%$ \\
\hline GHGs & $-19.6 \%$ & $-19.3 \%$ & $-19.6 \%$ & $-19.3 \%$ & $-24.6 \%$ & $-79.7 \%$ & $-148.2 \%$ & $-46.7 \%$ & $-100.6 \%$ & $-79.8 \%$ & $-46.4 \%$ \\
\hline
\end{tabular}

Table continued on next page. See page preceding Table 3.9 for acronym definitions. 
Table 3.10 (continued)

Long-Term Technology

Changes in Per-Mile, Fuel-Cycle Energy Use and Greenhouse Gas Emissions for Passenger Cars

(Percentage relative to conventional gasoline vehicles fueled with conventional gasoline)

\begin{tabular}{|c|c|c|c|c|c|c|c|c|c|c|c|}
\hline & $\begin{array}{l}\text { GI SIDI } \\
\text { HEV: } \\
\text { FRFG2, } \\
\text { ETBE }\end{array}$ & $\begin{array}{l}\text { GI SIDI } \\
\text { HEV: } \\
\text { FRFG2, } \\
\text { EtOH }\end{array}$ & $\begin{array}{l}\text { GI SI } \\
\text { HEV: } \\
\text { CNG }\end{array}$ & $\begin{array}{l}\text { GI SI } \\
\text { HEV: } \\
\text { LNG }\end{array}$ & $\begin{array}{l}\text { GI SI } \\
\text { HEVs: } \\
\text { LPG, } \\
\text { crude }\end{array}$ & $\begin{array}{c}\text { GI SI } \\
\text { HEVs: } \\
\text { LPG, NG }\end{array}$ & $\begin{array}{c}\text { GI SIDI } \\
\text { HEVs: } \\
\text { M90, NG }\end{array}$ & $\begin{array}{l}\text { GI SIDI } \\
\text { HEVs: } \\
\text { M90, FG }\end{array}$ & $\begin{array}{c}\text { GI SIDI } \\
\text { HEVs: } \\
\text { M90 LFG }\end{array}$ & $\begin{array}{c}\text { GI SIDI } \\
\text { HEVs: } \\
\text { E90, corn }\end{array}$ & $\begin{array}{c}\text { GI SIDI } \\
\text { HEVs: } \\
\text { E90, WB }\end{array}$ \\
\hline Total energy & $-47.4 \%$ & $-47.4 \%$ & $-43.5 \%$ & $-41.7 \%$ & $-46.2 \%$ & $-46.7 \%$ & $-37.0 \%$ & $-34.1 \%$ & $-43.2 \%$ & $-36.3 \%$ & $-8.7 \%$ \\
\hline Fossil fuels & $-47.4 \%$ & $-47.4 \%$ & $-44.1 \%$ & $-41.4 \%$ & $-46.2 \%$ & $-46.6 \%$ & $-36.7 \%$ & $-68.4 \%$ & $-77.4 \%$ & $-71.1 \%$ & $-89.7 \%$ \\
\hline Petroleum & $-47.4 \%$ & $-47.4 \%$ & $-99.6 \%$ & $-97.5 \%$ & $-36.1 \%$ & $-98.8 \%$ & $-87.3 \%$ & $-87.3 \%$ & $-87.7 \%$ & $-89.3 \%$ & $-88.0 \%$ \\
\hline $\mathrm{CH} 4$ & $-42.7 \%$ & $-41.7 \%$ & $33.0 \%$ & $32.8 \%$ & $-50.1 \%$ & $-45.8 \%$ & $-52.8 \%$ & $-55.6 \%$ & $-276.8 \%$ & $-45.7 \%$ & $-69.8 \%$ \\
\hline $\mathrm{N} 2 \mathrm{O}$ & $-16.9 \%$ & $-11.2 \%$ & $-50.0 \%$ & $-49.4 \%$ & $-3.0 \%$ & $-3.0 \%$ & $-2.1 \%$ & $-3.4 \%$ & $-1.6 \%$ & $168.3 \%$ & $42.9 \%$ \\
\hline $\cos 2$ & $-47.4 \%$ & $-47.2 \%$ & $-55.0 \%$ & $-55.0 \%$ & $-49.1 \%$ & $-50.1 \%$ & $-48.8 \%$ & $-86.9 \%$ & $-127.1 \%$ & $-70.3 \%$ & $-103.8 \%$ \\
\hline \multirow[t]{2}{*}{ GHGs } & $-46.4 \%$ & $-46.2 \%$ & $-52.1 \%$ & $-52.1 \%$ & $-48.3 \%$ & $-49.1 \%$ & $-48.1 \%$ & $-84.3 \%$ & $-129.4 \%$ & $-63.9 \%$ & $-99.3 \%$ \\
\hline & $\begin{array}{c}\text { GI SIDI } \\
\text { HEVs: } \\
\text { E90, HB }\end{array}$ & $\begin{array}{c}\text { GC SIDI } \\
\text { HEV: } \\
\text { FRFG2, } \\
\text { MTBE, } \\
\text { US mix } \\
\end{array}$ & $\begin{array}{l}\text { GC SIDI } \\
\text { HEV: } \\
\text { FRFG2, } \\
\text { ETBE, } \\
\text { US mix } \\
\end{array}$ & $\begin{array}{c}\text { GC SIDI } \\
\text { HEV: } \\
\text { FRFG2, } \\
\text { EtOH, } \\
\text { US mix } \\
\end{array}$ & $\begin{array}{c}\text { GC SIDI } \\
\text { HEV: } \\
\text { FRFG2, } \\
\text { MTBE, } \\
\text { CA mix }\end{array}$ & $\begin{array}{c}\text { GC SIDI } \\
\text { HEV: } \\
\text { FRFG2, } \\
\text { ETBE, } \\
\text { CA mix }\end{array}$ & $\begin{array}{c}\text { GC SIDI } \\
\text { HEV: } \\
\text { FRFG2, } \\
\text { EtOH, } \\
\text { CA mix } \\
\end{array}$ & $\begin{array}{l}\text { GC SI } \\
\text { HEV: } \\
\text { CNG, } \\
\text { US mix }\end{array}$ & $\begin{array}{l}\text { GC SI } \\
\text { HEV: } \\
\text { CNG, } \\
\text { CA mix }\end{array}$ & $\begin{array}{l}\text { GC SI } \\
\text { HEV: } \\
\text { LNG, } \\
\text { US mix }\end{array}$ & $\begin{array}{l}\text { GC SI } \\
\text { HEV: } \\
\text { LNG, } \\
\text { CA mix }\end{array}$ \\
\hline Total energy & $-16.4 \%$ & $-43.7 \%$ & $-43.8 \%$ & $-43.7 \%$ & $-43.9 \%$ & $-44.0 \%$ & $-43.9 \%$ & $-40.8 \%$ & $-41.0 \%$ & $-39.5 \%$ & $-39.7 \%$ \\
\hline Fossil fuels & $-87.4 \%$ & $-48.2 \%$ & $-47.1 \%$ & $-47.3 \%$ & $-55.5 \%$ & $-54.8 \%$ & $-54.9 \%$ & $-45.7 \%$ & $-53.0 \%$ & $-43.8 \%$ & $-51.1 \%$ \\
\hline Petroleum & $-89.1 \%$ & $-60.7 \%$ & $-60.4 \%$ & $-61.4 \%$ & $-61.0 \%$ & $-60.7 \%$ & $-61.7 \%$ & $-99.4 \%$ & $-99.6 \%$ & $-97.8 \%$ & $-98.1 \%$ \\
\hline $\mathrm{CH} 4$ & $-65.9 \%$ & $-43.6 \%$ & $-43.7 \%$ & $-39.7 \%$ & $-49.9 \%$ & $-50.0 \%$ & $-47.3 \%$ & $10.2 \%$ & $3.9 \%$ & $10.1 \%$ & $3.8 \%$ \\
\hline $\mathrm{N} 2 \mathrm{O}$ & $185.5 \%$ & $-28.2 \%$ & $-39.4 \%$ & $-35.0 \%$ & $-29.9 \%$ & $-40.5 \%$ & $-36.4 \%$ & $-61.7 \%$ & $-63.4 \%$ & $-6.1 .3 \%$ & $-63.0 \%$ \\
\hline $\operatorname{co} 2$ & $-93.1 \%$ & $-46.1 \%$ & $-46.5 \%$ & $-45.8 \%$ & $-56.0 \%$ & $-56.2 \%$ & $-55.8 \%$ & $-51.4 \%$ & $-61.3 \%$ & $-51.4 \%$ & $-61.4 \%$ \\
\hline GHGs & $-85.6 \%$ & $-45.6 \%$ & $-46.3 \%$ & $-45.4 \%$ & $-55.3 \%$ & $-55.6 \%$ & $-55.1 \%$ & $-49.6 \%$ & $-59.3 \%$ & $-49.7 \%$ & $-59.3 \%$ \\
\hline
\end{tabular}

Table continued on next page. See page preceding Table 3.9 for acronym definitions. 
Table 3.10 (continued)

\section{Long-Term Technology}

Changes in Per-Mile, Fuel-Cycle Energy Use and Greenhouse Gas Emissions for Passenger Cars (Percentage relative to conventional gasoline vehicles fueled with conventional gasoline)

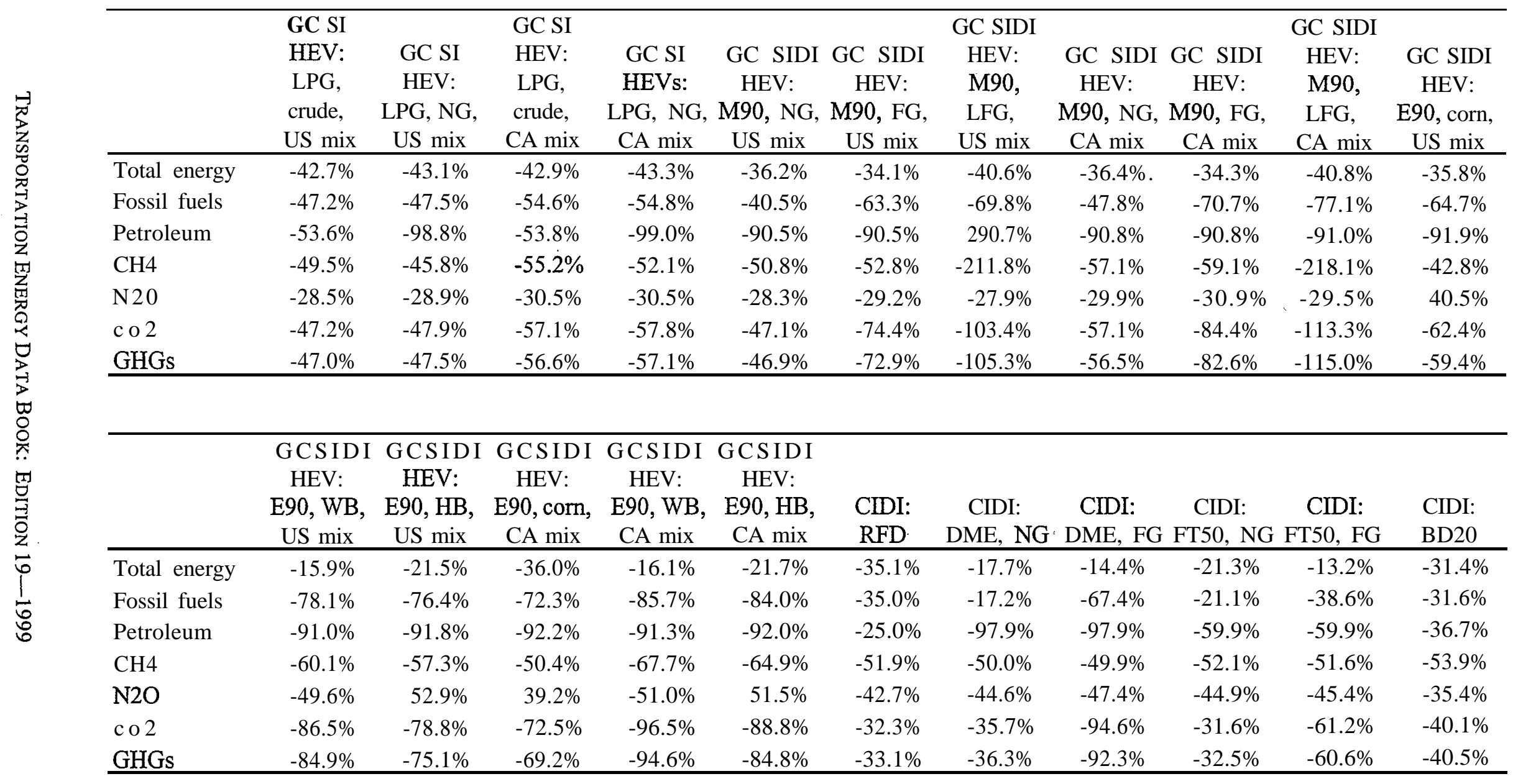

Table continued on next page. See page preceding Table 3.9 for acronym definitions. 
Table 3.10 (continued)

Long-Term Technology

Changes in Per-Mile, Fuel-Cycle Energy Use and Greenhouse Gas Emissions for Passenger Cars

(Percentage relative to conventional gasoline vehicles fueled with conventional gasoline)

\begin{tabular}{|c|c|c|c|c|c|c|c|c|c|c|c|}
\hline & $\begin{array}{c}\text { G I CID } \\
\text { HEV: } \\
\text { RFD }\end{array}$ & $\begin{array}{c}\text { I G I CIDI } \\
\text { HEV: } \\
\text { DME, NG }\end{array}$ & $\begin{array}{l}\text { I G I CIDI } \\
\text { HEV: } \\
\text { DME, FG F }\end{array}$ & $\begin{array}{c}\text { G I CIDI } \\
\text { HEV: } \\
\text { FT50, NG }\end{array}$ & $\begin{array}{l}\text { G I CIDI } \\
\text { HEV: } \\
\text { FT50, FG }\end{array}$ & $\begin{array}{c}\text { G I CIDI } \\
\text { HEV: } \\
\text { BD20 }\end{array}$ & $\begin{array}{c}\text { GC CIDI } \\
\text { HEV: } \\
\text { RFD, } \\
\text { US mix }\end{array}$ & $\begin{array}{c}\text { GC CIDI } \\
\text { HEV: } \\
\text { RFD, } \\
\text { CA mix }\end{array}$ & $\begin{array}{c}\text { GC CIDI } \\
\text { HEV: } \\
\text { DME, } \\
\text { NG, } \\
\text { US mix }\end{array}$ & $\begin{array}{l}\text { GC CIDI } \\
\text { HEV: } \\
\text { DME, FC } \\
\text { US mix }\end{array}$ & $\begin{array}{c}\text { GC CIDI } \\
\text { HEV: } \\
\text { DME, } \\
\text { G, NG, } \\
\text { CA mix }\end{array}$ \\
\hline Total energy & $-57.7 \%$ & $-46.3 \%$ & $-44.2 \%$ & $-43.3 \%$ & $-48.7 \%$ & $-55.3 \%$ & $-53.5 \%$ & $-53.3 \%$ & $-42.2 \%$ & $-40.7 \%$ & $-42.4 \%$ \\
\hline Fossil fuels & $-57.6 \%$ & $-46.0 \%$ & $-78.7 \%$ & $-43.1 \%$ & $-57.6 \%$ & $-55.4 \%$ & $-65.2 \%$ & $-57.8 \%$ & $-46.5 \%$ & $-70.5 \%$ & $-53.9 \%$ \\
\hline Petroleum & $-51.1 \%$ & $-98.6 \%$ & $-98.6 \%$ & $-73.8 \%$ & $-73.8 \%$ & $-58.7 \%$ & $-67.3 \%$ & $-67.1 \%$ & $-98.6 \%$ & $-98.6 \%$ & $-98.9 \%$ \\
\hline $\mathrm{CH} 4$ & $-68.1 \%$ & $-66.3 \%$ & $-66.3 \%$ & $-67.9 \%$ & $-68.2 \%$ & $-69.4 \%$ & $-69.6 \%$ & $-63.3 \%$ & $-60.1 \%$ & $-60.1 \%$ & $-66.4 \%$ \\
\hline $\mathrm{N} 2 \mathrm{O}$ & $-43.5 \%$ & $-44.8 \%$ & $-46.6 \%$ & $-45.3 \%$ & $-45.0 \%$ & $-38.8 \%$ & $-59.0 \%$ & $-57.3 \%$ & $-58.1 \%$ & $-59.5 \%$ & $-59.8 \%$ \\
\hline $\cos 2$ & $-55.8 \%$ & $-58.1 \%$ & $-96.5 \%$ & $-74.7 \%$ & $-55.4 \%$ & $-60.9 \%$ & $-64.5 \%$ & $-54.5 \%$ & $-53.2 \%$ & $-81.3 \%$ & $-63.2 \%$ \\
\hline GHGs & $-56.0 \%$ & $-58.1 \%$ & $-94.6 \%$ & $-74.0 \%$ & $-55.6 \%$ & $-60.7 \%$ & $-64.5 \%$ & $-54.8 \%$ & $-53.5 \%$ & $-80.2 \%$ & $-63.2 \%$ \\
\hline & $\begin{array}{c}\text { G C } \\
\text { HEV: } \\
\text { DME, F } \\
\text { CA mix }\end{array}$ & $\begin{array}{c}\text { GC CIDI } \\
\text { CIDI HEVs: } \\
\text { FT50, } \\
\text { G, NG, } \\
\text { US mix } \\
\end{array}$ & $\begin{array}{l}\text { GC CIDI } \\
\text { HEVs: } \\
\text { FT50, FG } \\
\text { US mix } \\
\end{array}$ & $\begin{array}{l}\text { GC CIDI } \\
\text { HEV: } \\
\text { FT50, } \\
\text { NG, } \\
\text { CA mix }\end{array}$ & $\begin{array}{l}\text { GCCIDI } \\
\text { HEV: } \\
\text { FT50, FG, } \\
\text { CA mix }\end{array}$ & $\begin{array}{l}\text { GCCIDI } \\
\text { HEV: } \\
\text { BD20, } \\
\text { US mix }\end{array}$ & $\begin{array}{l}\text { GCCIDI } \\
\text { HEV: } \\
\text { BD20, } \\
\text { CA mix } \\
\end{array}$ & $\begin{array}{c}\text { EV: } \\
\text { US mix } \\
\end{array}$ & $\begin{array}{c}\text { EV: } \\
\text { NE mix }\end{array}$ & $\begin{array}{c}\text { EV: } \\
\text { CA mix }\end{array}$ & $\begin{array}{c}\text { H2 FCV: } \\
\text { NG, } \\
\text { C. gaseous }\end{array}$ \\
\hline Total energy & $-40.9 \%$ & $-43.9 \%$ & $-40.1 \%$ & $-44.1 \%$ & $-40.3 \%$ & $-48.8 \%$ & $-49.0 \%$ & $-38.4 \%$ & $-39.7 \%$ & $-39.0 \%$ & $-59.7 \%$ \\
\hline Fossil fuels & $-77.8 \%$ & $-48.3 \%$ & $-56.7 \%$ & $-55.7 \%$ & $-64.0 \%$ & $-53.4 \%$ & $-60.7 \%$ & $-53.4 \%$ & $-57.2 \%$ & $-77.9 \%$ & $-60.3 \%$ \\
\hline Petroleum & $-98.9 \%$ & $-80.5 \%$ & $-80.5 \%$ & $-80.7 \%$ & $-80.7 \%$ & $-69.4 \%$ & $-69.7 \%$ & $-98.7 \%$ & $-98.2 \%$ & $-99.6 \%$ & $-99.7 \%$ \\
\hline $\mathrm{CH} 4$ & $-66.3 \%$ & $-61.4 \%$ & $-61.2 \%$ & $-67.7 \%$ & $-67.5 \%$ & $-62.3 \%$ & $-68.6 \%$ & $-48.8 \%$ & $-45.9 \%$ & $-69.7 \%$ & $-62.6 \%$ \\
\hline $\mathrm{N} 2 \mathrm{O}$ & $-61.1 \%$ & $-58.3 \%$ & $-58.5 \%$ & $-59.9 \%$ & $-60.2 \%$ & $-53.7 \%$ & $-55.4 \%$ & $-89.3 \%$ & $-90.2 \%$ & $-94.9 \%$ & $-98.2 \%$ \\
\hline $\cos 2$ & $-91.3 \%$ & $-51.2 \%$ & $-65.4 \%$ & $-61.2 \%$ & $-75.4 \%$ & $-55.3 \%$ & $-65.2 \%$ & $-46.3 \%$ & $-56.7 \%$ & $-79.4 \%$ & $-68.6 \%$ \\
\hline GHGs & $-89.9 \%$ & $-51.7 \%$ & $-65.1 \%$ & $-61.4 \%$ & $-74.8 \%$ & $-55.5 \%$ & $-65.1 \%$ & $-47.2 \%$ & $-57.0 \%$ & $-79.4 \%$ & $-69.0 \%$ \\
\hline
\end{tabular}

Table continued on next page. See page preceding Table 3.9 for acronym definitions. 
Table 3.10 (continued)

Long-Term Technology

Changes in Per-Mile, Fuel-Cycle Energy Use and Greenhouse Gas Emissions for Passenger Cars (Percentage relative to conventional gasoline vehicles fueled with conventional gasoline)

\begin{tabular}{lcccccc}
\hline & H2 FCV: & \multicolumn{3}{c}{} & \\
& NG, & & H2 FCV: & & FCV: & F C \\
& station & H2 FCV: & solar, & H2 FCV: & MeOH, & MeOH, \\
& gaseous & solar gas & liquid & NG, liquid & NG & FG \\
\hline Total energy & $-53.2 \%$ & $-65.5 \%$ & $-71.8 \%$ & $-58.8 \%$ & $-52.3 \%$ & $-49.7 \%$ \\
Fossil fuels & $-52.9 \%$ & $-93.1 \%$ & $-98.3 \%$ & $-58.5 \%$ & $-52.0 \%$ & $-80.3 \%$ \\
Petroleum & $-99.7 \%$ & $-99.9 \%$ & $-98.1 \%$ & $-97.8 \%$ & $-98.6 \%$ & $-98.6 \%$ \\
CH4 & $-47.1 \%$ & $-86.9 \%$ & $-98.8 \%$ & $-63.3 \%$ & $-68.0 \%$ & $-70.5 \%$ \\
N20 & $-96.7 \%$ & $-98.1 \%$ & $-99.4 \%$ & $-97.8 \%$ & $-79.2 \%$ & $-80.4 \%$ \\
c o 2 & $-63.7 \%$ & $-93.4 \%$ & $-98.3 \%$ & $-67.7 \%$ & $-62.8 \%$ & $-96.7 \%$ \\
GHGs & $-63.8 \%$ & $-93.3 \%$ & $-98.3 \%$ & $-68.1 \%$ & $-63.3 \%$ & $-95.6 \%$ \\
\hline & & & & & & \\
& & & & & & \\
\hline & FCV: & & EtOH & EtOH & EtOH & NG \\
& MeOH, & FCV: & FCV: & FCV: & FCV: & FCV: \\
\hline Total energy & $-57.8 \%$ & $-50.0 \%$ & $-37.7 \%$ & $-7.1 \%$ & $-15.6 \%$ & $-52.0 \%$ \\
Fossil fuels & $-88.3 \%$ & $-50.0 \%$ & $-77.4 \%$ & $-97.3 \%$ & $-94.8 \%$ & $-52.5 \%$ \\
Petroleum & $-98.9 \%$ & $-50.0 \%$ & $-96.5 \%$ & $-94.9 \%$ & $-96.2 \%$ & $-99.7 \%$ \\
CH4 & $-267.5 \%$ & $-53.0 \%$ & $-70.7 \%$ & $-92.8 \%$ & $-89.3 \%$ & $-9.4 \%$ \\
N20 & $-78.7 \%$ & $-78.7 \%$ & $182.0 \%$ & $8.6 \%$ & $205.8 \%$ & $-79.0 \%$ \\
c o 2 & $-132.5 \%$ & $-50.0 \%$ & $-76.4 \%$ & $-113.1 \%$ & $-101.4 \%$ & $-61.6 \%$ \\
GHGs & $-135.8 \%$ & $-50.6 \%$ & $-71.2 \%$ & $-110.1 \%$ & $-95.1 \%$ & $-60.3 \%$ \\
\hline
\end{tabular}

Source:

Wang, Michael Q., GREET Model Results, Argonne National Laboratory, Argonne,

Note:

$$
\text { IL, August } 1999 .
$$

See page preceding Table 3.9 for acronym definitions. 


\section{Chapter 4 \\ Criteria Pollutants}

Summary Statistics from Tables in this Chapter

\begin{tabular}{|c|c|c|}
\hline , Source & & \\
\hline \multirow[t]{8}{*}{ Table 4.1} & Transportation's share of U.S. emissions, 1997 & \\
\hline & c o & $76.6 \%$ \\
\hline & $N O_{X}$ & $49.2 \%$ \\
\hline & v o c & $39.9 \%$ \\
\hline & $P M-10$ & $2.2 \%$ \\
\hline & $P M-2.5$ & $7.4 \%$ \\
\hline & $\mathrm{SO}_{2}$ & $6.8 \%$ \\
\hline & $\mathrm{NH}_{3}$ & $7.6 \%$ \\
\hline \multirow[t]{3}{*}{ Table 4.10} & Transportation's share of lead emissions & \\
\hline & 1970 & $82.3 \%$ \\
\hline & 1997 & $13.3 \%$ \\
\hline \multirow[t]{4}{*}{ Table 4.11} & Automobile emission standards, 1998 (grams per mile) & \\
\hline & $H C$ & 0.25 \\
\hline & $\mathrm{CO}$ & 3.4 \\
\hline & $N O_{X}$ & 0.4 \\
\hline
\end{tabular}


Table 4.1

Total National Emissions of the Criteria Air Pollutants by Sector, 1997 (millions of short tons/percentage)

\begin{tabular}{|c|c|c|c|c|c|c|c|}
\hline Sector & c o & $\mathrm{NO}_{\mathrm{x}}$ & Voc c & PM-10 & PM-2.5 & SO, & $\mathrm{NH}_{3}$ \\
\hline \multirow[t]{2}{*}{ Highway vehicles } & 50.26 & 7.04 & 5.23 & 0.27 & 0.21 & 0.32 & 0.24 \\
\hline & $57.5 \%$ & $29.8 \%$ & $27.2 \%$ & $0.8 \%$ & $2.5 \%$ & $1.6 \%$ & $7.6 \%$ \\
\hline \multirow[t]{2}{*}{ Aircraft } & 1.01 & 0.18 & 0.19 & 0.04 & 0.03 & 0.01 & 0.00 \\
\hline & $1.2 \%$ & $0.8 \%$ & $1.0 \%$ & $0.1 \%$ & $0.3 \%$ & $0.1 \%$ & $0.0 \%$ \\
\hline \multirow[t]{2}{*}{ Railroads } & 0.12 & 0.95 & 0.05 & 0.03 & 0.00 & 0.11 & 0.00 \\
\hline & $0.1 \%$ & $4.0 \%$ & $0.3 \%$ & $0.1 \%$ & $0.0 \%$ & $0.6 \%$ & $0.1 \%$ \\
\hline \multirow[t]{2}{*}{ Vessels } & 0.09 & 0.24 & 0.05 & 0.03 & 0.02 & 0.25 & 0.00 \\
\hline & $0.1 \%$ & $1.0 \%$ & $0.3 \%$ & $0.1 \%$ & $0.3 \%$ & $1.2 \%$ & $0.0 \%$ \\
\hline \multirow[t]{2}{*}{ Other off-highway } & 15.54 & 3.20 & 2.14 & 0.37 & 0.36 & 0.69 & 0.00 \\
\hline & $17.8 \%$ & $13.6 \%$ & $11.2 \%$ & $1.1 \%$ & $4.3 \%$ & $3.4 \%$ & $0.0 \%$ \\
\hline \multirow[t]{2}{*}{ Transportation total } & 67.01 & 11.60 & 7.66 & 0.73 & 0.62 & 1.38 & 0.24 \\
\hline & $76.6 \%$ & $49.2 \%$ & $39.9 \%$ & $2.2 \%$ & $7.4 \%$ & $6.8 \%$ & $7.6 \%$ \\
\hline \multirow[t]{2}{*}{ Stationary source fuel combustion } & 4.82 & 10.72 & 0.86 & 1.10 & 0.85 & 17.26 & 0.03 \\
\hline & $5.5 \%$ & $45.5 \%$ & $4.5 \%$ & $3.3 \%$ & $10.2 \%$ & $84.7 \%$ & $1.0 \%$ \\
\hline \multirow[t]{2}{*}{ Industrial processes } & 4.81 & 0.81 & 9.39 & 0.98 & 0.52 & 1.67 & 0.29 \\
\hline & $5.5 \%$ & $3.5 \%$ & $48.9 \%$ & $2.9 \%$ & $6.3 \%$ & $8.2 \%$ & $9.0 \%$ \\
\hline \multirow[t]{2}{*}{ Waste disposal and recycling total } & 1.24 & 0.10 & 0.45 & 0.30 & 0.25 & 0.05 & 0.10 \\
\hline & $1.4 \%$ & $0.4 \%$ & $2.3 \%$ & $0.9 \%$ & $3.1 \%$ & $0.2 \%$ & $3.1 \%$ \\
\hline \multirow[t]{2}{*}{ Miscellaneous } & 9.57 & 0.35 & 0.86 & 30.47 & 6.07 & 0.01 & 2.52 \\
\hline & $10.9 \%$ & $1.5 \%$ & $4.5 \%$ & $90.7 \%$ & $\mathbf{7 3 . 0 \%}$ & $0.1 \%$ & $79.2 \%$ \\
\hline \multirow[t]{2}{*}{ Total of all sources } & 87.45 & 23.58 & 19.22 & 33.58 & 8.31 & 20.37 & 3.18 \\
\hline & $100.0 \%$ & $100.0 \%$ & $100.0 \%$ & $100.0 \%$ & $100.0 \%$ & $100.0 \%$ & $100.0 \%$ \\
\hline
\end{tabular}

Source:

All other-U. S. Environmental Protection Agency, National Air Pollutant Emission Trends, 1900-1997, 1998, Appendix A. (Additional resources: http://www.epa.gov/oar/oaqps)

Note:

$\mathrm{CO}=$ Carbon monoxide. $\mathrm{NO},=$ Nitrogen oxides. PM-10 $=$ Particulate matter less than 10 microns.

PM-2.5 = Particulate matter less than 2.5 microns. $\mathrm{SO},=$ Sulfur dioxide. $\mathrm{VOC}=$ Volatile organic compounds. $\mathrm{NH},=$ Ammonia. 
The transportation sector accountedfor two-thirds of the nation 's carbon monoxide (CO) emissions in 1997. Highway vehicles are by far the source of the greatest amount of CO. For details on the highway emissions of $\mathrm{CO}$, see Table 4.3.

Table 4.2

Total National Emissions of Carbon Monoxide, 1970-97”, (million short tons)

\begin{tabular}{|c|c|c|c|c|c|c|c|}
\hline Source category & 1970 & 1980 & 1990 & 1995 & 1996 & 1997 & $\begin{array}{c}\text { Percent } \\
\text { of total, } \\
1997\end{array}$ \\
\hline Highway vehicles & 88.03 & 78.05 & 57.85 & 54.11 & 53.26 & 50.26 & $57.5 \%$ \\
\hline Aircraft & 0.51 & 0.74 & 0.90 & 0.94 & 0.95 & 1.01 & $1.2 \%$ \\
\hline Railroads & 0.07 & 0.10 & 0.12 & 0.11 & 0.11 & 0.12 & $0.1 \%$ \\
\hline Vessels $^{b}$ & 0.01 & 0.04 & 0.08 & 0.08 & 0.09 & 0.09 & $0.1 \%$ \\
\hline Other off-highway & 10.70 & 12.88 & 14.27 & 15.13 & 15.27 & 15.54 & $17.8 \%$ \\
\hline Transportation total & 99.32 & 91.81 & 73.22 & 70.38 & 69.67 & 67.01 & $76.6 \%$ \\
\hline Stationary fuel combustion total & 4.63 & 7.30 & 5.51 & 5.93 & 5.98 & 4.82 & $5.5 \%$ \\
\hline Industrial processes total & 9.84 & 6.95 & 4.77 & 4.61 & 4.62 & 4.81 & $5.5 \%$ \\
\hline Waste disposal and recycling total & 7.06 & 2.30 & 1.08 & 1.19 & 1.20 & 1.24 & $1.4 \%$ \\
\hline Miscellaneous total & 7.91, & 8.34 & 11.21 & 7.05 & 9.46 & 9.57 & $10.9 \%$ \\
\hline Total of all sources & 128.76 & 116.70 & 95.80 & 89.15 & 90.93 & 87.45 & $100.0 \%$ \\
\hline
\end{tabular}

Source:

U. S. Environmental Protection Agency, National Air Pollutant Emission Trends, 1900-1997, 1998, pp. A-1-A-5, and annual. (Additional resources: http://www.epa/oar/oaqps)

Note:

Emission estimation methodology changes indicated by shaded areas. Transportation methodologies changed in 1970, while all others changed in 1990.

"The sums of subcategories may not equal total due to rounding.

'Recreational marine vessels. 
Table 4.3

Emissions of Carbon Monoxide from Highway Vehicles, 1970-97"

(million short tons)

\begin{tabular}{|c|c|c|c|c|c|c|c|c|c|c|c|c|c|}
\hline Source category & 1970 & 1975 & 1980 & 1985 & 1990 & 1991 & 1992 & 1993 & 1994 & 1995 & 1996 & 1997 & $\begin{array}{l}\text { Percent of } \\
\text { total, } 1997\end{array}$ \\
\hline \multicolumn{14}{|c|}{ Gasoline powered } \\
\hline Light vehicles \& motorcycles & 64.03 & 59.28 & 53.56 & 49.45 & 37.41 & 40.27 & 39.37 & 39.16 & 37.06 & 33.70 & 28.73 & 27.04 & $53.8 \%$ \\
\hline Light trucks ${ }^{\mathrm{b}}$ & 16.57 & 15.77 & 16.14 & 18.96 & 13.82 & 15.01 & 14.57 & 15.20 & 17.35 & 14.83 & 19.27 & 18.36 & $36.5 \%$ \\
\hline Heavy vehicles & 6.71 & 7.14 & 7.19 & 7.72 & 5.36 & 5.46 & 4.57 & 4.48 & 5.53 & 4.12 & 3.77 & 3.35 & $6.7 \%$ \\
\hline Total & 87.31 & 82.19 & 76.89 & 76.13 & 56.58 & 60.74 & 58.51 & 58.84 & 59.93 & 52.65 & 51.77 & 48.75 & $97.0 \%$ \\
\hline \multicolumn{14}{|c|}{ Diesel powered } \\
\hline Light vehicles & c & 0.03 & 0.02 & 0.02 & 0.03 & 0.03 & 0.03 & 0.03 & 0.03 & 0.03 & 0.03 & 0.03 & $0.1 \%$ \\
\hline Light trucks ${ }^{\mathrm{b}}$ & c & c & 0.00 & 0.00 & 0.01 & 0.01 & 0.01 & 0.01 & 0.01 & 0.01 & 0.01 & 0.01 & $0.0 \%$ \\
\hline Heavy vehicles & 0.72 & 0.92 & 1.14 & 1.24 & 1.23 & 1.30 & 1.32 & 1.33 & 1.41 & 1.41 & 1.45 & 1.47 & $2.9 \%$ \\
\hline Total & 0.72 & 0.95 & 1.16 & 1.26 & 1.27 & 1.33 & 1.35 & 1.37 & 1.45 & 1.45 & 1.49 & 1.51 & $3.0 \%$ \\
\hline \multicolumn{14}{|c|}{ Total } \\
\hline Highway vehicle total & 88.03 & 83.13 & 78.05 & 77.39 & 57.85 & 62.07 & 59.86 & 60.20 & 61.38 & 54.11 & 53.26 & 50.26 & $100.0 \%$ \\
\hline Percent diesel & $0.8 \%$ & $1.1 \%$ & $1.5 \%$ & $1.6 \%$ & $2.2 \%$ & $2.1 \%$ & $2.3 \%$ & $2.3 \%$ & $2.4 \%$ & $2.7 \%$ & $2.8 \%$ & $3.0 \%$ & \\
\hline
\end{tabular}

Source:

U. S. Environmental Protection Agency, National Air Pollutant Emission Trends, 1900-1997, 1998, p. A-3 and annual. (Additional resources: http://www.epa.gov/oar/oaqps)

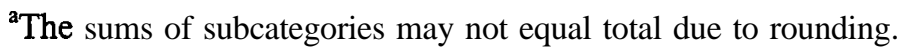

'Less than 8,500 pounds.

'Data are not available. 
The transportation sector accountedfor nearly half of the nation 's nitrogen oxide (NOx) emissions in 1997, with the majority coming from highway vehicles. For details on the highway emissions of NOx, see Table 4.5.

Table 4.4

Total National Emissions of Nitrogen Oxides, 1970-97" (million short tons)

\begin{tabular}{lrrrrrrr}
\hline & & & & & & & $\begin{array}{c}\text { Percent } \\
\text { of total, }\end{array}$ \\
Source category & 1970 & 1980 & 1990 & 1995 & 1996 & 1997 & 1997 \\
\hline \multicolumn{1}{c}{ Highway vehicles } & 7.39 & 8.62 & 7.04 & 7.32 & 7.25 & 7.04 & $29.8 \%$ \\
$\quad$ Railroads & 0.50 & 0.73 & 0.93 & 0.99 & 0.92 & 0.95 & $4.0 \%$ \\
$\quad$ Other off-highway & 1.69 & 3.29 & 3.31 & 3.52 & 3.56 & 3.61 & $15.3 \%$ \\
Transportation total & 9.57 & 12.64 & 11.28 & 11.83 & 11.72 & 11.60 & $49.2 \%$ \\
Stationary fuel combustion total & 10.06 & 11.32 & 10.89 & 10.83 & 10.52 & 10.72 & $45.5 \%$ \\
Industrial processes total & 0.78 & 0.56 & 0.80 & 0.77 & 0.78 & 0.81 & $3.5 \%$ \\
Waste disposal and recycling total & 0.44 & 0.11 & 0.09 & 0.10 & 0.10 & 0.10 & $0.4 \%$ \\
Miscellaneous total & 0.33 & 0.25 & 0.37 & 0.24 & 0.34 & 0.35 & $1.5 \%$ \\
Total of all sources & 21.18 & 24.87 & 23.43 & 23.77 & 23.46 & 23.58 & $\mathbf{1 0 0 . 0 \%}$ \\
\hline
\end{tabular}

\section{Source:}

U. S. Environmental Protection Agency, National Air Pollutant Emission Trends, 1900-1997, 1998, pp. A-6-A-10, and annual. (Additional resources: http://www.epa/oar/oaqps)

\section{Note:}

Emission estimation methodology changes indicated by shaded areas. Transportation methodologies changed in 1970, while all others changed in 1990.

"The sums of subcategories may not equal total due to rounding. 
Table 4.5

Emissions of Nitrogen Oxides from Highway Vehicles, 1970-97" (million short tons)

\begin{tabular}{|c|c|c|c|c|c|c|c|c|c|c|c|c|c|}
\hline Source category & 1970 & 1975 & 1980 & 1985 & 1990 & 1991 & 1992 & 1993 & 1994 & 1995 & 1996 & 1997 & $\begin{array}{l}\text { Percent of } \\
\text { total, } 1997\end{array}$ \\
\hline \multicolumn{14}{|c|}{ Gasoline powered } \\
\hline Light vehicles \& motorcycles & 4.16 & 4.73 & 4.42 & 3.81 & 3.22 & 3.46 & 3.61 & 3.68 & 3.57 & 3.44 & 2.98 & 2.88 & $40.9 \%$ \\
\hline Light trucks ${ }^{\mathrm{b}}$ & 1.28 & 1.46 & 1.41 & 1.53 & 1.26 & 1.34 & 1.36 & 1.42 & 1.66 & 1.52 & 1.95 & 1.90 & $27.0 \%$ \\
\hline Heavy vehicles & 0.28 & 0.32 & 0.30 & 0.33 & 0.33 & 0.33 & 0.31 & 0.32 & 0.35 & 0.33 & 0.33 & 0.33 & $4.6 \%$ \\
\hline Total & 5.71 & 6.51 & 6.13 & 5.67 & 4.80 & 5.13 & 5.28 & 5.42 & 5.58 & 5.30 & 5.26 & 5.10 & $72.5 \%$ \\
\hline Light vehicles & c & 0.02 & 0.03 & 0.03 & \multicolumn{2}{|c|}{ Diesel 0.04 powleodd } & 0.04 & 0.04 & 0.04 & 0.04 & 0.04 & 0.04 & $0.5 \%$ \\
\hline Light trucks ${ }^{b}$ & c & c & 0.01 & 0.01 & 0.01 & 0.01 & 0.01 & 0.01 & 0.01 & 0.01 & 0.01 & 0.01 & $0.2 \%$ \\
\hline Heavy vehicles & 1.68 & 2.12 & 2.46 & 2.39 & 2.19 & 2.20 & 2.12 & 2.05 & 2.04 & 1.98 & 1.94 & 1.89 & $26.8 \%$ \\
\hline Total & 1.68 & 2.14 & 2.49 & 2.42 & 2.24 & 2.24 & 2.16 & 2.09 & 2.09 & 2.03 & 1.99 & 1.93 & $27.5 \%$ \\
\hline \multicolumn{14}{|c|}{ Total } \\
\hline Highway vehicle total & 7.39 & 8.65 & 8.62 & 8.09 & 7.04 & 7.37 & 7.44 & 7.51 & 7.67 & 7.32 & 7.25 & 7.04 & $100.0 \%$ \\
\hline Percent diesel & $22.7 \%$ & $24.8 \%$ & $28.9 \%$ & $30.0 \%$ & $31.8 \%$ & $30.4 \%$ & $29.1 \%$ & $27.9 \%$ & $27.3 \%$ & $27.7 \%$ & $27.4 \%$ & $27.5 \%$ & \\
\hline
\end{tabular}

Source:

U. S. Environmental Protection Agency, National Air Pollutant Emission Trends, 1900-1997, 1998, p. A-10 and annual. (Additional resources: http://www.epa.gov/oar/oaqps)

"The sums of subcategories may not equal total due to rounding.

${ }^{\mathrm{b}}$ Less than 8,500 pounds.

'Data are not available. 
The transportation sector accountedfor nearly $40 \%$ of the nation 's volatile organic compound (VOC) emissions in 1997, with the majority coming from highway vehicles. For details on the highway emissions of VOC, see Table 4.7.

Table 4.6

Total National Emissions of Volatile Organic Compounds, 1970-97” (million short tons)

\begin{tabular}{lrrrrrrr}
\hline & & & & & & & $\begin{array}{c}\text { Percent } \\
\text { of total, }\end{array}$ \\
Source category & 1970 & 1980 & 1990 & 1995 & 1996 & 1997 & 1997 \\
\hline \multicolumn{1}{c}{ Highway vehicles } & 12.97 & 8.98 & 6.31 & 5.70 & 5.49 & 5.23 & $27.2 \%$ \\
$\quad$ Off-highway & 1.71 & 2.14 & 2.50 & 2.41 & 2.40 & 2.43 & $12.6 \%$ \\
Transportation total & 14.69 & 11.12 & 8.82 & 8.11 & 7.89 & 7.66 & $39.9 \%$ \\
Stationary fuel combustion total & $\mathbf{0 . 7 2}$ & 1.05 & 1.01 & 1.07 & 1.08 & 0.86 & $4.5 \%$ \\
Industrial processes total & 12.33 & 12.10 & 9.01 & 9.71 & 9.05 & 9.39 & $48.9 \%$ \\
Waste disposal and recycling total & 1.98 & 0.76 & 0.99 & 1.07 & 0.43 & 0.45 & $2.3 \%$ \\
Miscellaneous total & $\mathbf{1 . 1 0}$ & 1.13 & 1.16 & 0.60 & 0.85 & 0.86 & $4.5 \%$ \\
Total of all sources & 30.82 & 26.17 & 20.98 & 20.56 & 19.29 & 19.22 & $\mathbf{1 0 0 . 0 \%}$ \\
\hline
\end{tabular}

\section{Source:}

U. S. Environmental Protection Agency, National Air Pollutant Emission Trends, 1900-1997, 1998, pp. A-1 1-A-17, and annual. (Additional resources: http://www.epa.gov/oar/oaqps)

Note:

Emission estimationmethodology changes indicated by shaded areas. Transportation methodologies changed in 1970, while all others changed in 1990.

"The sum of subcategories may not equal total due to rounding. The EPA's definition of volatile organic compounds excludes methane, ethane, and certain other nonphotochemically reactive organic compounds. 
Table 4.7

Emissions of Volatile Organic Compounds from Highway Vehicles, 1970-97"

(thousand short tons)

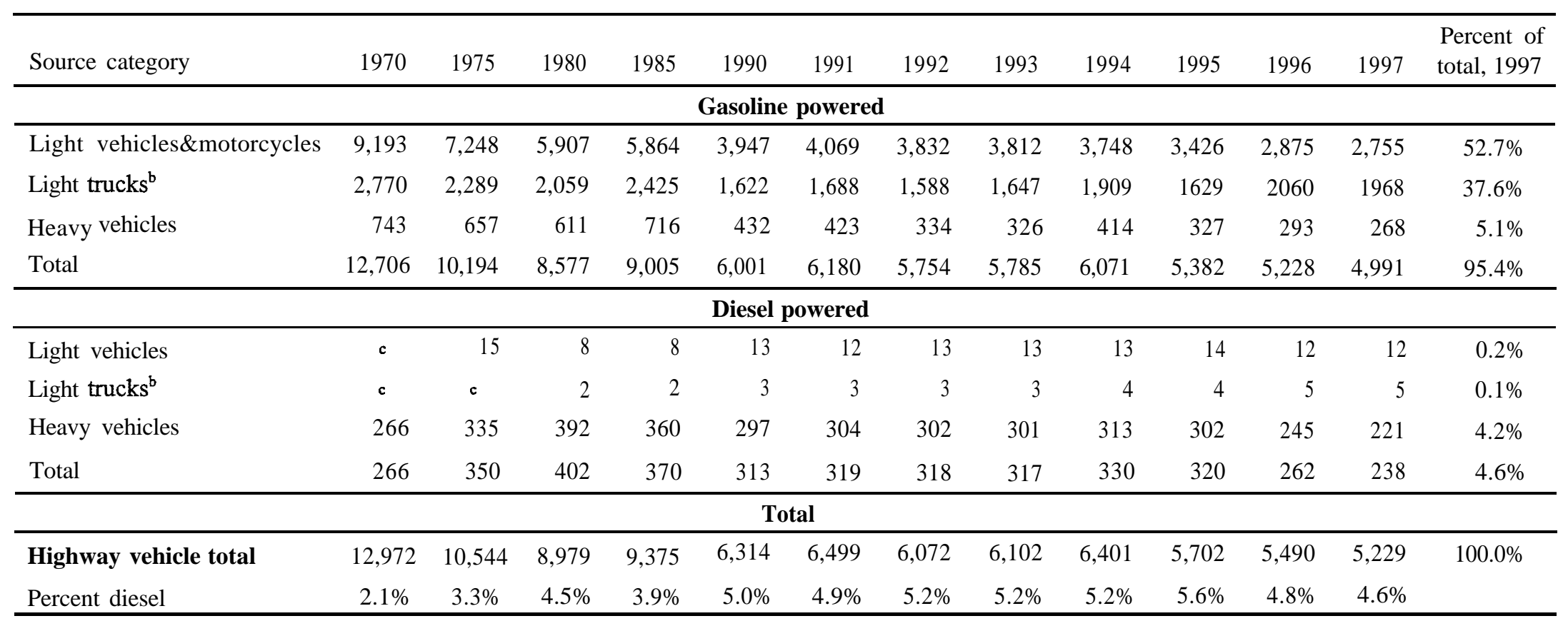

\section{Source:}

U. S. Environmental Protection Agency, National Air Pollutant Emission Trends, 1900-1997, 1998, p. A-16 and annual. (Additional resources: http://www.epa.gov/oar/oaqps)

\footnotetext{
"The sums of subcategories may not equal total due to rounding.

bess than 8,500 pounds.

'Data are not available.
} 
The transportation sector accounted for only $2 \%$ of the nation 's particulate matter (PM-1 0$)$ emissions in 1997. For details on the highway emissions of PM-IO, see Table 4.9.

Table 4.8

Total National Emissions of Particulate Matter (PM-lo), 1970-97ª (million short tons)

\begin{tabular}{lrrrrrrrr}
\hline & & & & & & \multicolumn{3}{c}{$\begin{array}{c}\text { Percent } \\
\text { of total, }\end{array}$} \\
Source category & 1970 & 1980 & 1990 & 1995 & 1996 & 1997 & 1997 \\
\hline \multicolumn{1}{c}{ Highway vehicles } & 0.44 & 0.40 & 0.34 & 0.29 & 0.27 & 0.27 & $0.8 \%$ \\
$\quad$ Off-highway & 0.26 & 0.57 & 0.50 & 0.46 & 0.46 & 0.47 & $1.4 \%$ \\
Transportation total & 0.70 & 0.96 & 0.83 & 0.75 & 0.73 & 0.73 & $2.2 \%$ \\
Stationary fuel combustion total & 2.87 & 2.45 & 1.20 & 1.18 & 1.19 & 1.10 & $3.3 \%$ \\
Industrial processes total & 7.67 & 2.75 & 1.04 & 0.95 & 0.94 & 0.98 & $2.9 \%$ \\
Waste disposal and recycling total & 1.00 & 0.27 & 0.27 & 0.29 & 0.29 & 0.30 & $0.9 \%$ \\
Miscellaneous total & 0.84 & 0.85 & 26.51 & 23.60 & 30.03 & 30.47 & $90.7 \%$ \\
Total of all sources & 13.08 & 7.29 & 29.84 & 26.76 & 33.19 & 33.58 & $\mathbf{1 0 0 . 0 \%}$ \\
\hline
\end{tabular}

\section{Source:}

U. S. Environmental Protection Agency, National Air Pollutant Emission Trends, 1900-1997, 1998, pp. A-22-A-26, and annual. (Additional resources: http:/www.epa.gov/oar/oaqps)

\section{Note:}

Emission estimation methodology changes indicated by shaded areas. Transportation methodologies changed in 1970, while all others changed in 1990.

"Fine particle matter less than 10 microns. The sums of subcategories may not equal total due to rounding. 
Table 4.9

Emissions of Particulate Matter (PM-10) from Highway Vehicles, 1970-97

(thousand short tons)

\begin{tabular}{|c|c|c|c|c|c|c|c|c|c|c|c|c|c|}
\hline Source category & 1970 & 1975 & 1980 & 1985 & 1990 & 1991 & 1992 & 1993 & 1994 & 1995 & 1996 & 1997 & $\begin{array}{l}\text { Percent of } \\
\text { total, } 1997\end{array}$ \\
\hline \multicolumn{14}{|c|}{ Gasoline powered } \\
\hline Light vehicles \& motorcycles & 22570 & 20772 & 12055 & 4377 & \multirow{2}{*}{\multicolumn{2}{|c|}{30613263}} & 3164 & 3165 & 3562 & 6232 & 4155 & 4056 & $21.0 \%$ \\
\hline Light trucks $^{\mathfrak{b}}$ & & & & & & & & & & & & & $15.0 \%$ \\
\hline Heavy vehicles & 13 & 15 & 15 & 14 & 10 & 10 & 9 & 10 & 10 & 9 & 9 & 9 & $3.4 \%$ \\
\hline Total & 308 & 294 & 190 & 134 & 101 & 105 & 104 & 106 & 107 & 103 & 105 & 105 & $39.3 \%$ \\
\hline \multicolumn{14}{|c|}{ Diesel powered } \\
\hline Light vehicles & c & 10 & 12 & 8 & 9 & 9 & 9 & 8 & 8 & 8 & 7 & 6 & $2.2 \%$ \\
\hline Light trucks ${ }^{b}$ & c & c & 2 & 1 & 1 & 2 & 2 & 2 & 2 & 2 & 2 & 2 & $0.7 \%$ \\
\hline Heavy vehicles & 136 & 166 & 194 & 219 & 224 & 234 & 228 & 205 & 204 & 181 & 168 & 154 & $57.7 \%$ \\
\hline Total & 136 & 177 & 209 & 228 & 235 & 245 & 239 & 215 & 213 & 190 & 177 & 163 & $60.7 \%$ \\
\hline \multicolumn{14}{|c|}{ Total } \\
\hline Highway vehicle total & 443 & 471 & 397 & 363 & 336 & 349 & 343 & 321 & 320 & 293 & 282 & 268 & $100.0 \%$ \\
\hline Percent diesel & $30.7 \%$ & $37.4 \%$ & $52.4 \%$ & $62.8 \%$ & $69.6 \%$ & $70.2 \%$ & $69.7 \%$ & $67.0 \%$ & $66.9 \%$ & $65.2 \%$ & $62.8 \%$ & $60.7 \%$ & \\
\hline
\end{tabular}

Source:

U. S. Enviromnental Protection Agency, National Air Pollutant Emission Trends, 19004997, 1998, p. A-25 and annual. (Additional resources: http://www.epa.gov/oar/oaqps)

${ }^{2}$ The sums of subcategories may not equal total due to rounding.

${ }^{b}$ Less than 8,500 pounds.

'Data are not available. 
The transportation sector accountedfor only $7 \%$ of the nation 's particulate matter (PM-2.5) emissions in 1997. For details on the highway emissions of PM-2.5, see Table 4.11.

Table 4.10

Total National Emissions of Particulate Matter (PM-2.5), 1990-97 (million short tons)

\begin{tabular}{|c|c|c|c|c|c|c|c|c|c|}
\hline Source category & 1990 & 1991 & 1992 & 1993 & 1994 & 1995 & 1996 & 1997 & $\begin{array}{c}\text { Percent } \\
\text { of total, } \\
1997\end{array}$ \\
\hline Highway vehicles & 0.28 & 0.29 & 0.28 & 0.26 & 0.26 & 0.23 & 0.22 & 0.21 & $2.5 \%$ \\
\hline Off-highway & 0.44 & 0.43 & 0.43 & 0.43 & 0.42 & 0.40 & 0.40 & 0.41 & $4.9 \%$ \\
\hline Transportation total & 0.71 & 0.72 & 0.71 & 0.68 & 0.68 & 0.63 & 0.62 & 0.62 & $7.4 \%$ \\
\hline Stationary fuel combustion total & 0.91 & 0.89 & 0.93 & 0.85 & 0.84 & 0.90 & 0.94 & 0.85 & $10.2 \%$ \\
\hline Industrial processes total & 0.56 & 0.57 & 0.58 & 0.50 & 0.50 & 0.50 & 0.50 & 0.52 & $6.3 \%$ \\
\hline Waste disposal and recycling total & 0.23 & 0.24 & 0.24 & 0.29 & 0.27 & 0.25 & 0.25 & 0.25 & $3.1 \%$ \\
\hline Miscellaneous total & 5.55 & 5.31 & 5.19 & 4.96 & 5.66 & 4.80 & 5.98 & 6.07 & $73.0 \%$ \\
\hline Total of all sources & 7.96 & 7.74 & 7.65 & 7.29 & 7.95 & 7.08 & 8.29 & 8.31 & $100.0 \%$ \\
\hline
\end{tabular}

Source:

U.S. Environmental Protection Agency, National Air Pollutant Emission Trends, 1900-1997, 1998, pp. A-27-A-31, and annual (Additional resources: http://www.epa.gov/oar/oaqps) 
Diesel vehicles are responsiblefor the majority ofhighway vehicle PM-2.5 emissions. Nearly two-thirds of the PM-2.5 emissions are from heavy diesel trucks.

Table 4.11

Emissions of Particulate Matter (PM-2.5) from Highway Vehicles, 1990-97ª (thousand short tons)

\begin{tabular}{|c|c|c|c|c|c|c|c|c|c|}
\hline Source category & 1990 & 1991 & 1992 & 1993 & 1994 & 1995 & 1996 & 1997 & $\begin{array}{c}\text { Percent } \\
\text { of total, } \\
1997\end{array}$ \\
\hline \multicolumn{10}{|c|}{ Gasoline powered } \\
\hline $\begin{array}{l}\text { Light vehicles \& } \\
\text { motorcycles }\end{array}$ & 37 & 38 & 38 & 38 & 36 & 36 & 32 & 32 & $15.5 \%$ \\
\hline Light trucks ${ }^{b}$ & 19 & 21 & 20 & 20 & 23 & 20 & 25 & 25 & $12.1 \%$ \\
\hline Heavy vehicles & 7 & 6 & 6 & 7 & 7 & 6 & 6 & 6 & $2.9 \%$ \\
\hline Total & 63 & 65 & 64 & 65 & 66 & 62 & 63 & 63 & $30.4 \%$ \\
\hline \multicolumn{10}{|c|}{ Diesel powered } \\
\hline Light vehicles & 8 & 8 & 8 & 7 & 7 & 7 & 6 & 6 & $2.9 \%$ \\
\hline Light trucks ${ }^{\mathrm{b}}$ & 1 & 1 & 2 & 1 & 2 & 2 & 2 & 2 & $1.0 \%$ \\
\hline Heavy vehicles & 203 & 212 & 206 & 183 & 182 & 161 & 149 & 136 & $65.7 \%$ \\
\hline Total & 212 & 221 & 216 & 192 & 190 & 169 & 157 & 144 & $69.6 \%$ \\
\hline \multicolumn{10}{|c|}{ Total } \\
\hline Highway vehicle total & 275 & 286 & 280 & 257 & 256 & 231 & 221 & 207 & $100.0 \%$ \\
\hline Percent diesel & $77.1 \%$ & $77.3 \%$ & $77.1 \%$ & $74.6 \%$ & $74.3 \%$ & $73.3 \%$ & $71.4 \%$ & $69.6 \%$ & \\
\hline
\end{tabular}

\section{Source:}

U.S. Environmental Protection Agency, National Air Pollutant Emission Trends, 1900-1997, 1998, p. A-30 and annual. (Additional resources: http://www.epa.gov/oar/oaqps)

a The sums of subcategories may not equal total due to rounding.

${ }^{\mathbf{b}}$ Less than 8,500 pounds. 
Historically the transportation sector, highway vehicles in particular, have been a major source of lead emissions in the U.S. Regulatory action in 1978 required a gradual reduction of the lead content of all gasoline over a period of many years. The transportation sector accounts for only $13 \%$ of lead emissions in 1997.

Table 4.12

National Lead Emission Estimates, 1970-97" (thousand short tons per year)

\begin{tabular}{|c|c|c|c|c|c|c|c|c|c|}
\hline Source category & 1970 & 1975 & 1980 & 1985 & 1990 & 1995 & 1996 & 1997 & $\begin{array}{c}\text { Percent } \\
\text { of total, } \\
1997 \\
\end{array}$ \\
\hline Highway vehicles & 171.96 & 130.21 & 60.50 & 18.05 & 0.42 & 0.02 & 0.02 & 0.02 & $0.5 \%$ \\
\hline Off-highway & 9.74 & 6.13 & 4.21 & 0.92 & 0.78 & 0.54 & 0.51 & 0.50 & $12.8 \%$ \\
\hline Transportation total & 181.70 & 136.34 & 64.71 & 18.97 & 1.20 & 0.56 & 0.53 & 0.52 & $13.3 \%$ \\
\hline Stationary source fuel combustion & 10.62 & 10.35 & 4.30 & 0.52 & 0.50 & 0.49 & 0.49 & 0.50 & $12.7 \%$ \\
\hline Industrial processes & 26.36 & 11.38 & 3.94 & 2.53 & 2.47 & 2.27 & 2.27 & 2.25 & $57.5 \%$ \\
\hline Waste disposal and recycling total & 2.20 & 1.60 & 1.21 & 0.87 & 0.80 & 0.60 & 0.62 & 0.65 & $16.5 \%$ \\
\hline Total of all sources & 220.87 & 159.66 & 74.15 & 22.89 & 4.98 & 3.92 & 3.91 & 3.92 & $100.0 \%$ \\
\hline
\end{tabular}

\section{Source:}

U. S. Environmental Protection Agency, National Air Pollutant Emission Trends, 1900-1997, 1998, pp. A-34-A-35, and annual. (Additional resources: http://www.epa.gov/oar/oaqps)

${ }^{2}$ The sums of subcategories may not equal due to rounding. 
Table 4.13

State-level Emissions for Criteria Pollutants, 1997 (thousand short tons)

\begin{tabular}{|c|c|c|c|c|c|}
\hline State & $\begin{array}{l}\text { Carbon } \\
\text { monoxide }\end{array}$ & $\begin{array}{c}\text { Nitrogen } \\
\text { oxides" }\end{array}$ & $\begin{array}{c}\text { Volatile } \\
\text { organic } \\
\text { compounds" }\end{array}$ & Sulfur dioxide & $\begin{array}{c}\text { Particulate } \\
\text { matter } \\
(\text { PM-1 0) }\end{array}$ \\
\hline Alabama & 2,392 & 627 & 427 & 811 & 585 \\
\hline Alaska & 486 & 42 & 64 & 5 & 183 \\
\hline Arizona & 1,627 & 453 & 297 & 256 & 302 \\
\hline Arkansas & 1,141 & 257 & 240 & 138 & 500 \\
\hline California & 6,000 & 1,236 & 1,494 & 200 & 1,600 \\
\hline Colorado & 1,259 & 414 & 293 & 141 & 476 \\
\hline Connecticut & 747 & 153 & 165 & 90 & 101 \\
\hline District of Columbia & 111 & 19 & 21 & 9 & 4 \\
\hline Delaware & 207 & 68 & 53 & 98 & 38 \\
\hline Florida & 4,610 & 916 & 859 & 879 & 764 \\
\hline Georgia & 3,917 & 691 & 595 & 639 & 1,017 \\
\hline Hawaii & 212 & 49 & 30 & 34 & 33 \\
\hline Idaho & 811 & 114 & 116 & 41 & 690 \\
\hline Illinois & 3,046 & 1,129 & 851 & 1,190 & 1,007 \\
\hline Indiana & 2,384 & 912 & 567 & 1,370 & 660 \\
\hline Iowa & 997 & 329 & 257 & 273 & 580 \\
\hline Kansas & 2,127 & 528 & 414 & 180 & 1,639 \\
\hline Kentucky & 1,412 & 690 & 406 & 806 & 336 \\
\hline Louisiana & 2.316 & 758 & 437 & 414 & 440 \\
\hline Maine & 529 & 95 & 109 & 101 & 156 \\
\hline Maryland & 1,160 & 331 & 243 & 387 & 208 \\
\hline Massachusetts & 1,212 & 275 & 294 & 255 & 285 \\
\hline Michigan & 2,996 & 839 & 705 & 653 & 530 \\
\hline Minnesota & 1,476 & 463 & 398 & 168 & 962 \\
\hline Mississippi & 1,565 & 351 & 339 & 235 & 479 \\
\hline Missouri & 2,002 & 523 & 444 & 506 & 1,350 \\
\hline Montana & 768 & 183 & 110 & 67 & 1,143 \\
\hline Nebraska & 785 & 252 & 205 & 102 & 632 \\
\hline Nevada & 545 & 135 & 98 & 65 & 150 \\
\hline New Hampshire & 359 & 80 & 77 & 164 & 54 \\
\hline New Jersey & 1,362 & 435 & 425 & 265 & 303 \\
\hline New Mexico & 938 & 297 & 152 & 207 & 4,948 \\
\hline New York & 3,116 & 667 & 767 & 663 & 818 \\
\hline N. Carolina & 2,759 & 643 & 685 & 610 & 480 \\
\hline N. Dakota & 317 & 239 & 99 & 308 & 412 \\
\hline Ohio & 3,812 & 1,185 & 709 & 1,966 & 663 \\
\hline Oklahoma & 1,733 & 470 & 350 & 239 & 999 \\
\hline Oregon & 1,758 & 215 & 258 & 44 & 661 \\
\hline Pennsylvania & 3,332 & 935 & 674 & 1,349 & 593 \\
\hline Rhode Island & 203 & 31 & 50 & 13 & 27 \\
\hline S. Carolina & 1,606 & 364 & 340 & 299 & 410 \\
\hline S. Dakota & 317 & 120 & 78 & 57 & 311 \\
\hline Tennessee & 2,391 & 797 & 610 & 840 & 384 \\
\hline Texas & 6,479 & 1,843 & 1,615 & 1,151 & 3,307 \\
\hline Utah & 1,029 & 233 & 170 & 83 & 248 \\
\hline Vermont & 232 & 43 & 48 & 17 & 79 \\
\hline Virginia & 2,082 & 564 & 492 & 486 & 445 \\
\hline Washington & 2,062 & 325 & 431 & 150 & 392 \\
\hline W. Virginia & 843 & 516 & 157 & 759 & 158 \\
\hline Wisconsin & 1,517 & 469 & 418 & 408 & 381 \\
\hline Wyoming & 363 & 275 & 68 & 179 & 659 \\
\hline Total & 87,451 & 23,575 & 19,204 & 20,369 & 33,581 \\
\hline
\end{tabular}

\section{Source:}

U.S. Environmental Protection Agency, National Air Pollutant Emission Trends, 19004997, 1998, p. A-38. Note: (Additional resources: http://www.epa.gov/oar/oaqps)

The sums of the States may not equal national totals due to rounding. 


\section{The Greenhouse Gases, Regulated Emissions, and Energy Use in Transportation (GREET) Model}

The energy and criteria pollutant estimates of the most recent version (Version 1.4) of the GREET model are displayed in the next two tables. The model estimates the full fuel-cycle emissions and energy use associated with various transportation fuels and advanced transportation technologies for light vehicles. It calculates fuel-cycle emissions of five criteria pollutants (volatile organic compounds, carbon monoxide, nitrogen oxides, sulfur oxides, and particulate matter measuring 10 microns or less) and three greenhouse gases (carbon dioxide, methane, and nitrous oxide). See Chapter 3 for the greenhouse gas data from GREET. The model also calculates the total fuel-cycle energy consumption, fossil fuel consumption, and petroleum consumption using various transportation fuels. The fuel cycles that are included in the GREET model are:

- petroleum to conventional gasoline, reformulated gasoline, conventional diesel, reformulated diesel, liquefied petroleum gas, and electricity via residual oil;

- natural gas to compressed natural gas, liquefied natural gas, liquefied petroleum gas, methanol, Fischer-Tropsch diesel, dimethyl ether, hydrogen, and electricity;

- coal to electricity;

,. uranium to electricity;

- renewable energy (hydropower, solar energy, and wind) to electricity;

- corn, woody biomass, and herbaceous biomass to ethanol;

. $\quad$ soybeans to biodiesel; and

- landfill gases to methanol.

Near-term technologies are ones which may be applied to 2000 model-year cars andlong-term technologies are ones which may be applied to 2010 model-year cars.

For additional information about the GREET model, contact:

Michael Q. Wang

Argonne National Laboratory

9700 South Cass Avenue, ES/362

Argonne, IL 60439-48 15

phone: 630-252-28 19

fax: $630-252-3443$

email: michael_wang@qmgate.anl.gov 


\section{Acronyms Used on Tables 4.14 and 4.15}

\section{Emissions acronyms}

v o c volatile organic compounds

c o carbon monoxide

NOx nitrogen oxides

PM10 particulate matter measuring 10 microns or less

s o x sulfur oxides

\section{, Technologies acronyms}

BD20 mixture of $20 \%$ biodiesel and $80 \%$ conventional diesel (by volume)

CARFG2 California Phase 2 reformulated gasoline

CD conventional diesel

CIDI compression ignition, direct injection

CNG compressed natural gas

CNGV compressed natural gas vehicle

Dedi. dedicated

DME dimethyl ether

E10 mixture of $10 \%$ ethanol and $90 \%$ gasoline (by volume)

E85 mixture of $85 \%$ ethanol and $15 \%$ gasoline (by volume)

E90 mixture of $90 \%$ ethanol and $10 \%$ gasoline (by volume)

ETBE ethyl tertiary butyl ether

EtOH ethanol

EtOHV ethanol vehicle

EV electric vehicle

FCV fuel-cell vehicle

FFV flexible fuel vehicle

FRFG2 federal Phase 2 reformulated gasoline

FG flared gas

FT50 mixture of 50\% Fischer-Tropsch diesel and 50\% conventional diesel (by volume)

GC grid-connected

GI grid-independent

GHGs greenhouse gases

GV gasoline vehicle

$\mathrm{H}_{2} \quad$ hydrogen

HB herbaceous biomass

HEV hybrid electric vehicle

LFG land-fill gas

LNG liquefied natural gas

LNGV liquefied natural gas vehicle

LPG liquefied petroleum gas

LPGV liquefied petroleum gas vehicle

M85 mixture of $85 \%$ methanol and $15 \%$ gasoline by volume

M90 mixture of $90 \%$ methanol and $10 \%$ gasoline by volume

$\mathrm{MeOH}$ methanol

$\mathrm{MeOHV}$ methanol vehicle

MTBE methyl tertiary butyl ether

NE northeast

NG natural gas

RFD reformulated diesel

SI spark ignition

SIDI spark-ignition, direct-injection

WB woody biomass

TRANSPORTATION ENERGY DATA BOOK: EDITION 19-1999 


\section{Table 4.14}

\section{Near-Term Technology}

Changes in Per-Mile, Fuel-Cycle Energy Use and Criteria Pollutant Emissions for Passenger Cars (Percentage relative to conventional gasoline vehicles fueled with conventional gasoline)

\begin{tabular}{|c|c|c|c|c|c|c|c|c|c|}
\hline & $\begin{array}{c}\text { GV: } \\
\text { FRFG2, } \\
\text { MTBE }\end{array}$ & $\begin{array}{c}\text { GV: } \\
\text { CARFG2, } \\
\text { ETBE } \\
\end{array}$ & $\begin{array}{c}\mathrm{GV}: \\
\text { CARFG2, } \\
\text { EtOH }\end{array}$ & $\begin{array}{c}\text { CIDI: } \\
\text { CD }\end{array}$ & $\begin{array}{l}\text { Bi-fuel } \\
\text { CNGV }\end{array}$ & $\begin{array}{l}\text { Dedi. } \\
\text { CNGV }\end{array}$ & $\begin{array}{c}\text { Dedi } \\
\text { LPGV: } \\
\text { crude }\end{array}$ & $\begin{array}{c}\text { Dedi. } \\
\text { LPGV: } \\
\text { NG }\end{array}$ & $\begin{array}{c}\text { M85 FFV: } \\
\text { NG }\end{array}$ \\
\hline Total energy & $0.0 \%$ & $0.8 \%$ & $0.4 \%$ & $-29.7 \%$ & $6.7 \%$ & $3.3 \%$ & $-8.6 \%$ & $-9.6 \%$ & $14.5 \%$ \\
\hline Fossil fuels & $0.0 \%$ & $-3.1 \%$ & $-2.6 \%$ & $-29.6 \%$ & $5.7 \%$ & $2.3 \%$ & $-8.6 \%$ & $-9.3 \%$ & $15.0 \%$ \\
\hline Petroleum & $-11.0 \%$ & $-10.1 \%$ & $-2.5 \%$ & $-26.7 \%$ & $-99.4 \%$ & $-99.4 \%$ & $-3.4 \%$ & $-98.2 \%$ & $-72.6 \%$ \\
\hline VOC: Total & $-15.1 \%$ & $-5.5 \%$ & $8.6 \%$ & $-60.9 \%$ & $-48.7 \%$ & $-71.2 \%$ & $-58.2 \%$ & $-64.1 \%$ & $-19.8 \%$ \\
\hline VOC: Urban & $-19.7 \%$ & $-19.4 \%$ & $5.3 \%$ & $-62.8 \%$ & $-45.8 \%$ & $-74.8 \%$ & $-63.1 \%$ & $-60.3 \%$ & $-20.3 \%$ \\
\hline CO: Total & $-19.1 \%$ & $-18.9 \%$ & $-19.4 \%$ & $-79.5 \%$ & $-34.8 \%$ & $-42.7 \%$ & $-39.3 \%$ & $-39.7 \%$ & $-37.4 \%$ \\
\hline CO: Urban & $-20.0 \%$ & $-19.9 \%$ & $-20.0 \%$ & $-80.5 \%$ & $-35.5 \%$ & $-43.5 \%$ & $-39.9 \%$ & $-39.9 \%$ & $-40.0 \%$ \\
\hline NOx: Total & $0.4 \%$ & $14.6 \%$ & $2.6 \%$ & $51.8 \%$ & $26.6 \%$ & $18.6 \%$ & $-17.7 \%$ & $-22.4 \%$ & $0.5 \%$ \\
\hline NOx: Urban & $-4.2 \%$ & $-2.9 \%$ & $-4.2 \%$ & $111.4 \%$ & $25.2 \%$ & $14.7 \%$ & $-9.1 \%$ & $-9.3 \%$ & $-12.0 \%$ \\
\hline PM10: Total & $-1.8 \%$ & $78.2 \%$ & $38.5 \%$ & $154.8 \%$ & $-35.9 \%$ & $-37.8 \%$ & $-34.1 \%$ & $-43.0 \%$ & $-26.5 \%$ \\
\hline PM10: Urban & $-1.4 \%$ & $-0.4 \%$ & $-1.3 \%$ & $258.5 \%$ & $-30.4 \%$ & $-32.3 \%$ & $-31.3 \%$ & $-31.3 \%$ & $-22.8 \%$ \\
\hline SOx: Total & $-28.9 \%$ & $-0.4 \%$ & $-16.3 \%$ & $-31.4 \%$ & $-37.5 \%$ & $-39.5 \%$ & $-57.3 \%$ & $-77.4 \%$ & $-58.7 \%$ \\
\hline SOx: Urban & $-82.7 \%$ & $-82.8 \%$ & $-83.0 \%$ & $-3.9 \%$ & $-96.1 \%$ & $-96.2 \%$ & $-98.0 \%$ & $-98.1 \%$ & $-73.7 \%$ \\
\hline
\end{tabular}

Table continued on next page. See previous pages for acronym definitions. 
Table 4.14 (continued)

Near-Term Technology

Changes in Per-Mile, Fuel-Cycle Energy Use and Criteria Pollutant Emissions for Passenger Cars

(Percentage relative to conventional gasoline vehicles fueled with conventional gasoline)

\begin{tabular}{|c|c|c|c|c|c|c|c|c|c|}
\hline & $\begin{array}{c}\text { E10 GV: } \\
\text { corn }\end{array}$ & $\begin{array}{c}\text { E85 FFV: } \\
\text { corn }\end{array}$ & $\begin{array}{c}\text { E85 FFV: } \\
\text { WB }\end{array}$ & $\begin{array}{c}\text { E85 FFV: } \\
\text { HB }\end{array}$ & $\begin{array}{c}\text { EV: } \\
\text { US mix }\end{array}$ & $\begin{array}{c}\text { EV: } \\
\text { NE mix }\end{array}$ & $\begin{array}{c}\text { EV: } \\
\text { CA mix }\end{array}$ & $\begin{array}{l}\text { GC SIDI } \\
\text { HEV: } \\
\text { ETBE, } \\
\text { CA mix }\end{array}$ & $\begin{array}{c}\text { GC SIDI } \\
\text { HEV: } \\
\text { EtOH, } \\
\text { CA mix }\end{array}$ \\
\hline Total energy & $2.1 \%$ & $17.9 \%$ & $90.4 \%$ & $79.1 \%$ & $-14.7 \%$ & $-14.0 \%$ & $-16.9 \%$ & $-35.9 \%$ & $-36.0 \%$ \\
\hline Fossil fuels & $-3.4 \%$ & $-41.8 \%$ & $-81.8 \%$ & $-72.7 \%$ & $-34.7 \%$ & $-46.0 \%$ & $-68.6 \%$ & $-52.9 \%$ & $-52.7 \%$ \\
\hline Petroleum & $-6.3 \%$ & $-73.8 \%$ & $-71.2 \%$ & $-73.2 \%$ & $-98.4 \%$ & $-96.9 \%$ & $-99.5 \%$ & $-64.9 \%$ & $-62.0 \%$ \\
\hline VOC: Total & $14.4 \%$ & $54.0 \%$ & $4.5 \%$ & $0.4 \%$ & $-88.0 \%$ & $-90.6 \%$ & $-95.2 \%$ & $-48.2 \%$ & $-36.7 \%$ \\
\hline VOC: Urban & $10.7 \%$ & $-16.0 \%$ & $-16.1 \%$ & $-16.1 \%$ & $-99.6 \%$ & $-99.4 \%$ & $-99.5 \%$ & $-50.8 \%$ & $-33.3 \%$ \\
\hline CO: Total & $-35.0 \%$ & $-37.4 \%$ & $-30.3 \%$ & $-31.7 \%$ & $-98.0 \%$ & $-98.0 \%$ & $-98.7 \%$ & $-54.7 \%$ & $-54.9 \%$ \\
\hline CO: Urban & $-35.9 \%$ & $-39.8 \%$ & $-39.8 \%$ & $-39.8 \%$ & $-99.9 \%$ & $-99.9 \%$ & $-99.9 \%$ & $-55.2 \%$ & $-55.2 \%$ \\
\hline NOx: Total & $9.9 \%$ & $101.9 \%$ & $125.7 \%$ & $139.8 \%$ & $64.5 \%$ & $11.5 \%$ & $-51.3 \%$ & $-21.6 \%$ & $-26.3 \%$ \\
\hline NOx: Urban & $0.8 \%$ & $-1.1 \%$ & $-2.4 \%$ & $-1.7 \%$ & $-94.7 \%$ & $-90.9 \%$ & $-93.2 \%$ & $-28.6 \%$ & $-29.1 \%$ \\
\hline PM10: Total & $57.2 \%$ & $615.2 \%$ & $139.9 \%$ & $124.4 \%$ & $48.5 \%$ & $10.5 \%$ & $-31.7 \%$ & $13.3 \%$ & $-2.1 \%$ \\
\hline PM10: Urban & $0.6 \%$ & $-15.0 \%$ & $-15.5 \%$ & $-15.2 \%$ & $-35.4 \%$ & $-33.4 \%$ & $-34.7 \%$ & $-5.8 \%$ & $-6.1 \%$ \\
\hline SOx: Total & $15.7 \%$ & $168.8 \%$ & $-151.8 \%$ & $-95.8 \%$ & $462.8 \%$ & $217.2 \%$ & $-13.9 \%$ & $-35.4 \%$ & $-41.6 \%$ \\
\hline SOx: Urban & $-6.7 \%$ & $-78.6 \%$ & $-79.1 \%$ & $-78.9 \%$ & $-96.9 \%$ & $-90.7 \%$ & $-99.0 \%$ & $-93.0 \%$ & $-93.1 \%$ \\
\hline
\end{tabular}

Source:

Wang, Michael Q., GREET Model Results, Argonne National Laboratory, Argonne, IL, August 1999.

Note:

See page preceding table for acronym definitions. 


\section{Table 4.15 \\ Long-Term Technology}

Changes in Per-Mile, Fuel-Cycle Energy Use and Criteria Pollutant Emissions for Passenger Cars

(Percentage relative to conventional gasoline vehicles fueled with conventional gasoline)

\begin{tabular}{|c|c|c|c|c|c|c|c|c|c|c|c|}
\hline & $\begin{array}{l}\text { Dedi. } \\
\text { CNGV }\end{array}$ & $\begin{array}{l}\text { Dedi. } \\
\text { LNGV }\end{array}$ & $\begin{array}{l}\text { Dedi. } \\
\text { LPGV: } \\
\text { crude }\end{array}$ & $\begin{array}{l}\text { Dedi. } \\
\text { LPGV: } \\
\text { NG }\end{array}$ & $\begin{array}{c}\text { Dedi. } \\
\text { MeOHV: } \\
\text { M90, NG }\end{array}$ & $\begin{array}{c}\text { Dedi. } \\
\text { MeOHV: } \\
\text { M90, FG }\end{array}$ & $\begin{array}{c}\text { Dedi. } \\
\text { MeOHV: } \\
\text { M90, } \\
\text { LFG }\end{array}$ & $\begin{array}{r}\text { Dedi. } \\
\text { EtOHV: } \\
\text { E90, corn }\end{array}$ & $\begin{array}{l}\text { Dedi. } \\
\text { EtOHV: } \\
\text { E90, WB }\end{array}$ & $\begin{array}{c}\text { Dedi. } \\
\text { EtOHV: } \\
\text { E90, HB }\end{array}$ & $\begin{array}{r}\text { SIDI: } \\
\text { FRFG2, } \\
\text { MTBE }\end{array}$ \\
\hline Total energy & $-8.6 \%$ & $-5.7 \%$ & $-16.8 \%$ & $-17.7 \%$ & $8.8 \%$ & $13.8 \%$ & $-1.8 \%$ & $10.0 \%$ & $57.7 \%$ & $44.4 \%$ & $-20.0 \%$ \\
\hline Fossil fuels & $-9.5 \%$ & $-5.1 \%$ & $-16.9 \%$ & $-17.4 \%$ & $9.4 \%$ & $-45.4 \%$ & $-61.0 \%$ & $-50.1 \%$ & $-82.2 \%$ & $-78.2 \%$ & $-20.0 \%$ \\
\hline Petroleum & $-99.4 \%$ & $960 \%$ & $-1.3 \%$ & $\%$ & $-78.1 \%$ & $-78.1 \%$ & $-78.7 \%$ & $-81.5 \%$ & $-79.3 \%$ & $-81.1 \%$ & $-20.0 \%$ \\
\hline VOC: Total & $-62.6 \%$ & $-55.8 \%$ & $-48.6 \%$ & $-56.2 \%$ & $-15.8 \%$ & $-156.0 \%$ & $-35.9 \%$ & $74.4 \%$ & $4.9 \%$ & $-0.1 \%$ & $-11.0 \%$ \\
\hline VOC: Urban & $-55.1 \%$ & $-56.8 \%$ & $-49.0 \%$ & $-45.3 \%$ & $-11.1 \%$ & $-11.1 \%$ & $-15.0 \%$ & $-8.9 \%$ & $-9.0 \%$ & $-9.0 \%$ & $-7.3 \%$ \\
\hline CO: Total & $-19.9 \%$ & $-18.2 \%$ & $-20.6 \%$ & $-21.3 \%$ & $1.5 \%$ & $-0.9 \%$ & $-14.9 \%$ & $2.1 \%$ & $11.7 \%$ & $9.5 \%$ & $-1.0 \%$ \\
\hline CO: Urban & $-19.4 \%$ & $-20.1 \%$ & $-19.9 \%$ & $-20.0 \%$ & $-0.2 \%$ & $-0.2 \%$ & $-5.6 \%$ & $0.0 \%$ & $0.0 \%$ & $.0 \%$ & $-0.1 \%$ \\
\hline NOx: Total & $26.5 \%$ & 67.79 & $-32.1 \%$ & $-39.9 \%$ & $-4.8 \%$ & $-74.3 \%$ & $-24.7 \%$ & $147.0 \%$ & $223.2 \%$ & $228.4 \%$ & $-16.6 \%$ \\
\hline NOx: Urban & $102.8 \%$ & $-11.5 \%$ & $-2.8 \%$ & $-3.7 \%$ & $-17.2 \%$ & $-17.2 \%$ & $-48.5 \%$ & $9.4 \%$ & $4.9 \%$ & $6.2 \%$ & $-5.2 \%$ \\
\hline PM10: Total & $-34.2 \%$ & $-30.4 \%$ & $-31.1 \%$ & $-39.0 \%$ & $-25.4 \%$ & $-36.7 \%$ & $-265.9 \%$ & $409.1 \%$ & $59.4 \%$ & $46.8 \%$ & $1.5 \%$ \\
\hline PM10: Urban & $-24.6 \%$ & $-26.0 \%$ & $-25.1 \%$ & $-25.2 \%$ & $-14.5 \%$ & $-14.5 \%$ & $-197.8 \%$ & $-12.2 \%$ & $-12.4 \%$ & $-12.3 \%$ & $11.9 \%$ \\
\hline SOx: Total & $-34.1 \%$ & $-76.7 \%$ & $-48.4 \%$ & $-71.8 \%$ & $-60.0 \%$ & $-60.4 \%$ & $50.8 \%$ & $120.0 \%$ & $-90.2 \%$ & $-70.0 \%$ & $-20.0 \%$ \\
\hline SOx: Urban & $-80.6 \%$ & $-98.3 \%$ & $-91.3 \%$ & $-91.5 \%$ & $-77.9 \%$ & $-77.9 \%$ & $-234.2 \%$ & $-82.9 \%$ & $-83.7 \%$ & $-83.3 \%$ & $-20.0 \%$ \\
\hline
\end{tabular}

Table continued on next page. See page preceding Table 4.14 for acronym definitions. 
Table 4.15 (continued)

Long-Term Technology

Changes in Per-Mile, Fuel-Cycle Energy Use and Criteria Pollutant Emissions for Passenger Cars

(Percentage relative to conventional gasoline vehicles fueled with conventional gasoline)

\begin{tabular}{|c|c|c|c|c|c|c|c|c|c|c|c|}
\hline & $\begin{array}{c}\text { SIDI : } \\
\text { FRFG2, } \\
\text { ETBE }\end{array}$ & $\begin{array}{c}\text { SIDI: } \\
\text { FRFG2 } \\
\text { EtOH }\end{array}$ & $\begin{array}{c}\text { SIDI: } \\
\text { CAR FG2, } \\
\text { ETBE }\end{array}$ & $\begin{array}{c}\text { SIDI: } \\
\text { CARFG2, } \\
\text { EtOH }\end{array}$ & $\begin{array}{c}\text { Dedi. } \\
\text { MeOH } \\
\text { SIDI: } \\
\text { NG }\end{array}$ & $\begin{array}{c}\text { Dedi. } \\
\text { MeOH } \\
\text { SDI: } \\
\text { M90, FG }\end{array}$ & $\begin{array}{c}\text { Dedi. } \\
\text { MeOH } \\
\text { SIDI: } \\
\text { M90, } \\
\text { LFG }\end{array}$ & $\begin{array}{c}\text { Dedi } \\
\text { EtOH } \\
\text { SIDI: } \\
\text { E90, corn }\end{array}$ & $\begin{array}{c}\text { Dedi. } \\
\text { EtOH } \\
\text { SIDI: } \\
\text { E90, WB }\end{array}$ & $\begin{array}{c}\text { Dedi. } \\
\text { EtOH } \\
\text { SIDI: } \\
\text { E90, HB }\end{array}$ & $\begin{array}{l}\text { GI SIDI } \\
\text { HEV: } \\
\text { FRFG2, } \\
\text { MTBE }\end{array}$ \\
\hline$\overline{\text { Total }}$ & $-20.0 \%$ & $-20.0 \%$ & $-20.0 \%$ & $-20.0 \%$ & $-6.7 \%$ & $-2.3 \%$ & $-16.1 \%$ & $-3.2 \%$ & $38.7 \%$ & $27.1 \%$ & $-47.4 \%$ \\
\hline Fossil fuels & $-20.0 \%$ & $-20.0 \%$ & $-19.0 \%$ & $-19.3 \%$ & $-6.2 \%$ & $-68.4 \%$ & $-68.1 \%$ & $-56.1 \%$ & $-84.4 \%$ & $-80.8 \%$ & $-47.4 \%$ \\
\hline Petro & $-20.0 \%$ & $-20.0 \%$ & $-17.2 \%$ & $-14.2 \%$ & $-82.1 \%$ & $-82.1 \%$ & $-82.6 \%$ & $-83.7 \%$ & $-81.8 \%$ & $-83.4 \%$ & $-47.4 \%$ \\
\hline VOC: & $-12.0 \%$ & $-8.7 \%$ & $-12.0 \%$ & $-8.7 \%$ & $-24.6 \%$ & $-148.0 \%$ & $-42.3 \%$ & $57.4 \%$ & $-3.8 \%$ & $-8.1 \%$ & $-28.0 \%$ \\
\hline VOC: Urban & $-7.4 \%$ & $-3.0 \%$ & $-7.4 \%$ & $-3.0 \%$ & $-15.8 \%$ & $-15.8 \%$ & $-19.3 \%$ & $-13.9 \%$ & $-14.0 \%$ & $-14.0 \%$ & $-32.2 \%$ \\
\hline CO: & $-1.1 \%$ & $-0.9 \%$ & $-1.1 \%$ & $-0.9 \%$ & $0.4 \%$ & $-1.8 \%$ & $-14.0 \%$ & $1.3 \%$ & $9.8 \%$ & $7.8 \%$ & $-2.4 \%$ \\
\hline CO: Urban & $-0.1 \%$ & $-0.1 \%$ & $-0.1 \%$ & $-0.1 \%$ & $-0.2 \%$ & $-0.2 \%$ & $-5.0 \%$ & $0.0 \%$ & $0.0 \%$ & $0.0 \%$ & $-0.1 \%$ \\
\hline NOx: & $-17.2 \%$ & $-16.7 \%$ & $-17.2 \%$ & $-16.7 \%$ & $-22.5 \%$ & $-83.6 \%$ & $-39.9 \%$ & $119.3 \%$ & $186.4 \%$ & $191.0 \%$ & $-39.3 \%$ \\
\hline NOx: & $-6.2 \%$ & $-5.3 \%$ & $-6.2 \%$ & $-5.3 \%$ & $-18.3 \%$ & $-18.3 \%$ & $-45.8 \%$ & $5.3 \%$ & $1.3 \%$ & $2.5 \%$ & $-12.4 \%$ \\
\hline PM10: Total & $-7.7 \%$ & $-4.4 \%$ & $-7.7 \%$ & $-4.4 \%$ & $-23.1 \%$ & $-33.1 \%$ & $-234.7 \%$ & $359.2 \%$ & $51.4 \%$ & $40.3 \%$ & $-12.2 \%$ \\
\hline PM10: Urban & $11.6 \%$ & $11.9 \%$ & $11.6 \%$ & $11.9 \%$ & $-2.1 \%$ & $-2.1 \%$ & $-163.4 \%$ & $0.0 \%$ & $-0.2 \%$ & $-0.1 \%$ & $4.8 \%$ \\
\hline SOx: Total & $-20.0 \%$ & $-20.0 \%$ & $-20.0 \%$ & $-20.0 \%$ & $-76.3 \%$ & $-76.7 \%$ & $21.2 \%$ & $93.6 \%$ & $-91.4 \%$ & $-73.6 \%$ & $-47.4 \%$ \\
\hline SOx: Urban & $-20.0 \%$ & $-20.0 \%$ & $-20.5 \%$ & $-20.5 \%$ & $-80.6 \%$ & $-80.6 \%$ & $-218.1 \%$ & $-85.0 \%$ & $-85.7 \%$ & $-85.3 \%$ & $-47.4 \%$ \\
\hline
\end{tabular}

Table continued on next page. See page preceding Table 4.14 for acronym definitions. 


\section{Table 4.15 (continued)}

\section{Long-Term Technology}

Changes in Per-Mile, Fuel-Cycle Energy Use and Criteria Pollutant Emissions for Passenger Cars (Percentage relative to conventional gasoline vehicles fueled with conventional gasoline)

\begin{tabular}{|c|c|c|c|c|c|c|c|c|c|c|c|}
\hline & $\begin{array}{l}\text { GI SIDI } \\
\text { HEV: } \\
\text { FRFG } 2, \\
\text { ETBE }\end{array}$ & $\begin{array}{l}\text { GI SIDI } \\
\text { HEV: } \\
\text { FRFG2, } \\
\text { EtOH }\end{array}$ & $\begin{array}{l}\text { GI SI } \\
\text { HEV: } \\
\text { CNG }\end{array}$ & $\begin{array}{l}\text { GI SI } \\
\text { HEV: } \\
\text { LNG }\end{array}$ & $\begin{array}{c}\text { GI SI } \\
\text { HEVs: } \\
\text { LPG, } \\
\text { crude }\end{array}$ & $\begin{array}{c}\text { GI SI } \\
\text { HEVs: } \\
\text { LPG, NG }\end{array}$ & $\begin{array}{l}\text { GI SIDI } \\
\text { HEVs: } \\
\text { M90, NG }\end{array}$ & $\begin{array}{l}\text { GI SIDI } \\
\text { HEVs: } \\
\text { M90, FG }\end{array}$ & $\begin{array}{l}\text { GI SIDI } \\
\text { HEVs: } \\
\text { M90 LFG }\end{array}$ & $\begin{array}{l}\text { GI SIDI } \\
\text { HEVs: } \\
\text { E90, corn }\end{array}$ & $\begin{array}{c}\text { GI SIDI } \\
\text { HEVs: } \\
\text { E90, WB }\end{array}$ \\
\hline Total energy & $-47.4 \%$ & $-47.4 \%$ & $-43.5 \%$ & $-41.7 \%$ & $-46.2 \%$ & $-46.7 \%$ & $-37.0 \%$ & $-34.1 \%$ & $-43.2 \%$ & $-36.3 \%$ & $-8.7 \%$ \\
\hline Fossil fuels & $-47.4 \%$ & $-47.4 \%$ & $-44.1 \%$ & $-41.4 \%$ & $-46.2 \%$ & $-46.6 \%$ & $-36.7 \%$ & $-68.4 \%$ & $-77.4 \%$ & $-71.1 \%$ & $-89.7 \%$ \\
\hline Petroleum & $-47.4 \%$ & $-47.4 \%$ & $-99.6 \%$ & $-97.5 \%$ & $-36.1 \%$ & $-98.8 \%$ & $-87.3 \%$ & $-87.3 \%$ & $-87.7 \%$ & $-89.3 \%$ & $-88.0 \%$ \\
\hline VOC: Total & $-30.2 \%$ & $-26.3 \%$ & $-65.5 \%$ & $-61.3 \%$ & $-55.2 \%$ & $-60.1 \%$ & $-35.0 \%$ & $-116.2 \%$ & $-46.7 \%$ & $16.3 \%$ & $-24.0 \%$ \\
\hline VOC: Urban & $-32.3 \%$ & $-23.6 \%$ & $-56.2 \%$ & $-57.2 \%$ & $-50.7 \%$ & $-48.3 \%$ & $-25.7 \%$ & $-25.7 \%$ & $-28.0 \%$ & $-24.3 \%$ & $-24.3 \%$ \\
\hline CO: Total & $-2.5 \%$ & $-2.1 \%$ & $-21.5 \%$ & $-20.4 \%$ & $-21.8 \%$ & $-22.2 \%$ & $-1.2 \%$ & $-2.7 \%$ & $-10.7 \%$ & $-0.7 \%$ & $4.9 \%$ \\
\hline CO: Urban & $-0.2 \%$ & $-0.1 \%$ & $-19.7 \%$ & $-20.1 \%$ & $-20.0 \%$ & $-20.0 \%$ & $-0.2 \%$ & $-0.2 \%$ & $-3.4 \%$ & $-0.1 \%$ & $-0.1 \%$ \\
\hline NOx: Total & $-40.8 \%$ & $-39.5 \%$ & $-15.4 \%$ & $10.1 \%$ & $-50.0 \%$ & $-55.1 \%$ & $-37.8 \%$ & $-78.0 \%$ & $-49.2 \%$ & $50.0 \%$ & $94.1 \%$ \\
\hline NOx: Urban & $-14.7 \%$ & $-12.5 \%$ & $53.5 \%$ & $-17.1 \%$ & $-11.1 \%$ & $-11.7 \%$ & $-21.0 \%$ & $-21.0 \%$ & $-39.1 \%$ & $-4.9 \%$ & $-7.6 \%$ \\
\hline PM1 0: Total & $-27.2 \%$ & $-21.8 \%$ & $-34.5 \%$ & $-32.1 \%$ & $-32.0 \%$ & $-37.1 \%$ & $-27.5 \%$ & $-34.1 \%$ & $-166.8 \%$ & $216.1 \%$ & $13.6 \%$ \\
\hline PM10: Urban & $4.4 \%$ & $4.8 \%$ & $-16.5 \%$ & $-17.4 \%$ & $-16.8 \%$ & $-16.8 \%$ & $-7.1 \%$ & $-7.1 \%$ & $-113.2 \%$ & $-5.6 \%$ & $-5.7 \%$ \\
\hline SOx: Total & $-47.4 \%$ & $-47.4 \%$ & $-59.3 \%$ & $-85.6 \%$ & $-66.6 \%$ & $-81.7 \%$ & $-76.8 \%$ & $-77.1 \%$ & $-12.7 \%$ & $27.4 \%$ & $-94.3 \%$ \\
\hline SOx: Urban & $-47.4 \%$ & $-47.4 \%$ & $-88.0 \%$ & $-98.9 \%$ & $-94.4 \%$ & $-94.5 \%$ & $-87.2 \%$ & $-87.2 \%$ & $-177.7 \%$ & $-90.1 \%$ & $-90.6 \%$ \\
\hline
\end{tabular}

Table continued on next page. See page preceding Table 4.14 for acronym definitions. 


\section{Table 4.15 (continued)}

\section{Long-Term Technology}

Changes in Per-Mile, Fuel-Cycle Energy Use and Criteria Pollutant Emissions for Passenger Cars

(Percentage relative to conventional gasoline vehicles fueled with conventional gasoline)

\begin{tabular}{|c|c|c|c|c|c|c|c|c|c|c|c|}
\hline & $\begin{array}{l}\text { GI SIDI } \\
\text { HEVs: } \\
\text { E90, HB }\end{array}$ & $\begin{array}{l}\text { GCSIDI } \\
\text { HEV : } \\
\text { I FRFG2, } \\
\text { MTBE, } \\
\text { US mix } \\
\end{array}$ & $\begin{array}{l}\text { GCSIDI } \\
\text { H E V : } \\
\text { FRFG2, } \\
\text { ETBE, } \\
\text { US mix } \\
\end{array}$ & $\begin{array}{l}\text { GCSIDI } \\
\text { HEV: } \\
\text { FRFG2, } \\
\text { EtOH, } \\
\text { US mix }\end{array}$ & $\begin{array}{l}\text { GCSIDI } \\
\text { HEV: } \\
\text { FRFG2, } \\
\text { MTBE, } \\
\text { x CAmix } \\
\end{array}$ & $\begin{array}{l}\text { GCSIDI } \\
\text { HEV: } \\
\text { FRFG2, } \\
\text { ETBE, } \\
\text { CAmix }\end{array}$ & $\begin{array}{c}\text { GCSIDI } \\
\text { HEV: } \\
\text { FRFG2, } \\
\text { EtOH, } \\
\text { CAmix }\end{array}$ & $\begin{array}{c}\text { GC SI } \\
\text { HEV: } \\
\text { CNG, } \\
\text { US mix }\end{array}$ & $\begin{array}{c}\text { GC SI } \\
\text { HEV: } \\
\text { CNG, } \\
\text { CA mix }\end{array}$ & $\begin{array}{c}\text { GC SI } \\
\text { HEV: } \\
\text { LNG, } \\
\text { US mix }\end{array}$ & $\begin{array}{l}\text { GC SI } \\
\text { HEV: } \\
\text { LNG, } \\
\text { CAmix }\end{array}$ \\
\hline Tot & $-16.4 \%$ & $-43.7 \%$ & $-43.8 \%$ & $-43.7 \%$ & $-43.9 \%$ & $-44.0 \%$ & $-43.9 \%$ & $-40.8 \%$ & $-41.0 \%$ & $-39.5 \%$ & $-39.7 \%$ \\
\hline Fossil fuels & $-87.4 \%$ & $-48.2 \%$ & $-47.1 \%$ & $-47.3 \%$ & $-55.5 \%$ & $-54.8 \%$ & $-54.9 \%$ & $-45.7 \%$ & $-53.0 \%$ & $-43.8 \%$ & $\%$ \\
\hline Petroleum & -8 & $-60.7 \%$ & -60 & $4 \%$ & $-61.0 \%$ & $-60.7 \%$ & $-61.7 \%$ & $-99.4 \%$ & $-99.6 \%$ & $-97.8 \%$ & $1 \%$ \\
\hline VOC: Total & $-26.9 \%$ & $-46.1 \%$ & $-47.9 \%$ & $-45.0 \%$ & $-48.2 \%$ & $-49.8 \%$ & $-47.0 \%$ & $-72.6 \%$ & $-74.8 \%$ & $-69.6 \%$ & $-71.7 \%$ \\
\hline VOC: Urban & $-24.4 \%$ & $-43.8 \%$ & $-43.9 \%$ & $-40.8 \%$ & $-43.8 \%$ & $-43.9 \%$ & $-40.8 \%$ & $-69.2 \%$ & $-69.2 \%$ & $-70.0 \%$ & $-70.0 \%$ \\
\hline CO: Total & $3.6 \%$ & $-30.9 \%$ & -31 & $-30.7 \%-$ & $-3 \quad 1.2 \%$ & $-31.3 \%$ & $-31.0 \%$ & $-44.3 \%$ & -44 & $-43.5 \%$ & \\
\hline CO: & $1 \%$ & $-30.1 \%$ & $-30.1 \%$ & $-30.1 \%$ & $-30.1 \%$ & $-30.1 \%$ & $-30.0 \%$ & $-43.8 \%$ & $-43.7 \%$ & $-44.1 \%$ & $-44.1 \%$ \\
\hline NOx: & $97.1 \%$ & $4.4 \%$ & & & $-37.8 \%$ & $-42.2 \%$ & $-38.3 \%$ & $21.7 \%$ & $-20.4 \%$ & $40.1 \%$ & $-2.0 \%$ \\
\hline $\mathrm{NOx}$ & $-6.8 \%$ & $-32.7 \%$ & $-34.6 \%$ & $-32.7 \%$ & $-31.1 \%$ & $-33.1 \%$ & $-31.1 \%$ & $14.9 \%$ & $16.5 \%$ & $-36.0 \%$ & $-34.5 \%$ \\
\hline PM1 & & $-2.9 \%$ & $-28.2 \%$ & $-19.1 \%$ & $-19.2 \%$ & $-37.6 \%$ & $-31.0 \%$ & $-18.6 \%$ & $-34.9 \%$ & $-16.9 \%$ & $-33.2 \%$ \\
\hline PM10: Urban & $-5.7 \%$ & $-6.3 \%$ & & $-6.3 \%$ & $-6.2 \%$ & $-6.7 \%$ & $-6.2 \%$ & $-21.2 \%$ & $-21.1 \%$ & $-21.9 \%$ & $-21.7 \%$ \\
\hline SOx: Total & $-82.6 \%$ & $81.0 \%$ & & & $-40.0 \%$ & $-44.3 \%$ & $-42.1 \%$ & $72.6 \%$ & $-48.5 \%$ & $53.6 \%$ & $-67.5 \%$ \\
\hline SOx: Urban & $-90.4 \%$ & $-59.2 \%$ & $-59.2 \%$ & $-59.2 \%$ & $-61.3 \%$ & $-61.4 \%$ & $-61.4 \%$ & $-88.1 \%$ & $-90.3 \%$ & $-96.0 \%$ & $-98.2 \%$ \\
\hline
\end{tabular}

Table continued on next page. See page preceding Table 4.14 for acronym definitions. 


\section{Table 4.15 (continued) \\ Long-Term Technology}

Changes in Per-Mile, Fuel-Cycle Energy Use and Criteria Pollutant Emissions for Passenger Cars

(Percentage relative to conventional gasoline vehicles fueled with conventional gasoline)

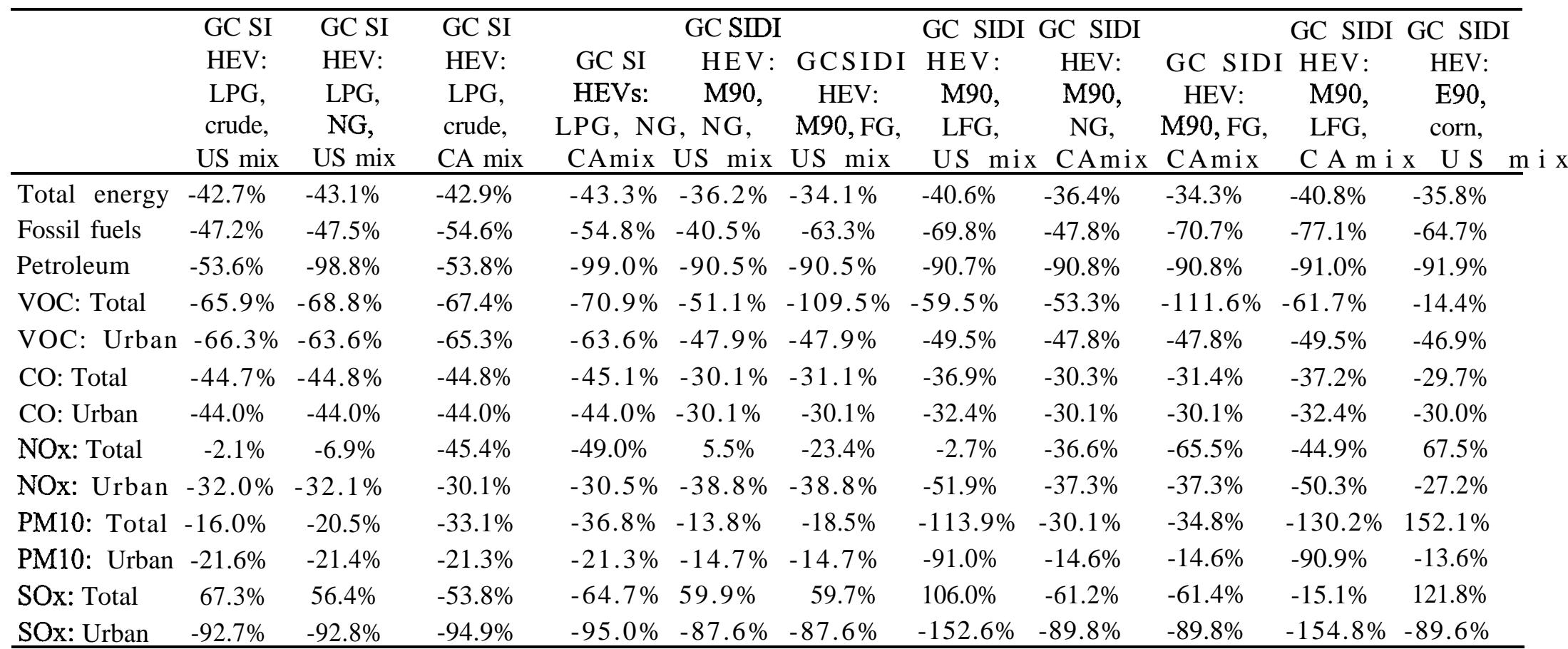

Table continued on next page. See page preceding Table 4.14 for acronym definitions. 
Table 4.15 (continued)

Long-Term Technology

Changes in Per-Mile, Fuel-Cycle Energy Use and Criteria Pollutant Emissions for Passenger Cars

(Percentage relative to conventional gasoline vehicles fueled with conventional gasoline)

\begin{tabular}{|c|c|c|c|c|c|c|c|c|c|c|c|}
\hline & $\begin{array}{l}\text { GC SIDI } \\
\text { HEV: } \\
\text { E90, W B } \\
\text { US mix }\end{array}$ & $\begin{array}{l}\text { GC SIDI } \\
\text { I HEV: } \\
\text { E90, H } \\
\text {, HB, } \\
x \text { US mix } \\
\end{array}$ & $\begin{array}{c}\text { GC SIDI } \\
\text { HEV: E90, } \\
\text { corn, } \\
\text { CA mix }\end{array}$ & $\begin{array}{c}\text { GC S ID } \\
\text { HEV: E90, } \\
\text { WB, } \\
\text { CA mix }\end{array}$ & $\begin{array}{l}\text { GC SIDI } \\
\text { DI HEV: } \\
\text { E90, } \\
\text { HB, } \\
\text { C A m i x }\end{array}$ & $\begin{array}{c}\text { CIDI: } \\
\text { R F D } \\
\end{array}$ & $\begin{array}{c}\text { CIDI: } \\
\text { DME, } \\
\text { NG } \\
\end{array}$ & $\begin{array}{c}\text { CIDI: } \\
\text { DME, FG }\end{array}$ & $\begin{array}{c}\text { CIDI: } \\
\text { FT50, } \\
\text { NG } \\
\end{array}$ & $\begin{array}{l}\text { CIDI: } \\
\text { FT50, FG }\end{array}$ & $\begin{array}{r}\text { CIDI: } \\
\text { BD20 } \\
\end{array}$ \\
\hline Total energy & $-15.9 \%$ & $-21.5 \%$ & $-36.0 \%$ & $-16.1 \%$ & $-21.7 \%$ & $-35.1 \%$ & $-17.7 \%$ & $-14.4 \%$ & $-21.3 \%$ & $-13.2 \%$ & $-31.4 \%$ \\
\hline Fossil fuels & $-78.1 \%$ & $-76.4 \%$ & $-72.3 \%$ & $-85.7 \%$ & $-84.0 \%$ & $-35.0 \%$ & $-17.2 \%$ & $-67.4 \%$ & $-21.1 \%$ & $-38.6 \%$ & $-31.6 \%$ \\
\hline Petroleum & $-91.0 \%$ & $-91.8 \%$ & $-92.2 \%$ & $-91.3 \%$ & $-92.0 \%$ & $-25.0 \%$ & $-97.9 \%$ & $-97.9 \%$ & $-59.9 \%$ & $-59.9 \%$ & $-36.7 \%$ \\
\hline VOC: Total & $-43.3 \%$ & $-45.4 \%$ & $-16.4 \%$ & $-45.3 \%$ & $-47.4 \%$ & $-61.9 \%$ & $-74.3 \%$ & $-192.5 \%$ & $-67.4 \%$ & $-135.9 \%$ & $-38.0 \%$ \\
\hline VOC: Urban & $-46.9 \%$ & $-46.9 \%$ & $-46.8 \%$ & $-46.9 \%$ & $-46.9 \%$ & $-63.4 \%$ & $-76.0 \%$ & $-76.0 \%$ & $-65.0 \%$ & $-65.0 \%$ & $-61.3 \%$ \\
\hline CO: Total & $-25.6 \%$ & $-26.6 \%$ & $-29.9 \%$ & $-25.9 \%$ & $-26.8 \%$ & $-2.4 \%$ & $-0.5 \%$ & $-3.1 \%$ & $-2.1 \%$ & $-3.1 \%$ & $-0.2 \%$ \\
\hline CO: Urban & $-30.0 \%$ & $-30.0 \%$ & $-30.0 \%$ & $-30.0 \%$ & $-30.0 \%$ & $-0.1 \%$ & $-0.3 \%$ & $-0.3 \%$ & $-0.2 \%$ & $-0.2 \%$ & $0.5 \%$ \\
\hline NOx: Total & $99.2 \%$ & $101.4 \%$ & $26.1 \%$ & $57.8 \%$ & $60.0 \%$ & $-24.4 \%$ & $-23.2 \%$ & $-90.0 \%$ & $-32.7 \%$ & $-64.7 \%$ & $17.7 \%$ \\
\hline NOx: Urban & $-29.1 \%$ & $-28.5 \%$ & $-25.5 \%$ & $-27.4 \%$ & $-26.9 \%$ & $43.1 \%$ & $32.6 \%$ & $32.6 \%$ & $38.2 \%$ & $38.2 \%$ & $78.5 \%$ \\
\hline PM10: Total & $6.5 \%$ & $1.2 \%$ & $140.2 \%$ & $-5.4 \%$ & $-10.6 \%$ & $-13.7 \%$ & $-36.0 \%$ & $-47.9 \%$ & $-28.6 \%$ & $-33.0 \%$ & $-7.0 \%$ \\
\hline PM10: Urban & $-13.6 \%$ & $-13.6 \%$ & $-13.4 \%$ & $-13.5 \%$ & $-13.5 \%$ & $-1.4 \%$ & $-12.0 \%$ & $-12.0 \%$ & $-8.3 \%$ & $-8.3 \%$ & $-0.8 \%$ \\
\hline SOx: Total & $34.3 \%$ & $42.7 \%$ & $11.7 \%$ & $-75.8 \%$ & $-67.4 \%$ & $-34.1 \%$ & $-82.2 \%$ & $-83.3 \%$ & $-58.4 \%$ & $-58.0 \%$ & $-32.6 \%$ \\
\hline SOx: Urban & $-90.0 \%$ & $-89.8 \%$ & $-91.8 \%$ & $-92.2 \%$ & $-92.0 \%$ & $6.8 \%$ & $-95.7 \%$ & $-95.7 \%$ & $-42.2 \%$ & $-42.2 \%$ & $-9.6 \%$ \\
\hline
\end{tabular}

Table continued on next page. See page preceding Table 4.14 for acronym definitions. 
Table 4.15 (continued)

Long-Term Technology

Changes in Per-Mile, Fuel-Cycle Energy Use and Criteria Pollutant Emissions for Passenger Cars

(Percentage relative to conventional gasoline vehicles fueled with conventional gasoline)

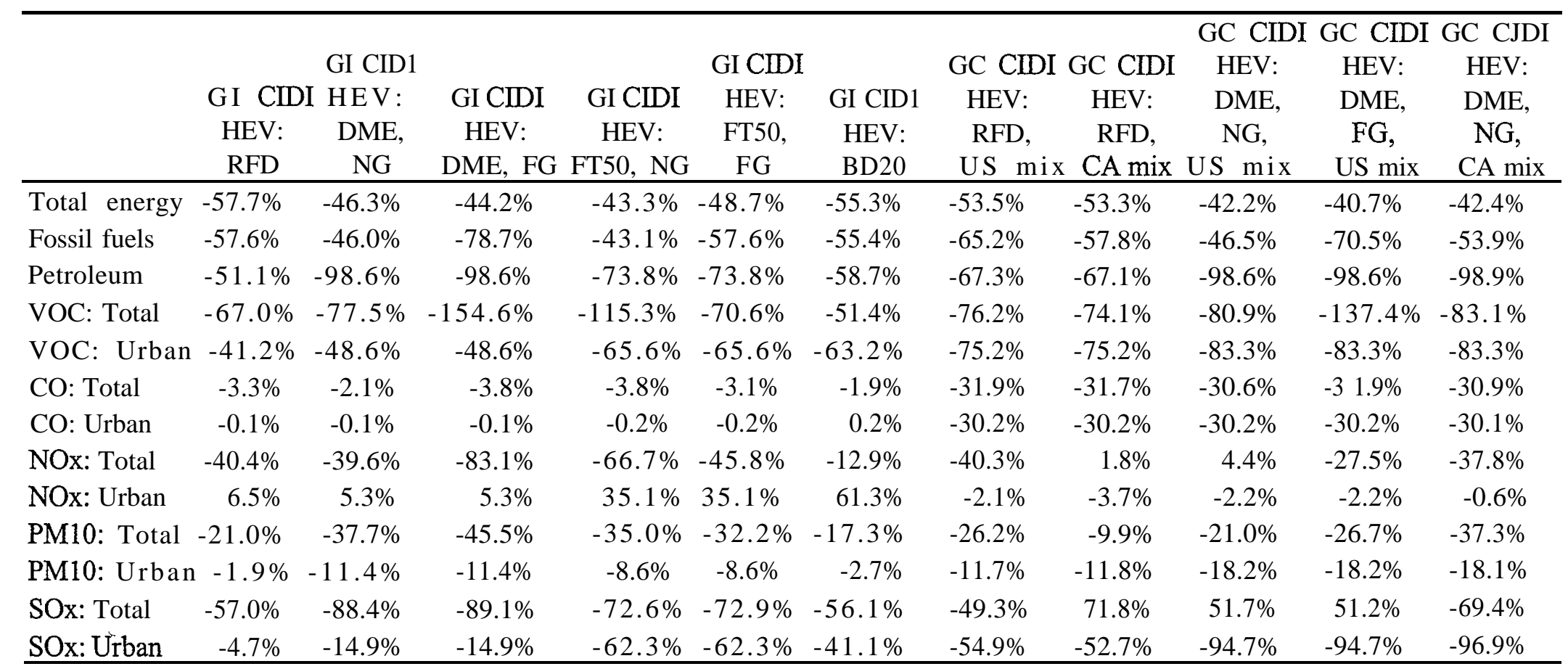

Table continued on next page. See page preceding Table 4.14 for acronym definitions. 
Table 4.15 (continued)

Long-Term Technology

Changes in Per-Mile, Fuel-Cycle Energy Use and Criteria Pollutant Emissions for Passenger Cars

(Percentage relative to conventional gasoline vehicles fueled with conventional gasoline)

\begin{tabular}{|c|c|c|c|c|c|c|c|c|c|c|c|}
\hline & $\begin{array}{c}\text { G C C I D } \\
\text { HEV: } \\
\text { DME, } \\
\text { F G, C A } \\
\text { mix }\end{array}$ & $\begin{array}{c}\text { GC } \\
\text { I CIDI } \\
\text { HEVs: } \\
\text { FT50, } \\
\text { NG, US } \\
\text { mix } \\
\end{array}$ & $\begin{array}{l}\text { GCCIDI } \\
\text { HEVs: } \\
\text { FT50, FG, } \\
\text { US mix }\end{array}$ & $\begin{array}{l}\text { I GCCIDI } \\
\text { HEV: } \\
\text { FT50, NG, } \\
\text { CA mix }\end{array}$ & $\begin{array}{c}\text { GC } \\
\text { CID1 } \\
\text { I HEV : } \\
\text { FT50, } \\
\text { F G, C A } \\
\text { mix } \\
\end{array}$ & $\begin{array}{c}\text { GCCID I } \\
\text { HEV: } \\
\text { B D } 20 \\
\text { US mix }\end{array}$ & $\begin{array}{c}\text { GCC ID I } \\
\text { HEV: } \\
\text { BD20, } \\
\text { CA mix }\end{array}$ & $\begin{array}{l}\text { EV: US } \\
\operatorname{mix}\end{array}$ & $\begin{array}{c}\mathrm{EV}: \mathrm{NE} \\
\operatorname{mix}\end{array}$ & $\begin{array}{c}\text { EV: CA } \\
\text { mix }\end{array}$ & $\begin{array}{r}\mathrm{H} 2 \mathrm{FCV} \\
\mathrm{NG}, \mathrm{C} . \\
\text { gaseous }\end{array}$ \\
\hline Total ener & $-40.9 \%$ & $-43.9 \%$ & $-40.1 \%$ & $-44.1 \%$ & $-40.3 \%$ & $-48.8 \%$ & $-49.0 \%$ & $-38.4 \%$ & $-39.7 \%$ & $-39.0 \%$ & $-59.7 \%$ \\
\hline Fossil fuels & $-77.8 \%$ & $-48.3 \%$ & $-56.7 \%$ & $-55.7 \%$ & $-64.0 \%$ & $-53.4 \%$ & & & & $9 \%$ & $-60.3 \%$ \\
\hline Petroleum & $-98.9 \%$ & $-80.5 \%$ & $-80.5 \%$ & $-80.7 \%$ & $-80.7 \%$ & $-69.4 \%$ & $-69.7 \%$ & $-98.7 \%$ & $-98.2 \%$ & $-99.6 \%$ & $7 \%$ \\
\hline VOC: Total & $-139.5 \%$ & $-76.1 \%$ & $-108.8 \%$ & $-78.2 \%$ & $-111.0 \%$ & $-62.0 \%$ & $-64.2 \%$ & -8 & $-92.5 \%$ & $7 \%$ & $8 \%$ \\
\hline VOC: Urban & $-83.3 \%$ & $-75.8 \%$ & $-75.8 \%$ & $-75.8 \%$ & $-75.8 \%$ & $-74.1 \%$ & $-74.0 \%$ & $-99.7 \%$ & $-99.5 \%$ & $7 \%$ & $-98.3 \%$ \\
\hline CO: Total & $-32.1 \%$ & $-31.4 \%$ & $-31.9 \%$ & $-31.7 \%$ & $-32.1 \%$ & $-30.5 \%$ & -30 & $-97.5 \%$ & $-97.3 \%$ & $5 \%$ & $-97.1 \%$ \\
\hline CO: Urban & $-30.1 \%$ & $-30.1 \%$ & $-30.1 \%$ & $-30.1 \%$ & $-30.1 \%$ & $-29.8 \%$ & & $-99.9 \%$ & $-99.8 \%$ & $9 \%$ & $.5 \%$ \\
\hline NOx: Total & $-69.6 \%$ & $-0.2 \%$ & $-15.5 \%$ & $-42.3 \%$ & $-57.6 \%$ & $23.9 \%$ & $-18.3 \%$ & $103.6 \%$ & $44.7 \%$ & $-36.8 \%$ & $-41.5 \%$ \\
\hline NOx: Urban & $-0.6 \%$ & $0.5 \%$ & & & $2.1 \%$ & $19.7 \%$ & & & $-60.2 \%$ & $-75.5 \%$ & $-23.4 \%$ \\
\hline PM10: Total & $-43.0 \%$ & $-17.0 \%$ & $-19.1 \%$ & $-33.3 \%$ & $-35.4 \%$ & $-6.2 \%$ & $-22.5 \%$ & $17.7 \%$ & $-8.2 \%$ & $-36.7 \%$ & $-47.4 \%$ \\
\hline PM10: Urban & $-18.1 \%$ & $-15.7 \%$ & $-15.7 \%$ & & $-15.6 \%$ & $-11.5 \%$ & & $-32.4 \%$ & $-30.5 \%$ & $-32.0 \%$ & $-32.2 \%$ \\
\hline SOx: Total & $-69.9 \%$ & $63.1 \%$ & $63.3 \%$ & $-58.0 \%$ & $-57.8 \%$ & $75.4 \%$ & $-45.7 \%$ & $377.4 \%$ & $147.0 \%$ & $-26.2 \%$ & $-66.0 \%$ \\
\hline SOx: Urban & $-96.9 \%$ & $-69.2 \%$ & $-69.2 \%$ & $-71.4 \%$ & $-71.4 \%$ & $-53.6 \%$ & $-55.8 \%$ & $-89.3 \%$ & $-78.7 \%$ & $-96.5 \%$ & $-99.0 \%$ \\
\hline
\end{tabular}

Table continued on next page. See page preceding Table 4.14 for acronym definitions. 


\section{Table 4.15 (continued) \\ Long-Term Technology}

Changes in Per-Mile, Fuel-Cycle Energy Use and Criteria Pollutant Emissions for Passenger Cars

(Percentage relative to conventional gasoline vehicles fueled with conventional gasoline)

\begin{tabular}{|c|c|c|c|c|c|c|c|c|c|c|c|c|}
\hline & $\begin{array}{c}\mathrm{H} 2 \\
\mathrm{FCV} \text { : } \\
\mathrm{NG}, \\
\text { station } \\
\text { gaseous }\end{array}$ & $\begin{array}{c}\mathrm{H} 2 \\
\text { FCV: } \\
\text { solar gas }\end{array}$ & $\begin{array}{c}\text { H2 FCV: } \\
\text { solar, } \\
\text { liquid }\end{array}$ & $\begin{array}{c}\text { H2 FCV: } \\
\text { NG, } \\
\text { liquid }\end{array}$ & $\begin{array}{c}\text { FCV: } \\
\text { MeOH, } \\
\text { NG }\end{array}$ & $\begin{array}{c}\text { FCV: } \\
\text { MeOH, } \\
\text { FG }\end{array}$ & $\begin{array}{l}\text { FCV: } \\
\text { MeOH, } \\
\text { LFG }\end{array}$ & $\begin{array}{c}\text { FCV: } \\
\text { FRFG2 }\end{array}$ & $\begin{array}{l}\text { EtOH } \\
\text { FCV: } \\
\text { Corn }\end{array}$ & $\begin{array}{l}\text { EtOH } \\
\text { FCV: } \\
\text { WB }\end{array}$ & $\begin{array}{l}\text { EtOH } \\
\text { FCV: } \\
\text { HB }\end{array}$ & $\begin{array}{l}\text { NG } \\
\text { FCV: } \\
\text { CNG }\end{array}$ \\
\hline Total & $-53.2 \%$ & $-65.5 \%$ & $-71.8 \%$ & $-58.8 \%$ & $-52.3 \%$ & $-49.7 \%$ & $-57.8 \%-$ & $-50.0 \%$ & $-37.7 \%$ & $-7.1 \%$ & $-15.6 \%$ & $-52.0 \%$ \\
\hline Fossil & $2.9 \%$ & $-93.1 \%$ & $-98.3 \%$ & $-58.5 \%$ & $-52.0 \%$ & $-80.3 \%$ & $-88.3 \%$ & $-50.0 \%$ & $-77.4 \%$ & $-97.3 \%$ & $3 \%$ & \\
\hline Petr & $9.7 \%$ & $-99.9 \%$ & $-98.1 \%$ & $-97.8 \%$ & $-98.6 \%$ & $-98.6 \%$ & $-98.9 \%$ & $-50.0 \%$ & -96 & $9 \%$ & $-96.2 \%$ & $\%$ \\
\hline VOC: Total & $-94.5 \%$ & $-95.8 \%$ & $-92.3 \%$ & $-89.8 \%$ & $-72.8 \%$ & $-145.1 \%$ & $6-83.2 \%$ & $-52.9 \%$ & $6-5.8 \%$ & $-53.0 \%$ & $-56.4 \%$ & $-88.5 \%$ \\
\hline VOC: Urban & $-94.9 \%$ & $-97.6 \%$ & $-97.8 \%$ & $-97.7 \%$ & $-73.6 \%$ & $-73.6 \%$ & $-75.6 \%$ & $-54.1 \%$ & $-72.0 \%$ & $-72.0 \%$ & $-72.1 \%$ & -8 \\
\hline CO: Total & $-95.9 \%$ & $-98.4 \%-$ & $-98.8 \%$ & $-96.7 \%$ & $-78.0 \%$ & $-79.3 \%$ & $-86.5 \%$ & $-78.5 \%$ & $-77.2 \%$ & $-71.1 \%$ & $-72.5 \%$ & $-78.9 \%$ \\
\hline CO: Urban & $-97.1 \%$ & $-99.3 \%$ & $-99.8 \%$ & $-99.8 \%$ & $-80.0 \%$ & $-80.0 \%$ & $-82.8 \%$ & $-79.9 \%$ & $-79.8 \%$ & $-79.9 \%$ & $-79.8 \%$ & $-79.6 \%$ \\
\hline NOx & $-30.8 \%$ & $-35.8 \%$ & $-71.0 \%$ & $-44.6 \%$ & $-63.3 \%$ & $-99.1 \%$ & $-73.5 \%$ & $-55.1 \%$ & $46.7 \%$ & $96.4 \%$ & $99.8 \%$ & $-39.1 \%$ \\
\hline NOx: & $93.3 \%$ & $7.2 \%$ & $-75.4 \%$ & $-73.0 \%$ & $-82.8 \%$ & $-82.8 \%$ & $-98.9 \%$ & $-72.1 \%$ & $-55.1 \%$ & $-59.5 \%$ & $-58.2 \%$ & $-17.5 \%$ \\
\hline PM10: & $-46.4 \%$ & $-46.4 \%-$ & $-43.6 \%$ & $-38.8 \%-$ & $-49.5 \%$ & $-55.4 \%-$ & $-173.5 \%$ & $-38.4 \%$ & $349.9 \%$ & $42.2 \%$ & $31.1 \%$ & $-46.7 \%$ \\
\hline PM 10: Urban & $-25.6 \%$ & $-31.4 \%$ & $-30.8 \%$ & $-30.6 \%$ & $-34.0 \%$ & $-34.0 \%-$ & $-128.6 \%$ & $-32.8 \%$ & $-31.6 \%$ & $-31.8 \%$ & $-31.7 \%$ & $-32.5 \%$ \\
\hline & $-91.7 \%$ & $-63.5 \%-$ & $-97.5 \%$ & $-90.6 \%$ & $-87.8 \%$ & $-88.0 \%$ & $-30.6 \%-$ & $-54.1 \%$ & $45.9 \%-$ & $-102.1 \%$ & $-87.9 \%$ & $-66.2 \%$ \\
\hline SOx: Urban & $-99.4 \%$ & $-98.8 \%$ & $-97.2 \%$ & $-97.1 \%$ & $-97.0 \%$ & $-97.0 \%-$ & $-177.6 \%$ & $-94.9 \%$ & $-95.8 \%$ & $-96.6 \%$ & $-96.1 \%$ & $-99.1 \%$ \\
\hline
\end{tabular}

Source:

Wang, Michael Q., GREET Model Results, Argonne National Laboratory, Argonne, IL, August 1999.

Note:

See page preceding Table 4.14 for acronym definitions.

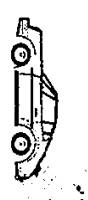


Table 4.16

Federal Exhaust Emission Certification Standards for Gasoline- and Diesel-Powered Light Vehicles $a, b$ (grams per mile)

\begin{tabular}{|c|c|c|c|c|c|c|c|c|c|c|c|c|c|}
\hline Engine Type \& Pollutant & $\begin{array}{l}\text { Prior to } \\
\text { control } \\
\end{array}$ & 1968-69 & $1970-71$ & 1972 & $1973-74$ & $1975-76$ & 1977-79 & 1980 & 1981 & $1982-86$ & $1987-93$ & \multicolumn{2}{|l|}{$1994+b$} \\
\hline \multicolumn{14}{|l|}{ Gasoline } \\
\hline Hydrocarbons (total) & 11 & $c$ & 2.2 & 3.4 & & 1.5 & & 0.41 & & & & 0.41 & $(e)$ \\
\hline Non-methane hydrocarbons & $d$ & 烈 & 然 & & & x & $g 8$ & sis & 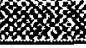 & 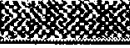 & 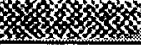 & 0.25 & $(0.31)$ \\
\hline Carbon monoxide & 80 & $c$ & 23 & 39 & & 15 & & 7.0 & 3.4 & & & 3.4 & $(4.2)$ \\
\hline Cold-temp. carbon monoxide $f$ & $d$ & & & & & 怒 & & & & & & 10 & $(e)$ \\
\hline Nitrogen oxides & 4 & & & & 3.0 & 3.1 & 2.0 & & 1.0 & & & 0.4 & $(0.6)$ \\
\hline Particulates & $d$ & 聯筑 & \% & & 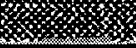 & Shy & 500 & & & & & 0.08 & $(0.10)$ \\
\hline \multicolumn{14}{|l|}{ Diesel } \\
\hline Hydrocarbons (total) & 11 & & & & & 1.5 & & 0.41 & & & & 0.41 & $(e)$ \\
\hline Non-methane hydrocarbons & $d$ & & & & & 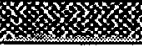 & & 坔 & 箘 & 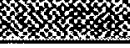 & $\gamma$ x & 0.25 & $(0.31)$ \\
\hline Carbon monoxide & 80 & & & & & 15 & & 7.0 & 3.4 & & & 3.4 & $(4.2)$ \\
\hline Nitrogen oxides & 4 & & & & & 3.1 & 2.0 & & 1.0 & & & 1.0 & $(1.25)$ \\
\hline Particulates & $d$ & \% & & 200 & & & & & x & 0.60 & 0.20 & 0.08 & $(0.10)$ \\
\hline \multicolumn{2}{|l|}{ Test Procedure } & 7-mode & & CVS-7 & & CVS-75 & & & & & & & \\
\hline \multirow{2}{*}{\multicolumn{2}{|c|}{$\begin{array}{c}\text { Useful Life (intermediate) } \boldsymbol{b} \\
\text { (full) }\end{array}$}} & 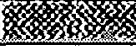 & & & 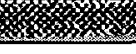 & 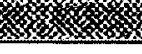 & 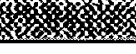 & & & & & \multirow{2}{*}{\multicolumn{2}{|c|}{\begin{tabular}{|l}
$5 \mathrm{yrs} / 50,000 \mathrm{mi}$ \\
$10 \mathrm{yrs} / 100,000 \mathrm{mi}$
\end{tabular}}} \\
\hline & & $5 \mathrm{yrs} / 50$, & $00 \mathrm{mi}$ & & & & & & & & & & \\
\hline
\end{tabular}

Source:

40 CFR 86.085-2; 40 CFR 86.090-2; 40 CFR 86.090-s; 40 CFR 86.094-s; 40 CFR 86.096-2; 40 CFR 86.096-s; 40 CFR 86.098-8;40 CFR 86.099-s; 40 CFR 86.082-2; 40 CFR 86.000-8. Lisa Snapp, Office of Air and Radiation, Environmental Protection Agency, Personal communication, April 1999.

${ }^{\mathrm{a}}$ The test procedure for measuring exhaust emissions has changed several times over the course of vehicle emissions regulation. The 7-mode procedure was used through model year 1971 and was replaced by the CVS-72 procedure beginning in model year 1972. The CVS-75 became the test procedure as of model year 1975 . While it may appear that the total hydrocarbon and carbon monoxide standards were relaxed in 1972-74, these standards were actually more stringent due to the more stringent nature of the CVS-72 test procedure. Additional standards for carbon monoxide and composite standards for non-methane hydrocarbons and nitrogen oxides tested over the new Supplemental Federal Test Procedure will be phased-in during model years 2000-02; these standards are not shown in this table.

${ }^{b}$ All emission standards must be met for a useful life of 5 years $/ 50,000$ miles. Beginning in with model year 1994, a second set of emission standards must also be met for a full useful life of 10 years/100,000 miles (these standards are shown in parentheses). Tier 1 exhaust standards were phased-in during 1994-96 at a rate of 40,80, and 100 percent, respectively.

cIn 1968-69, exhaust emission standards were issued in parts per million (ppm) rather than grams per mile and are, therefore, incompatible with this table.

d Noestimate available.

No standard set.

${ }^{f}$ The cold CO emission standard is measured at 20 degrees $F$ (rather than 75 degrees $F$ ) and is applicable for a 5-year/50,000-mile useful life. 
Table 4.17

Federal Exhaust Emission Certification Standards for Gasoline- and Diesel-Powered Light Trucks (Category LDT1) $a, b, c$ (grams per mile)

\begin{tabular}{|c|c|c|c|c|c|c|c|c|c|c|c|c|}
\hline Engine Type \& Pollutant & $\begin{array}{l}\text { Prior to } \\
\text { control }\end{array}$ & 1968-69 & $1970-71$ & $1972 \quad 1973-74$ & $1975 \quad 1976-78$ & 1979-81 & $1982-83$ & $1984 \quad 1985-86$ & 1987 & $1988-93$ & 1994 & $1995+$ \\
\hline \multicolumn{13}{|l|}{ Gasoline } \\
\hline Hydrocarbons (total) & 11 & $d$ & 2.2 & 3.4 & 2.0 & 1.7 & & 0.80 & & & $f \quad(0.80)$ & \\
\hline Non-methane hydrocarbons & $e$ & $8 \%$ \% & s. & 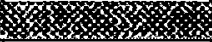 & 83 & & 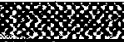 & 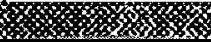 & & & $0.25(0.31)$ & \\
\hline Carbon monoxide & 80 & $d$ & 23 & 39 & 20 & 18 & & 10 & & & \begin{tabular}{|ll}
3.4 & $(4.2)$ \\
\end{tabular} & \\
\hline Cold-temp. carbon monoxide $g$ & $e$ & & & & & 8 & & 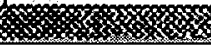 & & 200 & $10 \quad(f)$ & . \\
\hline Nitrogen oxides & 4 & K & & 3.0 & 3,1 & 2.3 & & & & 1.2 & $0.4 \quad(0.6)$ & \\
\hline Particulates & $e$ & 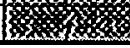 & 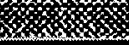 & Kz- & zom & r & 然 & 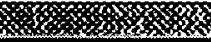 & & zon & sis & $0.08 \quad(0.10)$ \\
\hline
\end{tabular}

\begin{tabular}{l|l}
\hline Diesel & \\
\hline Hydrocarbons (total) & \\
\hline Non-methane hydrocarbons & \\
\hline Carbon monoxide & \\
\hline Nitrogen oxides & \\
\hline Particulates &
\end{tabular}

Nitrogen oxides

\begin{tabular}{ll}
\hline LDT1 Weight Critera $h$ & 4 \\
\hline
\end{tabular}

\section{Test Procedure $b$}

Useful Life (intermediate) $c$ (full)

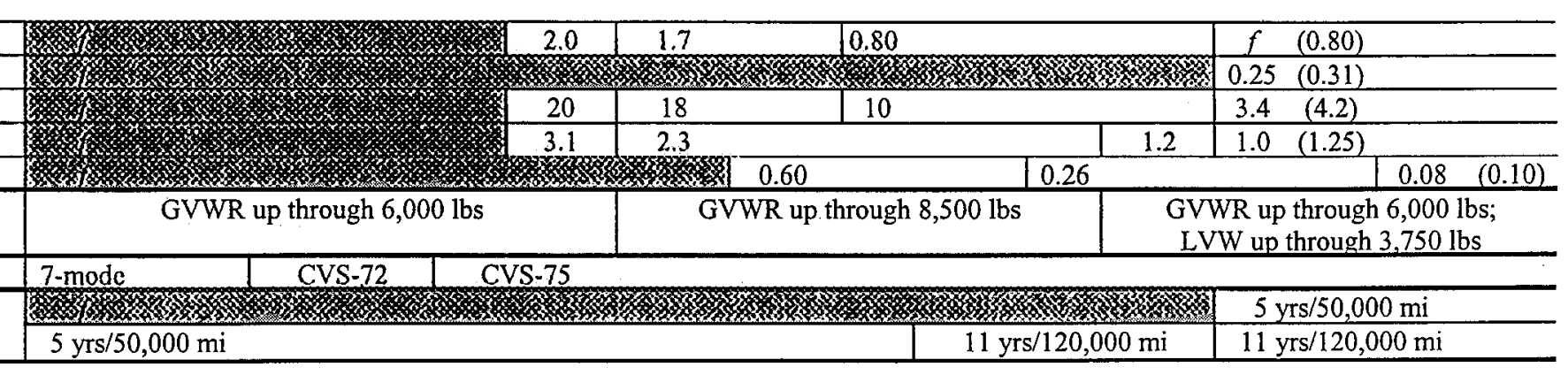

Source:

40 CFR 86.082-2; 40 CFR 86.085-2; 40 CFR 86.090-2; 40 CFR 86.090-9; 40 CFR 86.091-9; 40 CFR 86.094-9; 40 CFR 86.096-2; 40 CFR 86.096-9; 40 CFR 86.099-9; 40 CFR 86.000-9; 40 CFR 86.001-9; 40 CFR 86.004-9. Lisa Snapp, Office of Air and Radiation, Environmental Protection Agency, Personal communication.

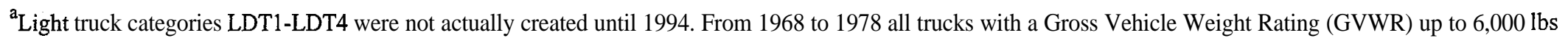
were classified as light trucks and were required to meet the same standards. As of 1979, the maximum weight was raised to 8,500 lbs GVWR. During 1988 through 1993, light trucks were divided into two subcategories that coincide with the current LDT1 and LDT2/3/4 categories.

The test procedure for measuring exhaust emissions has changed several times over the course of vehicle emissions regulation. The 7-mode procedure was used through model year 1971 and was replaced by the CVS-72 procedure beginning in model year 1972. The CVS-75 became the test procedure as of model year 1975 . While it may appear that the total hydrocarbon and carbon monoxide standards were relaxed in 1972-74, these standards were actually more stringent due to the more stringent nature of the CVS-72 test procedure. Additional standards for carbon monoxide and composite standards for non-methane hydrocarbons and nitrogen oxides tested over the new Supplemental Federal Test Procedure will be phased-in during model years 2000-02; these standards are not shown in this table.

'Emission standards had to be met for a useful life of 5 years $/ 50,000$ miles through model year 1983, and a full useful life of 11 years 120,000 miles was defined for 1985-93 (several useful life options were available for 1984). Beginning in model year 1994, emission standards were established for an intermediate useful life of 5 years/50,000 miles as well as a full useful life of 11 years 120,000 miles (these standards are shown in parentheses). Hydrocarbon standards, however, were established only for full useful life. Tier 1 exhaust standards, except PM standards, were phased-in during 1994-96 at a rate of 40, 80, and 100 percent, respectively. PM standards were phased-in at a rate of 40, 80, and 100 .percent during 1995-97.

In 1968-69, exhaust emission standards were issued in parts per million (ppm) rather than grams per mile and are, therefore, incompatible with this table.

No estimate available.

No standard set.

g The cold CO emission standard is measured at 20 degrees F (rather than 75 degrees F) and is applicable for a 5-year/50,000-mile useful life.

hross vehicle weight rating (GVWR) is the maximum design loaded weight. Loaded vehicle weight (LVW) is the curb weight (nominal vehicle weight) plus 300 Ibs. 
Table 4.18

Federal Exhaust Emission Certification Standards for Gasoline- and Diesel-Powered Light Trucks (Category LDT2) $a, b, c$ (grams per mile)

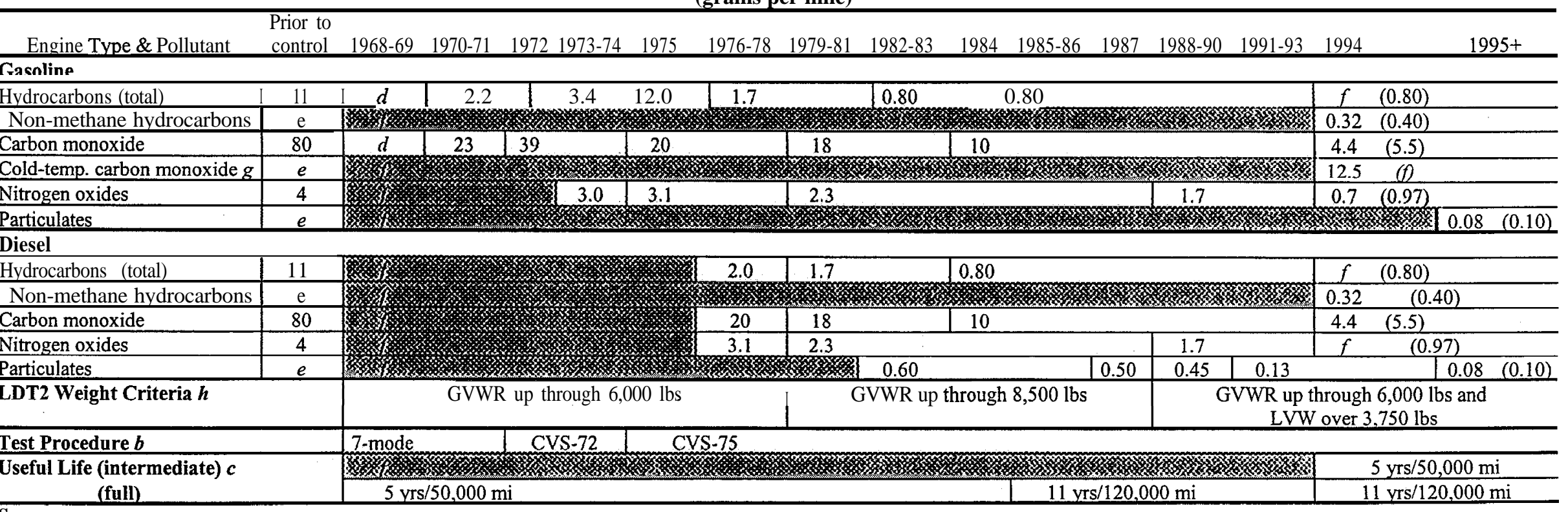

\section{Source:} (full)

$5 \mathrm{yrs} / 50,000 \mathrm{mi}$

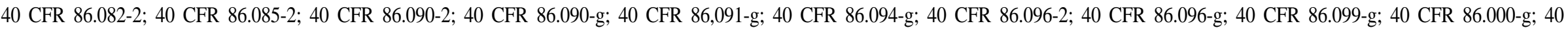
CFR 86.001-g; 40 CFR 86.004-g. Lisa Snapp, Office of Air and Radiation, Environmental Protection Agency, Personal communication, April 1999.

${ }^{\text {a }}$ Light truck categories LDT1-LDT4 were not actually created until 1994. From 1968 to 1978 all trucks with a Gross Vehicle Weight Rating (GVWR) up to 6,000 lbs were classified as light trucks and were required to meet the same standards. As of 1979, the maximum weight was raised to 8,500 lbs GVWR. During 1988-93, light trucks were divided into two subcategories that coincide with the current LDT1 and LDT2/3/4 categories. The standards for LDT1, LDT3, and LDT4 are given in tables 4-30a, 4-40c, and 4$30 \mathrm{~d}$.

${ }^{\mathbf{b}}$ The test procedure for measuring exhaust emissions has changed several times over the course of vehicle emissions regulation. The 7-mode procedure was used through model year 1971 and was replaced by the CVS-72 procedure beginning in model year 1972. The CVS-75 became the test procedure as of model year 1975 . While it may appear that the total hydrocarbon and carbon monoxide standards were relaxed in 1972-74, these standards were actually more stringent due to the more stringent nature of the CVS-72 test procedure. Additional standards for carbon monoxide and composite standards for non-methane hydrocarbons and nitrogen oxides tested over the new Supplemental Federal Test Procedure will be phased-in during model years 2000-02; these standards are not shown in this table.

${ }^{c}$ Emission standards had to be met for a useful life of 5 years/50,000 miles through model year 1983, and a full useful life of 11 years 120,000 miles was defined for 1985-93 (several

useful life options were available for 1984). Beginning in model year 1994, emission standards were established for an intermediate useful life of 5 years/50,000 miles as well as a full useful life of 11 years/120,000 miles (these standards are shown in parentheses). Hydrocarbon standards, however, were established only for full useful life. Tier 1 exhaust standards, except PM standards, were phased-in during 1994-96 at a rate of 40, 80, and 100 percent, respectively. PM standards were phased-in at a rate of 40, 80, and 100 percent during 1995-97.

In 1968-69, exhaust emission standards were issued in parts per million (ppm) rather than grams per mile and are, therefore, incompatible with this table.

No estimate available.

No standard set.

${ }^{\mathrm{g}}$ The cold CO emission standard is measured at 20 degrees F (rather than 75 degrees F) and is applicable for a 5-year/50,000-mile useful life

${ }^{\mathrm{h}}$ Gross vehicle weight rating (GVWR) is the maximum design loaded weight. Loaded vehicle weight (LVW) is the curb weight (nominal vehicle weight) plus 300 lbs. 
Table 4.19

Federal Exhaust Emission Certification Standards for Gasoline- and Diesel-Powered Light Trucks (Category LDT3) a,b,c (grams per mile)

\begin{tabular}{|c|c|c|c|c|c|c|c|c|c|c|c|c|c|c|c|}
\hline Engine Type \& Pollutant & $\begin{array}{l}\text { Prior to } \\
\text { control } \\
\end{array}$ & $1968-69$ & $1970-71$ & $1972 \quad 1973-74$ & 1975 & $1976-78$ & $1979-81 \quad 1982-83$ & 1984 & $1985-86$ & 1987 & $1988-89$ & 1990 & $1991-95$ & $1996+$ & \\
\hline \multicolumn{16}{|l|}{ Gasol ine } \\
\hline Hydrocarbons (total) & 11 & $d$ & 2.2 & 3.4 & 2.0 & & 1.7 & 0.80 & & & & & & $f$ & $(0.80)$ \\
\hline Non-methane hydrocarbons & $e$ & & & & & & & & & & & & & 0.32 & $(0.46)$ \\
\hline Carbon monoxide & 80 & $d$ & 23 & 39 & 20 & & 18 & 10 & & & & & & 4.4 & $(6.4)$ \\
\hline Cold-temp. carbon monoxide $g$ & $e$ & & & $\infty$ & & & xis & & & & & 稀 & 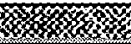 & 12.5 & $\theta$ \\
\hline Nitrogen oxides & 4 & 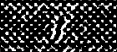 & & 3.0 & 3.1 & & 2,3 & & & & 2.3 & 1.7 & & 0.7 & $(0.98)$ \\
\hline Particulates & $e$ & 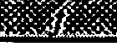 & 2 & 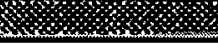 & 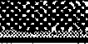 & s. & 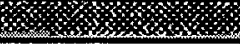 & 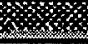 & 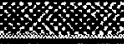 & 32 & ris & 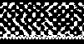 & 位 & $f$ & $(0.10)$ \\
\hline \multicolumn{16}{|l|}{ Diesel } \\
\hline Hydrocarbons (total) & 11 & & & & & 2.0 & 1.7 & 0.80 & & & & & & $f$ & $(0.80)$ \\
\hline Non-methane hydrocarbons & $e$ & $x$ & & & & & 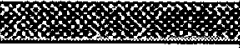 & & & & & & & 0.32 & $(0.46)$ \\
\hline Carbon monoxide & 80 & 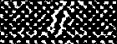 & & & & 20 & 18 & 10 & & & & & & 4.4 & $(6.4)$ \\
\hline Nitrogen oxides & 4 & 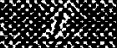 & & & & 3.1 & 2.3 & & & & 2.3 & 1.7 & & $f$ & $(0.98)$ \\
\hline Particulates & $e$ & 28 & & & & & 0.60 & & & 0.50 & 0.45 & & 0.13 & $f$ & $(0.10)$ \\
\hline \multirow{2}{*}{\multicolumn{2}{|c|}{ LDT3 Weight Criteria $h$}} & \multirow{2}{*}{\multicolumn{5}{|c|}{ GVWR up through $6,000 \mathrm{lbs}$}} & \multirow{2}{*}{\multicolumn{4}{|c|}{ GVWR up through $8,500 \mathrm{lbs}$}} & & ny $\mathrm{AL}$ & & \multicolumn{2}{|c|}{$\begin{array}{l}\text { ALW up through } \\
5,750 \mathrm{lbs} \\
\end{array}$} \\
\hline & & & & & & & & & & & \multicolumn{5}{|c|}{ GVWR $6,001-8,500 \mathrm{lbs}$} \\
\hline Test Procedure $b$ & & 7-mode & & CVS-72 & & $\mathrm{S}-75$ & & & & & & & & & \\
\hline \multirow{2}{*}{\multicolumn{2}{|c|}{$\begin{array}{l}\text { Useful Life (intermediate) } c \\
\text { (full) }\end{array}$}} & \multirow{2}{*}{\multicolumn{7}{|c|}{$\frac{5 \mathrm{yrs} / 50,000 \mathrm{mi}}{2}$}} & \multirow{2}{*}{\multicolumn{3}{|c|}{$11 \mathrm{yrs} / 120,000 \mathrm{mi}$}} & & 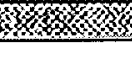 & \multirow{2}{*}{\multicolumn{2}{|c|}{$\begin{array}{l}5 \mathrm{yrs} / 50,000 \mathrm{mi} \\
11 \mathrm{yrs} / 120,000 \\
\end{array}$}} \\
\hline & & & & & & & & & & & & & & & \\
\hline
\end{tabular}

\section{Source:}

40 CFR 86.082-2; 40 CFR 86.085-2; 40 CFR 86.090-2; 40 CFR 86.090-9; 40 CFR 86.091-9; 40 CFR 86.094-9; 40 CFR 86.096-2; 40 CFR 86.096-g; 40 CFR 86.099-g; 40 CFR 86.000-g; 40 CFR 86.001-g; 40 CFR 86.004-g. Lisa Snapp, Office of Air and Radiation, Environmental Protection Agency, Personal communication, April 1999.

${ }^{2}$ Light truck categories LDT1-LDT4 were not actually created until 1994. From 1968 to 1978 all trucks with a Gross Vehicle Weight Rating (GVWR) up to 6,000 lbs were classified as light trucks and were required to meet the same standards. As of 1979, the maximum weight was raised to 8,500 lbs GVWR. During 1988-93, light trucks were divided into two subcategories that coincide with the current LDT1 and LDT2/3/4 categories.

${ }^{b}$ The test procedure for measuring exhaust emissions has changed several times over the course of vehicle emissions regulation. The 7-mode procedure was used through model year 1971 and was replaced by the CVS-72 procedure beginning in model year 1972. The CVS-75 became the test procedure as of model year 1975 . While it may appear that the total hydrocarbon and carbon monoxide standards were relaxed in 1972-74, these standards were actually more stringent due to the more stringent nature of the CVS-72 test procedure. Additional standards for carbon monoxide and composite standards for non-methane hydrocarbons and nitrogen oxides tested over the new

Supplemental Federal Test Procedure will be phased-in during model years 2002-04; these standards are not shown in this table.

'Emission standards had to be met for a full useful life of 5 years $/ 50,000$ miles through model year 1983, and a full useful life of 11 years 120,000 miles was defined for 1985-93 (several useful life options were available for 1984). Beginning in model year 1996, emission standards were established for an intermediate useful life of 5 years/50,000 miles as well as a full useful life of 11 years $/ 120,000$ miles (these standards are shown in parentheses). This applied to all pollutants except hydrocarbons and particulates for all LDT3s and NOx for diesel-powered LDT3s, which were only required to meet full useful life standards. Tier 1 exhaust standards were phased-in during 1996-97 at a rate of 50 and 100 percent, respectively.

In 1968-69, exhaust emission standards were issued in parts per million (ppm) rather than grams per mile and are, therefore, incompatible with this table.

Noestimate available.

fo standard set.

${ }^{\mathbf{g}}$ The cold CO emission standard is measured at 20 degrees $\mathrm{F}$ (rather than 75 degrees $\mathrm{F}$ ) and is applicable for a 5-year/50,000-mile useful life.

${ }^{h}$ Gross vehicle weight rating (GVWR) is the maximum design loaded weight. Loaded vehicle weight (LVW) is the curb weight (nominal vehicle weight) plus 300 lbs. 
Table 4.20

Federal Exhaust Emission Certification Standards for Gasoline- and Diesel-Powered Light Trucks (Category LDT4) $a, b, c$ (grams per mile)

Prior to

Engine Type \& Pollutant control $1968-69 \quad 1970-71 \quad 1972 \quad 1973-74 \quad 1975 \quad 1976-78 \quad 1979-81 \quad 1982-83 \quad 1984 \quad 1985-86 \quad 1987 \quad 1988-89 \quad 1990 \quad 1991-95 \quad 1996+$ Gasoline

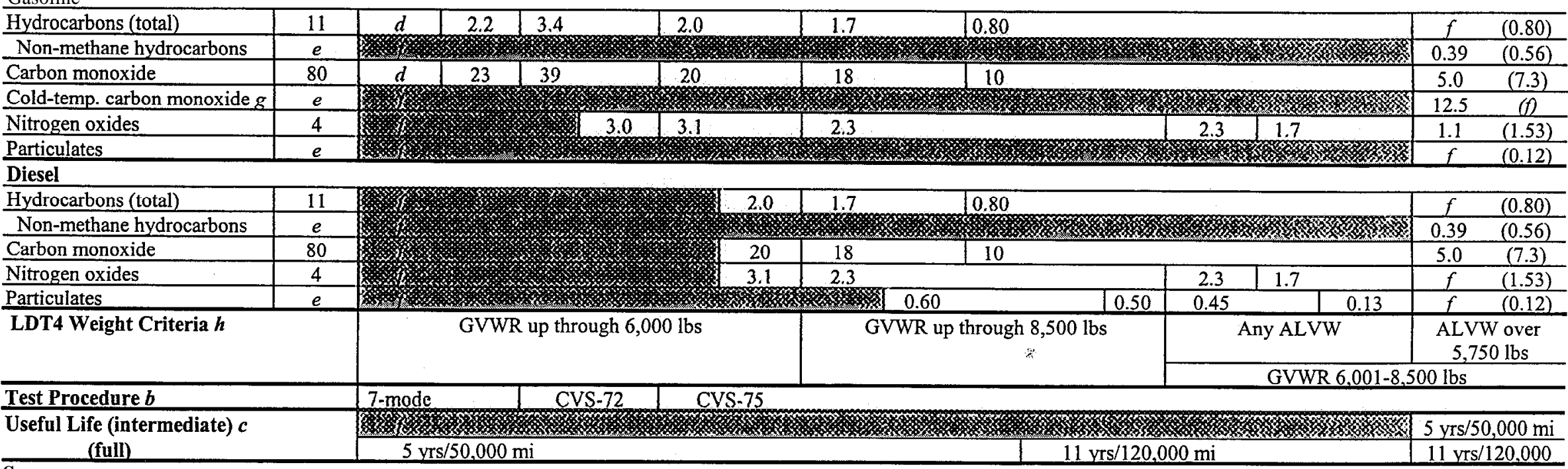

\section{Source:}

40 CFR 86.082-2; 40 CFR 86.085-2; 40 CFR 86.090-2; 40 CFR 86.090-g; 40 CFR 86.091-g; 40 CFR 86.094-g; 40 CFR 86.096-2; 40 CFR 86.096-g; 40 CFR 86.099-g;

40 CFR 86.000-g; 40 CFR 86.001-g; 40 CFR 86.004-g. Lisa Snapp, Office of Air and Radiation, Environmental Protection Agency, Personal communication, April 1999.

${ }^{\text {a }}$ Light truck categories LDT1-LDT4 were not actually created until 1994. From 1968 to 1978 all trucks with a Gross Vehicle Weight Rating (GVWR) up to 6,000 lbs were classified as light trucks and were required to meet the same standards. As of 1979, the maximum weight was raised to 8,500 lbs GVWR. During 1988-93, light trucks were divided into two subcategories that coincide with the current LDT1 and LDT2/3/4 categories.

${ }^{b}$ The test procedure for measuring exhaust emissions has changed several times over the course of vehicle emissions regulation. The 7-mode procedure was used through model year 1971 and was replaced by the CVS-72 procedure beginning in model year 1972. The CVS-75 became the test procedure as of model year 1975. While it may appear that the total hydrocarbon and carbon monoxide standards were relaxed in 1972-74, these standards were actually more stringent due to the more stringent nature of the CVS-72 test procedure. Additional standards for carbon monoxide and composite standards for non-methane hydrocarbons and nitrogen oxides tested over the new

Supplemental Federal Test Procedure will be phased-in during model years 2002-04; these standards are not shown in this table.

'Emission standards had to be met for a full useful life of 5 years $/ 50,000$ miles through model year 1983, and a full useful life of 11 years 120,000 miles was defined for 1985-93 (several useful life options were available for 1984). Beginning in model year 1996, emission standards were established for an intermediate useful life of 5 years $/ 50,000$ miles as well as a full useful life of 11 years/120,000 miles (these standards are shown in parentheses). This applied to all pollutants except hydrocarbons and particulates for all LDT3s and NOx for diesel-powered LDT3s, which were only required to meet full useful life standards. Tier 1 exhaust standards were phased-in during 1996-97 at a rate of 50 and 100 percent, respectively.

In 1968-69, exhaust emission standards were issued in parts per million (ppm) rather than grams per mile and are, therefore, incompatible with this table.

Noestimate available.

fostandard set.

${ }^{\mathfrak{g}}$ The cold CO emission standard is measured at 20 degrees $\mathrm{F}$ (rather than 75 degrees $\mathrm{F}$ ) and is applicable for a 5-year/50,000-mile useful life. curb weight.

hross vehicle weight rating (GVWR) is the maximum design loaded weight. Adjusted loaded vehicle weight (ALVW) is the numerical average of the GVWR and the 
Table 4.21

Federal Exhaust Emission Certification Standards for Gasoline- and Diesel-Powered Light Heavy Trucks

(Grams per brake horsepower-hour)

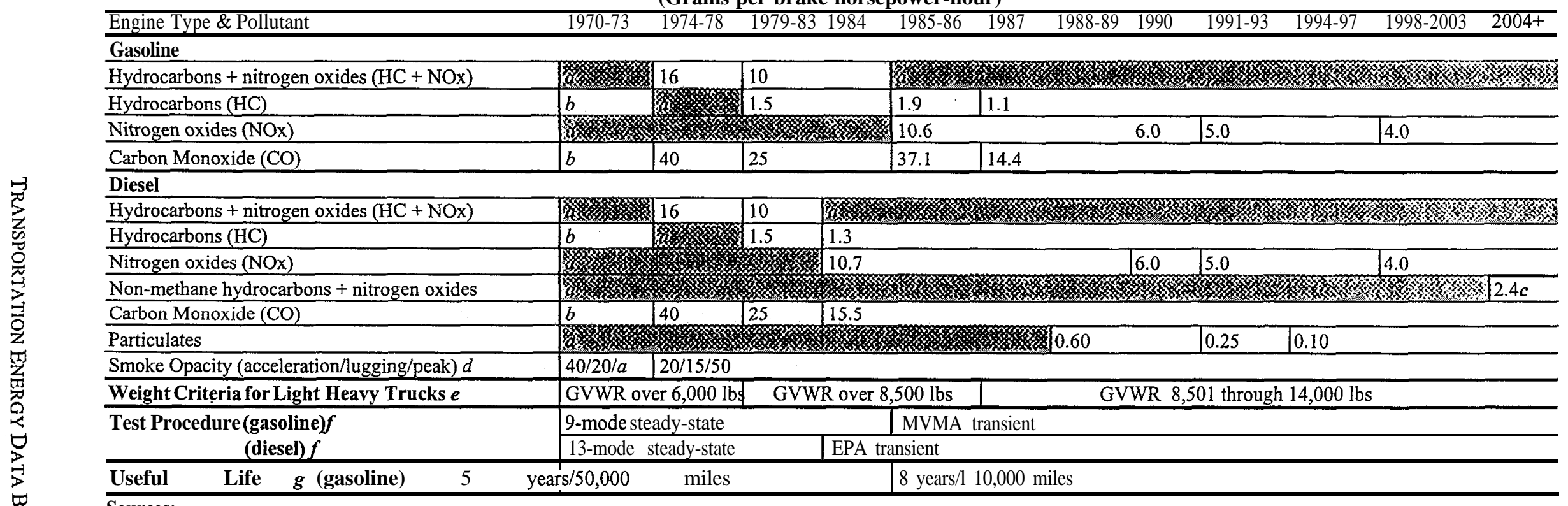

Sources:

40 CFR 86.082-2; 40 CFR 86.085-2; 40 CFR 86.088-10; 40 CFR 86.090-2; 40 CFR 86.090-10; 40 CFR 86.090-1 1; 40 CFR 86.091-10; 40 CFR 86.091-1 1; 40 CFR 86.093-1 1; 40 CFR 86.094-1 1; 40 CFR 86.096-2; 40 CFR 86.096-10; 40 CFR 86.096-1 1; 40 CFR 86.098-10; 40 CFR 86.098-I 1; 40 CFR 86.099-10; 40 CFR 86,099-1 1; 40 CFR 86.004-1 1; 40 CFR 86.004-15. Lisa Snapp, Office of Air and Radiation, Environmental Protection Agency, Personal communication, April 1999. Rob French, Office of Air and Radiation, Environmental Protection Agency, Personal communication, April 1999.

\section{${ }^{\mathrm{a}}$ No standard set.}

${ }^{\mathrm{b}}$ Although emission standards for hydrocarbons and carbon monoxide were in effect for these years, they were not measured in grams/brake horsepower-hour and are, therefore, incompatible with this table.

'Vehicles can meet a composite non-methane hydrocarbon and nitrogen oxide standard of 2.5 , if they meet a non-methane hydrocarbon standard of no more than 0.5 .

${ }^{\mathrm{d}}$ Smoke opacity is expressed in percentage for acceleration, lugging, and peak modes (acceleration/lugging/peak). Lugging is when a vehicle is carrying a load.

${ }^{\mathfrak{e}}$ Gross vehicle weight rating (GVWR) is the maximum design loaded weight.

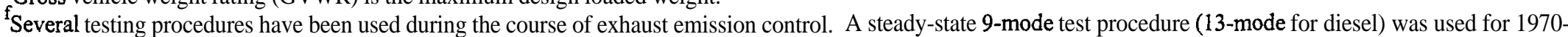
83 standards. For 1984, either the steady-state tests or the EPA transient test procedure could be used. For diesels, the EPA transient test was required from 1985 to the present. For gasoline-powered vehicles, either the EPA or MVMA (Motor Vehicle Manufacturers Association) transient test procedure could be used during 1985-86, and the MVMA procedure was required thereafter.

${ }^{g}$ Emissions standards apply to the useful life of the vehicle. Useful life was 5 years/50,000 miles through 1983 , and 8 years/1 10,000 miles for model year 1985 and after. 1984 was a transitional year in which vehicles could meet the older standard (and test procedure) or the newer one. Useful life requirement for gasoline-powered trucks meeting NOx standards for 1998 and after is 10 years/1 10,000 miles. The useful life requirements for heavy diesel truck standards are more complex and vary by vehicle weight, pollutant, test procedure, and year. Consult the U.S. Code of Federal Regulations for further information. 
Table 4.22

Federal Exhaust Emission Certification Standards for Gasoline- and Diesel-Powered Heavy Heavy Trucks (Grams per brake horsepower-hour)

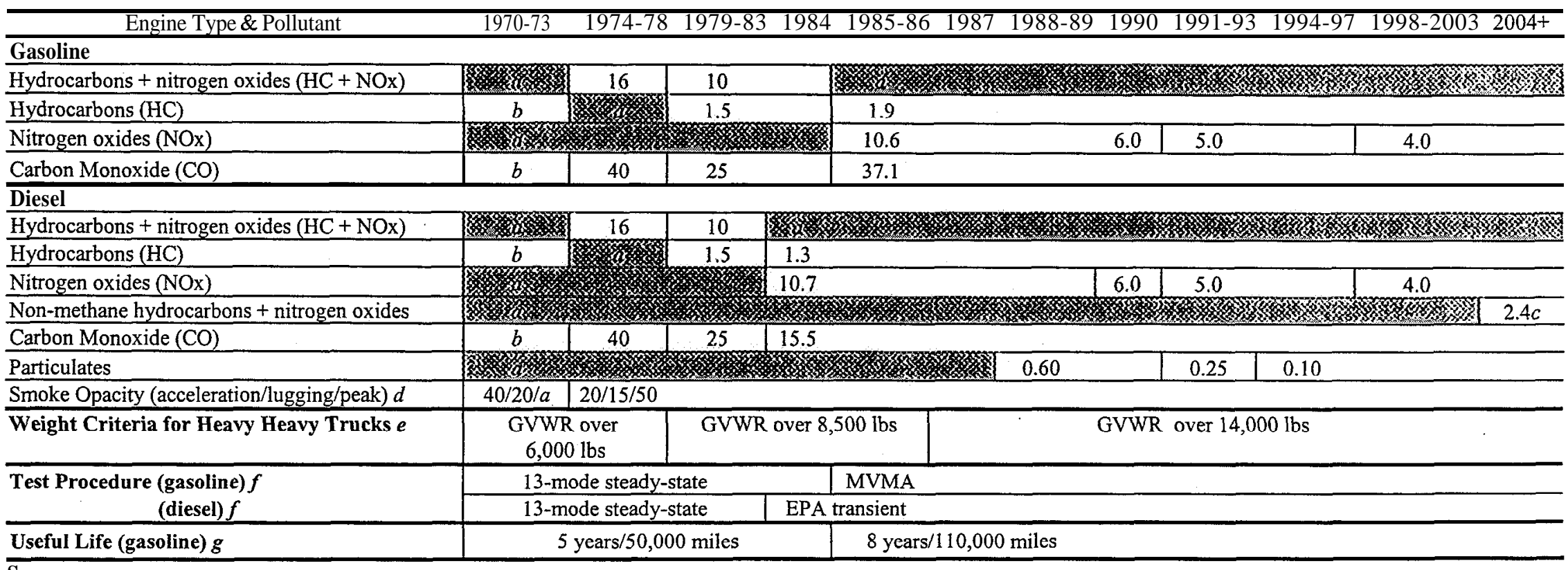

\section{Sources:}

40 CFR 86.082-2; 40 CFR 86.085-2; 40 CFR 86.088-10; 40 CFR 86.090-2; 40 CFR 86.090-10; 40 CFR 86.090-I 1; 40 CFR 86.091-10; 40 CFR 86.091-I 1; 40 CFR 86.093-1 1; 40 CFR 86.094-I 1; 40 CFR 86.096-2; 40 CFR 86.096-10; 40 CFR 86.096-1 1; 40 CFR 86.098-10; 40 CFR 86.098-1 1; 40 CFR 86.099-10; 40 CFR 86.099-1 1; 40 CFR 86.004-1 1; 40 CFR 86.004-15. Lisa Snapp, Office of Air and Radiation, Environmental Protection Agency, Personal communication, April 1999. Rob French, Office of Air and Radiation, Environmental Protection Agency, Personal communication, April 1999.

${ }^{\mathrm{a}} \mathrm{No}$ standard set

${ }^{\mathrm{b}}$ Although emission standards for hydrocarbons and carbon monoxide were in effect for these years, they were not measured in grams/brake horsepower-hour and are, therefore, incompatible with this table.

'Vehicles can meet a composite non-methane hydrocarbons and nitrogen oxides standard of 2.5 , if they meet a non-methane hydrocarbon standard of no more than 0.5 .

${ }^{\mathrm{d}}$ Smoke opacity is expressed in percentage for acceleration, lugging, and peak modes (acceleration/lugging/peak). Lugging is when a vehicle is carrying a load.

eGross vehicle weight rating (GVWR) is the maximum design loaded weight.

Several testing procedures have been used during the course of exhaust emission control. A steady-state 9-mode test procedure (13-mode for diesel) was used for 197083 standards. For 1984, either the steady-state tests or the EPA transient test procedure could be used. For diesels, the EPA transient test was required from 1985 to the present. For gasoline-powered vehicles, either either the EPA or MVMA (Motor Vehicle Manufacturers Association) transient test procedure could be used during 1985-86, and the MVMA procedure was required thereafter.

${ }^{g}$ Emissions standards apply to the useful life of the vehicle. Useful life was 5 years $/ 50,000$ miles through 1983 , and 8 years/l 10,000 miles for model year 1985 and after. 1984 was a transitional year in which vehicles could meet the older standard (and test procedure) or the newer one. Useful life requirement for gasoline-powered trucks meeting NOx standards for 1998 and after is IO years/l 10,000 miles. The useful life requirements for heavy diesel truck standards are more complex and vary by vehicle weight, pollutant, test procedure, and year. Consult the U.S. Code of Federal Regulations for further information. 
Table 4.23

California Passenger Cars and Light Trucks Federal Emission Certification Standards (grams/mile)

\begin{tabular}{|c|c|c|c|c|c|c|c|c|c|c|c|c|c|c|c|}
\hline \multirow{3}{*}{$\begin{array}{c}\text { Vehicle } \\
\text { Type }\end{array}$} & \multirow{3}{*}{$\begin{array}{l}\text { Emission } \\
\text { Category }\end{array}$} & \multicolumn{14}{|c|}{ Vehicle Useful Life } \\
\hline & & \multicolumn{7}{|c|}{5 Years / 50,000 Miles } & \multicolumn{7}{|c|}{10 Years / 100,000 Miles } \\
\hline & & $\mathrm{THC}$ & $\mathrm{NMHC}^{\mathrm{b}}$ & $\mathrm{NMOG}^{\mathrm{c}}$ & $\mathrm{CO}$ & $\mathrm{NO}_{\mathrm{x}}$ & $\mathrm{PM}$ & $\mathrm{HCHO}$ & THC" & $\mathrm{NMHC}^{\mathrm{b}}$ & $\mathrm{NMOG}^{\mathrm{c}}$ & $\mathrm{CO}$ & $\mathrm{NO}_{\mathrm{x}}$ & $\mathrm{PM}$ & $\mathrm{HCHO}$ \\
\hline \multirow[t]{6}{*}{ Passenger car } & Tier 0 & - & 0.39 & - & 7.0 & 0.4 & $0.08^{\mathrm{d}}$ & $0.015^{\mathrm{e}}$ & & & & & & & \\
\hline & Tier 1 & & 0.25 & - & 3.4 & 0.4 & $0.08^{d}$ & 0.015 & - & 0.31 & - & 4.2 & 0.6 & - & - \\
\hline & TLEV & - & & 0.125 & 3.4 & 0.4 & - & 0.015 & - & - & 0.156 & 4.2 & 0.6 & $0.08^{d}$ & 0.018 \\
\hline & LEV & - & & 0.075 & 3.4 & 0.2 & - & 0.015 & - & - & 0.090 & 4.2 & 0.3 & $0.08^{\mathrm{d}}$ & 0.018 \\
\hline & ULEV & & & 0.040 & 1.7 & 0.2 & - & 0.008 & - & - & 0.055 & 2.1 & 0.3 & $0.04^{\mathrm{d}}$ & 0.011 \\
\hline & ZEV & 0.0 & 0.00 & 0.000 & 0.0 & 0.0 & 0.00 & 0.000 & 0.00 & 0.00 & 0.000 & 0.0 & 0.0 & 0.00 & 0.000 \\
\hline \multirow[t]{6}{*}{ LDT1 } & Tier 0 & - & 0.39 & - & 9.0 & 0.4 & $0.08^{\mathrm{d}}$ & $0.015^{\prime}$ & & & & & & & \\
\hline & Tier 1 & - & 0.25 & - & 3.4 & 0.4 & $0.08^{\mathrm{d}}$ & 0.015 & - & 0.31 & - & 4.2 & 0.6 & - & - \\
\hline & TLEV & & & 0.125 & 3.4 & 0.4 & - & 0.015 & - & - & 0.156 & 4.2 & 0.6 & $0.08^{d}$ & 0.018 \\
\hline & LEV & & & 0.075 & 3.4 & 0.2 & - & 0.015 & - & - & 0.090 & 4.2 & 0.3 & $0.08^{d}$ & 0.018 \\
\hline & ULEV & & & 0.040 & 1.7 & 0.2 & - & 0.008 & - & - & 0.055 & 2.1 & 0.3 & $0.04^{\mathrm{d}}$ & 0.011 \\
\hline & ZEV & 0.0 & 0.00 & 0.000 & 0.0 & 0.0 & 0.00 & 0.000 & 0.00 & 0.00 & 0.000 & 0.0 & 0.0 & 0.00 & 0.000 \\
\hline \multirow[t]{5}{*}{ LDT2 } & Tier 0 & & 0.50 & - & 9.0 & 1.0 & $0.08^{\mathrm{d}}$ & $0.018^{\prime}$ & & & & & & & \\
\hline & Tier 1 & & 0.32 & - & 4.4 & 0.7 & $0.08^{\mathrm{d}}$ & $0.018^{\prime}$ & - & 0.40 & - & 5.5 & 0.97 & - & - \\
\hline & TLEV & & & 0.160 & 4.4 & 0.7 & - & 0.018 & - & - & 0.200 & 5.5 & 0.9 & $0.10^{d}$ & 0.023 \\
\hline & LEV & & & 0.100 & 4.4 & 0.4 & - & 0.018 & - & - & 0.130 & 5.5 & 0.5 & $0.10^{\mathrm{d}}$ & 0.023 \\
\hline & ULEV & & & 0.050 & 2.2 & 0.4 & - & 0.009 & - & - & 0.070 & 2.8 & 0.5 & $0.05^{\mathrm{d}}$ & 0.013 \\
\hline
\end{tabular}

Source:

U.S. Environmental Protection Agency, Office of Mobile Sources, EPA 420-B-98-001. (Additional resources: http://www.epa.gov/OMSWWW)

Note:

LDT1 $=$ light truck up through 3,750 lbs. loaded vehicle weight; LDT2 = light truck greater than 3,750 Ibs. loaded vehicle weight.

a THCE for methanol vehicles. Does not apply to CNG vehicles.

${ }^{b}$ THCE for Tier 0 methanol vehicles. NMHCE for other alcohol vehicles.

${ }^{c}$ NMHC for diesel-fueled vehicles.

${ }^{\mathrm{d}}$ Diesel-fueled vehicles only.

${ }^{\mathbf{e}}$ Ethanol- and methanol-fueled vehicles only. 
California's Low-Emission Vehicle regulationsprovidefor reduced emission vehicles to be available to consumers. Vehicles meeting these standards have even lower emissions than the basic standards for all new vehicles sold in California. Currently, there is a wide array of TLEVS and LEVS, and a few ULEVS and ZEVS on the market. For a listing of the available low emission vehicles, see the California Air Resources Board web site referenced below.

Table 4.24

California Vehicle Emission Reduction for Passenger Cars and Light Trucks

\begin{tabular}{llcc}
\hline & \multicolumn{3}{c}{$\begin{array}{c}\text { Emission reduction from the } \\
\text { basic California standards" }\end{array}$} \\
\cline { 2 - 4 } & HC & c o & NOx \\
\hline Transitional Low-Emission Vehicle (TLEV) & $50 \%$ & $=$ & $=$ \\
Low-Emission Vehicle (LEV) & $70 \%$ & $=$ & $50 \%$ \\
Ultra-Low-Emission Vehicle (ULEV) & $85 \%$ & $50 \%$ & $50 \%$ \\
Zero-Emission Vehicles (ZEV) & $100 \%$ & $100 \%$ & $100 \%$ \\
\hline
\end{tabular}

Source:

California Air Resources Board web site, http://www.arb.ca.gov/msprog/ccbg/ccbg.htm (Additional resources: http://www.arb.ca.gov)

Note:

$=$ indicates equivalent emissions to vehicles meeting the basic California standard.

${ }^{\mathrm{a} S e e}$ Table 4.23 . 
The California Air Resources Board adopted requirements in 1991 for fleet mixture in order to meet the emission standards. By the year 2001, it is proposed that $90 \%$ of each vehicle manufacturer's fleet be low-emission vehicles. A March 1996 amendment to the plan allows the marketplace to determine the number of zero emission vehicles from 1998 to 2002.

Table 4.25

California Air Resources Board Requirements for Meeting Emission Standards

\begin{tabular}{cccccc}
\hline Year & $\begin{array}{c}\text { Conventional } \\
\text { vehicles }\end{array}$ & $\begin{array}{c}\text { Transitional } \\
\text { low-emission } \\
\text { vehicles }\end{array}$ & $\begin{array}{c}\text { Low-emission } \\
\text { vehicles }\end{array}$ & $\begin{array}{c}\text { Ultra-low- } \\
\text { emission } \\
\text { vehicles }\end{array}$ & $\begin{array}{c}\text { Zero } \\
\text { emission } \\
\text { vehicles }\end{array}$ \\
\hline 1993 & $100 \%$ & & & & \\
1994 & $90 \%$ & $10 \%$ & & & \\
1995 & $85 \%$ & $15 \%$ & & & \\
1996 & $80 \%$ & $20 \%$ & $25 \%$ & $2 \%$ & \\
1997 & $73 \%$ & & $48 \%$ & $2 \%$ & \\
1998 & $48 \%$ & & $73 \%$ & $2 \%$ & \\
1999 & $25 \%$ & & $90 \%$ & $2 \%$ & \\
2000 & & & $90 \%$ & $5 \%$ & \\
2001 & & & $75 \%$ & $10 \%$ & \\
2002 & & & & & \\
2003 & & & & & \\
\hline
\end{tabular}

Source:

California Air Resources Board, Mobile Sources Division, El Monte, CA, 1996. (Additional resources: http://www.arb.ca.gov) 


\section{Chapter 5}

\section{Transportation and the Economy}

Summary Statistics from Tables/Figures in this Chapter

Source

Figure 5.1

Share of gasoline cost attributed to taxes, 1998

$\begin{array}{lc}\text { Canada } & 47 \% \\ \text { France } & 80 \% \\ \text { Germany } & 74 \% \\ \text { Japan } & 60 \% \\ \text { United Kingdom } & 76 \% \\ \text { United States } & 36 \%\end{array}$

Table 5.4 Retail prices for motor fuel in the U.S., 1998 (current cents per gallon)

Gasoline, average for all types $\quad 111.5$

Diesel fuel $\quad 112.0$

Table $5.10 \quad$ Average price of a new car, 1997 (current dollars) 20,447

$\begin{array}{ll}\text { Domestic } & 18,624\end{array}$

Import $\quad 29,708$

Table $5.11 \quad$ Automobile operating costs, 1998

Variable costs (constant 1997 dollars per 10,000 miles) $\quad 1,058$

Fixed costs (constant 1997 dollars per 10,000 miles) $\quad 4,315$

Table 5.17 Transportation share of total employment

1960

$13.5 \%$

1980

$11.3 \%$

1997

$11.1 \%$ 
Table 5.1

Gasoline Prices for Selected Countries, 1978-98

\begin{tabular}{|c|c|c|c|c|c|c|c|c|c|c|c|}
\hline & \multicolumn{9}{|c|}{ Current dollars per gallon } & \multicolumn{2}{|c|}{$\begin{array}{l}\text { Average annual } \\
\text { percentage change }\end{array}$} \\
\hline & $1978 ”$ & 1982 & $1986 ”$ & $1990^{b}$ & $1992^{b}$ & $1994^{\mathrm{b}}$ & $1996^{b}$ & $1997^{b}$ & $1998^{b}$ & $1978-98$ & $1990-98$ \\
\hline China & $\bar{d}$ & d & $\bar{d}$ & $\bar{d}$ & d & $\mathrm{d}$ & $093 "$ & d & $\mathrm{d}$ & $\bar{d}$ & $\mathrm{~d}$ \\
\hline India & d & d & d & 1.92 & 2.59 & 2.28 & 2.25 & $2.65 ”$ & d & $d$ & $d$ \\
\hline Japan & $2.00 ”$ & $2.60 ”$ & $2.79^{\prime}$ & 3.05 & $3.78 "$ & 4.14 & 3.77 & 3.28 & 2.94 & $1.9 \%$ & $-0.5 \%$ \\
\hline France & 2.15 & 2.56 & 2.58 & 3.40 & 3.69 & 3.31 & 4.41 & 4.22 & 3.82 & $2.9 \%$ & $1.5 \%$ \\
\hline United Kingdom & 1.22 & 2.42 & 2.07 & 2.55 & 3.28 & 2.86 & 3.47 & 4.25 & 3.90 & $6.0 \%$ & $5.5 \%$ \\
\hline Germany & 1.75 & 2.17 & 1.88 & 2.72 & 3.84 & 3.34 & 4.32 & 3.87 & 3.33 & $3.3 \%$ & $2.6 \%$ \\
\hline \multirow[t]{4}{*}{ UnitedanadStates ${ }^{e}$} & $0.69^{\prime}$ & $1.37^{\prime}$ & $1.31 ”$ & $1.92 "$ & $2.11^{\prime}$ & 1.57 & 1.80 & 1.92 & 1.55 & $4.1 \%$ & $-2.6 \%$ \\
\hline & $0.66^{\prime}$ & 1.32 & $0.93 ”$ & $1.04^{\prime}$ & $1.07 ”$ & 1.24 & 1.28 & 1.42 & 1.27 & $3.3 \%$ & $2.5 \%$ \\
\hline & \multicolumn{9}{|c|}{ Constant 1997 dollars ${ }^{i}$ per gallon } & \multicolumn{2}{|c|}{$\begin{array}{l}\text { Average annual } \\
\text { percentage change }\end{array}$} \\
\hline & $1978 ”$ & $1982 ”$ & $1986 "$ & $1990^{\mathrm{b}}$ & $1992^{b}$ & $1994^{\mathrm{b}}$ & $1996^{\mathrm{b}}$ & $1997^{b}$ & $1998^{b}$ & $1978-98$ & 1990-98 \\
\hline China & d & d & $\mathrm{d}$ & $d$ & $d$ & $\mathrm{~d}$ & 0.95 & $\bar{d}$ & 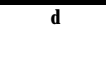 & $d$ & $\mathrm{~d}$ \\
\hline India & $d$ & $d$ & d & 2.36 & 2.95 & 2.46 & 2.30 & 2.65 & d & $\mathrm{d}$ & d \\
\hline Japan & 4.92 & $4.32^{\prime}$ & 4.07” & $3.75 "$ & $4.31 ”$ & 4.47 & 3.85 & $3.28^{\prime}$ & 2.88 & $-2.6 \%$ & $-3.2 \%$ \\
\hline France & 5.29 & 4.25 & 3.77 & 4.18 & 4.21 & 3.57 & 4.50 & 4.22 & 3.74 & $-1.7 \%$ & $-1.4 \%$ \\
\hline United Kingdom & 3.00 & 4.02 & 3.02 & 3.14 & 3.74 & 3.09 & 3.54 & 4.25 & 3.82 & $1.2 \%$ & $2.5 \%$ \\
\hline Germany & 4.31 & 3.60 & 2.74 & 3.35 & 4.38 & 3.61 & 4.41 & 3.87 & 3.26 & $-1.4 \%$ & $-0.3 \%$ \\
\hline Canada & $1.70 "$ & 2.27 & $1.91 ”$ & $2.36^{\prime}$ & $2.41^{\prime}$ & 1.70 & 1.84 & 1.92 & 1.52 & $-0.6 \%$ & $-5.4 \%$ \\
\hline United States $^{e}$ & $1.62^{\prime}$ & 2.19 & $1.36 ”$ & $1.28^{\prime}$ & $1.22^{\prime}$ & 1.34 & 1.31 & 1.42 & 1.24 & $-1.3 \%$ & $-0.4 \%$ \\
\hline
\end{tabular}

U.S. Department of Energy, Energy Information Administration, International Energy Annual 1997, Washington, DC, April 1999, Table 7.2 and annual.

Note:

(Additional resources: http://www.eia.doe.gov)

Comparisons between prices and price trends in different countries require care. They are of limited validity because of fluctuations in exchange rates; differences in product quality, marketing practices, and market structures; and the extent to which the standard categories of sales are representative of total national sales for a given period.

a Prices represent the retail prices (including taxes) for premium leaded gasoline. Prices are representative for each country based on quarterly data averaged for the year.

${ }^{\mathbf{b}}$ Prices represent the retail prices (including taxes) for premium gasoline on January 1 of the year, or the available time period closest to January 1.

c Regular gasoline.

${ }^{\circledR}$ Data are not available.

e These estimates are for international comparisons only and do not necessarily correspond to gasoline price estimates in other sections of the book.

'Adjusted by the U.S. Consumer Price Inflation Index. 
Figure 5.1. Gasoline Prices for Selected Countries, 1990 and 1998

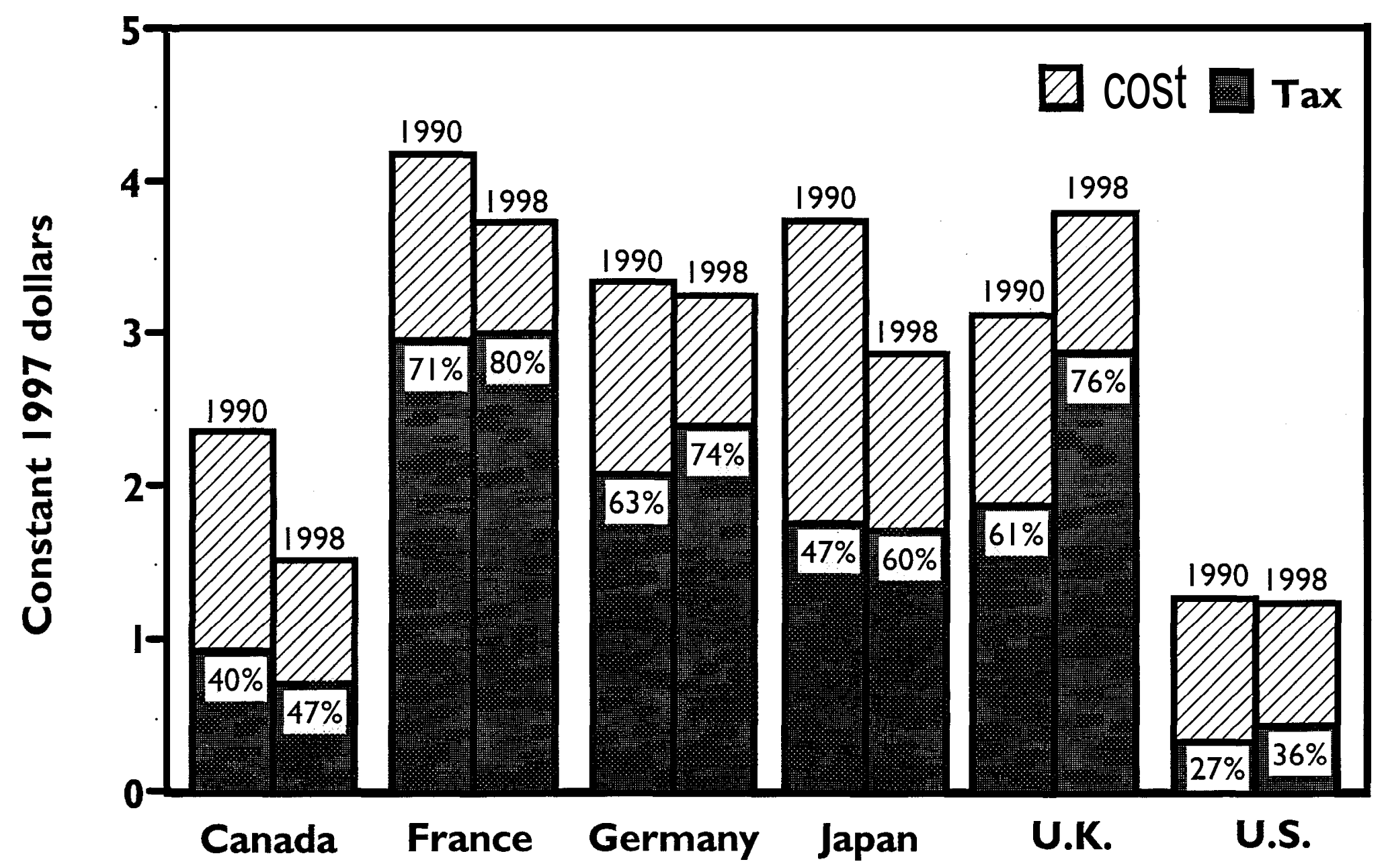

Source:

Table 5.1 and International Energy Agency, Energy Prices and Taxes, Fourth Quarter 1998, Paris, France, 1999. (Additional resources: http://www.iea.org) 
Table 5.2

Diesel Fuel Prices for Selected Countries, 1978-98

\begin{tabular}{|c|c|c|c|c|c|c|c|c|c|c|c|}
\hline & \multicolumn{9}{|c|}{ Current dollars per gallon } & \multicolumn{2}{|c|}{$\begin{array}{l}\text { Average annual } \\
\text { percentage change }\end{array}$} \\
\hline & $1978 "$ & 1982" & $1986 "$ & $1990^{b}$ & $1992^{\mathrm{b}}$ & $1994^{b}$ & $1996^{b}$ & $1997^{\mathrm{b}}$ & $1998^{b}$ & $1978-98$ & $1990-98$ \\
\hline China & $\bar{c}$ & \multirow{2}{*}{ c } & $\overline{\mathbf{c}}$ & $\bar{c}$ & c & $\overline{\mathbf{c}}$ & 0.88 & $\bar{c}$ & $\bar{c}$ & $\bar{c}$ & $\bar{c}$ \\
\hline India & \multirow{2}{*}{$\begin{array}{l}\mathrm{c} \\
\mathrm{c}\end{array}$} & & c & 0.78 & \multirow{2}{*}{$\begin{array}{r}0.73 \\
\mathbf{c}\end{array}$} & 0.74 & 0.92 & 1.11 & c & c & c \\
\hline Japan & & 1.78 & 1.90 & 1.75 & & 2.48 & 2.51 & 2.34 & 2.40 & \multirow{2}{*}{$\begin{array}{c}\text { c } \\
3.7 \%\end{array}$} & $4.0 \%$ \\
\hline France & 1.30 & 1.88 & 1.69 & 1.78 & & 2.10 & 3.10 & 3.08 & 2.71 & & $5.4 \%$ \\
\hline United Kingdom & 1.24 & 2.05 & 1.71 & 2.04 & c & 2.46 & 3.26 & 3.78 & 3.92 & $5.9 \%$ & $8.5 \%$ \\
\hline Germany & 1.48 & 1.81 & 1.51 & 2.72 & 2.81 & 2.16 & 3.02 & 2.91 & 2.43 & \multirow{2}{*}{$2.5 \%$} & $-1.4 \%$ \\
\hline Canada & & 1.27 & 1.27 & 1.55 & 1.78 & 1.47 & 1.43 & 1.56 & 1.46 & & $-0.7 \%$ \\
\hline \multirow[t]{3}{*}{ United States $^{\mathbf{d}}$} & 0.54 & 1.16 & 0.94 & 0.99 & 1.06 & 0.96 & 1.15 & 1.29 & 1.12 & $3.7 \%$ & $1.6 \%$ \\
\hline & \multicolumn{9}{|c|}{ Constant 1997 dollars' per gallon } & \multicolumn{2}{|c|}{$\begin{array}{l}\text { Average annual } \\
\text { percentage change }\end{array}$} \\
\hline & $1978 ”$ & $1982 ”$ & $1986 "$ & $1990^{\mathrm{b}}$ & $1992^{b}$ & $1994^{b}$ & $1996^{b}$ & $1997^{\mathrm{b}}$ & $1998^{\mathrm{b}}$ & $1978-98$ & $1990-98$ \\
\hline China & c & 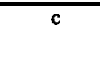 & $\mathbf{c}$ & $\mathbf{c}$ & $\mathbf{c}$ & $\mathbf{c}$ & 0.90 & $\mathbf{c}$ & $\mathbf{c}$ & $\mathbf{c}$ & $\mathbf{c}$ \\
\hline Japan & c & 2.95 & 2.77 & 2.15 & c & 2.68 & 2.56 & 2.34 & 2.35 & c & $1.1 \%$ \\
\hline France & 3.20 & 3.12 & 2.47 & 2.19 & \multirow[b]{2}{*}{ c } & 2.27 & 3.16 & 3.08 & 2.66 & $-0.9 \%$ & $2.5 \%$ \\
\hline United Kingdom & 3.05 & 3.40 & 2.50 & 2.51 & & 2.66 & 3.33 & 3.78 & 3.84 & $1.2 \%$ & $5.5 \%$ \\
\hline Germany & 3.64 & 3.00 & 2.20 & 3.35 & 3.20 & 2.33 & 3.08 & 2.91 & 2.38 & $-2.1 \%$ & $-4.2 \%$ \\
\hline Canada & c & 2.11 & 1.85 & 1.91 & 2.03 & 1.59 & 1.46 & 1.56 & 1.43 & c & $-3.6 \%$ \\
\hline United States $^{\mathbf{d}}$ & 1.33 & 1.93 & 1.37 & 1.22 & 1.21 & 1.04 & 1.17 & 1.29 & 1.10 & $-0.9 \%$ & $-1.3 \%$ \\
\hline
\end{tabular}

\section{Source:}

U.S. Department of Energy, Energy Information Administration, International Energy Annual 1997, Washington, DC, April 1999, Table 7.2 and annual.

Note: (Additional resources: http://www.eia.doe.gov)

Comparisons between prices and price trends in different countries require care. They are of limited validity because of fluctuations in exchange rates; differences in product quality, marketing practices, and market structures; and the extent to which the standard categories of sales are representative of total national sales for a given period.

a Prices represent the retail prices (including taxes) for diesel fuel. Prices are representative for each country based on quarterly data averaged for the year.

${ }^{b}$ Prices represent the retail prices (including taxes) for diesel fuel on January 1 of the year, or the available time period closest to January 1.

c Data are not available.

d These estimates are for international comparisons only and do not necessarily correspond to gasoline price estimates in other sections of the book.

'Adjusted by the U.S. Consumer Price Inflation Index. 


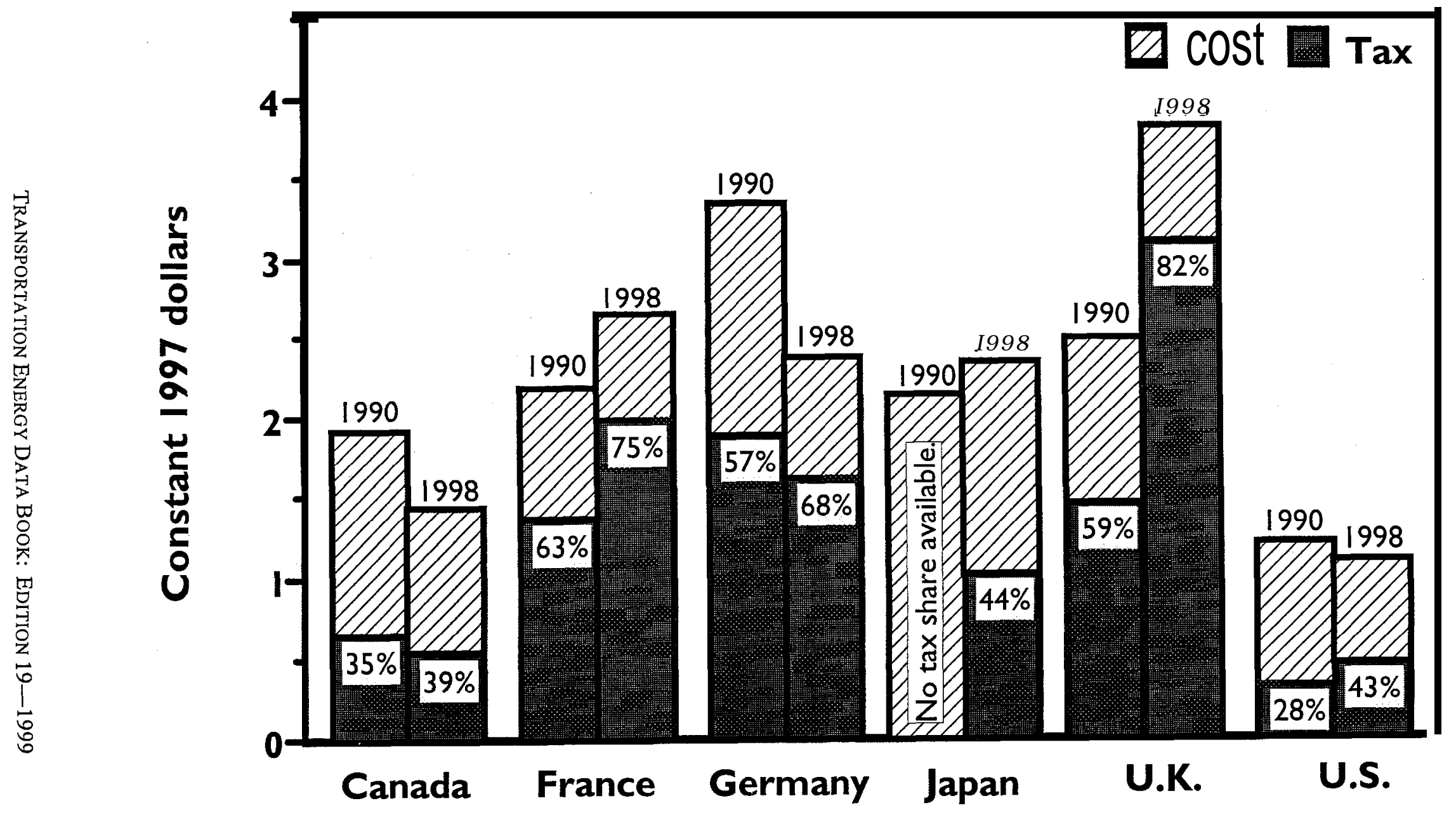

Source:

Table 5.2 and International Energy Agency, Energy Prices and Taxes, Fourth Quarter 1998, Paris, France, 1999. (Additional resources: http://www.iea.org) 
Though the cost of crude oil certainly influences the price of gasoline, it is not the only factor which determines the price at the pump. Processing cost, transportation cost, and taxes also play a major part of the cost of a gallon of gasoline. The average price of a barrel of crude oil (in constant 1997 dollars) declined by 55\% from 1990 to 1998, while the average price of a gallon of gasoline declined $27 \%$ in this same time period.

Table 5.3

Prices for a Barrel of Crude Oil and a Gallon of Gasoline, 1978-98

\begin{tabular}{|c|c|c|c|c|c|}
\hline \multirow[b]{2}{*}{ Year } & \multicolumn{2}{|c|}{$\begin{array}{c}\text { Crude oil" } \\
\text { (dollars per barrel) } \\
\end{array}$} & \multicolumn{2}{|c|}{$\begin{array}{c}\text { Gasoline }^{\mathrm{b}} \\
\text { (cents per gallon) }\end{array}$} & \multirow{2}{*}{$\begin{array}{l}\text { Ratio of } \\
\text { gasoline } \\
\text { to crude } \\
\quad \text { oil }\end{array}$} \\
\hline & Current & Constant 1997" & Current & Constant 1997' & \\
\hline 1978 & 12.46 & 30.65 & 65.2 & 160.39 & 0.45 \\
\hline 1979 & 17.72 & 39.16 & 88.2 & 194.92 & 0.48 \\
\hline 1980 & 28.07 & 54.74 & 122.1 & 238.10 & 0.55 \\
\hline 1981 & 35.24 & 62.37 & 135.3 & 239.48 & 0.62 \\
\hline 1982 & 31.87 & 52.90 & 128.1 & 212.65 & 0.59 \\
\hline 1983 & 28.99 & 46.67 & 122.5 & 197.23 & 0.56 \\
\hline 1984 & 28.63 & 44.38 & 119.8 & 185.69 & 0.57 \\
\hline 1985 & 26.75 & 39.86 & 119.6 & 178.20 & 0.53 \\
\hline 1986 & 14.55 & 21.24 & 93.1 & 135.93 & 0.37 \\
\hline 1987 & 17.90 & 25.24 & 95.7 & 134.94 & 0.27 \\
\hline 1988 & 14.67 & 19.95 & 96.3 & 130.97 & 0.36 \\
\hline 1989 & 17.97 & 23.18 & 106.0 & 136.74 & 0.40 \\
\hline 1990 & 22.22 & 27.33 & 121.7 & 149.69 & 0.43 \\
\hline 1991 & 19.06 & 22.49 & 119.6 & 141.13 & 0.38 \\
\hline 1992 & 18.43 & 21.01 & 119.0 & 135.66 & 0.37 \\
\hline 1993 & 16.41 & 18.22 & 117.3 & 130.20 & 0.33 \\
\hline 1994 & 15.59 & 16.84 & 117.4 & 126.79 & 0.32 \\
\hline 1995 & 17.23 & 18.09 & 120.5 & 126.53 & 0.34 \\
\hline 1996 & 20.71 & 21.12 & 128.8 & 131.38 & 0.38 \\
\hline 1997 & 19.04 & 19.04 & 129.1 & 129.10 & 0.35 \\
\hline 1998 & 12.57 & 12.32 & 111.5 & 109.27 & 0.27 \\
\hline \multicolumn{6}{|c|}{ Average annualpercentage change } \\
\hline $1978-98$ & $0.0 \%$ & $-4.5 \%$ & $2.7 \%$ & $-1.9 \%$ & \\
\hline $1988-98$ & $-1.5 \%$ & $-4.7 \%$ & $1.5 \%$ & $-1.8 \%$ & \\
\hline
\end{tabular}

Sources:

Crude oil - U.S. Department of Energy, Energy Information Administration, Monthly Energy Review, March 1999, Washington, DC, Table 9.1, p. 111.

Gasoline - U.S. Department of Energy, Energy Information Administration, Monthly Energy Review, March 1999, Washington, DC, Table 9.4, p. 114.

(Additional resources: http://www.eia.doe.gov)

"Refiner acquisition cost of composite (domestic and imported) crude oil.

bAverage for all types. These prices were collected from a sample of service stations in 85 urban areas selected to represent all urban consumers. Urban consumers make up about $80 \%$ of the total U.S. population.

"Adjusted by the Consumer Price Inflation Index. 
Table 5.4

Retail Prices for Motor Fuel, 1978-98

(cents per gallon, including tax)

\begin{tabular}{|c|c|c|c|c|c|c|c|c|}
\hline \multirow[b]{2}{*}{ Year } & \multicolumn{2}{|c|}{ Diesel fuel $^{\mathbf{a}}$} & \multicolumn{2}{|c|}{$\begin{array}{c}\text { Unleaded regular gasoline } \\
\text { (87 to } 88.9 \text { octane) }\end{array}$} & \multicolumn{2}{|c|}{$\begin{array}{l}\text { Unleaded premium gasoline } \\
\text { (91 octane and above) }\end{array}$} & \multicolumn{2}{|c|}{$\begin{array}{l}\text { Average for all } \\
\text { gasoline types } b\end{array}$} \\
\hline & Current & $\begin{array}{c}\text { Constant } \\
1997 "\end{array}$ & Current & $\begin{array}{l}\text { Constant } \\
1997 " \\
\end{array}$ & Current & $\begin{array}{c}\text { Constant } \\
1997 \\
\end{array}$ & Current & $\begin{array}{c}\text { Constant } \\
1997 "\end{array}$ \\
\hline 1978 & d & d & 67.0 & 164.8 & $\bar{d}$ & $\mathrm{~d}$ & 65.2 & 160.4 \\
\hline 1979 & d & d & 90.3 & 199.6 & d & d & 88.2 & 194.9 \\
\hline 1980 & 101 & 197 & 124.5 & 242.8 & d & $\mathrm{d}$ & 122.1 & 238.1 \\
\hline 1981 & 118 & 209 & 137.8 & 243.9 & 147.0 & 260.2 & 135.3 & 239.1 \\
\hline 1982 & 116 & 193 & 129.6 & 215.1 & 141.5 & 234.9 & 128.1 & 212.7 \\
\hline 1983 & 120 & 193 & 124.1 & 199.8 & 138.3 & 222.7 & 122.5 & 197.2 \\
\hline 1984 & 122 & 189 & 121.2 & 187.9 & 136.6 & 211.7 & 119.8 & 185.7 \\
\hline 1985 & 122 & 182 & 120.2 & 179.1 & 134.0 & 199.7 & 119.6 & 178.2 \\
\hline 1986 & 94 & 137 & 92.7 & 135.3 & 108.5 & 158.4 & 93.1 & 135.9 \\
\hline 1987 & 96 & 135 & 94.8 & 133.7 & 109.3 & 154.1 & 95.7 & 134.9 \\
\hline 1988 & 95 & 129 & 94.6 & 128.7 & 110.7 & 150.6 & 96.3 & 131.0 \\
\hline 1989 & 102 & 132 & 102.1 & 131.7 & 119.7 & 154.4 & 106.0 & 136.7 \\
\hline 1990 & 107 & 122 & 116.4 & 143.2 & 134.9 & 165.9 & 121.7 & 149.7 \\
\hline 1991 & 91 & 107 & 114.0 & 134.5 & 132.1 & 155.9 & 119.6 & 141.1 \\
\hline 1992 & 106 & 121 & 112.7 & 128.5 & 131.6 & 150.0 & 119.0 & 135.7 \\
\hline 1993 & 98 & 109 & 110.8 & 123.0 & 130.2 & 144.5 & 117.3 & 130.2 \\
\hline 1994 & 96 & 104 & 111.2 & 120.1 & 130.5 & 140.9 & 117.4 & 126.8 \\
\hline 1995 & 97 & 102 & 114.7 & 120.4 & 133.6 & 104.3 & 120.5 & 126.5 \\
\hline 1996 & 115 & 117 & 123.1 & 125.6 & 141.3 & 144.1 & 128.8 & 131.4 \\
\hline 1997 & 129 & 129 & 123.4 & 123.4 & 141.6 & 141.6 & 129.1 & 129.1 \\
\hline 1998 & 112 & 110 & 111.9 & 109.7 & 125.0 & 122.5 & 111.5 & 109.3 \\
\hline \multicolumn{9}{|c|}{ Average annual percentage change } \\
\hline 1978-98 & $0.6 \% "$ & $-3.2 \%$ & $2.6 \%$ & $-2.0 \%$ & $-0.9 \%$ & $-4.3 \%$ & $2.7 \%$ & $-1.9 \%$ \\
\hline $1988-98$ & $1.7 \%$ & $-1.6 \%$ & $1.7 \%$ & $-1.6 \%$ & $1.2 \%$ & $-2.0 \%$ & $1.5 \%$ & $-1.8 \%$ \\
\hline
\end{tabular}

\section{Source:}

Gasoline - U.S. Department of Energy, Energy Information Administration, Monthly Energy Review, March 1999, Washington, DC, Table 9.4, p. 114.

Diesel - U.S. Department of Energy, Energy Information Administration, International Energy Annual 1997, Washington, DC, April 1999, Table 7.2. (Additional resources: http://www.eia.doe.gov)

'Collected from a survey of prices on January 1 of the current year.

${ }^{\mathbf{b}}$ These prices were collected from a sample of service stations in 85 urban areas selected to represent all urban consumers. Urban consumers make up about $80 \%$ of the total U.S. population.

'Adjusted by the Consumer Price Inflation Index.

¿Data are not available.

'Average annual percentage change is from the earliest year possible to 1998 . 
The fuel prices shown here are refiner sales prices of transportation fuels to end users, excluding tax. Sales to end users are those made directly to the ultimate consumer, including bulk consumers. Bulk sales to utility, industrial, and commercial accounts previously included in the wholesale catego y are now counted as sales to end users.

Table 5.5

Prices for Selected Transportation Fuels, 1978-98

(cents per gallon, excluding tax)

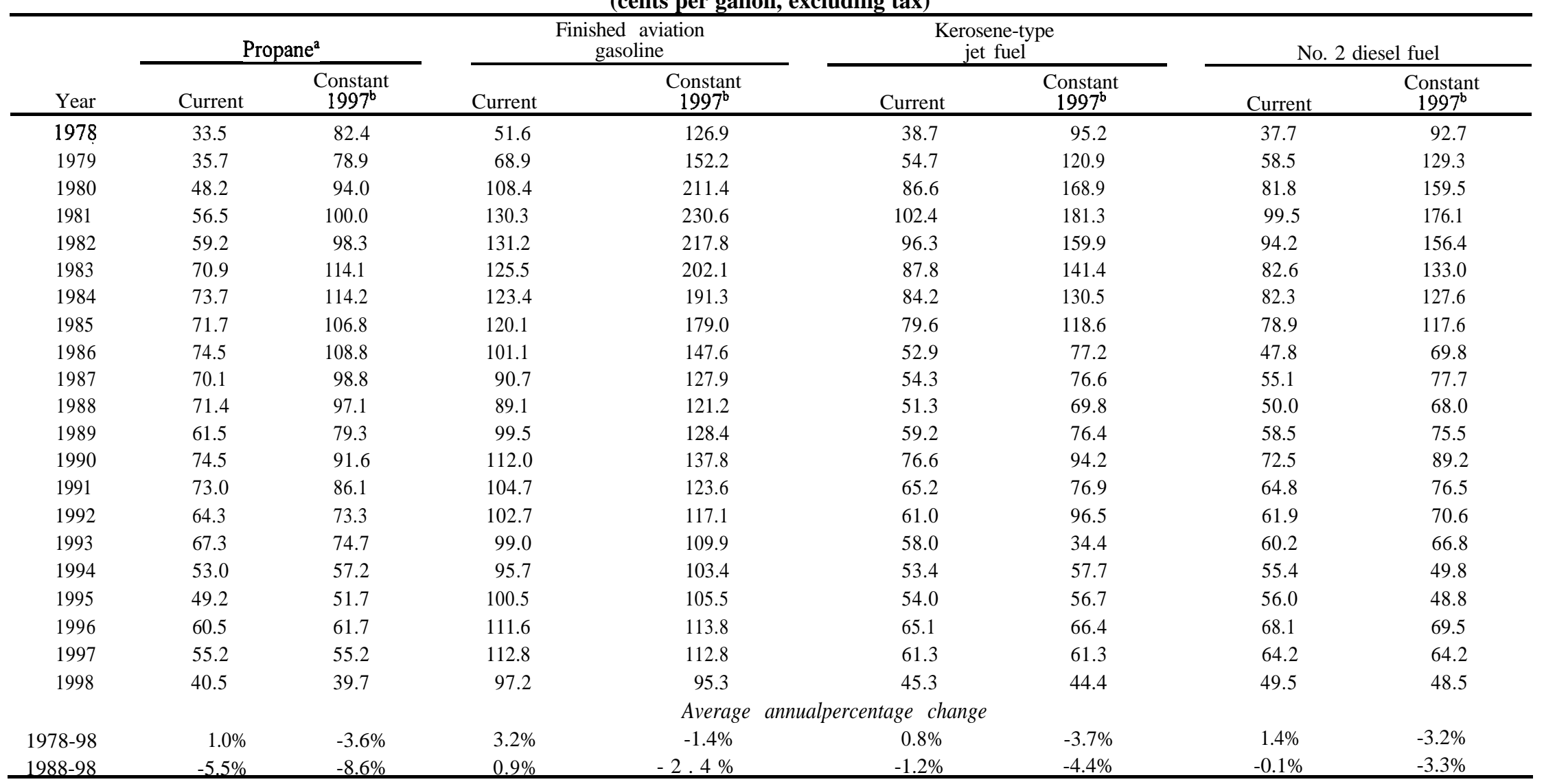

\section{Source:}

U.S. Department of Energy, Energy Information Administration, Monthly Energy Review, March 1999, Washington, DC, Table 9.7, p. 117.

(Additional resources: http://www.eia.doe.gov)

"Consumer grade.

${ }^{\mathbf{b}}$ Adjusted by the Consumer Price Inflation Index. 
Table 5.6

State Taxes on Motor Fuels, 1999

(dollars per gallon or gasoline equivalent gallon)

(Footnotes for this table appear on next page)

\begin{tabular}{|c|c|c|c|c|c|c|c|}
\hline$\overline{\text { State }}$ & Gasoline & Diesel fuel & Gasohol & $\mathrm{CNG}$ & Propane & Methanol & Ethanol \\
\hline Alabama & 0.16 & 0.17 & 0.16 & a & a & $0.16^{\mathrm{b}}$ & $0.16^{b}$ \\
\hline Alaska & 0.08 & 0.08 & $0.08 ”$ & 0.08 & 0.00 & $0.08^{\mathrm{b}}$ & $0.08^{\mathrm{b}}$ \\
\hline Arizona & 0.18 & 0.18 & 0.00 & $0.10^{\mathrm{d}}$ & 0.18 & 0.18 & 0.00 \\
\hline Arkansas & 0.185 & 0.185 & 0.185 & $0.05^{\mathrm{e}}$ & 0.165 & 0.185 & 0.185 \\
\hline California & 0.18 & 0.18 & 0.18 & $0.07 ”$ & 0.06 & 0.09 & 0.09 \\
\hline Colorado & 0.22 & 0.205 & 0.22 & 0.205 & 0.205 & 0.205 & 0.205 \\
\hline Connecticut & 0.39 & 0.18 & 0.38 & $0.18^{f}$ & $0.18^{f}$ & $0.37^{\mathrm{b}}$ & $0.37^{\mathrm{b}}$ \\
\hline Delaware & 0.23 & 0.22 & 0.23 & 0.22 & 0.22 & 0.22 & 0.23 \\
\hline District of Columbia & 0.20 & 0.20 & 0.20 & 0.20 & 0.20 & 0.20 & 0.20 \\
\hline Florida & 0.04 & 0.04 & 0.04 & $\mathrm{a}$ & $\mathrm{a}$ & $0.04^{\mathrm{b}}$ & $0.04^{\mathrm{b}}$ \\
\hline Georgia & 0.075 & 0.075 & 0.075 & 0.075 & 0.075 & 0.075 & 0.075 \\
\hline Hawaii (Honolulu) ${ }^{\mathrm{g}}$ & 0.325 & 0.325 & 0.325 & 0.325 & 0.22 & 0.325 & 0.325 \\
\hline Idaho & 0.25 & 0.25 & 0.25 & $0.197^{\mathrm{h}}$ & 0.181 & $0.25^{\mathrm{b}}$ & $0.25^{\mathrm{b}}$ \\
\hline Illinois & 0.19 & 0.215 & 0.19 & 0.19 & 0.19 & $0.19^{\mathrm{b}}$ & $0.19^{\mathrm{b}}$ \\
\hline Indiana & 0.15 & 0.16 & 0.15 & $\mathrm{a}$ & $\mathrm{a}$ & 0.15 & 0.15 \\
\hline Iowa & 0.20 & 0.225 & 0.19 & $0.16^{\mathrm{e}}$ & 0.20 & $0.19^{b}$ & $0.19^{\mathrm{b}}$ \\
\hline Kansas & 0.18 & 0.20 & 0.18 & 0.17 & 0.17 & 0.20 & 0.20 \\
\hline Kentucky & 0.15 & 0.12 & 0.15 & 0.12 & 0.15 & 0.15 & 0.15 \\
\hline Louisiana & 0.20 & 0.20 & 0.20 & 0.16 & $0.16^{\prime}$ & $0.20^{\mathrm{b}}$ & $0.20^{\mathrm{b}}$ \\
\hline Maine & 0.19 & 0.20 & 0.19 & 0.18 & 0.18 & 0.18 & 0.18 \\
\hline Maryland & 0.235 & 0.2425 & 0.235 & 0.235 & 0.235 & 0.235 & 0.235 \\
\hline Massachusetts & 0.21 & 0.21 & 0.21 & 0.0 & 0.097 & 0.21 & 0.21 \\
\hline Michigan & 0.15 & 0.15 & 0.18 & 0.0 & 0.15 & $0.15^{\mathrm{b}}$ & $0.025^{b}$ \\
\hline Minnesota & 0.20 & 0.20 & 0.20 & 0.001739' & 0.15 & NA & $0.20^{\mathrm{b}}$ \\
\hline Mississippi & 0.18 & 0.18 & 0.18 & $0.18^{\mathrm{e}}$ & 0.17 & $0.18^{b}$ & $0.18^{\mathrm{b}}$ \\
\hline Missouri & 0.17 & 0.17 & 0.17 & $\mathrm{a}$ & $\mathrm{a}$ & $0.17^{b}$ & $0.17^{\mathrm{b}}$ \\
\hline Montana & 0.27 & 0.2775 & 0.27 & $0.07^{\mathrm{k}}$ & a & 0.27 & 0.27 \\
\hline Nebraska & 0.253 & 0.253 & 0.253 & 0.253 & 0.253 & 0.253 & $0.253^{\mathrm{b}}$ \\
\hline Nevada & 0.23 & 0.27 & 0.23 & $0.23^{\mathrm{e}}$ & $0.23^{\mathrm{e}}$ & 0.23 & 0.23 \\
\hline New Hampshire & 0.18 & 0.18 & 0.18 & 0.18 & 0.18 & $0.18^{\mathrm{b}}$ & $0.18^{\mathrm{b}}$ \\
\hline New Jersey & 0.105 & 0.135 & 0.105 & 0.0525 & 0.0525 & $0.105^{b}$ & $0.105^{b}$ \\
\hline New Mexico & 0.22 & 0.18 & 0.22 & $0.06^{\prime}$ & $0.06^{\prime}$ & $0.22^{\mathrm{b}}$ & $0.22^{b}$ \\
\hline New York & $0.08^{\prime}$ & $0.10^{\prime}$ & $0.08^{\prime}$ & $0.08^{\prime}$ & $0.08^{\prime}$ & $0.08^{\prime}$ & $0.08^{\prime}$ \\
\hline North Carolina & 0.217 & 0.217 & 0.217 & 0.217 & 0.217 & 0.217 & 0.217 \\
\hline North Dakota & 0.20 & 0.20 & 0.20 & 0.20 & 0.20 & $0.20^{\mathrm{b}}$ & $0.20^{\mathrm{b}}$ \\
\hline Ohio & 0.22 & 0.22 & 0.22 & 0.22 & 0.22 & $0.22 "$ & $0.22^{\mathrm{b}}$ \\
\hline Oklahoma & 0.16 & 0.13 & 0.16 & $\mathrm{a}$ & 0.16 & $0.16^{\mathrm{b}}$ & $0.16^{\mathrm{b}}$ \\
\hline Oregon & 0.24 & 0.24 & 0.24 & 0.24 & 0.24 & 0.24 & 0.24 \\
\hline Pennsylvania & $0.12^{\mathrm{m}}$ & $0.12^{\mathrm{m}}$ & $0.12^{\mathrm{m}}$ & $0.12^{\mathrm{m}}$ & $0.12^{\mathrm{m}}$ & $0.12^{\mathrm{m}}$ & $0.12^{\mathrm{m}}$ \\
\hline Rhode Island & 0.28 & 0.28 & 0.28 & 0.0 & 0.28 & 0.28 & 0.28 \\
\hline
\end{tabular}


Table 5.6 (continued)

State Taxes on Motor Fuels, 1999

(dollars per gallon or gasoline equivalent gallon)

\begin{tabular}{lccccccc}
\hline State & Gasoline & Diesel fuel & Gasohol & CNG & Propane & Methanol & Ethanol \\
\hline South Carolina & 0.16 & 0.16 & 0.16 & 0.16 & 0.16 & 0.16 & 0.16 \\
South Dakota & 0.18 & 0.18 & 0.16 & 0.06 & 0.16 & 0.06 & 0.06 \\
Tennessee & 0.20 & 0.17 & 0.17 & 0.13 & 0.17 & 0.17 & 0.17 \\
Texas & 0.20 & 0.20 & 0.20 & 0.15 & 0.15 & $\mathbf{0 . 2 0 ^ { \mathrm { b } }}$ & $\mathbf{0 . 2 0 ^ { \mathrm { b } }}$ \\
Utah & 0.19 & 0.19 & 0.19 & $\mathbf{0 . 1 9}$ & $\mathbf{0 . 1 9 ^ { \mathrm { m } }}$ & 0.19 & 0.19 \\
\hline Vermont & 0.16 & 0.17 & 0.16 & 0.16 & $\mathrm{a}$ & 0.16 & 0.16 \\
Virginia & 0.175 & 0.16 & 0.175 & 0.10 & 0.10 & $\mathbf{0 . 1 7 5 ^ { \mathrm { b } }}$ & $\mathbf{0 . 1 7 5 ^ { \mathrm { b } }}$ \\
Washington & 0.23 & 0.23 & 0.23 & $\mathrm{a}$ & $\mathrm{a}$ & 0.23 & 0.23 \\
West Virginia & 0.205 & 0.205 & 0.205 & 0.205 & 0.205 & 0.205 & 0.205 \\
Wisconsin & 0.254 & 0.254 & 0.254 & 0.203 & 0.186 & 0.254 & 0.254 \\
Wyoming & 0.08 & 0.08 & 0.00 & 0.00 & 0.00 & $\mathbf{0 . 0 8}$ & $\mathbf{0 . 0 8}$ \\
\hline
\end{tabular}

Source:

Energy Futures, Inc., The Clean Fuels and Electric Vehicles Report, Boulder, CO, February 1999, pp. 150-1 51.

${ }^{a}$ Annual flat fee.

${ }^{b}$ Blends with gasoline only.

'November-February tax rate is $\$ 0.02$.

${ }^{\mathrm{d}}$ Per 1.25 therm.

${ }^{\mathrm{e}}$ Per $100 \mathrm{ft}^{3}$.

${ }^{f} \mathrm{CNG}$, LNG, and LPG are exempt from motor fuel taxes when used as vehicle fuel until July 1, 2001.

${ }^{\mathrm{g}}$ For County of Honolulu; for County of Maui LPG tax is $\$ 0.20 /$ gal. and all other fuels are taxed at $\$ 0.1$ 8/gal.; other counties have all fuels taxed at $\$ 0.26 / \mathrm{gal}$.

her therm.

'Optional: flat fee may be paid instead.

${ }^{j}$ Per cubic foot; LNG is taxed at $\$ 0.12 /$ gal.

ker $120 \mathrm{ft}^{3}$.

'Plus a petroleum business tax; the amount varies but is usually in the ballpark of \$0.12-\$0.14.

mPlus 0.1035 oil franchise tax. 
As of November 1998, only five states offered tax exemptions to encourage the use of gasohol for transportation purposes. This list is quite short compared to the 30 states which offered gasohol tax exemptions fifteen years ago. Still, the Federal Government encourages gasohol use via a difference in the Federal tax rates of gasoline and gasohol.

Table 5.7

State Tax Exemptions for Gasohol, November 1998

\begin{tabular}{lc}
\hline State & $\begin{array}{c}\text { Exemption } \\
\text { (Cents/gallon of gasohol) }\end{array}$ \\
\hline Alaska & 8.0 \\
Connecticut & 1.0 \\
Idaho & 2.5 \\
Iowa & 1.0 \\
South Dakota & 2.0 \\
\hline
\end{tabular}

Source:

U.S. Department of Transportation, Federal Highway Administration, "Monthly Motor Fuel Reported by the States, August 1998," January 1999, Washington, DC, Table MF121T. (Additional resources: http://www.fhwa.dat.gov)

Table 5.8

Federal Excise Taxes on Motor Fuels

\begin{tabular}{llc}
\hline Fuel & & Cents per gallon \\
\hline Gasoline & & 18.40 \\
Diesel” & & 24.40 \\
Gasohol & 10\% Ethanol & 13.00 \\
& 7.7\% Ethanol & 14.24 \\
& 5.7\% Ethanol & 15.32 \\
Gasohol & 10\% Methanol & 12.40 \\
& 7.7\% Methanol & 13.78 \\
& 5.7\% Methanol & 14.98 \\
Methanol & Qualified & 12.85 \\
& Partially exempt & 9.20 \\
Ethanol & Qualified $^{\mathrm{b}}$ & 12.85 \\
& Partially exempt" & 9.25 \\
CNG & & $48.54 / \mathrm{mcf}^{\mathbf{d}}$ \\
LNG & & 11.90 \\
LPG & & 13.60 \\
\hline
\end{tabular}

Source:

Energy Futures, Inc., The Clean Fuels and Electric Vehicles Report, Boulder, CO, February 1999, pp. 150-151.

${ }^{a}$ Reduced diesel rates are specified for marine fleets, trains and certain intercity buses. Diesel rates are also reduced for diesel/alcohol blends. Diesel used exclusively in state and local government fleets, non-profit organization vehicles, school buses and qualified local buses is exempt from Federal taxes.

${ }^{\text {b }}$ Qualified - contains at least 85 percent methanol or ethanol or other alcohol produced from a substance other than petroleum or natural gas.

"Partially exempt $->85$ percent alcohol and produced from natural gas.

'Thousand cubic feet. 
Table 5.9

States With Ethanol Tax Incentives

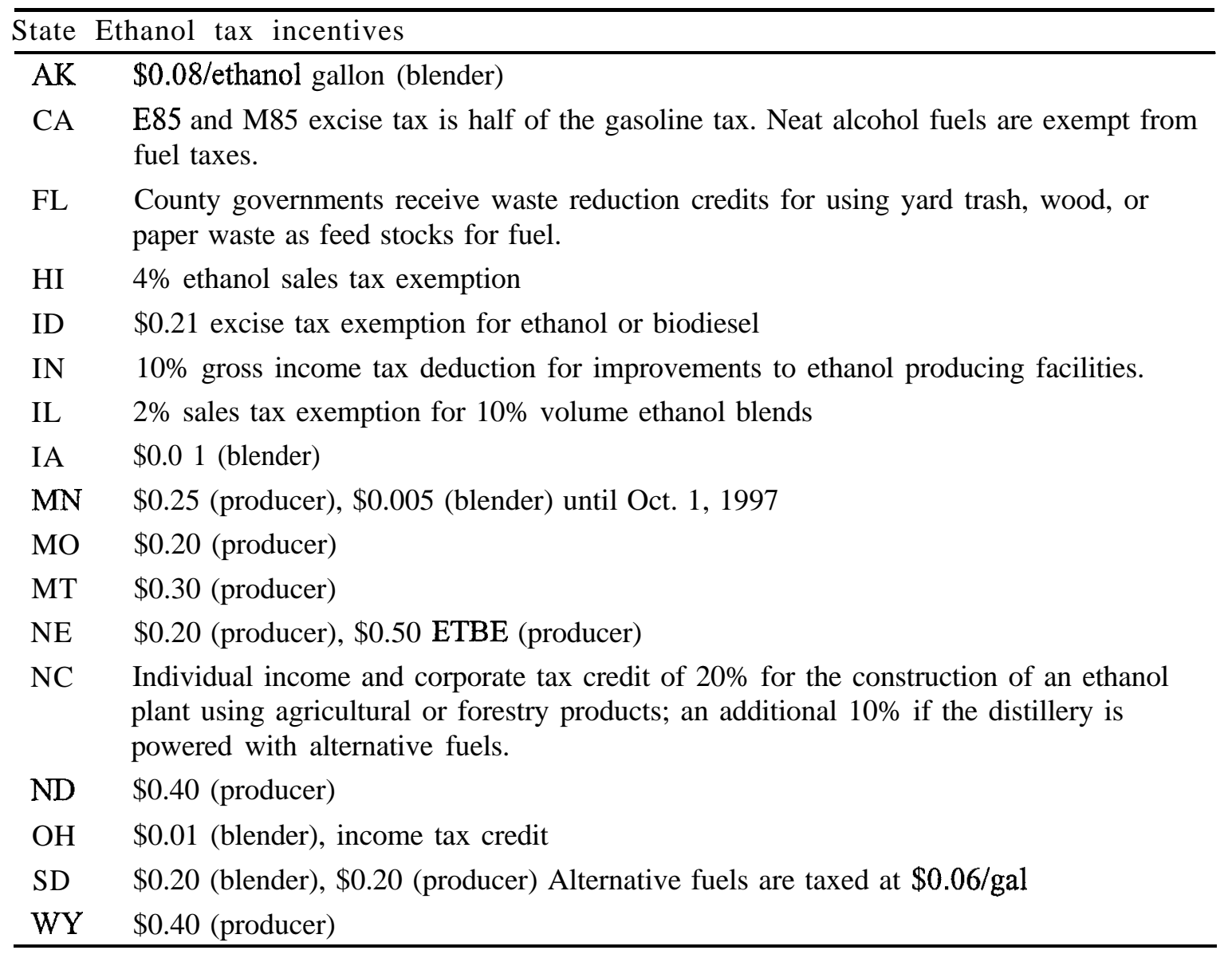

\section{Source:}

U.S. Department of Energy, Clean Cities Guide to Alternative Fuel Vehicle Incentives and Laws, 2nd edition, Washington, DC, November 1996.

(Additional resources: http://www.ccities.doe.gov) 
In current dollars, import cars, on average, were less expensive than domestic cars until 1982. Since then, import prices have nearly tripled, while domestic prices have nearly doubled \{current dollars).

Table 5.10

Average Price of a New Car, 1970-97

\begin{tabular}{|c|c|c|c|c|c|c|}
\hline \multirow[b]{2}{*}{ Year } & \multicolumn{2}{|c|}{ Domestic" } & \multicolumn{2}{|c|}{ Import } & \multicolumn{2}{|c|}{ Total } \\
\hline & $\begin{array}{l}\text { Current } \\
\text { dollars }\end{array}$ & $\begin{array}{c}\text { Constant } \\
1997 \\
\text { dollars }^{\text {b }}\end{array}$ & $\begin{array}{l}\text { Current } \\
\text { dollars }\end{array}$ & $\begin{array}{c}\text { Constant } \\
1997 \\
\text { dollars }^{\mathbf{b}}\end{array}$ & $\begin{array}{l}\text { Current } \\
\text { dollars }\end{array}$ & $\begin{array}{c}\text { Constant } \\
1997 \\
\text { dollars }^{b}\end{array}$ \\
\hline 1970 & 3,708 & 15,314 & 2,648 & 10,936 & 3,542 & 14,328 \\
\hline 1971 & 3,919 & 15,519 & 2,769 & 10,965 & 3,742 & 14,818 \\
\hline 1972 & 4,034 & 15,491 & 2,994 & 11,497 & 3,879 & 14,895 \\
\hline 1973 & 4,181 & 15,093 & 3,344 & 12,072 & 4,052 & 14,628 \\
\hline 1974 & 4,524 & 14,748 & 4,206 & 13,712 & 4,440 & 14,474 \\
\hline 1975 & 5,084 & 15,150 & 4,384 & 13,064 & 4,950 & 14,751 \\
\hline 1976 & 5,506 & 15,527 & 4,923 & 13,883 & 5,418 & 15,279 \\
\hline 1977 & 5,985 & 15,860 & 5,072 & 13,441 & 5,814 & 15,407 \\
\hline 1978 & 6,478 & 15,936 & 5,934 & 14,598 & 6,379 & 15,692 \\
\hline 1979 & 6,889 & 15,225 & 6,704 & 14,816 & 6,847 & 15,132 \\
\hline 1980 & 7,609 & 14,838 & 7,482 & 14,590 & 7,574 & 14,769 \\
\hline 1981 & 8,912 & 15,774 & 8,896 & 15,746 & 8,910 & 15,771 \\
\hline 1982 & 9,865 & 16,376 & 9,957 & 16,529 & 9,890 & 16,417 \\
\hline 1983 & 10,516 & 16,931 & 10,868 & 17,497 & 10,606 & 17,076 \\
\hline 1984 & 11,172 & 17,317 & 12,354 & 19,149 & 11,450 & 17,748 \\
\hline 1985 & 11,589 & 17,268 & 12,853 & 19,151 & 11,902 & 17,734 \\
\hline 1986 & 12,526 & 18,288 & 13,815 & 20,170 & 12,894 & 18,825 \\
\hline 1987 & 12,922 & 18,220 & 14,470 & 20,403 & 13,386 & 18,874 \\
\hline 1988 & 13,542 & 18,417 & 15,378 & 20,914 & 14,065 & 19,128 \\
\hline 1989 & 14,193 & 18,309 & 15,829 & 20,419 & 14,645 & 18,892 \\
\hline 1990 & 14,886 & 18,310 & 17,164 & 21,112 & 15,472 & 19,031 \\
\hline 1991 & 15,773 & 18,612 & 17,019 & 20,082 & 16,083 & 18,978 \\
\hline 1992 & 16,389 & 18,683 & 19,601 & 22,345 & 18,141 & 20,681 \\
\hline 1993 & 16,673 & 18,507 & 21,477 & 23,839 & 17,678 & 19,623 \\
\hline 1994 & 17,575 & 18,981 & 23,211 & 25,068 & 18,657 & 20,150 \\
\hline 1995 & 17,174 & 18,033 & 23,995 & 25,195 & 18,360 & 19,278 \\
\hline 1996 & 18,199 & 18,563 & 27,695 & 28,249 & 19,620 & 20,012 \\
\hline \multirow[t]{2}{*}{1997} & 18,624 & 18,624 & 29,708 & 29,708 & 20,447 & 20,447 \\
\hline & \multicolumn{6}{|c|}{ Average annualpercentage change } \\
\hline $1970-97$ & $6.2 \%$ & $0.7 \%$ & $9.4 \%$ & $3.8 \%$ & $6.7 \%$ & $1.3 \%$ \\
\hline $1987-97$ & $3.7 \%$ & $0.2 \%$ & $7.5 \%$ & $3.8 \%$ & $4.3 \%$ & $0.8 \%$ \\
\hline
\end{tabular}

Source:

American Automobile Manufacturers Association, Motor Vehicle Facts and Figures 1998, Detroit, MI, 1998, p.64.

(Additional resources: http://www.aama.com)

"Includes transplants.

"Adjusted by the Consumer Price Inflation Index. 
The total cost of operating an automobile is the sum of the fixed cost (depreciation, insurance, finance charge, and license fee) and the variable cost, which is

related to the amount of travel. The cost of operating a car in 1998 (constant 1990 cents) was approximately 44 cents per mile. Gas and oil accountedfor only

$11.5 \%$ of total cost per mile in 1998, which was the same as 1996 and is the lowest in the series history.

Table 5.11

Automobile Operating Cost per Mile, 1975-98

\begin{tabular}{|c|c|c|c|c|c|c|c|c|}
\hline \multirow[b]{2}{*}{$\begin{array}{l}\text { Model } \\
\text { year }^{\mathbf{c}}\end{array}$} & \multicolumn{4}{|c|}{ Variable costs (constant 1997 cents Per mile") } & \multicolumn{3}{|c|}{ Constant 1997 dollars per 10,000 miles $^{2}$} & \multirow{2}{*}{$\begin{array}{l}\text { Total cost per } \\
\text { mile }^{\mathbf{b}}(\text { constan } \\
\left.1997 \text { cents }^{2}\right)\end{array}$} \\
\hline & Gas and oil & $\begin{array}{l}\text { Percentage gas and } \\
\text { oil of total cost }\end{array}$ & Maintenance & Tires & Variable cost & Fixed cost & Total cost & \\
\hline 1975 & 14.36 & $26.3 \%$ & 2.89 & 1.97 & 1,922 & 3,534 & 5,456 & 54.56 \\
\hline 1977 & 10.89 & $20.4 \%$ & 2.73 & 1.75 & 1,537 & 3,813 & 5,350 & 53.50 \\
\hline 1979 & 9.08 & $17.1 \%$ & 2.43 & 1.44 & 1,295 & 4,002 & 5,297 & 52.97 \\
\hline 1980 & 11.43 & $21.0 \%$ & 2.18 & 1.25 & 1,486 & 3,964 & 5,450 & 54.50 \\
\hline 1981 & 11.10 & $19.6 \%$ & 2.09 & 1.27 & 1,446 & 4,204 & 5,650 & 56.50 \\
\hline 1982 & 11.19 & $20.8 \%$ & 1.66 & 1.05 & 1,389 & 3,981 & 5,370 & 53.70 \\
\hline 1983 & 10.69 & $19.9 \%$ & 1.67 & 1.09 & 1,346 & 4,035 & 5,381 & 53.81 \\
\hline 1984 & 9.59 & $19.8 \%$ & 1.61 & 0.98 & 1,218 & 3,636 & 4,855 & 48.55 \\
\hline $1985^{d}$ & 9.18 & $22.6 \%$ & 1.83 & 0.97 . & 1,198 & 2,855 & 4,053 & 40.53 \\
\hline 1986 & 6.54 & $15.1 \%$ & 2.00 & 0.98 & 952 & 3,368 & 4,320 & 43.20 \\
\hline 1987 & 6.77 & $14.7 \%$ & 2.26 & 1.13 & 1,015 & 3,587 & 4,602 & 46.02 \\
\hline 1988 & 7.07 & $13.6 \%$ & 2.18 & 1.09 & 1,074 & 4,121 & 5,195 & 51.95 \\
\hline 1989 & 6.71 & $13.6 \%$ & 2.45 & 1.03 & 1,019 & 3,909 & 4,928 & 49.28 \\
\hline 1990 & 6.64 & $13.2 \%$ & 2.58 & 1.11 & 1,033 & 4,005 & 5,038 & 50.38 \\
\hline 1991 & 7.91 & $15.4 \%$ & 2.60 & 1.06 & 1,156 & 3,993 & 5,150 & 51.50 \\
\hline 1992 & 6.84 & $13.1 \%$ & 2.51 & 1.03 & 1,037 & 4,180 & 5,218 & 52.18 \\
\hline 1993 & 6.66 & $13.3 \%$ & 2.66 & 1.00 & 1,032 & 3,978 & 5,011 & 50.11 \\
\hline 1994 & 6.05 & $12.0 \%$ & 2.70 & 1.19 & 994 & 4,045 & 5,038 & 50.38 \\
\hline 1995 & 6.30 & $12.3 \%$ & 2.73 & 1.47 & 1,050 & 4,086 & 5,136 & 51.36 \\
\hline 1996 & 6.02 & $11.5 \%$ & 2.86 & 1.43 & 1,030 & 4,216 & 5,246 & 52.46 \\
\hline 1997 & 6.60 & $12.4 \%$ & 2.80 & 1.40 & 1,080 & 4,228 & 5,308 & 53.08 \\
\hline 1998 & 6.17 & $11.5 \%$ & 3.04 & 1.37 & 1,058 & 4,315 & 5,373 & 53.73 \\
\hline \multicolumn{9}{|c|}{ Average annual percentage change } \\
\hline $1975-84$ & $-4.4 \%$ & & $-6.3 \%$ & $-7.5 \%$ & $-4.9 \%$ & $0.3 \%$ & $-1.3 \%$ & $-1.3 \%$ \\
\hline $1988-98$ & $-1.4 \%$ & & $3.4 \%$ & $2.3 \%$ & $-0.1 \%$ & $0.5 \%$ & $0.3 \%$ & $0.3 \%$ \\
\hline
\end{tabular}

Source:

American Automobile Association, Your Driving Costs, 1998 Edition, Heathrow, FL, and annual. (Additional resources: http://www.aaa.com, http://www.runzheimer.com)

a Adjusted by the Consumer Price Inflation Index.

${ }^{b}$ Based on 10,000 miles per year.

c Data for 1976 and 1978 are not available.

d Fixed and total operating costs preceding 1985 are not comparable with 1985 and later data. Fixed cost depreciation from 1975-84 was based on four years or 60,000 miles. After 1984, the depreciation was based on six years or 60,000 miles. 
Table 5.12

Fixed Automobile Operating Costs per Year, 1975-98 (constant 1997 dollars) ${ }^{\mathrm{a}}$

\begin{tabular}{|c|c|c|c|c|c|c|c|c|}
\hline Model year & Fire \& theft ${ }^{b}$ & Collision' & $\begin{array}{c}\text { Property } \\
\text { damage \& } \\
\text { liability" }\end{array}$ & $\begin{array}{l}\text { License, } \\
\text { registration } \\
\& \text { taxes } \\
\end{array}$ & Depreciation & $\begin{array}{c}\begin{array}{c}\text { Finance } \\
\text { charge }\end{array} \\
\end{array}$ & Total & $\begin{array}{c}\text { Average fixed } \\
\text { cost } \\
\text { per day } \\
\end{array}$ \\
\hline 1975 & 158 & 420 & 563 & 89 & 2,304 & e & 3,534 & 9.69 \\
\hline 1977 & 212 & 498 & 663 & 196 & 2,245 & c & 3,813 & 10.44 \\
\hline 1978 & 140 & 339 & 563 & 182 & 2,199 & e & 3,424 & 9.37 \\
\hline 1979 & 164 & 371 & 533 & 199 & 2,082 & 654 & 4,002 & 10.96 \\
\hline 1980 & 137 & 335 & 484 & 160 & 2,024 & 825 & 3,964 & 10.86 \\
\hline 1981 & 135 & 319 & 450 & 156 & 2,278 & 867 & 4,204 & 11.52 \\
\hline 1982 & 88 & 254 & 403 & 90 & 2,251 & 895 & 3,981 & 10.91 \\
\hline 1983 & 129 & 324 & 357 & 164 & 2,162 & 898 & 4,035 & 11.06 \\
\hline 1984 & 124 & 310 & 349 & 164 & 1,871 & 818 & 3,636 & 9.97 \\
\hline 1985 & 137 & 295 & 317 & 171 & 1,867 & 849 & 3,637 & 9.97 \\
\hline 1986 & 126 & 279 & 339 & 190 & 1,927 & 930 & 3,790 & 10.38 \\
\hline 1987 & 123 & 276 & 355 & 197 & 2,123 & 847 & 3,923 & 10.74 \\
\hline 1988 & 117 & 276 & 386 & 189 & 2,426 & 768 & 4,163 & 11.41 \\
\hline 1989 & 141 & 316 & 399 & 195 & 2,701 & 808 & 4,559 & 12.49 \\
\hline 1990 & 135 & 304 & 391 & 203 & 2,899 & 836 & 4,769 & 13.06 \\
\hline \multirow[t]{2}{*}{1991} & 136 & 304 & 417 & 199 & 3,001 & 919 & 4,976 & 13.63 \\
\hline & 129 & 298 & 425 & 204 & 3,169 & 948 & 5,173 & 14.17 \\
\hline 19921993 & 119 & 258 & 427 & 203 & 3,200 & 773 & 4,979 & 13.64 \\
\hline 1994 & 98 & 222 & 432 & 220 & 3,227 & 751 & 4,951 & 13.56 \\
\hline 1995 & 100 & 222 & 431 & 222 & 3,254 & 765 & 4,993 & 13.68 \\
\hline 1996 & 111 & 252 & 435 & 234 & 3,272 & 794 & 5,097 & 13.96 \\
\hline 1997 & 106 & 302 & 401 & 220 & 3,268 & 793 & 5,090 & 13.95 \\
\hline 1998 & 113 & 257 & 469 & 219 & 3,228 & 786 & 5,072 & 13.90 \\
\hline
\end{tabular}

Source:

Note:

(tp:/www.runzheimer.com)

The data in this table are costs per year, while the data on the previous table are costs per mile.

adjusted by the Consumer Price Inflation Index.

b $\$ 50$ deductible 1975 through 1977; \$100 deductible 1978 through 1992; \$250 deductible for 1993 through 1996.

c \$100 deductible through 1977; \$250 deductible 1978 through 1992; \$500 deductible for 1993 through 1996.

d Coverage: $\$ 100,000 / \$ 300,000$.

e Data are not available. 
Table 5.13

Economic Indicators, 1970-98

(billion dollars)

\begin{tabular}{|c|c|c|c|c|c|}
\hline \multirow[b]{2}{*}{ Year } & \multicolumn{2}{|c|}{$\begin{array}{l}\text { Gross National } \\
\text { Product }\end{array}$} & \multicolumn{2}{|c|}{$\begin{array}{l}\text { Total transportation } \\
\text { outlays }\end{array}$} & \multirow[b]{2}{*}{$\begin{array}{c}\text { Transportation as a } \\
\text { percent of GNP }\end{array}$} \\
\hline & Current & $\begin{array}{c}\text { Constant } \\
\text { 1997" }\end{array}$ & Current & $\begin{array}{l}\text { Constant } \\
\text { 1997" }\end{array}$ & \\
\hline 1970 & $1,015.5$ & $4,194.0$ & 195.2 & 806.2 & $19.2 \%$ \\
\hline 1980 & $2,732.0$ & $5,327.4$ & 542.9 & $1,058.7$ & $19.9 \%$ \\
\hline 1990 & $5,567.8$ & $6,848.4$ & 963.8 & $1,185.5$ & $17.3 \%$ \\
\hline \multirow[t]{2}{*}{1997} & $8,102.9$ & $8,102.9$ & $1,319.7$ & $1,391.7$ & $17.2 \%$ \\
\hline & \multicolumn{2}{|c|}{$\begin{array}{l}\text { Personal Consumption } \\
\text { Expenditures }\end{array}$} & \multicolumn{2}{|c|}{$\begin{array}{c}\text { Transportation Personal } \\
\text { Consumption Expenditures }\end{array}$} & $\begin{array}{l}\text { Transportation PCE as } \\
\text { a percent of total PCE }\end{array}$ \\
\hline 1970 & 640.0 & $2,643.2$ & 81.5 & 336.6 & $12.7 \%$ \\
\hline 1980 & $1,732.6$ & $3,378.6$ & 238.5 & 465.1 & $13.8 \%$ \\
\hline 1990 & $3,761.2$ & $4,626.3$ & 453.9 & 558.3 & $12.1 \%$ \\
\hline 1998 & $5,806.0$ & $5,689.9$ & 653.8 & 640.7 & $11.3 \%$ \\
\hline
\end{tabular}

Sources:

GNP - U.S. Department of Commerce, Bureau of Economic Analysis, Survey of Current Business, April 1999,

Table 1.9, p. D-4, and annual. (Additional resources: http://www.bea.doc.gov)

Transportation outlays - Eno Transportation Foundation, Transportation in America 1998, Sixteenth Edition, Lansdowne, VA, 1998, p. 38.

PCE - U.S. Department of Commerce, Bureau of Economic Analysis, Survey of Current Business, March 1999,

Table 2.2 and annual. (Additional resources: http://www.bea.doc.gov/bea/scbinf.html)

Table 5.14

Consumer Price Indices, 1970-98

$(1970=1.000)$

\begin{tabular}{cccccc}
\hline Year & $\begin{array}{c}\text { Consumer } \\
\text { Price Index }\end{array}$ & $\begin{array}{c}\text { Transportation } \\
\text { Consumer } \\
\text { Price Index }^{c}\end{array}$ & $\begin{array}{c}\text { New car } \\
\text { Consumer } \\
\text { Price Index }\end{array}$ & $\begin{array}{c}\text { Used car } \\
\text { Consumer } \\
\text { Price Index }\end{array}$ & $\begin{array}{c}\text { Gross National } \\
\text { Product }\end{array}$ \\
\hline 1970 & 1.000 & 1.000 & 1.000 & 1.000 & 1.000 \\
1980 & 2.122 & 2.216 & 1.667 & 1.995 & 2.690 \\
1990 & 3.365 & 3.213 & 2.283 & 3.769 & 5.483 \\
1998 & 4.199 & 3.776 & 2.706 & 4.827 & 8.361 \\
\hline
\end{tabular}

Source:

Bureau of Labor Statistics, Consumer Price Index Table 1A for 1998, and annual. [GNP-see above.] (Additional resources: http://stats.bls.gov/cpihome.htm)

a Adjusted by the implicit GNP price deflator.

b Transportation Personal Consumption Expenditures include user operating expenses (new and used auto purchases, gas and oil, repair, greasing, washing, parking, storage, rental, other motor vehicles, insurance premiums, tires, tubes and other parts); purchased intercity transportation; and purchased local transportation.

c Transportation Consumer Price Index includes new and used cars, gasoline, auto insurance rates, intracity mass transit, intracity bus fare, and airline fares. 
Table 5.15

Motor Vehicle Manufacturing Employment Statistics, 1972-97

\begin{tabular}{|c|c|c|c|c|c|c|c|c|c|}
\hline & Year & $\begin{array}{c}\text { Motor vehicle } \\
\text { manufacturing } \\
\text { employees } \\
\text { (thousands) }\end{array}$ & $\begin{array}{c}\text { Sales of } \\
\text { domestic } \\
\text { automobiles" } \\
\text { (thousands) }\end{array}$ & $\begin{array}{c}\text { Sales of } \\
\text { domestic } \\
\text { light trucks } \\
\text { (thousands) }\end{array}$ & $\begin{array}{c}\text { Employees per } \\
\text { hundred } \\
\text { vehicles sold } \\
\end{array}$ & $\begin{array}{c}\text { Expenditure per } \\
\text { new domestic } \\
\text { vehicle" }\end{array}$ & $\begin{array}{c}\text { Total domestic vehicle } \\
\text { expenditures }{ }^{d} \\
\text { (millions) }\end{array}$ & $\begin{array}{l}\text { Employees per } \\
\text { million dollar } \\
\text { expenditure } \\
\text { (current) }\end{array}$ & $\begin{array}{l}\text { Employees per } \\
\text { million dollar } \\
\text { expenditure } \\
\text { (constant } 19973\end{array}$ \\
\hline & 1972 & 415 & 9,327 & 2,096 & 3.6 & $\$ 4,034$ & $\$ 46,080$ & 9.0 & 34.6 \\
\hline & 1973 & 462 & 9,676 & 2,512 & 3.8 & $\$ 4,181$ & $\$ 50,958$ & 9.1 & 32.9 \\
\hline & 1974 & 416 & 7,454 & 2,163 & 4.3 & $\$ 4,524$ & $\$ 43,507$ & 9.6 & 31.3 \\
\hline 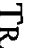 & 1975 & 375 & 7,053 & 2,053 & 4.1 & $\$ 5,084$ & $\$ 46,295$ & 8.1 & 24.1 \\
\hline & 1976 & 416 & 8,611 & 2,720 & 3.7 & $\$ 5,506$ & $\$ 62,388$ & 6.7 & 18.9 \\
\hline 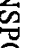 & 1977 & 442 & 9,109 & 3,108 & 3.6 & $\$ 5,985$ & $\$ 73,119$ & 6.0 & 15.9 \\
\hline ? & 1978 & 470 & 9,312 & 3,473 & 3.7 & $\$ 6,478$ & $\$ 82,821$ & 5.7 & 14.0 \\
\hline 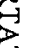 & 1979 & 463 & 8,341 & 2,844 & 4.1 & $\$ 6,889$ & $\$ 77,053$ & 6.0 & 13.3 \\
\hline 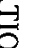 & 1980 & 368 & 6,581 & 1,959 & 4.3 & $\$ 7,609$ & $\$ 64,981$ & 5.7 & 11.1 \\
\hline 2 & 1981 & 359 & 6,209 & 1,745 & 4.5 & $\$ 8,912$ & $\$ 70,886$ & 5.1 & 9.0 \\
\hline 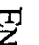 & 1982 & 318 & 5,759 & 2,062 & 4.1 & $\$ 9,865$ & $\$ 77,154$ & 4.1 & 6.8 \\
\hline 至 & 1983 & 349 & 6,795 & 2,518 & 3.7 & $\$ 10,516$ & $\$ 97,936$ & 3.6 & 5.8 \\
\hline & 1984 & 392 & 7,952 & 3,257 & 3.5 & $\$ 11,172$ & $\$ 125,227$ & 3.1 & 4.8 \\
\hline & 1985 & 409 & 8,205 & 3,691 & 3.4 & $\$ 11,589$ & $\$ 137,863$ & 3.0 & 4.5 \\
\hline & 1986 & 400 & 8,215 & 3,671 & 3.4 & $\$ 12,526$ & $\$ 148,884$ & 2.7 & 3.9 \\
\hline & 1987 & 381 & 7,081 & 3,785 & 3.5 & $\$ 12,922$ & $\$ 140,410$ & 2.7 & 3.8 \\
\hline ద & 1988 & 357 & 7,526 & 4,195 & 3.0 & $\$ 13,542$ & $\$ 158,725$ & 2.2 & 3.0 \\
\hline 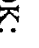 & 19891990 & 350329 & $\not 6,893$ & $\mathcal{9}, 968$ & 3.13 .0 & $\$ 14, \$ 8 B$ & $\$ 158,699$ & 2.22 .0 & 2.82 .5 \\
\hline$\theta$ & 1991 & 316 & 6,137 & 3,595 & 3.2 & $\$ 15,773$ & $\$ 153,503$ & 2.1 & 2.5 \\
\hline & 1992 & 314 & 6,277 & 4,233 & 3.0 & $\$ 16,389$ & $\$ 172,248$ & 1.8 & 2.1 \\
\hline & 1993 & 319 & 6,742 & 4,987 & 2.7 & $\$ 16,673$ & $\$ 195,558$ & 1.6 & 1.8 \\
\hline & 1994 & 340 & 7,255 & 5,638 & 2.6 & $\$ 17,575$ & $\$ 226,594$ & 1.5 & 1.6 \\
\hline & 1995 & 355 & 7,129 & 5,663 & 2.8 & $\$ 17,767$ & $\$ 227,275$ & 1.6 & 1.7 \\
\hline 1 & 19961997 & 342341 & $6,9177,254$ & $6,0886,626$ & 2.62 .6 & $\$ 18,199 \$ 18,624$ & $\$ 242, \$ 84,710$ & 1.4 1.4 & 1.4 1.4 \\
\hline & \multicolumn{9}{|c|}{ Average annualpercentage change } \\
\hline & $1972-97$ & $-0.8 \%$ & $-1.2 \%$ & $4.5 \%$ & $-1.3 \%$ & $6.3 \%$ & $6.9 \%$ & $-7.2 \%$ & $-12.0 \%$ \\
\hline & $1987-97$ & $-1.1 \%$ & $-0.2 \%$ & $5.1 \%$ & $-2.9 \%$ & $3.7 \%$ & $5.7 \%$ & $-6.4 \%$ & $-9.5 \%$ \\
\hline
\end{tabular}

Source:

Employees - American Automobile Manufacturers Association, Economic Indicators, Third Quarter,1998, Detroit, MI, 1998 , p. 18.

Sales and expenditures - American Automobile Manufacturers Association, Motor Vehicle Facts and Figures 1998, Detroit, MI, 1998, pp. 21, 24, 64, and annual.

${ }^{\mathbf{a}}$ Vehicles produced in North America.

b Less than 10,000 pounds gross vehicle weight.

c Autos and light trucks.

d Estimated as vehicle sales multiplied by average expenditure.

e Adjusted by the implicit Gross National Product price deflator. 
Employees of motor vehicle and related industries comprise $7.5 \%$ of the laborforce. For employment in the entire transportation industry, see the next table.

Table 5.16

Employees of Motor Vehicle and Related Industries, 1990 and 1995

\begin{tabular}{|c|c|c|c|c|c|c|c|}
\hline \multirow[b]{2}{*}{ Industry } & \multicolumn{3}{|c|}{1990} & \multicolumn{3}{|c|}{1995} & \multirow[b]{2}{*}{$\begin{array}{l}\text { Percent } \\
\text { change } \\
1990-95\end{array}$} \\
\hline & Employees & $\begin{array}{l}\text { Percent of } \\
\text { total motor } \\
\text { vehicle }\end{array}$ & $\begin{array}{l}\text { Percent of } \\
\text { total US. } \\
\text { employment" }\end{array}$ & Employees & $\begin{array}{l}\text { Percent of } \\
\text { total motor } \\
\text { vehicle }\end{array}$ & $\begin{array}{l}\text { Percent of } \\
\text { total U.S. } \\
\text { employment" }\end{array}$ & \\
\hline $\begin{array}{l}\text { Motor vehicle and equipment } \\
\text { manufacturing }\end{array}$ & $1,055,595$ & $15.0 \%$ & $1.1 \%$ & $1,180,923$ & $15.6 \%$ & $1.2 \%$ & $11.9 \%$ \\
\hline Motor vehicles and equipment & 707,160 & $10.0 \%$ & $0.8 \%$ & 815,214 & $10.8 \%$ & $0.8 \%$ & $15.3 \%$ \\
\hline Travel trailers and campers & 14,301 & $0.2 \%$ & $0.0 \%$ & $\mathrm{~b}$ & $\mathrm{~b}$ & $\mathrm{~b}$ & $\mathrm{~b}$ \\
\hline $\begin{array}{l}\text { Transportation equipment not } \\
\text { elsewhere classified }\end{array}$ & 17,263 & $0.2 \%$ & $0.0 \%$ & 51,891 & $0.7 \%$ & $0.1 \%$ & $200.6 \%$ \\
\hline Automotive stampings & 111,548 & $1.6 \%$ & $0.1 \%$ & 118,213 & $1.6 \%$ & $0.1 \%$ & $6.0 \%$ \\
\hline $\begin{array}{l}\text { Carburetors, pistons, piston } \\
\text { rings, and valves }\end{array}$ & 19,674 & $0.3 \%$ & $0.0 \%$ & 18,677 & $0.2 \%$ & $0.0 \%$ & $-5.1 \%$ \\
\hline Vehicular lighting equipment & 15.586 & $0.2 \%$ & $0.0 \%$ & 17,056 & $0.2 \%$ & $0.0 \%$ & $9.4 \%$ \\
\hline Storage batteries & 23,518 & $0.3 \%$ & $0.0 \%$ & 22,426 & $0.3 \%$ & $0.0 \%$ & $-4.6 \%$ \\
\hline $\begin{array}{l}\text { Electrical equipment for } \\
\text { internal combustion engines }\end{array}$ & 61,675 & $0.9 \%$ & $0.1 \%$ & 55,857 & $0.7 \%$ & $0.1 \%$ & $-9.4 \%$ \\
\hline Tires and inner tubes & 68,505 & $1.0 \%$ & $0.1 \%$ & 65,481 & $0.9 \%$ & $0.1 \%$ & $-4.4 \%$ \\
\hline $\begin{array}{l}\text { Cold-rolled steel sheet, strip, } \\
\text { and bars }\end{array}$ & 16,365 & $0.2 \%$ & $0.0 \%$ & 16,108 & $0.2 \%$ & $0.0 \%$ & $-1.6 \%$ \\
\hline $\begin{array}{l}\text { Road construction and } \\
\text { maintenance }\end{array}$ & 261,461 & $3.7 \%$ & $0.3 \%$ & $b$ & b & b & $b$ \\
\hline $\begin{array}{l}\text { Motor freight transportation and } \\
\text { related services }\end{array}$ & $1,662,836$ & $23.6 \%$ & $1.8 \%$ & $1,900,899$ & $25.2 \%$ & $1.9 \%$ & $14.3 \%$ \\
\hline $\begin{array}{l}\text { Trucking and courier services, } \\
\text { except by air or by the U.S. } \\
\text { Postal Service }\end{array}$ & $1,458,847$ & $20.7 \%$ & $1.6 \%$ & $1,691,277$ & $22.4 \%$ & $1.7 \%$ & $15.9 \%$ \\
\hline $\begin{array}{l}\text { Petroleum refining and wholesale } \\
\text { distribution }\end{array}$ & 264,820 & $3.8 \%$ & $0.3 \%$ & 250,087 & $3.3 \%$ & $0.2 \%$ & $-5.6 \%$ \\
\hline Passenger transportation & 672,271 & $9.5 \%$ & $0.7 \%$ & 796,595 & $10.6 \%$ & $0.8 \%$ & $18.5 \%$ \\
\hline Automotive sales and servicing & $3,135,783$ & $44.5 \%$ & $3.4 \%$ & $3,418,206$ & $45.3 \%$ & $3.4 \%$ & $9.0 \%$ \\
\hline $\begin{array}{l}\text { Total of motor vehicle and related } \\
\text { industries }\end{array}$ & $7,052,766$ & $100.0 \%$ & $7.5 \%$ & $7,546,710$ & $100.0 \%$ & $7.5 \%$ & $7.0 \%$ \\
\hline U.S. Total" & $93,476,087$ & & $100.0 \%$ & $100,334,745$ & & $100.0 \%$ & $7.3 \%$ \\
\hline
\end{tabular}

\section{Source:}

American Automobile Manufacturers Association, Motor Vehicle Facts and Figures 1998, Detroit, MI, 1998, p. 74, and annual. (Additional resources: http://www.aama.com)

${ }^{a}$ Data for employees of establishments totally exempt from FICA are excluded, as are self-employed persons, domestic service workers, railroad employees, agricultural production workers and most government employees.

'Data are not available. 
Table 5.17

Employment in Transportation and Related Industries, 1960-97 (persons in thousands)

\begin{tabular}{|c|c|c|c|c|c|c|c|c|c|}
\hline & 1960 & 1965 & 1970 & 1975 & 1980 & 1985 & 1990 & 1996 & 1997 \\
\hline \multicolumn{10}{|l|}{ Transportation Service } \\
\hline Air transport & 191 & 229 & 351 & 362 & 453 & 537 & 789 & 818 & 860 \\
\hline Bus, intercity & 41 & 42 & 43 & 39 & 38 & 36 & 20 & 24 & 25 \\
\hline Local transport & 101 & 83 & 77 & 69 & 79 & 90 & 136 & 257 & 264 \\
\hline Railroads & 885 & 735 & 627 & 538 & 532 & 346 & 285 & 281 & 300 \\
\hline Oil pipeline & 23 & 20 & 18 & 17 & 21 & 19 & 20 & 16 & 16 \\
\hline Taxi & 121 & 110 & 107 & 83 & 53 & 38 & 33 & 36 & 34 \\
\hline Trucking \& truck materials & 770 & 882 & 998 & 996 & 1,189 & 1,285 & 1,534 & 2,293 & 2,414 \\
\hline Water & 232 & 230 & 215 & 190 & 213 & 214 & 173 & 182 & 185 \\
\hline Total & 2,364 & 2,331 & 2,436 & 2,294 & 2,578 & 2,565 & 2,990 & 3,907 & 4,098 \\
\hline \multicolumn{10}{|c|}{ Transportation Equipment Manufacturing } \\
\hline Aircraft \& parts & 646 & 624 & 669 & 514 & 652 & 647 & 709 & 404 & 444 \\
\hline Motor vehicles, equipment, tires & 829 & 945 & 914 & 892 & 904 & 964 & 886 & 1,305 & 1,295 \\
\hline Railroad equipment & 43 & 56 & 51 & 52 & 71 & 34 & 34 & 37 & 36 \\
\hline Ship \& boat building \& repair & 141 & 160 & 170 & 194 & 221 & 193 & 189 & 182 & 179 \\
\hline Other transportation equipment & 33 & 57 & 111 & 115 & 149 & 130 & 46 & 76 & 75 \\
\hline Total & 1,692 & 1,842 & 1,915 & 1,767 & 1,997 & 1,968 & 1,864 & 2,004 & 2,029 \\
\hline \multicolumn{10}{|l|}{$\underline{\text { Transportation Related Industries }}$} \\
\hline Automotive/accessory retail dealers & 807 & 902 & 996 & 1,076 & 1,048 & 1,185 & 1,292 & 1,330 & 1,345 \\
\hline Automotive wholesalers & 215 & 255 & 320 & 367 & 418 & 433 & 451 & 498 & 494 \\
\hline Automotive service \& garages & 251 & 324 & 384 & 400 & 571 & 730 & 926 & 1,256 & 1,249 \\
\hline Gasoline service stations & 461 & 522 & 614 & 616 & 561 & 611 & 641 & 646 & 649 \\
\hline Highway \& street construction & 294 & 324 & 331 & 297 & 268 & 264 & 245 & 240 & 246 \\
\hline Petroleum" & 311 & 292 & 333 & 390 & 533 & 568 & 521 & 487 & 484 \\
\hline \multicolumn{10}{|l|}{ Other industries } \\
\hline Truck drivers \& deliverymen & 1,477 & 1,521 & 1,565 & 1,796 & 1,931 & 2,050 & 2,148 & 2,542 & 2,490 \\
\hline Freight handlers & 365 & 411 & 456 & 613 & 622 & 574 & 504 & 551 & 539 \\
\hline Total & 4,181 & 4,551 & 4,999 & 5,545 & 5,952 & 6,415 & 6,728 & 7,550 & 7,496 \\
\hline \multicolumn{10}{|l|}{ Government Transportation Employees } \\
\hline U.S. Department of Transportation & 38 & 45 & 66 & 75 & 72 & 61 & 65 & 63 & 63 \\
\hline Highways, state $\&$ local & 499 & 550 & 568 & 569 & 532 & 549 & 569 & 543 & 525 \\
\hline U.S. Postal Service ${ }^{\mathrm{b}}$ & 83 & 83 & 103 & 98 & 92 & 104 & 115 & 119 & 125 \\
\hline Other' & 18 & 16 & 12 & 13 & 13 & 11 & 11 & 11 & 11 \\
\hline Total & 638 & 694 & 749 & 755 & 709 & 725 & 760 & 736 & 724 \\
\hline Total transportation employment & 8,875 & 9,418 & 10,099 & 10,361 & 11,236 & 11,673 & 12,342 & 14,197 & 14,347 \\
\hline Total employed civilians & 65,778 & 71,088 & 78,627 & 85,783 & 99,303 & 107,150 & 117,914 & 126,708 & 129,558 \\
\hline Transportation percent of total & $13.5 \%$ & $13.2 \%$ & $12.8 \%$ & $12.1 \%$ & $11.3 \%$ & $10.9 \%$ & $10.5 \%$ & $11.2 \%$ & $11.1 \%$ \\
\hline
\end{tabular}

Source:

Eno Transportation Foundation, Transportation in America 1998, Lansdowne, VA, 1998, p. 61. demand.

${ }^{a}$ Estimated by assuming transport share of total petroleum industry employment is same as transport share of petroleum domestic

${ }^{b}$ Estimated share (approximately 14\%) of total employees engaged in transportation work.

c Agencies include Civil Aeronautics Board (sunset in 1985), Federal Maritime Commission, Federal Energy Regulatory Commission, Interstate Commerce Commission, Railroad Retirement Board, and Panama Canal Commission. 


\section{Chapter 6}

\section{Highway Vehicles and Characteristics}

Summary Statistics from Tables in this Chapter

\begin{tabular}{|c|c|c|}
\hline Source & & \\
\hline Table 6.1 & U.S. share of world automobile registrations, 1996 & $26.7 \%$ \\
\hline Table 6.2 & U.S. share of world truck \& bus registrations, 1996 & $41.3 \%$ \\
\hline Table 6.3 & Number of automobiles, 1997 (Polk - in thousands) & 124,673 \\
\hline Table 6.3 & Number of trucks, 1997 (Polk - in thousands) & 76,398 \\
\hline \multirow[t]{7}{*}{ Table 6.5} & Vehicle miles traveled, 1997 & (million miles) \\
\hline & Automobiles & $1,501,820$ \\
\hline & Motorcycles & 10,076 \\
\hline & Two-axle, four-tire trucks & 850,296 \\
\hline & Other single-unit trucks & 66,845 \\
\hline & Combination truck-s & 124,500 \\
\hline & Buses & 6,836 \\
\hline \multirow[t]{4}{*}{ Table 6.8} & Average age of vehicles, 1997 & (years) \\
\hline & Automobiles & 8.7 \\
\hline & Trucks & 8.3 \\
\hline & Average lifetime of vehicles & $(\mathrm{y}$ e $\mathrm{ar}$ \\
\hline Table 6.9 & Automobiles, 1990 model year & 13.7 \\
\hline Table 6.10 & Trucks, 1979-89 model years & 16.0 \\
\hline
\end{tabular}


Table 6.1

Automobile Registrations for Selected Countries, 1950-96 (thousands)

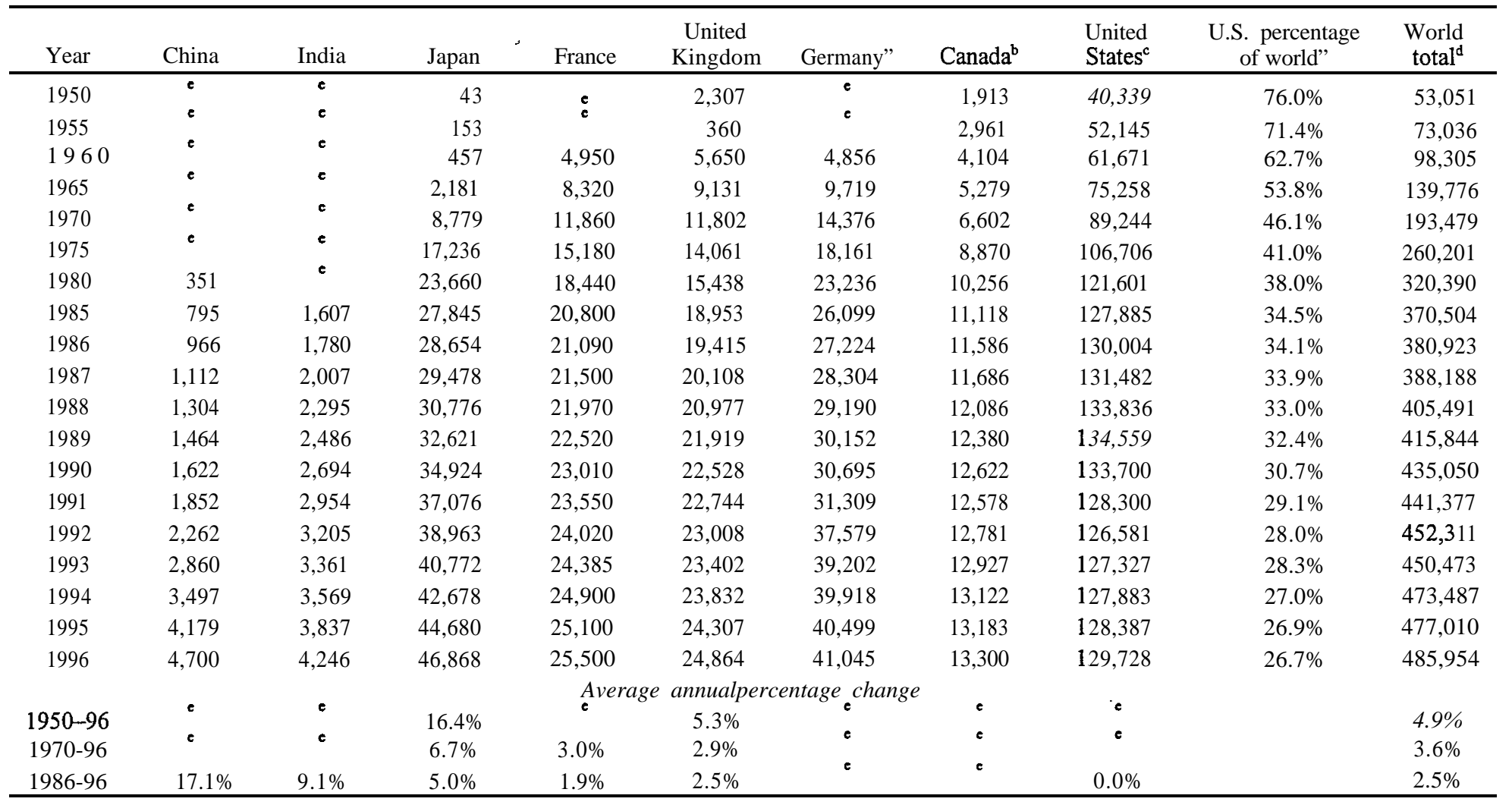

Source:

Motor Vehicle Manufacturers Association, World Motor Vehicle Data, 1998 Edition, Detroit, MI, 1998, pp. 8, 23, 28, 42, 85, 98, 169, 206, 230 and annual. (Additional resources: http://www.aama.com)

\footnotetext{
a Data for 1991 and prior include West Germany only. Kraftwagen are included with automobiles.

${ }^{b}$ Data from 1991 and later are not comparable to prior data.

c Data from 1985 and later are not comparable to prior data.

d World totals were recalculated from 1985-94 based on change in U.S. data.

${ }^{\boldsymbol{e}}$ Data are not available
} 
Table 6.2

Truck and Bus Registrations for Selected Countries, 1950-96 (thousands)

\begin{tabular}{|c|c|c|c|c|c|c|c|c|c|c|}
\hline Year & China & India & Japan & France & $\begin{array}{l}\text { United } \\
\text { Kingdom }\end{array}$ & Germany" & Canada $^{\mathrm{b}}$ & $\begin{array}{l}\text { United } \\
\text { States }^{\mathrm{c}}\end{array}$ & $\begin{array}{l}\text { U.S. percentage } \\
\text { of world }\end{array}$ & $\begin{array}{l}\text { World } \\
\text { total }^{\mathrm{d}}\end{array}$ \\
\hline 1950 & $\bar{c}$ & $\bar{c}$ & 183 & & 1,060 & \multirow{2}{*}{ e } & 643 & 8,823 & $50.9 \%$ & 17,349 \\
\hline 1955 & e & e & 318 & & 1,244 & & 952 & 10,544 & $46.1 \%$ & 22,860 \\
\hline 1960 & e & e & 896 & 1,540 & 1,534 & 786 & 1,056 & 12,186 & $42.6 \%$ & 28,583 \\
\hline 1965 & \multirow{2}{*}{ e } & e & 4,119 & 1,770 & 1,748 & 1,021 & 1,232 & 15,100 & $39.6 \%$ & 38,118 \\
\hline 1970 & & \multirow[t]{3}{*}{ 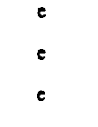 } & 8,803 & 1,850 & 1,769 & 1,228 & 1,481 & 19,175 & $36.2 \%$ & 52,899 \\
\hline 1975 & 811 & & 10,854 & 2,210 & 1,934 & 1,337 & 2,158 & 26,243 & $38.8 \%$ & 67,698 \\
\hline 1980 & 1,480 & & 14,197 & 2,550 & 1,920 & 1,617 & 2,955 & 34,195 & $37.7 \%$ & 90,592 \\
\hline 1985 & 2,402 & 1,045 & 18,313 & 3,310 & 3,278 & 1,723 & 3,149 & 43,804 & $37.4 \%$ & 117,038 \\
\hline 1986 & 2,884 & 1,090 & 19,319 & 3,980 & 3,336 & 1,760 & 3,213 & 45,697 & $38.6 \%$ & 118,373 \\
\hline 1987 & 3,247 & 1,229 & 20,424 & 4,200 & 3,452 & 1,801 & 3,576 & 47,428 & $37.4 \%$ & 126,890 \\
\hline 1988 & 3,716 & 1,383 & 21,674 & 4,370 & 3,621 & 1,846 & 3,766 & 50,557 & $37.6 \%$ & 134,294 \\
\hline 1989 & 4,118 & 1,457 & 22,472 & 4,570 & 3,754 & 1,914 & 3,889 & 52,797 & $37.4 \%$ & 141,184 \\
\hline 1990 & 4,496 & 1,536 & 22,773 & 4,748 & 3,774 & 1,989 & 3,931 & 55,097 & $37.2 \%$ & 148,073 \\
\hline 1991 & 4,721 & 1,687 & 22,839 & 4,910 & 3,685 & 2,114 & 3,402 & 59,837 & $38.9 \%$ & 153,695 \\
\hline 1992 & 5,177 & 1,872 & 22,694 & 5,040 & 3,643 & 2,672 & 3,413 & 63,781 & $39.6 \%$ & 161,219 \\
\hline 1993 & 5,316 & 1,967 & 22,490 & 5,065 & 3,604 & 2,842 & 3,409 & 66,736 & $40.1 \%$ & 166,614 \\
\hline 1994 & 5,922 & 2,083 & 22,333 & 5,140 & 3,605 & 2,960 & 3,466 & 70,162 & $45.1 \%$ & 155,591 \\
\hline 1995 & 6,221 & 2,221 & 22,173 & 5,195 & 3,635 & 3,062 & 3,485 & 73,143 & $43.1 \%$ & 169,749 \\
\hline 1996 & 6,750 & 2,506 & 21,933 & 5,255 & 3,621 & 3,122 & 3,515 & 76,637 & $41.3 \%$ & 185,404 \\
\hline $\begin{array}{l}1950-96 \\
1970-96 \\
1986-96\end{array}$ & $\begin{array}{l}e \\
\text { e } \\
8.9 \%\end{array}$ & $\begin{array}{c}\text { e } \\
\text { e } \\
8.7 \%\end{array}$ & $\begin{array}{r}11.0 \% \\
3.6 \% \\
1.3 \%\end{array}$ & $\begin{array}{l}4.1 \% \\
2.8 \%\end{array}$ & $\begin{array}{c}\text { annualper } \\
2.7 \% \\
2.8 \% \\
0.8 \%\end{array}$ & $\begin{array}{c}\text { ntage chang } \\
\text { e } \\
\text { с }\end{array}$ & $\begin{array}{l}\text { e } \\
\text { e } \\
\text { c }\end{array}$ & $\begin{array}{c}\text { e } \\
\text { e } \\
5.3 \%\end{array}$ & & $\begin{array}{l}5.3 \% \\
4.9 \% \\
4.6 \%\end{array}$ \\
\hline
\end{tabular}

Source:

Motor Vehicle Manufacturers Association, World Motor Vehicle Data, 1998 Edition, Detroit, MI, 1998, pp. 8, 23, 28, 42, 85, 98, 169, 206, 230 and annual. (Additional resources: http://www.aama.com)

\footnotetext{
${ }^{\text {a }}$ Data for 1991 and prior include West Germany only. Kraftwagen are included with automobiles (Table 1.1)

${ }^{b}$ Data from 1991 and later are not comparable to prior data.

${ }^{\mathbf{c}}$ Data from 1985 and later are not comparable to prior data.

d World totals were recalculated from 1985-94 based on change in U.S. data.

e Data are not available.
} 


\section{VEHICLES IN USE}

Both the Federal Highway Administration (FHWA) and The Polk Company report figures on the automobile and truck population each year. The two estimates, however, differ by as much as $25.6 \%$ for trucks (1992). The differences can be attributed to several factors:

- The FHWA data include all vehicles which have been registered at any time throughout the calendar year. Therefore, the data include vehicles which were retired during the year and may double count vehicles which have been registered in different states or the same states to different owners. The Polk Company data include only those vehicles which are registered on July 1 of the given year.

- The classification of mini-vans, station wagons on truck chasses, and utility vehicles as passenger cars or trucks causes important differences in the two estimates. The Polk Company data included passenger vans in the automobile count until 1980; since 1980 all vans have been counted as trucks. Recently, the Federal Highway Administration adjusted their definition of automobiles and trucks. Starting in 1993, some minivans and sport utility vehicles that were previously included with automobiles were included with trucks. This change produced a dramatic change in the individual percentage differences of cars and trucks. The difference in total vehicles has been less than $5 \%$ each year since 1990 and does not appear to be significantly affected by the FHWA reclassifications.

- The FHWA data include all non-military Federal vehicles, while The Polk Company data include only those Federal vehicles which are registered within a state. Federal vehicles are not required to have State registrations, and, according to the General Services Administration, most Federal Vehicles are not registered.

According to The Polk Company statistics, the number of passenger cars in use in the U.S. declined from 1991 to 1992. This is the first decline in vehicle stock since the figures were first reported in 1924. However, the data should be viewed with caution. A redesign of Polk's approach in 1992 allowed a national check for duplicate registrations, which was not possible in earlier years. Polk estimates that, due to processing limitations, its vehicle population counts may have been inflated by as much as $1 \frac{1}{1} 2$ percent. Assuming that percentage is correct, the number of passenger cars in use would have declined from 1991 to 1992 under the previous Polk method. The growing popularity of light trucks being used as passenger vehicles could also have had an impact on these figures. 
Table 6.3

Automobiles and Trucks in Use, 1970-97

(thousands)

\begin{tabular}{|c|c|c|c|c|c|c|c|c|c|}
\hline \multirow[b]{2}{*}{ Year } & \multicolumn{3}{|c|}{ Automobiles } & \multicolumn{3}{|c|}{ Trucks } & \multicolumn{3}{|c|}{ Total } \\
\hline & FHWA & $\begin{array}{l}\text { The Polk } \\
\text { Company }\end{array}$ & $\begin{array}{l}\text { Percentage } \\
\text { difference }\end{array}$ & FHWA & $\begin{array}{l}\text { The Polk } \\
\text { Company }\end{array}$ & $\begin{array}{l}\text { Percentage } \\
\text { difference }\end{array}$ & FHWA & $\begin{array}{l}\text { The Polk } \\
\text { Company }\end{array}$ & $\begin{array}{l}\text { Percentage } \\
\text { difference }\end{array}$ \\
\hline 1970 & 89,243 & 80,448 & $10.9 \%$ & 18,797 & 17,688 & $6.3 \%$ & 108,040 & 98,136 & $10.1 \%$ \\
\hline 1971 & 92,718 & 83,138 & $11.5 \%$ & 19,871 & 18,462 & $7.6 \%$ & 112,589 & 101,600 & $10.8 \%$ \\
\hline 1972 & 97,082 & 86,439 & $12.3 \%$ & 21,308 & 19,773 & $7.8 \%$ & 118,390 & 106,212 & $11.5 \%$ \\
\hline 1973 & 101,985 & 89,805 & $13.6 \%$ & 23,244 & 21,412 & $8.6 \%$ & 125,229 & 111,217 & $12.6 \%$ \\
\hline 1974 & 104,856 & 92,608 & $13.2 \%$ & 24,630 & 23,312 & $5.7 \%$ & 129,487 & 115,920 & $11.7 \%$ \\
\hline 1975 & 106,706 & 95,241 & $12.0 \%$ & 25,781 & 24,813 & $3.9 \%$ & 132,487 & 120,054 & $10.4 \%$ \\
\hline 1976 & 110,189 & 97,818 & $12.6 \%$ & 27,876 & 26,560 & $5.0 \%$ & 138,065 & 124,378 & $11.0 \%$ \\
\hline 1977 & 112,288 & 99,904 & $12.4 \%$ & 29,314 & 28,222 & $3.9 \%$ & 141,602 & 128,126 & $10.5 \%$ \\
\hline 1978 & 116,573 & 102,957 & $13.2 \%$ & 31,336 & 30,565 & $2.5 \%$ & 147,909 & 133,522 & $10.8 \%$ \\
\hline 1979 & 118,429 & 104,677 & $13.1 \%$ & 32,914 & 32,583 & $1.0 \%$ & 151,343 & 137,260 & $10.3 \%$ \\
\hline 1980 & 121,601 & 104,564 & $16.3 \%$ & 33,667 & 35,268 & $-4.5 \%$ & 155,267 & 139,832 & $11.0 \%$ \\
\hline 1981 & 123,098 & 105,839 & $16.3 \%$ & 34,644 & 36,069 & $-4.0 \%$ & 157,743 & 141,908 & $11.2 \%$ \\
\hline 1982 & 123,702 & 106,867 & $15.8 \%$ & 35,382 & 36,987 & $-4.3 \%$ & 159,084 & 143,854 & $10.6 \%$ \\
\hline 1983 & 126,444 & 108,961 & $16.0 \%$ & 36,723 & 38,143 & $-3.7 \%$ & 163,166 & 147,104 & $10.9 \%$ \\
\hline 1984 & 128,158 & 112,019 & $14.4 \%$ & 37,507 & 40,143 & $-6.6 \%$ & 165,665 & 152,162 & $8.9 \%$ \\
\hline 1985 & 127,885 & 114,662 & $11.5 \%$ & 43,210 & 42,387 & $1.9 \%$ & 171,095 & 157,049 & $8.9 \%$ \\
\hline 1986 & 130,004 & 117,268 & $10.9 \%$ & 45,103 & 44,826 & $0.6 \%$ & 175,106 & 162,094 & $8.0 \%$ \\
\hline 1987 & 131,482 & 119,849 & $9.7 \%$ & 46,826 & 47,344 & $-1.1 \%$ & 178,308 & 167,193 & $6.6 \%$ \\
\hline 1988 & 133,836 & 121,519 & $10.1 \%$ & 49,941 & 50,221 & $-0.6 \%$ & 183,777 & 171,740 & $7.0 \%$ \\
\hline 1989 & 134,559 & 122,758 & $9.6 \%$ & 52,172 & 53,202 & $-1.9 \%$ & 186,731 & 175,960 & $6.1 \%$ \\
\hline 1990 & 133,700 & 123,276 & $8.5 \%$ & 54,470 & 56,023 & $-2.8 \%$ & 188,171 & 179,299 & $4.9 \%$ \\
\hline 1991 & 128,300 & 123,268 & $4.1 \%$ & 59,206 & 58,179 & $1.8 \%$ & 187,505 & 181,447 & $3.3 \%$ \\
\hline 1992 & $126,58 \mathrm{I}$ & 120,347 & $5.2 \%$ & 63,136 & 61,172 & $3.2 \%$ & 189,717 & 181,519 & $4.5 \%$ \\
\hline 1993 & 127,327 & 121,055 & $5.2 \%$ & 66,082 & 65,260 & $1.3 \%$ & 193,409 & 186,315 & $3.8 \%$ \\
\hline 1994 & 127,883 & 121,997 & $4.8 \%$ & 69,491 & 66,717 & $4.2 \%$ & 197,375 & 188,714 & $4.6 \%$ \\
\hline 1995 & 128,387 & 123,242 & $4.2 \%$ & 72,458 & 70,199 & $3.2 \%$ & 200,845 & 193,441 & $3.8 \%$ \\
\hline 1996 & 129,728 & 124,613 & $4.1 \%$ & 75,940 & 73,681 & $3.1 \%$ & 205,669 & 198,294 & $3.7 \%$ \\
\hline 1997 & 129.749 & 124.673 & $41 \%$ & 77.307 & 76.398 & $12 \%$ & 207.056 & 701.071 & 30 \\
\hline
\end{tabular}

Source:

FHWA - U.S. Department of Transportation, Federal Highway Administration, Highway Statistics 1997, Washington, DC, 1998, Table VM-1, p. V-89, and annual. (Additionalresources:http://www.fhwa.dot.gov)

Polk - The Polk Company, Detroit, Michigan. FURTHER REPRODUCTION PROHIBITED. (Additional resources: http://www.polk.com) 
The data on automobile stock by size class are estimations based on historical sales data. This method assumes a constant scrappage rate for all size classes. The data on trucks by weight class are based on estimates from the 1992 Truck Inventory and Use Survey (latest available survey).

Table 6.4

Vehicle Stock and New Sales in United States, 1997 Calendar Year

\begin{tabular}{|c|c|c|c|c|c|}
\hline & \multicolumn{2}{|c|}{ Vehicle stock } & \multicolumn{3}{|c|}{ New sales } \\
\hline & Thousands & Percentage & $\begin{array}{c}\text { Domestic } \\
\text { (thousands) }\end{array}$ & $\begin{array}{c}\text { Import }^{\mathrm{b}} \\
\text { (thousands) }\end{array}$ & $\begin{array}{c}\text { Total } \\
\text { (thousands) }\end{array}$ \\
\hline Autos & 124,673 & $100.0 \%$ & $6,917(83.6 \%)$ & $1,355(16.4 \%)$ & $8,272(100.0 \%)$ \\
\hline Two seaters & 2,241 & $1.8 \%$ & $37(45.2 \%)$ & $45(54.8 \%)$ & $82(100.0 \%)$ \\
\hline Minicompact & 1,240 & $1.0 \%$ & $0(0.0 \%)$ & $41(100.0 \%)$ & $41(100.0 \%)$ \\
\hline Subcompact & 27,544 & $22.1 \%$ & $1,217 \quad(80.4 \%)$ & $296(19.6 \%)$ & $1,514(100.0 \%)$ \\
\hline Compact & 40,690 & $32.6 \%$ & $2,463(83.7 \%)$ & $478(16.3 \%)$ & $2,941(100.0 \%)$ \\
\hline Midsize & 35,566 & $28.5 \%$ & $2,072(8 \quad 1.7 \%)$ & $464(18.3 \%)$ & $2,536(100.0 \%)$ \\
\hline Large & 17,392 & $14.0 \%$ & $1,127 \quad(97.3 \%)$ & $32(2.7 \%)$ & $1,159(100.0 \%)$ \\
\hline Autos & 124,673 & $100.0 \%$ & c & c & c \\
\hline Business fleet autos ${ }^{d}$ & 9,225 & $7.4 \%$ & $\mathbf{c}$ & $\mathbf{c}$ & c \\
\hline Personal autos & 115.448 & $92.6 \%$ & c & c & $\mathbf{c}$ \\
\hline Motorcycles & $3,826 "$ & $100.0 \%$ & $\mathbf{c}$ & $\mathbf{c}$ & $\mathbf{c}$ \\
\hline Recreational vehicles & c & c & $438(\mathbf{1 0 0 . 0 \% )}$ & $0(0.0 \%)$ & $438(\mathbf{1 0 0 . 0 \%})$ \\
\hline Trucks & 76,398 & $100.0 \%$ & $6,633(\mathbf{9 1 . 8 \%})$ & $593(8.2 \%)$ & 7,227 (100.0\%) \\
\hline Light $(\mathrm{O}-10,000 \mathrm{Ibs})$ & 71,279 & $93.3 \%$ & $6,226(91.6 \%)$ & $571(8.4 \%)$ & $6,798(100.0 \%)$ \\
\hline Medium (1O,OOl-26,000 lbs) & 2,521 & $3.3 \%$ & $116(84.7 \%)$ & $21(15.3 \%)$ & $137(100.0 \%)$ \\
\hline Heavy-heavy (26,001 Ibs and over) & 2,598 & $3.4 \%$ & $291(99.7 \%)$ & $1(0.3 \%)$ & $292(100.0 \%)$ \\
\hline Trucks & 76,398 & $100.0 \%$ & c & c & c \\
\hline Business fleet trucks $\leq 19,500 \mathrm{lbs}^{\mathrm{d}}$ & 6,644 & $8.7 \%$ & $\mathbf{c}$ & c & c \\
\hline Personal trucks $\leq 19,500 \mathrm{lbs}$ & 66,240 & $86.7 \%$ & $\mathbf{c}$ & $\mathbf{c}$ & $\mathbf{c}$ \\
\hline Trucks $>19,500 \mathrm{lbs}$. & 3,314 & $4.6 \%$ & $\mathbf{c}$ & c & c \\
\hline
\end{tabular}

Source:

See Appendix A for Table 6.4. (Additional resources: http://www.aama.com, http://www.polk.com)

\footnotetext{
a Total auto and truck vehicle stock as of July 1 from The Polk Company (FURTHER REPRODUCTION PROHIBITED).

b Includes domestic-sponsored imports.

c Data are not available.

d In fleets of four or more vehicles.

e Includes mostly on-highway motorcycles. Many states do not require registration for off-highway vehicles.
} 
Table 6.5

Highway Vehicle Miles Traveled by Vehicle Type, 1970-97 (million miles)

\begin{tabular}{|c|c|c|c|c|c|c|c|}
\hline Year & Automobiles & Motorcycles & $\begin{array}{c}\text { Two-axle, } \\
\text { four-tire } \\
\text { trucks }\end{array}$ & $\begin{array}{l}\text { Other } \\
\text { single-unit } \\
\text { trucks }\end{array}$ & $\begin{array}{c}\text { Combination } \\
\text { trucks }\end{array}$ & Buses $^{\mathbf{a}}$ & Total \\
\hline 1970 & 916,700 & 2,979 & 123,286 & 27,081 & 35,134 & 4,544 & $1,109,724$ \\
\hline 1971 & 966,330 & 3,607 & 137,870 & 28,985 & 37,217 & 4,802 & $1,178,811$ \\
\hline 1972 & $1,021,365$ & 4,331 & 156,622 & 31,414 & 40,706 & 5,348 & $1,259,786$ \\
\hline 1973 & $1,045,981$ & 5,194 & 176,833 & 33,661 & 45,649 & 5,792 & $1,313,110$ \\
\hline 1974 & $1,007,251$ & 5,445 & 182,757 & 33,441 & 45,966 & 5,684 & $1,280,544$ \\
\hline 1975 & $1,033,950$ & 5,629 & 200,700 & 34,606 & 46,724 & 6,055 & $1,327,664$ \\
\hline 1976 & $1,078,215$ & 6,003 & 225,834 & 36,390 & 49,680 & 6,258 & $1,402,380$ \\
\hline 1977 & $1,109,243$ & 6,349 & 250,591 & 39,339 & 55,682 & 5,823 & $1,467,027$ \\
\hline 1978 & $1,146,508$ & 7,158 & 279,414 & 42,747 & 62,992 & 5,885 & $1,544,704$ \\
\hline 1979 & $1,113,640$ & 8,637 & 291,905 & 42,012 & 66,992 & 5,947 & $1,529,133$ \\
\hline 1980 & $1,111,596$ & 10,214 & 290,935 & 39,813 & 68,678 & 6,059 & $1,527,295$ \\
\hline 1981 & $1,133,332$ & 10,690 & 296,343 & 39,568 & 69,134 & 6,241 & $1,555,308$ \\
\hline 1982 & $1,161,713$ & 9,910 & 306,141 & 40,658 & 70,765 & 5,823 & $1,595,010$ \\
\hline 1983 & $1,195,054$ & 8,760 & 327,643 & 42,546 & 73,586 & 5,199 & $1,652,788$ \\
\hline 1984 & $1,227,043$ & 8,784 & 358,006 & 44,419 & 77,377 & 4,640 & $1,720,269$ \\
\hline 1985 & $1,246,798$ & 9,086 & 390,961 & 45,441 & 78,063 & 4,478 & $1,774,826$ \\
\hline 1986 & $1,270,167$ & 9,397 & 423,915 & 45,637 & 81,038 & 4,717 & $1,834,872$ \\
\hline 1987 & $1,315,982$ & 9,506 & 456,870 & 48,022 & 85,495 & 5,330 & $1,921,204$ \\
\hline 1988 & $1,370,271$ & 10,024 & 502,207 & 49,434 & 88,551 & 5,475 & $2,025,962$ \\
\hline 1989 & $1,401,221$ & 10,371 & 536,475 & 50,870 & 91,879 & 5,670 & $2,096,487$ \\
\hline 1990 & $1,408,266$ & 9,557 & 574,571 & 51,901 & 94,341 & 5,726 & $2,144,362$ \\
\hline 1991 & $1,358,185$ & 9,178 & 649,394 & 52,898 & 96,645 & 5,750 & $2,172,050$ \\
\hline 1992 & $1,371,569$ & 9,557 & 706,863 & 53,874 & 99,510 & 5,778 & $2,247,151$ \\
\hline 1993 & $1,374,709$ & 9,906 & 745,750 & 56,772 & 103,116 & 6,125 & $2,296,378$ \\
\hline 1994 & $1,406,089$ & 10,240 & 764,634 & 61,284 & 108,932 & 6,409 & $2,357,588$ \\
\hline 1995 & $1,438,294$ & 9,797 & 790,029 & 62,705 & 115,451 & 6,420 & $2,422,696$ \\
\hline 1996 & $1,469,854$ & 9,920 & 816,540 & 64,072 & 118,899 & 6,563 & $2,485,848$ \\
\hline 1997 & $1,501,820$ & 10,076 & 850,296 & 66,845 & 124,500 & 6,836 & $2,560,373$ \\
\hline \multicolumn{8}{|c|}{ Average annualpercentage change } \\
\hline $1970-97$ & $1.8 \%$ & $4.6 \%$ & $7.4 \%$ & $3.4 \%$ & $4.8 \%$ & $1.5 \%$ & $3.1 \%$ \\
\hline $1987-97$ & $1.3 \%$ & $0.6 \%$ & $6.4 \%$ & $3.4 \%$ & $3.8 \%$ & $2.5 \%$ & $2.9 \%$ \\
\hline
\end{tabular}

Source:

U.S. Department of Transportation, Federal Highway Administration, Highway Statistics 1997, Washington, DC, 1998, Table VM-1, p. V-89, and annual.

(Additional resources: http://www.fhwa.dot.gov)

"The data do not correspond with vehicle-miles of travel presented in the "Bus" section of this chapter due to differing data sources. 
Table 6.6

Automobiles in Operation and Vehicle Travel by Age, 1970 and 1997

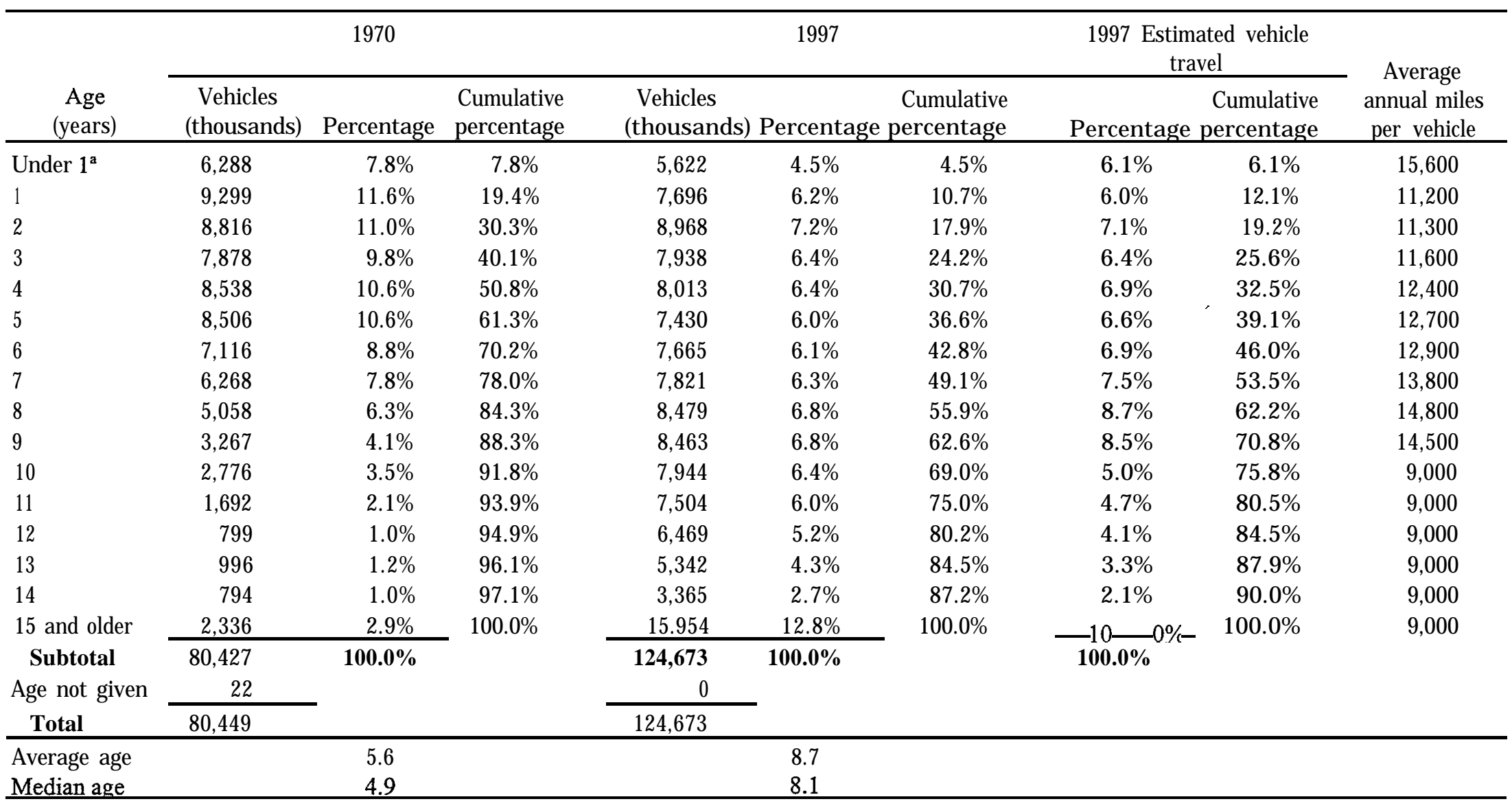

Source:

The Polk Company, Detroit, MI. FURTHER REPRODUCTION PROHIBITED.

Vehicle travel - Average annual miles per auto by age were multiplied by the number of vehicles in operation by age to estimate the vehicle travel. Average annual miles per auto by age - generated by ORNL. from the Nationwide Personal Transportation Survey web site: http://www-cta.ornl.gov/npts. (Additional resources: http://www.polk.com, http://www-cta.ornl.gov/npts)

\footnotetext{
"Automobiles sold as of July 1 of each year.
} 
Table 6.7

Trucks in Operation and Vehicle Travel by Age, 1970 and 1997

\begin{tabular}{|c|c|c|c|c|c|c|c|c|c|}
\hline \multirow[b]{2}{*}{$\begin{array}{c}\text { Age } \\
\text { (years) }\end{array}$} & \multicolumn{3}{|c|}{1970} & \multicolumn{3}{|c|}{1997} & \multicolumn{2}{|c|}{$\begin{array}{c}1997 \text { Estimated } \\
\text { vehicle travel }\end{array}$} & \multirow{2}{*}{$\begin{array}{l}\text { Average annual } \\
\text { miles per } \\
\text { vehicle }\end{array}$} \\
\hline & $\begin{array}{c}\text { Vehicles } \\
\text { (thousands) }\end{array}$ & Percentage & $\begin{array}{l}\text { Cumulative } \\
\text { percentage }\end{array}$ & $\begin{array}{c}\text { Vehicles } \\
\text { (thousands) }\end{array}$ & Percentage & $\begin{array}{l}\text { Cumulative } \\
\text { percentage }\end{array}$ & Percentage & $\begin{array}{l}\text { Cumulative } \\
\text { percentage }\end{array}$ & \\
\hline Under 1" & 1,262 & $7.1 \%$ & $7.1 \%$ & 4,624 & $6.1 \%$ & $6.1 \%$ & $6.7 \%$ & $6.7 \%$ & 14,288 \\
\hline 1 & 1,881 & $10.6 \%$ & $17.8 \%$ & 5,828 & $7.6 \%$ & $13.7 \%$ & $9.8 \%$ & $16.5 \%$ & 16,439 \\
\hline 2 & 1,536 & $8.7 \%$ & $26.5 \%$ & 6,362 & $8.3 \%$ & $22.0 \%$ & $11.9 \%$ & $28.5 \%$ & 18,388 \\
\hline 3 & 1,428 & $8.1 \%$ & $34.6 \%$ & 5,733 & $7.5 \%$ & $29.5 \%$ & $10.3 \%$ & $38.8 \%$ & 17,601 \\
\hline 4 & 1,483 & $8.4 \%$ & $43.0 \%$ & 4,838 & $6.3 \%$ & $35.8 \%$ & $8.3 \%$ & $47.0 \%$ & 16,775 \\
\hline 5 & 1,339 & $7.6 \%$ & $50.5 \%$ & 4,015 & $5.3 \%$ & $41.1 \%$ & $6.6 \%$ & $53.6 \%$ & 16,020 \\
\hline 6 & 1,154 & $6.5 \%$ & $57.1 \%$ & 3,912 & $5.1 \%$ & $46.2 \%$ & $5.8 \%$ & $59.4 \%$ & 14,574 \\
\hline 7 & 975 & $5.5 \%$ & $62.6 \%$ & 3,802 & $5.0 \%$ & $51.2 \%$ & $5.3 \%$ & $64.8 \%$ & 13,710 \\
\hline 8 & 826 & $4.7 \%$ & $67.3 \%$ & 4,340 & $5.7 \%$ & $56.9 \%$ & $5.9 \%$ & $70.6 \%$ & 13,255 \\
\hline 9 & 621 & $3.5 \%$ & $70.8 \%$ & 4,203 & $5.5 \%$ & $62.4 \%$ & $5.2 \%$ & $75.9 \%$ & 12,237 \\
\hline 10 & 658 & $3.7 \%$ & $74.5 \%$ & 3,633 & $4.8 \%$ & $67.1 \%$ & $3.0 \%$ & $78.9 \%$ & 8,224 \\
\hline 11 & 583 & $3.3 \%$ & $77.8 \%$ & 3,741 & $4.9 \%$ & $72.0 \%$ & $3.1 \%$ & $82.1 \%$ & 8,224 \\
\hline 12 & 383 & $2.2 \%$ & $80.0 \%$ & 3,111 & $4.1 \%$ & $76.1 \%$ & $2.6 \%$ & $84.7 \%$ & 8,224 \\
\hline 13 & 417 & $2.4 \%$ & $82.3 \%$ & 2,624 & $3.4 \%$ & $79.5 \%$ & $2.2 \%$ & $86.9 \%$ & 8,224 \\
\hline 14 & 414 & $2.3 \%$ & $84.7 \%$ & $-1,596$ & $2.1 \%$ & $81.6 \%$ & $1.3 \%$ & $88.2 \%$ & 8,224 \\
\hline 15 and older & 2,710 & $15.3 \%$ & $100.0 \%$ & 14.036 & $18.4 \%$ & $100.0 \%$ & $-11-8 \%$ & $100.0 \%$ & 8,224 \\
\hline Subtotal & 17,670 & $100.0 \%$ & & 76,398 & $100.0 \%$ & & $100.0 \%$ & & \\
\hline Age not given & 15 & & & 0 & & & & & \\
\hline Total & 17,685 & & & 76,398 & & & & & \\
\hline $\begin{array}{l}\text { Average age } \\
\text { Median age }\end{array}$ & & $\begin{array}{l}7.3 \\
5.9 \\
\end{array}$ & & & $\begin{array}{l}8.3 \\
7.8 \\
\end{array}$ & & & & \\
\hline
\end{tabular}

Source:

The Polk Company, Detroit, MI. FURTHER REPRODUCTION PROHIBITED.

Vehicle travel-The average annual vehicle-miles per truck by age were multiplied by the number of trucks in operation by age to estimate the vehicle travel. Average annual miles per truck by age were generated by ORNL from the 1992 Truck Inventory and Use Survey public use tape provided by U.S. Department of Commerce, Bureau of the Census, Washington, DC, 1995. (Additional resources: http://www.polk.com, http://www.census.gov)

"Trucks sold as of July 1 of each year. 
The average age of automobiles was lower than the average age of trucks until 1995. Since then, the average automobile age continues to grow, while the average truck age has held about the same. The increasingpopularity of light trucks aspersonalpassenger vehicles may have had an influence on the average age of trucks.

Table 6.8

Average Age of Automobiles and Trucks in Use, 1970-97 (years)

\begin{tabular}{|c|c|c|c|c|c|}
\hline \multirow{2}{*}{$\begin{array}{l}\text { Calendar } \\
\text { year }\end{array}$} & \multicolumn{2}{|c|}{ Automobiles } & & \multicolumn{2}{|c|}{ Trucks } \\
\hline & Mean $^{a}$ & Median $^{\mathrm{b}}$ & & Mean $^{a}$ & Median $^{\mathrm{b}}$ \\
\hline 1970 & 5.6 & 4.9 & & 7.3 & 5.9 \\
\hline 1971 & 5.7 & 5.1 & & 7.4 & 6.1 \\
\hline 1972 & 5.7 & 5.1 & & 7.2 & 6.0 \\
\hline 1973 & 5.7 & 5.1 & & 6.9 & 5.8 \\
\hline 1974 & 5.7 & 5.2 & & 7.0 & 5.6 \\
\hline 1975 & 6.0 & 5.4 & & 6.9 & 5.8 \\
\hline 1976 & 6.2 & 5.5 & & 7.0 & 5.8 \\
\hline 1977 & 6.2 & 5.6 & & 6.9 & 5.7 \\
\hline 1978 & 6.3 & 5.7 & & 6.9 & 5.8 \\
\hline 1979 & 6.4 & 5.9 & & 6.9 & 5.9 \\
\hline 1980 & 6.6 & 6.0 & & 7.1 & 6.3 \\
\hline 1981 & 6.9 & 6.0 & & 7.5 & 6.5 \\
\hline 1982 & 7.2 & 6.2 & & 7.8 & 6.8 \\
\hline 1983 & 7.4 & 6.5 & & 8.1 & 7.2 \\
\hline 1984 & 7.5 & 6 & 7 & 8.2 & 7.4 \\
\hline 1985 & 7.6 & 6.9 & & 8.1 & 7.6 \\
\hline 1986 & 7.6 & 7.0 & & 8.0 & 7.7 \\
\hline 1987 & 7.6 & 6.9 & & 8.0 & 7.8 \\
\hline 1988 & 7.6 & 6.8 & & 7.9 & 7.1 \\
\hline 1989 & 7.6 & 6.5 & & 7.9 & 6.7 \\
\hline 1990 & 7.8 & 6.5 & & 8.0 & 6.5 \\
\hline 1991 & 7.9 & 6.7 & & 8.1 & 6.8 \\
\hline 1992 & 8.1 & 7.0 & & 8.4 & 7.2 \\
\hline 1993 & 8.3 & 7.3 & & 8.6 & 7.5 \\
\hline 1994 & 8.4 & 7.5 & & 8.4 & 7.5 \\
\hline 1995 & 8.5 & 7.7 & & 8.4 & 7.6 \\
\hline 1996 & 8.6 & 7.9 & . & 8.3 & 7.7 \\
\hline 1997 & 8.7 & 8.1 & & 8.3 & 7.8 \\
\hline
\end{tabular}

Source:

The Polk Company, Detroit, MI. FURTHER REPRODUCTION PROHIBITED. (Additional resources: http://www.polk.com)

"Mean is the sum of the products of units multiplied by age, divided by the total units.

bMedian is a value in an ordered set of values below and above which there are an equal number of values. 
1990 model year (MY) automobiles will be in service an average of three years longer than their 1970 counterparts. The average lifetime of autos increased by 1.4 years from MY 1970 to MY 1980, then rose another 1.6 years by MY 1990.

Table 6.9

Scrappage and Survival Rates for Automobiles 1970, 1980 and 1990 Model Years

\begin{tabular}{|c|c|c|c|c|c|c|}
\hline \multirow{2}{*}{$\begin{array}{c}\text { Vehicle } \\
\text { age } \\
\text { (years) }\end{array}$} & \multicolumn{2}{|c|}{1970 model year } & \multicolumn{2}{|c|}{1980 model year } & \multicolumn{2}{|c|}{1990 model year } \\
\hline & $\begin{array}{c}\text { Scrappage } \\
\text { rate }^{\mathbf{a}}\end{array}$ & $\begin{array}{c}\text { Survival } \\
\text { rate }^{\mathrm{b}}\end{array}$ & $\begin{array}{c}\text { Scrappage } \\
\text { rate }^{\mathrm{a}}\end{array}$ & $\begin{array}{c}\text { Survival } \\
\text { rate }^{\mathrm{b}}\end{array}$ & $\begin{array}{c}\text { Scrappage } \\
\text { rate }^{\mathrm{a}}\end{array}$ & $\begin{array}{c}\text { Survival } \\
\text { rate }^{\mathrm{b}}\end{array}$ \\
\hline 0 & 0.000000 & 1.000000 & 0.000000 & 1.000000 & 0.000000 & 1.000000 \\
\hline 1 & 0.006050 & 0.993950 & 0.005553 & 0.994447 & 0.005255 & 0.994745 \\
\hline 2 & 0.009650 & 0.984359 & 0.007636 & 0.986854 & 0.007538 & 0.987246 \\
\hline 3 & 0.014590 & 0.969997 & 0.011011 & 0.975988 & 0.010522 & 0.976858 \\
\hline 4 & 0.022892 & 0.947792 & 0.013567 & 0.962746 & 0.014414 & 0.962778 \\
\hline 5 & 0.030522 & 0.918864 & 0.020498 & 0.943011 & 0.019623 & 0.943885 \\
\hline 6 & 0.040956 & 0.881231 & 0.034718 & 0.910272 & 0.025096 & 0.920197 \\
\hline 7 & 0.057029 & 0.830975 & 0.047366 & 0.867156 & 0.032690 & 0.890116 \\
\hline 8 & 0.084560 & 0.760708 & 0.055299 & 0.819204 & 0.042014 & 0.852719 \\
\hline 9 & 0.118527 & 0.670543 & 0.071153 & 0.760915 & 0.053468 & 0.807126 \\
\hline 10 & 0.151858 & 0.568716 & 0.092931 & 0.690202 & 0.066230 & 0.753669 \\
\hline 11 & 0.166996 & 0.473743 & 0.117300 & 0.609241 & 0.081338 & 0.692367 \\
\hline 12 & 0.171955 & 0.392280 & 0.158696 & 0.512557 & 0.096959 & 0.625236 \\
\hline 13 & 0.201774 & 0.313128 & 0.187663 & 0.416369 & 0.114297 & 0.553773 \\
\hline 14 & 0.198887 & 0.250851 & 0.208822 & 0.329422 & 0.131169 & 0.481135 \\
\hline 15 & 0.233611 & 0.192250 & 0.228359 & 0.254196 & 0.149005 & 0.409444 \\
\hline 16 & 0.271810 & 0.139994 & 0.238412 & 0.193592 & 0.166710 & 0.341186 \\
\hline 17 & 0.283363 & 0.100325 & 0.250547 & 0.145088 & 0.183826 & 0.278467 \\
\hline 18 & 0.283078 & 0.071925 & 0.261438 & 0.107157 & 0.199477 & 0.222919 \\
\hline 19 & 0.287708 & 0.051232 & 0.270527 & 0.078168 & 0.211449 & 0.175783 \\
\hline 20 & 0.292908 & 0.036226 & 0.277234 & 0.056497 & 0.223461 & 0.136502 \\
\hline $\begin{array}{l}\text { Average } \\
\text { lifetime }\end{array}$ & \multicolumn{2}{|c|}{10.7 years } & \multicolumn{2}{|c|}{12.1 years } & \multicolumn{2}{|c|}{13.7 years } \\
\hline
\end{tabular}

\section{Source:}

Miaou, Shaw-Pin, "Factors Associated with Aggregated Car Scrappage Rate in the United States: 1966-1992," Oak Ridge National Laboratory, Oak Ridge, TN, January 1995.

(Additional resources: http://www-cta.ornl.gov)

\footnotetext{
aThe probability that a 1970/80/90 model year automobile will be retired from use within a given year.
}

'The probability that a 1970/80/90 model year automobile will be in use at the end of a given year. 
Table 6.10

Scrappage and Survival Rates for Trucks

\begin{tabular}{|c|c|c|c|c|c|c|c|c|}
\hline \multirow[b]{3}{*}{$\begin{array}{l}\text { Vehicle age } \\
\text { (years) }\end{array}$} & \multicolumn{6}{|c|}{ All trucks } & \multirow{2}{*}{\multicolumn{2}{|c|}{$\begin{array}{c}\text { Lig h t trucks } \\
(1978-89) "\end{array}$}} \\
\hline & \multicolumn{2}{|c|}{$(1966-73)$} & \multicolumn{2}{|c|}{$(1973-78)$} & \multicolumn{2}{|c|}{$(1978-89)$} & & \\
\hline & $\begin{array}{c}\text { Scrappage } \\
\text { rate }\end{array}$ & $\begin{array}{c}\text { Survival } \\
\text { rate }\end{array}$ & $\begin{array}{c}\text { Scrappage } \\
\text { rate }\end{array}$ & $\begin{array}{c}\text { Survival } \\
\text { rate }\end{array}$ & $\begin{array}{c}\text { Scrappage } \\
\text { rate }\end{array}$ & $\begin{array}{c}\text { Survival } \\
\text { rate }\end{array}$ & $\begin{array}{c}\text { Scrappage } \\
\text { rate }\end{array}$ & $\begin{array}{c}\text { Survival } \\
\text { rate }\end{array}$ \\
\hline 0 & 0.00000 & 1.00000 & 0.00000 & 1.00000 & 0.00000 & 1.00000 & 0.00000 & 1.00000 \\
\hline 1 & 0.00582 & 0.99418 & 0.00505 & 0.99495 & $0.003 \quad 12$ & 0.99688 & 0.00249 & 0.99751 \\
\hline 2 & 0.00814 & 0.98608 & 0.00698 & 0.98801 & 0.00461 & 0.99228 & 0.00383 & 0.99369 \\
\hline 3 & 0.01129 & 0.97495 & 0.00958 & 0.97854 & 0.00676 & 0.98557 & 0.00583 & 0.98790 \\
\hline 4 & 0.01550 & 0.95983 & 0.01306 & 0.96576 & 0.00980 & 0.97591 & 0.00877 & 0.97923 \\
\hline 5 & 0.02101 & 0.93967 & 0.01762 & 0.94873 & 0.01399 & 0.96226 & 0.01296 & 0.96654 \\
\hline 6 & 0.02798 & 0.91337 & 0.02347 & 0.92647 & 0.01957 & 0.94343 & 0.01869 & 0.94848 \\
\hline 7 & 0.03649 & 0.88005 & 0.03073 & 0.89800 & 0.02663 & 0.91830 & 0.02606 & 0.92376 \\
\hline 8 & 0.04638 & 0.83923 & 0.03943 & 0.86260 & 0.03507 & 0.88609 & 0.03488 & 0.89154 \\
\hline 9 & 0.05730 & 0.79114 & 0.04940 & 0.81999 & 0.04445 & 0.84671 & 0.04454 & 0.85182 \\
\hline 10 & 0.06863 & 0.73685 & 0.06026 & 0.77058 & 0.05408 & 0.80092 & 0.05416 & 0.80569 \\
\hline 11 & 0.07970 & 0.67812 & 0.07147 & 0.71551 & 0.06320 & 0.75030 & 0.06285 & 0.75505 \\
\hline 12 & 0.08987 & 0.61718 & 0.08239 & 0.65656 & 0.07121 & 0.69687 & 0.07006 & 0.70215 \\
\hline 13 & 0.09872 & 0.55625 & 0.09247 & 0.59585 & 0.07776 & 0.64268 & 0.07562 & 0.64905 \\
\hline 14 & 0.10605 & 0.49726 & 0.10130 & 0.53548 & 0.08285 & 0.58944 & 0.07967 & 0.59734 \\
\hline 15 & 0.11189 & 0.44162 & 0.10871 & 0.47727 & 0.08662 & 0.53838 & 0.08251 & 0.54805 \\
\hline 16 & 0.11638 & 0.39023 & 0.11468 & 0.42254 & 0.08932 & 0.49029 & 0.08443 & 0.50178 \\
\hline 17 & 0.11976 & 0.34349 & 0.11936 & 0.37210 & 0.09122 & 0.44557 & 0.08571 & 0.45877 \\
\hline 18 & 0.12225 & 0.30150 & 0.12294 & 0.32636 & 0.09253 & 0.40434 & 0.08655 & 0.41907 \\
\hline 19 & 0.12406 & 0.26410 & 0.12562 & 0.28536 & 0.09343 & 0.36656 & 0.08710 & 0.38257 \\
\hline 20 & 0.12536 & 0.23099 & 0.12761 & 0.24894 & 0.09403 & 0.33209 & 0.08745 & 0.34911 \\
\hline 21 & 0.12629 & 0.20182 & 0.12906 & 0.21681 & 0.09444 & 0.30073 & 0.08768 & 0.31850 \\
\hline 22 & 0.12696 & 0.17620 & 0.13012 & 0.18860 & 0.09471 & 0.27225 & 0.08783 & 0.29052 \\
\hline 23 & 0.12743 & 0.15374 & 0.13089 & 0.16392 & 0.09490 & 0.24641 & 0.08793 & 0.26498 \\
\hline 24 & 0.12776 & 0.13410 & 0.13144 & 0.14237 & 0.09502 & 0.22300 & 0.08799 & 0.24166 \\
\hline 25 & 0.12799 & 0.11694 & 0.13183 & 0.12360 & 0.09510 & 0.20179 & 0.08803 & 0.22039 \\
\hline Average lifetime & \multicolumn{2}{|c|}{14.0 years } & \multicolumn{2}{|c|}{14.6 years } & \multicolumn{2}{|c|}{15.8 years } & \multicolumn{2}{|c|}{16.0 years } \\
\hline
\end{tabular}

\section{Source:}

Miaou, Shaw-Pin, "Study of Vehicle Scrappage Rates," Oak Ridge National Laboratory, Oak Ridge, TN, August 1990.

(Additional resources: http://www-cta.ornl.gov)

"Average scrappage and survival rates for all vehicles registered within this time period. 


\section{Chapter 7 \\ Light Vehicles and Characteristics}

Summary Statistics from Tables in this Chapter

\begin{tabular}{|c|c|c|}
\hline \multicolumn{3}{|l|}{ Source } \\
\hline Table 7.1 & Passenger cars, 1997 & \\
\hline & Registrations (thousands) & 129,749 \\
\hline & Vehicle miles (million miles) & $1,501,820$ \\
\hline & Fuel economy (miles per gallon) & 21.5 \\
\hline \multirow[t]{4}{*}{ Table 7.8} & Two-axle, four tire trucks, 1997 & \\
\hline & Registrations (thousands) & 70,224 \\
\hline & Vehicle miles (million miles) & 850,296 \\
\hline & Fuel economy (miles per gallon) & 17.2 \\
\hline \multirow[t]{7}{*}{ Table 7.3} & Automobile sales, 1998 sales period & \\
\hline & Minicompact & 12,159 \\
\hline & Subcompact & $1,491,233$ \\
\hline & Compact & $2,309,330$ \\
\hline & Midsize & $3,106,787$ \\
\hline & Large & $1,050,405$ \\
\hline & Two-seater & 101,023 \\
\hline \multirow[t]{3}{*}{ Table 7.8} & Light truck share of total light vehicle sales & \\
\hline & 1976 & $19.8 \%$ \\
\hline & 1998 & $46.9 \%$ \\
\hline \multirow[t]{7}{*}{ Table 7.12} & Light truck sales, 1998 sales period & \\
\hline & Small pickup & 460,097 \\
\hline & Large pickup & $2,377,916$ \\
\hline & Small van & $1,223,670$ \\
\hline & Large van & 380,829 \\
\hline & Small utility & 762,490 \\
\hline & Large utility & $1,932,648$ \\
\hline \multirow[t]{5}{*}{ Table 7.14} & Corporate average fuel economy & (mpg) \\
\hline & Automobile standard, MY 1998 & 27.5 \\
\hline & Automobile fuel economy, MY 1998 & 28.8 \\
\hline & Light truck standard, MY 1998 & 20.7 \\
\hline & Light truck fuel economy, MY 1998 & 20.8 \\
\hline Table 7.19 & Average fuel economy loss from 55 to $70 \mathrm{mph}$ & $17.1 \%$ \\
\hline
\end{tabular}


The Federal Highway Administration released revised historical data back to 1985 in their "Highway Statistics Summary to 1995" report. As a result, the data in this table have been revised. The data in this table from 1985-on DO NOT include minivans, pickups, or sport utility vehicles.

Table 7.1

Summary Statistics for Passenger Cars, 1970-97

\begin{tabular}{|c|c|c|c|c|}
\hline Year & $\begin{array}{r}\text { Registrations" } \\
\text { (thousands) }\end{array}$ & $\begin{array}{l}\text { Vehicle travel } \\
\text { (million miles) }\end{array}$ & $\begin{array}{c}\text { Fuel use } \\
\text { (million gallons) }\end{array}$ & $\begin{array}{l}\text { Fuel economy } \\
\text { (miles per gallon) }\end{array}$ \\
\hline 1970 & 89,244 & 916,700 & 67,820 & 13.5 \\
\hline 1971 & 92,718 & 966,330 & 71,346 & 13.5 \\
\hline 1972 & 97,082 & $1,021,365$ & 75,937 & 13.5 \\
\hline 1973 & 101,985 & $1,045,981$ & 78,233 & 13.4 \\
\hline 1974 & 104,856 & $1,007,251$ & 74,229 & 13.6 \\
\hline 1975 & 106,706 & $1,033,950$ & 74,140 & 13.9 \\
\hline 1976 & 110,189 & $1,078,215$ & 78,297 & 13.8 \\
\hline 1977 & 112,288 & $1,109,243$ & 79,060 & 14.0 \\
\hline 1978 & 116,573 & $1,146,508$ & 80,652 & 14.2 \\
\hline 1979 & 118,429 & $1,113,640$ & 76,588 & 14.5 \\
\hline 1980 & 121,601 & $1,111,596$ & 69,981 & 15.9 \\
\hline 1981 & 123,098 & $1,133,332$ & 69,112 & 16.4 \\
\hline 1982 & 123,702 & $1,161,713$ & 69,116 & 16.8 \\
\hline 1983 & 126,444 & $1,195,054$ & 70,322 & 17.0 \\
\hline 1984 & 128,158 & $1,227,043$ & 70,663 & 17.4 \\
\hline 1985" & 127,885 & $1,246,798$ & 71,518 & 17.4 \\
\hline 1986 & 130,004 & $1,270,167$ & 73,174 & 17.4 \\
\hline 1987 & 131,482 & $1,315,982$ & 73,308 & 18.0 \\
\hline 1988 & 133,836 & $1,370,271$ & 73,345 & 18.7 \\
\hline 1989 & 134,559 & $1,401,221$ & 73,913 & 19.0 \\
\hline 1990 & 133,700' & $1,408,266$ & 69,568 & 20.2 \\
\hline 1991 & 128,300 & $1,358,185$ & 64,318 & 21.1 \\
\hline 1992 & 126,581 & $1,371,569$ & 65,436 & 21.0 \\
\hline 1993 & 127,327 & $1,374,709$ & 67,047 & 20.5 \\
\hline 1994 & 127,883 & $1,406,089$ & 67,874 & 20.7 \\
\hline 1995 & 128,387 & $1,438,294$ & 68,072 & 21.1 \\
\hline 1996 & 129,728 & $1,469,854$ & 69,221 & 21.2 \\
\hline \multirow[t]{2}{*}{1997} & 129,749 & $1,501,820$ & 69,867 & 21.5 \\
\hline & \multicolumn{4}{|c|}{ Average annual percentage change } \\
\hline 1970-97 & $1.4 \%$ & $1.8 \%$ & $0.1 \%$ & $1.7 \%$ \\
\hline $1987-97$ & $-0.1 \%$ & $1.3 \%$ & $-0.5 \%$ & $1.8 \%$ \\
\hline
\end{tabular}

Source:

U.S. Department of Transportation, Federal Highway Administration, Highway Statistics 1997, Washington, DC, 1998, Table VM-1, p. V-94, and annual.

(Additional resources: http://www.fhwa.dot.gov)

a This number differs from R.L. Polk's estimates of "number of automobiles in use." See Table 6.3.

${ }^{\mathrm{b}}$ Fuel economy for automobile population.

c Beginning in this year the data were revised to exclude minivans, pickups and sport utility vehicles which may have been previously included. 
Table 7.2

New Retail Automobile Sales in the United States, 1970-97

\begin{tabular}{|c|c|c|c|c|c|c|c|}
\hline \multirow[b]{2}{*}{$\begin{array}{c}\text { Calendar } \\
\text { year }\end{array}$} & Domestic $^{a}$ & Import $^{\mathrm{b}}$ & Total & \multirow[b]{2}{*}{$\begin{array}{c}\text { Percentage } \\
\text { imports }\end{array}$} & \multirow{2}{*}{$\begin{array}{l}\text { Percentage } \\
\text { transplants }^{c} \\
\text { on model } \\
\text { year basis }\end{array}$} & \multirow{2}{*}{$\begin{array}{l}\text { Percentage } \\
\text { imports and } \\
\text { transplants }\end{array}$} & \multirow[b]{2}{*}{$\begin{array}{c}\text { Percentage } \\
\text { diesel }\end{array}$} \\
\hline & \multicolumn{2}{|c|}{ (thousands) } & & & & & \\
\hline 1970 & 7,119 & 1,285 & 8,404 & $15.3 \%$ & d & d & $d$ \\
\hline 1971 & 8,681 & 1,568 & 10,249 & $15.3 \%$ & d & d & $0.06 \%$ \\
\hline 1972 & 9,327 & 1,623 & $1 \mathbf{0 , 9 5 0}$ & $14.8 \%$ & d & d & $0.05 \%$ \\
\hline 1973 & 9,676 & 1,763 & 11,439 & $15.4 \%$ & d & d & $0.06 \%$ \\
\hline 1974 & 7,454 & 1,399 & 8,853 & $15.8 \%$ & d & d & $0.20 \%$ \\
\hline 1975 & 7,053 & 1,571 & 8,624 & $18.2 \%$ & d & d & $0.31 \%$ \\
\hline 1976 & 8,611 & 1,499 & 10,110 & $14.8 \%$ & $0.0 \%$ & $14.8 \%$ & $0.22 \%$ \\
\hline 1977 & 9,109 & 2,074 & 11,183 & $18.5 \%$ & $0.0 \%$ & $18.5 \%$ & $0.34 \%$ \\
\hline 1978 & 9,312 & 2,002 & 11,314 & $17.7 \%$ & $0.0 \%$ & $17.7 \%$ & $1.02 \%$ \\
\hline 1979 & 8,341 & 2,332 & 10,673 & $21.8 \%$ & $1.3 \%$ & $23.1 \%$ & $2.54 \%$ \\
\hline 1980 & 6,581 & 2,398 & 8,979 & $26.7 \%$ & $2.1 \%$ & $28.8 \%$ & $4.31 \%$ \\
\hline 1981 & 6,209 & 2,327 & 8,536 & $27.3 \%$ & $1.8 \%$ & $29.1 \%$ & $6.10 \%$ \\
\hline 1982 & 5,759 & 2,223 & 7,982 & $27.9 \%$ & $1.4 \%$ & $29.3 \%$ & $4.44 \%$ \\
\hline 1983 & 6,795 & 2,387 & 9,182 & $26.0 \%$ & $1.3 \%$ & $27.3 \%$ & $2.09 \%$ \\
\hline 1984 & 7,952 & 2,439 & 10,391 & $23.5 \%$ & $2.0 \%$ & $25.5 \%$ & $1.45 \%$ \\
\hline 1985 & 8,205 & 2,838 & 11,043 & $25.7 \%$ & $2.2 \%$ & $27.9 \%$ & $0.82 \%$ \\
\hline 1986 & 8,215 & 3,238 & 11,453 & $28.3 \%$ & $2.8 \%$ & $31.1 \%$ & $0.37 \%$ \\
\hline 1987 & 7,081 & 3,197 & 10,278 & $31.1 \%$ & $5.2 \%$ & $36.3 \%$ & $0.16 \%$ \\
\hline 1988 & 7,526 & 3,099 & 10,626 & $29.2 \%$ & $5.8 \%$ & $35.0 \%$ & $0.02 \%$ \\
\hline 1989 & 7,073 & 2,825 & 9,898 & $28.5 \%$ & $7.3 \%$ & $35.8 \%$ & $0.13 \%$ \\
\hline 1990 & 6,897 & 2,404 & 9,301 & $25.8 \%$ & $11.2 \%$ & $37.0 \%$ & $0.08 \%$ \\
\hline 1991 & 6,137 & 2,038 & 8,175 & $24.9 \%$ & $13.7 \%$ & $38.6 \%$ & $0.10 \%$ \\
\hline 1992 & 6,277 & 1,937 & 8,213 & $23.6 \%$ & $14.1 \%$ & $37.7 \%$ & $0.06 \%$ \\
\hline 1993 & 6,742 & 1,776 & 8,518 & $20.9 \%$ & $14.9 \%$ & $35.8 \%$ & $0.03 \%$ \\
\hline 1994 & 7,255 & 1,735 & 8,990 & $19.3 \%$ & $16.5 \%$ & $35.8 \%$ & $0.04 \%$ \\
\hline 1995 & 7,129 & 1,506 & 8,635 & $17.4 \%$ & $18.9 \%$ & $36.3 \%$ & $0.04 \%$ \\
\hline 1996 & 7,254 & 1,273 & 8,527 & $14.9 \%$ & d & d & $0.10 \%$ \\
\hline \multirow[t]{2}{*}{1997} & 6,917 & 1,355 & 8,272 & $16.4 \%$ & d & d & $0.09 \%$ \\
\hline & \multicolumn{6}{|c|}{ Average annual percentage change } & \\
\hline $1970-97$ & $-0.1 \%$ & $0.2 \%$ & $-0.1 \%$ & & & & \\
\hline $1987-97$ & $-0.2 \%$ & $-8.2 \%$ & $-2.1 \%$ & & & & \\
\hline
\end{tabular}

Source:

Domestic and import data - American Automobile Manufacturers Association, Motor Vehicle Facts and Figures 1998, Detroit, MI, 1998, p. 15, and annual. 1997 data from Economic Indicators, 4th Quarter 1997.

Diesel data - H. A. Stark (ed), Ward's Communications, Inc., Ward's Automotive Yearbook, Detroit, MI, 1998, p. 40, and annual.

Transplant data - Oak Ridge National Laboratory, Light-Duty Vehicle MPG and Market Shares Data System, Oak Ridge, TN, 1996. (Additional resources: http://www.aama.com, http://www.wardsauto.com)

a North American built.

b Does not include import tourist deliveries.

c A transplant is an automobile which was built in the U.S. by a foreign firm. Also included are joint ventures which are built in the U.S.

${ }^{d}$ Data are not available. 
Table 7.3

Period Sales, Market Shares, and Sales-Weighted Fuel Economies

of New Domestic and Import Automobiles, Selected Sales Periods 1976-98"

\begin{tabular}{|c|c|c|c|c|c|c|c|c|c|c|c|c|}
\hline & 1976 & 1980 & 1984 & 1988 & 1990 & 1992 & 1993 & 1994 & 1995 & 1996 & 1997 & $\overline{1998}$ \\
\hline \multicolumn{13}{|l|}{ MINICOMPACT } \\
\hline Total sales, units & - & 428,346 & 41,368 & 84,186 & 76,698 & 107,634 & 84,345 & 57,198 & 44,752 & 34,234 & 39,519 & 12,159 \\
\hline Market share, \% & & 4.7 & 0.4 & 0.8 & 0.8 & 1.3 & 1.0 & 0.6 & 0.5 & 0.4 & 0.5 & 0.2 \\
\hline \multicolumn{13}{|l|}{ SUBCOMPACT } \\
\hline Total sales, units & $2,625,929$ & $3,441,480$ & $2,510,929$ & $1,983,353$ & $2,030,226$ & $2,074,351$ & $1,944,892$ & $2,015,280$ & $1,518,209$ & $1,315,281$ & $1,510,050$ & $1,491,233$ \\
\hline Market share, \% & 27.1 & 37.8 & 24.6 & 19.1 & 22.0 & 25.6 & 23.2 & 22.6 & 17.4 & 15.2 & 18.3 & 18.5 \\
\hline Fuel economy, mpg & 23.5 & 27.3 & 30.5 & 31.7 & 31.3 & 31.8 & 31.9 & 31.3 & 31.7 & 32.1 & 32.6 & 31.3 \\
\hline \multicolumn{13}{|l|}{ COMPACT } \\
\hline Total sales, units & $2,839,603$ & 599,423 & $2,768,056$ & $4,199,638$ & $3,156,481$ & $2,451,498$ & $2,655,378$ & $3,077,203$ & $3,289,735$ & $3,492,957$ & $2,937,064$ & $2,309,330$ \\
\hline Market share, \% & 29.3 & 6.6 & 27.1 & 40.5 & 34.2 & 30.2 & 31.7 & 34.5 & 37.7 & 40.4 & 35.6 & 28.6 \\
\hline Fuel economy, mpg & 17.1 & 22.3 & 30.6 & 29.8 & 28.9 & 28.7 & 29.3 & 29.8 & 30.2 & 30.4 & 30.0 & 30.8 \\
\hline \multicolumn{13}{|l|}{ MIDSIZE } \\
\hline Total sales, units & $1,815,505$ & $3,073,103$ & $3,059,647$ & $2,550,964$ & $2,511,503$ & $2,249,553$ & $2,445,842$ & $2,359,898$ & $2,498,521$ & $2,487,880$ & $2,531,196$ & $3,106,787$ \\
\hline Market share, \% & 18.7 & 33.8 & 30.0 & 24.6 & 27.2 & 27.7 & 29.2 & 26.5 & 28.6 & 28.8 & 30.6 & 38.5 \\
\hline Fuel economy, mpg & 15.3 & 21.3 & 24.1 & 26.9 & 25.9 & 25.8 & 25.7 & 25.6 & 25.9 & 26.4 & 26.3 & 26.9 \\
\hline \multicolumn{13}{|l|}{ LARGE } \\
\hline Total sales, units & $2,206,102$ & $1,336,190$ & $1,502,097$ & $1,368,717$ & $1,279,092$ & $1,140,775$ & $1,186,991$ & $1,339,863$ & $1,320,608$ & $1,259,266$ & $1,162,290$ & $1,050,405$ \\
\hline Market share, \% & 22.8 & 14.7 & 14.7 & 13.2 & 13.9 & 14.1 & 14.2 & 15.0 & 15.1 & 14.6 & 14.1 & 13.0 \\
\hline Fuel economy, mpg & 13.9 & 19.3 & 20.2 & 24.2 & 23.5 & 23.7 & 24.0 & 24.2 & 24.1 & 24.2 & 24.5 & 24.6 \\
\hline \multicolumn{13}{|l|}{ TWO SEATER } \\
\hline Total sales, units & 199,716 & 215,964 & 328,968 & 186,127 & 170,465 & 83,192 & 70,480 & 67,020 & 53,045 & 62,231 & 80,921 & 101,023 \\
\hline Market share, $\%$ & 2.1 & 2.4 & 3.2 & 1.8 & 1.8 & 1.0 & 0.8 & 0.8 & 0.6 & 0.7 & 1.0 & 1.3 \\
\hline Fuel economy, mpg & 20.1 & 21.0 & 26.5 & 27.3 & 28.0 & 25.9 & 24.8 & 23.9 & 24.7 & 25.4 & 26.3 & 25.4 \\
\hline \multicolumn{13}{|l|}{ TOTAL } \\
\hline Total sales, units & $9,686,855$ & $9,094,506$ & $10,21 \quad 1,06$ & $10,372,98$ & $9,224,465$ & $8,107,003$ & $8,387,928$ & $8,916,462$ & $8,724,870$ & $8,651,849$ & $8,261,040$ & $8,070,937$ \\
\hline Market share, $\%$ & 100 & 100 & 100 & 100 & 100 & 100 & 100 & 100 & 100 & 100 & 100 . & 100 \\
\hline Fuel economy, mpg & 17.2 & 23.2 & 26.3 & 28.5 & 27.6 & 27.7 & 27.8 & 27.8 & 28.0 & 28.3 & 28.3 & 28.3 \\
\hline
\end{tabular}

\section{Source:}

Oak Ridge National Laboratory, Light-Duty Vehicle MPG and Market Shares System, Oak Ridge, TN, 1999. (Additional resources: http://www-cta.ornl.gov)

a "Sales period" is October 1 of the current year through September 30 of the next year. These figures represent only those sales that could be matched to corresponding EPA fuel economy values. 
Table 7.4

Sales-Weighted Engine Size of New Domestic and Import Automobiles by Size Class, Sales Periods 1976-98"

( liters ${ }^{\mathrm{b}}$ )

\begin{tabular}{cccccccc}
\hline Sales period" & Minicompact & Subcompact & Compact & Midsize & Large & Two seater & Fleet \\
\hline 1976 & $\mathbf{c}$ & 2.67 & 5.00 & 5.85 & 6.79 & $\mathbf{2 . 8 9}$ & $\mathbf{4 . 8 9}$ \\
1977 & 1.98 & 2.73 & 4.79 & 5.47 & 6.02 & 2.81 & 4.56 \\
1978 & 2.06 & 2.67 & 3.95 & 4.89 & 6.17 & 3.01 & 4.33 \\
1979 & 1.86 & 2.39 & 3.74 & 4.41 & 5.56 & 2.77 & 3.78 \\
1980 & 1.90 & 2.10 & 3.03 & 3.90 & 5.12 & 2.79 & 3.22 \\
1981 & 1.57 & 2.04 & 2.20 & 3.63 & 5.00 & 2.49 & 2.9 \\
1982 & 1.53 & 2.08 & 2.12 & 3.47 & 4.73 & 2.41 & 2.89 \\
1983 & 1.60 & 2.19 & 2.20 & 3.45 & 4.95 & 2.52 & 2.98 \\
1984 & 2.17 & 2.22 & 2.21 & 3.40 & 4.87 & 2.50 & 2.97 \\
1985 & 1.95 & 2.29 & 2.27 & 3.37 & 4.65 & 2.47 & 2.92 \\
1986 & 1.45 & 2.19 & 2.21 & 3.19 & 4.38 & 2.83 & 2.76 \\
1987 & 1.48 & 2.19 & 2.20 & 2.99 & 4.36 & 2.57 & 2.68 \\
1988 & 1.52 & 2.05 & 2.21 & 3.00 & 4.32 & 2.75 & 2.66 \\
1989 & 2.54 & 2.08 & 2.11 & 3.01 & 4.31 & 2.81 & 2.68 \\
1990 & 2.42 & 1.96 & 2.25 & 3.13 & 4.33 & 2.57 & 2.72 \\
1991 & 2.17 & 1.97 & 2.23 & 3.16 & 4.40 & 2.67 & 2.72 \\
1992 & 1.89 & 2.01 & 2.33 & 3.16 & 4.34 & 3.01 & 2.76 \\
1993 & 1.96 & 2.07 & 2.28 & 3.16 & 4.27 & 3.47 & 2.78 \\
1994 & 2.21 & 2.27 & 2.23 & 3.15 & 4.17 & 3.82 & 2.79 \\
1995 & 2.42 & 2.26 & 2.23 & 3.12 & 4.12 & 3.76 & 2.79 \\
1996 & 2.49 & 2.23 & 2.19 & 2.98 & 4.09 & 3.67 & 2.71 \\
1997 & 2.62 & 2.13 & 2.28 & 3.02 & 4.03 & 3.08 & 2.74 \\
1998 & 3.15 & 2.29 & 2.17 & 2.94 & 3.98 & 3.51 & 2.75 \\
$1976-98$ & $2.2 \% \mathbf{d}$ & $-0.7 \%$ & $-3.7 \%$ & $-3.1 \%$ & $-2.4 \%$ & $0.9 \%$ & $-2.6 \%$ \\
$1987-98$ & $7.6 \%$ & $1.1 \%$ & $0.2 \%$ & $-0.2 \%$ & $-0.8 \%$ & $2.5 \%$ & $0.3 \%$ \\
\hline & & & & & & & \\
& & Average annual percentage change & & & \\
195 & & & & & &
\end{tabular}

Source:

Oak Ridge National Laboratory, Light-Duty Vehicle MPG and Market Shares System, Oak Ridge, TN, 1999. (Additional resources: http://www-cta.ornl.gov)

a "Sales period" is October 1 of the current year through September 30 of the next year.

a 1 liter $=61.02$. cubic inches.

b There were no minicompact automobiles sold in 1976.

d Average annual percentage change begins with 1977. 
Table 7.5

Sales-Weighted Curb Weight of New Domestic and Import Automobiles by Size Class, Sales Periods 1976-98”

(pounds)

\begin{tabular}{|c|c|c|c|c|c|c|c|}
\hline $\begin{array}{c}\text { Sales } \\
\text { period" }\end{array}$ & Minicompact & Subcompact & Compact & Midsize & Large & $\begin{array}{l}\text { Two } \\
\text { seater }\end{array}$ & Fleet \\
\hline 1976 & $\mathrm{~b}$ & 2,577 & 3,609 & 4,046 & 4,562 & 2,624 & 3,608 \\
\hline 1977 & 2,228 & 2,586 & 3,550 & 3,900 & 4,026 & 2,608 & 3,424 \\
\hline 1978 & 2,200 & 2,444 & 3,138 & 3,427 & 3,956 & 2,763 & 3,197 \\
\hline 1979 & 2,120 & 2,367 & 3,048 & 3,287 & 3,763 & 2,699 & 3,000 \\
\hline 1980 & 2,154 & 2,270 & 2,813 & 3,081 & 3,667 & 2,790 & 2,790 \\
\hline 1981 & 1,920 & 2,370 & 2,382 & 2,996 & 3,672 & 2,744 & 2,744 \\
\hline 1982 & 2,002 & 2,302 & 2,422 & 2,992 & 3,703 & 2,525 & 2,730 \\
\hline 1983 & 2,072 & 2,334 & 2,441 & 3,027 & 3,779 & 2,663 & 2,788 \\
\hline 1984 & 2,376 & 2,380 & 2,454 & 2,990 & 3,734 & 2,559 & 2,788 \\
\hline 1985 & 2,211 & 2,392 & 2,464 & 2,954 & 3,575 & 2,539 & 2,743 \\
\hline 1986 & 2,120 & 2,415 & 2,432 & 2,857 & 3,451 & 2,575 & 2,675 \\
\hline 1987 & 1,960 & 2,423 & 2,474 & 2,857 & 3,483 & 2,602 & 2,689 \\
\hline 1988 & 1,933 & 2,346 & 2,558 & 2,880 & 3,487 & 2,693 & 2,717 \\
\hline 1989 & 2,576 & 2,357 & 2,517 & 2,985 & 3,496 & 2,735 & 2,760 \\
\hline 1990 & 2,651 & 2,368 & 2,637 & 3,065 & 3,594 & 2,656 & 2,828 \\
\hline 1991 & 2,584 & 2,406 & 2,652 & 3,085 & 3,650 & 2,707 & 2,848 \\
\hline 1992 & 2,395 & 2,444 & 2,674 & 3,131 & 3,670 & 2,770 & 2,879 \\
\hline 1993 & 2,449 & 2,478 & 2,659 & 3,142 & 3,615 & 2,967 & 2,894 \\
\hline 1994 & 2,719 & 2,571 & 2,639 & 3,171 & 3,657 & 3,035 & 2,921 \\
\hline 1995 & 2,831 & 2,552 & 2,647 & 3,179 & 3,648 & 2,947 & 2,937 \\
\hline 1996 & 2,847 & 2,533 & 2,667 & 3,203 & 3,671 & 2,985 & 2,950 \\
\hline 1997 & 2,997 & 2,489 & 2,737 & 3,241 & 3,653 & 2,863 & 2,977 \\
\hline 1998 & 3,004 & 2,584 & 2,703 & 3,198 & 3,675 & 2,956 & 3,002 \\
\hline \multicolumn{8}{|c|}{ Average annual percentage change } \\
\hline 1976-98 & $1.4 \% "$ & $0.0 \%$ & $-1.3 \%$ & $-1.1 \%$ & $-1.0 \%$ & $0.5 \%$ & $-0.8 \%$ \\
\hline $1987-98$ & $4.5 \%$ & $1.0 \%$ & $0.6 \%$ & $1.1 \%$ & $0.5 \%$ & $0.9 \%$ & $1.0 \%$ \\
\hline
\end{tabular}

Source:

Oak Ridge National Laboratory, Light-Duty Vehicle MPG and Market Shares System, Oak Ridge, TN, 1999. (Additional resources: http://www-cta.ornl.gov)

a "Sales period" is October 1 of the current year through September 30 of the next year.

${ }^{\text {a }}$ There were no miuicompact automobiles sold in 1976.

b Average annual percentage change begins with 1977. 
Table 7.6

Sales-Weighted Interior Space of New Domestic and Import Automobiles by Size Class, Sales Periods 1976-98"

(cubic feet)

\begin{tabular}{|c|c|c|c|c|c|c|}
\hline $\begin{array}{c}\text { Sales } \\
\text { period }^{\mathrm{a}}\end{array}$ & $\begin{array}{l}\text { Minicompact } \\
(<85)\end{array}$ & $\begin{array}{l}\text { Subcompact } \\
(85-99)\end{array}$ & $\begin{array}{l}\text { Compact } \\
(100-109)\end{array}$ & $\begin{array}{c}\text { Midsize } \\
(110-119) \\
\end{array}$ & $\begin{array}{c}\text { Large } \\
(>120)\end{array}$ & Fleet $^{\mathrm{b}}$ \\
\hline 1977 & 78.8 & 89.8 & 107.1 & 113.0 & 128.0 & 107.9 \\
\hline 1978 & 79.4 & “ 89.8 & 105.3 & 112.9 & 128.5 & 107.9 \\
\hline 1979 & 80.0 & 90.2 & 105.8 & 113.4 & 130.1 & 106.9 \\
\hline 1980 & 82.4 & 89.9 & 105.4 & 113.5 & 130.8 & 104.9 \\
\hline 1981 & 83.3 & 90.2 & 103.6 & 113.7 & 130.6 & 105.5 \\
\hline 1982 & 83.1 & 91.3 & 102.9 & 113.9 & 130.4 & 106.0 \\
\hline 1983 & 82.7 & 93.3 & 103.0 & 113.1 & 131.3 & 107.3 \\
\hline 1984 & 77.0 & 93.8 & 103.0 & 113.3 & 130.4 & 108.0 \\
\hline 1985 & 77.8 & 94.1 & 103.1 & 113.5 & 129.7 & 107.9 \\
\hline 1986 & 80.1 & 94.5 & 102.8 & 113.8 & 127.6 & 107.0 \\
\hline 1987 & 81.6 & 93.1 & 103.0 & 113.9 & 127.5 & 106.9 \\
\hline 1988 & 81.0 & 93.5 & 103.3 & 113.6 & 127.2 & 107.0 \\
\hline 1989 & 75.0 & 93.3 & 102.7 & 113.8 & 127.4 & 107.5 \\
\hline 1990 & 79.9 & 93.9 & 103.2 & 113.8 & 127.8 & 107.3 \\
\hline 1991 & 79.6 & 94.4 & 103.2 & 113.8 & 128.3 & 107.1 \\
\hline 1992 & 79.1 & 94.0 & 104.2 & 114.0 & 129.2 & 107.5 \\
\hline 1993 & 79.2 & 94.5 & 104.0 & 114.0 & 128.9 & 108.0 \\
\hline 1994 & 79.4 & 94.4 & 103.8 & 113.8 & 128.8 & 108.0 \\
\hline 1995 & 78.5 & 93.8 & 103.9 & 114.3 & 128.1 & 108.7 \\
\hline 1996 & 76.7 & 94.9 & 103.4 & 114.2 & 128.0 & 108.8 \\
\hline 1997 & 77.2 & 95.6 & 103.2 & 114.6 & 128.0 & 108.7 \\
\hline 1998 & 66.9 & 97.0 & 102.2 & 114.4 & 127.7 & 109.2 \\
\hline \multicolumn{7}{|c|}{ Average annual percentage change } \\
\hline 1977-98 & $-0.8 \%$ & $0.4 \%$ & $-0.2 \%$ & $0.1 \%$ & $0.0 \%$ & $0.1 \%$ \\
\hline $1987-98$ & $-1.9 \%$ & $0.4 \%$ & $0.1 \%$ & $0.1 \%$ & $0.0 \%$ & $0.2 \%$ \\
\hline
\end{tabular}

Source:

Oak Ridge National Laboratory, Light-Duty Vehicle MPG and Market Shares System, Oak Ridge, TN, 1999. (Additional resources: http://www-cta.ornl.gov)

a "Sales period" is October 1 of the current year through September 30 of the next year.

b Interior volumes of two-seaters are not reported to EPA. 
Figure 7.1. Engine Size, Curb Weight, and Interior Space of New Domestic and Import Automobiles, 1976-98

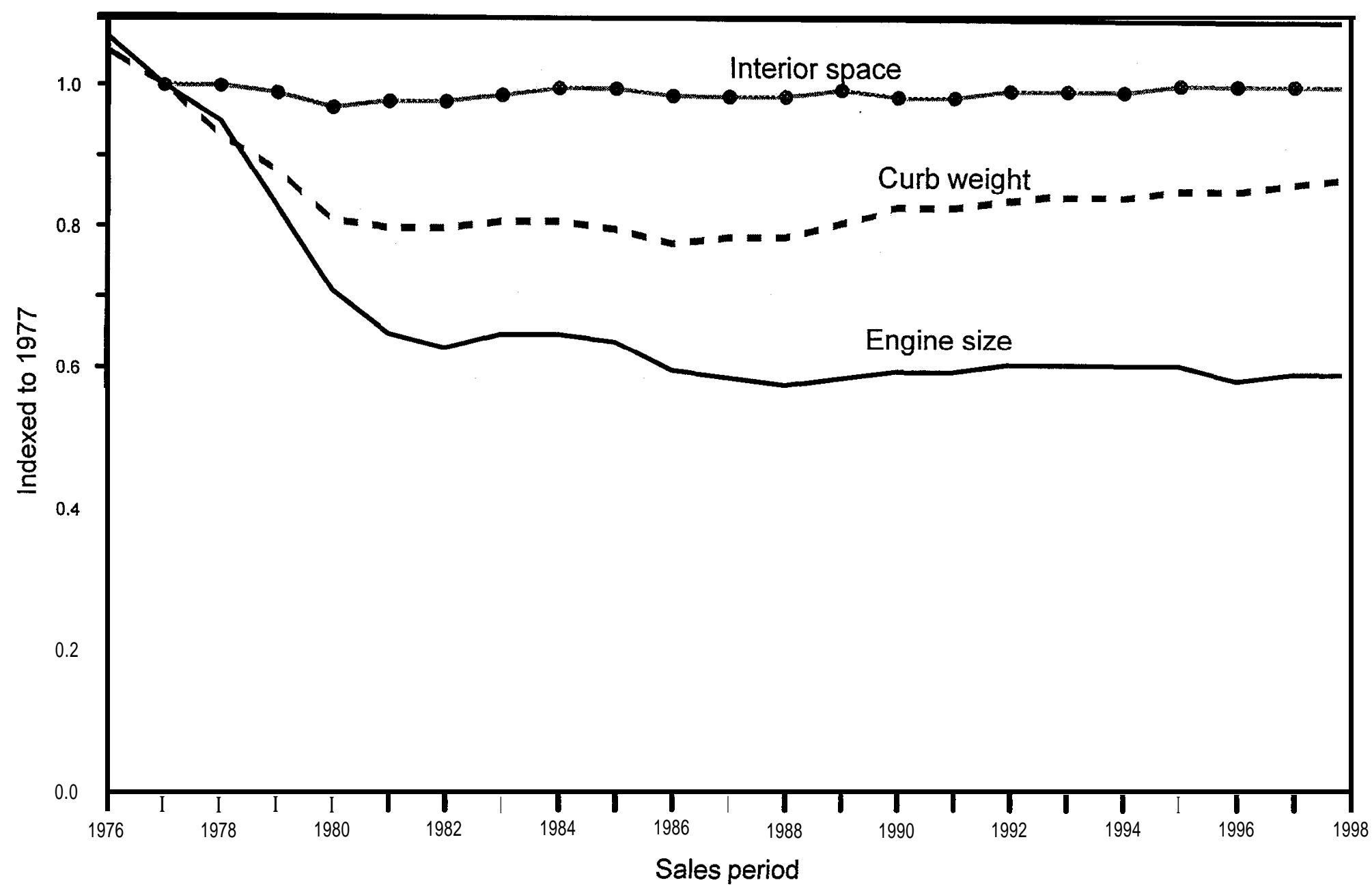

Source: See Tables 7.4, 7.5, and 7.6. 
The average auto lost over 300 pounds from 1978 to 1985, but gained a few pounds back since then. Much of the weight reduction was due to the declining use of conventional steel and iron and the increasing use of aluminum and plastics. Conventional steel, however, remained the predominant component of automobiles in 1998 with a $43 \%$ share of total materials. As conventional steel use has been decreasing, use of high-strength steel has increased.

Table 7.7

Average Material Consumption for a Domestic Automobile, 1978, 1985, and 1998

\begin{tabular}{lrrrrrrr}
\hline & \multicolumn{2}{c}{1978} & \multicolumn{2}{c}{1985} & & \multicolumn{2}{c}{1998} \\
\cline { 2 - 4 } \cline { 8 - 9 } Material & Pounds & Percentage & Pounds & Percentage & & Pounds & Percentage \\
\hline Conventional steel & $1,880.0$ & $53.8 \%$ & $1,481.5$ & $46.5 \%$ & & $1,408.5$ & $43.2 \%$ \\
High-strength steel & 127.5 & $3.6 \%$ & 217.5 & $6.8 \%$ & & 319.0 & $9.8 \%$ \\
Stainless steel & 25.0 & $0.7 \%$ & 29.0 & $0.9 \%$ & & 49.0 & $1.5 \%$ \\
Other steels & 56.0 & $1.6 \%$ & 54.5 & $1.7 \%$ & & 33.5 & $1.0 \%$ \\
Iron & 503.0 & $14.4 \%$ & 468.0 & $14.7 \%$ & & 359.0 & $11.0 \%$ \\
Aluminum & 112.0 & $3.2 \%$ & 138.0 & $4.3 \%$ & & 219.0 & $6.7 \%$ \\
Rubber & 141.5 & $4.1 \%$ & 136.0 & $4.3 \%$ & & 139.5 & $4.3 \%$ \\
Plastics/composites & 176.0 & $5.0 \%$ & 211.5 & $6.6 \%$ & & 243.5 & $7.5 \%$ \\
Glass & 88.0 & $2.5 \%$ & 85.0 & $2.7 \%$ & & 95.0 & $2.9 \%$ \\
Copper & 39.5 & $1.1 \%$ & 44.0 & $1.4 \%$ & & 46.0 & $1.4 \%$ \\
Zinc die castings & 28.0 & $0.8 \%$ & 18.0 & $0.5 \%$ & & 13.5 & $0.4 \%$ \\
Powder metal parts & 16.0 & $0.5 \%$ & 19.0 & $0.6 \%$ & & 32.5 & $1.0 \%$ \\
Fluids \& lubricants & 189.0 & $5.4 \%$ & 184.0 & $5.8 \%$ & & 198.5 & $6.1 \%$ \\
Other materials & 112.5 & $3.2 \%$ & 101.5 & $3.2 \%$ & & 105.0 & $3.2 \%$ \\
& & & & & & $3,261.5$ & $100.0 \%$ \\
Total & $3,494.0$ & $100.0 \%$ & $3,187.5$ & $100.0 \%$ & &
\end{tabular}

Source:

H. A. Stark (ed.), Ward's Communications, Inc., Wards Automotive Yearbook, Detroit, MI, 1998, p. 21, and annual. (Additional resources: http://www.wardsauto.com)

${ }^{a}$ Includes cold-rolled and pre-coated steel. 
Table 7.8

Light Vehicle Market Shares by Size Class, Sales Period 1976-98”,

\begin{tabular}{|c|c|c|c|c|c|c|c|c|c|c|c|c|}
\hline & 1976 & 1977 & 1978 & 1979 & 1980 & 1981 & 1982 & 1983 & 1984 & 1985 & 1986 & 1987 \\
\hline Minicompact & $0.0 \%$ & $6.5 \%$ & $6.7 \%$ & $4.3 \%$ & $3.8 \%$ & $3.9 \%$ & $2.7 \%$ & $2.1 \%$ & $0.3 \%$ & $0.3 \%$ & $1.2 \%$ & $1.0 \%$ \\
\hline Subcompact & $21.7 \%$ & $15.5 \%$ & $15.0 \%$ & $24.4 \%$ & $30.4 \%$ & $31.2 \%$ & $26.6 \%$ & $23.2 \%$ & $18.2 \%$ & $15.7 \%$ & $15.9 \%$ & $13.6 \%$ \\
\hline Compact & $23.5 \%$ & $21.8 \%$ & $12.0 \%$ & $6.7 \%$ & $5.3 \%$ & $5.4 \%$ & $10.8 \%$ & $12.6 \%$ & $20.0 \%$ & $23.2 \%$ & $23.6 \%$ & $27.1 \%$ \\
\hline Midsize & $15.0 \%$ & $15.6 \%$ & $26.1 \%$ & $26.9 \%$ & $27.2 \%$ & $27.9 \%$ & $28.3 \%$ & $24.5 \%$ & $22.1 \%$ & $20.5 \%$ & $19.1 \%$ & $16.9 \%$ \\
\hline Large & $18.3 \%$ & $20.0 \%$ & $17.6 \%$ & $15.4 \%$ & $11.8 \%$ & $12.1 \%$ & $10.1 \%$ & $9.6 \%$ & $10.9 \%$ & $10.0 \%$ & $9.4 \%$ & $9.3 \%$ \\
\hline Two seater & $1.7 \%$ & $1.7 \%$ & $1.5 \%$ & $1.7 \%$ & $1.9 \%$ & $2.0 \%$ & $2.2 \%$ & $2.0 \%$ & $2.4 \%$ & $2.5 \%$ & $1.8 \%$ & $1.6 \%$ \\
\hline Small pickup & $1.4 \%$ & $2.1 \%$ & $2.2 \%$ & $3.3 \%$ & $4.6 \%$ & $4.3 \%$ & $5.3 \%$ & $8.6 \%$ & $7.3 \%$ & $7.5 \%$ & $7.8 \%$ & $7.7 \%$ \\
\hline Large pickup & $13.1 \%$ & $13.2 \%$ & $13.4 \%$ & $12.0 \%$ & $9.9 \%$ & $8.8 \%$ & $9.1 \%$ & $9.3 \%$ & $8.8 \%$ & $9.3 \%$ & $8.5 \%$ & $8.8 \%$ \\
\hline Small van & $0.2 \%$ & $0.2 \%$ & $0.2 \%$ & $0.1 \%$ & $0.1 \%$ & $0.1 \%$ & $0.1 \%$ & $0.1 \%$ & $1.6 \%$ & $2.9 \%$ & $4.1 \%$ & $4.9 \%$ \\
\hline Large van & $4.8 \%$ & $3.2 \%$ & $4.8 \%$ & $4.3 \%$ & $2.9 \%$ & $3.0 \%$ & $3.4 \%$ & $4.7 \%$ & $3.9 \%$ & $3.5 \%$ & $3.3 \%$ & $3.2 \%$ \\
\hline Small utility & $0.0 \%$ & $0.0 \%$ & $0.1 \%$ & $0.4 \%$ & $0.7 \%$ & $0.4 \%$ & $0.3 \%$ & $1.6 \%$ & $2.9 \%$ & $3.2 \%$ & $3.8 \%$ & $4.6 \%$ \\
\hline Large utility & $0.3 \%$ & $0.3 \%$ & $0.3 \%$ & $0.4 \%$ & $1.5 \%$ & $1.1 \%$ & $1.2 \%$ & $1.6 \%$ & $1.6 \%$ & $1.5 \%$ & $1.5 \%$ & $1.4 \%$ \\
\hline Total light vehicles & sold 12,07 & 6513,04 & $014,03^{\prime}$ & 813,589 & $11,311,0$ & $11,029,9$ & $11,012,7$ & $0,345,47$ & $, 823,99$ & $5,203,880$ & 633,934 & 014,173 \\
\hline Cars & $80.2 \%$ & $81.0 \%$ & $79.0 \%$ & $79.4 \%$ & $80.4 \%$ & $82.5 \%$ & $80.6 \%$ & $74.0 \%$ & $73.9 \%$ & $72.1 \%$ & $71.0 \%$ & $69.5 \%$ \\
\hline$\underline{\text { Light trucks }}$ & $19.8 \%$ & $19.0 \%$ & $21.0 \%$ & $20.6 \%$ & $19.6 \%$ & $17.5 \%$ & $19.4 \%$ & $26.0 \%$ & $26.1 \%$ & $27.9 \%$ & $29.0 \%$ & $30.5 \%$ \\
\hline
\end{tabular}

\begin{tabular}{|c|c|c|c|c|c|c|c|c|c|c|c|}
\hline & 1988 & 1989 & 1990 & 1991 & 1992 & 1993 & 1994 & 1995 & 1996 & 1997 & 1998 \\
\hline Minicompact & $0.6 \%$ & $0.1 \%$ & $0.6 \%$ & $0.6 \%$ & $0.9 \%$ & $0.6 \%$ & $0.4 \%$ & $0.3 \%$ & $0.2 \%$ & $0.3 \%$ & $0.1 \%$ \\
\hline Subcompact & $13.1 \%$ & $13.1 \%$ & $14.8 \%$ & $17.5 \%$ & $16.6 \%$ & $14.5 \%$ & $13.8 \%$ & $10.4 \%$ & $8.8 \%$ & $10.2 \%$ & $9.8 \%$ \\
\hline Compact & $27.8 \%$ & $24.7 \%$ & $23.0 \%$ & $19.8 \%$ & $19.6 \%$ & $19.8 \%$ & $21.0 \%$ & $22.4 \%$ & $23.5 \%$ & $19.9 \%$ & $15.2 \%$ \\
\hline Midsize & $16.9 \%$ & $19.7 \%$ & $18.3 \%$ & $18.8 \%$ & $18.0 \%$ & $18.2 \%$ & $16.1 \%$ & $17.0 \%$ & $16.7 \%$ & $17.1 \%$ & $20.4 \%$ \\
\hline Large & $9.1 \%$ & $9.4 \%$ & $9.3 \%$ & $9.4 \%$ & $9.1 \%$ & $8.8 \%$ & $9.2 \%$ & $9.0 \%$ & $8.5 \%$ & $7.9 \%$ & $6.9 \%$ \\
\hline Two seater & $1.2 \%$ & $1.1 \%$ & $1.2 \%$ & $1.1 \%$ & $0.7 \%$ & $0.5 \%$ & $0.5 \%$ & $0.4 \%$ & $0.4 \%$ & $0.5 \%$ & $0.7 \%$ \\
\hline Small pickup & $6.8 \%$ & $5.9 \%$ & $4.9 \%$ & $4.9 \%$ & $4.7 \%$ & $2.5 \%$ & $2.5 \%$ & $2.4 \%$ & $3.9 \%$ & $3.5 \%$ & $3.0 \%$ \\
\hline Large pickup & $9.6 \%$ & $10.6 \%$ & $11.5 \%$ & $11.0 \%$ & $11.6 \%$ & $14.0 \%$ & $15.0 \%$ & $14.9 \%$ & $13.7 \%$ & $13.9 \%$ & $15.6 \%$ \\
\hline Small van & $5.6 \%$ & $5.8 \%$ & $6.8 \%$ & $7.1 \%$ & $7.7 \%$ & $8.4 \%$ & $8.6 \%$ & $8.6 \%$ & $8.3 \%$ & $8.2 \%$ & $8.0 \%$ \\
\hline Large van & $3.2 \%$ & $3.2 \%$ & $2.9 \%$ & $2.5 \%$ & $2.8 \%$ & $2.9 \%$ & $2.8 \%$ & $2.7 \%$ & $2.5 \%$ & $2.6 \%$ & $2.5 \%$ \\
\hline Small utility ${ }^{\mathrm{b}}$ & $4.6 \%$ & $5.0 \%$ & $5.4 \%$ & $6.3 \%$ & $6.9 \%$ & $7.1 \%$ & $7.1 \%$ & $8.4 \%$ & $9.3 \%$ & $11.6 \%$ & $5.0 \%$ \\
\hline Large utility & $1.5 \%$ & $1.5 \%$ & $1.4 \%$ & $1.1 \%$ & $1.3 \%$ & $2.8 \%$ & $3.0 \%$ & $3.5 \%$ & $4.3 \%$ & $4.3 \%$ & $12.7 \%$ \\
\hline \multicolumn{12}{|c|}{ Total light vehicles sold $15,115,985 \_14,939,837$} \\
\hline$\overline{\text { Cars }}$ & $68.6 \%$ & $68.1 \%$ & $67.1 \%$ & $67.1 \%$ & $64.9 \%$ & $62.4 \%$ & $60.9 \%$ & $59.5 \%$ & $58.1 \%$ & $55.9 \%$ & $53.1 \%$ \\
\hline Light trucks & $31.4 \%$ & $31.9 \%$ & $32.9 \%$ & $32.9 \%$ & $35.1 \%$ & $37.6 \%$ & $39.1 \%$ & $40.5 \%$ & $41.9 \%$ & $44.1 \%$ & $46.9 \%$ \\
\hline
\end{tabular}

\section{Source:}

Oak Ridge National Laboratory, Light-Duty Vehicle MPG and Market Shares System, Oak Ridge, TN, 1999. (Additional resources: http://www-cta.ornl.gov)

a "Sales period" is October 1 of the current year through September 30 of the next year.

b The definition of small and large utility changed in 1998 . 
Table 7.9

Sales-Weighted Wheelbase of New Automobiles and Light Trucks, Sales Period 1976-98” (inches)

\begin{tabular}{cccc}
\hline $\begin{array}{c}\text { Sales } \\
\text { period" }\end{array}$ & Automobiles & $\begin{array}{c}\text { Light } \\
\text { trucks }\end{array}$ & $\begin{array}{c}\text { Automobiles and } \\
\text { light trucks } \\
\text { combined }\end{array}$ \\
\hline 1976 & 110.78 & 118.87 & 112.03 \\
1977 & 109.75 & 117.79 & 111.05 \\
1978 & 107.67 & 116.23 & 108.65 \\
1979 & 105.77 & 116.27 & 107.93 \\
1980 & 103.61 & 114.54 & 105.76 \\
1981 & 102.97 & 114.86 & 105.10 \\
1982 & 103.01 & 114.87 & 105.60 \\
1983 & 103.76 & 113.73 & 106.10 \\
1984 & 103.50 & 113.87 & 106.21 \\
1985 & 102.96 & 113.98 & 106.02 \\
1986 & 102.27 & 113.40 & 105.48 \\
1987 & 102.11 & 113.27 & 105.52 \\
1988 & 102.21 & 111.79 & 105.21 \\
1989 & 102.66 & 112.23 & 105.71 \\
1990 & 103.13 & 111.41 & 105.85 \\
1991 & 103.27 & 111.09 & 105.82 \\
1992 & 103.60 & 112.68 & 106.78 \\
1993 & 104.03 & 112.57 & 107.21 \\
1994 & 104.31 & 113.23 & 107.75 \\
1995 & 104.95 & 113.37 & 108.31 \\
1996 & 105.04 & 113.36 & 108.53 \\
1997 & 105.36 & 113.36 & 108.89 \\
1998 & 105.55 & 114.53 & 109.76 \\
$1976-98$ & $-0.2 \%$ & $-0.2 \%$ & $-0.1 \%$ \\
$1988-98$ & $0.3 \%$ & $0.2 \%$ & $0.4 \%$ \\
\hline & & &
\end{tabular}

Source:

Oak Ridge National Laboratory, Light-Duty Vehicle MPG and Market Shares System, Oak Ridge, TN, 1999.

(Additional resources: http://www-cta.ornl.gov)

a "Sales period" is October 1 of the current year through September 30 of the next year. 
The Federal Highway Administrationreleased revised historical databack to 1985 which better reflected two-axle, four-tire trucks: The definition of this category includes vans, pickup trucks, and sport utility vehicles.

Table 7.10

Summary Statistics for Two-Axle, Four-Tire Trucks, 1970-97

\begin{tabular}{|c|c|c|c|c|}
\hline Year & $\begin{array}{c}\text { Registrations } \\
\text { (thousands) }\end{array}$ & $\begin{array}{l}\text { Vehicle travel } \\
\text { (million miles) }\end{array}$ & $\begin{array}{c}\text { Fuel use } \\
\text { (million gallons) }\end{array}$ & $\begin{array}{l}\text { Fuel economy } \\
\text { (miles per gallon) }\end{array}$ \\
\hline 1970 & 14,211 & 123,286 & 12,313 & 10.0 \\
\hline 1971 & 15,181 & 137,870 & 13,484 & 10.2 \\
\hline 1972 & 16,428 & 156,622 & 15,150 & 10.3 \\
\hline 1973 & 18,083 & 176,833 & 16,828 & 10.5 \\
\hline 1974 & 19,335 & 182,757 & 16,657 & 11.0 \\
\hline 1975 & 20,418 & 200,700 & 19,081 & 10.5 \\
\hline 1976 & 22,301 & 225,834 & 20,828 & 10.8 \\
\hline 1977 & 23,624 & 250,591 & 22,383 & 11.2 \\
\hline 1978 & 25,476 & 279,414 & 24,162 & 11.6 \\
\hline 1979 & 27,022 & 291,905 & 24,445 & 11.9 \\
\hline 1980 & 27,876 & 290,935 & 23,796 & 12.2 \\
\hline 1981 & 28,928 & 296,343 & 23,697 & 12.5 \\
\hline 1982 & 29,792 & 306,141 & 22,702 & 13.5 \\
\hline 1983 & 31,214 & 327,643 & 23,945 & 13.7 \\
\hline 1984 & 32,106 & 358,006 & 25,604 & 14.0 \\
\hline $1985 "$ & 37,214 & 390,961 & 27,363 & 14.3 \\
\hline 1986 & 39,382 & 423,915 & 29,074 & 14.6 \\
\hline 1987 & 41,107 & 456,870 & 30,598 & 14.9 \\
\hline 1988 & 43,805 & 502,207 & 32,653 & 15.4 \\
\hline 1989 & 45,945 & 536,475 & 33,271 & 16.1 \\
\hline 1990 & 48,275 & 574,571 & 35,611 & 16.1 \\
\hline 1991 & 53,033 & 649,394 & 38,217 & 17.0 \\
\hline 1992 & 57,091 & 706,863 & 40,929 & 17.3 \\
\hline 1993 & 59,994 & 745,750 & 42,851 & 17.4 \\
\hline 1994 & 62,904 & 764,634 & 44,112 & 17.3 \\
\hline 1995 & 65,738 & 790,029 & 45,605 & 17.3 \\
\hline 1996 & 69,134 & 816,540 & 47,354 & 17.2 \\
\hline 1997 & 70,224 & 850,296 & 49,370 & 17.2 \\
\hline & \multicolumn{4}{|c|}{ Average annual percentage change } \\
\hline $1970-97$ & $6.1 \%$ & $7.4 \%$ & $5.3 \%$ & $2.0 \%$ \\
\hline 1987-97 & $5.5 \%$ & $6.4 \%$ & $5.0 \%$ & $1.4 \%$ \\
\hline
\end{tabular}

Source:

U.S. Department of Transportation, Federal Highway Administration, Highway Statistics 1997, Washington, DC, 1998, Table VM-1, p. V-94, and annual.

(Additional resources: http://www.fhwa.dot.gov)

a Beginning in this year the data were revised to include all vans (including mini-vans), pickups and sport utility vehicles. 
Table 7.11

New Retail Sales of Trucks 10,000 Pounds GVW and Less in the United States, 1970-97

\begin{tabular}{|c|c|c|c|c|c|c|c|}
\hline \multirow[b]{2}{*}{$\begin{array}{c}\text { Calendar } \\
\text { year }\end{array}$} & \multirow[b]{2}{*}{$\begin{array}{l}\text { Light truck } \\
\text { sales" } \\
\text { (thousands) }\end{array}$} & \multicolumn{6}{|c|}{ Percentages } \\
\hline & & Import $^{\mathrm{b}}$ & Transplants" & Diesel $^{d}$ & $\begin{array}{c}\text { Four-wheel drive } \\
\text { of domestic } \\
\text { light trucks }\end{array}$ & $\begin{array}{l}\text { Light trucks of } \\
\text { light-duty } \\
\text { vehicle sales" }\end{array}$ & $\begin{array}{l}\text { Light trucks } \\
\text { of total } \\
\text { truck sales }\end{array}$ \\
\hline 1970 & 1,463 & $4.5 \%$ & $i$ & $\mathrm{~g}$ & $f$ & $14.8 \%$ & $80.4 \%$ \\
\hline 1971 & 1,757 & $4.8 \%$ & $f$ & g & f & $14.6 \%$ & $83.4 \%$ \\
\hline 1972 & 2,239 & $6.4 \%$ & f & $\mathrm{g}$ & f & $17.0 \%$ & $83.3 \%$ \\
\hline 1973 & 2,745 & $8.5 \%$ & $f$ & g & $f$ & $19.4 \%$ & $84.2 \%$ \\
\hline 1974 & 2,338 & $7.5 \%$ & $f$ & g & $18.0 \%$ & $20.9 \%$ & $84.2 \%$ \\
\hline 1975 & 2,281 & $10.0 \%$ & $f$ & $\mathrm{~g}$ & $23.4 \%$ & $20.9 \%$ & $87.9 \%$ \\
\hline 1976 & 2,956 & $8.0 \%$ & $0.0 \%$ & g & $23.8 \%$ & $22.6 \%$ & $89.8 \%$ \\
\hline 1977 & 3,430 & $9.4 \%$ & $0.0 \%$ & g & $24.6 \%$ & $23.5 \%$ & $89.7 \%$ \\
\hline 1978 & 3,808 & $8.8 \%$ & $0.0 \%$ & $1.0 \%$ & $28.5 \%$ & $25.2 \%$ & $89.2 \%$ \\
\hline 1979 & 3,311 & $14.1 \%$ & $0.0 \%$ & $1.2 \%$ & $29.4 \%$ & $23.7 \%$ & $88.7 \%$ \\
\hline 1980 & 2,440 & $19.7 \%$ & $0.9 \%$ & $3.6 \%$ & $20.7 \%$ & $21.4 \%$ & $88.9 \%$ \\
\hline 1981 & 2,189 & $20.3 \%$ & $0.0 \%$ & $3.1 \%$ & $18.6 \%$ & $20.4 \%$ & $89.8 \%$ \\
\hline 1982 & 2,470 & $16.5 \%$ & $0.0 \%$ & $8.5 \%$ & $16.8 \%$ & $23.6 \%$ & $92.8 \%$ \\
\hline 1983 & 2,984 & $15.6 \%$ & $0.0 \%$ & $6.7 \%$ & $28.5 \%$ & $24.5 \%$ & $93.6 \%$ \\
\hline 1984 & 3,863 & $15.7 \%$ & $2.0 \%$ & $4.8 \%$ & $27.0 \%$ & $27.1 \%$ & $93.0 \%$ \\
\hline 1985 & 4,458 & $17.2 \%$ & $2.6 \%$ & $3.8 \%$ & $29.1 \%$ & $28.8 \%$ & $93.6 \%$ \\
\hline 1986 & 4,594 & $20.1 \%$ & $2.3 \%$ & $3.7 \%$ & $27.0 \%$ & $28.6 \%$ & $94.3 \%$ \\
\hline 1987 & 4,610 & $17.9 \%$ & $1.7 \%$ & $2.3 \%$ & $32.0 \%$ & $31.0 \%$ & $93.9 \%$ \\
\hline 1988 & 4,800 & $12.6 \%$ & $2.4 \%$ & $2.3 \%$ & $32.1 \%$ & $31.1 \%$ & $93.2 \%$ \\
\hline 1989 & 4,610 & $10.9 \%$ & $2.6 \%$ & $2.9 \%$ & $31.4 \%$ & $31.8 \%$ & $93.3 \%$ \\
\hline 1990 & 4,548 & $13.2 \%$ & $3.4 \%$ & $3.1 \%$ & $31.6 \%$ & $32.8 \%$ & $93.9 \%$ \\
\hline 1991 & 4,123 & $12.8 \%$ & $4.5 \%$ & $3.2 \%$ & $34.4 \%$ & $33.5 \%$ & $94.5 \%$ \\
\hline 1992 & 4,629 & $8.6 \%$ & $5.5 \%$ & $3.3 \%$ & $31.6 \%$ & $36.0 \%$ & $94.4 \%$ \\
\hline 1993 & 5,351 & $6.8 \%$ & $7.1 \%$ & $3.7 \%$ & $32.6 \%$ & $38.6 \%$ & $94.2 \%$ \\
\hline 1994 & 6,033 & $6.5 \%$ & $8.1 \%$ & $3.9 \%$ & $34.4 \%$ & $40.2 \%$ & $94.0 \%$ \\
\hline 1995 & 6,053 & $6.5 \%$ & $7.5 \%$ & $4.1 \%$ & $39.1 \%$ & $41.2 \%$ & $93.4 \%$ \\
\hline 1996 & 6,519 & $6.6 \%$ & & $3.7 \%$ & $35.7 \%$ & $43.3 \%$ & $94.1 \%$ \\
\hline 1997 & 6,797 & $8.4 \%$ & & $4.8 \%$ & $39.6 \%$ & $46.6 \%$ & $94.1 \%$ \\
\hline \multicolumn{8}{|c|}{ Average annualpercentage change } \\
\hline 1970-97 & $5.9 \%$ & & & & & & \\
\hline $1985-97$ & $4.0 \%$ & & & & & & \\
\hline
\end{tabular}

Source:

Four-wheel drive - 1970-88: H. A. Stark (ed.), Ward's Communications, Inc., Ward's Automotive Yearbook, Detroit, MI, 1989, p. 168, and annual. 1989-97: H. A. Stark (ed.), Ward's Communications, Inc., Ward's Automotive Yearbook, Factory Installation Reports, Detroit, MI, 1998, p. 300, and annual.

Transplants - Oak Ridge National Laboratory, Light-Duty Vehicle MPG and Market Shares System, Oak Ridge, TN, 1996. All other - American Automobile Manufacturers Association, Motor Vehicle Facts and Figures 1998, Detroit, MI, 1998, pp. 8,

15, 24, and annual. (Additional resources: http:/www.aama.com, http://www.wardsauto.com)

${ }^{a}$ Includes all trucks of 10,000 pounds gross vehicle weight and less sold in the U.S.

bxcluding transplants.

c Based on model year data. A transplant is a light truck which was built in the U.S. by a foreign firm. Also included are joint ventures built in the U.S.

${ }^{\mathrm{d}}$ Based on model year factory installations. Column was revised.

e Light-duty vehicles include automobiles and light trucks.

${ }^{f}$ Data are not available.

g Indicates less than 1 percent. 
Table 7.12

Period Sales, Market Shares, and Sales-Weighted Fuel Economies

of New Domestic and Import Light Trucks, Selected Sales Periods 1976-98"

\begin{tabular}{|c|c|c|c|c|c|c|c|c|c|c|c|c|}
\hline & 1976 & 1980 & 1984 & 1988 & 1990 & 1992 & 1993 & 1994 & 1995 & 1996 & 1997 & 1998 \\
\hline \multicolumn{13}{|l|}{ SMALL PICKUP } \\
\hline Total sales, units & 170,351 & 516,412 & $1,012,298$ & $1,026,551$ & 678,488 & 586,752 & 332,470 & 365,322 & 356,856 & $574,930^{\mathrm{b}}$ & 520,834 & 460,097 \\
\hline Market share, $\%$ & 7.1 & 23.3 & 28.0 & 21.6 & 15.0 & 13.4 & 6.6 & 6.4 & 6.0 & 9.2 & 8.0 & 6.4 \\
\hline Fuel economy, mpg & 23.9 & 25.5 & 27.2 & 26.1 & 25.2 & 25.0 & 24.9 & 25.3 & 25.6 & 25.6 & 24.6 & 24.5 \\
\hline \multicolumn{13}{|l|}{ LARGE PICKUP } \\
\hline Total sales, units & $1,586,020$ & $1,115,248$ & $1,218,972$ & $1,453,255$ & $1,573,729$ & $1,452,192$ & $1,877,806$ & $2,199,224$ & $2,183,793$ & $2,042,179$ & $2,051,144$ & $2,377,916$ \\
\hline Market share, \% & 66.4 & 50.3 & 33.7 & 30.6 & 34.9 & 33.1 & 37.1 & 38.4 & 36.8 & 32.7 & 31.4 & 33.3 \\
\hline Fuel economy, mpg & 15.1 & 17 & 17.5 & 18.5 & 18.9 & 18.9 & 19.6 & 20.1 & 19.4 & 18.9 & 19.4 & 19.1 \\
\hline \multicolumn{13}{|l|}{ SMALL VAN } \\
\hline Total sales, units & 18,651 & 13,649 & 222,798 & 851,384 & 932,693 & 968,361 & $1,129,459$ & $1,263,933$ & $1,257,116$ & $1,229,650$ & $1,215,776$ & $1,223,670$ \\
\hline Market share, $\%$ & 0.8 & 0.6 & 6.2 & 18.0 & 20.7 & 22.0 & 22.3 & 22.1 & 21.2 & 19.7 & 18.6 & 17.1 \\
\hline Fuel economy, mpg & 19.5 & 19.6 & 25.0 & 22.9 & 23.1 & 22.5 & 22.9 & 22.1 & 22.8 & 22.8 & 22.9 & 23.3 \\
\hline \multicolumn{13}{|l|}{ LARGE VAN } \\
\hline Total sales, units & 574,745 & 328,065 & 545,595 & 486,981 & 398,877 & 350,013 & 388,435 & 407,737 & 401,056 & 370,126 & 386,563 & 380,829 \\
\hline Market share, $\%$ & 24.1 & 14.8 & 15.1 & 10.3 & 8.8 & 8.0 & 7.7 & 7.1 & 6.8 & 5.9 & 5.9 & 5.3 \\
\hline Fuel economy, mpg & 15.4 & 16.3 & 16.3 & 17.0 & 16.9 & 16.9 & 17.3 & 17.4 & 17.1 & 17.2 & 17.8 & 18.2 \\
\hline \multicolumn{13}{|l|}{ SMALL UTILITYY } \\
\hline Total sales, units & 4,716 & 75,875 & 398,000 & 701,005 & 738,294 & 867,934 & 948,797 & $1,042,584$ & $1,225,131$ & $1,378,696$ & $1,715,259$ & 762,490 \\
\hline Market share, $\%$ & 0.2 & 3.4 & 11.0 & 14.8 & 16.4 & 19.8 & 18.8 & 18.2 & 20.6 & 22.1 & 26.3 & 10.7 \\
\hline Fuel economy, mpg & 15.5 & 16.9 & 23.0 & 22.4 & 21.9 & 20.9 & 21.3 & 20.7 & 20.8 & 21.1 & 19.6 & 22.9 \\
\hline \multicolumn{13}{|l|}{ LARGE UTILITY' } \\
\hline Total sales, units & 32,427 & 167,288 & 215,271 & 223,824 & 192,544 & 167,199 & 378,710 & 445,601 & 509,914 & 641,280 & 637,140 & $1,932,648$ \\
\hline Market share, $\%$ & 1.4 & 7.5 & 6.0 & 4.7 & 4.3 & 3.8 & 7.5 & 7.8 & 8.6 & 10.3 & 9.8 & 27.1 \\
\hline Fuel economy, mpg & 14.7 & 14.6 & 15.7 & 16.2 & 16.1 & 16.9 & 17.5 & 17.8 & 17.4 & 18.2 & 18.2 & 18.9 \\
\hline \multicolumn{13}{|l|}{ TOTAL } \\
\hline Total sales, units & $2,386,910$ & $2,216,537$ & $3,612,934$ & $4,743,000$ & $4,514,625$ & $4,392,451$ & $5,055,677$ & $5,724,401$ & $5,933,866$ & $6,236,861$ & $6,526,716$ & $7,137,650$ \\
\hline Market share, $\%$ & 100.0 & 100.0 & 100.0 & 100.0 & 100.0 & 100.0 & 100.0 & 100.0 & 100.0 & 100.0 & 100.0 & 100.0 \\
\hline Fuel economy, mpg & 15.6 & 18.1 & 20.0 & 20.7 & 20.5 & 20.4 & 20.5 & 20.4 & 20.2 & 20.4 & 20.1 & 20.3 \\
\hline
\end{tabular}

Oak Ridge National Laboratory, Light-Duty Vehicle MPG and Market Shares System, Oak Ridge, TN, 1999. (Additional resources: http://www-cta.ornl.gov)

a "Sales period" is October 1 of the current year through September 30 of the next year These figures represent only those sales that could be matched to corresponding EPA fuel economy values.

${ }^{\mathrm{b}}$ Some four-wheel drive pickups previously classified as large pickups were correctly reclassified as small pickups.

c The definition of small and large utility changed in 1998. 
Table 7.13

Sales-Weighted Engine Size of New Domestic and Import Light Trucks by Size Class Sales Periods 1976-98

(liters" )

\begin{tabular}{|c|c|c|c|c|c|c|c|c|}
\hline $\begin{array}{l}\text { Model } \\
\text { year }\end{array}$ & $\begin{array}{l}\text { Small } \\
\text { pickup }\end{array}$ & $\begin{array}{c}\text { Large } \\
\text { pickup }\end{array}$ & $\begin{array}{c}\text { Small } \\
\text { van }\end{array}$ & $\begin{array}{c}\text { Large } \\
\text { van }\end{array}$ & $\begin{array}{l}\text { Small } \\
\text { utility }\end{array}$ & $\begin{array}{l}\text { Large } \\
\text { utility }\end{array}$ & Fleet & \\
\hline 1976 & 1.91 & 5.57 & 1.97 & 5.39 & 5.39 & 4.97 & 5.23 & \\
\hline 1977 & 2.01 & 5.48 & 1.97 & 5.32 & 5.46 & 4.95 & 5.03 & \\
\hline 1978 & 2.03 & 5.45 & 1.97 & 5.29 & 5.09 & 5.40 & 5.02 & \\
\hline 1979 & 2.05 & 5.15 & 1.97 & 5.13 & 4.52 & 5.30 & 4.62 & \\
\hline 1980 & 2.05 & 5.05 & 1.97 & 5.03 & 4.29 & 5.39 & 4.33 & \\
\hline 1981 & 2.14 & 4.82 & 1.97 & 4.84 & 3.94 & 5.15 & 4.15 & \\
\hline 1982 & 2.34 & 4.99 & 1.79 & 4.92 & 3.88 & 5.27 & 4.24 & \\
\hline 1983 & 2.35 & 4.97 & 1.87 & 5.06 & 3.05 & 5.34 & 4.00 & \\
\hline 1984 & 2.38 & 4.95 & 2.23 & 5.06 & 2.81 & 5.39 & 3.87 & \\
\hline 1985 & 2.38 & 4.77 & 2.65 & 5.12 & 2.83 & 5.37 & 3.77 & \\
\hline 1986 & 2.43 & 4.68 & 2.78 & 5.13 & 2.78 & 5.55 & 3 & 6 \\
\hline 1987 & 2.44 & 4.69 & 2.96 & 5.21 & 2.80 & 5.42 & 3.65 & \\
\hline 1988 & 2.56 & 4.68 & 3.15 & 5.21 & 3.14 & 5.51 & 3.82 & \\
\hline 1989 & 2.64 & 4.70 & 3.11 & 5.22 & 3.50 & 5.45 & 3.93 & \\
\hline 1990 & 2.90 & 4.49 & 3.29 & 5.21 & 3.38 & 5.48 & 3.93 & \\
\hline 1991 & 2.91 & 4.57 & 3.29 & 5.23 & 3.62 & 5.40 & 3.94 & \\
\hline 1992 & 3.07 & 4.57 & 3.32 & 5.28 & 3.69 & 5.47 & 4.00 & \\
\hline 1993 & 3.25 & 4.32 & 3.30 & 5.21 & 3.80 & 5.58 & 4.02 & \\
\hline 1994 & 3.10 & 4.45 & 3.48 & 5.31 & 3.77 & 5.54 & 4.10 & \\
\hline 1995 & 2.95 & 4.44 & 3.40 & 5.15 & 3.75 & 5.49 & 4.06 & \\
\hline 1996 & 2.83 & 4.72 & 3.41 & 5.21 & 3.68 & 5.11 & 4.12 & \\
\hline 1997 & 2.90 & 4.62 & 3.36 & 5.04 & 3.98 & 4.97 & 4.14 & \\
\hline 1998 & 2.84 & 4.64 & 3.37 & 5.02 & 2.83 & 4.74 & 4.16 & \\
\hline \multicolumn{9}{|c|}{ Average annual percentage change } \\
\hline $1976-98$ & $1.8 \%$ & $-0.8 \%$ & $2.5 \%$ & $-0.3 \%$ & $-2.9 \%$ & $-0.2 \%$ & $-1.0 \%$ & \\
\hline $1987-98$ & $1.0 \%$ & $-0.1 \%$ & $0.7 \%$ & $-0.4 \%$ & $-1.0 \%$ & $-1.5 \%$ & $0.9 \%$ & \\
\hline
\end{tabular}

Source:

Oak Ridge National Laboratory, Light-Duty Vehicle MPG and Market Shares System, Oak Ridge, TN, 1999. (Additional resources: http://www-cta.ornl.gov)

\footnotetext{
a 1 liter $=61.02$ cubic inches.
} 
The number of franchised dealerships which sell new light-duty vehicles (cars and light trucks) has declined $26 \%$ since 1970, though new vehicle sales have increased. The average number of vehicles sold per dealer in 1997 was 664 vehicles per dealer - more than double the 1970 number.

Table 7.14

New Light-Duty Vehicle Dealerships and Sales, 1970-97

\begin{tabular}{|c|c|c|c|}
\hline Year & $\begin{array}{c}\text { Number of } \\
\text { franchised new } \\
\text { light-duty vehicle } \\
\text { dealerships" }\end{array}$ & $\begin{array}{l}\text { New } \\
\text { light-duty } \\
\text { vehicle sales } \\
\text { (thousands) } \\
\end{array}$ & $\begin{array}{c}\text { Light-duty } \\
\text { vehicle sales } \\
\text { per dealer }\end{array}$ \\
\hline 1970 & 30,800 & 9,867 & 320 \\
\hline 1971 & 30,300 & 12,006 & 396 \\
\hline 1972 & 30,100 & 13,189 & 438 \\
\hline 1973 & 30,100 & 14,184 & 471 \\
\hline 1974 & 30,000 & 11,191 & 373 \\
\hline 1975 & 29,600 & 10,905 & 368 \\
\hline 1976 & 29,300 & 13,066 & 446 \\
\hline 1977 & 29,100 & 14,613 & 502 \\
\hline 1978 & 29,000 & 15,122 & 521 \\
\hline 1979 & 28,500 & 13,984 & 491 \\
\hline 1980 & 27,900 & 11,419 & 409 \\
\hline 1981 & 26,350 & 10,725 & 407 \\
\hline 1982 & 25,700 & 10,452 & 407 \\
\hline 1983 & 24,725 & 12,166 & 492 \\
\hline 1984 & 24,725 & 14,254 & 577 \\
\hline 1985 & 24,725 & 15,501 & 627 \\
\hline 1986 & 24,825 & 16,047 & 646 \\
\hline 1987 & 25,150 & 14,888 & 592 \\
\hline 1988 & 25,025 & 15,426 & 616 \\
\hline 1989 & 25,000 & 14,508 & 580 \\
\hline 1990 & 24,825 & 13,849 & 558 \\
\hline 1991 & 24,200 & 12,298 & 508 \\
\hline 1992 & 23,500 & 12,842 & 546 \\
\hline 1993 & 22,950 & 13,869 & 604 \\
\hline 1994 & 22,850 & 15,023 & 657 \\
\hline 1995 & 22,800 & 14,688 & 644 \\
\hline 1996 & 22,750 & 15,046 & 661 \\
\hline 1997 & 22,700 & 15,069 & 664 \\
\hline & \multicolumn{3}{|c|}{ Average annual percentage change } \\
\hline 1970-97 & $-1.1 \%$ & $1.6 \%$ & $2.7 \%$ \\
\hline $1987-97$ & $-1.0 \%$ & $0.1 \%$ & $1.2 \%$ \\
\hline
\end{tabular}

Source:

Number of dealers and transaction price - National Automobile Dealers Association, Automotive Executive Magazine, 1998. (Additional resources: http://www.nada.org/nadadata)

Light-duty vehicle sales - See tables 7.2 and 7.9.

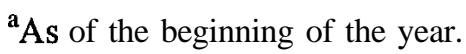


Table 7.15

Corporate Average Fuel Economy (CAFE)

Standards versus Sales-Weighted Fuel Economy Estimates

for Automobiles and Light Trucks, 1978-98"

(miles per gallon)

\begin{tabular}{|c|c|c|c|c|c|c|c|c|}
\hline \multirow{3}{*}{$\begin{array}{c}\text { Model } \\
\text { Year }\end{array}$} & \multicolumn{4}{|c|}{ Automobiles } & \multicolumn{4}{|c|}{ Light Trucks $^{\mathrm{b}}$} \\
\hline & \multirow{2}{*}{$\begin{array}{c}\text { CAFE } \\
\text { Standards }\end{array}$} & \multicolumn{3}{|c|}{ CAFE Estimates $^{c}$} & \multirow{2}{*}{$\begin{array}{c}\text { CAFE } \\
\text { Standards }\end{array}$} & \multicolumn{3}{|c|}{ CAFE Estimates" } \\
\hline & & Domestic & Import & Combined & & Domestic & Import & Combined \\
\hline '1978 & 18.0 & 18.7 & 27.3 & 19.9 & $\bar{d}$ & $\bar{e}$ & $\mathrm{e}$ & e \\
\hline 1979 & 19.0 & 19.3 & 26.1 & 20.3 & d & 17.7 & 20.8 & 18.2 \\
\hline 1980 & 20.0 & 22.6 & 29.6 & 24.3 & d & 16.8 & 24.3 & 18.5 \\
\hline 1981 & 22.0 & 24.2 & 31.5 & 25.9 & d & 18.3 & 27.4 & 20.1 \\
\hline 1982 & 24.0 & 25.0 & 31.1 & 26.6 & 17.5 & 19.2 & 27.0 & 20.5 \\
\hline 1983 & 26.0 & 24.4 & 32.4 & 26.4 & 19.0 & 19.6 & 27.1 & 20.7 \\
\hline 1984 & 27.0 & 25.5 & 32.0 & 26.9 & 20.0 & 19.3 & 26.7 & 20.6 \\
\hline 1985 & 27.5 & 26.3 & 31.5 & 27.6 & 19.5 & 19.6 & 26.5 & 20.7 \\
\hline 1986 & 26.0 & 26.9 & 31.6 & 28.2 & 20.0 & 20.0 & 25.9 & 21.5 \\
\hline 1987 & 26.0 & 27.0 & 31.2 & 28.4 & 20.5 & 20.5 & 25.2 & 21.7 \\
\hline 1988 & 26.0 & 27.4 & 31.5 & 28.0 & 20.5 & 20.6 & 24.6 & 21.3 \\
\hline 1989 & 26.5 & 27.2 & 30.8 & 28.4 & 20.5 & 20.4 & 23.5 & 21.0 \\
\hline 1990 & 27.5 & 26.9 & 29.9 & 27.9 & 20.0 & 20.3 & 23.0 & 20.8 \\
\hline 1991 & 27.5 & 27.3 & 30.1 & 28.4 & 20.2 & 20.9 & 23.0 & 21.3 \\
\hline 1992 & 27.5 & 27.0 & 29.2 & 27.9 & 20.2 & 20.5 & 22.7 & 20.8 \\
\hline 1993 & 27.5 & 27.8 & 29.6 & 28.4 & 20.4 & 20.7 & 22.8 & 21.0 \\
\hline 1994 & 27.5 & 27.5 & 29.7 & 28.3 & 20.5 & 20.5 & 22.0 & 20.8 \\
\hline 1995 & 27.5 & 27.7 & 30.3 & 28.6 & 20.6 & 20.3 & 21.5 & 20.5 \\
\hline 1996 & 27.5 & 28.1 & 29.6 & 28.5 & 20.7 & 20.5 & 22.1 & 20.8 \\
\hline 1997 & 27.5 & 27.8 & 30.1 & 28.7 & 20.7 & 20.1 & 22.1 & 20.5 \\
\hline 1998 & 27.5 & 28.0 & 29.9 & 28.7 & 20.7 & 20.4 & 23.0 & 20.9 \\
\hline 1999 & 27.5 & 28.2 & 28.6 & 28.3 & 20.7 & & $\mathbf{e}$ & 20.8 \\
\hline
\end{tabular}

Source:

U.S. Department of Transportation, NHTSA, "Summary of Fuel Economy Performance," Washington, DC, March 1998. (Additional resources: http://www.nhtsa.dot.gov)

"Only vehicles with at least 75 percent domestic content can be counted in the average domestic fuel economy for a manufacturer.

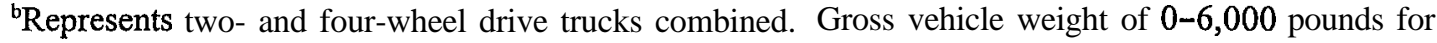
model year 1978-1979 and 0-8,500 pounds for subsequent years.

${ }^{c}$ All CAFE calculations are sales-weighted.

dStandards were set for two-wheel drive and four-wheel drive light trucks separately, but no combined standard was set in this year.

'Data are not available. 
Table 7.16

Corporate Average Fuel Economy (CAFE) Fines Collected, 1983-97 (thousands)

\begin{tabular}{rrr}
\hline $\begin{array}{r}\text { Model } \\
\text { year }\end{array}$ & $\begin{array}{c}\text { Current } \\
\text { dollars }\end{array}$ & $\begin{array}{c}1997 \text { constant } \\
\text { dollars }^{\mathrm{b}}\end{array}$ \\
\hline 1983 & 58 & 93 \\
1984 & 5,958 & 9,235 \\
1985 & 15,565 & 23,192 \\
1986 & 29,872 & 43,613 \\
1987 & 31,261 & 44,078 \\
1988 & 44,519 & 60,546 \\
1989 & 47,381 & 61,121 \\
1990 & 48,449 & 59,592 \\
1991 & 42,243 & 49,847 \\
1992 & 38,287 & 43,647 \\
1993 & 28,688 & 31,844 \\
1994 & 31,478 & 33,996 \\
1995 & 40,788 & 42,827 \\
1996 & 19,302 & 19,688 \\
1997 & 35,991 & 35,991 \\
\hline Total & 404,547 & 559,310 \\
\hline
\end{tabular}

Source:

U.S. Department of Transportation, National Highway Traffic Safety Administration, Office of Vehicle Safety Compliance, Washington, DC, March, 1999.

(Additional resources: http://www.nhtsa.dot.gov)

Table 7.17

Tax Receipts from the Sale of Gas Guzzlers, 1980-97

\begin{tabular}{ccc}
\multicolumn{3}{c}{ (thousands) } \\
\hline $\begin{array}{c}\text { Fiscal } \\
\text { year }\end{array}$ & $\begin{array}{c}\text { Current } \\
\text { dollars }\end{array}$ & $\begin{array}{c}1997 \text { constant } \\
\text { dollars }^{\mathrm{b}}\end{array}$ \\
\hline 1980 & 740 & 1,443 \\
1981 & 780 & 1,381 \\
1982 & 1,720 & 2,855 \\
1983 & 4,020 & 6,472 \\
1984 & 8,820 & 13,671 \\
1985 & 39,790 & 59,287 \\
1986 & 147,660 & 215,584 \\
1987 & 145,900 & 205,719 \\
1988 & 116,780 & 158,821 \\
1989 & 109,640 & 141,436 \\
1990 & 103,200 & 126,936 \\
1991 & 118,400 & 139,712 \\
1992 & 144,200 & 164,388 \\
1993 & 111,600 & 123,876 \\
1994 & 64,100 & 69,228 \\
1995 & 73,500 & 77,175 \\
1996 & 52,600 & 53,652 \\
1997 & 48.200 & 48.200 \\
\hline
\end{tabular}

Source:

Motor Vehicle Manufacturers Association, Motor Vehicle Facts and Figures 1998, Detroit, MI, 1998, p. 84.

(Additional resources: http://www.aama.com)

${ }^{\mathbf{a}}$ These are fines which are actually collected. Fines which are assessed in a certain year may not have been collected in that year.

${ }^{\mathbf{b}}$ Adjusted using the Consumer Price Inflation Index.

c No CAFE fines have been collected for MY 1996. 
Consumers must pay the Gas Guzzler Tax when purchasing an automobile that has an Environmental Protection Agency (EPA)f ue economy rating less than that stipulated in the table below. The Gas Guzzler Tax doubled in 1991 after remaining constant from 1986 to 1990. The tax has not changed since 1991.

Table 7.18

The Gas Guzzler Tax on New Cars (dollars per vehicle)

\begin{tabular}{|c|c|c|c|c|c|c|c|c|}
\hline $\begin{array}{l}\text { Vehicle fuel } \\
\text { economy } \\
\text { (mpg) }\end{array}$ & 1980 & 1981 & 1982 & 1983 & 1984 & 1985 & $1986-90$ & $1991+$ \\
\hline Over 22.5 & 0 & 0 & 0 & 0 & 0 & 0 & 0 & 0 \\
\hline $22.0-22.5$ & 0 & 0 & 0 & 0 & 0 & 0 & 500 & 1,000 \\
\hline $21.5-22.0$ & 0 & 0 & 0 & 0 & 0 & 0 & 500 & 1,000 \\
\hline $21.0-21.5$ & 0 & 0 & 0 & 0 & 0 & 0 & 650 & 1,300 \\
\hline $20.5-21.0$ & 0 & 0 & 0 & 0 & 0 & 500 & 650 & 1,300 \\
\hline $20.0-20.5$ & 0 & 0 & 0 & 0 & 0 & 500 & 850 & 1,700 \\
\hline $19.5-20.0$ & 0 & 0 & 0 & 0 & 0 & 600 & 850 & 1,700 \\
\hline $19.0-19.5$ & 0 & 0 & 0 & 0 & 450 & 600 & 1,050 & 2,100 \\
\hline $18.5-19.0$ & 0 & 0 & 0 & 350 & 450 & 800 & 1,050 & 2,100 \\
\hline $18.0-18.5$ & 0 & 0 & 200 & 350 & 600 & 800 & 1,300 & 2,600 \\
\hline $17.5-18.0$ & 0 & 0 & 200 & 500 & 600 & 1,000 & 1,300 & 2,600 \\
\hline $17.0-17.5$ & 0 & 0 & 350 & 500 & 750 & 1,000 & 1,500 & 3,000 \\
\hline $16.5-17.0$ & 0 & 200 & 350 & 650 & 750 & 1,200 & 1,500 & 3,000 \\
\hline $16.0-16.5$ & 0 & 200 & 450 & 650 & 950 & 1,200 & 1,850 & 3,700 \\
\hline $15.5-16.0$ & 0 & 350 & 450 & 800 & 950 & 1,500 & 1,850 & 3,700 \\
\hline $15.0-15.5$ & 0 & 350 & 600 & 800 & 1,150 & 1,500 & 2,250 & 4,500 \\
\hline $14.5-15.0$ & 200 & 450 & 600 & 1,000 & 1,150 & 1,800 & 2,250 & 4,500 \\
\hline $14.0-14.5$ & 200 & 450 & 750 & 1,000 & 1,450 & 1,800 & 2,700 & 5,400 \\
\hline $13.5-14.0$ & 300 & 550 & 750 & 1,250 & 1,450 & 2,200 & 2,700 & 5,400 \\
\hline $13.0-13.5$ & 300 & 550 & 950 & 1,250 & 1,750 & 2,200 & 3,200 & 6,400 \\
\hline $12.5-13.0$ & 550 & 650 & 950 & 1,550 & 1,750 & 2,650 & 3,200 & 6,400 \\
\hline Under 12.5 & 550 & 650 & 1,200 & 1,550 & 2,150 & 2,650 & 3,850 & 7,700 \\
\hline
\end{tabular}

Source:

Internal Revenue Service, Form 6 197, (Rev. 1-9 1 ), "Gas Guzzler Tax."

(Additional resources: http://www.irs.ustreas.gov) 


\section{New Data by Vehicle Speed}

ORNL has developed fuel consumption and emissions lookup tables for the Federal Highway Administration, for use in their TRAF series of traffic models (NETSIM, CORSIM, FRESIM), although more generic uses are also possible. To develop the data-based models, vehicles are tested both on-road and on a chassis dynamometer. Engine parameters are measured on-road under real-world driving conditions that cover the vehicle's entire operating envelope. Emissions and fuel consumption are then measured on the chassis dynamometer as functions of engine conditions. The two data sets are merged to produce the final three-dimensional maps as functions of vehicle speed and acceleration. Eight well-functioning, late-model vehicles, and one 1997 model vehicle, have been tested thus far in fully warmed-up conditions.

Similar continuing work is planned for the Department of Energy as well as FHWA, which will include more well-functioning, late-model vehicles, pre-control (1960's) vehicles, malfunctioning high-emitter vehicles, light-duty diesel vehicles (cars and pickup trucks), alternative fuel vehicles, and possibly heavy-duty diesel vehicles. ORNL will also be developing cold-start algorithms to enhance the existing models, since emissions and fuel economy generally improve as vehicles warm up to normal operating temperatures.

For further information regarding this study please contact:

Scott Sluder

Fuels, Combustion, and Engine Technology

P.O. Box 2009, Building 9108

Oak Ridge, TN 3783 1-8087
Phone: 423-241-9133

Fax: 423-241-1747

email: sluders@ornl.gov 
Table 7.19

Vehicle Specifications for Tested Vehicles

\begin{tabular}{lcccccc}
\hline & Curb & & Fuel & & \multicolumn{2}{c}{ EPA fuel economy } \\
\cline { 6 - 7 } \multicolumn{1}{c}{ Vehicle } & weight & Engine & $\begin{array}{c}\text { delivery } \\
\text { system" }\end{array}$ & $\begin{array}{c}\text { Trans- } \\
\text { mission }\end{array}$ & City & Highway \\
\hline 1988 Chevrolet Corsica & 2,665 & 2.8 liter V6 & PFI & M5 & 19 & $\mathbf{2 9}$ \\
1994 Olds Cutlass Supreme & 3,290 & 3.4 liter V6 & PFI & L4 & 17 & 26 \\
1994 Oldsmobile 88 & 3,433 & 3.8 literV6 & PFI & L4 & 19 & 29 \\
1994 Mercury Villager & 4,020 & 3.0 liter V6 & PFI & L4 & 17 & 23 \\
1995 Geo Prizm & 2,359 & 1.6 liter I-4 & PFI & L3 & 26 & 30 \\
1994 Jeep Grand Cherokee & 3,820 & 4.0 liter I-6 & PFI & L4 & 15 & 20 \\
1994 Chevrolet Pickup & 4,020 & 5.7 liter V8 & TBI & L4 & 14 & 18 \\
1993 Subaru Legacy & 2,800 & 2.2 liter H4 & PFI & L4 & 22 & 29 \\
1997 Toyota Celica & 2,395 & 1.8 liter 14 & PFI & L4 & 27 & 34 \\
\hline
\end{tabular}

Source:

West, B.H., R.N. McGill, J.W. Hodgson, S.S. Sluder, and D.E. Smith, Development and Verification of LightDuty Modal Emissions and Fuel Consumption Values for Traffic Models, Washington, DC, April 1997 and additional project data, April 1998.

a $\mathrm{PFI}=$ port fuel injection. $\mathrm{TBI}=$ throttle- body fuel injection. 
The two earlier studies by the Federal Highway Administration (FHWA) indicate maximum fuel efficiency was achieved at speeds of 35 to $40 \mathrm{mph}$. The recent FHWA study indicates greater fuel efficiency at higher speeds. Note that the 1973 study did not include light trucks.

Table 7.20

Fuel Economy by Speed, 1973, 1984, and 1997 (miles per gallon)

\begin{tabular}{cccc}
\hline $\begin{array}{c}\text { Speed } \\
\text { (miles per hour })\end{array}$ & $\begin{array}{c}1973 ” \\
(13 \text { vehicles })\end{array}$ & $\begin{array}{c}1984^{\mathrm{b}} \\
\text { vehicles })\end{array}$ & $\begin{array}{c}1997^{\prime} \\
(9 \text { vehicles })\end{array}$ \\
\hline 15 & $\mathrm{~d}$ & 21.1 & 24.4 \\
20 & $\mathrm{~d}$ & 25.5 & 27.9 \\
25 & $\mathrm{~d}$ & 30.0 & 30.5 \\
30 & 21.1 & 31.8 & 31.7 \\
35 & 21.1 & 33.6 & 31.2 \\
40 & 21.1 & 33.6 & 31.0 \\
45 & 20.3 & 33.5 & 31.6 \\
50 & 19.5 & 31.9 & 32.4 \\
55 & 18.5 & 30.3 & 32.4 \\
60 & 17.5 & 27.6 & 31.4 \\
65 & 16.2 & 24.9 & 29.2 \\
70 & 14.9 & 22.5 & 26.8 \\
75 & $\mathrm{~d}$ & 20.0 & 24.8 \\
& & Fuel economy loss & \\
$55-65 \mathrm{mph}$ & $12.4 \%$ & $17.8 \%$ & $9.7 \%$ \\
$65-70 \mathrm{mph}$ & $8.0 \%$ & $9.6 \%$ & $8.2 \%$ \\
$55-70 \mathrm{mph}$ & $19.5 \%$ & $25.7 \%$ & $17.1 \%$ \\
\hline
\end{tabular}

Source:

1973- U.S. Department of Transportation, Federal Highway Administration, Office of Highway Planning, The Effect of Speed on Automobile Gasoline Consumption Rates, Washington, DC, October 1973.

1984 - U.S. Department of Transportation, Federal Highway Administration, Fuel Consumption and Emission Values for Traffic Models, Washington, DC, May 1985.

1997 - West, B.H., R.N. McGill, J.W. Hodgson, S.S. Sluder, and D.E. Smith, Development and Verification of Light-Duty Modal Emissions and Fuel ConsumptionValues for Traffic Models, FHWA Report (in press), Washington, DC, April 1997, and additional project data, April 1998.

(Additional resources: http://www.fkwa-tsis.com)

"Model years 1970 and earlier automobiles.

'Model years 1981-84 automobiles and light trucks.

${ }^{c}$ Model years 1988-97 automobiles and light trucks.

'Data are not available.' 
Figure 7.2. Fuel Economy by Speed, 1973, 1984, and 1997

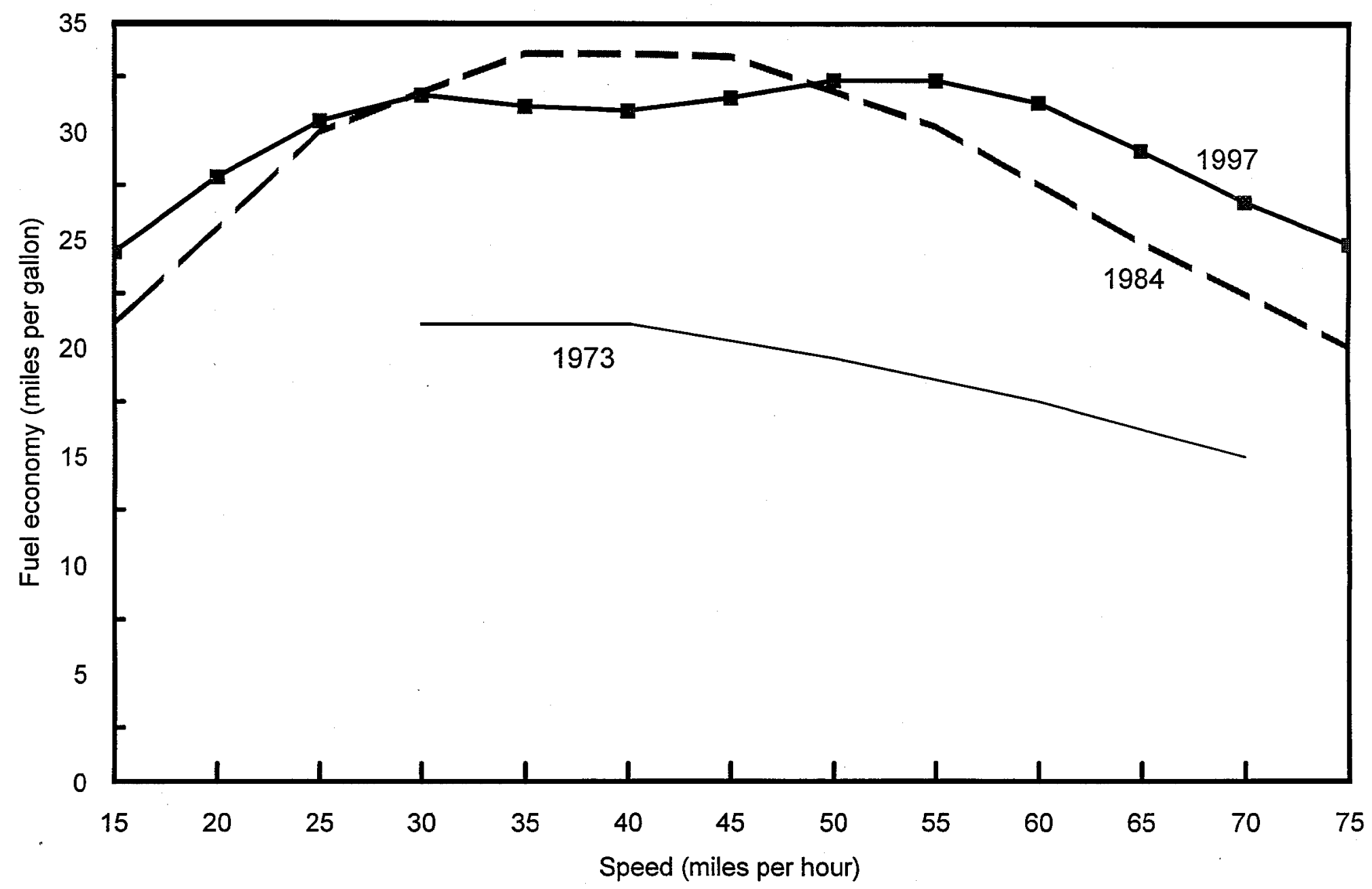

Source: See Table 7.20. 
Table 7.21

Steady Speed Fuel Economy for Tested Vehicles (miles per gallon)

\begin{tabular}{|c|c|c|c|c|c|c|c|c|c|}
\hline $\begin{array}{l}\text { Speed } \\
\text { (mph) }\end{array}$ & $\begin{array}{c}1988 \\
\text { Chevrolet } \\
\text { Corsica }\end{array}$ & $\begin{array}{c}1993 \\
\text { Subaru } \\
\text { Legacy }\end{array}$ & $\begin{array}{c}1994 \\
\text { Oldsmobile } \\
\text { Olds } 88\end{array}$ & $\begin{array}{c}1994 \\
\text { Oldsmobile } \\
\text { Cutlass }\end{array}$ & $\begin{array}{c}1994 \\
\text { Chevrolet } \\
\text { Pickup }\end{array}$ & $\begin{array}{l}1994 \text { Jeep } \\
\text { Grand } \\
\text { Cherokee }\end{array}$ & $\begin{array}{c}1994 \\
\text { Mercury } \\
\text { Villager }\end{array}$ & $\begin{array}{c}1995 \\
\text { Geo } \\
\text { Prizm }\end{array}$ & $\begin{array}{c}1997 \\
\text { Toyota } \\
\text { Celica }\end{array}$ \\
\hline 5 & 10.0 & 14.5 & 10.5 & 5.1 & 7.9 & 8.2 & 12.3 & 18.1 & 19.1 \\
\hline 10 & 16.8 & 24.7 & 14.9 & 7.9 & 18.0 & 11.2 & 19.0 & 23.1 & 34.1 \\
\hline 15 & 17.7 & 31.9 & 22.2 & 11.4 & 16.3 & 17.5 & 22.4 & 38.9 & 41.7 \\
\hline 20 & 21.7 & 34.4 & 26.3 & 12.5 & 19.9 & 24.7 & 25.8 & 39.4 & 46.0 \\
\hline 25 & 23.9 & 37.4 & 28.3 & 15.6 & 22.7 & 21.8 & 30.8 & 41.7 & 52.6 \\
\hline 30 & 28.7 & 39.7 & 29.0 & 19.0 & 26.3 & 21.6 & 30.3 & 40.0 & 50.8 \\
\hline 35 & 28.6 & 38.0 & 30.9 & 21.2 & 24.3 & 25.0 & 26.1 & 39.1 & 47.6 \\
\hline 40 & 29.2 & 37.0 & 33.2 & 23.0 & 26.7 & 25.5 & 29.0 & 38.9 & 36.2 \\
\hline 45 & 28.8 & 33.7 & 32.4 & 23.0 & 27.3 & 25.4 & 27.8 & 42.3 & 44.1 \\
\hline 50 & 31.2 & 33.7 & 34.2 & 27.3 & 26.3 & 24.8 & 30.1 & 39.1 & 44.8 \\
\hline 55 & 29.1 & 37.7 & 34.6 & 29.1 & 25.1 & 24.0 & 31.7 & 37.7 & 42.5 \\
\hline 60 & 28.2 & 35.9 & 32.5 & 28.2 & 22.6 & 23.2 & 27.3 & 36.7 & 48.4 \\
\hline 65 & 28.7 & 33.4 & 30.0 & 25.0 & 21.8 & 21.3 & 25.3 & 34.1 & 43.5 \\
\hline 70 & 26.1 & 31.0 & 26.7 & 22.9 & 20.1 & 20.0 & 23.9 & 31.7 & 39.2 \\
\hline 75 & 23.7 & 28.8 & 24.0 & 21.6 & 18.1 & 19.1 & 22.4 & 28.3 & 36.8 \\
\hline \multicolumn{10}{|c|}{ Fuel economy loss } \\
\hline 55-65 mph & $1.4 \%$ & $11.4 \%$ & $13.3 \%$ & $14.1 \%$ & $13.1 \%$ & $11.3 \%$ & $20.2 \%$ & $9.5 \%$ & $-2.4 \%$ \\
\hline $65-75 \mathrm{mph}$ & $17.4 \%$ & $13.8 \%$ & $20.0 \%$ & $13.6 \%$ & $17.0 \%$ & $10.3 \%$ & $11.5 \%$ & $17.0 \%$ & $15.4 \%$ \\
\hline $55-75 \mathrm{mph}$ & $18.6 \%$ & $23.6 \%$ & $30.6 \%$ & $25.8 \%$ & $27.9 \%$ & $20.4 \%$ & $29.3 \%$ & $24.9 \%$ & $13.4 \%$ \\
\hline
\end{tabular}

\section{Source:}

B.H. West, R.N. McGill, J.W. Hodgson, S.S. Sluder, D.E. Smith, Development and Verification of Light-Duty Modal Emissions and Fuel Consumption Values for Traffic Models, Washington, DC, April 1997, and additional project data, April 1998. (Additional resources: http://www.fhwa-tsis.com)

Note:

For specifications of the tested vehicles, please see page 7-20. 
The Environmental Protection Agency (EPA) tests new vehicles to determine fuel economy ratings. The city and highway fuel economies that are posted on the windows of new vehicles are determined by testing the vehicle during these driving cycles. The driving cycles simulate the performance of an engine while driving in the city and on the highway. Once the urban cycle is completed, the engine is stopped, then started again for the 8.5 minute hot start cycle.

Figure 7.3. Urban Driving Cycle

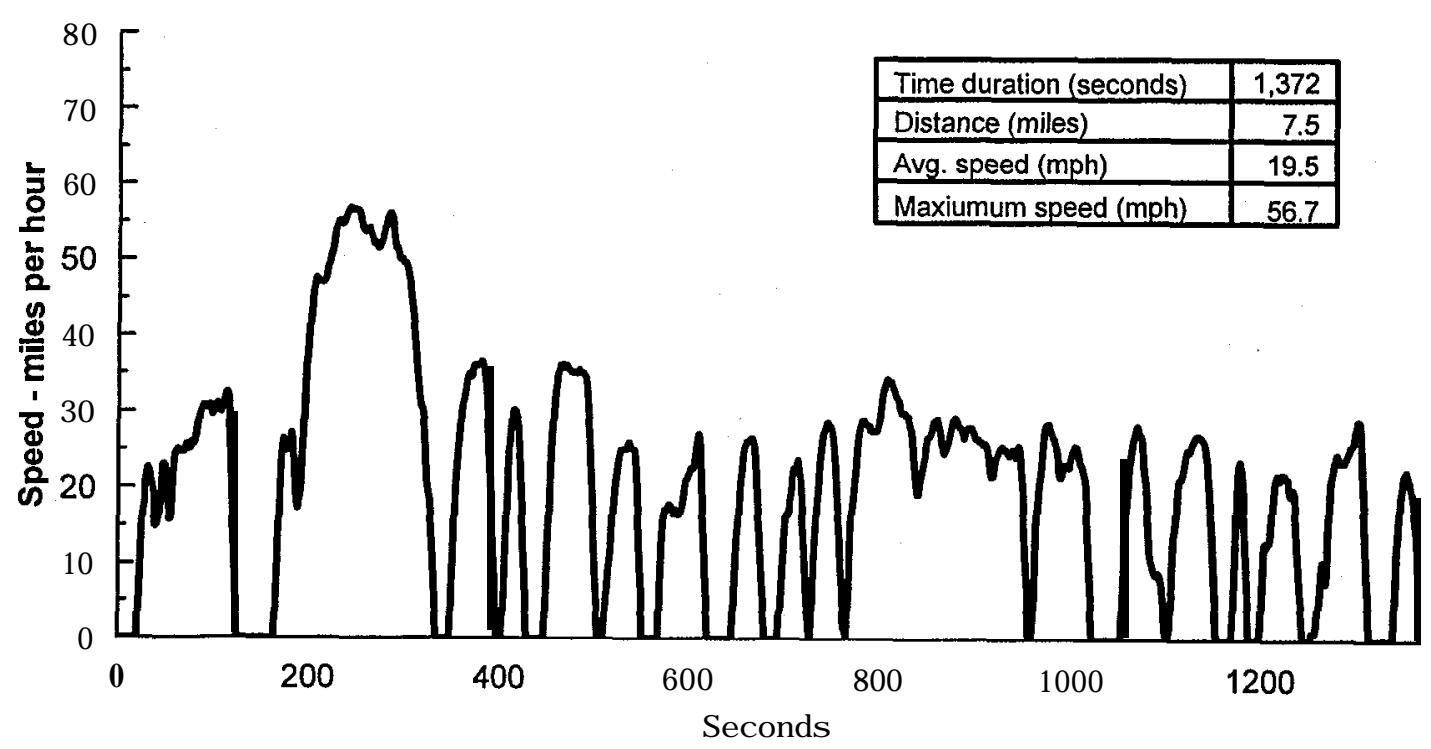

Figure 7.4. Highway Driving Cycle

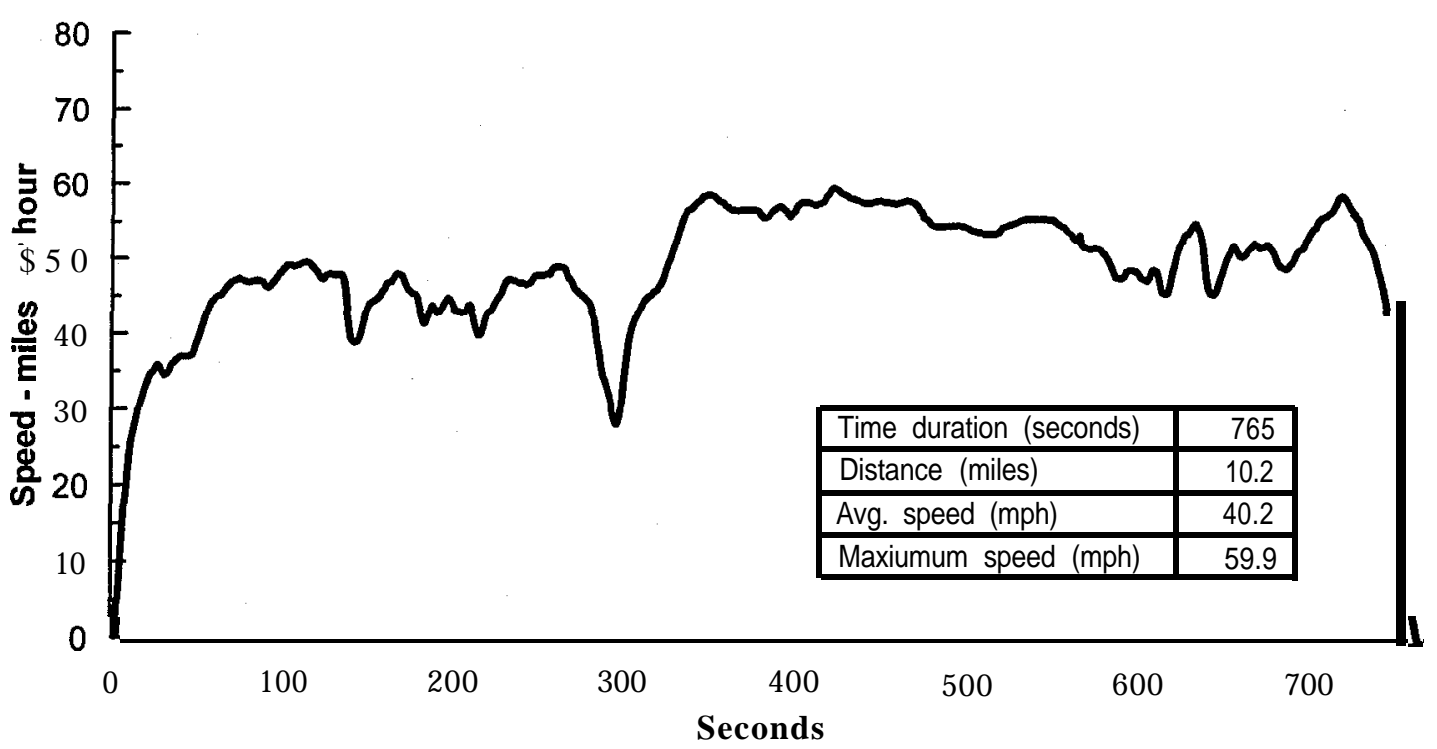

Source:

Code of Federal Regulations, 40CFR, "Subpart B - Fuel Economy Regulations for 1978 and Later Model Year Automobiles - Test Procedures," July 1, 1988 edition, p. 676. 
The New York Test Cycle was developed in the 1970's in order to simulate driving in downtown congested areas. The Representative Number Five Test Cycle was developed recently to better represent actual on-road driving by combining modern urban and freeway driving.

Figure 7.5. New York City Driving Cycle

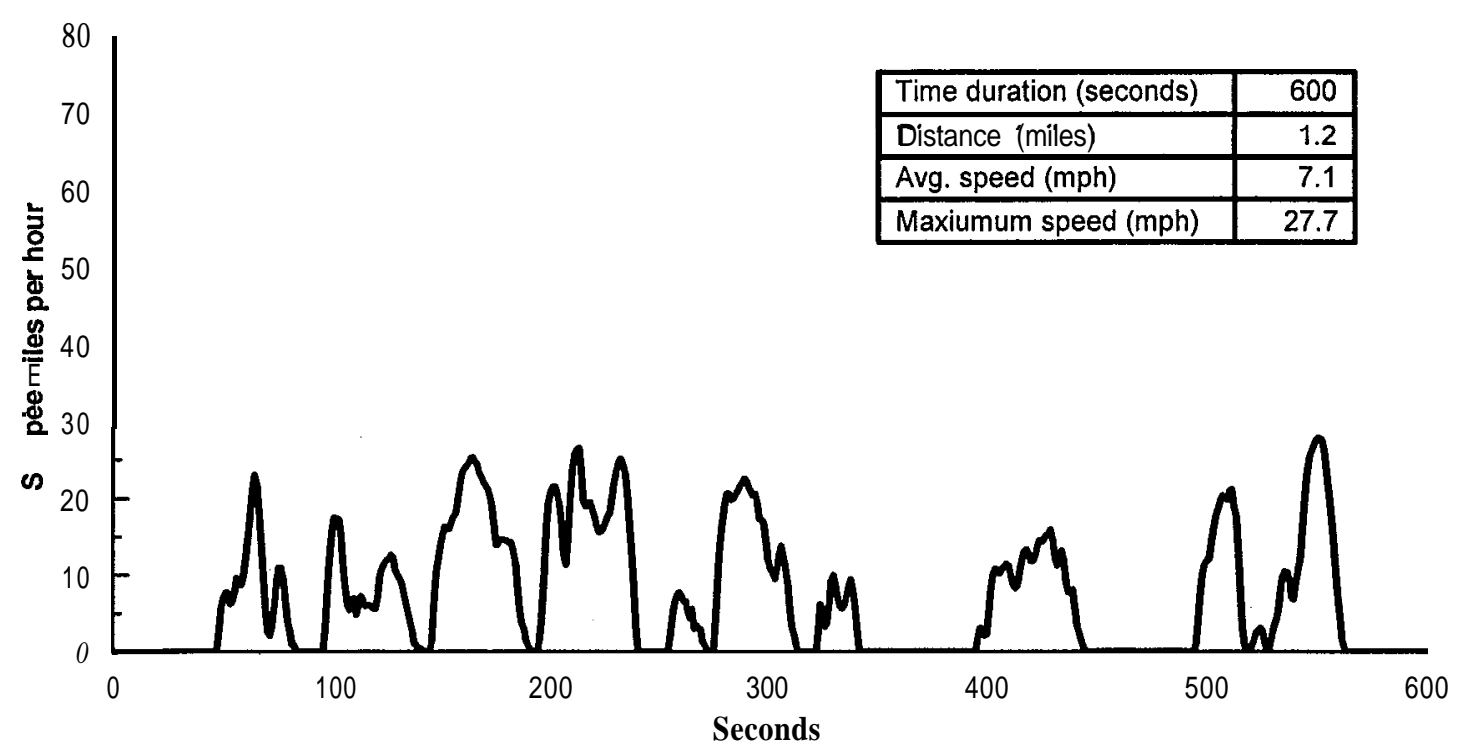

Figure 7.6. Representative Number Five Driving Cycle

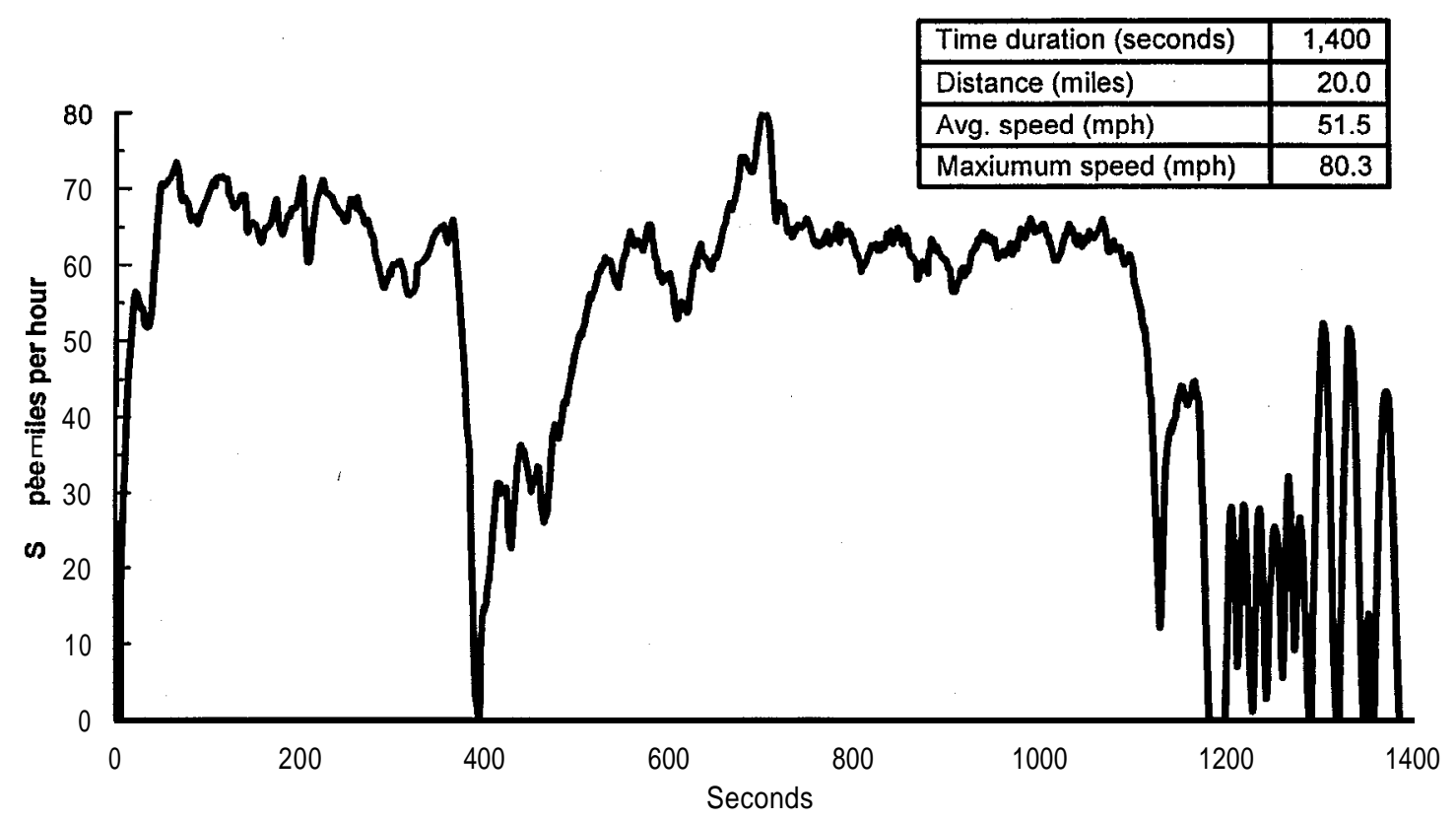

Source:

Data obtained from Michael Wang, Argonne National Laboratory, Argonne, IL, 1997. 
The US06 driving cycle was developed as a supplement to the Federal Test Procedure. It is a short-duration cycle (600 seconds) which represents hard-acceleration driving.

Figure 7.7. US06 Driving Cycle

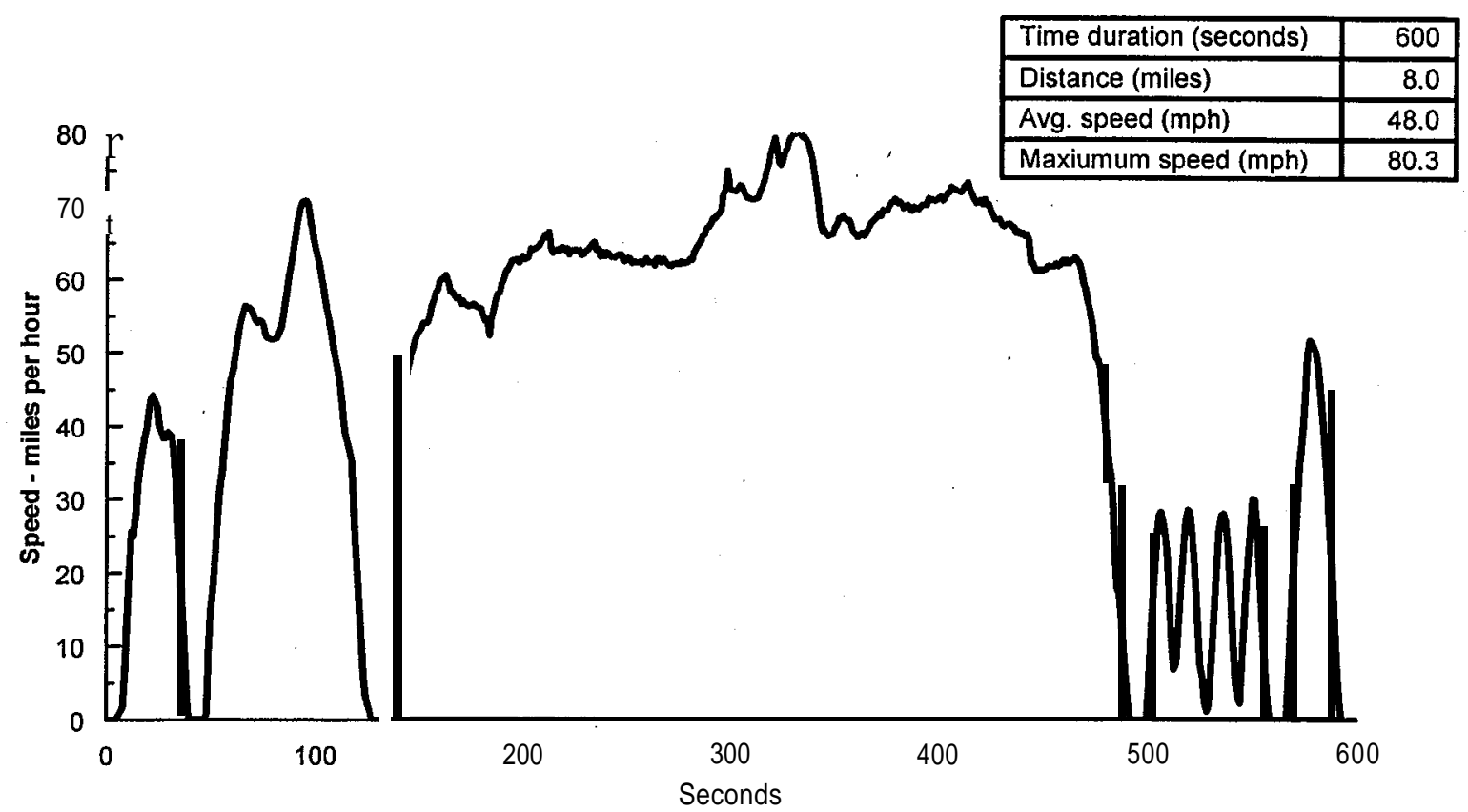

Source:

Data obtained from Michael Wang, Argonne National Laboratory, Argonne, IL, 1997. 


\section{Chapter 8}

\section{Heavy Vehicles and Characteristics}

Summary Statistics from Tables in this Chapter

Source

Table 8.1 Heavy single-unit trucks, 1997

Registration (thousands)

5,293

Vehicle miles (millions)

66,845

Fuel economy (miles per gallon)

Table 8.1 Combination trucks, 1997

Registration (thousands)

Vehicle miles (millions)

124,500

Fuel economy (miles per gallon)

Table 8.3 Trucks by size, 1992 Truck Inventory \& Use Survey

Light $(0-10,000 \mathrm{lbs})$

$93.3 \%$

Medium (10,001-26,000 lbs)

Heavy (26,001 lbs and over)

Table 8.11 Freight Shipments, 1993 Commodity Flow Survey

Value (million dollars)

$6,123,832$

Tons (thousands)

$12,157,105$

Ton-miles (millions)

$3,627,919$

Table 8.13 Bus passenger miles, 1997

(millions)

Transit

20,357

Intercity

29,600

School

82,900 
Table 8.1

Summary Statistics for Other Single-Unit and Combination Trucks, 1970-97”

\begin{tabular}{|c|c|c|c|c|c|c|c|c|}
\hline \multirow[b]{2}{*}{ Year } & \multicolumn{4}{|c|}{ Other single-unit trucks ${ }^{b}$} & \multicolumn{4}{|c|}{ Combination trucks ${ }^{\mathrm{c}}$} \\
\hline & $\begin{array}{c}\text { Registrations } \\
\text { (thousands) }\end{array}$ & $\begin{array}{l}\text { Vehicle travel } \\
\text { (million miles) }\end{array}$ & $\begin{array}{c}\text { Fuel use } \\
\text { (million gallons) }\end{array}$ & $\begin{array}{c}\text { Fuel economy } \\
\text { (miles per gallon) }\end{array}$ & $\begin{array}{c}\text { Registrations } \\
\text { (thousands) }\end{array}$ & $\begin{array}{l}\text { Vehicle travel } \\
\text { (million miles) }\end{array}$ & $\begin{array}{c}\text { Fuel use } \\
\text { (million gallons) }\end{array}$ & $\begin{array}{c}\text { Fuel economy } \\
\text { (miles per gallon) }\end{array}$ \\
\hline 1970 & 3,681 & 27,081 & 3,968 & 6.8 & 905 & 35,134 & 7,348 & 4.8 \\
\hline 1971 & 3,770 & 28,985 & 4,217 & 6.9 & 919 & 37,217 & 7,595 & 4.9 \\
\hline 1972 & 3,918 & 31,414 & 4,844 & 6.5 & 961 & 40,706 & 8,120 & 5.0 \\
\hline 1973 & 4,131 & 33,661 & 5,294 & 6.4 & 1,029 & 45,649 & 9,026 & 5.1 \\
\hline 1974 & 4,211 & 33,441 & 5,261 & 6.4 & 1,085 & 45,966 & 9,080 & 5.1 \\
\hline 1975 & 4,232 & 34,606 & 5,420 & 6.4 & 1,131 & 46,724 & 9,177 & 5.1 \\
\hline 1976 & 4,350 & 36,390 & 5,706 & 6.4 & 1,225 & 49,680 & 9,703 & 5.1 \\
\hline 1977 & 4,450 & 39,339 & 6,268 & 6.3 & 1,240 & 55,682 & 10,814 & 5.1 \\
\hline 1978 & 4,518 & 42,747 & 6,955 & 6.1 & 1,342 & 62,992 & 12,165 & 5.2 \\
\hline 1979 & 4,505 & 42,012 & 7,050 & 6.0 & 1,386 & 66,992 & 12,864 & 5.2 \\
\hline 1980 & 4,374 & 39,813 & 6,923 & 5.8 & 1,417 & 68,678 & 13,037 & 5.3 \\
\hline 1981 & 4,455 & 39,568 & 6,867 & 5.8 & 1,261 & 69,134 & 13,509 & 5.1 \\
\hline 1982 & 4,325 & 40,658 & 6,803 & 6.0 & 1,265 & 70,765 & 13,583 & 5.2 \\
\hline 1983 & 4,204 & 42,546 & 6,965 & 6.1 & 1,304 & 73,586 & 13,796 & 5.3 \\
\hline 1984 & 4,061 & 44,419 & 7,240 & 6.1 & 1,340 & 77,377 & 14,188 & 5.5 \\
\hline 1985 & 4,593 & 45,441 & 7,399 & 6.1 & 1,403 & 78,063 & 14,005 & 5.6 \\
\hline 1986 & 4,313 & 45,637 & 7,386 & 6.2 & 1,408 & 81,038 & 14,475 & 5.6 \\
\hline 1987 & 4,188 & 48,022 & 7,523 & 6.4 & 1,530 & 85,495 & 14,990 & 5.7 \\
\hline 1988 & 4,470 & 49,434 & 7,701 & 6.4 & 1,667 & 88,551 & 15,224 & 5.8 \\
\hline 1989 & 4,519 & 50,870 & 7,779 & 6.5 & 1,707 & 91,879 & 15,733 & 5.8 \\
\hline 1990 & 4,487 & 51,901 & 8,357 & 6.2 & 1,709 & 94,341 & 16,133 & 5.8 \\
\hline 1991 & 4,481 & 52,898 & 8,172 & 6.5 & 1,691 & 96,645 & 16,809 & 5.7 \\
\hline 1992 & 4,370 & 53,874 & 8,237 & 6.5 & 1,675 & 99,510 & 17,216 & 5.8 \\
\hline 1993 & 4,408 & 56,772 & 8,488 & 6.7 & 1,680 & 103,116 & 17,748 & 5.8 \\
\hline 1994 & 4,906 & 61,284 & 9,032 & 6.8 & 1,681 & 108,932 & 18,653 & 5.8 \\
\hline 1995 & 5,024 & 62,705 & 9,216 & 6.8 & 1,696 & 115,451 & 19,777 & 5.8 \\
\hline 1996 & 5,266 & 64,072 & 9,409 & 6.8 & 1,747 & 118,899 & 20,192 & 5.9 \\
\hline 1997 & 5,293 & 66,845 & 9,573 & 7.0 & 1,790 & 124,500 & 20,294 & 6.1 \\
\hline \multicolumn{9}{|c|}{ Average annualpercentage change } \\
\hline 1970-97 & $1.4 \%$ & $3.4 \%$ & $3.3 \%$ & $0.1 \%$ & $2.6 \%$ & $4.8 \%$ & $3.8 \%$ & $0.9 \%$ \\
\hline $1987-97$ & $2.4 \%$ & $3.4 \%$ & $2.4 \%$ & $0.9 \%$ & $1.6 \%$ & $3.8 \%$ & $3.1 \%$ & $0.7 \%$ \\
\hline
\end{tabular}

Source:

U. S. Department of Transportation, Federal Highway Administration, Highway Statistics 1997, Washington, DC, 1998, Table VM1 and annual. (Additional resources: http://www.fhwa.dot.gov)

${ }^{\mathbf{a}}$ The Federal Highway Administration changed the combination truck travel methodology in 1993.

${ }^{b}$ Other single-unit trucks are defined as all single-unit trucks with more than two axles or more than four tires.

${ }^{\mathbf{c}}$ The fuel economy for combination trucks is not the same as the fuel economy for Class 8 trucks. Fuel economy for Class 8 trucks is shown in Table 8.5 . 
Table 8.2

New Retail Truck Sales by Gross Vehicle Weight, 1970-97"

\begin{tabular}{|c|c|c|c|c|c|c|c|c|c|}
\hline Calendar year & $\begin{array}{c}\text { Class } 1 \\
6,000 \text { Ibs. } \\
\text { or less } \\
\end{array}$ & $\begin{array}{c}\text { Class } 2 \\
6,001- \\
10,000 \mathrm{lbs} .\end{array}$ & $\begin{array}{c}\text { Class } 3 \\
10,001- \\
14,000 \mathrm{lbs} . \\
\end{array}$ & $\begin{array}{c}\text { Class } 4 \\
14,001- \\
16,000 \mathrm{lbs} .\end{array}$ & $\begin{array}{c}\text { Class } 5 \\
16,001- \\
19.500 \mathrm{lbs}\end{array}$ & $\begin{array}{c}\text { Class } 6 \\
19,501- \\
26.000 \mathrm{lbs} \\
\end{array}$ & $\begin{array}{r}\text { Class } 7 \\
26,001- \\
33,000 \mathrm{lbs} \\
\end{array}$ & $\begin{array}{c}\text { Class } 8 \\
33,001 \text { lbs. } \\
\text { and over }\end{array}$ & Total \\
\hline \multicolumn{10}{|c|}{ Domestic sales (import data are not available) } \\
\hline $1970^{b}$ & 1,049 & 408 & 6 & 12 & 58 & 133 & 36 & 89 & 1,791 \\
\hline 1971 & 1,185 & 488 & 6 & 15 & 46 & 140 & 34 & 99 & 2,013 \\
\hline 1972 & 1,498 & 599 & 55 & 11 & 29 & 182 & 35 & 126 & 2,535 \\
\hline 1973 & 1,754 & 758 & 50 & 3 & 16 & 236 & 37 & 155 & 3,009 \\
\hline 1974 & 1,467 & 696 & 21 & 3 & 14 & 207 & 31 & 148 & 2,587 \\
\hline $\begin{array}{l}1975 \\
1976\end{array}$ & $\begin{array}{l}1,101 \\
1,318\end{array}$ & $\begin{array}{r}952 \\
1,401\end{array}$ & $\begin{array}{l}23 \\
43\end{array}$ & $\begin{array}{l}1 \\
\mathbf{c}\end{array}$ & $\begin{array}{l}9 \\
9\end{array}$ & $\begin{array}{l}159 \\
153\end{array}$ & $\begin{array}{l}23 \\
22\end{array}$ & $\begin{array}{l}83 \\
97\end{array}$ & $\begin{array}{l}2,351 \\
3,043\end{array}$ \\
\hline 1977 & 1,306 & 1,803 & 36 & 3 & 5 & 163 & 28 & 141 & 3,485 \\
\hline 1978 & 1,334 & 2,140 & 73 & 6 & 3 & 156 & 41 & 162 & 3,915 \\
\hline 1979 & 1,271 & 1,574 & 15 & 3 & 3 & 146 & 50 & 174 & 3,236 \\
\hline 1980 & 985 & 975 & 4 & c & 2 & 90 & 58 & 117 & 2,231 \\
\hline 1981 & 896 & 850 & 1 & c & 2 & 72 & 51 & 100 & 1,972 \\
\hline 1982 & 1,102 & 961 & 1 & c & 1 & 44 & 62 & 76 & 2,248 \\
\hline 1983 & 1,314 & 1,207 & c & c & 1 & 47 & 59 & 82 & 2,710 \\
\hline 1984 & 2,031 & 1,224 & 6 & c & 5 & 55 & 78 & 138 & 3,538 \\
\hline 1985 & 2,408 & $1 ; 280$ & 11 & c & 5 & 48 & 97 & 134 & 3,983 \\
\hline \multicolumn{10}{|c|}{ Domestic and import sales } \\
\hline 1986 & 3,380 & 1,214 & 12 & c & 6 & 45 & 101 & 113 & 4,870 \\
\hline 1987 & 3,435 & 1,175 & 14 & 2 & 8 & 44 & 103 & 131 & 4,912 \\
\hline 1988 & 3,467 & 1,333 & 14 & 21 & 8 & 54 & 103 & 148 & 5,149 \\
\hline 1989 & 3,313 & 1,297 & 19 & 27 & 7 & 39 & 93 & 145 & 4,942 \\
\hline 1990 & 3,451 & 1,097 & 21 & 27 & 5 & 38 & 85 & 121 & 4,846 \\
\hline 1991 & 3,246 & 876 & 21 & 24 & 3 & 22 & 73 & 99 & 4,365 \\
\hline 1992 & 3,608 & 1,021 & 26 & 26 & 4 & 28 & 73 & 119 & 4,903 \\
\hline 1993 & 4,119 & 1,232 & 27 & 33 & 4 & 27 & 81 & 158 & 5,681 \\
\hline 1994 & 4,527 & 1,506 & 35 & 44 & 4 & 20 & 98 & 186 & 6,421 \\
\hline 1995 & 4,422 & 1,631 & 40 & 53 & 4 & 23 & 106 & 201 & 6,481 \\
\hline 1996 & 4,829 & 1,690 & 52 & 59 & 7 & 19 & 104 & 170 & 6,930 \\
\hline 1997 & 5,085 & 1,712 & 53 & 57 & 9 & 18 & 114 & 178 & 7,226 \\
\hline \multicolumn{10}{|c|}{ Average annualpercentage change } \\
\hline $1970-85$ & $5.7 \%$ & $7.9 \%$ & $4.1 \%$ & & $-15.1 \%$ & $-6.6 \%$ & $6.8 \%$ & $2.8 \%$ & $5.5 \%$ \\
\hline $1986-97$ & $3.8 \%$ & $3.2 \%$ & $14.5 \%$ & & $3.8 \%$ & $-8.0 \%$ & $1.1 \%$ & $4.2 \%$ & $3.7 \%$ \\
\hline
\end{tabular}

American Automobile Manufacturers Association, Motor Vehicle Facts and Figures 1998, Detroit, MI, 1998, p. 24, and annual. (Additional resources: http://www.aama.com)

\footnotetext{
${ }^{a}$ Sales include domestic-sponsored imports.

b Data for 1970 is based on new truck registrations.

${ }^{c}$ Less than 500 trucks.
} 
There are many different definitions of light trucks used in the transportation industry. When using gross vehicle weight $(G V W)$ to define light trucks, some data sources use cutoffs of 8,500 lbs. GVW and others use 10,000 lbs. $G V W$. The sales data for Class $2 a$ and $2 b$ trucks are estimated by using data from different sources.

Table 8.3

New Retail Sales of Light Trucks by Gross Vehicle Weight, 1970-97

\begin{tabular}{|c|c|c|c|c|c|c|c|}
\hline & $\begin{array}{c}\text { Class } 1 \\
\text { Imports }\end{array}$ & $\begin{array}{c}\text { Class 1 } \\
\text { Domestic }\end{array}$ & $\begin{array}{c}\text { Total } \\
\text { Class } 1\end{array}$ & Class 2a & Class 2b & $\begin{array}{c}\text { Total } \\
\text { Class } 2 \\
\end{array}$ & Total \\
\hline Year & & $\begin{array}{c}6,000 \text { lbs. } \\
\text { or less } \\
\end{array}$ & & $\begin{array}{c}6,000- \\
8,500 \mathrm{lbs} .\end{array}$ & $\begin{array}{c}8,500- \\
10,000 \text { lbs. }\end{array}$ & $\begin{array}{c}6,000- \\
10,000 \text { lbs. }\end{array}$ & $\begin{array}{c}10,000 \text { lbs. } \\
\text { or less }\end{array}$ \\
\hline 1970 & 1,049 & 66 & 1,115 & 265 & 143 & 408 & 1,523 \\
\hline 1971 & 1,185 & 84 & 1,269 & 317 & 171 & 488 & 1,757 \\
\hline 1972 & 1,498 & 143 & 1,641 & 389 & 210 & 599 & 2,240 \\
\hline 1973 & 1,754 & 233 & 1,987 & 493 & 265 & 758 & 2,745 \\
\hline 1974 & 1,467 & 175 & 1,642 & 452 & 244 & 696 & 2,338 \\
\hline 1975 & 1,101 & 228 & 1,329 & 619 & 333 & 952 & 2,281 \\
\hline 1976 & 1,318 & 236 & 1,554 & 911 & 490 & 1,401 & 2,955 \\
\hline 1977 & 1,306 & 322 & 1,628 & 1,172 & 631 & 1,803 & 3,431 \\
\hline 1978 & 1,334 & 335 & 1,669 & 1,391 & 749 & 2,140 & 3,809 \\
\hline 1979 & 1,271 & 467 & 1,738 & 1,023 & 551 & 1,574 & 3,312 \\
\hline 1980 & 985 & 503 & 1,488 & 634 & 341 & 975 & 2,463 \\
\hline 1981 & 896 & 444 & 1,340 & 553 & 298 & 850 & 2,190 \\
\hline 1982 & 1,102 & 408 & 1,510 & 625 & 336 & 961 & 2,471 \\
\hline 1983 & 1,314 & 466 & 1,780 & 785 & 422 & 1,207 & 2,987 \\
\hline 1984 & 2,031 & 684 & 2,715 & 796 & 428 & 1,224 & 3,939 \\
\hline 1985 & 2,408 & 883 & 3,291 & 785 & 423 & 1,208 & 4,499 \\
\hline 1986 & 2,351 & 1,029 & 3,380 & 1,043 & 171 & 1,214 & 4,594 \\
\hline 1987 & 2,531 & 904 & 3,435 & 444 & 731 & 1,175 & 4,610 \\
\hline 1988 & 2,747 & 720 & 3,467 & 1,137 & 196 & 1,333 & 4,800 \\
\hline 1989 & 2,691 & 622 & 3,313 & 1,135 & 162 & 1,297 & 4,610 \\
\hline 1990 & 2,696 & 755 & 3,451 & 351 & 746 & 1,097 & 4,548 \\
\hline 1991 & 2,533 & 713 & 3,246 & 812 & 65 & 876 & 4,123 \\
\hline 1992 & 2,955 & 653 & 3,608 & 440 & 581 & 1,021 & 4,629 \\
\hline 1993 & 3,375 & 744 & 4,119 & 641 & 592 & 1,232 & 5,351 \\
\hline 1994 & 3,646 & 881 & 4,527 & 1,020 & 468 & 1,506 & 6,033 \\
\hline 1995 & 4,031 & 391 & 4,422 & 1,289 & 342 & 1,631 & 6,053 \\
\hline 1996 & 4,398 & 431 & 4,829 & 424 & 1,266 & 1,690 & 6,519 \\
\hline 1997 & 4,559 & 526 & 5,085 & 1,053 & 659 & 1,712 & 6,797 \\
\hline \multicolumn{8}{|c|}{ Average annualpercentage change } \\
\hline $1970-97$ & $5.6 \%$ & $8.0 \%$ & $5.8 \%$ & $5.2 \%$ & $5.8 \%$ & $5.5 \%$ & $5.7 \%$ \\
\hline $1987-97$ & $6.1 \%$ & $-5.3 \%$ & $4.0 \%$ & $9.0 \%$ & $-1.0 \%$ & $3.8 \%$ & $4.0 \%$ \\
\hline
\end{tabular}

\section{Source:}

Methodology by John D. Maples, Trancon, Inc.

1970-85: Estimated using Tables 3.18 and 3.19, Transportation Energy Data Book Edition 15, ORNL-6856.

Assumes all imports and transplants are Class 1. Assumes Class 2a is $65 \%$ of Class 2 sales and Class $2 \mathrm{~b}$ is $35 \%$ (average of available data).

1986-97: American Automobile Manufacturers Association (AAMA), Motor Vehicle Facts and Figures 1998, U.S.

Sales of Trucks by Gross Vehicle Weight Rating and Body Type, pg. 24, and annual. U.S. Department of

Transportation, National Highway Traffic Safety Administration (NHTSA), "Summary of Fuel Economy

Performance, 1999," and annual. NHTSA's sales for trucks $\leq 8,500 \mathrm{lbs}$. GVW were subtracted from AAMA

sales for trucks $\leq 10,000 \mathrm{lbs}$. GVW to estimate Class $2 \mathrm{~b}$ sales. 


\section{Truck Inventory and Use Survey}

The Truck Inventory and Use Survey (TIUS) provides data on the physical and operational characteristics of the Nation's truck population. It is based on a probability sample of private and commercial trucks registered (or licensed) in each state. Data for 1992 have been released in a report, as well as on CD-ROM. Copies may be obtained by contacting the U.S. Bureau of the Census, Transportation Characteristics Surveys Branch (30 1)457-2797. Internet site http://www. census.gov/svsd/www/tiusview.html is the location of the TIUS on-line.

The 1987 and 1992 surveys, in addition to trucks, included minivans, vans, station wagons on truck chassis, and jeep-like vehicles. The 1977 and 1982 surveys did not include those vehicle types. The estimated number of trucks that were within the scope of the 1992 TIUS and registered in the U.S. as of July 1, 1992, was 59.2 million. These trucks were estimated to have been driven a total of 786.3 billion miles during 1992, an increase of $33.7 \%$ from 1987. The average annual miles traveled per truck was estimated at 11,900 miles.

In the 1992 TIUS, there are several ways to classify a truck by weight. The survey respondent was asked the average weight of the vehicle or vehicle-trailer combination when carrying a typical payload; the empty weight (truck minus cargo) of the vehicle as it was usually operated; and the maximum gross weight at which the vehicle or vehicle-trailer combination was operated. The Census Bureau also collected information on the Gross Vehicle Weight Class of the vehicles (decoded from the vehicle identification number) and the registered weight of the vehicles from the State registration files. Some of these weights are only provided in categories, while others are exact weights. Since all these weights could be quite different for a single truck, the tabulations by weight can be quite confusing. For illustration of this, see Tables 8.4 and 8.5 . The first set of data are based on the Gross Vehicle Weight Class of the vehicle when it was manufactured; the data on Table 8.5 are based on the average weight as reported by the respondent. There is a $22.8 \%$ difference in the number of Class 1 trucks. In most tables, the Gross Vehicle Weight Class was used. However, on the tables comparing different survey estimates, average weight must be used, as the older surveys did not include data on the Gross Vehicle Weight rating.

The 1997 survey data are available on the web site for selected states; nationwide data are expected in late 1999. The name of the 1997 survey was changed to the Vehicle Inventory and Use Survey due to future possibilities of including additional vehicle types. 
These tables illustrate the difference between two weight variables in the Truck Inventory and Use Survey. The manufacturer's gross vehicle weight class is likely to be a more accurate representation,

Table 8.4

Truck Statistics by Gross Vehicle Weight Class, 1992

\begin{tabular}{lrrrrrr}
\hline $\begin{array}{c}\text { Manufacturer's } \\
\text { gross vehicle weight } \\
\text { class }\end{array}$ & $\begin{array}{c}\text { Number of Percentage of } \\
\text { trucks }\end{array}$ & $\begin{array}{c}\text { Average } \\
\text { trucks }\end{array}$ & $\begin{array}{c}\text { Average } \\
\text { per truck }\end{array}$ & $\begin{array}{c}\text { Gallons of } \\
\text { fuel } \\
\text { economy }\end{array}$ & $\begin{array}{c}\text { fuel used } \\
\text { (millions) }\end{array}$ & $\begin{array}{c}\text { Percentage } \\
\text { of fuel use }\end{array}$ \\
\hline $6,000 \mathrm{lbs}$ and less & $37,068,163$ & $62.61 \%$ & 12,739 & 17.23 & 27,397 & $44.76 \%$ \\
$6,001-10,000 \mathrm{lbs}$ & $\mathbf{1 7 , 5 1 9 , 2 1 6}$ & $29.59 \%$ & 11,610 & 13.00 & 15,646 & $25.56 \%$ \\
$10,001-14,000 \mathrm{lbs}$ & 349,301 & $5.90 \%$ & 15,814 & 9.48 & 583 & $0.95 \%$ \\
$14,001-16,000 \mathrm{lbs}$ & 127,219 & $0.21 \%$ & 14,420 & 9.19 & 200 & $0.33 \%$ \\
$16,001-19,500 \mathrm{lbs}$ & 209,158 & $0.35 \%$ & 4,876 & 8.21 & 124 & $0.20 \%$ \\
$19,501-26,000 \mathrm{lbs}$ & $1,859,529$ & $3.14 \%$ & 11,746 & 7.26 & 3,008 & $4.91 \%$ \\
$26,001-33,000 \mathrm{lbs}$ & 197,985 & $0.33 \%$ & 30,074 & 6.64 & 897 & $1.46 \%$ \\
33,001 lbs and up & $\mathbf{1 , 8 7 0 , 1 8 3}$ & $3.16 \%$ & 39,832 & 5.58 & 13,353 & $21.82 \%$ \\
\hline Total & $\mathbf{5 9 , 2 0 0 , 7 5 5}$ & $\mathbf{1 0 0 . 0 0 \%}$ & $\mathbf{1 3 , 2 8 1}$ & $\mathbf{1 2 . 8 5}$ & $\mathbf{6 1 , 2 0 6}$ & $\mathbf{1 0 0 . 0 0 \%}$ \\
\hline
\end{tabular}

Source:

U.S. Department of Commerce, Bureau of the Census, 1992 Truck Inventory and Use Survey, Microdata File on CD, 1995. (Additional resources: http://www.census.gov/svsd/www.tiusview.html)

Table 8.5

Percentage of Trucks by Size Class, 1977, 1982, 1987, and 1992 (percentage)

\begin{tabular}{|c|c|c|c|c|c|}
\hline Size class & $\begin{array}{l}\text { Average weight as } \\
\text { reported by respondent }\end{array}$ & $\begin{array}{c}1977 \\
\text { TIUS }\end{array}$ & $\begin{array}{c}1982 \\
\text { TIUS }\end{array}$ & $\begin{array}{l}1987 \\
\text { TIUS }\end{array}$ & $\begin{array}{l}1992 \\
\text { TIUS }\end{array}$ \\
\hline Class 1 & $6,000 \mathrm{lbs}$ and less & $66.0 \%$ & $77.8 \%$ & $85.4 \%$ & $85.4 \%$ \\
\hline Class 2 & $6,001-10,000 \mathrm{lbs}$ & $17.9 \%$ & $11.6 \%$ & $6.5 \%$ & $7.9 \%$ \\
\hline Class 3 & $10,000-14,000 \mathrm{lbs}$ & $3.1 \%$ & $1.6 \%$ & $1.2 \%$ & $1.2 \%$ \\
\hline Class 4 & $14,001-16,000 \mathrm{lbs}$ & $1.3 \%$ & $0.9 \%$ & $0.5 \%$ & $0.5 \%$ \\
\hline Class 5 & $16,001-19,500 \mathrm{lbs}$ & $2.1 \%$ & $1.0 \%$ & $0.6 \%$ & $0.5 \%$ \\
\hline Class 6 & $19,501-26,000 \mathrm{lbs}$ & $3.4 \%$ & $2.4 \%$ & $1.7 \%$ & $1.2 \%$ \\
\hline Class 7 & $26,001-33,000 \mathrm{lbs}$ & $1.5 \%$ & $1.0 \%$ & $0.8 \%$ & $0.7 \%$ \\
\hline Class 8 & $33,001 \mathrm{lbs}$ and over & $4.6 \%$ & $3.8 \%$ & $3.3 \%$ & $2.8 \%$ \\
\hline
\end{tabular}

\section{Source:}

Estimates are based on data provided on the following public use tapes: U.S. Department of Commerce, Bureau of the Census, 1977 Census of Transportation, Truck Inventory and Use Survey, Washington, DC, 1980; U.S. Department of Commerce, Bureau of the Census, 1982 Census of Transportation, Truck Inventory and Use Survey, Washington, DC, 1985; U.S. Department of Commerce, Bureau of the Census, 1987 Census of Transportation, Truck Inventory and Use Survey, Washington, DC, 1990; and U.S. Department of Commerce, Bureau of the Census, 1992 Census of Transportation, Truck Inventory and Use Survey, Washington, DC, 1995. (Additional resources: http://www.census.gov/svsd/www/tiusview.html) 
Table 8.6

Truck Fuel Economy by Size Class, 1977, 1982, 1987, and 1992

(miles per gallon)

\begin{tabular}{|c|c|c|c|c|c|}
\hline Size class & $\begin{array}{l}\text { Average weight as } \\
\text { reported by respondent }\end{array}$ & $\begin{array}{c}1977 \\
\text { TIUS }\end{array}$ & $\begin{array}{r}1982 \\
\text { TIUS }\end{array}$ & $\begin{array}{l}1987 \\
\text { TIUS }\end{array}$ & $\begin{array}{r}1992 \\
\text { TIUS }\end{array}$ \\
\hline Class 1 & $6,000 \mathrm{lbs}$ and less & 13.2 & 14.2 & 15.0 & 16.1 \\
\hline Class 2 & $6,001-10,000 \mathrm{lbs}$ & 11.5 & 11.1 & 10.9 & 12.2 \\
\hline Class 3 & $10,000-14,000 \mathrm{lbs}$ & 9.4 & 8.1 & 8.1 & 9.2 \\
\hline Class 4 & $14,001-16,000 \mathrm{lbs}$ & 6.9 & 7.5 & 7.5 & 8.5 \\
\hline Class 5 & $16,001-19,500 \mathrm{lbs}$ & 7.6 & 7.2 & 7.1 & 8.1 \\
\hline Class 6 & $19,501-26,000 \mathrm{lbs}$ & 6.1 & 6.9 & 6.4 & 7.2 \\
\hline Class 7 & $26,001-33,000 \mathrm{lbs}$ & 5.3 & 6.2 & 6.1 & 6.8 \\
\hline Class 8 & $33,001 \mathrm{lbs}$ and over & 4.8 & 5.2 & 5.3 & 5.5 \\
\hline
\end{tabular}

Source:

Estimates are based on data provided on the following public use tapes: U.S. Department of Commerce, Bureau of the Census, 1977 Census of Transportation, Truck Inventory and Use Survey, Washington, DC, 1980; U.S. Department of Commerce, Bureau of the Census, 1982 Census of Transportation, Truck Inventory and Use Suwey, Washington, DC, 1985; U.S. Department of Commerce, Bureau of the Census, 1987 Census of Transportation, Truck Inventory and Use Suwey, Washington, DC, 1990; and U.S. Department of Commerce, Bureau of the Census, 1992 Census of Transportation, Truck Inventory and Use Survey, Washington, DC, 1995. (Additional resources: http://www.census.gov/svsd/www/tiusview.html)

Table 8.7

Percentage of Trucks by Fleet Size and Primary Refueling Facility, 1992

\begin{tabular}{lccccc}
\hline & \multicolumn{4}{c}{ Primary refueling facility } & \\
\cline { 2 - 5 } \multicolumn{1}{c}{$\begin{array}{c}\text { Truck } \\
\text { fleet size }\end{array}$} & $\begin{array}{c}\text { Central } \\
\text { company-owned } \\
\text { fueling facility }\end{array}$ & $\begin{array}{c}\text { Single contract fueling } \\
\text { facility } \\
\text { located off-site }\end{array}$ & $\begin{array}{c}\text { Public fueling } \\
\text { stations }\end{array}$ & Other & Total \\
\hline 1 & $7.91 \%$ & $2.52 \%$ & $84.55 \%$ & $5.02 \%$ & $100 \%$ \\
$2-5$ & $16.41 \%$ & $4.44 \%$ & $72.51 \%$ & $6.64 \%$ & $100 \%$ \\
$6-9$ & $31.40 \%$ & $7.73 \%$ & $55.53 \%$ & $5.33 \%$ & $100 \%$ \\
$10-24$ & $43.90 \%$ & $9.44 \%$ & $43.70 \%$ & $2.96 \%$ & $100 \%$ \\
$25-99$ & $56.98 \%$ & $7.39 \%$ & $33.50 \%$ & $2.13 \%$ & $100 \%$ \\
$100-499$ & $58.34 \%$ & $7.50 \%$ & $31.18 \%$ & $2.98 \%$ & $100 \%$ \\
$500-999$ & $57.93 \%$ & $7.26 \%$ & $30.89 \%$ & $3.92 \%$ & $100 \%$ \\
$1,000-4,999$ & $60.71 \%$ & $3.28 \%$ & $32.65 \%$ & $3.36 \%$ & $100 \%$ \\
$5,000-9,999$ & $58.90 \%$ & $5.05 \%$ & $29.09 \%$ & $6.96 \%$ & $100 \%$ \\
10,000 \& up & $59.96 \%$ & $4.68 \%$ & $25.69 \%$ & $9.66 \%$ & $100 \%$ \\
\hline Total & $33.26 \%$ & $5.76 \%$ & $56.15 \%$ & $4.83 \%$ & $\mathbf{1 0 0 \%}$ \\
\hline
\end{tabular}

Source:

U.S. Department of Commerce, Bureau of the Census, 1992 Truck Inventory and Use Survey, Microdata File on CD, 1995. (Additional resources: http:/www.census.gov/svsd/www/tiusview.html) 
Though diesel engines are generally more efjcient than gasoline engines, variations in patterns of use and weight distributions within a weight category can cause the fuel economies to be more similar. Data in the Total row give a good indication that the gasoline trucks are mainly lighter vehicles and diesels are used in heavier applications.

Table 8.8

Truck Fuel Economy by Fuel Type and Size Class, 1992 (miles per gallon)

\begin{tabular}{cccc}
\hline Size class & $\begin{array}{c}\text { Manufacturer's } \\
\text { gross vehicle } \\
\text { weight class }\end{array}$ & $\begin{array}{c}\text { Gasoline } \\
\text { trucks }\end{array}$ & $\begin{array}{c}\text { Diesel } \\
\text { trucks }\end{array}$ \\
\hline Class 1 & $6,000 \mathrm{lbs}$ and less & 17.2 & 18.8 \\
Class 2 & $6,001-10,000 \mathrm{lbs}$ & 12.9 & 15.0 \\
Class 3 & $10,001-14,000 \mathrm{lbs}$ & 9.3 & 9.5 \\
Class 4 & $14,001-16,000 \mathrm{lbs}$ & 8.3 & 10.1 \\
Class 5 & $16,001-19,500 \mathrm{lbs}$ & 7.6 & 10.0 \\
Class 6 & $19,501-26,000 \mathrm{lbs}$ & 7.3 & 7.3 \\
Class 7 & $26,001-33,000 \mathrm{lbs}$ & 6.1 & 6.7 \\
Class 8 & $33,001 \mathrm{lbs}$ and up & 5.5 & 5.5 \\
\hline Total & & 15.4 & 6.5 \\
\hline
\end{tabular}

\section{Source:}

U.S. Department of Commerce, Bureau of the Census, 1992 Truck Inventory and Use Survey, Microdata File on CD, 1995. (Additional resources: http://www.census.gov/svsd/www/tiusview.html) 
Table 8.9

Truck Statistics by Size, 1992

\begin{tabular}{|c|c|c|c|c|}
\hline & \multicolumn{3}{|c|}{ Manufacturer's gross vehicle weight class } & \multirow[b]{2}{*}{ Total } \\
\hline & $\begin{array}{c}\text { Light } \\
(<10,000 \mathrm{lbs}) \\
\end{array}$ & $\begin{array}{c}\text { Medium } \\
(10,001- \\
26,000 \mathrm{lbs}) \\
\end{array}$ & $\begin{array}{c}\text { Heavy } \\
(>26,000 \mathrm{lbs})\end{array}$ & \\
\hline Trucks & 54587,379 & 685,679 & $3,927,697$ & $59,200,755$ \\
\hline Trucks (\%) & $92.21 \%$ & $1.16 \%$ & $6.63 \%$ & $100 \%$ \\
\hline Miles per truck & 12,377 & 12,219 & 26,044 & 13,281 \\
\hline Total miles (\%) & $85.92 \%$ & $1.07 \%$ & $13.01 \%$ & $100 \%$ \\
\hline Fuel use (\%) & $70.32 \%$ & $1.48 \%$ & $28.20 \%$ & $100 \%$ \\
\hline \multirow[t]{2}{*}{ Fuel economy (mpg) } & 15.70 & 9.24 & 5.93 & 12.85 \\
\hline & \multicolumn{4}{|c|}{ Range of operation } \\
\hline Under 50 miles & $75.84 \%$ & $68.55 \%$ & $56.47 \%$ & $74.49 \%$ \\
\hline 50-100 miles & $11.33 \%$ & $14.40 \%$ & $14.55 \%$ & $11.57 \%$ \\
\hline 100-200 miles & $3.31 \%$ & $4.43 \%$ & $6.53 \%$ & $3.53 \%$ \\
\hline 200-500 miles & $2.14 \%$ & $1.68 \%$ & $6.33 \%$ & $2.41 \%$ \\
\hline Over 500 miles & $2.17 \%$ & $1.36 \%$ & $7.51 \%$ & $2.51 \%$ \\
\hline Off-road & $5.21 \%$ & $9.59 \%$ & $8.61 \%$ & $5.48 \%$ \\
\hline \multirow[t]{2}{*}{ Total } & $100 \%$ & $100 \%$ & $100 \%$ & $100 \%$ \\
\hline & \multicolumn{4}{|c|}{ Primary refueling facility } \\
\hline Central company-owned & $15.83 \%$ & $23.56 \%$ & $36.73 \%$ & $32.06 \%$ \\
\hline Single off-site contract & $3.51 \%$ & $4.34 \%$ & $6.30 \%$ & $5.65 \%$ \\
\hline Pubic station & $77.05 \%$ & $66.72 \%$ & $51.86 \%$ & $57.37 \%$ \\
\hline Other & $3.61 \%$ & $5.39 \%$ & $5.10 \%$ & $4.93 \%$ \\
\hline Total & $100 \%$ & $100 \%$ & $100 \%$ & $100 \%$ \\
\hline
\end{tabular}

Source:

U.S. Department of Commerce, Bureau of the Census, 1992 Truck Inventory and Use Survey, Microdata File on CD, 1995. (Additional resources: http://www.census.gov/svsd/www/tiusview.html) 
Table 8.10

Percentage of Trucks by Major Use and Primary Refueling Facility, 1992

\begin{tabular}{|c|c|c|c|c|c|}
\hline \multirow[b]{2}{*}{ Major Use } & \multicolumn{4}{|c|}{ Primary refueling facility } & \multirow[b]{2}{*}{ Total } \\
\hline & $\begin{array}{c}\text { Central } \\
\text { company-owned } \\
\text { fueling facility }\end{array}$ & $\begin{array}{l}\text { Single contract } \\
\text { fueling facility } \\
\text { located off-site }\end{array}$ & $\begin{array}{c}\text { Public fueling } \\
\text { stations }\end{array}$ & Other & \\
\hline Agricultural services & $32.66 \%$ & $2.73 \%$ & $51.68 \%$ & $12.93 \%$ & $100 \%$ \\
\hline Forestry or Lumbering Activities & $26.34 \%$ & $6.43 \%$ & $63.71 \%$ & $3.52 \%$ & $100 \%$ \\
\hline Construction work & $35.79 \%$ & $4.93 \%$ & $56.71 \%$ & $2.57 \%$ & $100 \%$ \\
\hline Contractor Activities or special trades & $16.62 \%$ & $4.93 \%$ & $77.01 \%$ & $1.44 \%$ & $100 \%$ \\
\hline Manufacturing, refining or processing activities & $37.54 \%$ & $11.21 \%$ & $49.05 \%$ & $2.20 \%$ & $100 \%$ \\
\hline Wholesale trade & $35.55 \%$ & $12.72 \%$ & $49.99 \%$ & $1.74 \%$ & $100 \%$ \\
\hline Retail trade & $31.35 \%$ & $8.18 \%$ & $58.67 \%$ & $1.81 \%$ & $100 \%$ \\
\hline Business and Personal services & $23.48 \%$ & $5.94 \%$ & $68.24 \%$ & $2.34 \%$ & $100 \%$ \\
\hline Utilities & $58.68 \%$ & $2.31 \%$ & $36.42 \%$ & $2.58 \%$ & $100 \%$ \\
\hline Mining or quarryng activities & $53.75 \%$ & $5.82 \%$ & $38.05 \%$ & $2.38 \%$ & $100 \%$ \\
\hline Daily rental & $49.95 \%$ & $2.79 \%$ & $44.75 \%$ & $2.50 \%$ & $100 \%$ \\
\hline Not in use & $14.42 \%$ & $3.64 \%$ & $46.70 \%$ & $35.24 \%$ & $100 \%$ \\
\hline For-hire transportation & $37.80 \%$ & $5.22 \%$ & $53.65 \%$ & $3.33 \%$ & $100 \%$ \\
\hline One-way rental & $5.28 \%$ & $0.07 \%$ & $93.05 \%$ & $1.60 \%$ & $100 \%$ \\
\hline Personal transportation & $1.51 \%$ & $0.68 \%$ & $93.14 \%$ & $4.67 \%$ & $100 \%$ \\
\hline Total & $32.06 \%$ & $5.65 \%$ & $57.37 \%$ & $4.93 \%$ & $100 \%$ \\
\hline
\end{tabular}

Source:

U.S. Department of Commerce, Bureau of the Census, 1992 Truck Inventory and Use Survey, Microdata File on CD, 1995. (Additional resources: http://www.census.gov/svsd/www/tiusview.html) 
Table 8.11

Percentage of Trucks by Size Ranked by Major Use, 1992

\begin{tabular}{|c|c|c|c|}
\hline Rank & 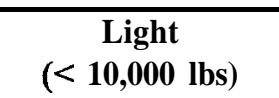 & $\begin{array}{r}\text { M e d i u m } \\
(10,001-26,000 \text { Ibs })\end{array}$ & $\begin{array}{c}\text { Heavy } \\
(>26,000 \text { lbs })\end{array}$ \\
\hline \multirow[t]{2}{*}{1} & Personal & Agriculture & For Hire \\
\hline & $73.54 \%$ & $21.12 \%$ & $18.21 \%$ \\
\hline \multirow[t]{2}{*}{2} & Construction & Construction & Construction \\
\hline & $7.57 \%$ & $20.59 \%$ & $18.17 \%$ \\
\hline \multirow[t]{2}{*}{3} & Services' & Services' & Agriculture \\
\hline & $5.12 \%$ & $12.32 \%$ & $17.42 \%$ \\
\hline \multirow[t]{2}{*}{4} & Agriculture & Retail & Wholesale \\
\hline & $4.99 \%$ & $9.05 \%$ & $8.73 \%$ \\
\hline \multirow[t]{2}{*}{5} & Retail & Utilities & Retail \\
\hline & $2.94 \%$ & $6.44 \%$ & $7.22 \%$ \\
\hline \multirow[t]{2}{*}{6} & Not in Use & Wholesale & Personal \\
\hline & $1.50 \%$ & $6.04 \%$ & $6.56 \%$ \\
\hline \multirow[t]{2}{*}{7} & Wholesale & For Hire & Services $^{\mathrm{a}}$ \\
\hline & $1.38 \%$ & $5.90 \%$ & $6.20 \%$ \\
\hline \multirow[t]{2}{*}{8} & Manufacturing & Personal & Manufacturing \\
\hline & $1.02 \%$ & $5.86 \%$ & $5.53 \%$ \\
\hline \multirow[t]{2}{*}{9} & Utilities & Manufacturing & Not in Use \\
\hline & $0.72 \%$ & $3.51 \%$ & $3.49 \%$ \\
\hline \multirow[t]{2}{*}{10} & Daily Rental & Not in Use & Utilities \\
\hline & $0.40 \%$ & $3.43 \%$ & $2.66 \%$ \\
\hline \multirow[t]{2}{*}{11} & Forestry & Daily Rental & Forestry \\
\hline & $0.31 \%$ & $2.89 \%$ & $2.16 \%$ \\
\hline \multirow[t]{2}{*}{12} & Mining & Forestry & Daily Rental \\
\hline & $0.27 \%$ & $1.48 \%$ & $1.70 \%$ \\
\hline \multirow[t]{2}{*}{13} & For Hire & Mining & Mining \\
\hline & $0.24 \%$ & $1.00 \%$ & $1.69 \%$ \\
\hline \multirow[t]{2}{*}{14} & One-Way Rental & One-Way Rental & One-Way Rental \\
\hline & $0.01 \%$ & $0.36 \%$ & $0.26 \%$ \\
\hline \multirow[t]{2}{*}{15} & Other & Other & Other \\
\hline & $0.00 \%$ & $0.00 \%$ & $0.00 \%$ \\
\hline
\end{tabular}

Source:

U.S. Department of Commerce, Bureau of the Census, 1992 Truck Inventory and Use Survey, Micro data File on CD, 1995. (Additional resources: http://www.census.gov/svsd/www/tiusview.html)

${ }^{\text {a }}$ Business and personal services. 


\section{Commodity Flow Survey}

The Commodity Flow Survey (CFS) is designed to provide data on the flow of goods and materials by mode of transport. The 1993 CFS is a continuation of statistics collected in the Commodity Transportation Survey from 1963 through 1977, and includes major improvements in methodology, sample size, and scope. A sample of 200,000 domestic establishments randomly selected from a universe of about 900,000 establishments engaged in mining, manufacturing, wholesale, auxiliary establishments (warehouses) of multi-establishment companies, and some selected activities in retail and service was used. Each selected establishment reported a sample of approximately 30 outbound shipments for a two-week period in each of the four calendar quarters of 1993. This produced a total sample of about 20 million shipments. For each sampled shipment, zip codes of origin and destination, 5-digit Standard Transportation Commodity Classification (STCC) code, weight, value, and modes of transport, were provided. Establishments were also asked to indicate whether the shipment was containerized, a hazardous material, or an export.

The 1993 CFS differs from previous surveys in its greatly expanded coverage of intermodalism. Earlier surveys reported only the principal mode. The 1993 survey reports all modes used for the shipment (for-hire truck, private truck, rail, inland water, deep sea water, pipeline, air, parcel delivery or U.S. Postal Service, other mode, unknown). Route distance for each mode for each shipment as imputed from a mode-distance table developed by Oak Ridge National Laboratory. Distance, in turn, was used to compute ton-mileage by mode of transport.

The CFS was conducted again in 1997, with few changes from the 1993 survey. These data are scheduled to be released in the Fall of 1999. For more information about the CFS, contact the Commodity Flow Survey Branch, Department of Commerce, Bureau of the Census, Services Division at (301) 457-2108, or visit the following Internet site: http://www.bts.gov/cfs . 
Table 8.12

1993 Commodity Flow Survey: Shipment Characteristics by Mode of Transportation

\begin{tabular}{|c|c|c|c|c|c|c|c|c|c|}
\hline Mode & $\begin{array}{c}\text { Value } \\
\text { (million } \\
\text { dollars) }\end{array}$ & $\begin{array}{c}\text { Tons } \\
\text { (thousands) }\end{array}$ & $\begin{array}{l}\text { Ton miles } \\
\text { (millions) }\end{array}$ & $\begin{array}{c}\text { Value } \\
\text { (percent) }\end{array}$ & $\begin{array}{c}\text { Tons } \\
\text { (percent) }\end{array}$ & $\begin{array}{c}\text { Ton } \\
\text { miles } \\
\text { (percent) }\end{array}$ & $\begin{array}{c}\text { Value per } \\
\text { ton } \\
\text { (dollars) }\end{array}$ & $\begin{array}{c}\text { Value per } \\
\text { pound } \\
\text { (dollars) }\end{array}$ & $\begin{array}{l}\text { Ton miles } \\
\text { per ton }\end{array}$ \\
\hline CFS plus ORNL & $\$ 6,123,832$ & $12,157,105$ & $3,627,919$ & $100.0 \%$ & $100.0 \%$ & $100.0 \%$ & $\$ 503.7$ & $\$ 0.25$ & 298 \\
\hline Parcel, postal, courier service & $\$ 563,277$ & 18,892 & 13,151 & $9.2 \%$ & $0.2 \%$ & $0.4 \%$ & $\$ 29,815.6$ & $\$ 14.91$ & 696 \\
\hline Truck (for-hire, private, both) & $\$ 4,403,495$ & $6,385,915$ & 869,536 & $71.9 \%$ & $52.5 \%$ & $24.0 \%$ & $\$ 689.6$ & $\$ 0.34$ & 136 \\
\hline Air (including truck and air) & $\$ 139,087$ & 3,139 & 4,009 & $2.3 \%$ & $0.0 \%$ & $0.1 \%$ & $\$ 44,309.3$ & $\$ 22.15$ & 1,277 \\
\hline Rail & $\$ 247,394$ & $1,544,148$ & 942,561 & $4.0 \%$ & $12.7 \%$ & $26.0 \%$ & $\$ 160.2$ & $\$ 0.08$ & 610 \\
\hline Water & $\$ 64,077$ & 518,912 & 271,981 & $1.0 \%$ & $4.3 \%$ & $7.5 \%$ & $\$ 123.5$ & $\$ 0.06$ & 524 \\
\hline Pipeline & $\$ 89,849$ & 483,645 & a & $1.5 \%$ & $4.0 \%$ & a & $\$ 185.8$ & $\$ 0.09$ & a \\
\hline Truck and rail & $\$ 83,082$ & 40,624 & 37,675 & $1.4 \%$ & $0.3 \%$ & $1.0 \%$ & $\$ 2,045.1$ & $\$ 1.02$ & 927 \\
\hline Rail and water & $\$ 3,636$ & 79,222 & 70,219 & $0.1 \%$ & $0.7 \%$ & $1.9 \%$ & $\$ 4.6$ & $\$ 0.00$ & 886 \\
\hline Other intermodal & $\$ 9,746$ & 69,661 & 114,811 & $0.2 \%$ & $0.6 \%$ & $3.2 \%$ & $\$ 14.0$ & $\$ 0.00$ & 1,648 \\
\hline $\begin{array}{l}\text { Other and unknown } \\
\text { ORNL estimates: }\end{array}$ & $\$ 242,691$ & 544,335 & 96,972 & $4.0 \%$ & $4.5 \%$ & $2.7 \%$ & $\$ 445.8$ & SO.22 & 178 \\
\hline Water (not in CFS) & $\$ 187,085$ & $1,609,309$ & 614,104 & $3.1 \%$ & $13.2 \%$ & $16.9 \%$ & $\$ 116.3$ & $\$ 0.06$ & 382 \\
\hline Pipeline (not in CFS) & $\$ 90,413$ & 859,303 & 592,900 & $1.5 \%$ & $7.1 \%$ & $16.3 \%$ & $\$ 105.2$ & $\$ 0.05$ & 690 \\
\hline Intermodal ${ }^{\mathrm{b}}$ total & $\$ 659,741$ & 208,399 & 235,856 & $10.8 \%$ & $1.7 \%$ & $6.5 \%$ & $\$ 3,165.8$ & $\$ 1.58$ & 1,132 \\
\hline
\end{tabular}

\section{Source:}

U.S. Department of Commerce, Bureau of the Census, 1993 Commodity Flow Survey: United States, TC92-CF-52, and Oak Ridge National Laboratory estimates, Washington, DC, 1996. (Additional resources: http:/www.bts.gov/cfs/cfs.html)

"Data do not meet publication standards.

${ }^{{ }^{b}}$ Intermodal is a combination of parcel, postal or courier; truck and rail; truck and water, rail and water; and other intermodal. It excludes truck and air which is added to air transportation. 
Table 8.13

Value, Tons, and Ton-Miles of Commodity Shipments, 1993

\begin{tabular}{|c|c|c|c|c|}
\hline Commodity description" & $\begin{array}{c}\text { Value } \\
\text { (million } \\
\text { dollars) }\end{array}$ & $\begin{array}{c}\text { Tons } \\
\text { (thousands) }\end{array}$ & $\begin{array}{l}\text { Value per ton } \\
\text { (dollars) }\end{array}$ & $\begin{array}{l}\text { Ton-miles } \\
\text { (millions) }\end{array}$ \\
\hline \multicolumn{5}{|l|}{ Energy ${ }^{b}$} \\
\hline Petroleum or coal products & $\$ 359,471$ & $1,885,833$ & $\$ 191$ & 287,081 \\
\hline Coal & $\$ 23,449$ & $1,129,945$ & $\$ 21$ & 487,791 \\
\hline \multicolumn{5}{|l|}{ Lumber and forest } \\
\hline Pulp, paper, or allied products & $\$ 195,002$ & 217,233 & $\$ 898$ & 100,721 \\
\hline Lumber or wood products, excluding furniture & $\$ 126,662$ & 663,351 & $\$ 191$ & 120,669 \\
\hline Forest products & $\$ 1,700$ & 30,520 & $\$ 56$ & 3,635 \\
\hline \multicolumn{5}{|l|}{ Mining } \\
\hline Metallic ores & $\$ 20,278$ & 149,562 & $\$ 136$ & 36,895 \\
\hline Nonmetallic minerals & $\$ 20,695$ & $1,786,381$ & $\$ 12$ & 155,417 \\
\hline \multicolumn{5}{|l|}{ Farm and food } \\
\hline Food or kindred products & $\$ 856,884$ & 859,764 & $\$ 997$ & 270,984 \\
\hline Farm products & $\$ 142,442$ & 636,630 & $\$ 224$ & 276,260 \\
\hline Fresh fish or other marine products & $\$ 11,062$ & 2,995 & $\$ 3,693$ & 1,746 \\
\hline \multicolumn{5}{|l|}{ Equipment, machinery, and instruments } \\
\hline Transportation equipment & $\$ 652,474$ & 87,617 & $\$ 7,447$ & 49,098 \\
\hline Machinery, excluding electrical & $\$ 442,770$ & 34,180 & $\$ 12,954$ & 19,112 \\
\hline Electrical machinery, equipment, or supplies & $\$ 411,030$ & 30,156 & $\$ 13,630$ & 19,591 \\
\hline $\begin{array}{l}\text { Instruments, photographic goods, optical goods, } \\
\text { watches, or clocks }\end{array}$ & $\$ 198,492$ & 8,600 & $\$ 23,080$ & 5,390 \\
\hline \multicolumn{5}{|l|}{ Industrial products } \\
\hline Chemicals or allied products & $\$ 532,907$ & 545,405 & $\$ 977$ & 236,856 \\
\hline Fabricated metal products & $\$ 237,316$ & 84,895 & $\$ 2,795$ & 30,489 \\
\hline Primary metal products & $\$ 228,610$ & 266,409 & $\$ 858$ & 97,266 \\
\hline Rubber or miscellaneous plastics products & $\$ 175,267$ & 52,349 & $\$ 3,348$ & 25,528 \\
\hline Clay, concrete, glass, or stone products & $\$ 91,365$ & 799,481 & $\$ 114$ & 84,032 \\
\hline \multicolumn{5}{|l|}{ Consumer goods } \\
\hline Apparel or other finished textile products & $\$ 291,203$ & 15,128 & $\$ 19,249$ & 9,967 \\
\hline Textile mill products & $\$ 102,189$ & 24,757 & $\$ 4,128$ & 11,341 \\
\hline Furniture or fixtures & $\$ 69,471$ & 16,568 & $\$ 4,193$ & 9,789 \\
\hline Tobacco products, excluding insecticides & $\$ 60,640$ & 3,225 & $\$ 18,803$ & 931 \\
\hline Leather or leather products & $\$ 50,645$ & 2,401 & $\$ 21,093$ & 2,182 \\
\hline \multicolumn{5}{|l|}{ Waste materials $\mathbf{s}^{\mathrm{i}}$} \\
\hline Waste or scrap materials & $\$ 18,258$ & 130,894 & $\$ 139$ & 27,591 \\
\hline Waste hazardous materials or substances & $\$ 558$ & 813 & $\$ 686$ & 314 \\
\hline \multicolumn{5}{|l|}{ Miscellaneous and other unknown } \\
\hline Miscellaneous products of manufacturing & $\$ 200,803$ & 20,731 & $\$ 9,686$ & 10,992 \\
\hline Miscellaneous freight shipments & $\$ 81,297$ & 20,830 & $\$ 3,903$ & 5,038 \\
\hline Ordnance or accessories & $\$ 17,174$ & 663 & $\$ 25,903$ & 629 \\
\hline $\begin{array}{l}\text { Containers, carriers or devices, shipping, returned } \\
\text { empty }\end{array}$ & $\$ 1,144$ & 702 & $\$ 1,630$ & 230 \\
\hline Commodity unknown & $\$ 21,941$ & 7,804 & $\$ 2,812$ & 2,522 \\
\hline
\end{tabular}

Source:

U.S. Department of Commerce, Bureau of the Census, 1993 Commodity Flow Survey, United States, TC92-CF-52, Washington, DC, 1996.

\section{Note:}

The sum of the data by commodity groups in this table is not equal to the total in previous table because it includes additional estimates of water and pipeline shipments by ORNL.

\footnotetext{
"Excludes data for printed matter because the data do not meet publication standards.

${ }^{b}$ Excludes data for pipeline shipments calculated by Oak Ridge National Laboratory (ORNL)that are included in previous table.

'Excludes data on municipal solid wastes.
} 
Table 8.14

Summary Statistics on Buses by Type, 1970-97

\begin{tabular}{|c|c|c|c|}
\hline Year & Transit motor bus" & Intercitv bus & School bus \\
\hline \multicolumn{4}{|c|}{ Number in operation } \\
\hline 1970 & 49,700 & 22,000 & 288,700 \\
\hline 1975 & 50,811 & 20,500 & 368,300 \\
\hline 1980 & 59,411 & 21,400 & 418,255 \\
\hline 1985 & 64,258 & 20,200 & 480,400 \\
\hline 1990 & 58,714 & 20,680 & 508,261 \\
\hline 1994 & 68,123 & 19,146 & 547,718 \\
\hline 1995 & 67,107 & 20,138 & $560,447$. \\
\hline 1996 & 69,020 & 20,649 & 569,395 \\
\hline 1997 & 69,513 & 20,910 & 568,113 \\
\hline \multicolumn{4}{|c|}{ Vehicle-miles (millions) } \\
\hline 1970 & 1,409 & 1,209 & 2,100 \\
\hline 1975 & 1,526 & 1,126 & 2,500 \\
\hline 1980 & 1,677 & 1,162 & 2,900 \\
\hline 1985 & 1,863 & 933 & 3,448 \\
\hline 1990 & 2,123 & 991 & 3,800 \\
\hline 1994 & 2,162 & 1,211 & 4,400 \\
\hline 1995 & 2,184 & 1,194 & 5,000 \\
\hline 1996 & 2,221 & 1,220 & 5,000 \\
\hline 1997 & 2,307 & 1,276 & 4,400 \\
\hline \multicolumn{4}{|c|}{ Passenger-miles (millions) } \\
\hline 1970 & 18,210 & 25,300 & $\mathrm{~b}$ \\
\hline 1975 & 18,300 & 25,400 & $\mathrm{~b}$ \\
\hline 1980 & 21,790 & 27,400 & b \\
\hline 1985 & 21,161 & 23,800 & $b$ \\
\hline 1990 & 20,981 & 23,000 & 74,200 \\
\hline 1994 & 18,832 & 28,100 & 85,000 \\
\hline 1995 & 18,818 & 28,100 & 95,000 \\
\hline 1996 & 19,096 & 28,800 & 99,000 \\
\hline 1997 & 20,357 & 29,600 & 82,900 \\
\hline \multicolumn{4}{|c|}{ Energy use (trillion Btu) } \\
\hline 1970 & 44.8 & 26.6 & 37.5 \\
\hline 1975 & 51.5 & 24.8 & 42.6 \\
\hline 1980 & 61.3 & 29.3 & 47.5 \\
\hline 1985 & 72.4 & 31.5 & 57.0 \\
\hline 1990 & 78.9 & 21.7 & 62.2 \\
\hline 1994 & $86.7 ”$ & 24.7 & 90.6 \\
\hline 1995 & 87.5 & 22.6 & $68.4^{\mathrm{d}}$ \\
\hline 1996 & 85.1 & 22.6 & 82.2 \\
\hline 1997 & 87.9 & 23.6' & 72.3 \\
\hline
\end{tabular}

Source:

See Appendix A for Table 7.13. (Additional resources: http://www.apta.com, http://www.fhwa.dot.gov, http://www.schoolbusfleet.com)

a Data for transit buses after 1983 are not comparable with prior data. Data for prior years were provided voluntarily and statistically expanded; in 1984 reporting became mandatory.

b Data are not available.

c Beginning in 1992, data became available on alternative fuel use by transit buses.

d Assumptions about fuel type changed in this year. See Appendix for details.

e Estimated using vehicle-miles 


\section{Chapter 9 \\ Alternative Fuel Vehicles and Characteristics}

Summary Statistics from Tables in this Chapter

\begin{tabular}{ccr}
\hline Source & & \\
\hline Table 9.1 & Light alternative fuel vehicles, 1997 & 303,306 \\
& LPG & 211,181 \\
& CNG & 57,534 \\
& LNG & 170 \\
& M85 & 21,034 \\
& E85 & 9,130 \\
& Electric & 4,257 \\
Table 9.2 & Heavy alternative fuel vehicles, 1997 & 66,684 \\
& LPG & 52,002 \\
& CNG & 13,318 \\
& LNG & 643 \\
& M85/M100 & 178 \\
& E95 & 347 \\
& Electric & 196 \\
& Number of alternative fuel refuel sites, 1998 & 7,271 \\
& LPG & 5,318 \\
& CNG & 1,268 \\
& LNG & 66 \\
& M85 & 91 \\
& E85 & 40 \\
& Electric & 486 \\
\hline
\end{tabular}

Fuel type abbreviations are used throughout this chapter.

$L P G=$ liquified petroleum gas

$\boldsymbol{C N G}=$ compressed natural gas

$M-85=85 \%$ methanol, $15 \%$ gasoline

$E-85=85 \%$ ethanol, $15 \%$ gasoline

$M-l 00=100 \%$ methanol

E-95 = $95 \%$ ethanol, $5 \%$ gasoline

$L N G=$ liquified natural gas 


\section{THE ALTERNATIVE FUELS DATA CENTER}

The Department of Energy (DOE) has established the Alternative Fuels Data Center (AFDC) in support of its work aimed at fulfilling the Alternative Motor Fuels Act (AMFA) directives. The AFDC is operated and managed by the National Renewable Energy Laboratory (NREL) in Golden, Colorado.

The purposes of the AFDC are:

- to gather and analyze information on the fuel consumption, emissions, operation, and durability of alternative fuel vehicles, and

- $\quad$ to provide unbiased, accurate information on alternative fuels and alternative fuel vehicles to government agencies, private industry, research institutions, and other interested organizations.

The data are collected for three specific vehicle types: (1) light vehicles, including automobiles, light trucks, and mini-vans; (2) heavy vehicles such as tractor-trailers and garbage trucks; and (3) urban transit buses. An Oracle Relational Database Management System is used to manage the data, along with a statistical software package capable of providing statistical, graphic, and textual information to users. Several tables and graphs in this chapter contain statistics which were generated by the AFDC. Future editions of the Transportation Energy Data Book will continue to present graphical and statistical information from the AFDC.

The Department of Energy is sponsoring the National Alternative Fuels Hotline for Transportation Technologies in order to assist the general public and interested organizations in improving their understanding of alternative transportation fuels. The Hotline can be reached by dialing 1-800-423-1DOE, or on the Internet at http://www.afdc.nrel.gov. 
There are more LPG vehicles in use than any other alternative fuel vehicle. The population of E85 vehicles, however,

has grown the most since 1992. For details on alternative fuel use by fuel type, see Table 2.10

Table 9.1

Estimates of Alternative Fuel Vehicles in Use, 1992-99

\begin{tabular}{|c|c|c|c|c|c|c|c|c|c|}
\hline Fuel type & 1992 & 1993 & 1994 & 1995 & 1996 & 1997 & 1998 & 1999 & $\begin{array}{c}\text { Percentage } \\
\text { change } \\
1992-99 \\
\end{array}$ \\
\hline LPG & 221,000 & 269,000 & 264,000 & 259,000 & 263,000 & 263,000 & 269,000 & 274,000 & $3.1 \%$ \\
\hline CNG & 23,191 & 32,714 & 41,227 & 50,218 & 60,144 & 70,852 & 85,730 & 96,017 & $22.5 \%$ \\
\hline LNG & 90 & 299 & 484 & 603 & 663 & 813 & 1,358 & 1,517 & $49.7 \%$ \\
\hline M85 & 4,850 & 10,263 & 15,484 & 18,319 & 20,265 & 21,040 & 21,578 & 21,829 & $24.0 \%$ \\
\hline M100 & 404 & 414 & 415 & 386 & 172 & 172 & 378 & 378 & $-0.9 \%$ \\
\hline E85 & 172 & 441 & 605 & 1,527 & 4,536 & 9,130 & 11,743 & 17,892 & $94.2 \%$ \\
\hline E95 & 38 & 27 & 33 & 136 & 361 & 347 & 14 & 14 & $-13.3 \%$ \\
\hline Electricity & 1,607 & 1,690 & 2,224 & 2,860 & 3,280 & 4,453 & 5,824 & 6,481 & $22.0 \%$ \\
\hline Total & 251,352 & 314,848 & 324,472 & 333,049 & 352,421 & 369,807 & 395,625 & 418,128 & $7.5 \%$ \\
\hline
\end{tabular}

\section{Source:}

U. S. Department of Energy, Energy Information Administration, Alternatives to Traditional Transportation Fuels,1997, Washington, DC, 1998, web site http://www.eia.doe.gov/cneaf/solar.renewables/alt_trans_fuel97/atf.html. (Additional resources: http://www.eia.doe.gov)

\footnotetext{
"Based on plans or projections.
} 
Table 9.2

Estimates of Light Alternative Fuel Vehicles, 1995, 1997, and 1999

\begin{tabular}{|c|c|c|c|c|c|c|c|c|c|}
\hline \multirow[b]{2}{*}{ Fuel type } & \multicolumn{3}{|c|}{ Private } & \multicolumn{3}{|c|}{ State and local govemment } & \multicolumn{3}{|c|}{ Federal Government } \\
\hline & 1995 & 1997 & 1999” & 1995 & 1997 & $1999 ”$ & 1995 & 1997 & $1999 "$ \\
\hline LPG & 166,000 & 168,000 & 175,000 & 42,000 & 43,000 & 44,000 & 139 & 181 & 695 \\
\hline CNG & 22,950 & 30,530 & 44,224 & 10,670 & 13,594 & 19,889 & 9,432 & 13,410 & 13,855 \\
\hline LNG & 49 & 58 & 58 & 47 & 25 & 25 & 47 & 87 & 239 \\
\hline M-85 & 5,198 & 9,875 & 12,750 & 3,569 & 6,982 & 7,596 & 9,552 & 4,177 & 1,477 \\
\hline M-100 & 0 & 0 & 0 & 0 & 0 & 0 & 0 & 0 & 0 \\
\hline E-85 & 54 & 2,483 & 3,201 & 1,084 & 3,759 & 4,605 & 389 & 2,888 & 10,086 \\
\hline E-95 & 1 & 0 & 0 & 0 & 0 . & 0 & 0 & 0 & 0 \\
\hline Electricity & 2,400 & 3,187 & 4,128 & 160 & 801 & 1,400 & 191 & 269 & 685 \\
\hline Total & 196,652 & 214,133 & 239,361 & 57,530 & 68,161 & 77,515 & 19,750 & 21,012 & 27,037 \\
\hline
\end{tabular}

Source:

U. S. Department of Energy, Energy Information Administration, Alternatives to Traditional Transportation Fuels, 1997, Washington, DC, 1998, web site http:/www.eia.doe.gov/cneaf/solar.renewables/alt_trans_fuel97/atf.html. (Additional resources: http://www.eia.doe.gov)

"Based on plans or projections. 
Table 9.3

Estimates of Heavy Alternative Fuel Vehicles, 1995, 1997, and 1999

\begin{tabular}{|c|c|c|c|c|c|c|c|c|c|}
\hline \multirow[b]{2}{*}{ Fuel type } & \multicolumn{3}{|c|}{ Private } & \multicolumn{3}{|c|}{ State and local government } & \multicolumn{3}{|c|}{ Federal government } \\
\hline & 1995 & 1997 & 1999” & 1995 & 1997 & $1999 "$ & 1995 & 1997 & $1999 "$ \\
\hline LPG & 41,000 & 42,000 & 44,000 & 10,000 & 10,000 & 11,000 & 2 & 2 & 2 \\
\hline $\mathrm{CNG}$ & 3,981 & 7,398 & 10,026 & 3,185 & 5,919 & 8,022 & 0 & 1 & 1 \\
\hline LNG & 34 & 140 & 262 & 426 & 497 & 920 & 0 & 6 & 13 \\
\hline M85 & 0 & 0 & 0 & 0 & 6 & 6 & 0 & 0 & 0 \\
\hline M100 & 0 & 0 & 0 & 386 & 172 & 378 & 0 & 0 & 0 \\
\hline E85 & 0 & 0 & 0 & 0 & 0 & 0 & 0 & 0 & 0 \\
\hline E95 & 1 & 0 & 0 & 134 & 347 & 14 & 0 & 0 & 0 \\
\hline Electricity & 26 & 40 & 40 & 83 & 146 & 214 & 0 & 10 & 14 \\
\hline Total & 45,042 & 49,578 & 54,328 & 14,214 & 17,087 & 20,554 & 2 & 19 & 30 \\
\hline
\end{tabular}

Source:

U. S. Department of Energy, Energy Information Administration, Alternatives to Traditional Transportation Fuels, 1997, Washington, DC, 1998, web site http://www.eia.doe.gov/cneaf/solar.renewables/alt_trans_fuel97/atf.html. (Additional resources: http://www.eia.doe.gov)

"Based on plans or projections. 
Table 9.4

Alternative Fuel Vehicles Available by Manufacturer, Model Year 1999”

\begin{tabular}{|c|c|c|c|}
\hline Model & Fuel & Tvpe & Emission class \\
\hline \multicolumn{4}{|c|}{ Chrysler Products: l-800-255-2616 } \\
\hline EPIC (CA, NY-lease only) & Electric-lead acid or NiMH & Minivan & ZEV \\
\hline Minivan & Electric-lead acid or NiMH & Minivan & ZEV \\
\hline Minivan & E-85 & Minivan & $\mathrm{N} / \mathrm{A}$ \\
\hline Ram Wagon & CNG dedicated & Large van & ULEV and ILEV \\
\hline Ram Van & CNG dedicated & Large van & WLEV and ILEV \\
\hline \multicolumn{4}{|c|}{ Ford Products: 1-800-ALT-FUEL } \\
\hline Ranger & Electric-lead acid & Light truck & ZEV \\
\hline Ranger & E-85 & Light truck & TLEV \\
\hline Contour (QVM) & CNG bi-fuel & Compact sedan & TLEV \\
\hline Crown Victoria & CNG Dedicated & Full-size sedan & ULEV/ILEV \\
\hline Econoline & $\begin{array}{l}\text { CNG/LPG dedicated } \\
\text { or bi-fuel }\end{array}$ & Full-size van & Various \\
\hline F-Series & $\begin{array}{l}\text { CNG/LPG dedicated } \\
\text { or bi-fuel }\end{array}$ & Light truck & Various \\
\hline Taurus & E-85 or M-85 gasoline & Mid-size sedan & TLEV \\
\hline \multicolumn{4}{|c|}{ General Motors Products: 1-800-25Electric, 313-556-7723 or 1-888-GM-AFT-4U(CNG) } \\
\hline EV1 (CA and AZ only) & Electric-lead acid or NiMH & Sedan two-seater & $\overline{\mathrm{ZEV}}$ \\
\hline Chevrolet S- 10 & Electric-lead acid & Compact pickup & ZEV \\
\hline Chevrolet Cavalier & CNG bi-fuel & Subcompact sedan & TLEV \\
\hline \multicolumn{4}{|l|}{ Honda: 1-888-CCHonda } \\
\hline Honda EV Plus & Electric-NiMH & Compact sedan & ZEV \\
\hline Civic GX & CNG dedicated & Subcompact sedan & $\begin{array}{l}\text { ULEV California } \\
\text { ILEV Federal }\end{array}$ \\
\hline \multicolumn{4}{|c|}{ Nissan: 1-310-771-3422 (Demonstration fleets only) } \\
\hline Altra EV & Electric lithium-ion & Compact sedan & ZEV \\
\hline \multicolumn{4}{|c|}{ Solectria Corporation: l-508-658-2231 } \\
\hline Flash & Electric-lead acid & Pickup truck & $\mathrm{ZEV}$ \\
\hline Force & Electric-lead acid or NiMH & Sedan & ZEV \\
\hline \multicolumn{4}{|c|}{ Toyota: 1-800-331-4331 (Press 3 for Alternative Fuel Information) (Fleet sales only) } \\
\hline RAV4-EV & Electric-lead acid/NiMH & Sports utilitv vehicle & ZEV \\
\hline
\end{tabular}

Source:

U.S. Department of Energy, National Alternative Fuels Data Center, web site, http://www.afdc.nrel.gov/vehicles/OEM_YEAR.html, February 1999.

(Additional resources: http://www.afdc.nrel.gov)

Note:

LEV=low emission vehicle. ILEV=inherently low emission vehicle. ULEV=ultra low emission vehicle. ZEV=zero emission vehicle. TLEV=transitional low emission vehicle.

${ }^{\mathrm{a}}$ In addition, Mazda (1-800-248-0459) and Volvo (1-800-970-0888) have experimental alternative fuel vehicles which are not yet on the market. 
This list includes public and private refuel sites; therefore, not all of these sites are available to the public.

Table 9.5

Number of Alternative Refuel Sites by State and Fuel Type, 1998

\begin{tabular}{|c|c|c|c|c|c|c|c|}
\hline State & $\begin{array}{l}\text { M85 } \\
\text { sites }\end{array}$ & $\begin{array}{l}\text { CNG } \\
\text { sites }\end{array}$ & $\begin{array}{l}\text { ES5 } \\
\text { sites }\end{array}$ & $\begin{array}{l}\text { LPG } \\
\text { sites }\end{array}$ & $\begin{array}{c}\text { LNG } \\
\text { sites }\end{array}$ & $\begin{array}{l}\text { Electric } \\
\text { sites }\end{array}$ & Total \\
\hline Alabama & 0 & 16 & 0 & 152 & 0 & 2 & 170 \\
\hline Alaska & 0 & 0 & 0 & 12 & 0 & 0 & 12 \\
\hline Arizona & 1 & 28 & 0 & 92 & 46 & 2 & 169 \\
\hline Arkansas & 0 & 6 & 0 & 144 & 0 & 0 & 150 \\
\hline California & 66 & 209 & 0 & 279 & 335 & 18 & 907 \\
\hline Colorado & 2 & 44 & 1 & 91 & 0 & 3 & 141 \\
\hline Connecticut & 0 & 27 & 0 & 25 & 1 & 0 & 53 \\
\hline Delaware & 0 & 6 & 0 & 8 & 0 & 0 & 14 \\
\hline District of Columbia & 0 & 4 & 0 & 0 & 1 & 0 & 5 \\
\hline Florida & 2 & 42 & 0 & 265 & 5 & 0 & 314 \\
\hline Georgia & 1 & 70 & 0 & 111 & 25 & 3 & 210 \\
\hline Hawaii & 0 & 0 & 0 & 0 & 3 & 0 & 4 \\
\hline Idaho & 0 & 7 & 1 & 44 & 1 & 1 & 54 \\
\hline Illinois & 1 & 24 & 3 & 188 & 2 & 0 & 218 \\
\hline Indiana & 0 & 38 & 1 & 137 & 1 & 3 & 180 \\
\hline Iowa & 0 & 5 & 4 & 133 & 1 & 0 & 143 \\
\hline Kansas & 0 & 5 & 1 & 58 & 0 & 1 & 65 \\
\hline Kentucky & 0 & 9 & 2 & 46 & 0 & 0 & 57 \\
\hline Louisiana & 0 & 15 & 0 & 55 & 0 & 0 & 70 \\
\hline Maine & 0 & 1 & 0 & 20 & 0 & 0 & 21 \\
\hline Maryland & 1 & 27 & 0 & 26 & 1 & 3 & 58 \\
\hline Massachusetts & 0 & 17 & 0 & 53 & 4 & 0 & 74 \\
\hline Michigan & 2 & 32 & 2 & 205 & 10 & 2 & 253 \\
\hline Minnesota & 0 & 14 & 9 & 169 & 0 & 1 & 193 \\
\hline Mississippi & 0 & 3 & 0 & 83 & 0 & 0 & 86 \\
\hline Missouri & 0 & 10 & 3 & 134 & 0 & 0 & 147 \\
\hline Montana & 0 & 11 & 0 & 82 & 0 & 1 & 94 \\
\hline Nebraska & 0 & 6 & 6 & 77 & 0 & 0 & 90 \\
\hline Nevada & 0 & 18 & 0 & 46 & 0 & 0 & 64 \\
\hline New Hampshire & 0 & 2 & 0 & 42 & 1 & 0 & 45 \\
\hline New Jersey & 0 & 22 & 0 & 45 & 0 & 0 & 67 \\
\hline New Mexico & 0 & 17 & 0 & 102 & 0 & 1 & 120 \\
\hline New York & 13 & 58 & 0 & 126 & 4 & 0 & 201 \\
\hline N. Carolina & 0 & 9 & 0 & 98 & 7 & 0 & 114 \\
\hline N. Dakota & 0 & 4 & 1 & 23 & 0 & 0 & 28 \\
\hline Ohio & 0 & 49 & 0 & 119 & 1 & 1 & 170 \\
\hline Oklahoma & 0 & 60 & 0 & 78 & 0 & 0 & 138 \\
\hline Oregon & 0 & 9 & 0 & 42 & 0 & 1 & 52 \\
\hline Pennsylvania & 0 & 59 & 0 & 184 & 1 & 1 & 245 \\
\hline Rhode Island & 0 & 4 & 0 & 6 & 0 & 0 & 10 \\
\hline S. Carolina & 0 & 4 & 0 & 94 & 1 & $\overline{0}$ & 99 \\
\hline S. Dakota & 0 & 4 & 5 & 45 & 0 & 0 & 54 \\
\hline Tennessee & 1 & 5 & 0 & 109 & 2 & 0 & 117 \\
\hline Texas & 0 & 73 & 0 & 890 & 2 & 15 & 980 \\
\hline Utah & 0 & 62 & 0 & 35 & 0 & 1 & 98 \\
\hline Vermont & 0 & 1 & 0 & 39 & 7 & 0 & 47 \\
\hline Virginia & 0 & 27 & 0 & 69 & 18 & 3 & 117 \\
\hline Washington & 1 & 28 & 0 & 131 & 6 & 1 & 167 \\
\hline W. Virginia & 0 & 39 & 0 & 24 & 0 & 0 & 63 \\
\hline Wisconsin & 0 & 20 & 1 & 225 & 0 & 0 & 246 \\
\hline Wyoming & 0 & 18 & 0 & 57 & 0 & 2 & 77 \\
\hline Total & 91 & 1,268 & 40 & 5,318 & 486 & 66 & 7,271 \\
\hline
\end{tabular}

Source:

U.S. Department of Energy, Alternative Fuels Data Center web site, http://www.afdc.nrel.gov/newrefuel/state_tot.cgi, February 1999. 
Clean Cities is a locally-based government/industrypartnership, coordinated by the U.S. Department of Energy to expand the use of alternatives to gasoline and dieselfuel. By combining the decision-making with voluntary action by partners, the "grass-roots" approach of Clean Cities departs from traditional "top-down" Federal programs. It establishes a plan, carried out at the local level, for creating a sustainable, nationwide alternative fuels market.

Table 9.6

List of Clean Cities as of 9/1/99 by Designation

1. Atlanta, GA $-9 / 8 / 93$

2. Denver, CO- $9 / 13 / 93$

3. Philadelphia, PA - 9/22/93

4. State of Deleware - 10/12/93

5. Las Vegas, $\mathrm{NV}-10 / 18 / 93$

6. Washington, DC $-10 / 21 / 93$

7. Boston, MA - 3/18/94

8. Austin, TX - 4/18/94

9. Florida Gold Coast $-5 / 3 / 94$

10. Chicago, IL $-5 / 13 / 94$

11. Albuquerque, NM - 6/1/94

12. Wisconsin - SE Area - 6/30/94

13. Colorado Springs, CO - 7/13/94

14. Long Beach, CA - 8/31/94

15. Lancaster, CA $-9 / 22 / 94$

16. Salt Lake City, UT - 1 0/3/94

17. White Plains, NY $-10 / 4 / 94$

18. Baltimore, MD - 10/7/94

19. State of $\mathrm{WV}-10 / 18 / 94$

20. Louisville, KY $-10 / 18 / 94$

21. Rogue Valley, OR - 1 1/10/94

22. San Francisco, CA - 10/21/94

23. Sacramento, CA - 10/21/94

24. South Bay (San Jose), CA - 10/21/94

25. East Bay, CA - 10/21/94

26. San Joaquin Valley, CA - 10/21/94

27. Western New York $-11 / 4 / 94$

28. Portland, OR - 1 1/10/94

29. St. Louis, MO - 1 1/1 8/94

30. Waterbury, CT $-11 / 21 / 94$

31. Connecticut Southwestern Area, - 1 1/21/94

32. Norwich, CT - $11 / 22 / 94$

33. New London, CT -1 1/22/94

34. Peoria, IL - $11 / 22 / 94$

35. Kansas - SW Area - 3/30/95

36. Central New York $-6 / 15 / 95$

37. Dallas/Ft. Worth, TX $-7 / 25 / 95$
38. Honolulu, HI - 8/29/95

39. Missoula, MT $-9 / 21 / 95$

40. New Haven, CT $-10 / 5 / 95$

41. Central Arkansas - $10 / 25 / 95$

42. Paso Del Norte $-11 / 17 / 95$

43. Pittsburgh, PA - 12/5/95

44. S. California Assn. Gov. - 3/1/96

45. Los Angeles, CA - 3/22/96

46. Coachella Valley, CA - 4/22/96

47. Weld/Larimer/Rocky Mountain National Park - 5/2 1/96

48. Central Oklahoma $-5 / 29 / 96$

49. Hampton Roads, VA -10/4/96

50. San Diego, CA $12 / 12 / 96$

51. Long Island, $\mathrm{NY}-1 \mathrm{O} / \mathrm{l} 8 / 96$

52. Detroit, MI/Toronto, $\mathrm{ON}-12 / 18 / 96$

53. Cincinatti, $\mathrm{OH}-1 / 29 / 97$

54. Evansville, IN $-1 / 30 / 97$

55. Houston, TX $-9 / 4 / 97$

56. Portland, ME $-9 / 4 / 97$

57. Tulsa, OK $-9 / 22 / 97$

58. Maricopa Assn. of Govts. - 10/8/97

59. Riverside, CA - 10/24/97

60. North Jersey, NJ $-10 / 31 / 97$

61. Corpus Christi, TX $-3 / 30 / 98$

62. Genesee Region, Rochester, NY - 5/28/98

63. Red River Valley/Grand Forks, ND - 8/10/98

64. Puget Sound, WA $-8 / 13 / 98$

65. Providence, RI - 9/14/98

66. Omaha, NE - 9/18/98

67. Kansas City, KS/MO - 1 1/18/98

68. Central Indiana CC Aliance, IN - 3/4/99

69. Ann Arbor, MI - 4/19/99

70. Capital District (Albany), NY - 4/26/99

71. South Shore, IN $-6 / 15 / 99$

72. Capital Clean Cities of CT $-6 / 21 / 99$

For more information, contact the Clean Cities Hotline at (800) CCITIES, or write to: U.S. Department of Energy, EE-33, Clean Cities Program, 1000 Independence Avenue SW, Washington, DC 20585.

Source:

U.S. Department of Energy, Alternative Fuel Information, Clean Cities: Guide to Alternative Fuel Vehicle Incentives \& Laws, Washington, DC, November 1996, and updates from web site, February 1999. (Additional resources: http://www.ccities.doe.gov) 
Figure 9.1 Map of Clean Cities as of 9/1/99

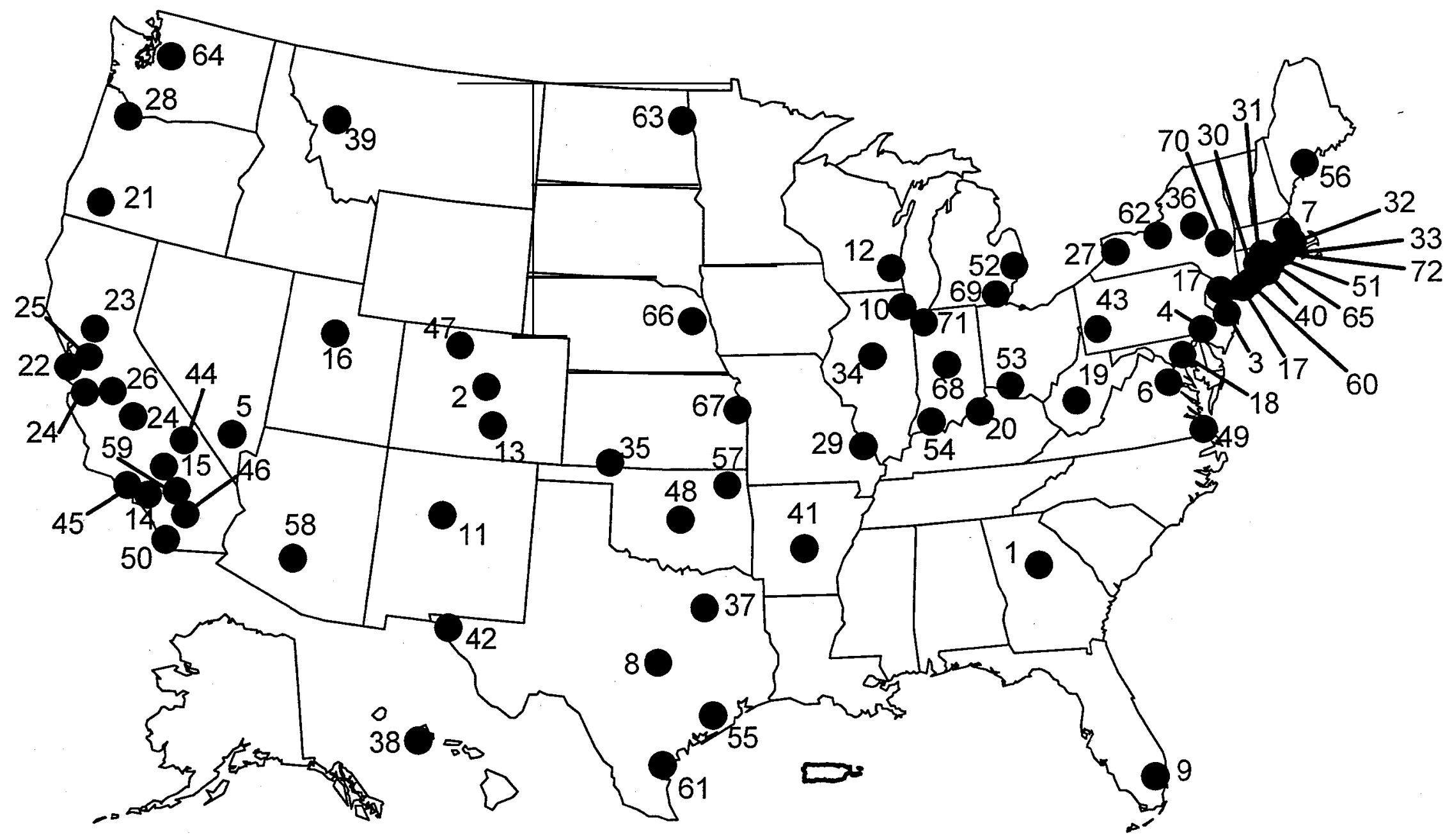

\section{Source:}

U.S. Department of Energy, Alternative Fuel Information, Clean Cities: Guide to Alternative Fuel Vehicle Incentives \& Laws, Washington, DC, November 1996, and updates from the web site, February 1999. (Additional resources: http://www.ccities.doe.gov) 


\section{U.S. ADVANCED BATTERY CONSORTIUM}

Electric and hybrid-electric vehicles are the subject of intense research and development because they are required to be sold in California (10\% in 2003) under the California LowEmission Vehicle (LEV) program. Other states, such as New York and Massachusetts, have indicated that they will also enforce the LEV program. One of the greatest advantages in using electric vehicles is that there are no tailpipe emissions. The U.S. Advanced Battery Consortium (USABC) was established in January 1991 to concentrate efforts on battery development for future electric vehicles. The USABC consists of the Big Three U.S. auto manufacturers (Chrysler, Ford, General Motors), the Electric Power Research Institute, and the U.S. Department of Energy. Five major U.S. electric utilities are also direct participants in USABC.

The USABC has established research contracts with several companies for the development of advanced batteries. Also, a series of Cooperative Research and Development Agreements (CRADAs) with several DOE National Laboratories have been established.

Table 9.7

U.S. Advanced Battery Consortium Research Agreements, Phase II

\begin{tabular}{r}
\hline $\begin{array}{r}\text { Research contracts } \\
\text { General Motors-Ovonic Joint } \\
\text { Venture }\end{array}$ \\
$\begin{array}{l}\text { Cost reduction program for nickel-metal hydride } \\
\text { battery and testing of nickel-metal hydride pilot } \\
\text { production modules (Completed 1998) }\end{array}$ \\
SAFT $\begin{array}{l}\text { Cost reduction program for nickel-metal hydride } \\
\text { battery (Completed 1998) }\end{array}$ \\
3M Hydro-Quebec \\
Phase II development of lithium-polymer battery \\
Duracell/VARTA $\begin{array}{l}\text { Phase II development of lithium-ion battery } \\
\text { (Completed 1999) }\end{array}$ \\
CRADAs for advanced battery testing \\
Argonne National Laboratory, Argonne, IL \\
Sandia National Laboratory, Albuquerque, NM \\
Idaho National Engineering and Environmental Laboratory, Idaho Falls, ID
\end{tabular}

Source:

U.S. Advanced Battery Consortium, February, 1999. 
Today's lead acid batteries provide 30-40 watt hours per kilogram, cost between \$50-150 per kilowatt hour, and have a two-to three-year lifetime. However, the batteries currently used in electric vehicles do not provide the energy or performance sufficient to make these vehicles competitive with gasoline-fueled vehicles. When attained, the Advanced Battery Technology goals will effectively double the range and performance of electric vehicles compared to the range and performance possible with today's battery technology.

Table 9.8 U.S. Advanced Battery Consortium Goals for Electric Vehicle Batteries

\begin{tabular}{|c|c|c|}
\hline Primary criteria & $\begin{array}{l}\text { Mid-term goals } \\
\text { (1997) }\end{array}$ & $\begin{array}{l}\text { Long-term goals }{ }^{\mathbf{a}} \\
\text { (2000) }\end{array}$ \\
\hline Power density ${ }^{\mathrm{b}} \mathrm{W} / \mathrm{L}$ & 250 & 460 \\
\hline Specific power ${ }^{b} \mathrm{~W} / \mathrm{kg}(80 \% \mathrm{DOD} / 30 \mathrm{sec})$ & 150 (200 desired $)$ & 300 \\
\hline Energy density ${ }^{b} \mathrm{Wh} / \mathrm{L}(\mathrm{C} / 3$ discharge rate $)$ & 135 & 230 \\
\hline Specific energy ${ }^{\mathrm{b}}$ Whikg $(\mathrm{C} / 3$ discharge rate $)$ & 80 (100 desired) & 150 \\
\hline Life (years) & 5 & 10 \\
\hline Cycle life ${ }^{\mathrm{b}}$ (cycles) (80\% DOD) & 800 & $\begin{array}{c}1000 \\
1800(@ 50 \% \text { DOD }) \\
2670(@ 30 \% \text { DOD })\end{array}$ \\
\hline Power and capacity degradation ${ }^{\mathrm{b}}(\%$ of rated spec) & $20 \%$ & $20 \%$ \\
\hline Ultimate price' $(\$ / \mathrm{kWh})(10,000$ units @ $40 \mathrm{kWh})$ & $<\$ 150$ & $<\$ 150($ desired to 75$)$ \\
\hline Operating environment & -30 to $65^{\circ} \mathrm{C}$ & -30 to $65^{\circ} \mathrm{C}$ \\
\hline Recharge time $\mathrm{b}^{\mathrm{b}}$ & $<6$ hours & $<6$ hours \\
\hline Continuous discharge in 1 hour (no failure) & $\begin{array}{l}75 \% \text { (of rated energy } \\
\text { canacitv) }\end{array}$ & $\begin{array}{l}75 \% \text { (of rated energy } \\
\text { canacitv) }\end{array}$ \\
\hline \multicolumn{3}{|l|}{ Secondary criteria } \\
\hline Efficiency $(\mathrm{C} / 3 \text { discharge } \& \mathrm{C} / 3 \text { charge })^{\mathrm{d}}$ & $75 \%$ & $80 \%$ \\
\hline Self discharge ${ }^{\mathrm{b}}$ & $<15 \%$ in 48 hours & $<20 \%$ in 12 days \\
\hline Maintenance & $\begin{array}{l}\text { No maintenance. Service } \\
\text { by qualified personnel } \\
\text { only. }\end{array}$ & $\begin{array}{l}\text { No maintenance. } \\
\text { Service by qualified } \\
\text { personnel only. }\end{array}$ \\
\hline Thermal loss ${ }^{b}$ & $\begin{array}{l}3.2 \mathrm{~W} / \mathrm{kWh} ; 15 \% \text { of } \\
\text { capacity; } 48 \text { hour period }\end{array}$ & $\begin{array}{l}\text { Covered by self } \\
\text { discharge }\end{array}$ \\
\hline Abuse resistance $^{\mathrm{b}}$ & $\begin{array}{l}\text { Tolerant } \\
\text { Minimized by on-board } \\
\text { controls }\end{array}$ & $\begin{array}{l}\text { Tolerant } \\
\text { Minimized by on-board } \\
\text { controls }\end{array}$ \\
\hline
\end{tabular}

Source:

U.S. Department of Energy, Office of Transportation Technologies, Washington, DC, February, 1998.

Note:

$\mathrm{W}=$ watt; kg-kilogram; $\mathrm{L}=$ liter; $\mathrm{DOD}=$ depth of discharge; $\mathrm{Wh}=$ watt-hour; $\mathrm{kWh}=$ kilowatt-hour.

"For interim commercialization (Reflects USABC revisions of September 1996).

'Specifics on criteria can be found in "USABC Electric Vehicle Battery Test Procedures Manual Revision 2" DOE/ID-10479, Rev. 2, January 1996.

'Cost to the Original Equipment Manufacturers.

${ }^{\mathrm{d}}$ Roundtrip charge/discharge efficiency. 


\section{Chapter 10 \\ Fleet Vehicles and Characteristics}

Summary Statistics from Tables/Figures in this Chapter

\begin{tabular}{clc}
\hline Source & & \\
\hline Figure 10.1 & Fleet automobiles, 1998 & $4,852,000$ \\
Figure 10.1 & Fleet Class 1-5 Trucks, 1998 & $4,224,000$ \\
Table 10.4 & Average annual miles per automobile & \\
& Business fleets & 29,200 \\
Uable 10.5 & Fedility fleets & 14,500 \\
& Government fleets & 13,700 \\
& Automobiles & 550,373 \\
& Buses & 113,366 \\
& Light trucks & 6,376 \\
& Medium trucks & 381,525 \\
& Heavy trucks & 32,281 \\
& & 16,825 \\
\hline
\end{tabular}


Significant changes have been made in recent years to fleet vehicle estimations. Newly available data improve the accuracy of fleet vehicle estimates but, at the same time, make it impossible to compare the data historically. Therefore, only the 1998 data arepresented here.

Figure 10.1. Fleet Vehicles in Service as of January 1, 1998

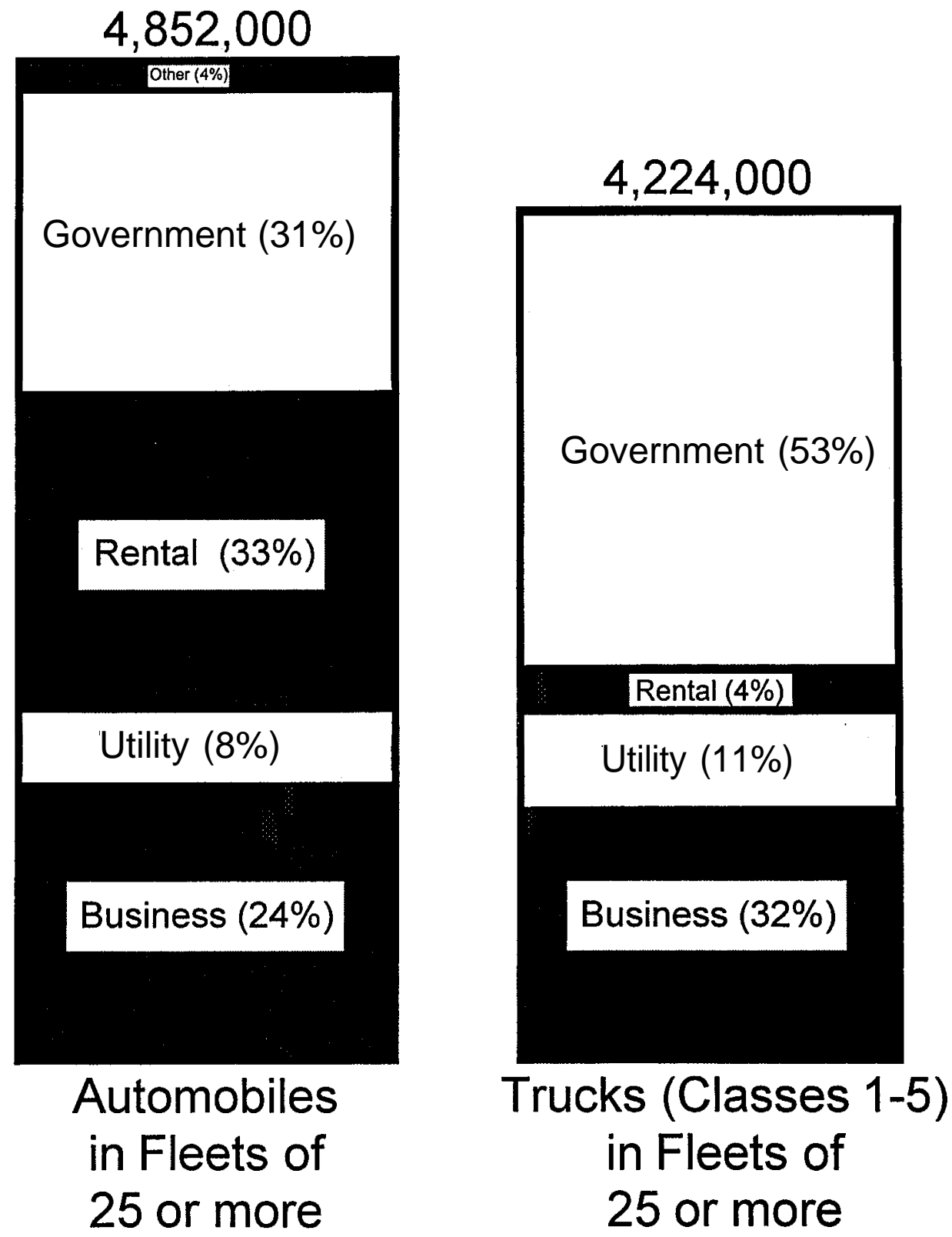

Source:

Bobit Publishing Company, Automotive Fleet Research Department, Automotive Fleet Factbook 1998,

Redondo Beach, CA, 1998. (Additional resources: http://www.fleet-central.com) Note:

Truck classes 1-5 are 19,500 lbs. and less. 
These are the top ten states in terms of fleets andjleet vehicles, according to Dwight's Energydata, Denver, CO. Autos and light trucks make up the largest share of fleet vehicles in each of the states. The average number of vehicles per fleet is the highest in Florida and California.

Table 10.1

Top Ten States with Fleets of Ten Vehicles or More, 1999

\begin{tabular}{lccccccc}
\hline & \multirow{2}{*}{$\begin{array}{c}\text { Fleets of ten } \\
\text { vehicles or }\end{array}$} & \multicolumn{5}{c}{ Fleet vehicles } & \multicolumn{2}{c}{$\begin{array}{c}\text { Average } \\
\text { vehicles per } \\
\text { States }\end{array}$} & more & Autos & Trucks & Trucks & Trucks & Tlass 1-2) & (class 3-5) & (class 6-8) & Total & fleet $^{\mathbf{2}}$ \\
\hline California & 12,005 & 474,627 & 443,869 & 205,883 & 321,332 & $1,457,716$ & 121 \\
Texas & 8,851 & 260,885 & 262,270 & 107,599 & 247,960 & 887,565 & 100 \\
New York & 6,706 & 227,144 & 191,415 & 78,215 & 182,015 & 685,495 & 102 \\
Pennsylvania & 5,973 & 166,880 & 148,086 & 52,840 & 179,086 & 552,865 & 93 \\
Florida & 5,986 & 233,209 & 208,919 & 88,230 & 199,408 & 735,752 & 123 \\
Illinois & 5,653 & 178,939 & 149,886 & 76,441 & 179,770 & 590,689 & 104 \\
Ohio & 5,418 & 177,830 & 130,846 & 60,350 & 163,627 & 538,071 & 99 \\
Michigan & 3,945 & 149,536 & 103,684 & 53,670 & 104,373 & 415,208 & 105 \\
New Jersey & 3,919 & 139,327 & 121,717 & 35,172 & 119,839 & 419,974 & 107 \\
North Carolina & 3,821 & 102,047 & 109,667 & 38,342 & 142,354 & 396,231 & 104 \\
\hline
\end{tabular}

\section{Source:}

Bobit Publishing Company, Automotive Fleet Industry Statistics web site:

$\mathrm{http} / \mathrm{wwww}$. fleet-central.com/AF/Resources/Stats/chart3 .htm. Original data source: Dwight's Energydata, Denver, CO. (Additional resources: http://www.fleet-central.com/AF)

"Calculated as the ratio of total fleet vehicles to total fleets. 
These fleet data, which were generatedfrom a 1991-92 ORNL study, are still the latest available data of this kind.

Table 10.2

Fleet Vehicle Composition by Vehicle Type, 1991

(percent)

\begin{tabular}{lccccc}
\hline \multicolumn{1}{c}{ Fleet type } & Cars & $\begin{array}{c}\text { Light trucks" } \\
\text { and vans }\end{array}$ & $\begin{array}{c}\text { Medium } \\
\text { trucks }^{\mathbf{b}}\end{array}$ & $\begin{array}{c}\text { Heavy } \\
\text { trucks }^{\mathrm{c}}\end{array}$ & Total \\
\hline Business & $24.2 \%$ & $21.1 \%$ & $45.8 \%$ & $8.9 \%$ & $100 \%$ \\
Utility & $22.6 \%$ & $39.0 \%$ & $15.0 \%$ & $23.4 \%$ & $100 \%$ \\
Government & $48.5 \%$ & $42.8 \%$ & $6.8 \%$ & $1.8 \%$ & $100 \%$ \\
\hline
\end{tabular}

Table 10.3

Average Length of Time Fleet Vehicles are Kept Before Sold to Others, 1991

(months)

\begin{tabular}{lcrc}
\hline & Business & Utility & Government \\
\hline Cars & 35 & 68 & 81 \\
Light trucks $^{\mathbf{a}}$ & 56 & 60 & 82 \\
Medium trucks $^{\mathbf{b}}$ & 83 & 86 & 96 \\
Heavy trucks $^{\mathrm{b}}$ & 103 & 132 & 117 \\
\hline
\end{tabular}

Table 10.4

Average Annual and Daily Vehicle-Miles of Travel for Fleet Vehicles, 1991

\begin{tabular}{|c|c|c|c|c|c|c|}
\hline \multirow[b]{2}{*}{ Vehicle type } & \multicolumn{2}{|c|}{ Business } & \multicolumn{2}{|c|}{ Utility } & \multicolumn{2}{|c|}{ Government } \\
\hline & $\begin{array}{l}\text { Miles/yea } \\
\text { (thousands) }\end{array}$ & $\begin{array}{l}\text { Miles/day } \\
\text { ir @250 } \\
\text { days/year }\end{array}$ & $\begin{array}{l}\text { Miles/year } \\
\text { (thousands) }\end{array}$ & $\begin{array}{c}\text { Miles/day } \\
\text { @250 } \\
\text { days/year }\end{array}$ & $\begin{array}{l}\text { Miles/year } \\
\text { (thousands) }\end{array}$ & $\begin{array}{c}\text { Miles/day } \\
@ 250 \\
\text { days/year }\end{array}$ \\
\hline Cars & 29.2 & 117 & 14.5 & 58 & 13.7 & 55 \\
\hline Light trucks $\mathrm{s}^{\mathrm{a}}$ & 26.6 & 106 & 17.5 & 70 & 13.9 & 56 \\
\hline Medium trucks ${ }^{b}$ & 17.5 & 70 & 11.8 & 47 & 11.9 & 48 \\
\hline Heavy trucks ${ }^{\mathfrak{c}}$ & 64.4 & 258 & 13.8 & 55 & 10.7 & 43 \\
\hline
\end{tabular}

Source:

Miaou, S. P., et. al., Fleet Vehicles in the United States: Composition, Operating Characteristics, and Fueling Practices, (ORNL-6717), Oak Ridge National Laboratory, Oak Ridge, TN, May 1992.

(Additional resources: http://www-cta.ornl.gov)

${ }^{2}$ In this study, light trucks are $<8,500 \mathrm{lbs}$ gross vehicle weight.

'In this study, medium trucks are between 8,500-26,000 lbs gross vehicle weight.

"In this study, heavy trucks are $>26,000$ lbs gross vehicle weight. 
Figure 10.2. Worldwide Federal Inventory, 1992-97

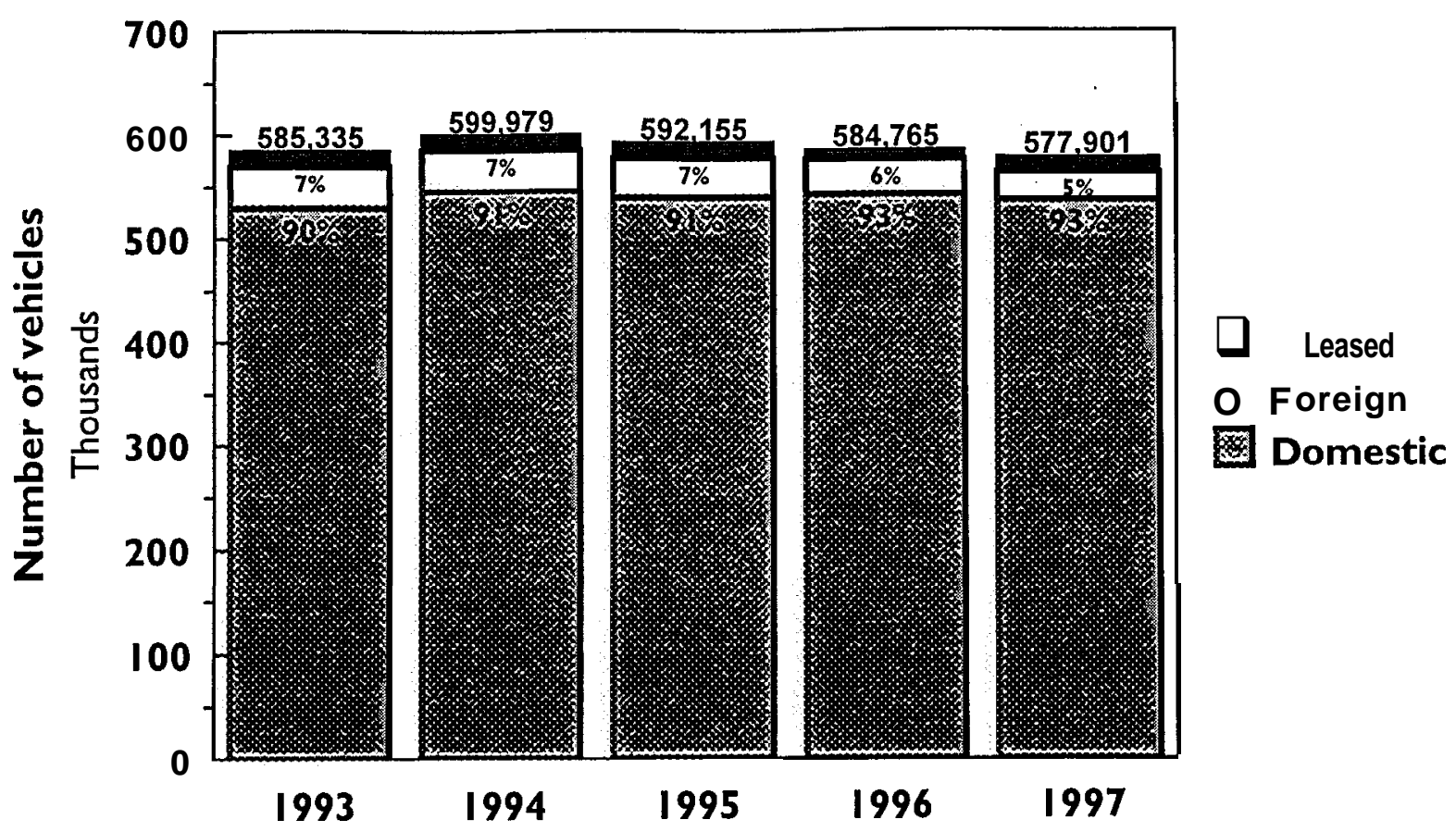

Source:

U.S. General Services Administration, Federal Vehicle Policy Division, FY 1997 Federal Fleet Report, Washington, DC, 1999, Tables 1 and 12.

(Additional resources: http://policyworks.gov/org/main/mt/homepage/mtv/mtvhp.htm)

Figure 10.3. Average Miles per Federal Vehicle by Vehicle Type, 1997

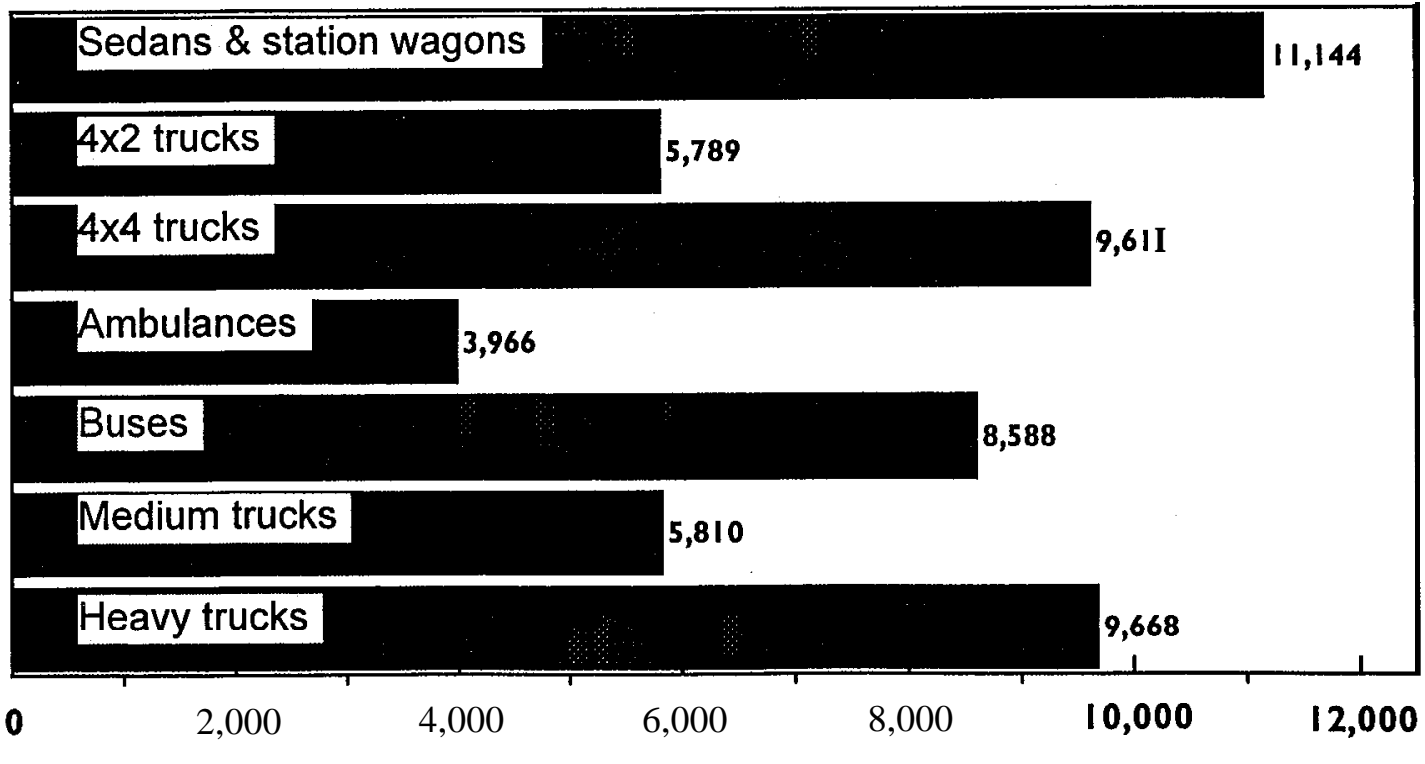

S o u r c e :

Average Miles per Vehicle

U.S. General Services Administrations, Federal Vehicle Policy Division, FY 1997 Federal Fleet Report, Washington, DC, 1999, Table 5.

(Additional resources: http://policyworks.gov/org/main/mt/homepage/mtv/mtvhp.htm) 
Table 10.5

Federal Government Vehicles by Agency, Fiscal Year 1997”

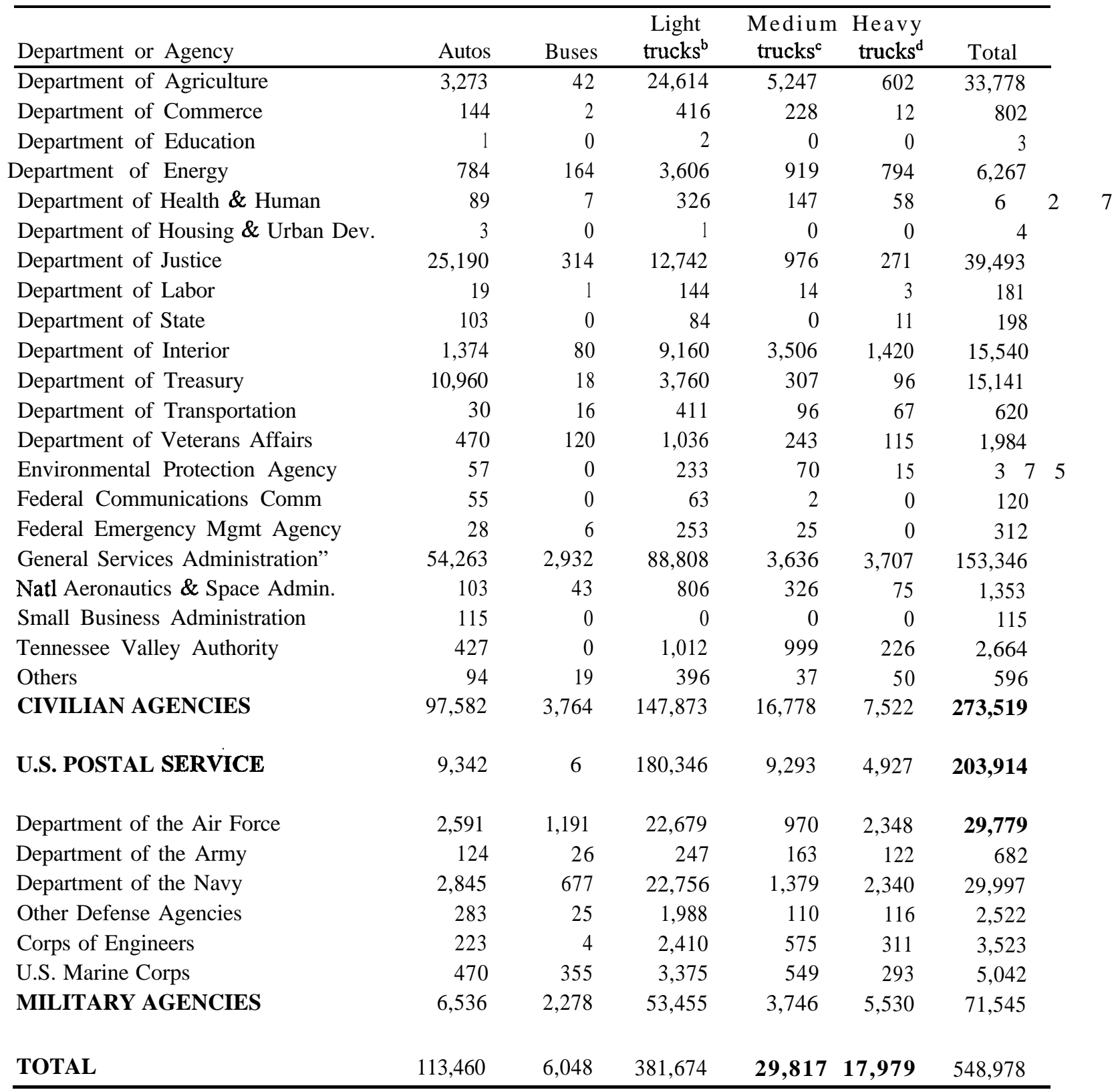

Source:

U.S. General Services Administration, Federal Supply Service, FY 1997 Federal Fleet Report, Washington, DC, 1999,

Table 14. (Additional resources: http://policyworks.gov/org/main/mt/homepage/mtv/mtvhp.htm)

a Federally-owned and commercially-leased vehicles.

b Less than 8,500 Ibs GVWR. Includes ambulances.

c 8,501-23,999 lbs GVWR.

d 24,000 lbs. Or more GVWR.

e GSA Fleet vehicles. 
Table 10.6

Federal Fleet Vehicle Acquisitions by Fuel Type, FY 1997"

\begin{tabular}{lc}
\hline & Vehicle \\
\hline Gasoline & 14,097 \\
Diesel & 489 \\
Natural gas & 172 \\
E-85 & 160 \\
Electricity & 139 \\
Other & 12 \\
M-85 & 9 \\
LPG & 1 \\
Biodiesel & 0 \\
Hydrogen & 0 \\
\hline Total & 15,079 \\
\hline
\end{tabular}

Source:

U.S. General Services Administrations, Federal Vehicle Policy Division, FY 1997 Federal Fleet Report, Washington, DC, 1999, Table 18.

(Additional resources: http://policyworks.gov/org/main/mt/homepage/mtv/mtvhp.htm)

Table 10.7

Fuel Consumed by Federal Government Fleets, FY 1997’

\begin{tabular}{lc}
\hline & $\begin{array}{c}\text { Thousand gasoline } \\
\text { equivalent gallons }\end{array}$ \\
\hline Gasoline & 280,051 \\
Diesel & 64,834 \\
NG & 4,076 \\
Electricity & 287 \\
Biodiesel & 186 \\
Methanol & 151 \\
M-85 & 137 \\
LPG & 37 \\
Ethanol & 19 \\
\hline Total & 349,780 \\
\hline
\end{tabular}

Source:

U.S. General Services Administrations, Federal Vehicle Policy Division, FY 1997 Federal Fleet Report, Washington, DC, 1999, Table 6.

(Additional resources: http://policyworks.gov/org/main/mt/homepage/mtv/mtvhp.htm)

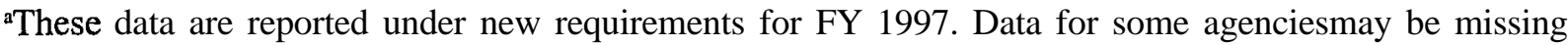
or incomplete. 
The Energy Policy Act of 1992 (EPACT) set alternative fuel vehicle acquisition requirements for Federal and State Governments, fuel providers and the private sector. Additional rule making has adjusted the original purchase requirements. State government andfuelproviders requirements began in 1997.

Table 10.8

Energy Policy Act Purchase Requirements of Light Alternative Fuel Vehicles

\begin{tabular}{lcccc}
\hline Year & F e d e r a & State & $\begin{array}{c}\text { Fuel } \\
\text { providers }\end{array}$ & Private $^{\mathrm{a}}$ \\
\hline 1993 & 5,000 & - & & - \\
1994 & 7,500 & - & - & - \\
1995 & 10,000 & - & - & \\
1996 & $25 \%$ & & & \\
1997 & $33 \%$ & $10 \%$ & $30 \%$ & \\
1998 & $50 \%$ & $15 \%$ & $50 \%$ & \\
1999 & $75 \%$ & $25 \%$ & $70 \%$ & \\
2000 & $75 \%$ & $50 \%$ & $90 \%$ & \\
2001 & $75 \%$ & $75 \%$ & $90 \%$ & $40 \%$ \\
2002 & $75 \%$ & $75 \%$ & $90 \%$ & $60 \%$ \\
2003 & $75 \%$ & $75 \%$ & $90 \%$ & $70 \%$ \\
2004 & $75 \%$ & $75 \%$ & $90 \%$ & $70 \%$ \\
2005 & $75 \%$ & $75 \%$ & $90 \%$ & \\
$2006-$ on & $75 \%$ & $75 \%$ & $90 \%$ & \\
\hline
\end{tabular}

\section{Source:}

Final rule for the alternative fuels transportation programs, Federal Register, Vol. 61, p. 10622, March 14, 1996.

Private alternative fueled vehicle acquisition requirements for private and local government fleets, Federal Register, vol. 62, p. 19701, April 23, 1997.

Note:

The Department of Energy has provided an Alternative Fuel Vehicles Acquisitions and Credits Database on the Internet to provide fleet managers with a convenient way to report their compliance with this mandate. (http://www.ott.doe.gov/credits)

"Additional rule making is required by January 1, 2000, for private AFV requirements to take effect. 


\section{Chapter 11 Household Vehicles and Characteristics}

Summary Statistics from Tables/Figures in this Chapter

\begin{tabular}{|c|c|c|}
\hline Source & & \\
\hline Table 11.1 & Vehicles pkicensed driver, 1997 & 1.10 \\
\hline Table 11.2 & Average household transportation expense, 1997 & $18.5 \%$ \\
\hline \multirow[t]{5}{*}{ Table 11.8} & Share of households owning 3 or more vehicles & \\
\hline & 1960 & $2.5 \%$ \\
\hline & 1970 & $5.5 \%$ \\
\hline & 1980 & $17.5 \%$ \\
\hline & 1990 & $17.3 \%$ \\
\hline Table 11.12 & Average annual miles per household vehicle, 1995 & 11,800 \\
\hline \multirow[t]{5}{*}{ Figure 11.1} & Average occupancy rates by vehicle type, 1995 & \\
\hline & Automobile & 1.6 \\
\hline & Pickup truck & 1.4 \\
\hline & Sports Utility & 1.7 \\
\hline & $\operatorname{Van}$ & 2.1 \\
\hline Table 11.13 & Share of workers who car pooled, 1990 & $13.4 \%$ \\
\hline \multirow[t]{3}{*}{ Figure 11.3} & Long-distance trips in the U.S., 1995 & \\
\hline & Trips & 1,001 million \\
\hline & Person-miles & 827 billion \\
\hline
\end{tabular}


Table 11.1

Population and Vehicle Profile, 1950-97

\begin{tabular}{|c|c|c|c|c|c|c|c|c|c|c|c|}
\hline Year & $\begin{array}{c}\text { Resident } \\
\text { population" } \\
\text { (thousands) }\end{array}$ & $\begin{array}{c}\text { Total } \\
\text { households } \\
\text { (thousands) }\end{array}$ & $\begin{array}{l}\text { Number of } \\
\text { vehicles in } \\
\text { operation } \\
\text { (thousands) }\end{array}$ & $\begin{array}{c}\text { Total } \\
\text { vehicle-miles } \\
\text { (millions) }\end{array}$ & $\begin{array}{c}\text { Number of } \\
\text { licensed } \\
\text { drivers } \\
\text { (thousands) }\end{array}$ & $\begin{array}{l}\text { Number of } \\
\text { civilian } \\
\text { employed } \\
\text { persons } \\
\text { (thousands) }\end{array}$ & $\begin{array}{l}\text { Vehicles } \\
\text { per } \\
\text { capita } \\
\end{array}$ & $\begin{array}{l}\text { Vehicle- } \\
\text { miles } \\
\text { per } \\
\text { capita } \\
\end{array}$ & $\begin{array}{l}\text { Licensed } \\
\text { drivers } \\
\text { per } \\
\text { household }\end{array}$ & $\begin{array}{l}\text { Vehicles } \\
\text { per } \\
\text { licensed } \\
\text { driver } \\
\end{array}$ & $\begin{array}{c}\text { Vehicles } \\
\text { per } \\
\text { civilian } \\
\text { employed } \\
\text { persons }\end{array}$ \\
\hline 1950 & 151,868 & 43,554 & 43,256 & 458,246 & 62,194 & 58,918 & 0.28 & 3,029 & 1.43 & 0.70 & 0.73 \\
\hline 1955 & 165,069 & 47,874 & 55,804 & 605,646 & 74,686 & 62,170 & 0.34 & 3,656 & 1.56 & 0.75 & 0.90 \\
\hline 1960 & 179,979 & 52,799 & 66,582 & 718,762 & 87,253 & 65,778 & 0.37 & 3,994 & 1.65 & 0.76 & 1.01 \\
\hline 1965 & 193,526 & 57,251 & 82,067 & 887,812 & 98,502 & 71,088 & 0.42 & 4,587 & 1.72 & 0.83 & 1.15 \\
\hline 1970 & 203,984 & 63,401 & 98,136 & $1,109,724$ & 111,543 & 78,678 & 0.48 & 5,440 & 1.76 & 0.88 & 1.25 \\
\hline 1975 & 215,465 & 71,120 & 120,054 & $1,327,664$ & 129,791 & 85,846 & 0.56 & 6,162 & 1.82 & 0.92 & 1.40 \\
\hline 1980 & 227,225 & 80,776 & 139,832 & $1,527,295$ & 145,295 & 99,303 & 0.62 & 6,722 & 1.80 & 0.96 & 1.41 \\
\hline 1981 & 229,466 & 82,368 & 141,908 & $1,555,308$ & 147,075 & 100,397 & 0.62 & 6,778 & 1.79 & 0.96 & 1.41 \\
\hline 1982 & 231,664 & 83,527 & 143,854 & $1,595,010$ & 150,234 & 99,526 & 0.62 & 6,885 & 1.80 & 0.96 & 1.45 \\
\hline 1983 & 233,792 & 83,918 & 147,104 & $1,652,788$ & 154,389 & 100,834 & 0.63 & 7,069 & 1.84 & 0.95 & 1.46 \\
\hline 1984 & 235,825 & 85,407 & 152,162 & $1,720,269$ & 155,424 & 105,005 & 0.65 & 7,295 & 1.82 & 0.98 & 1.45 \\
\hline 1985 & 237,924 & 86,789 & 157,048 & $1,774,826$ & 156,868 & 107,150 & 0.66 & 7,460 & 1.81 & 1.00 & 1.47 \\
\hline 1986 & 240,133 & 88,458 & 162,094 & $1,834,872$ & 159,487 & 109,597 & 0.68 & 7,641 & 1.80 & 1.02 & 1.48 \\
\hline 1987 & 242,289 & 89,479 & 167,193 & $1,921,204$ & 161,975 & 112,440 & 0.69 & 7,929 & 1.81 & 1.03 & 1.49 \\
\hline 1988 & 244,499 & 91,061 & 171,741 & $2,025,962$ & 162,853 & 114,968 & 0.70 & 8,286 & 1.79 & 1.05 & 1.49 \\
\hline 1989 & 246,819 & 92,830 & 175,960 & $2,096,487$ & 165,555 & 117,342 & 0.71 & 8,494 & 1.78 & 1.06 & 1.50 \\
\hline 1990 & 249,440 & 93,347 & 179,299 & $2,144,362$ & 167,015 & 118,793 & 0.72 & 8,597 & 1.79 & 1.07 & 1.51 \\
\hline 1991 & 252,124 & 94,312 & 181,438 & $2,172,050$ & 168,995 & 117,718 & 0.72 & 8,615 & 1.79 & 1.07 & 1.54 \\
\hline 1992 & 255,002 & 95,689 & 181,519 & $2,247,151$ & 173,125 & 118,492 & 0.71 & 8,782 & 1.81 & 1.05 & 1.53 \\
\hline 1993 & 257,753 & 96,391 & 186,315 & $2,296,378$ & 173,149 & 120,259 & 0.72 & 8,909 & 1.80 & 1.08 & 1.55 \\
\hline 1994 & 260,292 & 97,107 & 188,714 & $2,357,588$ & 175,403 & $123,060 "$ & 0.73 & 9,057 & 1.81 & 1.08 & 1.53 \\
\hline 1995 & 262,761 & 98,990 & 193,441 & $2,422,696$ & 176,628 & $124,900^{\mathrm{b}}$ & 0.74 & 9,220 & 1.78 & 1.10 & 1.55 \\
\hline 1996 & 265,179 & 99,627 & 198,294 & $2,485,848$ & 179,539 & $126,708^{b}$ & 0.75 & 9,374 & 1.80 & 1.10 & 1.56 \\
\hline \multirow[t]{2}{*}{1997} & 267,636 & 101,018 & 201,071 & $2,560,373$ & 182,709 & $129,558^{b}$ & 0.75 & 9,567 & 1.81 & 1.10 & 1.55 \\
\hline & \multicolumn{11}{|c|}{ Average annual percentage change } \\
\hline 1950-97 & $1.2 \%$ & $1.8 \%$ & $3.3 \%$ & $3.7 \%$ & $2.3 \%$ & $1.7 \%$ & $2.1 \%$ & $2.5 \%$ & $0.5 \%$ & $1.0 \%$ & $1.6 \%$ \\
\hline$\underline{1987-97}$ & $1.0 \%$ & $1.2 \%$ & $1.9 \%$ & $2.9 \%$ & $1.2 \%$ & $1.4 \%$ & $0.8 \%$ & $1.9 \%$ & $0.0 \%$ & $0.7 \%$ & $0.4 \%$ \\
\hline
\end{tabular}

Resident population, total households, and civilian employed persons - U.S. Department of Commerce, Bureau of the Census, Statistical Abstract of the United States-1998, 118 th edition, Washington, DC, 1998, pp. 8, 61, 403, and annual. (Additional resources: http://www.census.gov)

Vehicles in operation - The Polk Company. FURTHER REPRODUCTION PROHIBITED. (Additional resources: http://www.polk.com)

Licensed drivers and vehicle-miles - U.S. Department of Transportation, Federal Highway Administration, Highway Statistics 1997, Tables DL- 1 C and VM-I, and annual. (Additional resources: http://www.fhwa.dot.gov)

${ }^{2}$ Estimates as of July 1 . Includes Armed Forces stationed in the United States.

bata are not comparable to earlier years due to changes in definitions and methodology. See original source for more details. 
Transportation (18.6\%) is second only to housing (30.6\%) as the largest expenditure for the average household. In 1996, approximately 17\% of

transportation expenditures were for purchasing gasoline and motor oil. There is an average of two vehicles per household.

Table 11.2

Average Annual Expenditures of Households by Income, 1997

\begin{tabular}{|c|c|c|c|c|c|c|c|c|c|c|}
\hline & \multirow[b]{2}{*}{$\begin{array}{c}\text { All } \\
\text { households }\end{array}$} & \multicolumn{9}{|c|}{ Income before taxes } \\
\hline & & $\begin{array}{l}\text { Less than } \\
\$ 5,000\end{array}$ & $\begin{array}{c}\$ 5,000- \\
\$ 9999\end{array}$ & $\begin{array}{c}\$ 10,000- \\
\$ 14949\end{array}$ & $\begin{array}{c}\$ 15,000- \\
\$ 19,999\end{array}$ & $\begin{array}{c}\$ 20,000- \\
\$ 29,999\end{array}$ & $\begin{array}{l}\$ 30,000- \\
\$ 39,999\end{array}$ & $\begin{array}{l}\$ 40,000- \\
\$ 49,999\end{array}$ & $\begin{array}{c}\$ 50,000- \\
\$ 69,999\end{array}$ & $\begin{array}{l}\$ 70,000 \\
\text { and over }\end{array}$ \\
\hline \multirow[t]{2}{*}{ Total expenditures } & $\$ 36,146$ & $" \$ 16,545$ & $\$ 15,154$ & $\$ 18,737$ & $\$ 23,940$ & $\$ 27,836$ & $\$ 34,376$ & $\$ 40,779$ & $\$ 48,417$ & $\$ 71,656$ \\
\hline & \multicolumn{10}{|c|}{ Percentage of total expenditures ${ }^{b}$} \\
\hline Food' & $14.5 \%$ & $17.5 \%$ & $18.1 \%$ & $16.7 \%$ & $16.5 \%$ & $15.7 \%$ & $15.1 \%$ & $14.2 \%$ & $13.7 \%$ & $12.5 \%$ \\
\hline Housing & $31.4 \%$ & $36.2 \%$ & $36.9 \%$ & $36.7 \%$ & $33.6 \%$ & $31.5 \%$ & $31.0 \%$ & $30.3 \%$ & $29.7 \%$ & $30.4 \%$ \\
\hline Apparel and services & $4.9 \%$ & $5.7 \%$ & $5.1 \%$ & $4.1 \%$ & $4.9 \%$ & $4.9 \%$ & $5.2 \%$ & $4.4 \%$ & $5.4 \%$ & $4.8 \%$ \\
\hline Transportation & $18.5 \%$ & $15.4 \%$ & $14.6 \%$ & $16.8 \%$ & $17.7 \%$ & $18.9 \%$ & $20.8 \%$ & $20.7 \%$ & $19.5 \%$ & $17.3 \%$ \\
\hline Vehicle purchases (net outlay) & $7.9 \%$ & $5.1 \%$ & $5.8 \%$ & $6.1 \%$ & $7.2 \%$ & $8.2 \%$ & $9.8 \%$ & $10.0 \%$ & $8.4 \%$ & $6.8 \%$ \\
\hline Gasoline and motor oil & $3.1 \%$ & $3.3 \%$ & $3.2 \%$ & $3.4 \%$ & $3.5 \%$ & $3.5 \%$ & $3.4 \%$ & $3.3 \%$ & $3.2 \%$ & $2.5 \%$ \\
\hline Other vehicle expenditures & $6.4 \%$ & $5.2 \%$ & $4.7 \%$ & $5.8 \%$ & $6.0 \%$ & $6.3 \%$ & $6.7 \%$ & $6.6 \%$ & $6.9 \%$ & $6.5 \%$ \\
\hline Public transportation & $1.1 \%$ & $1.2 \%$ & $1.0 \%$ & $0.8 \%$ & $0.9 \%$ & $0.9 \%$ & $0.8 \%$ & $0.9 \%$ & $1.0 \%$ & $1.5 \%$ \\
\hline Health care & $5.3 \%$ & $5.1 \%$ & $8.0 \%$ & $8.3 \%$ & $7.6 \%$ & $6.9 \%$ & $5.3 \%$ & $5.0 \%$ & $4.6 \%$ & $3.7 \%$ \\
\hline Entertainment & $5.2 \%$ & $4.5 \%$ & $4.3 \%$ & $4.4 \%$ & $4.4 \%$ & $4.6 \%$ & $4.4 \%$ & $5.0 \%$ & $5.5 \%$ & $6.0 \%$ \\
\hline Others $^{d}$ & $9.7 \%$ & $13.8 \%$ & $10.8 \%$ & $9.5 \%$ & $10.7 \%$ & $10.6 \%$ & $9.3 \%$ & $9.6 \%$ & $9.0 \%$ & $9.5 \%$ \\
\hline Households (thousands) & 84,991 & 4,178 & 8,904 & 9,096 & 7,424 & 12,415 & 10,392 & 7,949 & 11,930 & 12,705 \\
\hline Percentage of households & $100 \%$ & $4.9 \%$ & $10.5 \%$ & $10.7 \%$ & $8.7 \%$ & $14.6 \%$ & $12.2 \%$ & $9.4 \%$ & $14.0 \%$ & $14.9 \%$ \\
\hline Average number of vehicles in $\mathrm{HH}$ & 2.0 & 1.0 & 0.9 & 1.3 & 1.7 & 1.8 & 2.2 & 2.4 & 2.7 & 3.0 \\
\hline
\end{tabular}

Source:

U.S. Department of Labor, Bureau of Labor Statistics, Consumer Expenditures in 1997, Washington, DC, February 1999, p. 9. (Additional resources: http://www.bls.gov)

\footnotetext{
${ }^{\text {a }}$ Public assistance monies are included in reported income. Data for those reporting income.

${ }^{\mathrm{b}}$ Percentages may not sum to totals due to rounding.

c Includes alcoholic beverages.

d Includes personal care, reading, education, tobacco and smoking supplies, cash contributions, and miscellaneous items.
} 
Table 11.3

Average Number of Vehicles and Vehicle Travel per Household, 1991 and 1994 RTECS

\begin{tabular}{lcccc}
\hline & \multicolumn{2}{c}{$\begin{array}{c}\text { Average } \\
\text { number of vehicles } \\
\text { per household }\end{array}$} & \multicolumn{2}{c}{$\begin{array}{c}\text { Average } \\
\text { vehicle-miles } \\
\text { per household }\end{array}$} \\
\hline Number of Drivers & $\mathbf{1 9 9 1}$ & $\mathbf{1 9 9 4}$ & $\mathbf{1 9 9 1}$ & $\mathbf{1 9 9 4}$ \\
\hline 1 & 1.2 & 1.2 & 10,900 & 12,300 \\
2 & 2.0 & 2.0 & 21,400 & 23,200 \\
3 & 2.6 & 2.8 & 30,700 & 33,100 \\
4 or more & 3.1 & 3.4 & 36,700 & 43,000 \\
\hline Household size & & & & \\
\hline 1 person & 1.2 & 1.2 & 10,600 & 11,600 \\
2 persons & 1.8 & 1.8 & 17,700 & 20,000 \\
3 persons & 2.0 & 2.1 & 22,300 & 25,200 \\
4 persons & 2.2 & 2.2 & 26,200 & 26,600 \\
5 persons & 2.1 & 2.2 & 23,600 & 26,300 \\
6 or more persons & 1.9 & 2.3 & 22,600 & 30.900 \\
\hline Household urban status & & & & \\
\hline Urban & 1.8 & 1.8 & 18,800 & 20,700 \\
Central city & 1.6 & 1.7 & 15,900 & 18,000 \\
Suburban & 1.9 & 1.9 & 20,400 & 22,300 \\
Rural & 1.9 & 1.9 & 19,500 & 22.500 \\
\hline Household composition & & & & \\
\hline With children & 2.0 & 2.0 & 22,800 & 24,800 \\
Without children & 1.7 & 1.7 & 16,500 & 18.900 \\
\hline Total & $\mathbf{1 . 8}$ & $\mathbf{1 . 8}$ & $\mathbf{1 8 , 9 0 0}$ & $\mathbf{2 1 , 1 0 0}$ \\
\hline & & & & \\
\hline & & & & \\
\hline & & & & \\
\hline
\end{tabular}

Source:

1991-U.S. Department of Energy, Energy Information Administration, Household

Vehicles Energy Consumption 1994, Washington, DC, 1996, pp. 48, 49.

1994-Personal Communication, U.S. Department of Energy, Energy Information Administration, Office of Markets and End Use, Energy End Use Division. (Additional resources: http://www.eia.doe.gov)

"Residential Transportation Energy Consumption Survey. 
Household vehicle ownership shows a dramatic increase from 1960 to 1990. In 1960, nearly 79\% of households owned less than two vehicles; by 1990, it declined to 45\%. Census data prior to 1990 indicated that the majority of households owned one vehicle; in 1990 that changed to two vehicles.

Table 11.4

Household Vehicle Ownership, 1960-90 Census (percentage)

\begin{tabular}{cccccc}
\hline & $\begin{array}{c}\text { No } \\
\text { vehicles }\end{array}$ & $\begin{array}{c}\text { One } \\
\text { vehicle }\end{array}$ & $\begin{array}{c}\text { Two } \\
\text { vehicles }\end{array}$ & $\begin{array}{c}\text { Three or } \\
\text { more } \\
\text { vehicles }\end{array}$ & $\begin{array}{c}\text { Total } \\
\text { vehicles" }\end{array}$ \\
\hline 1960 & $21.53 \%$ & $56.94 \%$ & $19.00 \%$ & $2.53 \%$ & $54,766,718$ \\
1970 & $17.47 \%$ & $47.71 \%$ & $29.32 \%$ & $5.51 \%$ & $79,002,052$ \\
1980 & $12.92 \%$ & $35.53 \%$ & $34.02 \%$ & $17.52 \%$ & $129,747,911$ \\
1990 & $11.53 \%$ & $33.74 \%$ & $37.35 \%$ & $17.33 \%$ & 152.380 .479 \\
\hline
\end{tabular}

\section{Source:}

U. S. Department of Transportation, Volpe National Transportation Systems Center, Journey-to- Work Trends in the United States and its Major Metropolitan Area, 1960-l 990, Cambridge, MA, 1994, p. 2-2. (Additional resources: http://www.census.gov)

"Compiled by the Census Bureau, these data on the total number of vehicles do not match the figures on Table 4.1. The figures on Table 4.1, from R.L. Polk and Company, are the preferred data. 


\section{Nationwide Personal Travel Survey}

The 1995 Nationwide Personal Travel Survey (NPTS) is a national survey designed to collect data on the nature and characteristics of personal travel. The definition of a trip in the NPTS is "any one-way travel from one addressto another by private motor vehicle, public transportation, bicycle, or walking." Excluded from the survey are jogging and walking for exercise, as is all bicycling and walking for individuals under 5 years of age. The survey collects detailed data on household trips, their purposes and the transportation modes used. The NPTS is sponsored by several agencies of the U.S. Department of Transportation and is conducted approximately every seven years. Since each of the surveys differ somewhat in terminology, survey procedure, and target population, one should be cautious when comparing statistics from one survey to the next. Improved methodologies used in the collection of the trip information in the 1995 NPTS make it impossible to compare these data with past NPTS survey data. Thus, the 1990 NPTS trip data have been adjusted to make it comparable with the latest survey. Both the original 1990 data and the adjusted 1990 data are shown in tables comparing trip information. The 1995 trip data should only be compared to the adjusted 1990 trip data, and the original trip 1990 data should be compared with previous surveys. Additional analyses can be done on the 1995 NPTS data through the Internet site: http://www-cta.ornl.gov/npts.

Table 11.5

Demographic Statistics

1969, 1977, 1983, 1990, and 1995 NPTS

\begin{tabular}{lcccccc}
\hline & & & & & & $\begin{array}{c}\text { Percent } \\
\text { change } \\
1969-95\end{array}$ \\
\hline Personpehousehold & 1969 & 1977 & 1983 & 1990 & 1995 & 1969 \\
Vehiclepehousehold & 3.16 & 2.83 & 2.69 & 2.56 & 2.63 & $-17 \%$ \\
Workerpehousehold & 1.16 & 1.59 & 1.68 & 1.77 & 1.78 & $53 \%$ \\
Vehicl psr worker & 1.21 & 1.23 & 1.21 & 1.27 & 1.33 & $10 \%$ \\
Average vehicle trip length (miles) & 0.96 & 1.29 & 1.39 & 1.40 & 1.34 & $40 \%$ \\
\hline
\end{tabular}

Source:

U.S. Department of Transportation, Federal Highway Administration, 1990 Nationwide Personal Transportation Survey: Summary of Travel Trends, FHWA-PL-92-027, Washington, DC, March 1992, Table 2. Data for 1995 were generated from the Internet site http://www-cta.ornl.gov/npts.

Note: (Additional resources: http://www.fhwa.dot.gov)

Average vehicle trip length for 1990 and 1995 is calculated using only those records with trip mileage information present. The 1969 survey does not include pickups and other light trucks as household vehicles. 
The 1995 NPTS data should be compared only to the 1990 adjusted data due to survey methodology improvements in collecting trip information. The original 1990 data are comparable to all previous surveys; however, comparisons should always be made with caution because of differing survey methodologies.

Table 11.6

Average Annual Vehicle-Miles, Vehicle Trips and

Trip Length per Household

$1969,1977,1983,1990$, and 1995 NPTS

\begin{tabular}{|c|c|c|}
\hline & Joumev-to-work" & A11 trins \\
\hline \multicolumn{3}{|c|}{ Average annual vehicle-miles per household } \\
\hline 1969 & 4,183 & 12,423 \\
\hline 1977 & 3,815 & 12,036 \\
\hline 1983 & 3,538 & 11,739 \\
\hline 1990 original & 4.853 & 15.100 \\
\hline 1990 adjusted & 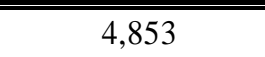 & 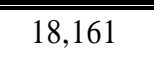 \\
\hline 1995 & 6.492 & 20.895 \\
\hline
\end{tabular}

Average annual vehicle trips per household

\begin{tabular}{lcc}
1969 & 445 & 1,396 \\
1977 & 423 & 1,442 \\
1983 & 414 & 1,486 \\
1990 original & 448 & 1,702 \\
\hline \hline 1990 adjusted & 448 & 2,077 \\
1995 & 553 & 2,321 \\
\hline \hline \multicolumn{3}{c}{ Average vehicle trip length (miles) } \\
1969 & 9.4 & 8.9 \\
1977 & 9.0 & 8.4 \\
1983 & 8.5 & 7.9 \\
1990 original & 11.0 & 9.0 \\
\hline \hline 1990 adjusted & 11.0 & 8.9 \\
1995 & 11.8 & 9.1 \\
\hline \hline
\end{tabular}

Source:

U.S. Department of Transportation, Federal Highway Administration, 1990 Nationwide Personal Transportation Survey: Summary of Travel Trends, FHWA-PL-92-027, Washington, DC, March 1992, Table 7. Data for 1995 were generated from the Internet site http://wwwcta.ornl.gov/npts. 1990 adjusted data - Oak Ridge National Laboratory, Oak Ridge, TN, August 1998. (Additional resources: http://www.fhwa.dot.gov, http://wwwcta.oml.gov/npts)

"It is believed that the methodology changes in the 1995 NPTS did not affect journey-to-work trips; therefore, no adjustment is necessary. 
The 1995 NPTS data should be compared only to the 1990 adjusted data due to survey methodology improvements in collecting trip information. The original 1990 data are comparable to allprevious surveys; however, comparisons should always be made with caution because of differing survey methodologies.

Table 11.7

Average Annual Person-Miles Traveled (PMT), Person Trips and Trip Length per Household by Selected Trip Purposes 1983, 1990, and 1995 NPTS

\begin{tabular}{lcccc}
\hline & Journey-to-work" & Shopping & $\begin{array}{c}\text { Social and } \\
\text { recreational }\end{array}$ & $\begin{array}{c}\text { All } \\
\text { purposes }^{\mathrm{b}}\end{array}$ \\
\hline \multicolumn{5}{c}{ Average annual PMT per household } \\
1983 & 4,586 & 2,567 & 8,964 & 22,802 \\
1990 original & 5,637 & 2,674 & 8,567 & 24,803 \\
\hline \hline 1990 adjusted & 5,637 & 3,343 & 11,308 & 30,316 \\
1995 & 7,740 & 4,659 & 10,571 & 34,459 \\
\hline \hline & Average annual person trips per household & \\
1983 & 537 & 474 & 728 & 2,628 \\
1990 original & 539 & 504 & 662 & 2,673 \\
\hline \hline 1990 adjusted & 539 & 630 & 874 & 3,262 \\
1995 & 676 & 775 & 953 & 3.828 \\
\hline \hline & Average person trip length (miles) & & \\
1983 & 8.5 & 5.4 & 12.3 & 9.7 \\
1990 original & 10.7 & 5.4 & 13.2 & 9.5 \\
\hline \hline 1990 adjusted & 10.7 & 5.4 & 13.2 & 9.1 \\
1995 & 11.6 & 6.1 & 11.3 & \\
\hline \hline
\end{tabular}

\section{Source:}

U.S. Department of Transportation, Federal Highway Administration, Nationwide Personal Transportation Study, Public Use Tapes, Washington, DC. Data for 1995 were generated from the Internet site http://www-cta.ornl.gov/npts. 1990 adjusted data - Oak Ridge National Laboratory, Oak Ridge, TN, August 1998. (Additional resources: http://www.fhwa.dot.gov, http://www-cta.ornl.gov/npts)

Note:

Average person trip length for 1990 and 1995 is calculated using only those records with trip mileage information present. "All purposes" includes unreported trip purposes.

"It is believed that the methodology changes in the 1995 NPTS did not affect journey-to-work trips; therefore, no adjustment is necessary.

'Includes trip purposes not shown on this table. 
Figure 11.1. Average Vehicle Occupancy by Vehicle Type, 1995 NPTS

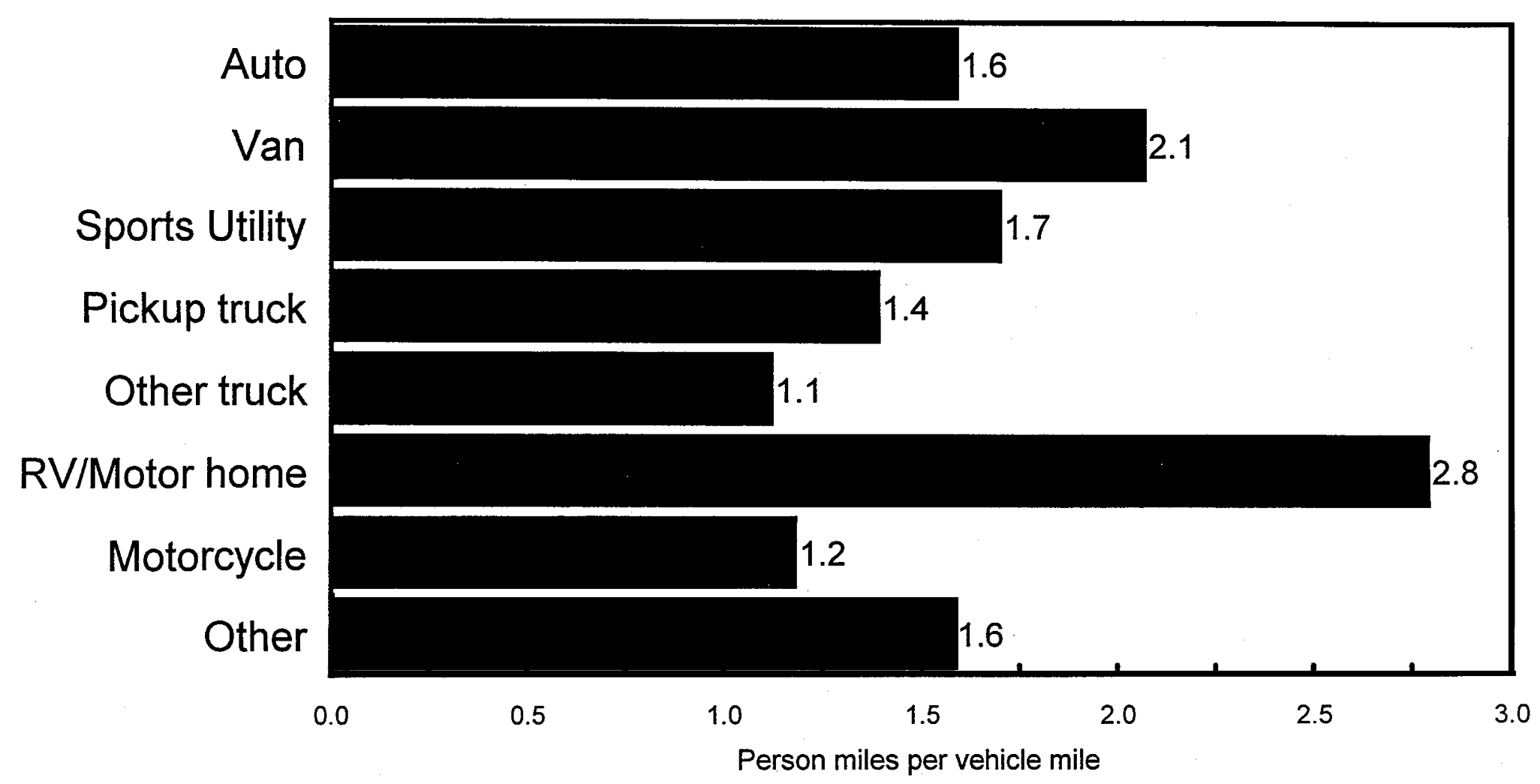

Source:

U.S. Department of Transportation, Federal Highway Administration, Nationwide Personal Transportation Survey, Washington, DC, 1997. (Additional resources: http://www.fhwa.dot.gov, http://www-cta.ornl.gov/npts) 
The average vehicle occupancy, calculated as person-miles per vehicle-mile, was nearly identical in 1990 and 1995 for every trip purpose. The highest vehicle occupancy levels were in 1977. The increased number of vehicles per household and the decrease in average household size could have contributed to the decline since then.

Figure 11.2. Average Vehicle Occupancy by Trip Purpose $1977,1983,1990$, and 1995 NPTS

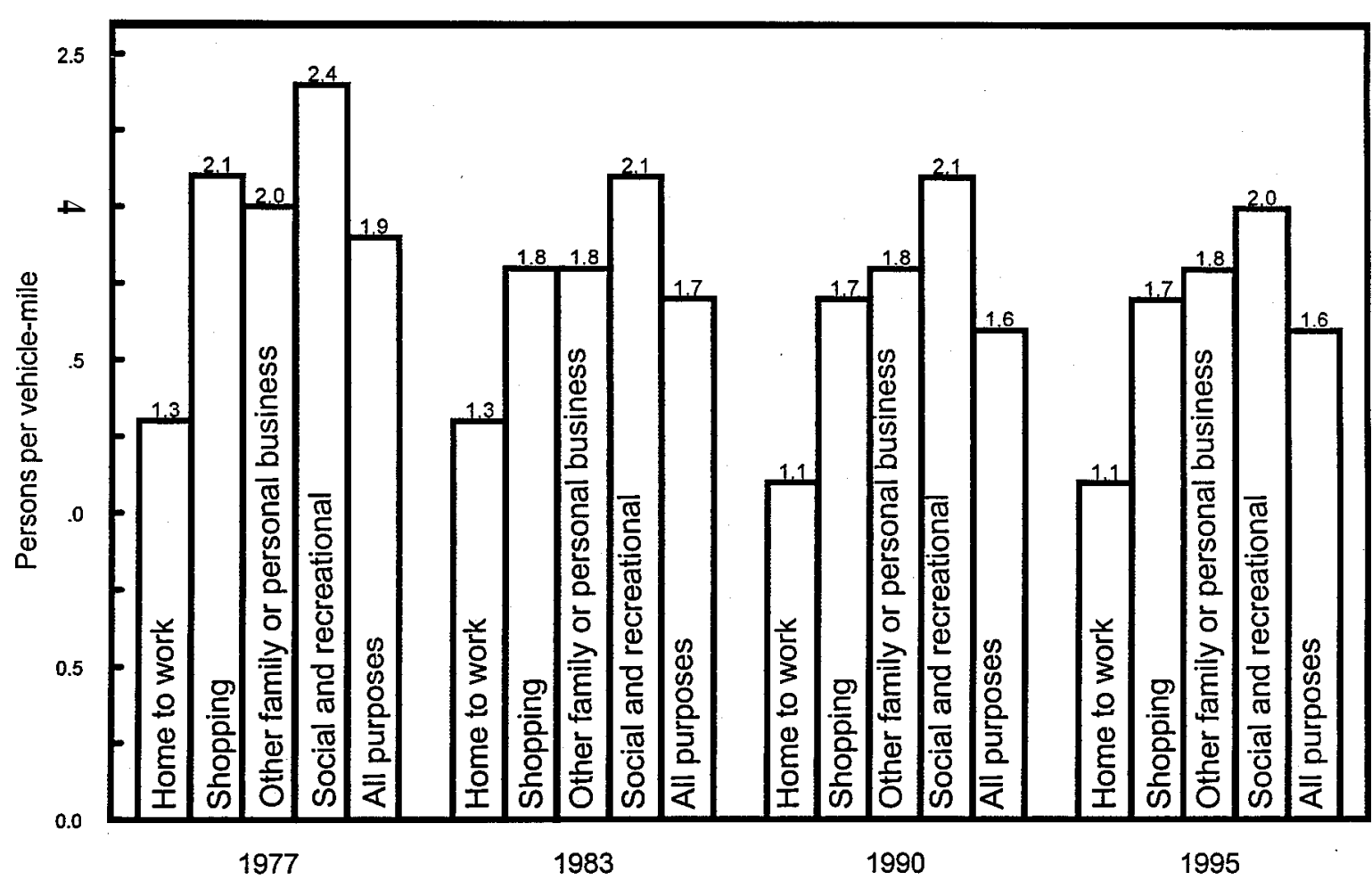

Source:

U.S. Department of Transportation, Federal Highway Administration, 1990 Nationwide Personal Transportation Survey: Summary of Travel Trends, FHWA-PL92027, Washington, DC, March 1992, Figure 6. Data from 1995 were generated from the public use file.

(Additional resources: http://www.fhwa.dot.gov, http://www-cta.ornl.gov/npts) 
Less than $27 \%$ of all household vehicle-miles are trips to or from work. Errands such as family and personal business and shopping (combined) make up a third of vehicle travel. One quarter of all trips 75 miles or longer (one way) were for the purpose of visiting friends or relatives.

Table 11.8

Vehicle-Miles by Trip Purpose, 1995 NPTS

\begin{tabular}{|c|c|c|c|c|c|c|}
\hline \multirow[b]{2}{*}{ Purpose of trip } & \multicolumn{2}{|c|}{ Daily trip vehicle-miles } & \multicolumn{2}{|c|}{ Long trip vehicle-miles" } & \multicolumn{2}{|c|}{ Total trip vehicle-miles } \\
\hline & (millions) & (percent) & (millions) & (percent) & (millions) & (percent) \\
\hline To or from work & 642,610 & $31.1 \%$ & 16,032 & $4.2 \%$ & 658,642 & $26.8 \%$ \\
\hline Work-related business & 137,867 & $6.7 \%$ & 56,613 & $14.7 \%$ & 194,480 & $7.9 \%$ \\
\hline Shopping & 277,860 & $13.4 \%$ & 13,377 & $3.5 \%$ & 291,237 & $11.9 \%$ \\
\hline Other family or personal business & 426,330 & $20.6 \%$ & 54,722 & $14.2 \%$ & 481,052 & $19.6 \%$ \\
\hline School/church & 78,313 & $3.8 \%$ & 11,874 & $3.1 \%$ & 90,187 & $3.7 \%$ \\
\hline Doctor/dentist & 30,613 & $1.5 \%$ & 5,016 & $1.3 \%$ & 35,629 & $1.5 \%$ \\
\hline Vacation & 20,318 & $1.0 \%$ & 38,765 & $10.0 \%$ & 59,083 & $2.4 \%$ \\
\hline Visit friends or relatives & 195,068 & $9.4 \%$ & 99,308 & $25.7 \%$ & 294,376 & $12.0 \%$ \\
\hline Other social or recreational & 256,169 & $12.4 \%$ & 85,989 & $22.3 \%$ & 342,158 & $13.9 \%$ \\
\hline Other & 2,797 & $0.1 \%$ & 4,281 & $1.1 \%$ & 7,078 & $0.3 \%$ \\
\hline Not ascertained & 422 & $0.0 \%$ & 20 & $0.0 \%$ & 442 & $0.0 \%$ \\
\hline All & $2,068,368$ & $100.0 \%$ & 385,997 & $100.0 \%$ & $2,454,365$ & $100.0 \%$ \\
\hline
\end{tabular}

\section{Source:}

U.S. Department of Transportation, Federal Highway Administration, Nationwide Personal Transportation Survey web site: http://wwwcta.ornl.gov/npts.

'Defined as a trip which is 75 miles or longer one way. 
As households owned more vehicles, the average annual miles for the most frequently driven vehicle increased. For example, the mostfrequently driven vehicle in five-vehicle households was driven $26 \%$ more per year than the one in two-vehicle households (21,177 miles vs. 16,804 miles).

Table 11.9

Average Annual Miles per Vehicle by Household Vehicle Ownership, 1995 NPTS

\begin{tabular}{cccccc}
\hline Vehicle a $^{\text {a }}$ & $\begin{array}{c}\text { One-vehicle } \\
\text { household }\end{array}$ & $\begin{array}{c}\text { Two-vehicle } \\
\text { household }\end{array}$ & $\begin{array}{c}\text { Three-vehicle } \\
\text { household }\end{array}$ & $\begin{array}{c}\text { Four-vehicle } \\
\text { household }\end{array}$ & $\begin{array}{c}\text { Five-vehicle } \\
\text { household }\end{array}$ \\
\hline$\# 1$ & 12,379 & 16,804 & 18,853 & 20,724 & 21,177 \\
$\# 2$ & & 8,322 & 9,806 & 11,311 & 12,880 \\
$\# 3$ & & 4,555 & 6,395 & 7,319 \\
$\# 4$ & & & 3,218 & $4,177$. \\
$\# 5$ & & & & & 2,321 \\
Average & $\mathbf{1 2 , 3 7 9}$ & $\mathbf{1 2 , 8 5 5}$ & $\mathbf{1 1 , 6 0 4}$ & $\mathbf{1 1 , 1 0 0}$ & $\mathbf{1 0 , 3 7 2}$ \\
\hline
\end{tabular}

Source:

Generated from the Department of Transportation, Federal Highway Administration, Nationwide Personal Transportation Survey Public Use Files, Washington, DC, 1998. (Additional resources: http://www-cta.ornl.gov/npts)

Table 11.10

Average Age of Vehicles by Household Vehicle Ownership, 1995 NPTS

\begin{tabular}{cccccc}
\hline Vehicle" & $\begin{array}{c}\text { One-vehicle } \\
\text { household }\end{array}$ & $\begin{array}{c}\text { Two-vehicle } \\
\text { household }\end{array}$ & $\begin{array}{c}\text { Three-vehicle } \\
\text { household }\end{array}$ & $\begin{array}{c}\text { Four-vehicle } \\
\text { household }\end{array}$ & $\begin{array}{c}\text { Five-vehicle } \\
\text { household }\end{array}$ \\
\hline$\# 1$ & 7.48 & 6.45 & 6.74 & 7.01 & 7.35 \\
$\# 2$ & - & 8.54 & 8.55 & 8.68 & 9.54 \\
$\# 3$ & & 12.25 & 11.36 & 11.89 \\
$\# 4$ & & & 14.52 & 14.60 \\
$\# 5$ & & & & & 17.81 \\
Average & 7.48 & 7.42 & 8.93 & 10.03 & $\mathbf{1 1 . 6 2}$ \\
\hline
\end{tabular}

Source:

Generated from the Department of Transportation, Federal Highway Administration, Nationwide Personal Transportation Survey Public Use Files, Washington, DC, 1998. (Additional resources: http://www-cta.ornl.gov/npts)

"Vehicles are ranked by descending annual miles driven. 
Historically, the data from the Nationwide Personal Transportation Study (NPTS) are based on estimates reported by survey respondents. For the 1995 survey, odometer data was also collected. The Residential Transportation Energy Consumption Survey (RTECS) data has always been collectedfrom odometer readings. These data indicate that respondents may overestimate the number of miles driven in a year.

Table 11.11

Average Annual Miles Per Household Vehicle by Vehicle Age

\begin{tabular}{|c|c|c|c|c|c|c|c|c|c|}
\hline \multirow[b]{2}{*}{$\begin{array}{l}\text { Vehicle age } \\
\text { (years) }\end{array}$} & \multicolumn{4}{|c|}{$\begin{array}{l}\text { National Personal } \\
\text { Transportation Study }\end{array}$} & \multicolumn{5}{|c|}{$\begin{array}{l}\text { Residential Transportation } \\
\text { Energy Consumption Survey }\end{array}$} \\
\hline & $\begin{array}{c}1983 \\
\text { self-reported } \\
\end{array}$ & $\begin{array}{c}1990 \\
\text { self-reported } \\
\end{array}$ & $\begin{array}{c}1995 \\
\text { self-reported } \\
\end{array}$ & $\begin{array}{c}1995 \\
\text { odometer } \\
\end{array}$ & $\begin{array}{c}1983 \\
\text { odometer }\end{array}$ & $\begin{array}{c}1985 \\
\text { odometer } \\
\end{array}$ & $\begin{array}{c}1988 \\
\text { odometer }\end{array}$ & $\begin{array}{c}1991 \\
\text { odometer }\end{array}$ & $\begin{array}{c}1994 \\
\text { odometer }\end{array}$ \\
\hline Under 1 & 8,200 & 19,600 & 15,900 & 15,600 & 13,400 & 12,700 & 12,900 & 13,400 & 15,220 \\
\hline 1 & 15,200 & 16,800 & 12,200 & 11,200 & 13,000 & 13,000 & 13,400 & 14,100 & 14,250 \\
\hline 2 & 16,800 & 16,600 & 12,200 & 11,300 & 12,700 & 12,600 & 12,600 & 12,600 & 13,740 \\
\hline 3 & 14,500 & 14,700 & 12,800 & 11,600 & 12,100 & 12,400 & 12,100 & 13,200 & 13,080 \\
\hline 4 & 13,000 & 13,600 & 13,200 & 12,400 & 11,300 & 11,100 & 11,500 & 13,300 & 12,500 \\
\hline 5 & 12,100 & 12,900 & 13,500 & 12,700 & 9,700 & 10,600 & 10,600 & 12,200 & 12,560 \\
\hline 6 & 11,300 & 13,200 & 14,100 & 12,900 & 9,700 & 10,000 & 10,800 & 11,200 & 12,290 \\
\hline 7 & 10,000 & 12,400 & 14,400 & 13,800 & 9,500 & 9,700 & 10,000 & 10,700 & 12,030 \\
\hline 8 & 9,800 & 12,600 & 15,500 & 14,800 & 8,700 & 8,900 & 10,300 & 11,400 & 10,915 \\
\hline 9 & 9,000 & 11,500 & 16,800 & 14,500 & 8,400 & 8,600 & 8,900 & 10,000 & 10,950 \\
\hline 10 and older & 7,300 & 9,200 & 8,900 & 9,000 & 8,700 & 8,400 & 7,500 & 7,200 & 9,780 \\
\hline All vehicles & 10,400 & 12,500 & 12,200 & 11,800 & 9,400 & 9,900 & 10,200 & 10,600 & 11,400 \\
\hline
\end{tabular}

\section{Source:}

Nationwide Personal Transportation Study-1983: D. Klinger and J. Richard Kuzmyak, COMSIS Corporation, Personal Travel in the United States. Volume 1: 1983-84 Nationwide Personal Travel Study, prepared for the U.S. Department of Transportation, Washington, DC, August 1986, Table 4-22, p.4-21. 1990: Generated from the 1990 Nationwide Personal Transportation Study Public Use Tape, March 1992. 1995: Generated from the Internet site: http://www-cta.ornl.gov/npts.

Residential Transportation Energy Consumption Survey-Personal communication with Energy Information Agency, Office of Markets and End Use, Energy End Use Division.

(Additional resources: http://www.fhwa.dot.gov, http://www.eia.doe.gov)

Note:

Data include all household vehicles. Data have been rounded to the nearest hundred. 
In 1995 the average journey-to-work speed was faster (miles per hour increased to 34.6), but the travel time still increased, due to an increase in the average travel distance. Journeys-towork using public transportation continued to take twice as long as private transportation, though there is only a slight difference in travel distance.

Table 11.12

Journey-to-Work Statistics 1983, 1990, and 1995 NPTS

\begin{tabular}{|c|c|c|c|c|}
\hline Year & $\begin{array}{c}\text { Private } \\
\text { transportation }\end{array}$ & $\begin{array}{c}\text { Public } \\
\text { transportation }\end{array}$ & Other & Total \\
\hline \multicolumn{5}{|c|}{ Average travel time (minutes) ${ }^{b}$} \\
\hline 1983 & 17.6 & 39.8 & 10.6 & 18.2 \\
\hline 1990 & 19.1 & 41.1 & 12.4 & 19.6 \\
\hline 1995 & 20.1 & 42.0 & 18.8 & 20.7 \\
\hline \multicolumn{5}{|c|}{ Average trip length (miles) } \\
\hline 1983 & 8.9 & 11.8 & 1.4 & 8.5 \\
\hline 1990 & 11.0 & 12.8 & 2.2 & 10.7 \\
\hline 1995 & 11.8 & 12.9 & 8.2 & 11.6 \\
\hline \multicolumn{5}{|c|}{ Average speed (miles per hour) } \\
\hline 1983 & 30.2 & 17.8 & 7.6 & 28.2 \\
\hline $1990 "$ & 34.7 & 18.2 & 7.6 & 33.3 \\
\hline 1995” & 35.4 & 19.3 & 25.9 & 34.6 \\
\hline
\end{tabular}

Source:

U.S. Department of Transportation, Federal Highway Administration, Nationwide Personal Transportation Study, Public Use Tapes, Washington, DC. Data for 1995 were generated from the Internet site http://www-cta.ornl.gov/npts. (Additionalresources: http://www.fhwa.dot.gov, http://www-cta.ornl.gov/npts)

aIt is believed that the methodology changes in the 1995 NPTS did not affect journey-to-work trips; therefore, no adjustment is necessary.

${ }^{b}$ Does not include time spent waiting for transportation.

'Does not include segmented trips. 
According to the U.S. Census data, the percentage of workers who car pooled has dropped from $19.7 \%$ in 1980 to $13.4 \%$ in 1990. Thepercent ofworkers usingpublic transit declinedfrom $6.4 \%$ to $5.3 \%$ during the same time period. The average travel time increased by 0.7 minutes from 1980 to 1990.

Table 11.13

Means of Transportation to Work, 1980 and 1990 Census

\begin{tabular}{|c|c|c|c|c|}
\hline \multirow[b]{2}{*}{ Means of transportation } & \multicolumn{2}{|c|}{1980 Census } & \multicolumn{2}{|c|}{1990 Census } \\
\hline & $\begin{array}{l}\text { Number } \\
\text { of workers }\end{array}$ & Percentage & $\begin{array}{l}\text { Number } \\
\text { of workers }\end{array}$ & Percentage \\
\hline Private vehicle & $81,258,496$ & $84.1 \%$ & $99,592,932$ & $86.5 \%$ \\
\hline Drove alone & $62,193,449$ & $64.4 \%$ & $84,215,298$ & $73.2 \%$ \\
\hline Car pooled & $19,065,047$ & $19.7 \%$ & $15,377,634$ & $13.4 \%$ \\
\hline Public transportation & $6,175,061$ & $6.4 \%$ & $6,069,589$ & $5.3 \%$ \\
\hline Bus or trolley bus ${ }^{\mathrm{a}}$ & $3,924,787$ & $4.1 \%$ & $3,445,000$ & $3.0 \%$ \\
\hline Streetcar or trolley car ${ }^{\mathbf{a}}$ & b & b & 78,130 & $0.1 \%$ \\
\hline Subway or elevated & $1,528,852$ & $1.6 \%$ & $1,755,476$ & $1.5 \%$ \\
\hline Railroad & 554,089 & $0.6 \%$ & 574,052 & $0.5 \%$ \\
\hline Ferryboat & $\mathrm{b}$ & $\mathrm{b}$ & 37,497 & $0.0 \%$ \\
\hline Taxicab & 167,133 & $0.2 \%$ & 179,434 & $0.2 \%$ \\
\hline Other means & 703,273 & $0.7 \%$ & 808,582 & $0.7 \%$ \\
\hline Motorcycle & 419,007 & $0.4 \%$ & 237,404 & $0.2 \%$ \\
\hline Bicycle & 468,348 & $0.5 \%$ & 466,856 & $0.4 \%$ \\
\hline Walked only & $5,413,248$ & $5.6 \%$ & $4,488,886$ & $3.9 \%$ \\
\hline Worked at home & $2,179,863$ & $2.3 \%$ & $3,406,025$ & $3.0 \%$ \\
\hline Total workers & $96,617,296$ & $100.0 \%$ & $115,070,274$ & $100.0 \%$ \\
\hline Average travel time (minutes) & 21.7 & & 22.4 & \\
\hline
\end{tabular}

Source:

Data provided by the Journey-to-Work and Migration Statistics Branch, Population Division, U.S. Bureau of the Census. (Additional resources: http://www.census.gov)

"This category was "Bus or streetcar" in 1980.

${ }^{b}$ Data are not available. 
Table 11.14

National and Metropolitan Area Comparisons of Journey-to-Work Statistics, 1990 Census

\begin{tabular}{lcc}
\hline & National & Metropolitan areas $^{\mathbf{a}}$ \\
\hline Workers per household & 1.25 & 1.31 \\
Workers per vehicle & 0.76 & 0.82 \\
Average travel time (minutes) & 22.38 & 25.20 \\
\hline Commute length (percentage) & & \\
\hline Less than 15 minutes & $15.87 \%$ & $11.45 \%$ \\
15-29 minutes & $51.64 \%$ & $49.22 \%$ \\
$30-39$ minutes & $14.66 \%$ & $17.48 \%$ \\
$40-59$ minutes & $9.01 \%$ & $11.77 \%$ \\
60 minutes or more & $5.86 \%$ & $7.52 \%$ \\
\hline Mode (percentage) & & $70.75 \%$ \\
\hline Drive alone & $73.19 \%$ & $12.69 \%$ \\
Percentage car pooled & $13.36 \%$ & $8.98 \%$ \\
Public transit & $5.27 \%$ & $0.21 \%$ \\
Motorcycle & $0.21 \%$ & $3.76 \%$ \\
Walk & $3.90 \%$ & $0.43 \%$ \\
Bicycle & $0.41 \%$ & $0.62 \%$ \\
Other & $0.70 \%$ & $2.57 \%$ \\
Work at home & $2.96 \%$ & $11.57 \%$ \\
\hline Time workers leave home (percentage) & & $25.49 \%$ \\
\hline 5:00 AM-6.59 AM & $10.28 \%$ & \\
7:00 AM-8:29 AM & $18.85 \%$ & \\
All other departures & & \\
\hline & & \\
\hline
\end{tabular}

Source:

U. S. Department of Transportation, Volpe National Transportation Systems Center, Journey-to- Work Trends in theUnited States and its Major Metropolitan Area, 1960-1990, FHWA-PL-94-012, Cambridge, MA, 1994, p. 2-6. (Additional resources: http://www.census.gov)

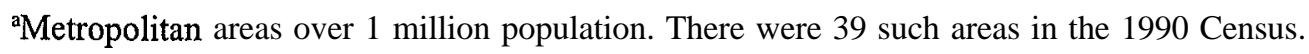




\section{American Travel Survey}

The American Travel Survey (ATS) was conducted by the Bureau of Transportation Statistics to obtain information about the long-distance travel of persons living in the United States. Approximately 80,000 randomly selected households were interviewed for the survey, which collected information about all trips of 100 miles or more, one-way, taken by household members in 1995. The ATS data provide detailed information on state-to-state travel, as well as travel to and from metropolitan areas by mode of transportation.

For additional information about the American Travel Survey, contact the Bureau of Transportation Statistics at (202) 366-3282 or visit the following Internet site: http://www.bts.gov/ats

Figure 11.3 Long-Distance Trips by Destination, 1995

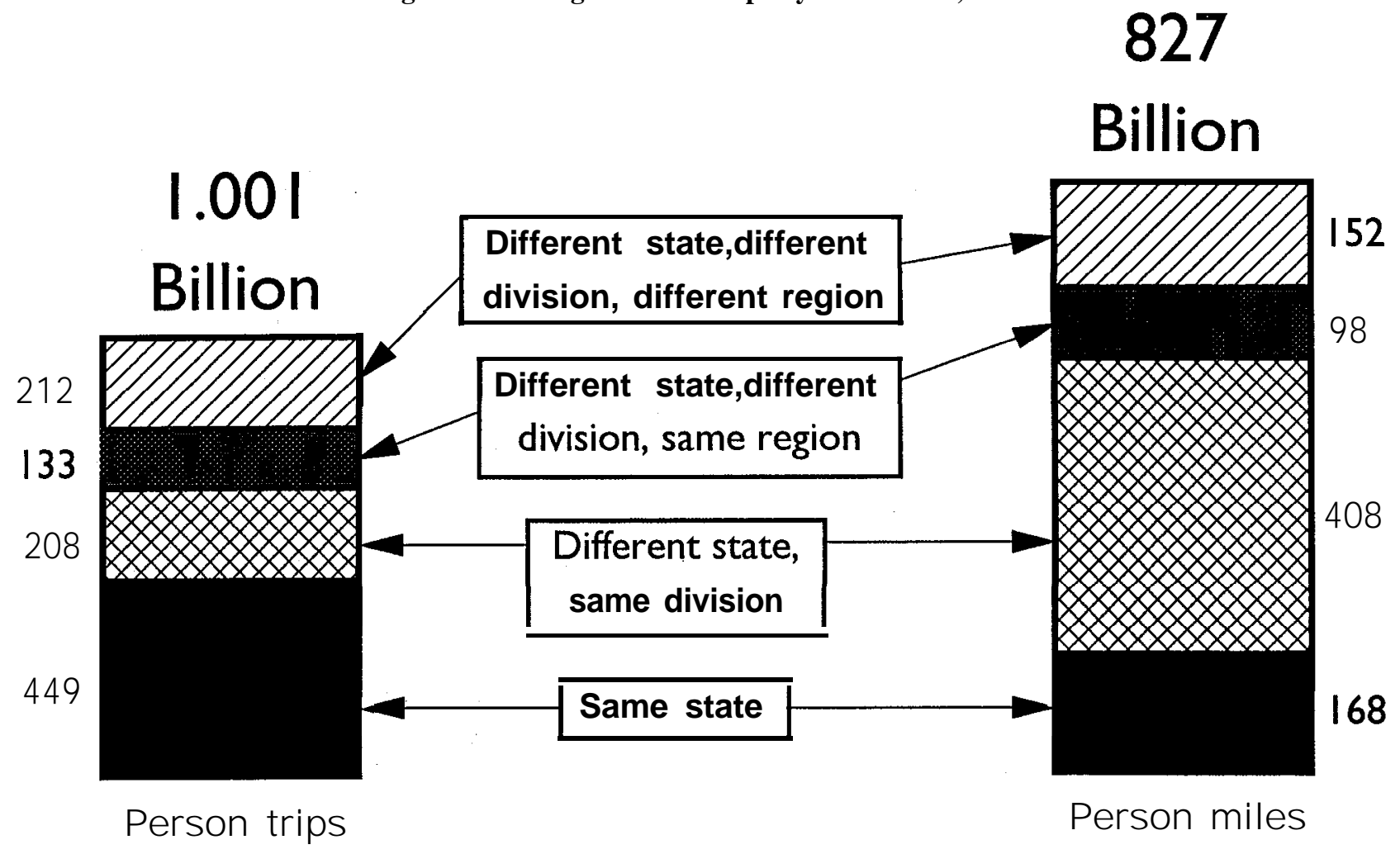

Source:

U.S. Department of Transportation, Bureau of Transportation Statistics, 1995 American Travel Survey Profile,

Washington, DC, October 1997, p. 2. (Additional resources: http://www.bts.gov/ats)

Note:

Definitions of divisions and regions are in Appendix C. 
Table 11.15

Long-Distance Trips" by Mode and Purpose, 1995

\begin{tabular}{|c|c|c|c|c|c|c|}
\hline \multirow[b]{3}{*}{ Principal means of transportation } & \multicolumn{6}{|c|}{ Main purpose of trip } \\
\hline & \multirow[b]{2}{*}{ Business } & \multicolumn{3}{|c|}{ Pleasure } & \multirow[b]{2}{*}{$\begin{array}{l}\text { Personal } \\
\text { business }\end{array}$} & \multirow[b]{2}{*}{ Total } \\
\hline & & $\begin{array}{l}\text { Visit friends } \\
\text { or relatives }\end{array}$ & Leisure & Total & & \\
\hline & \multicolumn{6}{|c|}{ Person trips (thousands) } \\
\hline Personal use vehicle & 151,697 & 283,153 & 254,186 & 537,339 & 124,791 & 813,858 \\
\hline Commercial airplane & 67,083 & 41,881 & 31,581 & 73,462 & 15,386 & 155,936 \\
\hline Intercity bus & 286 & 1,830 & 690 & 2,519 & 439 & 3,244 \\
\hline Charter or tour bus & 1,281 & 1,198 & 9,253944 & 10,451 & 2,514 & 14,247 \\
\hline Train & 1,342 & 2,004 & & 2,948 & 704 & 4,994 \\
\hline Ship, boat, or ferry & 68 & 43 & 483 & 525 & 20 & 614 \\
\hline \multirow[t]{2}{*}{ Total } & 224,835 & 330,755 & 299,355 & 630,110 & 146,338 & $1,001,319$ \\
\hline & \multicolumn{6}{|c|}{ Percentage } \\
\hline Personal use vehicle & 18.6 & 34.8 & 31.2 & 66.0 & 15.3 & 100.0 \\
\hline Commercial airplane & 43.0 & 26.9 & 20.3 & 47.1 & 9.9 & 100.0 \\
\hline Intercity bus & 8.8 & 56.4 & 21.3 & 77.7 & 13.5 & 100.0 \\
\hline Charter or tour bus & 9.0 & 8.4 & 64.9 & 73.4 & 17.6 & 100.0 \\
\hline Train & 26.9 & 40.1 & 18.9 & 59.0 & 14.1 & 100.0 \\
\hline Ship, boat, or ferry & 11.1 & 7.0 & 78.7 & 85.5 & 3.3 & 100.0 \\
\hline Total & 22.5 & 33.0 & 29.9 & 62.9 & 14.6 & 100.0 \\
\hline
\end{tabular}

\section{Source:}

U.S. Department of Transportation, Bureau of Transportation Statistics, 1995 American Travel Survey Profile, Washington, DC, October 1997 , p. 13. (Additional resources: http://www.bts.gov/ats)

${ }^{\mathrm{a} A}$ long-distance trip is any trip of 100 miles or more, one way. 
Figure 11.4. Long-Distance Household Trips by Mode and Trip Distance, 1995

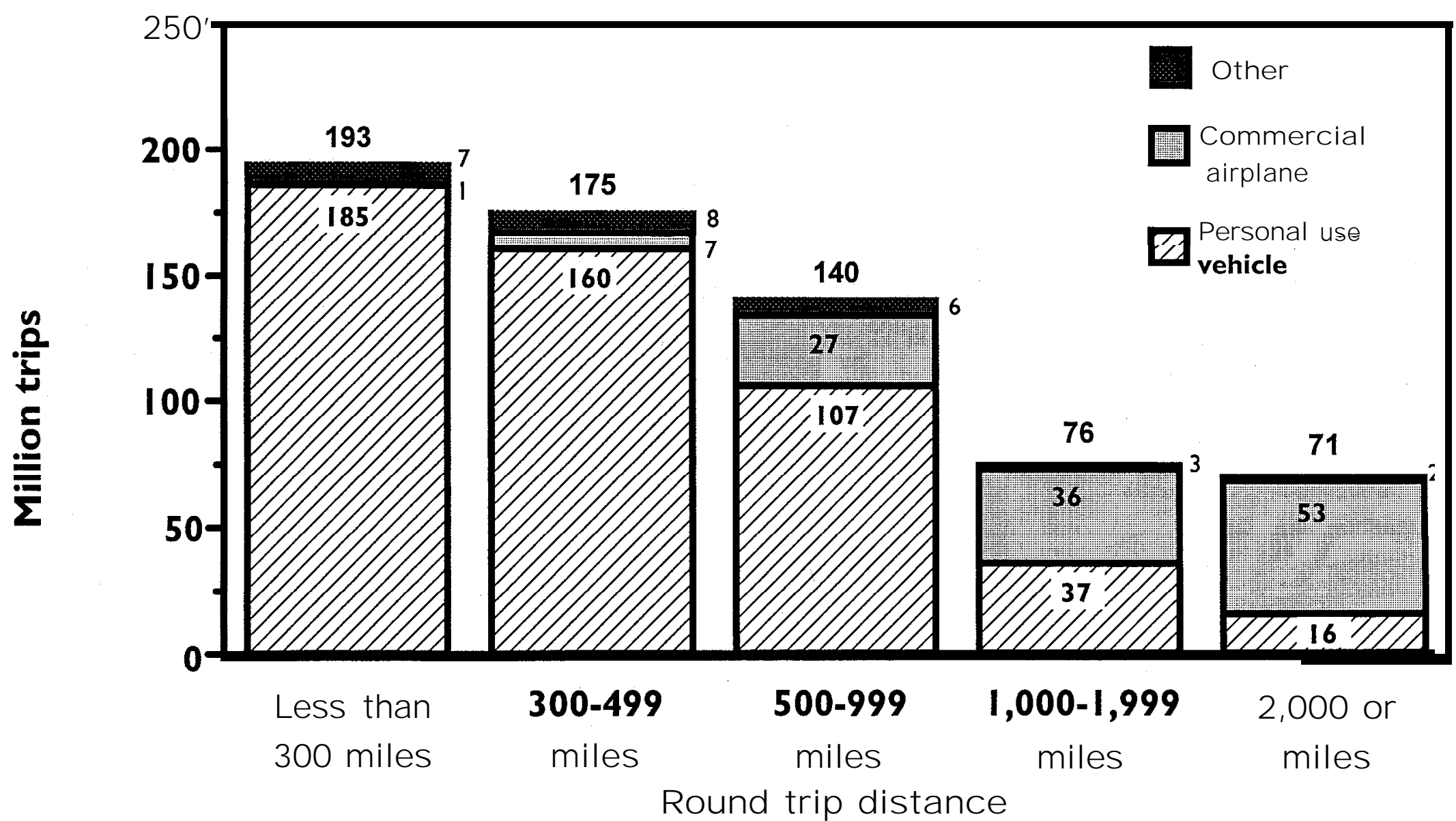

Source:

U.S. Department of Transportation, Bureau of Transportation Statistics, 1995 American Travel Survey Profile, Washington, DC, October 1997 , p. 3. (Additional resources: http://www.bts.gov/ats) 
Figure 11.5. Shares of Long-Distance Person Trips by Mode and Household Income, 1995

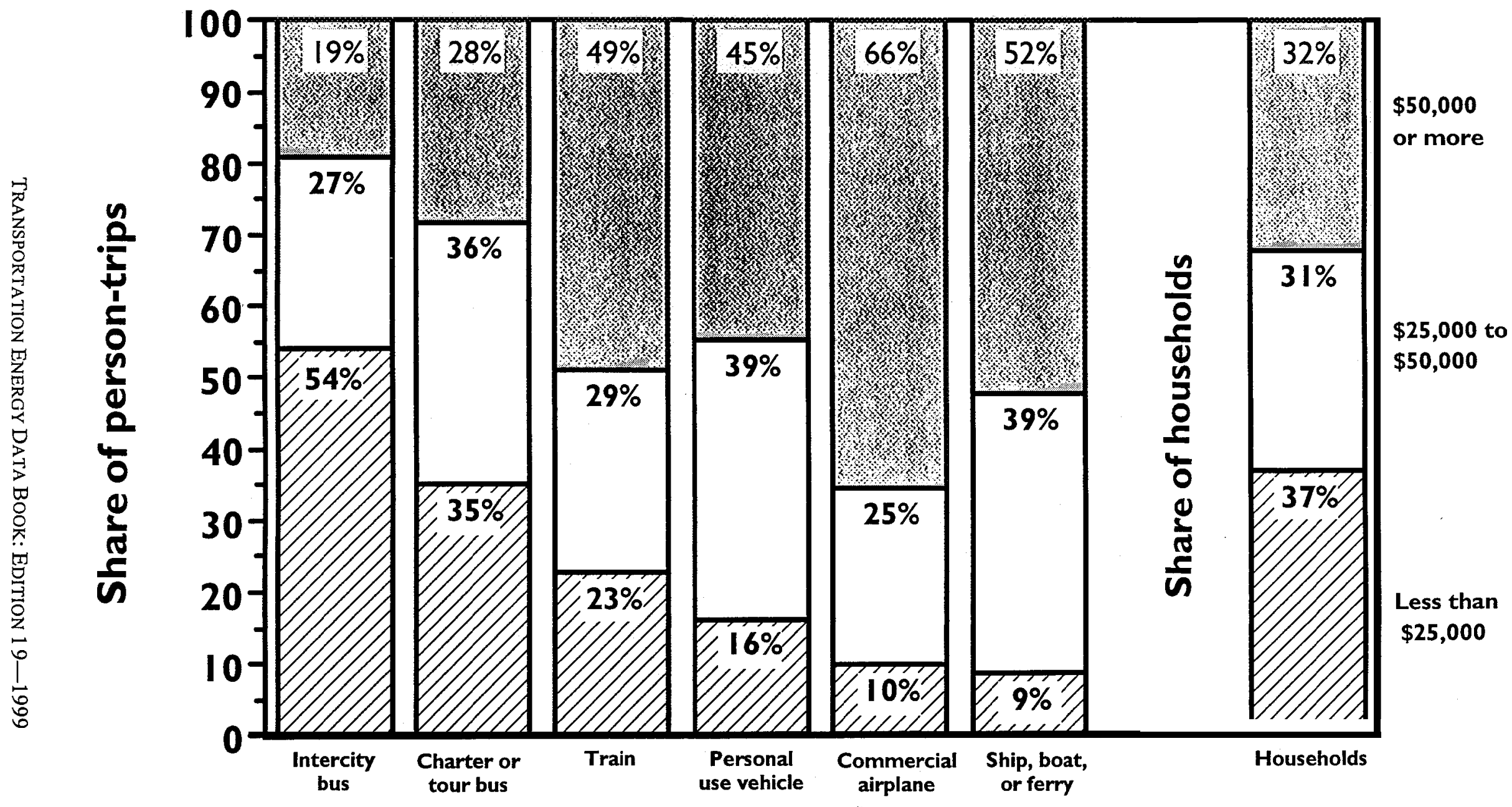

Source:

U.S. Department of Transportation, Bureau of Transportation Statistics, 1995 American Travel Survey Profile, Washington, DC, October 1997 , p. 8.

U.S. Department of Commerce, Bureau of the Census, Statistical Abstract of the United States, 117 ${ }^{\text {th }}$ Edition, Washington, DC, 1997 , p. 465.

(Additional resources: http://www.bts.gov/ats, http://www.census.gov) 


\section{Chapter 12 \\ Nonhighway Modes}

Summary Statistics from Tables in this Chapter

\begin{tabular}{|c|c|c|}
\hline \multicolumn{3}{|l|}{ Source } \\
\hline & Passenger-miles, 1997 & (millions) \\
\hline Table $12 . I$ & Domestic and international air carrier & 621,733 \\
\hline Table 12.2 & General aviation & 12,500 \\
\hline Table 12.10 & Amtrak & 5,166 \\
\hline \multirow[t]{2}{*}{ Table 12.11} & Transit rail & 13,095 \\
\hline & Freight ton-miles, 1997 & (millions) \\
\hline Table 12.4 & Domestic waterborne commerce & 707,000 \\
\hline \multirow[t]{2}{*}{ Table 12.7} & Class I railroad & $1,348,926$ \\
\hline & Passenger energy use, 1997 & (trillion Btus) \\
\hline Table 12.1 & Domestic and international air carrier & $2,494.5$ \\
\hline Table 12.2 & General aviation & 121.1 \\
\hline Table 12.10 & Amtrak energy use & 12.7 \\
\hline \multirow[t]{2}{*}{ Table $12.1 \mathrm{I}$} & Transit rail & 42.6 \\
\hline & Freight energy use, 1997 & (trillion Btus) \\
\hline Table 12.4 & Domestic waterborne commerce & 293.2 \\
\hline Table 12.7 & Class I railroad & 499.7 \\
\hline
\end{tabular}


Table 12.1

Summary Statistics for U.S. Domestic and International Certificated Route Air Carriers (Combined Totals), 1970-97

Revenue

aircraft-miles

Average

Revenue

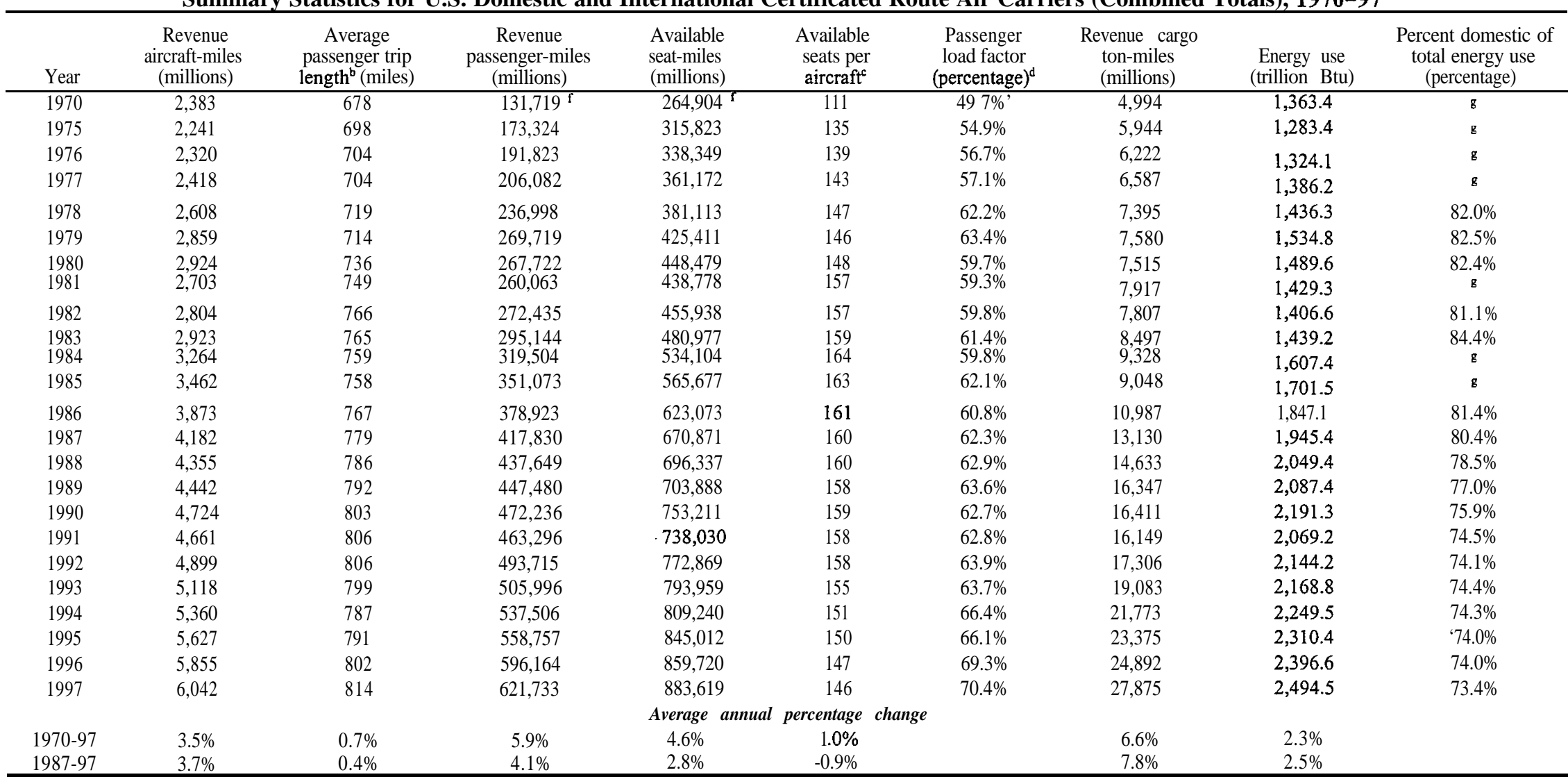

Source:

U.S. Department of Transportation, Bureau of Transportation Statistics, Air Carrier Traffic Statistics Monthly, December 1997/1996, Washington, DC, pp. 1-2, and annual.

1970-81 Energy Use - Department of Transportation, Civil Aeronautics Board, Fuel Cost and Consumption, Washington, DC, 1981, and annual.

1982-97 Energy Use - Department of Transportation, Research and Special Programs Administration, "Fuel Cost and Consumption Tables," Washington, DC, monthly. Annual totals are derived

by summing monthly totals for domestic and international air carriers. (Additional resources: http://www.bts.gov, http://www.faa.gov)

"Data are for all U.S. air carriers reporting on Form 41.

'Scheduled services of domestic operations only. The average passenger trip length for international operations is more than three and a half times longer than for domestic operations.

'Available seats per aircraft is calculated as the ratio of available seat-miles to revenue aircraft-miles.

dPassenger load factor is calculated as the ratio of revenue passenger-miles to available seat-miles for scheduled and nonscheduled services.

'Energy use includes fuel purchased abroad for international flights.

'Scheduled services only.

Data are not available. 
Table 12.2

Summary Statistics for General Aviation, 1970-97

\begin{tabular}{|c|c|c|c|c|}
\hline Calendar year & $\begin{array}{c}\text { Total number } \\
\text { of aircraft }\end{array}$ & $\begin{array}{c}\text { Hours flown } \\
\text { (thousands) }\end{array}$ & $\begin{array}{l}\text { Intercity passenger travel } \\
\text { (billion passenger-miles) }\end{array}$ & $\begin{array}{l}\text { Energy use } \\
\text { (trillion btu) }\end{array}$ \\
\hline 1970 & $131,700 "$ & $26,030^{b}$ & 9.1 & 94.4 \\
\hline 1971 & $131,100 "$ & $25,512^{\mathrm{b}}$ & 9.2 & 91.6 \\
\hline 1972 & $145,000 ”$ & $26,974^{\mathrm{b}}$ & 10.0 & 103.4 \\
\hline 1973 & $148,000 ”$ & 28,599 & 10.7 & 90.4 \\
\hline 1974 & 161,502 & 29,758 & 11.2 & 101.4 \\
\hline 1975 & 168,475 & 30,298 & 11.4 & 121.5 \\
\hline 1976 & 177,964 & 31,950 & 12.1 & 130.3 \\
\hline 1977 & 184,294 & 33,679 & 12.8 & 149.7 \\
\hline 1978 & 199,178 & 36,844 & 14.1 & 159.4 \\
\hline 1979 & 210,339 & 40,432 & 15.5 & 167.2 \\
\hline 1980 & 211,045 & 41,016 & 14.7 & 169.0 \\
\hline 1981 & 213,226 & 40,704 & 14.6 & 162.4 \\
\hline 1982 & 209,779 & 36,457 & 13.1 & 170.5 \\
\hline 1983 & 213,293 & 35,249 & 12.7 & 143.9 \\
\hline 1984 & 220,943 & 36,119 & 13.0 & 148.9 \\
\hline 1985 & 196,500 & 31,456 & 12.3 & 144.0 \\
\hline 1986 & 205,300 & 31,782 & 12.4 & 148.0 \\
\hline 1987 & 202,700 & 30,883 & 12.1 & 139.1 \\
\hline 1988 & 196,200 & 31,114 & 12.6 & 148.6 \\
\hline 1989 & 205,000 & 32,332 & 13.1 & 134.0 \\
\hline 1990 & 198,000 & 32,096 & 13.0 & 131.9 \\
\hline 1991 & 196,874 & 29,862 & 12.1 & 120.4 \\
\hline 1992 & 185,650 & 26,747 & 10.8 & 104.7 \\
\hline 1993 & 177,120 & 24,455 & 9.9 & 97.5 \\
\hline 1994 & 172,935 & 24,092 & 9.8 & 95.3 \\
\hline 1995 & 188,089 & 26,612 & 10.4 & 106.6 \\
\hline 1996 & 191,129 & 26,909 & 10.6 & 111.1 \\
\hline 1997 & 192,414 & 27,713 & 12.5 & 121.1 \\
\hline \multicolumn{5}{|c|}{ Average Annual Percentage Change } \\
\hline $1970-97$ & $1.4 \%$ & $0.2 \%$ & $1.2 \%$ & $0.9 \%$ \\
\hline $1987-97$ & $-0.5 \%$ & $-1.1 \%$ & $0.3 \%$ & $-1.4 \%$ \\
\hline
\end{tabular}

Sources:

Intercity passenger-miles - Eno Foundation for Transportation, Transportation in America 1998, Fifteenth edition, Lansdowne, VA, 1998, p. 47, and annual.

All other- U.S. Department of Transportation, Federal Aviation Administration, General Aviation Activity and Avionics Survey: Calendar Year 1997, pp. 1-7, 1-14, 5-3, and annual.

(Additional resources: http://www.faa.gov)

"Active fixed-wing general aviation aircraft only.

bInclude rotocraft. 
In the early seventies, domestic waterborne commerce accounted for over $60 \%$ of total tonnage, but

by 1994 foreign tonnage grew to more than half of all waterborne tonnage.

Table 12.3

Tonnage Statistics for Domestic and

International Waterborne Commerce, 1970-97

(million tons shipped)

\begin{tabular}{ccccc}
\hline Year & $\begin{array}{c}\text { Foreign and } \\
\text { domestic total }\end{array}$ & Foreign tota $^{\mathrm{a}}$ & Domestic total $^{\mathrm{b}}$ & $\begin{array}{c}\text { Percent domestic } \\
\text { of total }^{\text {Dor }}\end{array}$ \\
\hline 1970 & 1,532 & 581 & 951 & $62.1 \%$ \\
1971 & 1,513 & 566 & 947 & $62.6 \%$ \\
1972 & 1,617 & 630 & 987 & $61.0 \%$ \\
1973 & 1,762 & 767 & 994 & $56.4 \%$ \\
1974 & 1,747 & 764 & 983 & $56.3 \%$ \\
1975 & 1,695 & 749 & 946 & $55.8 \%$ \\
1976 & 1,835 & 856 & 979 & $53.4 \%$ \\
1977 & 1,908 & 935 & 973 & $51.0 \%$ \\
1978 & 2,021 & 946 & 1,075 & $53.2 \%$ \\
1979 & 2,073 & 993 & 1,080 & $52.1 \%$ \\
1980 & 1,999 & 921 & 1,077 & $53.9 \%$ \\
1981 & 1,942 & 887 & 1,054 & $54.3 \%$ \\
1982 & 1,777 & 820 & 957 & $53.9 \%$ \\
1983 & 1,708 & 751 & 957 & $56.0 \%$ \\
1984 & 1,836 & 803 & 1,033 & $56.3 \%$ \\
1985 & 1,788 & 774 & 1,014 & $56.7 \%$ \\
1986 & 1,874 & 837 & 1,037 & $55.3 \%$ \\
1987 & 1,967 & 891 & 1,076 & $54.7 \%$ \\
1988 & 2,088 & 976 & 1,112 & $53.3 \%$ \\
1989 & 2,140 & 1,038 & 1,103 & $51.5 \%$ \\
1990 & 2,164 & 1,042 & 1,122 & $51.8 \%$ \\
1991 & 2,092 & 1,014 & 1,079 & $51.6 \%$ \\
1992 & 2,132 & 1,037 & 1,095 & $51.4 \%$ \\
1993 & 2,128 & 1,060 & 1,068 & $50.2 \%$ \\
1994 & 2,215 & 1,116 & 1,099 & $49.6 \%$ \\
1995 & 2,240 & 1,147 & 1,093 & $48.8 \%$ \\
1996 & 2,284 & 1,183 & 1,101 & $48.2 \%$ \\
1997 & 2,333 & 1,220 & 1,113 & $47.8 \%$ \\
$1970-97$ & $1.6 \%$ & $2.8 \%$ & $0.6 \%$ & \\
$1987-97$ & $1.7 \%$ & $3.2 \%$ & $0.3 \%$ & \\
\hline Average & annualpercentage & change & \\
& & & & \\
\hline
\end{tabular}

Source:

U.S. Department of the Army, Corps of Engineers, Waterborne Commerce of the United States, Calendar Year 1997, Part 5: National Summaries, New Orleans, Louisiana, 1999, Table 1-1, p. 1-3, and annual.

(Additional resources: http://www.wrc-ndc.usace.army.mil/ndc)

"All movements between the U.S. and foreign countries and between Puerto Rico and the Virgin Islands and foreign countries are classified as foreign trade.

bAll movements between U.S. ports, continental and noncontiguous, and on the inland rivers, canals, and connecting channels of the U.S., Puerto Rico, and the Virgin Islands, excluding the Panama Canal. Beginning in 1996, fish was excluded for internal and intra port domestic traffic. 
Table 12.4

Summary Statistics for Domestic Waterborne Commerce, 1970-97

\begin{tabular}{|c|c|c|c|c|c|c|}
\hline Year & $\begin{array}{c}\text { Number of } \\
\text { vessels }^{\mathbf{a}}\end{array}$ & $\begin{array}{c}\text { Ton- } \\
\text { miles } \\
\text { (billions) }\end{array}$ & $\begin{array}{c}\text { Tons shipped } \\
\text { (millions) }\end{array}$ & $\begin{array}{l}\text { Average } \\
\text { length of } \\
\text { haul } \\
\text { (miles) }\end{array}$ & $\begin{array}{c}\text { Energy } \\
\text { intensity } \\
\text { (Btu/ton-mile) }\end{array}$ & $\begin{array}{c}\text { Energy use } \\
\text { (trillion Btu) }\end{array}$ \\
\hline 1970 & 25,832 & 596 & 949 & 628.2 & 545 & 324.8 \\
\hline 1971 & 26,063 & 593 & 944 & 628.1 & 506 & 300.0 \\
\hline 1972 & 27,347 & 604 & 985 & 612.8 & 522 & 315.1 \\
\hline 1973 & 28,431 & 585 & 990 & 590.7 & 576 & 337.0 \\
\hline 1974 & 29,328 & 586 & 979 & 599.1 & 483 & 283.3 \\
\hline 1975 & 31,666 & 566 & 944 & 599.9 & 549 & 311.0 \\
\hline 1976 & 33,204 & 592 & 976 & 606.3 & 468 & 277.3 \\
\hline 1977 & 35,333 & 599 & 969 & 618.0 & 458 & 274.3 \\
\hline 1978 & 35,723 & 827 & 1,072 & 771.6 & 383 & 316.6 \\
\hline 1979 & 36,264 & 829 & 1,076 & 770.0 & 457 & 378.7 \\
\hline 1980 & 38,792 & 922 & 1,074 & 856.4 & 358 & 329.8 \\
\hline 1981 & 42,079 & 929 & 1,051 & 884.0 & 360 & 334.5 \\
\hline 1982 & 42,079 & 886 & 954 & 929.0 & 310 & 274.9 \\
\hline 1983 & 41,784 & 920 & 953 & 964.6 & 319 & 293.7 \\
\hline 1984 & 41,784 & 888 & 1,029 & 862.5 & 346 & 307.3 \\
\hline 1985 & 41,672 & 893 & 1,011 & 883.5 & 446 & 398.6 \\
\hline 1986 & 40,308 & 873 & 1,033 & 845.3 & 463 & 404.0 \\
\hline 1987 & 40,000 & 895 & 1,072 & 835.0 & 402 & 370.7 \\
\hline 1988 & 39,192 & 890 & 1,106 & 804.3 & 361 & 321.3 \\
\hline 1989 & 39,209 & 816 & 1,097 & 743.2 & 403 & 328.6 \\
\hline 1990 & 39,233 & 834 & 1,118 & 745.7 & 388 & 323.2 \\
\hline 1991 & 39,233 & 848 & 1,074 & 789.9 & 386 & 327.5 \\
\hline 1992 & 39,210 & 857 & 1,090 & 785.7 & 398 & 341.0 \\
\hline 1993 & 39,064 & 790 & 1,063 & 742.7 & 389 & 307.0 \\
\hline 1994 & 39,064 & 815 & 1,093 & 745.5 & 369 & 300.7 \\
\hline 1995 & 39,641 & 808 & 1,086 & 743.6 & 374 & 302.2 \\
\hline 1996 & 41,104 & 765 & 1,093 & 699.4 & 412 & 314.9 \\
\hline 1997 & 41,419 & 707 & 1,106 & 639.5 & 415 & 293.2 \\
\hline \multicolumn{7}{|c|}{ Average annual percentage change } \\
\hline $1970-97$ & $1.8 \%$ & $0.6 \%$ & $0.6 \%$ & $0.1 \%$ & $-1.0 \%$ & $-0.4 \%$ \\
\hline $1987-97$ & $0.3 \%$ & $-2.3 \%$ & $0.3 \%$ & $-2.6 \%$ & $-0.3 \%$ & $-2.3 \%$ \\
\hline
\end{tabular}

\section{Source:}

Number of vessels -

1970-92, 1995-96 - U.S. Department of the Army, Corps of Engineers, "Summary of U.S. Flag

Passenger and cargo vessels, 1996," New Orleans, LA, 1998, and annual.

1993-94 - U.S. Dept of the Army, Corps of Engineers, The U.S. Waterway System-Facts,

Navigation Data Center, New Orleans, Louisiana, January 1996.

Ton-miles, tons shipped, average length of haul - U.S. Department of the Army, Corps of Engineers,

Waterborne Commerce of the United States, Calendar Year 1997, Part 5: National Summaries,

New Orleans, LA, 1999, Table 1-4, pp. 1-6, 1-7, and annual.

Energy use - See Appendix A for Table 2.7.

(Additional resources: http://www.wrc-ndc.usace.army.mil/ndc)

${ }^{\mathrm{a}} \mathrm{Grand}$ total for self-propelled and non-self-propelled.

'These figures are not consistent with the figures on Table 6.4 because intra-territory tons are not included in this table. Irma-territory traffic is traffic between ports in Puerto Rico and the Virgin Islands. 
Fifty-sixpercent of all domestic marine cargo in 1997 were energy-relatedproducts (petroleum, coal, coke). The majority of the energy-relatedproducts were shipped internally and locally (63\%). Barge traffic accounted for $96 \%$ of all internal and local waterborne commerce.

Table 12.5

Breakdown of Domestic Marine Cargo by Commodity Class, 1997

\begin{tabular}{|c|c|c|c|c|c|c|c|c|c|}
\hline \multirow[b]{2}{*}{ Commodity class } & \multicolumn{2}{|c|}{ Coastwise } & \multicolumn{2}{|c|}{ Lakewise } & \multicolumn{2}{|c|}{ Internal and local } & \multicolumn{3}{|c|}{ Total domestic $^{\mathrm{a}}$} \\
\hline & $\begin{array}{c}\text { Tons } \\
\text { shipped } \\
\text { (millions) }\end{array}$ & $\begin{array}{c}\text { Average } \\
\text { haul }^{b} \\
\text { (miles) }\end{array}$ & $\begin{array}{c}\text { Tons } \\
\text { shipped } \\
\text { (millions) }\end{array}$ & $\begin{array}{c}\text { Average } \\
\text { haul }^{\mathrm{b}} \\
\text { (miles) }\end{array}$ & $\begin{array}{c}\text { Tons } \\
\text { shipped } \\
\text { (millions) }\end{array}$ & $\begin{array}{c}\text { Average } \\
\text { haul }^{\mathrm{b}} \\
\text { (miles) }\end{array}$ & $\begin{array}{c}\text { Tons } \\
\text { shipped } \\
\text { (millions) }\end{array}$ & Percentage & $\begin{array}{c}\text { Average } \\
\text { haul }^{\mathrm{b}} \\
\text { (miles) }\end{array}$ \\
\hline Petroleum and products & 190 & 1,371 & 2 & 312 & 198 & 190 & 391 & $35.3 \%$ & 766 \\
\hline Chemicals and related products & 17 & 2,161 & c & 321 & 64 & 483 & 81 & $7.3 \%$ & 834 \\
\hline Crude materials & 18 & 623 & 93 & 513 & 135 & 346 & 246 & $22.3 \%$ & 429 \\
\hline Coal and coke & 15 & 669 & 23 & 526 & 194 & 378 & 232 & $21.0 \%$ & 412 \\
\hline Primary manufactured goods & 8 & 718 & 4 & 294 & 26 & 790 & 37 & $3.4 \%$ & 727 \\
\hline Food and farm products & 8 & 1,819 & c & 908 & 86 & 992 & 94 & $8.5 \%$ & 1,059 \\
\hline Manufactured equipment & 7 & 1,566 & c & c & 12 & 93 & 19 & $1.7 \%$ & 653 \\
\hline Waste and scrap & c & 1,000 & 0 & 0 & 6 & 60 & 6 & $0.5 \%$ & 60 \\
\hline Unknown & $\mathbf{c}$ & 2,015 & c & c & c & c & c & $0.0 \%$ & 1,607 \\
\hline Total & 263 & 1,329 & 123 & 506 & 720 & 410 & 1,106 & $100.0 \%$ & 639 \\
\hline Barge traffic (million tons) & 114 & & 11 & & 691 & & 816 & & \\
\hline Percentage by barge & $43.2 \%$ & & $8.8 \%$ & & $95.9 \%$ & & $73.7 \%$ & & \\
\hline
\end{tabular}

\section{Source:}

U.S. Department of the Army, Corps of Engineers, Waterborne Commerce of the United States, Calendar Year 1997, Part 5: National Summaries, New Orleans, Louisiana, 1999, Tables 2-1, 2-2, and 2-3, pp. 2-1-2-8, and annual.

(Additional resources: http://www.wrc-ndc.usace.army.mil/ndc)

Note:

Coastwise applies to domestic traffic receiving a carriage over the ocean or between the Great Lakes ports and seacoast ports when having a carriage over the ocean.

Lakewise applies to traffic between United States ports on the Great Lakes. Internal applies to traffic between ports or landings wherein the entire movement takes place on inland waterways. Local applies to movements of freight within the confines of a port.

"Does not include intra-territory tons.

${ }^{b}$ Calculated as ton-miles divided by tons shipped.

Negligible. 
The Interstate Commerce Commission designates Class I railroads on the basis of annual gross revenues. In 1997, nine railroads were given this classification.

Table 12.6

Class I Railroad Freight Systems in the United States

Ranked by Revenue Ton-Miles, 1997

\begin{tabular}{lcc}
\hline \multicolumn{1}{c}{ Railroad } & $\begin{array}{c}\text { Revenue ton-miles } \\
\text { (billions) }\end{array}$ & Percent \\
\hline Union Pacific Railroad Company & 452 & $33.5 \%$ \\
Burlington Northern and Sante Fe Railway Company & 425 & $31.5 \%$ \\
CSX Transportation & 166 & $12.3 \%$ \\
Norfolk Southern Corporation & 136 & $10.1 \%$ \\
Consolidated Rail Corporation (Conrail) & 98 & $7.3 \%$ \\
Illinois Central Railroad Company & 22 & $1.6 \%$ \\
Soo Line Railroad Company & 21 & $1.6 \%$ \\
Kansas City Southern Railway Company & 19 & $1.4 \%$ \\
Grand Trunk Western Railroad Inc. & 10 & $0.7 \%$ \\
& & \\
Total & $\mathbf{1 , 3 4 9}$ & $\mathbf{1 0 0 . 0 \%}$ \\
\hline
\end{tabular}

Source:

Association of American Railroads, Railroad Facts, 1998 Edition, Washington, DC, October 1998, p. 66. (Additional resources: http:/www.aar.org) 
Table 12.7

Summary Statistics for Class I Freight Railroads, 1970-97

\begin{tabular}{|c|c|c|c|c|c|c|c|c|c|}
\hline Year & $\begin{array}{l}\text { Number o } \\
\text { locomotives } \\
\mathrm{i} \quad \mathrm{n} \quad \mathrm{s}\end{array}$ & $\begin{array}{l}\text { of } \begin{array}{l}\text { Number of } \\
\text { es } \\
\text { freight cars } \\
\text { service }^{a} \text { (thousands) }\end{array} \\
\end{array}$ & $\begin{array}{c}\text { Train-miles } \\
\text { (millions) }\end{array}$ & $\begin{array}{l}\text { Car-miles } \\
\text { (millions) }\end{array}$ & $\begin{array}{c}\text { Tons } \\
\text { originated' } \\
\text { (millions) } \\
\end{array}$ & $\begin{array}{c}\text { Average } \\
\text { length of haul } \\
\text { (miles) }\end{array}$ & $\begin{array}{l}\text { Revenue } \\
\text { ton-miles } \\
\text { (millions) }\end{array}$ & $\begin{array}{c}\text { Energy } \\
\text { intensity } \\
\text { (Btu/ton-mile) }\end{array}$ & $\begin{array}{c}\text { Energy use } \\
\text { (trillion Btu) }\end{array}$ \\
\hline 1970 & $27,077^{d}$ & 1,424 & 427 & 29,890 & 1,485 & 515 & 764,809 & 691 & 528.1 \\
\hline 1971 & $27,160^{d}$ & 1,422 & 430 & 29,181 & 1,391 & 507 & 739,723 & 717 & 530.2 \\
\hline 1972 & 27,044 & 1,411 & 451 & 30,309 & 1,448 & 511 & 776,746 & 714 & 554.4 \\
\hline 1973 & 27,438 & 1,395 & 469 & 31,248 & 1,532 & 531 & $85 \quad 1,809$ & 677 & 577.1 \\
\hline 1974 & 27,627 & 1,375 & 469 & 30,719 & 1,531 & 527 & 850,961 & 681 & 579.1 \\
\hline 1975 & 27,855 & 1,359 & 403 & 27,656 & 1,395 & 541 & 754,252 & 687 & 518.3 \\
\hline 1976 & 27,233 & 1,332 & 425 & 28,530 & 1,407 & 540 & 794,059 & 680 & 540.3 \\
\hline 1977 & 27,298 & 1,287 & 428 & 28,749 & 1,395 & 549 & 826,292 & 669 & 552.7 \\
\hline 1978 & 26,959 & 1,226 & 433 & 29,076 & 1,390 & 617 & 858,105 & 641 & 550.4 \\
\hline 1979 & 27,660 & 1,217 & 438 & 29,436 & 1,502 & 611 & 913,669 & 618 & 564.8 \\
\hline 1980 & 28,094 & 1,168 & 428 & 29,277 & 1,492 & 616 & 918,621 & 597 & 548.7 \\
\hline \multirow[t]{2}{*}{1981} & 27,421 & 1,111 & 408 & 27,968 & 1,453 & 626 & 910,169 & 572 & 521.0 \\
\hline & 26,795 & 1,039 & 345 & 23,952 & 1,269 & 629 & 797,759 & 553 & 440.8 \\
\hline 19821983 & 25,448 & 1,007 & 346 & 24,358 & 1,293 & 641 & 828,275 & 525 & 435.1 \\
\hline 1984 & 24,117 & 948 & 369 & 26,409 & 1,429 & 645 & 921,542 & 510 & 470.0 \\
\hline 1985 & 22,548 & 867 & 347 & 24,920 & 1,320 & 664 & 876,984 & 497 & 436.1 \\
\hline 1986 & 20,790 & 799 & 347 & 24,414 & 1,306 & 664 & 867,722 & 486 & 421.5 \\
\hline \multirow[t]{2}{*}{1987} & 19,647 & 749 & 361 & 25,627 & 1,372 & 688 & 943,747 & 456 & 430.3 \\
\hline & 19,364 & 725 & 379 & 26,339 & 1,430 & 697 & 996,182 & 443 & 441.4 \\
\hline 19881989 & 19,015 & 682 & 383 & 26,196 & 1,403 & 723 & $1,013,841$ & 437 & 442.6 \\
\hline 1990 & 18,835 & 659 & 380 & 26,159 & 1,425 & 726 & $1,033,969$ & 420 & 434.7 \\
\hline \multirow[t]{3}{*}{19921991} & 18,344 & 633 & 375 & 25,628 & 1,383 & 751 & $1,038,875$ & 391 & 405.8 \\
\hline & 18,004 & 605 & 390 & 26,128 & 1,399 & 763 & $1,066,781$ & 393 & 419.2 \\
\hline & 18,161 & 587 & 405 & 26,883 & 1,397 & 794 & $1,109,309$ & 389 & 431.6 \\
\hline 19931994 & 18,505 & 591 & 441 & 28,485 & 1,470 & 817 & $1,200,701$ & 388 & 465.4 \\
\hline 1995 & 18,812 & 583 & 458 & 30,383 & 1,550 & 843 & $1,305,688$ & 372 & 485.9 \\
\hline 1996 & 19,269 & 571 & 469 & 31,715 & 1,611 . & 842 & $1,355,975$ & 368 & 499.4 \\
\hline \multirow[t]{2}{*}{1997} & 19,684 & 568 & 475 & 31,660 & 1,585 & 851 & $1,348,926$ & 370 & 499.7 \\
\hline & \multicolumn{9}{|c|}{ Average annualpercentage change } \\
\hline 1970-97 & $-1.2 \%$ & $-3.3 \%$ & $0.4 \%$ & $0.2 \%$ & $0.2 \%$ & $1.9 \%$ & $2.1 \%$ & $-2.3 \%$ & $-0.2 \%$ \\
\hline $1987-97$ & $0.0 \%$ & $-2.7 \%$ & $2.8 \%$ & $2.1 \%$ & $1.5 \%$ & $2.1 \%$ & $3.6 \%$ & $-2.1 \%$ & $1.5 \%$ \\
\hline
\end{tabular}

Source:

Association of American Railroads, Railroad Facts, 1998 Edition, Washington, DC, October 1998, pp. 27, 28, 33, 34, 36, 48, 50, 60. (Additional resources: http://www.aar.org)

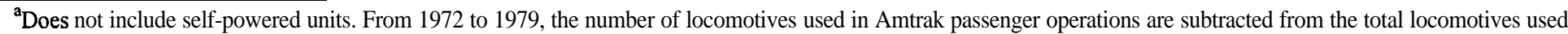
in passenger and freight service to calculate the number of Class I locomotives in service.

boes not include private or shipper-owned cars.

' Tons originated is a more accurate representation of total tonnage than revenue tons. Revenue tons often produces double-counting of loads switched between rail companies.

${ }^{\mathrm{d}}$ Data represent total locomotives used in freight and passenger service. Separate estimates are not available. 
The "other" category, which consists primarily of intermodal traffic, has grown 119\% in carloads from 1974 to 1997. Coal now accounts for one quarter of all carloads.

T a b l e 12.8

Railroad Revenue Carloads by Commodity Group, 1974 and 1997

\begin{tabular}{|c|c|c|c|c|c|}
\hline \multirow[b]{2}{*}{ Commodity group } & \multicolumn{2}{|c|}{$\begin{array}{l}\text { Carloads } \\
\text { (thousands) }\end{array}$} & \multirow{2}{*}{$\frac{\text { Percent }}{1974}$} & distribution & \multirow{2}{*}{$\begin{array}{c}\text { Percentage } \\
\text { change } \\
1974-97\end{array}$} \\
\hline & 1974 & 1997 & & 1997 & \\
\hline Coal & 4,544 & 6,703 & $17.0 \%$ & $26.8 \%$ & $47.5 \%$ \\
\hline Farm products & 3,021 & 1,408 & $11.3 \%$ & $5.6 \%$ & $-53.4 \%$ \\
\hline Chemicals and allied products & 1,464 & 1,705 & $5.5 \%$ & $6.8 \%$ & $16.5 \%$ \\
\hline Nonmetallic minerals & 821 & 1,160 & $3.1 \%$ & $4.6 \%$ & $41.3 \%$ \\
\hline Food and kindred products & 1,777 & 1,295 & $6.6 \%$ & $5.2 \%$ & $-27.1 \%$ \\
\hline Lumber and wood products & 1,930 & 669 & $7.2 \%$ & $2.7 \%$ & $-65.3 \%$ \\
\hline Metallic ores & 1,910 & 327 & $7.1 \%$ & $1.3 \%$ & $-82.9 \%$ \\
\hline Stone, clay and glass & 2,428 & 485 & $9.1 \%$ & $1.9 \%$ & $-80.0 \%$ \\
\hline Pulp, paper, and allied products & 1,180 & 582 & $4.4 \%$ & $2.3 \%$ & $-50.7 \%$ \\
\hline Petroleum products & 877 & 503 & $3.3 \%$ & $2.0 \%$ & $-42.6 \%$ \\
\hline Primary metal products & 1,366 & 633 & $5.1 \%$ & $2.5 \%$ & $-53.7 \%$ \\
\hline Waste and scrap material & 889 & 608 & $3.3 \%$ & $2.4 \%$ & $-31.6 \%$ \\
\hline Transportation equipment & 1,126 & 1,380 & $4.2 \%$ & $5.5 \%$ & $22.6 \%$ \\
\hline Others & 3,451 & 7,558 & $12.9 \%$ & $30.2 \%$ & $119.0 \%$ \\
\hline Total & 26,784 & 25,016 & $100.0 \%$ & $100.0 \%$ & $-6.6 \%$ \\
\hline
\end{tabular}

Source:

1974 - Association of American Railroads, Railroad Facts, 1976 Edition, Washington, DC, 1975, p. 26. 1997 - Association of American Railroads, Railroad Facts, 1998 Edition, Washington, DC,

October 1998, p. 25.

(Additional resources: http://www.aar.org) 
The number of trailers and containers moved by railroads has increased more than four-fold from 1965 to 1997. Containerization has increased in recent years, evidenced by the 128\% increase in the number of containersfrom 1988 to 1997. According to the 1993 Commodity Flow Survey, $4.5 \%$ of all freight ton-miles are rail intermodal shipments (truck/rail or rail/water). See Table 8.11 for details.

Table 12.9

Intermodal Rail Traffic, 1965-97

\begin{tabular}{cccc}
\hline Year & $\begin{array}{c}\text { Trailers \& } \\
\text { containers }\end{array}$ & Trailers & Containers \\
\hline 1965 & $1,664,929$ & $\mathrm{a}$ & $\mathrm{a}$ \\
1970 & $2,363,200$ & $\mathrm{a}$ & $\mathrm{a}$ \\
1975 & $2,238,117$ & $\mathrm{a}$ & $\mathrm{a}$ \\
1980 & $3,059,402$ & $\mathrm{a}$ & $\mathrm{a}$ \\
1981 & $3,150,522$ & $\mathrm{a}$ & $\mathrm{a}$ \\
1982 & $3,396,973$ & $\mathrm{a}$ & $\mathrm{a}$ \\
1983 & $4,090,078$ & $\mathrm{a}$ & $\mathrm{a}$ \\
1984 & $4,565,743$ & $\mathrm{a}$ & $\mathrm{a}$ \\
1985 & $4,590,952$ & $\mathrm{a}$ & $\mathrm{a}$ \\
1986 & $4,997,229$ & $\mathrm{a}$ & $\mathrm{a}$ \\
1987 & $5,503,819$ & $\mathrm{a}$ & $\mathrm{a}$ \\
1988 & $5,779,547$ & $3,481,020$ & $2,298,527$ \\
1989 & $5,987,355$ & $3,496,262$ & $2,491,093$ \\
1990 & $6,206,782$ & $3,451,953$ & $2,754,829$ \\
1991 & $6,246,134$ & $3,201,560$ & $3,044,574$ \\
1992 & $6,627,841$ & $3,264,597$ & $3,363,244$ \\
1993 & $7,156,628$ & $3,464,126$ & $3,692,502$ \\
1994 & $8,128,228$ & $3,752,502$ & $4,375,726$ \\
1995 & $8,070,309$ & $3,519,664$ & $4,550,645$ \\
$1996^{\mathrm{b}}$ & $8,153,942$ & $3,320,312$ & $4,833,630$ \\
$1997^{\mathrm{b}}$ & $8,695,860$ & $3,453,081$ & $5,242,779$ \\
$1965-97$ & $5.3 \%$ & $\mathrm{a}$ & $\mathrm{a}$ \\
$1988-97$ & $4.6 \%$ & $-0.1 \%$ & $9.6 \%$ \\
\hline \multicolumn{4}{c}{ Average annualpercentage change } \\
\hline
\end{tabular}

Source:

Association of American Railroads, Railroad Facts, 1.998 edition, Washington, DC, October 1998 p.26. (Additional resources: http://www.aar.org)

\footnotetext{
${ }^{\mathbf{a}}$ Data are not available.

b The Grand Trunk Western Railroad and the Soo Line Railroad Company data are excluded.
} 
Table 12.10

Summary Statistics for the National Railroad Passenger Corporation (Amtrak), 1971-97

\begin{tabular}{|c|c|c|c|c|c|c|c|c|}
\hline Year & $\begin{array}{c}\text { Number of } \\
\text { locomotives } \\
\text { in service }\end{array}$ & $\begin{array}{c}\text { Number of } \\
\text { passenger cars }\end{array}$ & $\begin{array}{l}\text { Train-miles } \\
\text { (thousands) }\end{array}$ & $\begin{array}{l}\text { Car-miles } \\
\text { (thousands) }\end{array}$ & $\begin{array}{c}\text { Revenue } \\
\text { passenger-miles } \\
\text { (millions) }\end{array}$ & $\begin{array}{l}\text { Average trip length } \\
\text { (miles) }\end{array}$ & $\begin{array}{l}\text { Energy intensity } \\
\text { (Btu per revenue } \\
\text { passenger-mile) }\end{array}$ & $\begin{array}{l}\text { Energy use } \\
\text { (trillion Btu) }\end{array}$ \\
\hline 1971 & $\mathrm{a}$ & 1,165 & 16,537 & 140,147 & 1,993 & 188 & & a \\
\hline 1972 & 285 & 1,571 & 26,302 & 213,261 & 3,039 & 183 & $\mathbf{a}$ & a \\
\hline 1973 & 352 & 1,777 & 27,151 & 239,775 & 3, 807 & 224 & 3,156 & 14. 3 \\
\hline 1974 & 457 & 1,848 & 29, 538 & 260,060 & 4, 259 & 233 & 3,240 & 13.8 \\
\hline 1975 & 355 & 1,913 & 30,166 & 253,898 & 3,753 & 224 & 3,677 & 13.8 \\
\hline 1976 & 379 & 2,062 & 30,885 & 263,589 & 4,268 & 229 & 3,397 & 14. 5 \\
\hline 1977 & 369 & 2,154 & 33,200 & 261,325 & 4,204 & 221 & 3,568 & 15.0 \\
\hline 1978 & 441 & 2,084 & 32,451 & 255,214 & 4,154 & 217 & 3,683 & 15.3 \\
\hline 1979 & 437 & 2,026 & 31, 379 & 255,129 & 4,867 & 226 & 3,472 & 16. 9 \\
\hline 1980 & 448 & 2,128 & 29,487 & 235,235 & 4,503 & 217 & 3, 176 & 14.3 \\
\hline 1981 & 398 & 1,830 & 30,380 & 222,753 & 4, 397 & 226 & 2,979 & 13.1 \\
\hline 1982 & 396 & 1,929 & 28,833 & 217,385 & 3, 993 & 220 & 3,156 & 12.6 \\
\hline 1983 & 388 & 1,880 & 28,805 & 223,509 & 4,227 & 223 & 2,957 & 12.5 \\
\hline 1984 & 387 & 1,844 & 29,133 & 234, 557 & 4,427 & 227 & 3,027 & 13.4 \\
\hline 1985 & 382 & 1,818 & 30,038 & 250,642 & 4,785 & 238 & 2,800 & 13.4 \\
\hline 1986 & 369 & 1,793 & 28,604 & 249,665 & 5,011 & 249 & 2,574 & 12.9 \\
\hline 1987 & 381 & 1,850 & 29,515 & 261,054 & 5,361 & 259 & 2,537 & 13.6 \\
\hline 1988 & 391 & 1,845 & 30,221 & 277,774 & 5,686 & 265 & 2,462 & 14.0 \\
\hline 1989 & 312 & 1,742 & 31,000 & 285,255 & 5,859 & 274 & 2,731 & 16.0 \\
\hline 1990 & 318 & 1,863 & 33,000 & 300,996 & 6,057 & 273 & 2,609 & 15.8 \\
\hline 1991 & 316 & 1,786 & 34,000 & 312,484 & 6,273 & 285 & 2,503 & 15.7 \\
\hline 1992 & 336 & 1,796 & 34,000 & 307,282 & 6,091 & 286 & 2,610 & 15. 9 \\
\hline 1993 & 360 & 1,853 & 34,936 & 302,739 & 6,199 & 280 & 2,646 & 16.4 \\
\hline 1994 & 411 & 1,874 & 34,940 & 305,600 & 5,869 & 276 & 2,351 & $13.8^{b}$ \\
\hline 1995 & 422 & 1,907 & 31,579 & 282,579 & 5,401 & 266 & 2,314 & $12.5^{\prime}$ \\
\hline 1996 & 348 & 1,501 & 30,542 & 277,750 & 5,066 & 257 & 2,389 & $12.1^{\prime}$ \\
\hline 1997 & 292 & 1,572 & 32,000 & 287,760 & 5,166 & 255 & 2,458 & $12.7^{\prime}$ \\
\hline \multicolumn{9}{|c|}{ Average annual percentage change } \\
\hline 1971- 97 & $0.1 \%{ }^{\mathrm{d}}$ & 1. $2 \%$ & 2. $6 \%$ & 2. $8 \%$ & 3. $7 \%$ & $1.2 \%$ & $-1.8 \%^{\mathrm{d}}$ & $-0.5 \%^{d}$ \\
\hline 1987- 97 & $-2.6 \%$ & $-1.6 \%$ & $0.8 \%$ & $1.0 \%$ & $-0.4 \%$ & $-0.2 \%$ & $-0.3 \%^{d}$ & $-0.7 \%{ }^{d}$ \\
\hline
\end{tabular}

Source:

1971-83- Association of American Railroads, Economics and Finance Department, Statistics of Class I Railroads, Washington, DC, and annual.

1984-88- Association of American Railroads, Railroad Facts, 1988 Edition, Washington, DC, December 1989, p. 61 , and annual.

1989-93- Personal communication with the Corporate Accounting Office of Amtrak, Washington, D.C.

1994-97- Number of locomotives in service, number of passenger cars, train-miles, car-miles, revenue passenger-miles, and average trip length - Association of American Railroads, Railroad Facts, 1998 Edition, Washington, DC, 1998, p. 77.

Energy use - Personal communication with the Amtrak, Washington, DC. (Additional resources: http://www.amtrak.com, http://www.aar.org)

a Data are not available.

${ }^{\circ}$ Energy use for 1994 on is not directly comparable to earlier years. Some commuter rail energy use may have been inadvertently included in earlier years.

c Estimated using train-miles.

Average annual percentage change is from earliest year available to 1997. 
Table 12.11

Summary Statistics for Rail Transit Operations, 1970-97"

\begin{tabular}{|c|c|c|c|c|c|c|c|}
\hline Year & $\begin{array}{c}\text { Number of } \\
\text { passenger vehicles }\end{array}$ & $\begin{array}{c}\begin{array}{c}\text { Vehicle-miles } \\
\text { (millions) }\end{array} \\
\end{array}$ & $\begin{array}{c}\text { Passenger trips } \\
\text { (millions) }^{\mathrm{b}}\end{array}$ & $\begin{array}{c}\text { Estimated passenger-miles } \\
\text { (millions) }\end{array}$ & $\begin{array}{l}\text { Average trip length } \\
\text { (miles) }^{d}\end{array}$ & $\begin{array}{c}\text { Energy intensity } \\
\text { (Btu/passenger-mile) }\end{array}$ & $\begin{array}{l}\text { Energy use } \\
\text { (trillion Btu) }\end{array}$ \\
\hline 1970 & 10,548 & 440.8 & 2,116 & 12,273 & & 2,453 & 30.1 \\
\hline 1971 & 10,550 & 440.4 & 2,000 & 11,600 & $\mathrm{f}$ & 2,595 & 30.1 \\
\hline 1972 & 10,599 & 417.8 & 1,942 & 11,264 & $f$ & 2,540 & 28.6 \\
\hline 1973 & 10,510 & 438.5 & 1,921 & 11,142 & & 2,460 & 27.4 \\
\hline 1974 & 10,471 & 458.8 & 1,876 & 10,881 & & 2,840 & 30.9 \\
\hline 1975 & 10,617 & 446.9 & 1,797 & 10,423 & & 2,962 & 31.1 \\
\hline 1976 & 10,625 & 428.1 & 1,744 & 10,115 & & 2,971 & 30.3 \\
\hline 1977 & 10,579 & 381.7 & 1,713 & 10,071 & 5.8 & 2,691 & 27.1 \\
\hline 1978 & 10,459 & 383.0 & 1,810 & 10,722 & 5.9 & 2,210 & 23.7 \\
\hline 1979 & 10,429 & 399.6 & 1,884 & 11,167 & 5.9 & 2,794 & 31.2 \\
\hline 1980 & 10,654 & 402.2 & 2,241 & 10,939 & 4.9 & 3,008 & 32.9 \\
\hline 1981 & 10,824 & 436.6 & 2,217 & 10,590 & 4.8 & 2,946 & 31.2 \\
\hline 1982 & 10,831 & 445.2 & 2,201 & 10,428 & 4.6 & 3,069 & 32.0 \\
\hline 1983 & 10,904 & 423.5 & 2,304 & 10,741 & 4.7 & 3,212 & 34.5 \\
\hline 1984 & 10,848 & 452.7 & 2,388 & 10,531 & 4.4 & 3,732 & 39.3 \\
\hline 1985 & 11,109 & 467.8 & 2,422 & 10,777 & 4.4 & 3,461 & 37.3 \\
\hline 1986 & 11,083 & 492.8 & 2,467 & 11,018 & 4.5 & 3,531 & 38.9 \\
\hline 1987 & 10,934 & 508.6 & 2,535 & 11,603 & 4.6 & 3,534 & 41.0 \\
\hline 1988 & 11,370 & 538.3 & 2,462 & 11,836 & 4.8 & 3,565 & 42.2 \\
\hline 1989 & 11,261 & 553.4 & 2,704 & 12,539 & 4.6 & 3,397 & 42.6 \\
\hline \multirow[t]{2}{*}{19911990} & 11,332 & 560.9 & 2,521 & 12,046 & 4.8 & 3,453 & 41.6 \\
\hline & 11,426 & 554.8 & 2,356 & 11,190 & 4.7 & 3,727 & 41.7 \\
\hline 1992 & 11,303 & 554.1 & 2,396 & 11,441 & 4.8 & 3,575 & 40.9 \\
\hline 1993 & 11,286 & 549.8 & 2,234 & 10,936 & 4.9 & 3,687 & 42.2 \\
\hline 1994 & 11,192 & 565.8 & 2,453 & 11,501 & 4.8 & 3,828 & 44.0 \\
\hline 1995 & 11,156 & 571.8 & 2,284 & 11,419 & 5.0 & 3,818 & 43.6 \\
\hline 1996 & 11,341 & 580.7 & 2,417 & 12,484 & 5.2 & 3,444 & 43.0 \\
\hline 1997 & 11,471 & 598.8 & 2,693 & 13,095 & 4.9 & 3,253 & 42.6 \\
\hline \multicolumn{8}{|c|}{ Average annual percentage change } \\
\hline $1970-97$ & $0.3 \%$ & $1.1 \%$ & $0.9 \%$ & $0.2 \%$ & $-0.8 \%{ }^{\mathrm{g}}$ & $1.1 \%$ & $1.3 \%$ \\
\hline $1987-97$ & $0.5 \%$ & $1.6 \%$ & $0.6 \%$ & $1.2 \%$ & $0.6 \%$ & $-0.8 \%$ & $0.4 \%$ \\
\hline
\end{tabular}

American Public Transit Association, 1999 Transit Fact Book, Washington, DC, February 1999, pp. 69, 71, 78, 83. (Additional resources: http://www.apta.com) Energy use - See Appendix A for Table 2.7.

"Heavy rail and light rail. Series not continuous between 1983 and 1984 because of a change in data source by the American Public Transit Association (APTA). Beginning in 1984, data provided by APTA are taken from mandatory reports filed with the Urban Mass Transit Administration (UMTA). Data for prior years were provided on a voluntary basis by APTA members and expanded statistically.

${ }^{\text {b } 1970-79 ~ d a t a ~ r e p r e s e n t s ~ t o t a l ~ p a s s e n g e r ~ r i d e s ; ~ a f t e r ~ 1979, ~ d a t a ~ r e p r e s e n t s ~ u n l i n k e d ~ p a s e n g e r ~ t r i p s . ~}$

'Estimated for years 1970-76 based on an average trip length of 5.8 miles.

${ }^{d}$ Calculated as the ratio of passenger-miles to passenger trips.

'Large system-to-system variations exist within this category.

Data are not available.

${ }^{\mathbf{g}}$ Average annual percentage change is calculated for years 1977-97. 


\section{APPENDIX A}

\section{SOURCES}

This appendix contains documentation of the estimation procedures used by ORNL. The reader can examine the methodology behind the estimates and form an opinion as to their utility.

The appendix is arranged by table number and subject heading. Only tables which contain ORNL estimations are documented in Appendix A; all other tables have sources listed at the bottom of the table. Since abbreviations are used throughout the appendix, a list of abbreviations is also included. 


\section{List of Abbreviations Used in Appendix A}

$\begin{array}{ll}\text { AAMA } & \text { American Automobile Manufacturers Association } \\ \text { AAR } & \text { Association of American Railroads } \\ \text { APTA } & \text { American Public Transit Association } \\ \text { Amtrak } & \text { National Railroad Passenger Corporation } \\ \text { Btu } & \text { British thermal unit } \\ \text { DOC } & \text { Department of Commerce } \\ \text { DOE } & \text { Department of Energy } \\ \text { DOT } & \text { Department of Transportation } \\ \text { EIA } & \text { Energy Information Administration } \\ \text { EPA } & \text { Environmental Protection Agency } \\ \text { FAA } & \text { Federal Aviation Administration } \\ \text { FHWA } & \text { Federal Highway Administration } \\ \text { gvw } & \text { gross vehicle weight } \\ \text { lpg } & \text { liquefied petroleum gas } \\ \text { mpg } & \text { miles per gallon } \\ \text { NHTSA } & \text { National Highway Traffic Safety Administration } \\ \text { NPTS } & \text { Nationwide Personal Transportation Study } \\ \text { ORNL } & \text { Oak Ridge National Laboratory } \\ \text { pmt } & \text { passenger-miles traveled } \\ \text { RECS } & \text { Residential Energy Consumption Survey } \\ \text { RTECS } & \text { Residential Transportation Energy Consumption Survey } \\ \text { TIUS } & \text { Truck Inventory and Use Survey } \\ \text { TSC } & \text { Transportation Systems Center } \\ \text { vmt } & \text { vehicle-miles traveled } \\ & \end{array}$


Table 2.5

Domestic Consumption of Transportation Energy by Mode and Fuel Type, 1997

Most of the source data were given in gallons. It was converted to Btu by using the conversion factors in Appendix B.

\section{Highway}

Automobiles

Total gallons of fuel taken from DOT, FHWA, Highway Statistics 1997, Table VM- 1. These were distributed as follows: 97.\% gasoline, $1.0 \%$ gasohol, and $1.3 \%$ diesel. Percentages were derived from the DOE, EIA, Office of Markets and End Use, Energy End Use Division, Household Vehicles Energy Consumptiqn 1994, August 1997, p. 46. Natural gas data are from the DOE, EIANatural Gas Annual 1997, Table 1; transit bus natural gas was subtracted from the total and the remainder was assumed to be light vehicle use. Automobiles were assumed to use $25 \%$ of light vehicle natural gas use. Methanol use was estimated using data from DOE, EIA, Alternatives to Traditional Transportation Fuels 1997, Washington, DC, 1998, Table 12.

\section{Motorcycles}

DOT, FHWA, Highway Statistics 1997, Table VM-1. For conversion purposes, fuel for all motorcycles was assumed to be gasoline.

\section{Buses}

\section{Transit:}

APTA, 1999 Transit Fact Book, 1999, Washington, DC.

Non-diesel fossil fuel consumption was assumed to be used by motor buses,

\section{Intercity:}

Eno Transportation Foundation, Transportation in America 1998, Sixteenth Edition, 1998, Lansdowne, VA, p. 56. For conversion purposes, fuel for all intercity buses was assumed to be diesel fuel. (1997 data were estimated using vehicle travel information.)

\section{School:}

Gasoline and Diesel - Eno Transportation Foundation, Transportation in America 1998, Sixteenth Edition, 1998, Lansdowne, VA, p. 56. For conversion purposes, fuel for school buses was assumed to be $60 \%$ diesel fuel and $40 \%$ gasoline.

Methanol - Methanol use was estimated using data from DOE, EIA, Alternatives to Traditional Transportation Fuels 1997, Washington DC, 1998. 
Trucks

Total:

Sum of light trucks and other trucks.

Light Trucks:

DOT, FHWA, Highway Statistics 1997, Table VM-1, for single-unit, 2-axle, 4-tire trucks. $96.2 \%$ of fuel assumed to be gasoline, $3.3 \%$ diesel, $0.3 \% \mathrm{lpg}$; percentages were generated from the 1992 TIUS Public Use Tape. Natural gas data are from the DOE, EIA Natural Gas Annual 1997, Table 1; transit bus natural gas was subtracted from the total and the remainder was assumed to be light vehicle use. Light trucks were assumed to use $75 \%$ of light vehicle natural gas use.

Other Trucks:

DOT, FHWA, Highway Statistics 1997, Table VM-1. Total gallons for other trucks was the difference between total and 2-axle, 4-tire trucks. These gallons were distributed as follows based on data from the 1992 TIUS Public Use Tape: $16.2 \%$ of fuel assumed to be gasoline, $83.3 \%$ diesel, and $0.5 \% \mathrm{lpg}$.

\section{Off Highway}

Diesel:

Data supplied by Marianne Mintz, Argonne National Laboratory, from the Public Use Data Base, National Energy Accounts, DOC, OBA-NEA- 10, August 1988.

\section{Gasoline:}

DOT, FHWA, Highway Statistics 1997, Table MF-24. Agriculture and Construction totals.

\section{Nonhighway}

Air

\section{General Aviation:}

DOT, FAA, General Aviation Activity and Avionics Survey: Annual Summary Report Calendar Year 1997, Table 5.1. Jet fuel was converted from gallons to Btu using 135,000 Btu/gallon (kerosene-type jet fuel).

\section{Domestic and International Air Carrier:}

DOT, Bureau of Transportation Statistics, "Fuel Cost and Consumption Tables;" annual figures were obtained by summing monthly totals. Because the data for international included fuel purchased abroad, the international total was divided in half to estimate domestic fuel use for international flights. 
Water

\section{Freight:}

Total - DOE, EIA, Fuel Oil and Kerosene Sales 1997, Table 23. Adjusted sales of distillate and residual fuel oil for vessel bunkering. (This may include some amounts of bunker fuels used for recreational purposes.)

\section{Recreational Boating:}

Fuel use by recreational boating was calculated using the methodology developed by D. L. Greene in the report, Off-Highway Use of Gasoline in the United States (DOT, FHWA, July 1986, p. 3-22). Results from Model 1 in the report indicated an average annual consumption of 205 gallons per boat. Total consumption in gallons was then calculated using the following equation: Total $=0.95$ (Gal/boat) (number ofboats). An estimate of number of recreational boats in operation is from the U.S. Coast Guard (numbered boats).

\section{Pipeline}

The sum of natural gas, crude petroleum and petroleum product, and coal slurry and water.

Natural Gas:

The amount of natural gas used to transport natural gas was defined as "pipeline fuel" as reported in DOE, EIA, Natural Gas Annual 1997, Table 1. Cubic feet were converted to Btu using $1,031 \mathrm{Btu} / \mathrm{ft}^{3}$. Electricity use was estimated using the following procedure as reported on p. 5- 110 of J. N. Hooker et al., End Use Energy Consumption DataBase: Transportation Sector. The energy consumption of a natural gas pipeline 'was taken to be the energy content of the fuel used to drive the pumps. Some $94 \%$ of the installed pumping horsepower was supplied by natural gas. The remaining $6 \%$ of the horse power was generated more efficiently, mostly by electric motors. The energy consumed by natural gas pipeline pumps that were electrically powered was not known. In order to estimate the electricity consumed, the Btu of natural gas pipeline fuel consumed was multiplied by a factor of 0.015 . From this computed value, electricity efficiency and generation loss must be taken into account. The electricity energy use in Btu must be converted to $\mathrm{kWhr}$, using the conversion factor $29.305 \times 10^{-5} \mathrm{kWhr} / \mathrm{Btu}$. Electricity generation and distribution efficiency was 29\%. When generation and distribution efficiency are taken into account, $1 \mathrm{kWhr}$ equals 11,765 Btu.

Crude petroleum and petroleum product:

J. N. Hooker, Oil Pipeline Energy Consumption and Efficiency, ORNL-5697, ORNL, Oak Ridge, TN, 198 1. (Latest available data.)

Coal slurry and water:

W. F. Banks, Systems, Science and Software, Energy Consumption in the Pipeline Industry, LaJolla, CA, October 1977. (Latest available data.) 
Rail

\section{Total:}

Sum of freight and passenger rail.

\section{Freight:}

AAR, Railroad Facts, 1998 Edition, Washington, DC, 1998, p. 60.

\section{Passenger:}

Transit and Commuter - APTA, 1999 Transit Fact Book, Washington, DC, 1999, p. 106- 108. Transit was defined as the sum of "heavy rail," "light rail," and "other."

Intercity - Personal communication with Amtrak, Washington, DC. (1997 data were estimated using train-mile information.) 
Table 2.7

Transportation Energy Consumption by Mode, 1970-97

\section{Highway}

Automobiles

Total gallons of fuel for automobiles was taken from DOT, FHWA, Highway Statistics Summary to 199.5, Table VM-20 1A; and Table VM- 1 in the 1996 annual edition. Fuel for automobiles was distributed between fuel types for conversion into Btu's as follows:

1970-80 - 94.7\% gasoline, 5.3\% diesel as reported in the DOE, EIA, Office of Energy Markets and End Use, Residential Energy Consumption Survey: Consumption Patterns of Household Vehicles, June 1979 to December 1980, p. 10.

198 1-82 - 94.1\% gasoline, 5.9\% diesel as reported in the DOE, EIA, Office of Energy Markets and End Use, Residential Energy Consumption Survey: Consumption Patterns of Household Vehicles, Supplement: January 1981 to September 1981, pp. 11, 13.

1983-84-97.5\% gasoline, 2.5\% diesel as reported in the DOE, EIA, Office of Markets and End Use, Energy End Use Division, Residential Transportation Energy Consumption Survey: Consumption Patterns of Household Vehicles, 1983, Jan., 1985, pp. 7, 9.

$1985-87-98.5 \%$ gasoline, $1.5 \%$ diesel as reported in the DOE, EIA, Office of Energy Markets and End Use, Residential Transportation Energy Consumption Survey: Consumption Patterns of Household Vehicles 1985, April 1987, pp. 25, 27.

1988-90 - 98.8\% gasoline and 1.2\% diesel as reported in the DOE, EIA, Office of Markets and End Use, Energy End Use Division, Household Vehicles Energy Consumption 1988, March 1990, p. 65.

199 1-93 - 97.8\% gasoline, $1.0 \%$ gasohol, and 1.2\% diesel as reported in the DOE, EIA, Office of Markets and End Use, Energy End Use Division, Household Vehicles Energy Consumption 1991, December 1993, p. 46.

1994-97 - 97.7\% gasoline, 1 .O\% gasohol, 1.3\% diesel as reported in the DOE, EIA, Office of Energy Markets and EndUse, Household Vehicles Energy Consumption, 1994, Washington, DC, August 1997, p. 46.

1993-97 - Methanol use was estimated using data from DOE, EIA, Alternatives to Traditional Transportation Fuels 1997, Washington, DC, 1998, Table 12.

1993-97 - Natural gas data are from the DOE, EIA Natural Gas Annual 1997, Table 1; transit bus natural gas was subtracted from the total and the remainder was assumed to be light vehicle use. Automobiles were assumed to use $25 \%$ of light vehicle natural gas use.

\section{Motorcycles}

Department of Transportation, Federal Highway Administration,Highway Statistics Summary to 1995, Table VM-201A; and Table VM-1 in the 1996 and 1997 annual editions. For conversion purposes, fuel for all motorcycles was assumed to be gasoline. 
Buses

Sum of transit, intercity and school.

\section{Transit:}

APTA, 1999 Transit Fact Book, January 1999, Washington, DC, pp. 106-108, and annual.

Non-diesel fossil fuel consumption was assumed to be used by motor buses. For the years 1988-92, motor bus gasoline use wasestimated as 5\% of "other" fuels, based on personal communication with the APTA Research and Statistics Department.

\section{Intercity:}

1970-84 - American Bus Association, Annual Report, Washington, DC, annual.

1985-97- Eno Transportation Foundation, Transportation in America 1998, Sixteenth Edition, 1998, Lansdowne, VA, p. 56. For conversion purposes, fuel for all intercity buses was assumed to be diesel fuel. (1997 data were estimated using vehicle travel information.)

\section{School:}

1970-84 - DOT, FHWA, Highway Statistics 1984, Washington, DC, Table VM-1, and annual.

1985-86 - DOT, Research and Special Programs Administration, National Transportation Statistics, Figure 2, p. 5, and annual.

1987-97- Eno Transportation Foundation, Transportation in America 1998, Sixteenth Edition, 1998, Lansdowne, VA, p. 56. For conversion purposes, fuel for school buses was assumed to be $60 \%$ diesel fuel and $40 \%$ gasoline. (1996 data were estimated using vehicle travel information.)

\section{Trucks}

Light Trucks:

Defined as 2-axle, 4-tire trucks. Total gallons of fuel was taken from DOT, FHWA, Highway Statistics Summary to 1995, Table VM-201A, and Table VM-1 of the 1996 and 1997 annual editions. Based on data from the 1982 TIUS Public Use Tape, fuel use for 1970-87 was distributed among fuel types as follows: $95.3 \%$ gasoline; $3.5 \%$ diesel; and $1.2 \%$ lpg. Fuel use for 1988-93 was distributed based on the 1987 TIUS: 96.6\% gasoline; 3.3\% diesel; and 0.1\% lpg. Fuel use for 1994-97 was distributed based on the 1992 TIUS: $96.4 \%$ gasoline; 3.3\% diesel; 0.3\% Ipg. Natural gas data are from the DOE, EIA Natural Gas Annual 1997, Table 1; transit bus natural gas was subtracted from the total and the remainder was assumed to be light vehicle use. Light trucks were assumed to use $75 \%$ of light vehicle natural gas use. 


\section{Other Trucks:}

Defined as the difference between total trucks and 2-axle, 4-tire trucks. Total gallons of fuel was taken from DOT, FHWA, Highway Statistics Summary to 1995, Table VM201A, and Table VM-1 of the 1996 and 1997 annual editions. Based on data from the 1982 TIUS Public Use Tape, fuel use for 1970-87 was distributed among fuel types as follows: $39.6 \%$ gasoline; 59.4\% diesel; and 1.0\% Ipg. Fuel use for 1988-93 was distributed based on the 1987 TIUS: $19.4 \%$ gasoline; $80.4 \%$ diesel; and $0.2 \% \mathrm{lpg}$. Fuel use for 1994-97 was distributed based on the 1992 TIUS: $16.2 \%$ gasoline; $83.3 \%$ diesel; and $0.5 \%$ Ipg.

\section{Total Highway}

Sum of autos, motorcycles, buses, light trucks, and other trucks.

\section{Nonhighway}

Air

Sum of fuel use by General Aviation and Certificated Route Air Carrier.

\section{General Aviation:}

1970-74 - DOT, TSC, National Transportation Statistics, Cambridge, MA, 1981.

1975-85 - DOT, FAA, FAA Aviation Forecasts, Washington, DC, annual.

1985-97 - DOT, FAA, General Aviation Activity and Avionics Survey: Annual Summary

Report, Calendar Year 1997, Table 5.1. Jet fuel was converted from gallons to Btu using 135,000 Btu/gallon (kerosene-type jet fuel).

\section{Certificated Route Air Carrier:}

1970-81 - DOT, Civil Aeronautics Board, Fuel Cost and Consumption, Washington, DC, annual.

1982-97- DOT, Bureau of Transportation Statistics, "Fuel Cost and Consumption Tables;" annual figures were obtained by summing monthly totals. These data are for domestic carriers, but include the international operations of those domestic carriers. The international operations total was divided in half to estimate domestic fuel use for international flights.

\section{Water}

Sum of vessel bunkering fuel (i.e., freight) and fuel used by recreational boats.

\section{Freight:}

Total - DOE, EIA, Fuel Oil and Kerosene Sales 1997, Table 23. Adjusted sales of distillate and residual fuel oil for vessel bunkering.(This may include some amounts of bunker fuels used for recreational purposes.)

\section{Recreational Boating:}

1970-84 - DOT, FHWA, Highway Statistics, Washington, DC, Table MF-24, annual. 
1985-97 - Fuel use by recreational boating was calculated using the methodology developed by D. L. Greene in the report, Off-Highway Use of Gasoline in the United States (DOT, FHWA, July 1986, p. 3-22). Results from Model 1 in the report indicated an average annual consumption of 205 gallons per boat. Total consumption in gallons was then calculated using the following equation: Total $=$ 0.95 (Gal/boat) (number of boats). An estimate of number of recreational boats in operation is from the U.S. Coast Guard (numbered boats).

\section{Pipeline}

The sum of natural gas, crude petroleum and petroleum product, and coal slurry and water.

Natural Gas:

The amount of natural gas used to transport natural gas was defined as "pipeline fuel" as reported in DOE, EIA, Natural Gas Annual 1997, Table 1. Cubic feet were converted to Btu using $1,031 \mathrm{Btu} / \mathrm{ft}^{3}$. Electricity use was estimated using the following procedure as reported on p. 5-110 of J. N. Hooker et al., End Use Energy Consumption DataBase: Transportation Sector. The energy consumption of a natural gas pipeline was taken to be the energy content of the fuel used to drive the pumps. Some $94 \%$ of the installed pumping horsepower was supplied by natural gas. The remaining $6 \%$ of the horse power was generated more efficiently, mostly by electric motors. The energy consumed by natural gas pipeline pumps that were electrically powered was not known. In order to estimate the electricity consumed, the Btu of natural gas pipeline fuel consumed was multiplied by a factor of 0.015 . From this computed value, electricity efficiency and generation loss must be taken into account. The electricity energy use in Btu must be converted to $\mathrm{kWhr}$, using the conversion factor $29.305 \times 10^{-5} \mathrm{kWhr} /$ Btu. Electricity generation and distribution efficiency was $29 \%$. When generation and distribution efficiency are taken into account, $1 \mathrm{kWhr}$ equals $11,765 \mathrm{Btu}$.

Crude petroleum and petroleum product.

J. N. Hooker, Oil Pipeline Energy Consumption and Efficiency, ORNL-5697, ORNL, Oak Ridge, Tennessee, 198 1. (Latest available data.)

Coal slurry and water:

W. F. Banks, Systems, Science and Software, Energy Consumption in the Pipeline Industry, LaJolla, California, October 1977. (Latest available data.)

Rail

Total:

Sum of freight and passenger rail.

\section{Freight:}

AAR, Railroad Facts, 1998 Edition, Washington, DC, p. 60. 


\section{Passenger:}

Transit and Commuter - APTA, 1999 Transit Fact Book, 1999, Washington, DC, p. 106-108, annual. Transit was defined as the sum of "heavy rail," "light rail," and "other."

Intercity - Personal communication with Amtrak, Washington, DC. (1995, 1996, and 1997 data were estimated using train-mile information.) 
Table 2.11

Passenger Travel and Energy Use in the United States, 1997

\section{Highway}

Automobiles

Number of Vehicles - DOT, FHWA, Highway Statistics 1997 Table VM- 1.

Vmt - DOT, FHWA, Highway Statistics 1997, Table VM-1.

Pmt - Calculated by ORNL (load factor times vmt).

Load Factor - DOT, FHWA, Office of Highway Information Management, 1995 NPTS, Public Use Tape, 1997.

Energy Use - Total gallons of fuel taken from DOT, FHWA, Highway Statistics 1997, Table VM-1. These were distributed as follows: $97.8 \%$ gasoline, $1.0 \%$ gasohol, and $1.2 \%$ diesel. Percentages were derived from the DOE, EIA, Office of Markets and End Use, Energy End Use Division, Household Vehicles Energy Consumption 1991, December 1993, p. 46. Natural gas data are from the DOE, EIA Natural Gas Annual 1997, Table 1; transit bus natural gas was subtracted from the total and the remainder was assumed to be light vehicle use. Automobiles were assumed to use $25 \%$ of light vehicle natural gas use. Methanol use was estimated using data from DOE, EIA, Alternatives to Traditional Transportation Fuels 1997 ,Washington, DC, 1998, Table 12.

\section{Personal Trucks}

Number of Vehicles - Based on the 1992 TIUS, 73.9\% of total 2-axle, 4-tire trucks and 15.5\% of total other trucks were for personal use. Therefore, $73.9 \%$ of total 2-axle, 4-tire trucks (as reported by DOT, FHWA in Highway Statistics 1997, Table VM-1) and 15.5\% of total other trucks were estimated to be for personal use.

Vmt $-68.8 \%$ of total vehicle miles traveled by 2-axle, 4-tire trucks (as reported by DOT, FHWA in Highway Statistics 1997, Table VM-1) and 7.1\% of total vehicle miles traveled by other trucks were for personal use. The percentages were derived by ORNL from the 1992 TIUS Micro Data File on CD.

Pmt - Calculated by ORNL as vmt multiplied by load factor.

Load Factor - DOT, FHWA, Office of Highway Information Management, 1995 NPTS, Public Use Tape, 1997.

Energy Use- Assuming that there is no difference in fuel economy (measured in miles per gallon) between personal-use trucks and non-personal use trucks, $66.0 \%$ of total fuel consumption by 2-axle, 4-tire trucks (as reported by DOT, FHWA in Highway Statistics 1996, Table VM-1) and 3.5\% of total other truck fuel consumption was for personal use. These percentages were derived by ORNL from the 1992 TIUS Public Use tape. Total truck energy use was the sum of light truck and other truck energy use.

Light Trucks: DOT, FHWA, Highway Statistics 1997, Table VM-1, for single-unit, 2axle, 4-tire trucks. $96.4 \%$ of fuel assumed to be gasoline, $3.3 \%$ diesel, $0.3 \% \mathrm{lpg}$; percentages were generated from the 1992 TIUS Micro Data File on CD. Natural gas data are from the DOE, EIA Natural Gas Annual 1997, Table 1; transit bus 
natural gas was subtracted from the total and the remainder was assumed to be light vehicle use. Light trucks were assumed to use $75 \%$ of light vehicle natural gas use.

Other Trucks: DOT, FHWA, Highway Statistics 1997, Table VM-1. Total gallons for other trucks was the difference between total and 2-axle, 4-tire trucks. These values were distributed based on data from the 1992 TIUS Public Use Tape: 16.2\% of fuel assumed to be gasoline, $83.3 \%$ diesel, and $0.5 \% \mathrm{lpg}$.

\section{Motorcycles}

Number of Vehicles and Vmt - DOT, FHWA, Highway Statistics 1997, Table VM- 1.

Pmt - Calculated by ORNL as vmt multiplied by load factor.

Load Factor - DOT, FHWA, Office of Highway Information Management, 1995 NPTS, Public Use Tape, 1997.

Energy Use - DOT, FHWA, Highway Statistics 1997, Table VM-1. For conversion purposes, fuel for all motorcycles was assumed to be gasoline.

\section{Buses}

\section{Transit:}

Number of Vehicles, Vmt, Pmt, and Energy Use - Motor bus only. APTA, 1999 Transit Fact Book, 1999, Washington, DC, pp. 122.

Load Factor - Calculated by ORNL as pmt/vmt.

\section{Intercity:}

Number of Vehicles - Estimated by ORNL as $18 \%$ of commercial bus registrations, DOT, FHWA, Highway Statistics 1997, Table MV- 10.

Pmt - Eno Transportation Foundation, Transportation in America, 1998, Sixteenth Edition, Lansdowne, VA, 1998, p. 47.

Vmt - Estimated using passenger travel and an average load factor of 23.2 persons/vehicle.

Load Factor -Estimated as 23.2 based on historical data.

Energy Use - Eno Transportation Foundation, Transportation in America 1998, Sixteenth Edition, 1998, Lansdowne, VA, p. 56. For conversion purposes, fuel for all intercity buses was assumed to be diesel fuel. (1997 data were estimated using vehicle travel information.)

\section{School:}

Number of Vehicles - School and other nonrevenue as reported in DOT, FHWA, Highway Statistics 1997, Table MV- 10.

Vmt, Pmt - National Safety Council, Accident Facts, 1998 Edition, Chicago, IL, pp. 94-95.

Load Factor - Calculated by ORNL as pmt/vmt.

Energy Use - Eno Transportation Foundation, Transportation in America 1998, Sixteenth Edition, 1998, Lansdowne, VA, p. 56. For conversion purposes, fuel for school buses was assumed to be $60 \%$ diesel fuel and $40 \%$ gasoline. (1997 data were estimated using vehicle travel information.) 


\section{Nonhighway}

Air

\section{Large Certified Route Air Carriers:}

Vmt, Pmt - DOT, Bureau of Transportation Statistics, Air Carrier Traffic Statistics Monthly, December 19970996, Washington, DC, p.2.

Load Factor - Calculated by ORNL as pmt/vmt.

Energy Use - DOT, Bureau of Transportation Statistics, "Fuel Cost and Consumption Tables;" annual figures were obtained by summing monthly totals for domestic only.

General Aviation:

Number of Vehicles, Vmt, Energy Use - DOT, FAA, General Aviation Activity and Avionics, Survey: Calendar Year 1997, pp. 1-7, 3-1 1, 5-3.

Pmt - Eno Transportation Foundation, Transportation in America 1998, SixteenthEdition, Lansdowne, VA, 1998, p. 47.

Load Factor - Calculated by ORNL as pmt/vmt.

\section{Recreational Boating}

Number of Vehicles - U.S. Coast Guard, Office of Boating Safety, Washington, DC, May 1999.

Energy Use - Fuel use by recreational boating was calculated using the methodology developed by D. L. Greene in the report, Off-Highway Use of Gasoline in the United States (DOT, FHWA, July 1986, p. 3-22). Results from Model 1 in the report indicated an average annual consumption of 205 gallons per boat. Total consumption in gallons was then calculated using the following equation: Total $=$ 0.95 (Gal/boat) (number of boats). An estimate of number of recreational boats in operation is from the U.S. Coast Guard (numbered boats).

\section{Rail}

\section{Intercity:}

Number of Vehicles, Vmt and Pmt -AAR, Railroad Facts, 1998 Edition, Washington, DC, p. 78.

Load Factor - Calculated by ORNL as pmt/vmt.

Energy Use - Personal communication with Amtrak, Washington, DC. (1997 data estimated using train-mile information.)

\section{Transit and Commuter:}

Number of Vehicles, Vmt and Pmt - APTA, 1999 Transit Fact Book, Washington, DC, 1998, pp. 124, 130, 132.

Load Factor - Calculated by ORNL as pmt/vmt.

Energy Use - APTA, 1999 Transit Fact Book, 1999 Washington, DC, pp. 106-108. Transit was defined as the sum of "heavy rail," "light rail," and "other." 
Table 2.13

Intercity Freight Movement and Energy Use in the

United States, 1997

\section{Highway}

\section{Trucks}

Vehicles - 0.3\% of total 2-axle, 4-tire trucks (as reported by DOT, FHWA inHighway Statistics 1997, Table VM-1) and $24 \%$ of total other trucks were engaged in intercity freight movement. These percentages were derived by ORNL from the 1992 TIUS Micro Data File on CD. Intercity freight trucks were defined as any truck whose:

- greatest share of miles were traveled more than 50 miles away from the vehicle's home base; and

- principal use was not personal or passenger transportation; and

- body type was not pickup, minivan, or utility vehicle.

$V m t-0.6 \%$ of total vehicle miles traveled by 2-axle, 4-tire trucks (as reported by DOT, FHWA in Highway Statistics 1997, Table VM-1) and 59.5\% of total vehicle miles traveled by other trucks were used in intercity freight movement. These percentages were derived by ORNL from the 1992 TIUS Micro Data File on CD.

Ton Miles, Tons Shipped and Average Length of Haul - Eno Transportation Foundation, Transportation in America 1998, Sixteenth Edition, Lansdowne, VA, 1997, pp. 44, 46, 71.

Energy Intensity - Energy use divided by ton-miles.

Energy Use $-0.9 \%$ of total fuel consumption by 2-axle, 4-tire trucks (as reported by DOT, FHWA in Highway Statistics 1997, Table VM-1) and. 67.2\% of total other truck fuel consumption were used in intercity freight movement. These percentages were derived by ORNL from the 1992 TIUS Micro Data File on CD.

\section{Nonhighway}

\section{Waterborne Commerce}

Vehicles - U.S. Department of the Army, Army Corps of Engineers, "Summary of U.S. Flag Passenger and Cargo Vessels, 1996," New Orleans, LA, 1998.

Ton Miles, Tons Shipped, and Average Length of Haul - U.S. Department of the Army, Corps of Engineers, Waterborne Commerce of the United States, Calendar Year 1997, Part 5: National Summaries, New Orleans, LA, 1999, pp. 1-6, 1-7.

Energy Intensity - Energy use divided by ton miles.

Energy Use - DOE, EIA, Fuel Oil and Kerosene Sales 1997, Table 23. Adjusted sales of distillate and residual fuel oil for vessel bunkering. (This may include some amounts of bunker fuels used for recreational purposes.)

Domestic freight energy use was calculated as:

Distillate fuel $-77.5 \%$ domestic

Residual fuel $-9.3 \%$ domestic.

Percentages were derived from the DOC, U.S. Foreign Trade, Bunker Fuels, "Oil and Coal Laden in the U.S. on Vessels Engaged in Foreign Trade," 1988. 


\section{Pipeline}

\section{Natural Gas:}

Tons shipped - DOE, EIA, Natural Gas Annual 1997, Washington, DC, 1998, Table 1. Total natural gas disposition divided by $44,870 \mathrm{ft}^{3} /$ ton.

Energy use - The amount of natural gas used to transport natural gas was defined as "pipeline fuel" as reported in DOE, EIA, Natural Gas Annual 1997, Table 1. Cubic feet were converted to Btu using 1,03 $1 \mathrm{Btu} / \mathrm{ft}^{3}$. Electricity use was estimated using the following procedure as reported on p. 5-1 10 of J. N. Hooker et al., End Use Energy Consumption DataBase: Transportation Sector. The energy consumption of a natural gas pipeline was taken to be the energy content of the fuel used to drive the pumps. Some $94 \%$ of the installed pumping horsepower was supplied by natural gas. The remaining $6 \%$ of the horse power was generated more efficiently, mostly by electric motors. The energy consumed by natural gas pipeline pumps that were electrically powered was not known. In order to estimate the electricity consumed, the Btu of natural gas pipeline fuel consumed was multiplied by a factor of 0.015 . From this computed value, electricity efficiency and generation loss must be taken into account. The electricity energy use in Btu must be converted to $\mathrm{kWhr}$, using the conversion factor $29.305 \times 10^{-5} \mathrm{kWhr} / \mathrm{Btu}$. Electricity generation and distribution efficiency was $29 \%$. When generation and distribution efficiency are taken into account, $1 \mathrm{kWhr}$ equals 11,765 Btu.

\section{Crude Oil and Petroleum Product:}

Ton Miles and Tons Shipped - Eno Transportation Foundation, Transportation in America 1998, Sixteenth Edition, Lansdowne, VA, 1998 pp. 44, 46.

Energy Use - W. F. Banks, Systems, Science, and Software, Inc., Energy Consumption in the Pipeline Industry, LaJolla, CA, 1977.

\section{Rail}

Vehicles, Vmt, Ton Miles, Average Length of Haul - AAR, Railroad Facts, 1998 Edition, Washington, DC, 1998, pp. 27, 34, 36, 50.

Tons shipped - AAR, Analysis of Class I Railroads 1997, 1998, p. 31.

Energy Use -AAR, Railroad Facts, 1998 Edition, Washington, DC, 1997, p. 60. 
Table 2.12

Energy Intensities of Passenger Modes, 1970-97

In reference to transportation, the energy intensity of a mode is the ratio of the energy inputs to a process to a measure of the useful outputs from that process; for example, Btu per pmt or Btu per ton-mile. The energy intensity ratios were calculated for each passenger mode using the following data sources:

\section{Highway}

Automobiles

Vmt - DOT, FHWA, Highway Statistics Summary to 1995, Table VM-201A, and Table VM-1 of the 1996 and 1997 editions.

Pmt - vmt multiplied by the load factor.

Energy Use - Total gallons of fuel for automobiles was taken from DOT, FHWA,

Highway Statistics Summary to 1995, Table VM-20 1 A; and Table VM- 1 in the 1996 and 1997 annual editions. Fuel for automobiles was distributed between fuel types for conversion into Btu's as follows:

1970-80 - 94.7\% gasoline, 5.3\% diesel as reported in the DOE, EIA, Office of Energy Markets and End Use, Residential Energy Consumption Survey: Consumption Patterns of Household Vehicles, June 1979 to December 1980, p. 10.

1981-82-94.1\% gasoline, 5.9\% diesel as reported in the DOE, EIA, Office of Energy Markets and End Use, Residential Energy Consumption Survey: Consumption Patterns of Household Vehicles, Supplement: January 1981 to September 1981, pp. $11,13$.

1983-84 - 97.5\% gasoline, 2.5\% diesel as reported in the DOE, EIA, Office of Markets and End Use, Energy End Use Division, Residential Transportation Energy Consumption Survey: Consumption Patterns of Household Vehicles, 1983, Jan., 1985, pp. 7, 9.

1985-87-98.5\% gasoline, $1.5 \%$ diesel as reported in the DOE, EIA, Office of Energy Markets and End Use, Residential Transportation Energy Consumption Survey: Consumption Patterns of Household Vehicles 198.5, April 1987, pp. 25, 27.

1988-90 - 98.8\% gasoline and 1.2\% diesel as reported in the DOE, EIA, Office of Markets and End Use, Energy End Use Division, Household Vehicles Energy Consumption 1988, March 1990, p. 65.

1991-93 - 97.8\% gasoline, $1.0 \%$ gasohol, and 1.2\% diesel as reported in the DOE, EIA, Office of Markets and End Use, Energy End Use Division, Household Vehicles Energy Consumption 1991, December 1993, p. 46.

1994-96 - 97.7\% gasoline, 1.0\% gasohol, $1.3 \%$ diesel as reported in the DOE, EIA, Office of Energy Markets and End Use, Household Vehicles Energy Consumption 1994, Washington, DC, August 1997, p. 46.

1993-97 - Methanol use was estimated using data from DOE, EIA, Alternatives to Traditional Transportation Fuels 1997, Washington, DC, 1998, Table 12.

1993-97 - Natural gas data are from the DOE, EIA Natural Gas Annual 1997, Table 1; transit bus natural gas was subtracted from the total and the remainder was assumed to be light vehicle use. Automobiles were assumed to use $25 \%$ of light vehicle natural gas use. 


\section{Light Trucks}

Vmt - DOT, FHWA, Highway Statistics Summary to 1995, Table VM-20 1 A, and Table VM-1 of the 1996 and 1997 editions. Light trucks were defined as 2-axle, 4-tire trucks.

Energy Use - Light trucks were defined as 2-axle, 4-tire trucks. Total gallons of fuel was taken from DOT, FHWA, Highway Statistics Summary to 1995, Table VM-201A, and Table VM-1 of the 1996 and 1997 annual edition. Based on data from the 1982 TIUS Public Use Tape, fuel use for 1970-87 was distributed among fuel types as follows: $95.3 \%$ gasoline; 3.5\% diesel; and 1.2\% Ipg. Fuel use for 1988-93 was distributed based on the 1987 TIUS: $96.6 \%$ gasoline; $3.3 \%$ diesel; and 0.1\% lpg. Fuel use for 1994-95 was distributed based on the 1992 TIUS: $96.2 \%$ gasoline; 3.3\% diesel; $0.3 \%$ Ipg; and $0.2 \%$ cng.

\section{Buses}

\section{Transit:}

Vmt, Pmt, Energy Use - APTA, 1999 Transit Fact Book, Washington, DC,1998, pp. 122, 106-108, and annual.

Non-diesel fossil fuel consumption was assumed to be used by motor buses. For the years 1988-94, motor bus gasoline use was estimated as 5\% of "other" fuels, based on personal communication with the APTA Research and Statistics Department.

\section{Intercity:}

Pmt - 1970-84 - American Bus Association, Annual Report, Washington, DC, annual.

1985-95 - Eno Transportation Foundation, Transportation in America 1997, Fifteenth Edition, Lansdowne, VA, 1997, p. 47.

Energy Use - 1970-84 - American Bus Association, Annual Report, Washington, DC, annual.

1985-97 - Eno Transportation Foundation, Transportation in America 1998, Sixteenth Edition, Lansdowne, VA, p. 56, and annual. For conversion purposes, fuel for all intercity buses was assumed to be diesel fuel. (1995, 1996, and 1997 data were estimated using vehicle travel information.)

\section{School:}

Vmt - 1970-84 - DOT, FHWA, Highway Statistics 1984, Washington, DC, Table VM-1, p. 175, and annual. 1985-87 - DOT, TSC, National Transportation Statistics, 1989, Figure 2, p. 7, and annual.

1988-97 - National Safety Council, Accident Facts, 1997 Edition , Chicago, IL, p. 95 , and annual.

Energy Use - 1970-84 - DOT, FHWA, Highway Statistics 1984, Washington, DC, Table VM- 1 , and annual.

1985-86 - DOT, TSC, National Transportation Statistics, Figure 2, p. 5, and annual. 1987-96 - Eno Transportation Foundation, Transportation in America 1998, Sixteenth Edition, Lansdowne, VA, p. 56, and annual. For conversion purposes, fuel for school buses was assumed to be $60 \%$ diesel fuel and $40 \%$ gasoline. $(1995,1996$, and 1997 data were estimated using vehicle travel information.) 


\section{Nonhighway}

Air

Certificated Air Carriers:

Pmt - DOT, Bureau of Transportation Statistics,\&-Carrier Traffic Statistics Monthly, December 1997/96, Washington, DC, p. 2.

Energy Use - 1970-81 - DOT, Civil Aeronautics Board, Fuel Cost and Consumption, Washington, DC, annual.

1982-97 - DOT, Bureau of Transportation Statistics, "Fuel Cost and Consumption Tables;" annual figures were obtained by summing monthly totals for domestic only.

\section{General Aviation:}

Pmt - Eno Transportation Foundation, Transportation In America 1998, Sixteenth Edition, Washington, DC, 1998, p.47.

Energy Use - 1970-74 - DOT, TSC, National Transportation Statistics, Cambridge, MA, 1981.

1975-85 - DOT, FAA, FAA Aviation Forecasts, Washington, DC, annual. 1985-97 - DOT, FAA, General Aviation Activity and Avionics Survey: Calendar Year 1997, Table 5.1. Jet fuel was converted from gallons to Btu using 135,000 Btu/gallon (kerosene-type jet fuel).

\section{Rail}

\section{Passenger (Amtrak) :}

Pmt - 1971-83 - AAR, Statistics of Class I Railroads, Washington, DC, annual. 1984-88, 1995-96 - AAR, Railroad Facts, 1987 Edition, Washington, DC, December 1987, p. 78, and annual. 1989-94 - Personal communication with Amtrak.

Energy Use - Personal communication with Amtrak. (1995, 1996, and 1997 data were estimated using train-mile information.)

\section{Transit:}

Pmt and Energy Use - APTA, 1999 Transit Fact Book, February 1998, Washington, DC, pp. 12, 106-108. Transit was defined as the sum of "heavy rail," "light rail." 
Table 2.14

Energy Intensities of Freight Modes, 1970-97

In reference to transportation, the energy intensity of a mode is the ratio of the energy inputs to a process to a measure of the useful outputs from that process; for example, Btu per pmt or Btu per ton-mile. The energy intensity ratios were calculated for each freight mode using the following data sources:

\section{Highway}

\section{Heavy Single-Unit and Combination Trucks}

Vmt - DOT, FHWA, Highway Statistics Summary to 1995, Table VM-20 1 A, and Table VM- 1 of the 1996 and 1997 editions. Heavy single-unit and combination trucks were defined as the difference between total trucks and 2-axle, 4-tire trucks.

Energy Use - Heavy single-unit and combination trucks were defined as the difference between total trucks and 2-axle, 4-tire trucks. Total gallons of fuel was taken from DOT, FHWA, Highway Statistics Summary to 199.5, Table VM-201A, and Table VM-1 of the 1996 and 1997 annual editions. Based on data from the 1982 TIUS Public Use Tape, fuel use for 1970-87 was distributed among fuel types as follows: $39.6 \%$ gasoline; $59.4 \%$ diesel; and $1.0 \%$ lpg. Fuel use for 1988-93 was distributed based on the 1987 TIUS: $19.4 \%$ gasoline; $80.4 \%$ diesel; and $0.2 \%$ lpg. Fuel use for 1994-97 was distributed based on the 1992 TIUS: $16.2 \%$ gasoline; $83.3 \%$ diesel; and $0.5 \% \mathrm{lpg}$.

\section{Nonhighway}

\section{Water}

Ton Miles - U.S. Department of the Army, Corps of Engineers, Waterborne Commerce of the United States, Calendar Year 1997, Part 5: National Summaries, New Orleans, LA, 1998, p. 1-6, and annual.

Energy Use - Calculated as the difference between total water freight energy use and foreign water freight energy use.

Total - DOE, EIA, Fuel Oil and Kerosene Sales 1997, Table 23. Adjusted sales of distillate and residual fuel oil for vessel bunkering.(This may include some amounts Rail of bunker fuels used for recreational purposes.)

Freight Car Miles, Ton Miles and Energy Use - AAR, Railroad Facts, 1998 Edition, Washington, DC, 1998, pp. 27, 36, 60, and annual. 
Table 6.4

Vehicle Stock and New Sales in the

United States, 1997 Calendar Year

\section{Highway}

\section{Automobiles}

Stock -The number of vehicles in use by EPA size class were derived as follows: Market Shares by EPA size class for new car sales from 1970-75 were taken from the DOT, NHTSA, Automotive Characteristics Historical DataBase, Washington, DC. Market shares for the years 1976-90 were found in Linda S. Williams and Patricia S. Hu, Highway Vehicle MPG and Market Shares Report: Model Year 1990, ORNL-6672, April 1991, and Table 7 and the ORNL MPG and Market Shares Database, thereafter. These data were assumed to represent the number of cars registered in each size class for each year. These percentages were applied to the automobiles in operation for that year as reported by R. L. Polk and Company (FURTHER REPRODUCTION PROHIBITED) and summed to calculate the total mix. This method assumed that all vehicles, large and small, were scrapped at the same rate.

Sales - Domestic, import, and total sales were from AAMA, Facts and Figures 1997, p. 19. The domestic sales were distributed by size class according to the following percentages: Two seater, 0.5\%; Minicompact, 0\%; Subcompact, 17.6\%; Compact 35.6\%; Midsize, $30.0 \%$; and Large, $16.3 \%$. The import sales were distributed by size class according to the following percentages: Two-seater, 3.3\%; Minicompact, 3 .O\%; Subcompact, $21.9 \%$; Compact, 35.3\%; Midsize, 34.2\%; and Large, 2.3\%. These percentages were derived from the ORNL MPG and Market Shares Database. Domestic-sponsored imports (captive imports) were included in the import figure only.

Business fleet autos - Bobit Publishing Company, Automotive Fleet Research Department, Automotive Fleet Factbook 1998, Redondo Beach, CA, 1998.

Personal autos - Difference between total vehicle stock and business fleet autos.

See Glossary for definition of Automobile Size Classifications.

\section{Motorcycles}

Stock - DOT, FHWA, Highway Statistics 1997, Table VM-1, 1998.

\section{Recreational Vehicles}

Sales - Ward's Automotive Yearbook 1998, U.S. Recreation Vehicle Shipments by Type, "Total," p. 215. 


\section{Trucks}

Stock - Vehicles in use by weight class were determined by applying the percentage in use by weight class as reported in DOC, Bureau of the Census, 1992 TIUS, (O-10,000 lbs, $93.3 \%$; 10,001-19,500 lbs, 2.1\%; 19,501-26,000 lbs, $1.2 \%$; 26,001 lbs and over, 3.4\%) to the total number of trucks in use as reported by R. L. Polk and Company (FURTHER REPRODUCTION PROHIBITED).

Sales - AAMA, Facts and Figures 1998, p. 24.

Business fleet trucks - Bobit Publishing Company, Automotive Fleet Research Department, Automotive Fleet Factbook 1998, Redondo Beach, CA, 1998.

Personal trucks - Difference between total stock and business fleet trucks. 
Table 8.14

Summary Statistics on Buses by Type, 1970-97

\section{Number in Operation}

Transit buses:

American Public Transit Association, 1999 Transit Fact Book, Washington, DC, 1999, p. 122, and annual.

\section{Intercity buses:}

1970-80 - American Bus Association, 1984 Annual Report, Washington, DC, and annual. 1985 - U.S. Department of Transportation, Transportation Systems Center, National Transportation Statistics, Cambridge, MA, August 1990, Figure 5, p. 8, and annual. 1990-97 - Estimated as 38\% of commercial buses (less transit motor buses). Commercial bus total found in Highway Statistics 1997, Table MV-10, and annual.

School buses:

U.S. Department of Transportation, Federal Highway Administration,Highway Statistics 1997, Washington, DC, 1998, Table MV-1,O and annual.

\section{Vehicle-miles and Passenger-miles}

Transit buses:

American Public Transit Association, 1999 Transit Fact Book, Washington, DC, 1999, pp. 122, and annual.

\section{Intercity buses:}

1970-80 - American Bus Association, Annual Report, Washington, DC, annual.

198.5-97 - Eno Transportation Foundation, Transportation in America 1998, Sixteenth edition, Lansdowne, VA, 1997, p. 47.

1990-97 vehicle travel - Estimated using passenger travel and an average load factor of 23.2 .

\section{School buses:}

1970-80 - U.S. Department of Transportation, Federal Highway Administration, Highway Statistics 1984, Washington, DC, Table VM-1, p. 175, and annual.

1985 - U.S. Department of Transportation, Research and Special Programs Administration, National Transportation Statistics, 1989, Figure 2, p. 7, and annual. 1990-97 - National Safety Council, Accident Facts, 1998 Edition, Chicago, IL, pp. 94-95, and annual. 


\section{Energy Use}

Transit buses:

American Public Transit Association, 1999 Transit Fact Book, Washington, DC, 1999, pp. 106-108. Non-diesel fossil fuel consumption was assumed to be used by motor buses. For the years 1988-92, motor bus gasoline use was estimated as 5\% of "other" fuels, based on personal communication with the APTA Research and Statistics Department.

\section{Intercity buses:}

1970-80 - American Bus Association, Annual Report, Washington, DC, annual.

1985-97 - Eno Transportation Foundation, Transportation in America 1998, Sixteenth edition, Lansdowne, VA, p. 56. For conversion purposes, fuel for all intercity buses was assumed to be diesel fuel. (1995, 1996, and 1997 data were estimated using vehicle travel information.)

School buses:

1970-80 - DOT, FHWA, Highway Statistics 1984, Washington, DC, Table VM-1, and annual.

1985-86-DOT, Research and Special Programs Administration,National Transportation Statistics, Figure 2, p. 5, and annual.

1987-97- Eno Transportation Foundation, Transportation in America 1998,Sixteenth edition, Lansdowne, VA, p. 56. For conversion purposes, fuel for school was assumed to be $60 \%$ diesel fuel and 40\% gasoline. (1995, 1996, and 1997 data were estimated using vehicle travel information.) 


\section{APPENDIX B \\ CONVERSIONS}

\section{A Note About Heating Values}

The heat content of a fuel is the quantity of energy released by burning a unit amount of that fuel. However, this value is not absolute and can vary according to several factors. For example, empirical formulae for determining the heating value of liquid fuels depend on the fuels' American Petroleum Institute (API) gravity. 'The API gravity varies depending on the percent by weight of the chemical constituents and impurities in the fuel, both of which are affected by the combination of raw materials used to produce the fuel and by the type of manufacturing process. Temperature and climatic conditions are also factors.

Because of these variations, the heating values in Table B. 1 may differ from values in other publications. The figures in this report are representative or average values, not absolute ones. The gross heating values used here agree with those used by the Energy Information Administration (EIA).

Heating values fall into two categories, gross and net. If the products of fuel combustion are cooled back to the initial fuel-air or fuel-oxidizer mixture temperature and the water formed during combustion is condensed, the energy released by the process is the higher (gross) heating value. If the products of combustion are cooled to the initial fuel-air temperature, but the water is considered to remain as a vapor, the energy released by the process is lower (net) heating value. Usually the difference between the gross and net heating values for fuels used in transportation is around 5 to 8 percent; however, it is important to be consistent in their use. 
Table B.l

Approximate Heat Content for Various Fuels

Automotive gasoline

Diesel motor fuel

Methanol

Ethanol

Gasohol

Aviation gasoline

Propane

Butane

Jet fuel (naphtha)

Jet fuel (kerosene)

Lubricants

Waxes

Asphalt and road oil

Petroleum coke

Natural gas

Wet

Dry

Compressed

Liquid

Crude petroleum

Fuel Oils

Residual

Distillate

Coal

Anthracite - Consumption

Bituminous and lignite - Consumption

Production average

Consumption average
125,000 Btu/gal(gross) $=115,400 \mathrm{Btu} /$ gal(net)

$138,700 \mathrm{Btu} / \mathrm{gal}$ (gross) $=128,700 \mathrm{Btu} / \mathrm{gal}($ net $)$

64,600 Btu/gal $($ gross) $=56,560 \mathrm{Btu} / \mathrm{gal}($ net $)$

$84,600 \mathrm{Btu} / \mathrm{gal}$ (gross) $=75,670 \mathrm{Btu} / \mathrm{gal}$ (net)

$120,900 \mathrm{Btu} / \mathrm{gal}$ (gross) $=112,417 \mathrm{Btu} / \mathrm{gal}($ net $)$

$120,200 \mathrm{Btu} / \mathrm{gal}($ gross $)=112,000 \mathrm{Btu} / \mathrm{gal}($ net $)$

91,300 Btu/gal (gross) = 83,500 Btu/gal (net)

103,000 Btu/gal $($ gross $)=93,000 \mathrm{Btu} / \mathrm{gal}($ net $)$

$127,500 \mathrm{Btu} / \mathrm{gal}(\mathrm{gross})=118,700 \mathrm{Btu} / \mathrm{gal}$ (net)

$135,000 \mathrm{Btu} / \mathrm{gal}(\mathrm{gross})=128,100 \mathrm{Btu} / \mathrm{gal}$ (net)

$144,400 \mathrm{Btu} / \mathrm{gal}(\mathrm{gross})=130,900 \mathrm{Btu} / \mathrm{gal}(\mathrm{net})$

$131,800 \mathrm{Btu} / \mathrm{gal}$ (gross) $=120,200 \mathrm{Btu} / \mathrm{gal}$ (net)

$158,000 \mathrm{Btu} / \mathrm{gal}(\mathrm{gross})=157,700 \mathrm{Btu} / \mathrm{gal}$ (net)

$143,400 \mathrm{Btu} / \mathrm{gal}(\mathrm{gross})=168,300 \mathrm{Btu} / \mathrm{gal}($ net $)$

$1,109 \mathrm{Btu} / \mathrm{ft}^{3}$

$1,027 \mathrm{Btu} / \mathrm{ft}^{3}$

20,551 Btu/pound

$960 \mathrm{Btu} /$ cubic foot

90,800 Btu/gal $($ gross $)=87,600 \mathrm{Btu} / \mathrm{gal}$ (net)

$138,100 \mathrm{Btu} / \mathrm{gal}(\mathrm{gross})=131,800 \mathrm{Btu} / \mathrm{gal}$ (net)

$149,700 \mathrm{Btu} / \mathrm{gal}$ (gross) $=138,400 \mathrm{Btu} / \mathrm{gal}$ (net)

$138,700 \mathrm{Btu} / \mathrm{gal}($ gross $)=131,800 \mathrm{Btu} / \mathrm{gal}($ net $)$ 
Table B.2

Fuel Equivalents

1 million bbl crude oil/day

1 billion bbl crude oil/year

1 quadrillion Btu/year

1 billion short tons coal/year

1 billion metric tons coal/year

1 trillion $\mathrm{ft}^{3}$ natural gas/year

1 exajoule/year
$=0.3650 \mathrm{billion} \mathrm{bbl}$ crude oil/year $=2.117$ quadrillion Btu/year

$=99.45$ million short tons coal/year

$=90.22$ million metric tons coal/year $=2.061$ trillion $\mathrm{ft}^{3}$ natural gas/year

$=2.233$ exajoulelyear

$=2.740$ million bbl crude oil/day

$=5.800$ quadrillion Btu/year

$=272.5$ million short tons coal/year

$=247.2$ million metric tons coal/year

$=5.648$ trillion $\mathrm{ft}^{3}$ natural gas/year

$=6.119$ exajoule/year

$=0.4724$ million bbl crude oil $/$ day

$=172.4$ million bbl crude oil/year

$=46.98$ million short tons coal/year

$=42.62$ million metric tons coal $/$ year

$=973.7$ billion $\mathrm{ft}^{3}$ natural gas/year

$=1.055 \times 10^{-3}$ exajoule $/$ year

$=0.9072$ billion metric tons coal/year

$=10.06$ million bbl crude oil $/$ day

$=3.670$ billion bbl crude oil/year

$=21.29$ quadrillion Btu/year

$=20.73$ trillion $\mathrm{ft}^{3}$ natural gas/year

$=22.46$ exajoulelyear

$=1.102$ billion short tons coal/year

$=9.122$ million bbl crude oi $1 /$ day

$=3.330$ billion bbl crude oil/year

$=19.31$ quadrillion btu/year

$=18.80$ trillion $\mathrm{ft}^{3}$ natural gas/year

$=20.37$ exajoules/year

$=0.4851$ million bbl crude oil $/$ day

$=0.1771 \mathrm{billion}$ bbl crude oil $/$ year

$=1.027$ quadrillion Btu/year

$=48.25$ million short tons coal/year

$=43.77$ million metric tons coal $/$ year

$=1.083 \times 10^{-3}$ exajoules $/$ year

$=0.4477$ million bbl crude oil $/$ day

$=0.1634$ billion bbl crude oil/year

$=947.9$ trillion $\mathrm{Btu} /$ year

$=44.53$ million short tons coal/year

$=40.40 \mathrm{million}$ metric tons coal/year

$=0.9229$ trillion $\mathrm{ft}$ ? natural gas/year 
Table B.3

Energy Unit Conversions.

\begin{tabular}{|c|c|c|c|}
\hline \multirow[t]{6}{*}{$1 \mathrm{Btu}$} & $=778.2 \mathrm{ft}-1 \mathrm{~b}$ & $1 \mathrm{kWhr}$ & $=3412$ Btu" \\
\hline & $=107.6 \mathrm{~kg}-\mathrm{m}$ & & $=2.655 \times 10^{6} \mathrm{ft}-\mathrm{lb}$ \\
\hline & $=1055 \mathrm{~J}$ & & $=3.671 \times 10^{5} \mathrm{~kg}-\mathrm{m}$ \\
\hline & $=39.30 \times 10 " \mathrm{hp}-\mathrm{h}$ & & $=3.600 \times 10^{6} \mathrm{~J}$ \\
\hline & $=39.85 \times 10^{-5}$ metric $\mathrm{hp}-\mathrm{h}$ & & $=1.341 \mathrm{hp}-\mathrm{h}$ \\
\hline & $=29.31 \times 10^{-5} \mathrm{kWhr}$ & & $=1.360$ metric $\mathrm{hp}-\mathrm{h}$ \\
\hline \multirow[t]{6}{*}{$1 \mathrm{~kg}-\mathrm{m}$} & $=92.95 \times 10^{-4} \mathrm{Btu}$ & 1 Joule & $=94.78 \times 10^{-5} \mathrm{Btu}$ \\
\hline & $=7.233 \mathrm{ft}-\mathrm{lb}$ & & $=0.7376 \mathrm{ft}-\mathrm{lb}$ \\
\hline & $=9.806 \mathrm{~J}$ & & $=0.1020 \mathrm{~kg}-\mathrm{m}$ \\
\hline & $=36.53 \times 10^{-7} \mathrm{hp}-\mathrm{h}$ & & $=37.25 \times 10^{-8} \mathrm{hp}-\mathrm{h}$ \\
\hline & $=37.04 \times 10^{-7}$ metric hp-h & & $=37.77 \times 10^{-8}$ metric hp-h \\
\hline & $=27.24 \times 10^{-7} \mathrm{kWhr}$ & & $=27.78 \times 10^{-8} \mathrm{kWhr}$ \\
\hline \multirow[t]{6}{*}{$1 \mathrm{hp}-\mathrm{h}$} & $=2544 \mathrm{Btu}$ & 1 metric $\mathrm{hp}-\mathrm{h}$ & $=2510 \mathrm{Btu}$ \\
\hline & $=1.98 \times 10^{6} \mathrm{ft}-\mathrm{lb}$ & & $=1.953 \times 10^{6} \mathrm{ft}-\mathrm{lb}$ \\
\hline & $=2.738 \times 10^{6} \mathrm{kgm}$ & & $=27.00 \times 10^{4} \mathrm{~kg}-\mathrm{m}$ \\
\hline & $=2.685 \times 10^{6} \mathrm{~J}$ & & $=2.648 \times 10^{6} \mathrm{~J}$ \\
\hline & $=1.014$ metric $\mathrm{hp}-\mathrm{h}$ & & $=0.9863 \mathrm{hp}-\mathrm{h}$ \\
\hline & $=0.7475 \mathrm{kWhr}$ & & $=0.7355 \mathrm{kWhr}$ \\
\hline
\end{tabular}

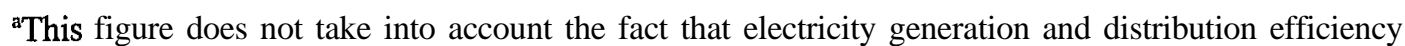
is approximately $29 \%$. If generation and distribution efficiency are taken into account, $1 \mathrm{kWhr}=11,765$ Btll. 
Table B.4

Distance and Velocity Conversions

\begin{tabular}{|c|c|c|c|}
\hline \multirow[t]{5}{*}{1 in. } & $=83.33 \times 10 " \mathrm{ft}$ & $1 \mathrm{ft}$ & $=12.0 \mathrm{in}$ \\
\hline & $=27.78 \times 10^{-3} \mathrm{yd}$ & & $=0.33 \mathrm{yd}$ \\
\hline & $=15.78 \times 10^{-6}$ mile & & $=189.4 \times 10^{-3} \mathrm{mile}$ \\
\hline & $=25.40 \times 10^{-3} \mathrm{~m}$ & & $=0.3048 \mathrm{~m}$ \\
\hline & $=0.2540 \times 10^{-6} \mathrm{~km}$ & & $=0.3048 \times 10 " \mathrm{~km}$ \\
\hline \multirow[t]{9}{*}{1 mile } & $=63360 \mathrm{in}$. & $1 \mathrm{~km}$ & $=39370 \mathrm{in}$ \\
\hline & $=5280 \mathrm{ft}$ & & $=3281 \mathrm{ft}$ \\
\hline & $=1760 \mathrm{yd}$ & & $=1093.6 \mathrm{yd}$ \\
\hline & $=1609 \mathrm{~m}$ & & $=0.6214$ mile \\
\hline & $=1.609 \mathrm{~km}$ & & $=1000 \mathrm{~m}$ \\
\hline & \multicolumn{3}{|c|}{$1 \mathrm{ft} / \mathrm{sec}=0.3048 \mathrm{~m} / \mathrm{s}=0.6818 \mathrm{mph}=1.0972 \mathrm{~km} / \mathrm{h}$} \\
\hline & \multicolumn{3}{|c|}{$1 \mathrm{~m} / \mathrm{sec}=3.281 \mathrm{ft} / \mathrm{s}=2.237 \mathrm{mph}=3.600 \mathrm{~km} / \mathrm{h}$} \\
\hline & \multicolumn{3}{|c|}{$1 \mathrm{~km} / \mathrm{h}=0.9114 \mathrm{ft} / \mathrm{s}=0.2778 \mathrm{~m} / \mathrm{s}=0.6214 \mathrm{mph}$} \\
\hline & \multicolumn{3}{|c|}{$1 \mathrm{mph}=1.467 \mathrm{ft} / \mathrm{s}=0.4469 \mathrm{~m} / \mathrm{s}=1.609 \mathrm{~km} / \mathrm{h}$} \\
\hline
\end{tabular}

T a b l e B . 5

Alternative Measures of Greenhouse Gases

1 pound methane, measured in carbon = units $\left(\mathrm{CH}_{4}\right)$

1 pound carbon dioxide, measured in = carbon units $(\mathrm{CO},-\mathrm{C})$

1 pound carbon monoxide, measured in = carbon units (CO-C)

1 pound nitrous oxide, measured in = nitrogen units $\left(\mathrm{N}_{2} \mathrm{O}-\mathrm{N}\right)$
1.333 pounds methane, measured at full molecular weight $\left(\mathrm{CH}_{4}\right)$

3.6667 pounds carbon dioxide, measured at full molecular weight $(\mathrm{CO}$,

2.333 pounds carbon monoxide, measured at full molecular weight $(\mathrm{CO})$

$=\quad 1.571$ pounds nitrous oxide, measured at full molecular weight $\left(\mathrm{N}_{2} \mathrm{O}\right)$ 
Table B.6

Volume and Flow Rate Conversions'

\begin{tabular}{|c|c|c|c|}
\hline \multirow[t]{6}{*}{1 U.S. gal } & $=231 \mathrm{in}^{3}$ & 1 liter & $=61.02$ in. $^{3}$ \\
\hline & $=0.1337 \mathrm{ft}^{3}$ & & $=3.531 \times 10^{-2} \mathrm{ft}^{3}$ \\
\hline & $=3.785$ liters & & $=0.2624$ U.S. gal \\
\hline & $=0.8321$ imperial gal & & $=0.2200$ imperial gal \\
\hline & $=0.0238 \mathrm{bbl}$ & & $=6.29 \times 10 " \mathrm{bbl}$ \\
\hline & $=0.003785 \mathrm{~m}^{3}$ & & $=0.001 \mathrm{~m}^{3}$ \\
\hline \multicolumn{4}{|c|}{ A U.S. gallon of gasoline weighs 6.2 pounds } \\
\hline \multirow[t]{6}{*}{1 imperial gal } & $=277.4$ in. $^{3}$ & l bbl & $=9702$ in. $^{3}$ \\
\hline & $=0.1606 \mathrm{ft}^{3}$ & & $=5.615 \mathrm{ft}^{3}$ \\
\hline & $=4.545$ liters & & $=158.97$ liters \\
\hline & $=1.201$ U.S. gal & & $=42$ U.S. gal \\
\hline & $=0.0286 \mathrm{bbl}$ & & $=34.97$ imperial gal \\
\hline & $=0.004546 \mathrm{~m}^{3}$ & & $=0.15897 \mathrm{~m}^{3}$ \\
\hline \multirow[t]{5}{*}{1 U.S. gal/hr } & $=3.209 \mathrm{ft}^{3} /$ day & & $=1171 \mathrm{ft}^{3} /$ year \\
\hline & $=90.84$ liter/day & & $=33157$ liter/year \\
\hline & $=19.97 \mathrm{imperial} \mathrm{gal} / \mathrm{day}$ & & $=7289 \mathrm{imperial} \mathrm{gal} / \mathrm{year}$ \\
\hline & $=0.5712 \mathrm{bbl} /$ day & & $=207.92 \mathrm{bbl} /$ year \\
\hline & \multicolumn{3}{|c|}{ For Imperial gallons, multiply above values by 1.201} \\
\hline \multirow[t]{4}{*}{1 liter $/ \mathrm{hr}$} & $=0.8474 \mathrm{ft}^{3} /$ day & & $=309.3 \mathrm{ft}^{3} /$ year \\
\hline & $=6.298$ U.S. gal/day & & = 2299 U.S. gal $/$ year \\
\hline & $=5.28 \mathrm{imperial} \mathrm{gal} / \mathrm{day}$ & & = $1927 \mathrm{imperial} \mathrm{gal/year}$ \\
\hline & $=0.1510 \mathrm{bbl} /$ day & & $=55.10 \mathrm{bbl} /$ year \\
\hline \multirow[t]{4}{*}{$1 \mathrm{bbl} / \mathrm{hr}$} & $=137.8 \mathrm{ft}^{3} /$ year & & $=49187 \mathrm{ft}^{3}$ year \\
\hline & $=1008$ U.S. gal/day & & $=3.679 \times 10^{5}$ U.S. gal $/$ year \\
\hline & $=839.3 \mathrm{imperial}$ gal $/ \mathrm{day}$ & & $=3.063 \times 10^{5} \mathrm{imperial}$ gal $/$ year \\
\hline & $=3815$ liter/day & & $=1.393 \times 10^{6}$ liter $/$ day \\
\hline
\end{tabular}

${ }^{a}$ The conversions for flow rates are identical to those for volume measures, if the time units are identical. 
Table B.7

Power Conversions

\begin{tabular}{lcccccc}
\hline & \multicolumn{7}{c}{ TO } \\
\cline { 2 - 6 } \multicolumn{1}{c}{ FROM } & Horsepower & Kilowatts & $\begin{array}{c}\text { Metric } \\
\text { horsepower }\end{array}$ & $\begin{array}{c}\text { Ft-lb } \\
\text { per sec }\end{array}$ & $\begin{array}{c}\text { Kilocalories } \\
\text { per sec }\end{array}$ & Btu per sec \\
\hline Horsepower & 1 & 0.7457 & 1.014 & 550 & 0.1781 & 0.7068 \\
Kilowatts & 1.341 & 1 & 1.360 & 737.6 & 0.239 & 0.9478 \\
Metric horsepower & 0.9863 & 0.7355 & 1 & 542.5 & 0.1757 & 0.6971 \\
Ft-lb per sec & $1.36 \times 10^{-3}$ & $1.356 \times 10^{-3}$ & $1.84 \times 10^{-3}$ & 1 & $0.3238 \times 10^{\prime \prime}$ & $1.285 \times 10^{-3}$ \\
Kilocalories per sec & 5.615 & 4.184 & 5.692 & 3088 & 1 & 3.968 \\
Btu per sec & 1.415 & 1.055 & 1.434 & 778.2 & 0.2520 & 1 \\
\hline
\end{tabular}


Table B.8

Mass Conversions

\begin{tabular}{llllll}
\hline & \multicolumn{5}{c}{ TO } \\
\cline { 2 - 6 } FROM & Pound & Kilogram & Short ton & Long ton & Metric ton \\
\hline Pound & 1 & 0.4536 & $5.0 \times 10^{-4}$ & $4.4643 \times 10^{-4}$ & $4.5362 \times 10^{-4}$ \\
Kilogram & 2.205 & 1 & $1.1023 \times 10^{-3}$ & $9.8425 \times 10^{-4}$ & $1.0 \times 10^{\prime}$ \\
Short ton & 2000 & 907.2 & 1 & 0.8929 & 0.9072 \\
Long ton & 2240 & 1016 & 1.12 & 1 & 1.016 \\
Metric ton & 2205 & 1000 & 1.102 & 0.9842 & 1 \\
\hline
\end{tabular}


Table B.9

Fuel Efficiency Conversions'

\begin{tabular}{|c|c|c|c|}
\hline MPG & Miles/liter & Kilometers/L & L/100 kilometers \\
\hline 10 & 2.64 & 4.25 & 23.52 \\
\hline 15 & 3.96 & 6.38 & 15.68 \\
\hline 20 & 5.28 & 8.50 & 11.76 \\
\hline 25 & 6.60 & 10.63 & 9.41 \\
\hline 30 & 7.92 & 12.75 & 7.84 \\
\hline 35 & 9.25 & 14.88 & 6.72 \\
\hline 40 & 10.57 & 17.00 & 5.88 \\
\hline 45 & 11.89 & 19.13 & 5.23 \\
\hline 50 & 13.21 & 21.25 & 4.70 \\
\hline 55 & 14.53 & 23.38 & 4.28 \\
\hline 60 & 15.85 & 25.51 & 3.92 \\
\hline 65 & 17.17 & 27.63 & 3.62 \\
\hline 70 & 18.49 & 29.76 & 3.36 \\
\hline 75 & 19.81 & 31.88 & 3.14 \\
\hline 80 & 21.13 & 34.01 & 2.94 \\
\hline 85 & 22.45 & 36.13 & 2.77 \\
\hline 90 & 23.77 & 38.26 & 2.61 \\
\hline 95 & 25.09 & 40.38 & 2.48 \\
\hline 100 & 26.42 & 42.51 & 2.35 \\
\hline 105 & 27.74 & 44.64 & 2.24 \\
\hline 110 & 29.06 & 46.76 & 2.14 \\
\hline 115 & 30.38 & 48.89 & 2.05 \\
\hline 120 & 31.70 & 51.01 & 1.96 \\
\hline 125 & 33.02 & 53.14 & 1.88 \\
\hline 130 & 34.34 & 55.26 & 1.81 \\
\hline 135 & 35.66 & 57.39 & 1.74 \\
\hline 140 & 36.98 & 59.51 & 1.68 \\
\hline 145 & 38.30 & 61.64 & 1.62 \\
\hline 150 & 39.62 & 63.76 & 1.57 \\
\hline
\end{tabular}

${ }^{\text {a}}$ To convert fuel efficiency from miles per gallon (mpg) to liters per hundred kilometers, divide mpg into 235.24. 
Table B.10

SI Prefixes and Their Values

\begin{tabular}{llll}
\hline & Value & Prefix & Symbol \\
\hline One million million millionth & $10^{-18}$ & atto & a \\
One thousand million millionth & $10^{-15}$ & femto & $\mathrm{f}$ \\
One million millionth & $10^{-12}$ & pico & $\mathrm{P}$ \\
One thousand millionth & $10^{-9}$ & nano & $\mathrm{n}$ \\
One millionth & $10^{-6}$ & micro & $\mu$ \\
One thousandth & $10^{-3}$ & milli & $\mathrm{m}$ \\
One hundredth & $10^{-2}$ & centi & $\mathrm{c}$ \\
One tenth & $10^{-1}$ & deci & \\
One & $10^{0}$ & & \\
Ten & $10^{\prime}$ & deca & \\
One hundred & $10^{2}$ & hecto & \\
One thousand & $10^{3}$ & kilo & $\mathrm{k}$ \\
One million & $10^{6}$ & mega & $\mathrm{M}$ \\
One billion" & $10^{9}$ & giga & $\mathrm{G}$ \\
One trillion" & $10^{12}$ & tera & $\mathrm{T}$ \\
One quadrillion" & $10^{15}$ & peta & $\mathrm{P}$ \\
One quintillion" & $10^{18}$ & exa & $\mathrm{E}$ \\
\hline
\end{tabular}

${ }^{a}$ Care should be exercised in the use of this nomenclature, especially in foreign correspondence, as it is either unknown or carries a different value in other countries. A "billion," for example, signifies a value of $10^{12}$ in most other countries. 
Table B.11

Metric Units and Abbreviations

\begin{tabular}{lll}
\hline \multicolumn{1}{c}{ Quantity } & Unit name & $\mathrm{Symbol}$ \\
\hline Energy & joule & $\mathrm{J}$ \\
Specific energy & joule/kilogram & $\mathrm{J} / \mathrm{kg}$ \\
Specific energy consumption & joule/kilogram•kilometer & $\mathrm{J} / \mathrm{kg} \cdot \mathrm{km}$ ) \\
Energy consumption & joule/kilometer & $\mathrm{J} / \mathrm{km}$ \\
Energy economy & kilometer/kilojoule & $\mathrm{km} / \mathrm{kJ}$ \\
Power & kilowatt & $\mathrm{K}$ \\
Specific power & watt/kilogram & $\mathrm{W} / \mathrm{kg}$ \\
Power density & watt/mete? & $\mathrm{W} / \mathrm{m}^{3}$ \\
Speed & kilometer/hour & $\mathrm{km} / \mathrm{h}$ \\
Acceleration & meter/second & $\mathrm{m} / \mathrm{s}^{2}$ \\
Range (distance) & kilometer & $\mathrm{km}$ \\
Weight & kilogram & $\mathrm{kg}$ \\
Torque & newton॰meter & $\mathrm{N} \bullet \mathrm{m}$ \\
Volume & meter ${ }^{3}$ & $\mathrm{~m}$ \\
Mass; payload & kilogram & $\mathrm{kg}$ \\
Length; width & meter & $\mathrm{m}$ \\
Brake specific fuel consumption & kilogram/joule & $\mathrm{kg} / \mathrm{J}$ \\
Fuel economy (heat engine) & liters/100 km & $\mathrm{L} / 100 \mathrm{~km}$ \\
\hline
\end{tabular}




\section{Conversion of Constant Dollar Values}

Many types of information in this data book are expressed in dollars. Generally, constant dollars are used--that is, dollars of a fixed value for a specific year, such as 1990 dollars. Converting current dollars to constant dollars, or converting constant dollars for one year to constant dollars for another year, requires conversion factors (Table B. 12 and B. 13). Table B. 12 shows conversion factors for the Consumer Price Index inflation factors. Table B. 13 shows conversion factors using the Gross National Product inflation factors.

Due to the size of the tables, the data in Tables B. 12 and B. 13 were changed to two decimal places starting with Edition 17. However, three decimal places were used to calculate all constant dollar values. 


\section{Table B.12}

Consumer Price Inflation (CPI) Index

\begin{tabular}{|c|c|c|c|c|c|c|c|c|c|c|c|c|c|c|c|c|c|c|c|c|c|c|c|c|c|c|c|c|c|}
\hline \multirow[b]{2}{*}{ 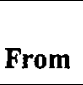 } & \\
\hline & & 71 & 2 & & & 75 & & 77 & 1978 & 9 & & & & & & & & & & & & & & & & & 6 & 1997 & \\
\hline 1970 & & & & & & & & & & & & & & & & & & & & & & & & & & 20 & $\overline{0}$ & 412 & 20 \\
\hline & & & & & & & & & & & & & & & & & & & & & & & & & & & 3.87 & & \\
\hline & & & & & & & & & & & & & & & & & & & & & & & & & & & & & \\
\hline & & & & & & 1 & & 36 & & & & & & 24 & 2.34 & - & & & & 79 & & & & & & & & & \\
\hline 77 & & 80 & 85 & 90 & O & 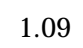 & 15 & 3 & 20 & 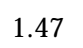 & 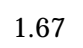 & 24 & 6 & 02 & 1 & 8 & 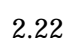 & 1 & 10 & 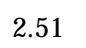 & 55 & 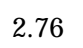 & 35 & 3 & 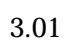 & 9 & 18 & 326 & 31 \\
\hline & & & & & & & & & & & & & & 1.85 & & & & & & & & & 2.0 & & & & .92 & & \\
\hline & & & & & & & & & & & & & & & & & & & & & & & & & & & 1.16 & & \\
\hline & & & & & & & & & & & & & & 1.05 & & 1.78 & & & 1.9 & & & & 2.32 & & 2.4 & & 2.59 & 2.0 & \\
\hline & & & & & & & & & & & & & & 1.53 & & 5 & & & & & U & 2 & 2. & & 2.27 & & 2.40 & 2.4 & 2.50 \\
\hline & & 6 & & & & 74 & & & & & & & & 37 & & 8 & & & 63 & & 30 & & 1.93 & 99 & 04 & & 16 & . & 25 \\
\hline & & & & & & & & & & & & & & & & & & & & & & & & & 80 & & & & \\
\hline & & & & & & & & & & & & & & & & & & & & & & & & & & & & & \\
\hline & & & & & & & & & & & & & & 1.03 & & 11 & & & & & & & 1.40 & & 1.54 & & 1.63 & 1.00 & 1.69 \\
\hline & & 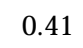 & & & & 54 & & & & & & & & 1.00 & & 8 & & & & & & & 1.41 & & 1.49 & 1.53 & 1.57 & 1.01 & 1.64 \\
\hline 04 & & 39 & & & & 52 & & 58 & ( & & & 88 & & 0 & 0 & 1.0 & & & 1 & & o & & 13 & 1. & . & 14 & 5 & 15 & 1 \\
\hline 85 & & م & & & & 50 & & 56 & 4 & & & 85 & 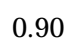 & 4 & ( & 1.00 & 1.02 & 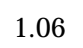 & 1.10 & 1.15 & 1.22 & 1 & 1.30 & & 1.0 & & & & 1. \\
\hline 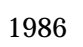 & & & & & & & & & & & & O & 0.88 & 0.91 & & 0.98 & & & 1.08 & & & & 1.28 & & & & & & 1. \\
\hline & & & & & & & & & & & & & & & & & & & & & & & & & & & & & \\
\hline 88 & & 4 & & & 12 & 6 & 48 & & 55 & & 70 & 77 & 32 & 0.84 & & 0.91 & 3 & 0.96 & 00 & 5 & 11 & 15 & 1.19 & 1. & .25 & .29 & .33 & 1.3 & 1.38 \\
\hline 89 & & 33 & & 36 & 40 & 3 & 46 & 19 & 0 & 9 & 0.67 & 0.73 & 78 & 0.80 & 84 & 0.87 & 0.88 & 0.92 & 0.95 & .00 & 1.05 & 0 & 1.13 & 7 & 1.20 & 1.23 & 1.27 & $1.2 \mathrm{~s}$ & 1.32 \\
\hline & & & & & & & & & & & & 0.70 & 0.74 & 0 & ‘ 0.80 & 0.82 & 0.84 & 0.87 & 0.91 & .95 & 1.00 & 1.04 & 1.07 & 1.11 & 1 & 1 & 1.2 & $?$ & 1.25 \\
\hline & & & & & & & & & & & & & 0.71 & 0.73 & & 0.79 & & 0.83 & 0.87 & 0.91 & 0 & 100 & 1 & 1.06 & 10 & 110 & $11:$ & 11 & 10 \\
\hline 992 & & & & & 0.35 & & 0.41 & & & & & & 9 & c & & 0.77 & & & & & & & 1.0 & & & & & & 1.16 \\
\hline & & & & & & & & & & & & & & 0.69 & & 0.75 & & & & & & & & 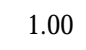 & & & & & 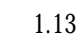 \\
\hline n & & & & & & & & & & & & & & & & & & & & & & & 0.5 & 0.98 & & 1.00 & 1.06 & 1.00 & 1.10 \\
\hline & & & & & & & & & & & & & & & & & & .75 & & 0.81 & & & 0.92 & 95 & 7 & 00 & 03 & 05 & .07 \\
\hline & & 0.26 & & & & & & & & & & & & 0.04 & & 0.09 & & & & & & & & & & 0.97 & 1.00 & 10 & 1.04 \\
\hline 997 & & 25 & & 28 & 31 & & 35 & 0.38 & 41 & 0.45 & & 0.57 & 0.60 & 0.62 & 0.65 & 0.67 & 0.68 & 0.71 & 0.74 & 0.77 & 0.81 & 0.85 & 0.87 & 0.90 & 0.92 & 0.95 & 0.98 & 1.00 & 1.02 \\
\hline 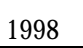 & & & & 10 & 0 & & su & 37 & $\mathrm{IO}$ & 45 & 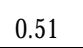 & 56 & 59 & .61 & 64 & 66 & 0.67 & .70 & 0 & 0.76 & 0.80 & 0.84 & 0.86 & 0.89 & 0.91 & 0.94 & 0.96 & 0.98 & 1.00 \\
\hline
\end{tabular}

\section{Source:}

Personal contact with the Bureau of Labor Statistics. 
Table B.13

Gross National Product (GNP) Implicit Price Deflator

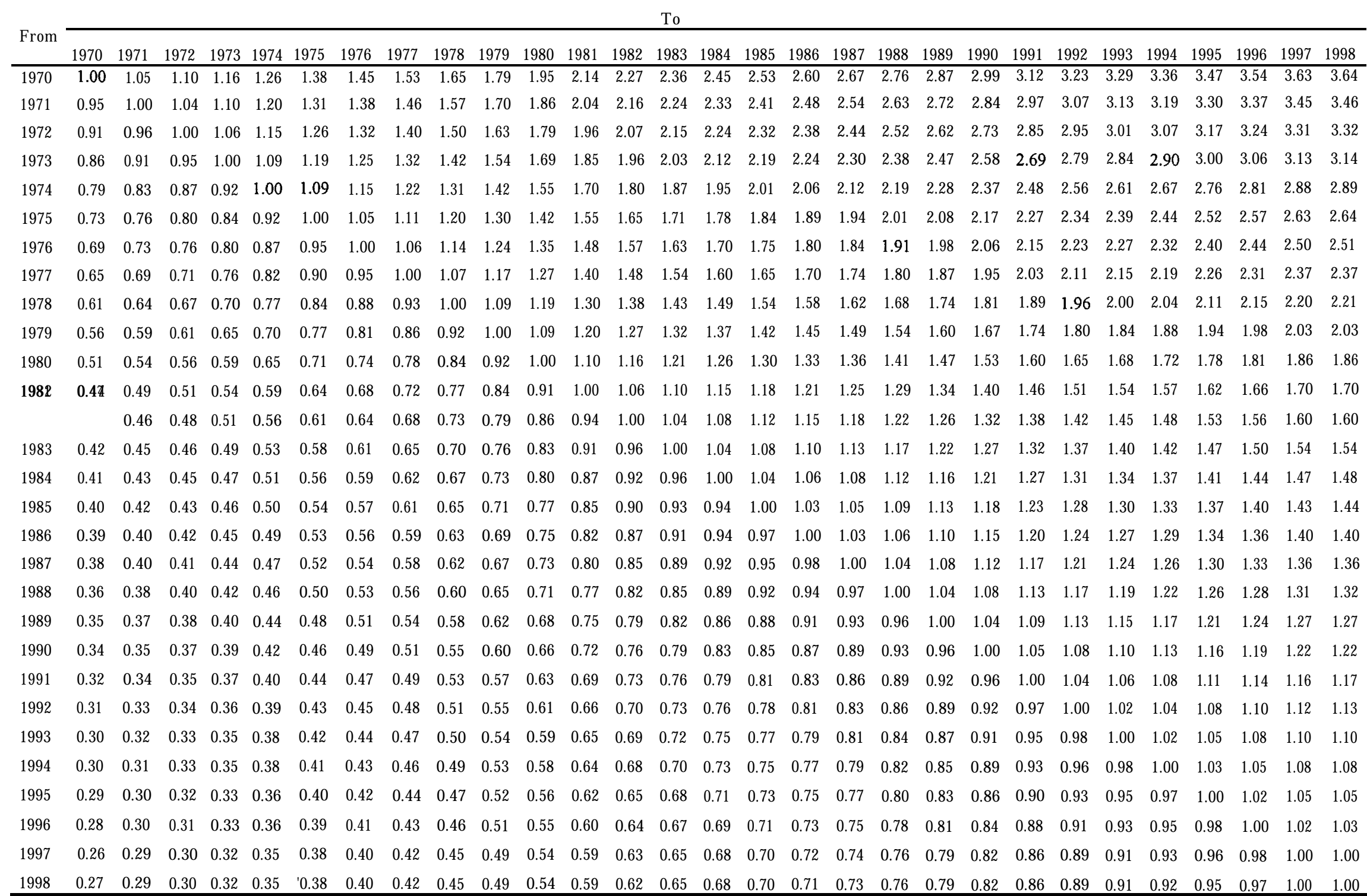

Source:

U.S. Department of Commerce, Bureau of Economic Analysis, Survey of Current Business, Washington, DC, monthly. 


\section{APPENDIX C}

\section{CENSUS DIVISIONS AND REGIONS}

Transportation EnERgy Data Book: Edition 19-1999 
Table C.I

Census Divisions and Regions

\begin{tabular}{|c|c|c|c|}
\hline \multicolumn{4}{|c|}{ Northeast Division } \\
\hline \multicolumn{2}{|c|}{ Mid-Atlantic region } & \multicolumn{2}{|c|}{ New England region } \\
\hline $\begin{array}{l}\text { New Jersey } \\
\text { New York }\end{array}$ & Pennsylvania & $\begin{array}{l}\text { Connecticut } \\
\text { Maine } \\
\text { Massachusetts }\end{array}$ & $\begin{array}{l}\text { New Hampshire } \\
\text { Rhode Island } \\
\text { Vermont }\end{array}$ \\
\hline \multicolumn{4}{|c|}{ South Division } \\
\hline $\begin{array}{l}\text { West South Central } \\
\text { region }\end{array}$ & $\begin{array}{l}\text { East South Central } \\
\text { region }\end{array}$ & & $\begin{array}{l}\text { Atlantic } \\
\text { ion }\end{array}$ \\
\hline $\begin{array}{l}\text { Arkansas } \\
\text { Louisiana } \\
\text { Oklahoma } \\
\text { Texas }\end{array}$ & $\begin{array}{l}\text { Alabama } \\
\text { Kentucky } \\
\text { Mississippi } \\
\text { Tennessee }\end{array}$ & $\begin{array}{l}\text { Delaware } \\
\text { Florida } \\
\text { Georgia } \\
\text { Maryland } \\
\text { North Carolina }\end{array}$ & $\begin{array}{l}\text { South Carolina } \\
\text { Virginia } \\
\text { Washington, DC } \\
\text { West Virginia }\end{array}$ \\
\hline \multicolumn{4}{|c|}{ West Division } \\
\hline \multicolumn{2}{|c|}{ Pacific region } & \multicolumn{2}{|c|}{ Mountain region } \\
\hline $\begin{array}{l}\text { Alaska } \\
\text { California } \\
\text { Hawaii }\end{array}$ & $\begin{array}{l}\text { Oregon } \\
\text { Washington }\end{array}$ & $\begin{array}{l}\text { Arizona } \\
\text { Colorado } \\
\text { Idaho } \\
\text { Montana }\end{array}$ & $\begin{array}{l}\text { Nevada } \\
\text { New Mexico } \\
\text { Utah } \\
\text { Wyoming }\end{array}$ \\
\hline \multicolumn{4}{|c|}{ Midwest Division } \\
\hline \multicolumn{2}{|c|}{ West North Central region } & \multicolumn{2}{|c|}{ East North Central region } \\
\hline $\begin{array}{l}\text { Iowa } \\
\text { Kansas } \\
\text { Minnesota } \\
\text { Missouri }\end{array}$ & $\begin{array}{l}\text { Nebraska } \\
\text { North Dakota } \\
\text { South Dakota }\end{array}$ & $\begin{array}{l}\text { Illinois } \\
\text { Indiana } \\
\text { Michigan }\end{array}$ & $\begin{array}{l}\text { Ohio } \\
\text { Wisconsin }\end{array}$ \\
\hline
\end{tabular}




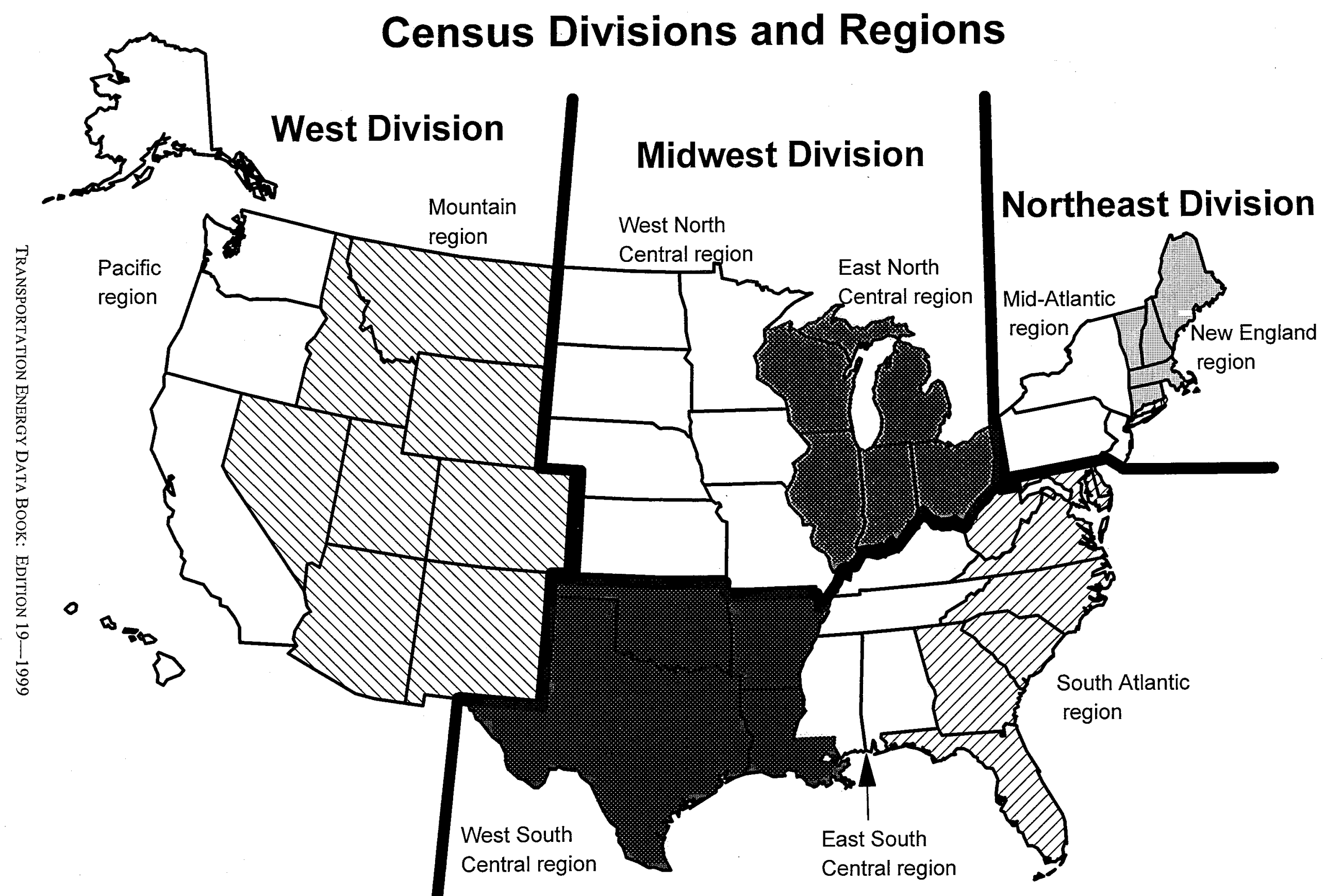

South Division 


\section{GLOSSARY}

Acceleration power - Measured in kilowatts. Pulse power obtainable from a battery used to accelerate a vehicle. This is based on a constant current pulse for 30 seconds at no less than $2 / 3$ of the maximum open-circuit-voltage, at $80 \%$ depth-of-discharge relative to the battery's rated capacity and at 20 " C ambient temperature.

Air Carrier - The commercial system of air transportation consisting of certificated air carriers, air taxis (including commuters), supplemental air carriers, commercial operators of large aircraft, and air travel clubs.

Certificated route air carrier: An air carrier holding a Certificate of Public Convenience and Necessity issued by the Department of Transportation to conduct scheduled interstate services. Nonscheduled or charter operations may also be conducted by these carriers. These carriers operate large aircraft (30 seats or more, or a maximum payload capacity of 7,500 pounds or more) in accordance with Federal Aviation Regulation part 121.

Domestic air operator: Commercial air transportation within and between the 50 States and the District of Columbia. Includes operations of certificated route air carriers, Pan American, local service, helicopter, intra-Alaska, intra-Hawaii, all-cargo carriers and other carriers. Also included are transborder operations conducted on the domestic route segments of U.S. air carriers. Domestic operators are classified based on their operating revenue as follows:

$$
\begin{aligned}
& \text { Majors - over } \$ 1 \text { billion } \\
& \text { Nationals - \$100-1 ,000 million } \\
& \text { Large Regionals - \$10-99.9 million } \\
& \text { Medium Regionals - \$0-9.99 million }
\end{aligned}
$$

International air operator: Commercial air transportation outside the territory of the United States, including operations between the U.S. and foreign countries and between the U.S. and its territories and possessions.

Supplemental air carrier: A class of air carriers which hold certificates authorizing them to perform passenger and cargo charter services supplementing the scheduled service of the certificated route air carriers. Supplemental air carriers are often referred to as nonscheduled air carriers or "nonskeds". 
Alcohol - The family name of a group of organic chemical compounds composed of carbon, hydrogen, and oxygen. The molecules in the series vary in chain length and are composed of a hydrocarbon plus a hydroxyl group. Alcohol includes methanol and ethanol.

\section{Amtrak - See Rail.}

, Anthropogenic - Human made. Usually used in the context of emissions that are produced as the result of human activities.

Automobile size classifications - Size classifications of automobiles are established by the Environmental Protection Agency (EPA) as follows:

Minicompact - less than 85 cubic feet of passenger and luggage volume.

Subcompact - between 85 to 100 cubic feet of passenger and luggage volume.

Compact - between 100 to 110 cubic feet of passenger and luggage volume.

Midsize - between 110 to 120 cubic feet of passenger and luggage volume.

Large - more than 120 cubic feet of passenger and luggage volume.

Two seater - automobiles designed primarily to seat only two adults.

Station wagons are included with the size class for the sedan of the same name.

\section{Aviation - Se G eneral aviation.}

Aviation gasoline - All special grades of gasoline for use in aviation reciprocating engines, as given in the American Society for Testing and Materials (ASTM) Specification D 910. Includes all refinery products within the gasoline range that are to be marketed straight or in blends as aviation gasoline without further processing (any refinery operation except mechanical blending). Also included are finished components in the gasoline range which will be used for blending or compounding into aviation gasoline.

Barges - Shallow, nonself-propelled vessels used to carry bulk commodities on the rivers and the Great Lakes.

Battery efficiency - Measured in percentage. Net DC energy delivered on discharge, as a percentage of the total DC energy required to restore the initial state-of-charge. The efficiency value must include energy losses resulting from self-discharge, cell equalization, thermal loss compensation, and all battery-specific auxiliary equipment.

Btu - The amount of energy required to raise the temperature of 1 pound of water 1 degree Fahrenheit at or near 39.2 degrees Fahrenheit. An average Btu content of fuel is the heat value per quantity of fuel as determined from tests of fuel samples. 
Bunker - A storage tank.

Bunkering fuels - Fuels stored in ship bunkers.

Bus -

Intercity bus: A standard size bus equipped with front doors only, high backed seats, luggage compartments separate from the passenger compartment and usually with restroom facilities, for high-speed long distance service.

Motor bus: Rubber-tired, self-propelled, manually-steered bus with fuel supply on board the vehicle. Motor bus types include intercity, school, and transit.

School and other nonrevenue bus: Bus services for which passengers are not directly charged for transportation, either on a per passenger or per vehicle basis.

Transit bus: A bus designed for frequent stop service with front and center doors, normally with a rear-mounted diesel engine, low-back seating, and without luggage storage compartments or restroom facilities. Includes motor bus and trolley coach.

Trolley coach: Rubber-tired electric transit vehicle, manually-steered, propelled by a motor drawing current, normally through overhead wires, from a central power source not on board the vehicle.

Calendar year - The period of time between January 1 and December 31 of any given year.

Captive imports - Products produced overseas specifically for domestic manufacturers.

Carbon dioxide $(\mathrm{CO}$,$) - A colorless, odorless, non-poisonous gas that is a normal part of the$ ambient air. Carbon dioxide is a product of fossil fuel combustion.

Carbon monoxide (CO) - A colorless, odorless, highly toxic gas that is a normal by-product of incomplete fossil fuel combustion. Carbon monoxide, one of the major air pollutants, can be harmful in small amounts if breathed over a certain period of time.

Car-mile (railroad) - A single railroad car moved a distance of one mile.

\section{Cargo ton-mile - See Ton-mile.}

Certificated route air carriers - See Air carriers.

\section{Class I freight railroad - See R ail.}


Clean Fuel Vehicle - Vehicle meeting the clean fuel vehicle exhaust emissions standards with no restriction on fuel type.

Coal slurry - Finely crushed coal mixed with sufficient water to form a fluid.

Combination trucks - Consist of a power unit (a truck tractor) and one or more trailing units (a semi-trailer or trailer). The most frequently used combination is popularly referred to as a "tractor-semitrailer" or "tractor trailer".

\section{Commercial sector - See Residential and Commercial sector.}

\section{Commuter railroad - See Rail.}

\section{Compact car - See Automobile size classifications.}

Constant dollars - A series of figures is expressed in constant dollars when the effect of change in the purchasing power of the dollar has been removed. Usually the data are expressed in terms of dollars of a selected year or the average of a set of years.

Consumer Price Index (CPI) - An index issued by the U.S. Department of Labor, Bureau of Labor Statistics. The CPI is designed to measure changes in the prices of goods and services bought by wage earners and clerical workers in urban areas. It represents the cost of a typical consumption bundle at current prices as a ratio to its cost at a base year.

Continuous discharge capacity - Measured as percent of rated energy capacity. Energy delivered in a constant power discharge required by an electric vehicle for hill climbing and/or highspeed cruise, specified as the percent of its rated energy capacity delivered in a one hour constant-power discharge.

Corporate Average Fuel Economy (CAFE) standards - CAFE standards were originally established by Congress for new automobiles, and later for light trucks, in Title V of the Motor Vehicle Information and Cost Savings Act (15 U.S.C. 1901, et seq.) with subsequent amendments. Under CAFE, automobile manufacturers are required by law to produce vehicle fleets with a composite sales-weighted fuel economy which cannot be lower than the CAFE standards in a given year, or for every vehicle which does not meet the standard, a fine of $\$ 5.00$ is paid for every one-tenth of a mpg below the standard.

Crude oil - A mixture of hydrocarbons that exists in the liquid phase in natural underground reservoirs and remains liquid at atmospheric pressure after passing through surface 
separating facilities. Crude oil production is measured at the wellhead and includes lease condensate.

Crude oil imports - The volume of crude oil imported into the 50 States and the District of Columbia, including imports from U.S. territories, but excluding imports of crude oil into the Hawaiian Foreign Trade Zone.

Curb weight - The weight of a vehicle including all standard equipment, spare tire and wheel, all fluids and lubricants to capacity, full tank of fuel, and the weight of major optional accessories normally found on the vehicle.

Current dollars - Represents dollars current at the time designated or at the time of the transaction. In most contexts, the same meaning would be conveyed by the use of the term "dollars".

\section{Disposable personal income - See Income.}

Distillate fuel oil - The lighter fuel oils distilled off during the refining process. Included are products known as ASTM grades numbers 1 and 2 heating oils, diesel fuels, and number 4 fuel oil. The major uses of distillate fuel oils include heating, fuel for on-and offhighway diesel engines, and railroad diesel fuel.

\section{Domestic air operator - See Air carrier.}

\section{Domestic water transportation - See Internal water transportation.}

Electric utilities sector - Consists of privately and publicly owned establishments which generate electricity primarily for resale.

Emission standards - Standards for the levels of pollutants emitted from automobiles and trucks. Congress established the first standards in the Clean Air Act of 1963. Currently, standards are set for four vehicle classes - automobiles, light trucks, heavy-duty gasoline trucks, and heavy-duty diesel trucks.

Energy capacity - Measured in kilowatt hours. The energy delivered by the battery, when tested at $\mathrm{C} / 3$ discharge rate, up to termination of discharge specified by the battery manufacturer. The required acceleration power must be delivered by the battery at any point up to $80 \%$ of the battery's energy capacity rating.

Energy efficiency - In reference to transportation, the inverse of energy intensiveness: the ratio of outputs from a process to the energy inputs; for example, miles traveled per gallon of fuel (mpg). 
Energy intensity - In reference to transportation, the ratio of energy inputs to a process to the useful outputs from that process; for example, gallons of fuel per passenger-mile or Btu per ton-mile.

Ethanol $\left(\mathbf{C}_{2} \mathbf{H}_{5} \mathbf{O H}\right)$ - Otherwise known as ethyl alcohol, alcohol, or grain-spirit. A clear, colorless, flammable oxygenated hydrocarbon with a boiling point of 78.5 degrees Celsius in the anhydrous state. In transportation, ethanol is used as a vehicle fuel by itself (E100), blended with gasoline (E85), or as a gaoline octane enhancer and oxygenate $(10 \%$ 'concentration).

\section{Fixed operating cost - See 0 perating cost.}

\section{Fleet vehicles -}

Private fleet vehicles: Ideally, a vehicle could be classified as a member of a fleet if it is:

a) operated in mass by a corporation or institution,

b) operated under unified control, or

c) used for non-personal activities.

However, the definition of a fleet is not consistent throughout the fleet industry. Some companies make a distinction between cars that were bought in bulk rather than singularly, or whether they are operated in bulk, as well as the minimum number of vehicles that constitute a fleet (i.e. 4 or 10).

Government fleet vehicles: Includes vehicles owned by all federal (GSA), state, county, city, and metro units of government, including toll road operations.

Foreign freight - Movements between the United States and foreign countries and between Puerto Rico, the Virgin Islands, and foreign countries. Trade between U.S. territories and possessions (e.g. Guam, Wake, American Samoa) and foreign countries is excluded. Traffic to or from the Panama Canal Zone is included.

Gas Guzzler Tax - Originates from the 1978 Energy Tax Act (Public Law 95-618). A new car purchaser is required to pay the tax if the car purchased has a combined city/highway fuel economy rating that is below the standard for that year. For model years 1986 and later, the standard is $22.5 \mathrm{mpg}$.

Gasohol - A mixture of $10 \%$ anhydrous ethanol and $90 \%$ gasoline by volume. There are other fuels that contain methanol and gasoline, but these fuels are not referred to as gasohol.

\section{Gasoline - See Motor gasoline.}


General aviation - That portion of civil aviation which encompasses all facets of aviation except air carriers. It includes any air taxis, commuter air carriers, and air travel clubs which do not hold Certificates of Public Convenience and Necessity.

Gross National Product - A measure of monetary value of the goods and services becoming available to the nation from economic activity. Total value at market prices of all goods and services produced by the nation's economy. Calculated quarterly by the Department of Commerce, the Gross National Product is the broadest available measure of the level of economic activity.

Gross vehicle weight (gvw) - The weight of the empty vehicle plus the maximum anticipated load weight.

\section{Heavy-heavy truck - See Truck size classifications.}

Household - Consists of all persons who occupy a housing unit, including the related family members and all unrelated persons, if any, who share the housing unit.

Housing unit - A house, apartment, a group of rooms, or a single room occupied or intended for occupancy as separate living quarters. Separate living quarters are those in which the occupants do not live and eat with any other persons in the structure and which have either (1) direct access from the outside of the building or through a common hallway intended to be used by the occupants of another unit or by the general public, or (2) complete kitchen facilities for the exclusive use of the occupants. The occupants may be a single family, one person living alone, two or more families living together, or any other group of related or unrelated persons who share living arrangements.

Hydrocarbon (HC) - A compound that contains only hydrogen and carbon. The simplest and lightest forms of hydrocarbon are gaseous. With greater molecular weights they are liquid, while the heaviest are solids.

\section{Income -}

Disposable personal income: Personal income less personal tax and non-tax payments.

National income - The aggregate earnings of labor and property which arise in the current production of goods and services by the nation's economy.

Personal income: The current income received by persons from all sources, net of contributions for social insurance.

Industrial sector - Construction, manufacturing, agricultural and mining establishments. 
Inertia weight - The curb weight of a vehicle plus 300 pounds.

\section{Intercity bus - See Bus.}

Internal water transportation - Includes all local (intraport) traffic and traffic between ports or landings wherein the entire movement takes place on inland waterways. Also termed internal are movements involving carriage on both inland waterways and the water of the Great Lakes, and inland movements that cross short stretches of open water that link inland systems.

\section{International air operator - See Air carrier.}

\section{International freight - See Foreign freight.}

Jet fuel - Includes both naphtha-type and kerosene-type fuels meeting standards for use in aircraft turbine engines. Although most jet fuel is used in aircraft, some is used for other purposes such as generating electricity in gas turbines.

Kerosene-type jet fuel: A quality kerosene product with an average gravity of 40.7 degrees API and $10 \%$ to $90 \%$ distillation temperatures of 217 to 261 degrees centigrade. Used primarily as fuel for commercial turbojet and turboprop aircraft engines. It is a relatively low freezing point distillate of the kerosene type.

Naphtha-type jet fuel: A fuel in the heavy naphtha boiling range with an average gravity of 52.8 degrees API and $10 \%$ to $90 \%$ distillation temperatures of 117 to 233 degrees centigrade used for turbojet and turboprop aircraft engines, primarily by the military. Excludes ramjet and petroleum.

Kerosene - A petroleum distillate in the 300 to 500 degrees Fahrenheit boiling range and generally having a flash point higher than 100 degrees Fahrenheit by the American Society of Testing and Material (ASTM) Method D56, a gravity range from 40 to 46 degrees API, and a burning point in the range of 150 to 175 degrees Fahrenheit. It is a clean-burning product suitable for use as an illuminant when burned in wick lamps. Includes grades of kerosene called range oil having properties similar to Number 1 fuel oil, but with a gravity of about 43 degrees API and an end point of 625 degrees Fahrenheit. Used in space heaters, cooking stoves, and water heaters.

Kerosene-type jet fuel - See Jet fuel.

Large car - See A utomobile size classifications. 
Lease Condensate - A liquid recovered from natural gas at the well or at small gas/oil separators in the field. Consists primarily of pentanes and heavier hydrocarbons (also called field condensate).

Light duty vehicles - Automobiles and light trucks combined.

Light truck - Unless otherwise noted, light trucks are defined in this publication as two-axle, fourtire trucks. The U.S. Bureau of Census classifies all trucks with a gross vehicle weight less than 10,000 pounds as light trucks (See Truck size classifications).

\section{Light-heavy truck - See Truck size classifications.}

Liquified petroleum gas (lpg) - Consists of propane and butane and is usually derived from natural gas. In locations where there is no natural gas and the gasoline consumption is low, naphtha is converted to Ipg by catalytic reforming.

Load factor - A term relating the potential capacity of a system relative to its actual performance. Is often calculated as total passenger miles divided by total vehicle miles.

Low-emission vehicle - A clean fuel vehicle meeting the low-emission vehicle standards.

\section{Medium truck - See Truck size classifications.}

Methanol $\left(\mathrm{CH}_{3} \mathbf{O H}\right)$ - A colorless poisonous liquid with essentially no odor and very little taste. It is the simplest alcohol and boils at 64.7 degrees Celsius. In transportation, methanol is used as a vehicle fuel by itself (M100), or blended with gasoline (M85). 
Midsize car - See Automobile size classifications.

\section{Minicompact car - See Automobile size classifications.}

Model year - In this publication, model year is referring to the "sales" model year, the period from October 1 to the next September 31 .

\section{Motor bus - See Bus.}

Motor Gasoline - A mixture of volatile hydrocarbons suitable for operation of an internal combustion engine whose major components are hydrocarbons with boiling points ranging from 78 to 217 degrees centigrade and whose source is distillation of petroleum and cracking, polymerization, and other chemical reactions by which the naturally occurring petroleum hydrocarbons are converted into those that have superior fuel properties.

\section{Naphtha-type jet fuel - See Jet fuel.}

\section{National income - See Income.}

Nationwide Personal Transportation Study (NPTS) - A nationwide home interview survey of households that provides information on the characteristics and personal travel patterns of the U.S. population. Surveys were conducted in 1969, 1977, 1983 and 1990 by the U.S. Bureau of Census for the U.S. Department of Transportation.

Natural gas - A mixture of hydrocarbon compounds and small quantities of various nonhydrocarbons existing in the gaseous phase or in solution with crude oil in natural underground reservoirs at reservoir conditions.

Natural Gas Plant Liquids - Products obtained from processing natural gas at natural gas processing plants, including natural gasoline plants, cycling plants, and fractionators. Products obtained include ethane, liquefied petroleum gases, (propanes, butane, propanebutane mixtures, and ethane-propane mixtures), isopentane, natural gasoline, unfractionated streams, plant condensate, and other minor quantities of finished products, such as motor gasoline, special naphthas, jet fuel, kerosene, and distillate fuel oil.

Nitrogen Oxides $\left(\mathbf{N O}_{\mathbf{x}}\right.$ ) - A product of combustion of fossil fuels whose production increases with the temperature of the process. It can become an air pollutant if concentrations are excessive. 
Oil Stocks - Oil stocks include crude oil (including strategic reserves), unfinished oils, natural gas plant liquids, and refined petroleum products.

\section{Operating cost -}

Fixed operating cost: In reference to passenger car operating cost, refers to those expenditures that are independent of the amount of use of the car, such as insurance costs, fees for license and registration, depreciation and finance charges.

Variable operating cost: In reference to passenger car operating cost, expenditures which are dependent on the amount of use of the car, such as the cost of gas and oil, tires, and other maintenance.

Organization for Economic Cooperation and Development (OECD) - Consists of Australia, Austria, Belgium, Canada, Czech Republic, Denmark, Finland, France, Germany, Greece, Hungary, Iceland, Ireland, Italy, Japan, Luxembourg, Mexico, Netherlands, New Zealand, Norway, Poland, Portugal, South Korea, Spain, Sweden, Switzerland, Turkey, United Kingdom, and United States. Total OECD includes the United States Territories (Guam, Puerto Rico, and the U.S. Virgin Islands). Total OECD excludes data for Czech Republic, Hungary, Mexico, Poland, and South Korea which are not yet available.

OECD Europe: Consists of Austria, Belgium, Czech Republic, Denmark, Finland, France, Germany, Greece, Hungary, Iceland, Ireland, Italy, Luxembourg, Netherlands, Norway, Poland, Portugal, Spain, Sweden, Switzerland, Turkey, and United Kingdom. OECD Europe excludes data for Czech Republic, Hungary, and Poland which are not yet available.

OECD Pacific: Consists of Australia, Japan, and New Zealand.

Organization for Petroleum Exporting Countries (OPEC) - Includes Saudi Arabia, Iran, Venezuela, Libya, Indonesia, United Arab Emirates, Algeria, Nigeria, Ecuador, Gabon, Iraq, Kuwait, and Qatar. Data for Saudi Arabia and Kuwait include their shares from the Partitioned Zone (formerly the Neutral Zone).

Arab OPEC - Consists of Algeria, Iraq, Kuwait, Libya, Qatar, Saudi Arabia and the United Arab Emirates.

\section{Other single-unit truck - See Single-unit truck.}

Oxygenate - A substance which, when added to gasoline, increases the amount of oxygen in that gasoline blend. Includes fuel ethanol, methanol, and methyl tertiary butyl ether (MTBE). 
Particulates - Carbon particles formed by partial oxidation and reduction of the hydrocarbon fuel. Also included are trace quantities of metal oxides and nitrides, originating from engine wear, component degradation, and inorganic fuel additives. In the transportation sector, particulates are emitted mainly from diesel engines.

Passenger-miles traveled (PMT) - One person traveling the distance of one mile. Total passenger-miles traveled, thus, give the total mileage traveled by all persons.

\section{Passenger rail - See Rail, "Amtrak" and "Transit Railroad".}

Persian Gulf countries: Consists of Bahrain, Iran, Iraq, Kuwait, Qatar, Saudi Arabia, and the United Emirates.

Personal Consumption Expenditures (PCE) - As used in the national accounts, the market value of purchases of goods and services by individuals and nonprofit institutions and the value of food, clothing, housing, and financial services received by them as income in kind. It includes the rental value of owner-occupied houses but excludes purchases of dwellings, which are classified as capital goods (investment).

\section{Personal income - See Income.}

Petroleum - A generic term applied to oil and oil products in all forms, such as crude oil, lease condensate, unfinished oil, refined petroleum products, natural gas plant liquids, and nonhydrocarbon compounds blended into finished petroleum products.

Petroleum consumption: A calculated demand for petroleum products obtained by summing domestic production, imports of crude petroleum and natural gas liquids, imports of petroleum products, and the primary stocks at the beginning of the period and then subtracting the exports and the primary stocks at the end of the period.

Petroleum exports: Shipments of petroleum products from the 50 States and the District of Columbia to foreign countries, Puerto Rico, the Virgin Islands, and other U.S. possessions and territories.

Petroleum imports: All imports of crude petroleum, natural gas liquids, and petroleum products from foreign countries and receipts from Guam, Puerto Rico, the Virgin Islands, and the Hawaiian Trade Zone. The commodities included are crude oil, unfinished oils, plant condensate, and refined petroleum products. 
Petroleum inventories: The amounts of crude oil, unfinished oil, petroleum products, and natural gas liquids held at refineries, at natural gas processing plants, in pipelines, at bulk terminals operated by refining and pipeline companies, and at independent bulk terminals. Crude oil held in storage on leases is also included; these stocks are know as primary stocks. Secondary stocks - those held by jobbers dealers, service station operators, and consumers -are excluded. Prior to 1975, stock held at independent bulk terminals were classified as secondary stocks.

Petroleum products supplied: For each petroleum product, the amount supplied is calculated by summing production, crude oil burned directly, imports, and net withdrawals from primary stocks and subtracting exports.

Processing Gain - The amount by which the total volume of refinery output is greater than the volume of input for given period of time. The processing gain arises when crude oil and other hydrocarbons are processed into products that are, on average, less dense than the input.

Processing Loss - The amount by which the total volume of refinery output is less than the volume of input for given period of time. The processing loss arises when crude oil and other hydrocarbons are processed into products that are, on average, more dense than the input.

Proved Reserves of Crude Oil - The estimated quantities of all liquids defined as crude oil, which geological and engineering data demonstrate with reasonable certainty to be recoverable in future years from known reservoirs under existing economic and operating conditions.

Quad - Quadrillion, $10^{15}$. In this publication, a Quad refers to Quadrillion Btu.

\section{Rail -}

Amtrak (American Railroad Tracks): Operated by the National Railroad Passenger Corporation of Washington, DC. This rail system was created by President Nixon in 1970, and was given the responsibility for the operation of intercity, as distinct from suburban, passenger trains between points designated by the Secretary of Transportation.

Class I freight railroad: Defined by the Interstate Commerce Commission each year based on annual operating revenue. A railroad is dropped from the Class I list if it fails to meet the annual earnings threshold for three consecutive years.

Commuter railroad: Those portions of mainline railroad (not electric railway) transportation operations which encompass urban passenger train service for local travel between a central city and adjacent suburbs. Commuter railroad service - using both locomotive-hauled and self-propelled railroad passenger cars - is characterized by multitrip tickets, specific station-to-station fares, and usually only one or two stations in the central business district. Also known as suburban railroad. 
Transit railroad: Includes "heavy" and "light" transit rail. Heavy transit rail is characterized by exclusive rights-of-way, multi-car trains, high speed rapid acceleration, sophisticated signaling, and high platform loading. Also known as subway, elevated railway, or metropolitan railway (metro). Light transit rail may be on exclusive or shared rights-of-way, high or low platform loading, multi-car trains or single cars, automated or manually operated. In generic usage, light rail includes streetcars, trolley cars, and tramways.

Residential and Commercial sector - Consists of housing units, non-manufacturing business establishments (e.g., wholesale and retail businesses), health and educational institutions, and government offices.

Residential Transportation Energy Consumption Survey (RTECS) - This survey was designed by the Energy Information Administration of the Department of Energy to provide information on how energy is used by households for personal vehicles. It has been conducted five times since 1979, the most recent being 1991.

Residual fuel oil - The heavier oils that remain after the distillate fuel oils and lighter hydrocarbons are boiled off in refinery operations. Included are products know as ASTM grade numbers 5 and 6 oil, heavy diesel oil, Navy Special Fuel Oil, Bunker C oil, and acid sludge and pitch used as refinery fuels. Residual fuel oil is used for the production of electric power, for heating, and for various industrial purposes.

Rural - Usually refers to areas with population less than 5,000.

Sales period - October 1 of the previous year to September 30 of the given year. Approximately the same as a model year.

Sales-weighted miles per gallon (mpg) - Calculation of a composite vehicle fuel economy based on the distribution of vehicle sales.

Scrappage rate - As applied to motor vehicles, it is usually expressed as the percentage of vehicles of a certain type in a given age class that are retired from use (lacking registration) in a given year.

School and other nonrevenue bus - See Bus. 
Single unit truck - Includes two-axle, four-tire trucks and other single unit trucks.

Two-axle, four tire truck: A motor vehicle consisting primarily of a single motorized device with two axles and four tires.

Other single-unit truck: A motor vehicle consisting primarily of a single motorized device with more than two axles or more than four tires.

Special fuels - Consist primarily of diesel fuel with small amount of liquified petroleum gas, as defined by the Federal Highway Administration.

Specific acceleration power - Measured in watts per kilogram. Acceleration power divided by the battery system weight. Weight must include the total battery system.

Specific energy - Measured in watt hours per kilogram. The rated energy capacity of the battery divided by the total battery system weight.

Subcompact car - See Automobile size classifications.

Supplemental air carrier - See Air carrier.

Test weight - The weight setting at which a vehicle is tested on a dynomometer by the U.S. Environmental Protection Agency (EPA). This weight is determined by the EPA using the inertia weight of the vehicle.

Ton-mile - The movement of one ton of freight the distance'of one mile. Ton-miles are computed by multiplying the weight in tons of each shipment transported by the distance hauled.

\section{Transmission types -}

A3 - Automatic three speed

A4 - Automatic four speed

A5 - Automatic five speed

L4 - Automatic lockup four speed

M5 - Manual five speed

\section{Transit bus - See Bus.}

Transit railroad - See Rail. 
Transportation sector - Consists of both private and public passenger and freight transportation, as well as government transportation, including military operations.

Truck Inventory and Use Survey (TIUS) - Survey designed to collect data on the characteristics and operational use of the nation's truck population. It is conducted every five years by the U.S. Bureau of the Census. Surveys were conducted in 1963, 1967, 1972, 1977, 1982, 1987, and 1992. The 1992 data have not yet been released.

Trolley coach - See Bus.

Truck size classifications - U.S. Bureau of the Census has categorized trucks by gross vehicle weight (gvw) as follows:

Light - Less than 10,000 pounds gvw (Also see Light Truck.)

Medium - 10,001 to 20,000 pounds gvw

Light-heavy - 20,001 to 26,000 pounds gvw

Heavy-heavy $-26,001$ pounds gvw or more.

Two-axle, four-tire truck - See Single-unit truck.

Two seater car - Se Automobile size classifications.

Ultra-low emission vehicle - A clean fuel vehicle meeting the more stringent Ultra-low emission standards.

Urban - Usually refers to areas with population of 5,000 or greater.

Variable operating cost - See 0 perating cost.

Vehicle-miles traveled (vmt) - One vehicle traveling the distance of one mile. Total vehicle miles, thus, is the total mileage traveled by all vehicles.

Zero-emission vehicle - A clean fuel vehicle meeting even more stringent zero-emission vehicle standards. 


\section{TITLE INDEX}

TRANSPORTATION ENERGY Data Book: EdITION 19-1 999 
Act

Energy Policy Act Purchase Requirements of Light Alternative Fuel Vehicles. . . . . . . . . . 10-8 Advanced

U.S. Advanced Battery Consortium Research Agreements, Phase II ................ 9-10

U.S. Advanced Battery Consortium Goals for Electric Vehicle Batteries $\ldots \ldots \ldots \ldots \ldots \ldots$. 9-11 Age

Automobiles in Operation and Vehicle Travel by Age, 1970 and 1997 . . . . . . . . . . . . . 6-8

Trucks in Operation and Vehicle Travel by Age, 1970 and 1997 . . . . . . . . . . . . . . . . 6-9

Average Age of Automobiles and Trucks in Use, 1970-97 . . . . . . . . . . . . . . . . . 6 6-10

Average Age of Vehicles by Household Vehicle Ownership, 1995 NPTS . . . . . . . . . . . $11-12$

Average Annual Miles per Household Vehicle by Vehicle Age .................. 11 1-1 3 Agency

Federal Government Vehicles by Agency, Fiscal Year $1997 \ldots \ldots \ldots \ldots \ldots \ldots \ldots \ldots \ldots$ 10 .6. . . . . Air

Total National Emissions of the Criteria Air Pollutants by Sector, 1997 . . . . . . . . . . . . 4-2

California Air Resources Board Requirements for Meeting Emission Standards ........ 4-37

Summary Statistics for U.S. Domestic and International Certificated

Route Air Carriers (Combined Totals), 1970-97 ......................... 12-2

Alternative

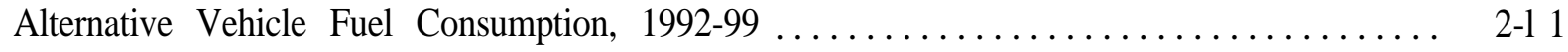

Estimates of Alternative Fuel Vehicles in Use, $1992-99 \ldots \ldots \ldots \ldots \ldots \ldots \ldots \ldots \ldots . \ldots \ldots$

Estimates of Light Alternative Fuel Vehicles, 1995, 1997, and $1999 \ldots \ldots \ldots \ldots \ldots \ldots \ldots$. . . . .

Estimates of Heavy Alternative Fuel Vehicles, 1995, 1997, and $1999 \ldots \ldots \ldots \ldots \ldots \ldots \ldots$. . . . . .

Alternative Fuel Vehicles Available by Manufacturer, Model Year 1999 . . . . . . . . . . . 9.6

Number of Alternative Refuel Sites by State and Fuel Type, $1998 \ldots \ldots \ldots \ldots \ldots \ldots \ldots \ldots$. . . . . . .

Energy Policy Act Purchase Requirements of Light Alternative Fuel Vehicles......... 10-8 Amtrak

Summary Statistics for the National Railroad Passenger Corporation (Amtrak), 1971-97 . . . 12-11 Annual

Average Annual and Daily Vehicle-Miles of Travel for Fleet Vehicles, $1991 \ldots \ldots \ldots \ldots \ldots$. . . . .

Average Annual Expenditures of Households by Income, 1997 . . . . . . . . . . . . . . 11 1-3

Average Annual Vehicle-Miles, Vehicle Trips and Trip Length

per Household 1969, 1977, 1983, 1990, and 1995 NPTS ................. 11-7

Average Annual Person-Miles Traveled (PMT), Person Trips and Trip Length

per Household by Selected Trip Purposes, 1983, 1990, and 1995 NPTS . . . . . . . . . $11-8$

Average Annual Miles per Vehicle by Household Vehicle Ownership, 1995 NPTS . . . . . . . 11-12

Average Annual Miles per Household Vehicle by Vehicle Age ................. 11 1-1 3 Area

National and Metropolitan Area Comparisons of Journey-to-Work Statistics, 1990 Census . . 11 1-1 6 Assessments

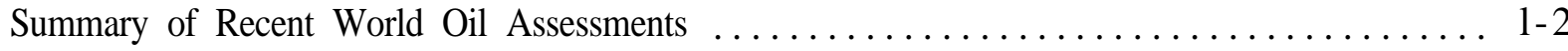


Automobile

Automobile Operating Cost per Mile, $1975-98 \ldots \ldots \ldots \ldots \ldots \ldots \ldots \ldots \ldots \ldots \ldots \ldots$ 5-14

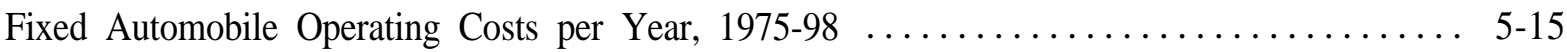

Automobile Registrations for Selected Countries, $1950-96 \ldots \ldots \ldots \ldots \ldots \ldots \ldots \ldots \ldots .6 .2$

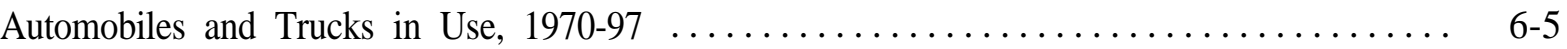

Automobiles in Operation and Vehicle Travel by Age, 1970 and 1997 . . . . . . . . . . . 6-8

Average Age of Automobiles and Trucks in Use, 1970-97 ................... 6-1 0

Scrappage and Survival Rates for Automobiles 1970, 1980 and 1990 Model Years ........ 6-1 1

New Retail Automobile Sales in the United States, 1970-97 . . . . . . . . . . . . . . . . . 7-3

Period Sales, Market Shares, and Sales-Weighted Fuel Economics of New

Domestic and Import Automobiles, Selected Sales Periods 1976-98 . . . . . . . . . . . 7-4

Sales-Weighted Engine Size of New Domestic and Import Automobiles

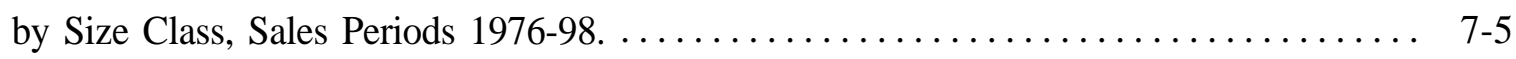

Sales-Weighted Curb Weight of New Domestic and Import Automobiles

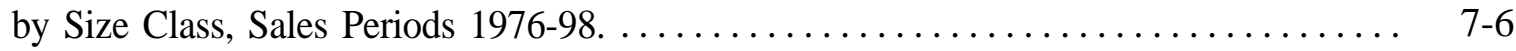

Sales-Weighted Interior Space of New Domestic and Import Automobiles

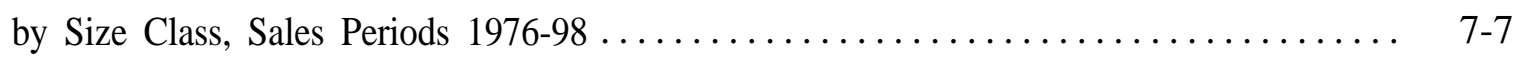

Engine Size, Curb Weight, and Interior Space of New Domestic and

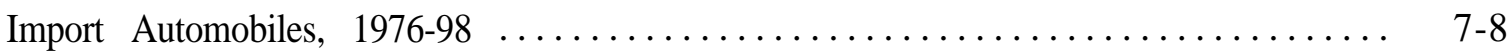

Average Material Consumption for a Domestic Automobile,

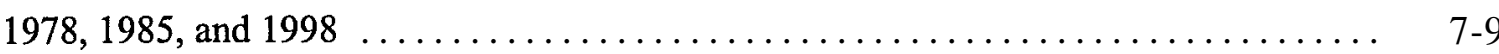

Sales-Weighted Wheelbase of New Automobiles and Light Trucks,

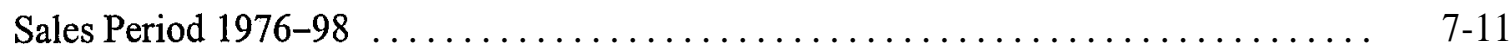

Corporate Average Fuel Economy (CAFE) Standards versus Sales-Weighted

Fuel Economy Estimates for Automobiles and Light Trucks, 1978-98 .......... 7-1 7 Available

Alternative Fuel Vehicles Available by Manufacturer, Model Year 1999 . . . . . . . . . . 9-6 Average

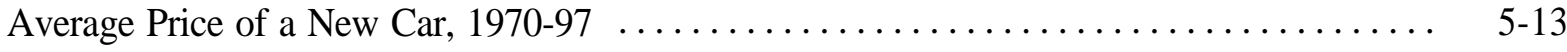

Average Age of Automobiles and Trucks in Use, 1970-97 . . . . . . . . . . . . . 6 6-10

Average Material Consumption for a Domestic Automobile, 1978, 1985, and 1998 ......... 7-9

Corporate Average Fuel Economy (CAFE) Standards versus Sales-Weighted

Fuel Economy Estimates for Automobiles and Light Trucks, 1978-98. ........... 7-17

Corporate Average Fuel Economy (CAFE) Fines Collected, 1983-97 . . . . . . . . . . 7-18

Average Length of Time Fleet Vehicles are Kept Before Sold to Others, $1991 \ldots \ldots \ldots \ldots$. . . 10-4

Average Annual and Daily Vehicle-Miles of Travel for Fleet Vehicles, $1991 \ldots \ldots \ldots \ldots \ldots$ 10-4

Average Miles per Federal Vehicle by Vehicle Type, 1997 . . . . . . . . . . . . . . . . . . $10-5$

Average Annual Expenditures of Households by Income, $1997 \ldots \ldots \ldots \ldots \ldots \ldots \ldots \ldots$. $11-3$

Average Number of Vehicles and Vehicle Travel per Household, 1991 and 1994 RTECS ..... 11 1-4

Average Annual Vehicle-Miles, Vehicle Trips and Trip Length per Household.

$1969,1977,1983,1990$, and 1995 NPTS . . . . . . . . . . . . . . . . . . 
Average (continued)

Average Annual Person-Miles Traveled (PMT), Person Trips and Trip Length

per Household by Selected Trip Purposes, 1983, 1990, and 1995 NPTS . . . . . . . . . . . 1 1-8

Average Vehicle Occupancy by Vehicle Type, 1995 NPTS . . . . . . . . . . . . . . . . . . 11 1-9

Average Vehicle Occupancy by Trip Purpose, 1977, 1983, 1990, and 1995 NPTS . . . . . . , . 1 1-10

Average Annual Miles per Vehicle by Household Vehicle Ownership, 1995 NPTS . . . . . . . . 11-12

Average Age of Vehicles by Household Vehicle Ownership, 1995 NPTS . . . . . . . . . . . 1 1-1 2

Average Annual Miles per Household Vehicle by Vehicle Age . . . . . . . . . . . . . . . 11 1-1 3 Aviation

Summary Statistics for General Aviation, $1970-97 \ldots \ldots \ldots \ldots \ldots \ldots \ldots \ldots \ldots \ldots \ldots$ Axle

Summary Statistics for Two-Axle, Four-Tire Trucks, $1970-97 \ldots \ldots \ldots \ldots \ldots \ldots \ldots \ldots$. . . . . . . 2 Barrel

Refinery Yield of Petroleum Products from a Barrel of Crude Oil, 1978-97 . . . . . . . . . . . . . 1-14

Prices for a Barrel of Crude Oil and a Gallon of Gasoline, 1978-98 . . . . . . . . . . . . 5 5-6 Battery

U.S. Advanced Battery Consortium Research Agreements, Phase II . . . . . . . . . . . . . 9-1 0

U.S. Advanced Battery Consortium Goals for Electric Vehicle Batteries . . . . . . . . . . . . 9-1 1 Board

California Air Resources Board Requirements for Meeting Emission Standards ........ 4-37 Breakdown

Breakdown of Domestic Marine Cargo by Commodity Class, $1997 \ldots \ldots \ldots \ldots \ldots \ldots \ldots$. . . . . . . 6 Bus

Truck and Bus Registrations for Selected Countries, $1950-96 . \ldots \ldots \ldots \ldots \ldots \ldots \ldots \ldots$ 6 3 Buses

Summary Statistics on Buses by Type, $1970-97 \ldots \ldots \ldots \ldots \ldots \ldots \ldots \ldots \ldots \ldots \ldots$ CAFE

Corporate Average Fuel Economy (CAFE) Standards versus Sales-Weighted

Fuel Economy Estimates for Automobiles and Light Trucks, 1978-98 . . . . . . . . . 7-17

Corporate Average Fuel Economy (CAFE) Fines Collected, 1983-97 ............. 7-18 Calendar

Vehicle Stock and New Sales in United States, 1997 Calendar Year ................. 6-6 California

California Passenger Cars and Light Trucks Emission Certification Standards ......... 4-35

California Vehicle Emission Reduction for Passenger Cars and Light Trucks. .......... 4-36

California Air Resources Board Requirements for Meeting Emission Standards ......... 4-37 Car

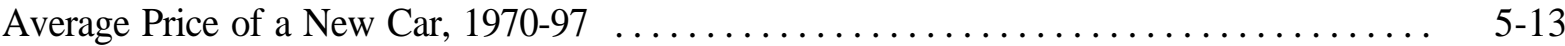
Carbon

International Man-Made Emissions of Carbon Dioxide, $1990-96 \ldots \ldots \ldots \ldots \ldots \ldots \ldots \ldots$ 3-3

International Man-Made Emissions of Carbon Dioxide by Source Category, 1990 and 1996 . . . 3-4 U.S. Carbon Dioxide Emissions from Fossil Energy Consumption by End-Use Sector, 1984-97 3-7 
Carbon (continued)

U.S. Carbon Dioxide Emissions from Energy Use in the Transportation Sector, 1980-97 .... . 3-8

Numerical Estimates of Global Warming Potentials Compared With Carbon Dioxide ........ 3-9

Total National Emissions of Carbon Monoxide, 1970-97 ................... 4-3

Emissions of Carbon Monoxide from Highway Vehicles, $1970-97 \ldots \ldots \ldots \ldots \ldots \ldots \ldots \ldots$. . . . . .

Cargo

Breakdown of Domestic Marine Cargo by Commodity Class, 1997 . . . . . . . . . . . . . . 12-6

Carloads

Railroad Revenue Carloads by Commodity Group, 1974 and 1997 . . . . . . . . . . . . . 12-9

Carriers

Summary Statistics for U.S. Domestic and International Certificated

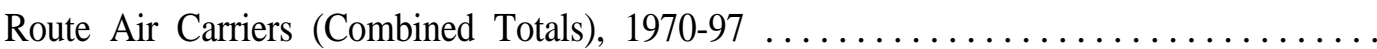

Cars

Changes in Per-Mile, Fuel-Cycle Energy Use and Greenhouse Gas Emissions

for Passenger Cars using Near-Term Technologies ...................... 3-12

Changes in Per-Mile, Fuel-Cycle Energy Use and Greenhouse Gas Emissions

for Passenger Cars using Long-Term Technologies $\ldots \ldots \ldots \ldots \ldots \ldots \ldots \ldots \ldots \ldots . . \ldots \ldots \ldots$

Changes in Per-Mile, Fuel-Cycle Energy Use and Criteria Pollutant Emissions

for Passenger Cars using Near-Term Technologies ................... 4-17

Changes in Per-Mile, Fuel-Cycle Energy Use and Criteria Pollutant Emissions

for Passenger Cars using Long-Term Technologies .................... 4-19

California Passenger Cars and Light Trucks Emission Certification Standards ........... 4-35

California Vehicle Emission Reduction for Passenger Cars and Light Trucks. .......... 4-36

Summary Statistics for Passenger Cars, 1970-97 ..................... $7-2$

The Gas Guzzler Tax on New Cars . . . . . . . . . . . . . . . . . . . . . . . 7-19

Census

Household Vehicle Ownership, 1960-90 Census ....................... 11 .5

Means of Transportation to Work, 1980 and 1990 Census . . . . . . . . . . . . . . 11-15

National and Metropolitan Area Comparisons of Journey-to-Work Statistics, 1990 Census . . 11-16 Certificated

Summary Statistics for U.S. Domestic and International Certificated

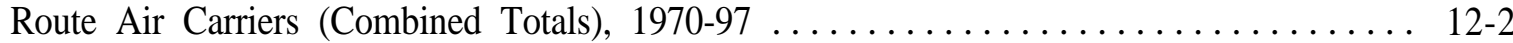

Certification

Federal Exhaust Emission Certification Standards for Gasoline- and

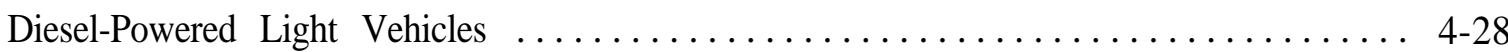

Federal Exhaust Emission Certification Standards for Gasoline- and

Diesel-Powered Light Trucks (Category LDT1) . .................. 4-29

Federal Exhaust Emission Certification Standards for Gasoline- and

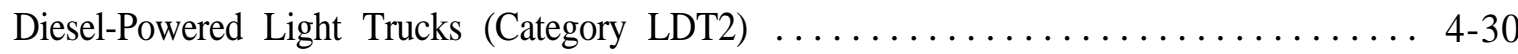

Federal Exhaust Emission Certification Standards for Gasoline- and

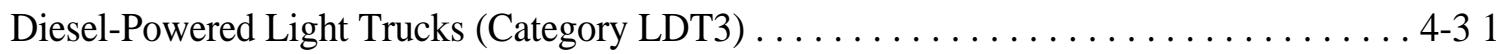


Certification (continued)

Federal Exhaust Emission Certification Standards for Gasoline- and

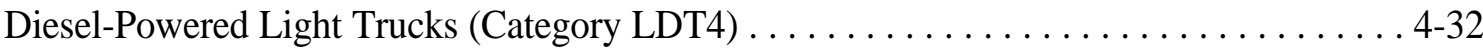

Federal Exhaust Emission Certification Standards for Gasoline- and

Diesel-Powered Light Heavy Trucks . . . . . . . . . . . . . . . . . . . 4-33

Federal Exhaust Emission Certification Standards for Gasoline- and

Diesel-Powered Heavy Heavy Trucks . . . . . . . . . . . . . . . . . . . . . . 4-34

California Passenger Cars and Light Trucks Emission Certification Standards . . . . . . . . . . 4-35 Cities

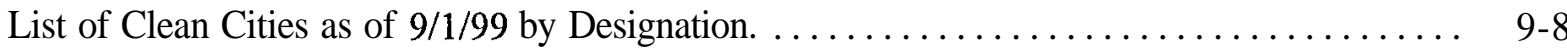

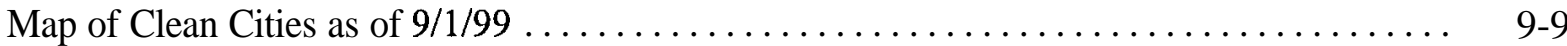

City

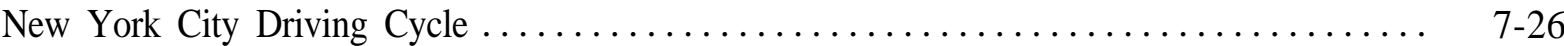

Class

Sales-Weighted Engine Size of New Domestic and Import Automobiles

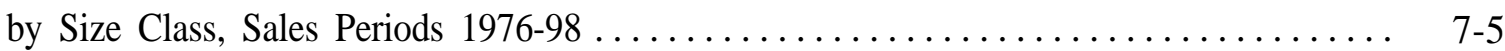

Sales-Weighted Curb Weight of New Domestic and Import Automobiles

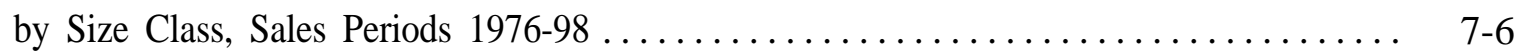

Sales-Weighted Interior Space of New Domestic and Import Automobiles

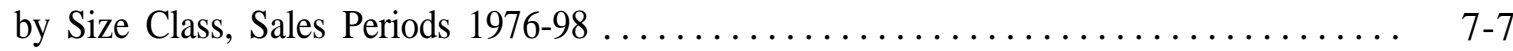

Light Vehicle Market Shares by Size Class, Sales Period 1976-98 . . . . . . . . . . . . . . 7-10

Sales-Weighted Engine Size of New Domestic and Import Light Trucks

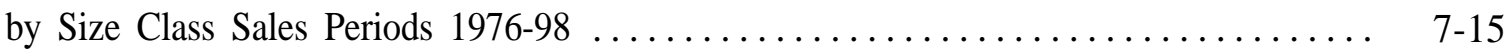

Truck Statistics by Gross Vehicle Weight Class, $1992 \ldots \ldots \ldots \ldots \ldots \ldots \ldots \ldots \ldots \ldots \ldots$. $8-6$

Percentage of Trucks by Size Class, 1977, 1982, 1987, and $1992 \ldots \ldots \ldots \ldots \ldots \ldots \ldots \ldots$

Truck Fuel Economy by Size Class, 1977, 1982, 1987, and $1992 \ldots \ldots \ldots \ldots \ldots \ldots \ldots . \quad 8$ - 7

Truck Fuel Economy by Fuel Type and Size Class, $1992 \ldots \ldots \ldots \ldots \ldots \ldots \ldots \ldots \ldots$. ${ }^{2} 8$

Breakdown of Domestic Marine Cargo by Commodity Class, $1997 \ldots \ldots \ldots \ldots \ldots \ldots \ldots \ldots$. $12-6$

Class I Railroad Freight Systems in the United StatesRanked by Revenue Ton-Miles, 1997 . . 12-7

Summary Statistics for Class I Freight Railroads, $1970-97 \ldots \ldots \ldots \ldots \ldots \ldots \ldots \ldots \ldots \ldots$ Clean

List of Clean Cities as of $9 / 1 / 99$ by Designation. . . . . . . . . . . . . . . . . .

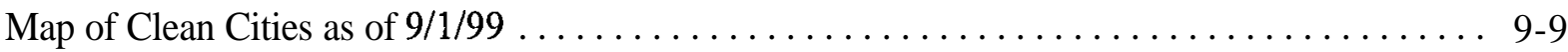

Collected

Corporate Average Fuel Economy (CAFE) Fines Collected, 1983-97 ............ 7-18 Commerce

Tonnage Statistics for Domestic and International Waterborne Commerce, 1970-97 . . . . . 12-4

Summary Statistics for Domestic Waterborne Commerce, $1970-97 \ldots \ldots \ldots \ldots \ldots \ldots \ldots$. 12-5 
Commodity

1993 Commodity Flow Survey: Shipment Characteristics by Mode of Transportation ....... 8-13

Value, Tons, and Ton-Miles of Commodity Shipments, $1993 \ldots \ldots \ldots \ldots \ldots \ldots \ldots \ldots \ldots$. 8 .14

Breakdown of Domestic Marine Cargo by Commodity Class, 1997 . . . . . . . . . . . . . 12-6

Railroad Revenue Carloads by Commodity Group, 1974 and 1997 . . . . . . . . . . . . . 12-9

Composition

Fleet Vehicle Composition by Vehicle Type, $1991 \ldots \ldots \ldots \ldots \ldots \ldots \ldots \ldots \ldots \ldots \ldots \ldots$

Compounds

Total National Emissions of Volatile Organic Compounds, $1970-97 \ldots \ldots \ldots \ldots \ldots \ldots$. . . . . . .

Emissions of Volatile Organic Compounds from Highway Vehicles, 1970-97 . . . . . . . . . . 4-8

Consortium

U.S. Advanced Battery Consortium Research Agreements, Phase II ............... 9-10

U.S. Advanced Battery Consortium Goals for Electric Vehicle Batteries ............. 9-11

Consumer

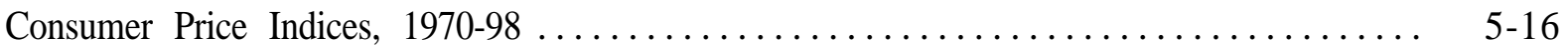

Consumption

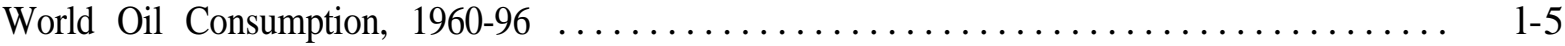

Share of OECD Petroleum Stocks to OECD Petroleum Consumption, 1973-96. . . . . . . . . 1-7

Share of Petroleum Stocks to Petroleum Consumption by Country, $1996 \ldots \ldots \ldots \ldots \ldots \ldots \ldots$. $1-8$

United States Petroleum Production and Consumption, 1973-98 ............... 1-15

United States Petroleum Production and Consumption, $1973-98 \ldots \ldots \ldots \ldots \ldots \ldots \ldots \ldots$ 1-16

Import Share of U.S. Petroleum Consumption, $1973-98 \ldots \ldots \ldots \ldots \ldots \ldots \ldots \ldots \ldots \ldots$ 1-17

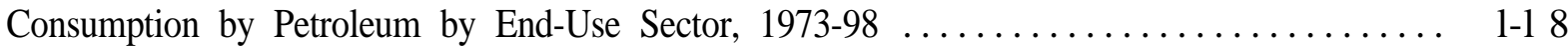

World Consumption of Primary Energy by Selected Country Groups, 1988-97 . . . . . . . 2-3

U. S. Consumption of Total Energy by End-Use Sector, $1970-98 \ldots \ldots \ldots \ldots \ldots \ldots \ldots \ldots \ldots$. . . . . .

Distribution of Energy Consumption by Source, 1973, 1980, and $1998 \ldots \ldots \ldots \ldots \ldots \ldots$. . . . . .

Domestic Consumption of Transportation Energy by Mode and Fuel Type, 1997 . . . . . . . . 2-6

Transportation Energy Consumption by Mode, 1970-97 ................. 2-8

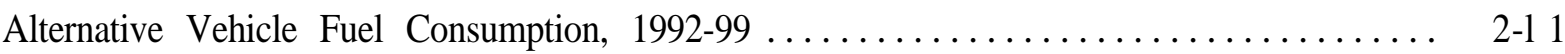

U.S. Carbon Dioxide Emissions from Fossil Energy Consumption by End-Use Sector, 1984-97 3-7

Average Material Consumption for a Domestic Automobile, 1978, 1985, and $1998 \ldots \ldots \ldots$..... 7-9

Corporate

Corporate Average Fuel Economy (CAFE) Standards versus Sales-Weighted

Fuel Economy Estimates for Automobiles and Light Trucks, 1978-98 . . . . . . . . . . . 7-17

Corporate Average Fuel Economy (CAFE) Fines Collected, $1983-97 \ldots \ldots \ldots \ldots \ldots \ldots$ 7-18 Corporation

Summary Statistics for the National Railroad Passenger Corporation (Amtrak), 1971-97 ... 12-11 cost

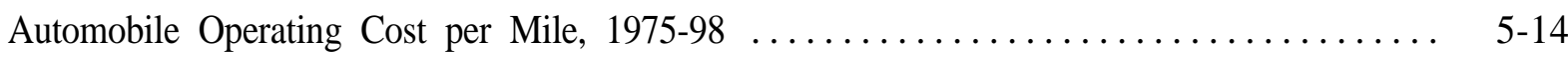
costs

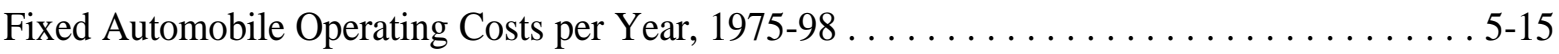


Countries

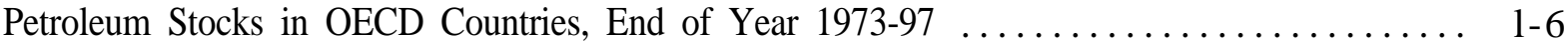

Gasoline Prices for Selected Countries, $1978-98 \ldots \ldots \ldots \ldots \ldots \ldots \ldots \ldots \ldots \ldots \ldots \ldots$. . . . . . . . . .

Diesel Fuel Prices for Selected Countries, $1978-98 \ldots \ldots \ldots \ldots \ldots \ldots \ldots \ldots \ldots \ldots \ldots$. $\ldots \ldots \ldots$

Automobile Registrations for Selected Countries, $1950-96 \ldots \ldots \ldots \ldots \ldots \ldots \ldots \ldots \ldots$. 6 .

Truck and Bus Registrations for Selected Countries, $1950-96 \ldots \ldots \ldots \ldots \ldots \ldots \ldots \ldots \ldots$. 6 . 3

Country

Share of Petroleum Stocks to Petroleum Consumption by Country, $1996 \ldots \ldots \ldots \ldots \ldots \ldots \ldots$. . . . 8

World Production of Primary Energy by Selected Country Groups, 1988-97 . . . . . . . . . 2-2

World Consumption of Primary Energy by Selected Country Groups, 1988-97 .......... 2-3 Criteria

Total National Emissions of the Criteria Air Pollutants by Sector, $1997 \ldots \ldots \ldots \ldots \ldots \ldots \ldots$. . . . . . 2

State-level Emissions for Criteria Pollutants, 1997. ........................ 4-14

Changes in Per-Mile, Fuel-Cycle Energy Use and Criteria Pollutant Emissions

for Passenger Cars using Near-Term Technologies . . . . . . . . . . . . . . . . . 7

Changes in Per-Mile, Fuel-Cycle Energy Use and Criteria Pollutant Emissions

for Passenger Cars using Long-Term Technologies ....................... 4-19

Crude

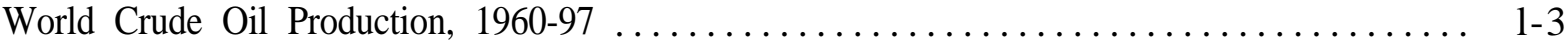

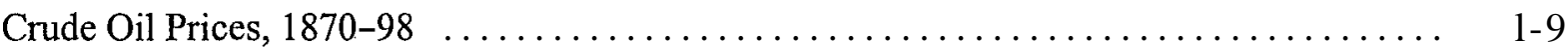

U.S. Refinery Input of Crude Oil and Petroleum Products, 1987-97 . . . . . . . . . . . 1-13

Refinery Yield of Petroleum Products from a Barrel of Crude Oil, 1978-97. . . . . . . . . . 1-14

Prices for a Barrel of Crude Oil and a Gallon of Gasoline, 1978-98 .................. 5-6 Curb

Sales-Weighted Curb Weight of New Domestic and Import Automobiles

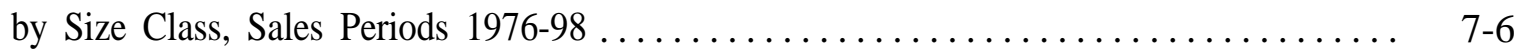

Engine Size, Curb Weight, and Interior Space of New Domestic and

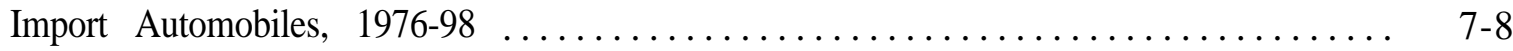

Daily

Average Annual and Daily Vehicle-Miles of Travel for Fleet Vehicles, $1991 \ldots \ldots \ldots \ldots \ldots$. . . $10-4$

Defending

Summary of 1996 Military Expenditures for Defending Oil Supplies

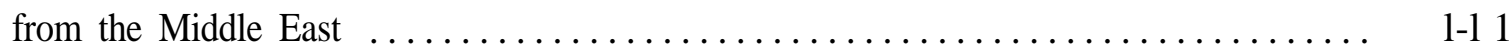

Demographic

Demographic Statistics, 1969, 1977, 1983, 1990, and 1995 NPTS $\ldots \ldots \ldots \ldots \ldots \ldots \ldots$. 11-6 Destination

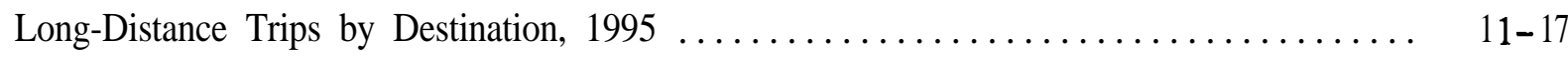
Diesel

Federal Exhaust Emission Certification Standards for Gasoline- and

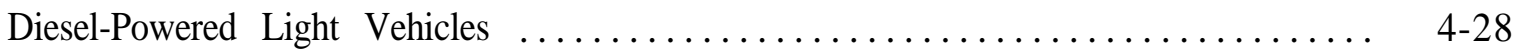

Federal Exhaust Emission Certification Standards for Gasoline- and

Diesel-Powered Light Trucks (Category LDT1) $\ldots \ldots \ldots \ldots \ldots \ldots \ldots \ldots \ldots \ldots \ldots . \ldots \ldots$ 4-29 
Diesel (continued)

Federal Exhaust Emission Certification Standards for Gasoline- and

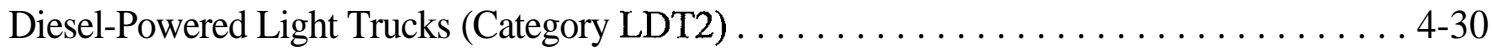

Federal Exhaust Emission Certification Standards for Gasoline- and

Diesel-Powered Light Trucks (Category LDT3) . . . . . . . . . . . . . . 4-3 1

Federal Exhaust Emission Certification Standards for Gasoline- and

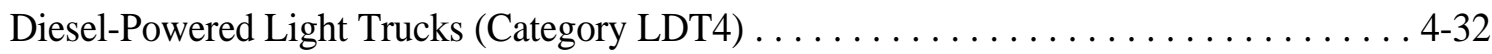

Federal Exhaust Emission Certification Standards for Gasoline- and

Diesel-Powered Light Heavy Trucks . . . . . . . . . . . . . . . . . . . 4-33

Federal Exhaust Emission Certification Standards for Gasoline- and

Diesel-Powered Heavy Heavy Trucks . . . . . . . . . . . . . . . . . . . 4-34

Diesel Fuel Prices for Selected Countries, 1978-98 . . . . . . . . . . . . . . . . . . . . . . 5-4

Dioxide

International Man-Made Emissions of Carbon Dioxide, $1990-96 \ldots \ldots \ldots \ldots \ldots \ldots \ldots \ldots$. . . . . . . .

International Man-Made Emissions of Carbon Dioxide by Source Category, 1990 and 1996 ... 3-4

U.S. Carbon Dioxide Emissions from Fossil Energy Consumption by End-Use Sector, 1984-97 3-7

U.S. Carbon Dioxide Emissions from Energy Use in the Transportation Sector, 1980-97 ... . 3-8

Numerical Estimates of Global Warming Potentials Compared With Carbon Dioxide ....... 3-9 Distance

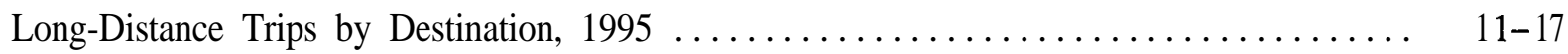

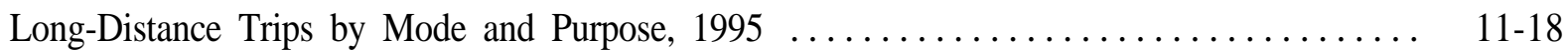

Long-Distance Household Trips by Mode and Trip Distance, 1995 . . . . . . . . . . . . . . 11-19

Shares of Long-Distance Person Trips by Mode and Household Income, $1995 \ldots \ldots \ldots \ldots$. 1 1-20 Distribution

Distribution of Energy Consumption by Source, 1973, 1980, and $1998 \ldots \ldots \ldots \ldots \ldots$ 2-5 Domestic

Domestic Consumption of Transportation Energy by Mode and Fuel Type, 1997 . . . . . . . . 2-6

Period Sales, Market Shares, and Sales-Weighted Fuel Economics of New

Domestic and Import Automobiles, Selected Sales Periods 1976-98. . . . . . . . . . . . . . . . . . 7-4

Sales-Weighted Engine Size of New Domestic and Import Automobiles

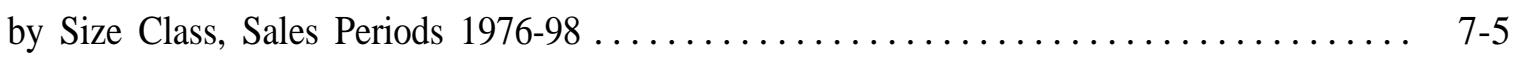

Sales-Weighted Curb Weight of New Domestic and Import Automobiles by Size Class, Sales Periods 1976-98 . . . . . . . . . . . . . . . . . . . . . 7-6

Sales-Weighted Interior Space of New Domestic and Import Automobiles

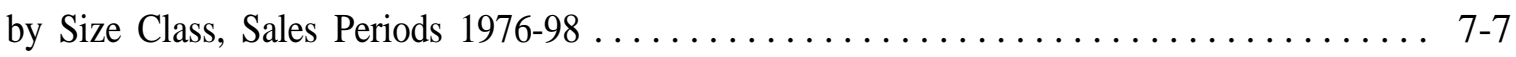

Engine Size, Curb Weight, and Interior Space of New Domestic and

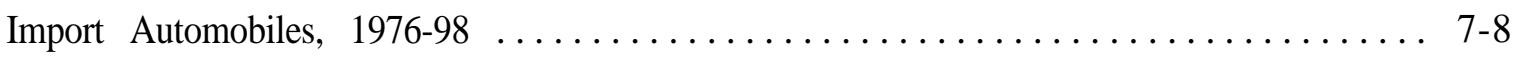

Average Material Consumption for a Domestic Automobile, 1978, 1985, and $1998 \ldots \ldots \ldots$.... 7-9

Period Sales, Market Shares, and Sales-Weighted Fuel Economics of New Domestic and Import Light Trucks, Selected Sales Period 1976-98 . . . . . . . . . . . . . 7-14

Sales-Weighted Engine Size of New Domestic and Import Light Trucks

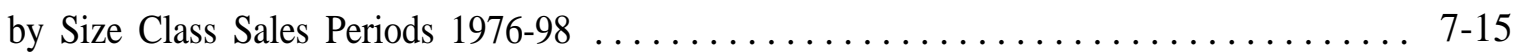


Domestic (continued)

Summary Statistics for U.S. Domestic and International Certificated

Route Air Carriers (Combined Totals), 1970-97 . . . . . . . . . . . . . . . . . 12-2

Tonnage Statistics for Domestic and International Waterborne Commerce, 1970-97 ........ 12-4

Summary Statistics for Domestic Waterborne Commerce, $1970-97$. . . . . . . . . . . . 12-5

Breakdown of Domestic Marine Cargo by Commodity Class, $1997 \ldots \ldots \ldots \ldots \ldots \ldots \ldots$. 12-6

Driving

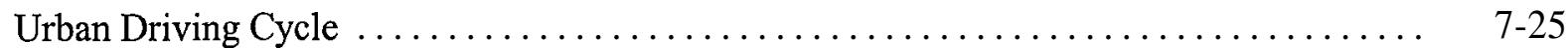

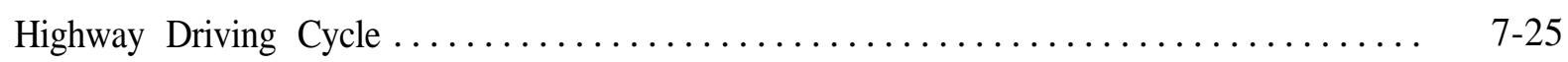

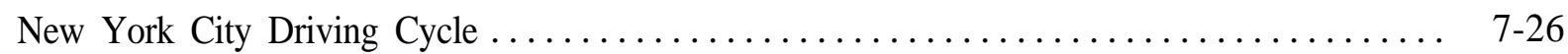

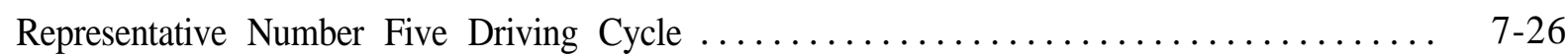

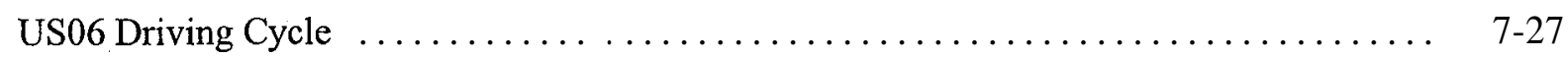

Economic

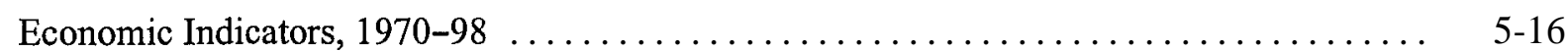

Economies

Period Sales, Market Shares, and Sales-Weighted Fuel Economies of New

Domestic and Import Automobiles, Selected Sales Periods 1976-98 . . . . . . . . . . . 7-4

Period Sales, Market Shares, and Sales-Weighted Fuel Economies of New

Domestic and Import Light Trucks, Selected Sales Period 1976-98 . . . . . . . . . . . 7-14

Economy

Corporate Average Fuel Economy (CAFE) Standards versus Sales-Weighted

Fuel Economy Estimates for Automobiles and Light Trucks, 1978-98 . . . . . . . . . 7-17

Corporate Average Fuel Economy (CAFE) Fines Collected, 1983-97 ............. 7-18

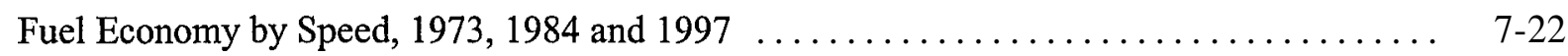

Steady Speed Fuel Economy for Tested Vehicles. .................... $7-24$

Truck Fuel Economy by Size Class, 1977, 1982, 1987, and $1992 \ldots \ldots \ldots \ldots \ldots \ldots \ldots$.

Truck Fuel Economy by Fuel Type and Size Class, $1992 \ldots \ldots \ldots \ldots \ldots \ldots \ldots \ldots \ldots$ Emission

National Lead Emission Estimates, $1970-97 \ldots \ldots \ldots \ldots \ldots \ldots \ldots \ldots \ldots \ldots \ldots \ldots \ldots$. . . . . . . . . . . . . .

Federal Exhaust Emission Certification Standards for Gasoline- and

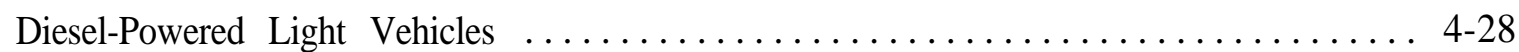

Federal Exhaust Emission Certification Standards for Gasoline- and

Diesel-Powered Light Trucks (Category LDT1) $\ldots \ldots \ldots \ldots \ldots \ldots \ldots \ldots \ldots \ldots . . \ldots \ldots$ 4-29

Federal Exhaust Emission Certification Standards for Gasoline- and

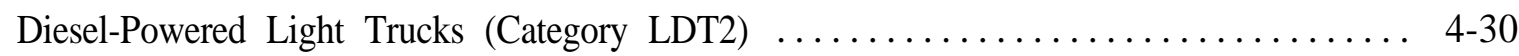

Federal Exhaust Emission Certification Standards for Gasoline- and

Diesel-Powered Light Trucks (Category LDT3) $\ldots \ldots \ldots \ldots \ldots \ldots \ldots \ldots \ldots \ldots \ldots \ldots \ldots$

Federal Exhaust Emission Certification Standards for Gasoline- and

Diesel-Powered Light Trucks (Category LDT4) $\ldots \ldots \ldots \ldots \ldots \ldots \ldots \ldots \ldots \ldots . . \ldots \ldots$. 32

Federal Exhaust Emission Certification Standards for Gasoline- and

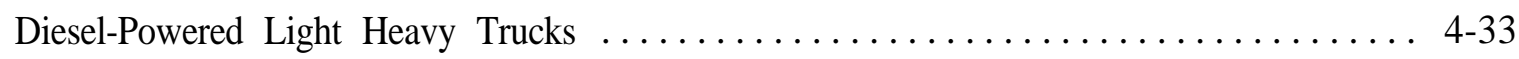


Emission (continued)

Federal Exhaust Emission Certification Standards for Gasoline- and

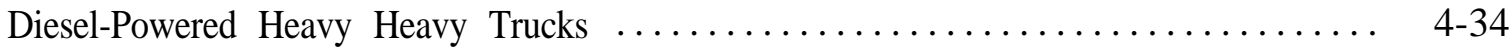

California Passenger Cars and Light Trucks Emission Certification Standards .......... 4-35

California Vehicle Emission Reduction for Passenger Cars and Light Trucks............ 4-36

California Air Resources Board Requirements for Meeting Emission Standards ......... 4-37

Emissions

International Man-Made Emissions of All Greenhouse Gases, 1990-96 . . . . . . . . . . . 3-2

International Man-Made Emissions of Carbon Dioxide, $1990-96 \ldots \ldots \ldots \ldots \ldots \ldots \ldots \ldots .3$

International Man-Made Emissions of Carbon Dioxide by Source Category, 1990 and 1996 ... 3-4

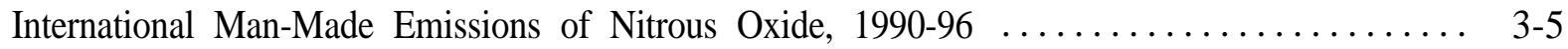

Estimated U.S. Emissions of Greenhouse Gases, $1990-97 \ldots \ldots \ldots \ldots \ldots \ldots \ldots \ldots \ldots \ldots$ 3-6

U.S. Carbon Dioxide Emissions from Fossil Energy Consumption by End-Use Sector, 1984-97 3-7

U.S. Carbon Dioxide Emissions from Energy Use in the Transportation Sector, 1980-97 . . . . 3-8

Changes in Per-Mile, Fuel-Cycle Energy Use and Greenhouse Gas Emissions

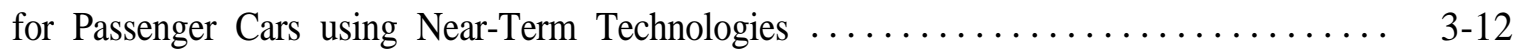

Changes in Per-Mile, Fuel-Cycle Energy Use and Greenhouse Gas Emissions

for Passenger Cars using Long-Term Technologies ...................... 3-1 3

Total National Emissions of the Criteria Air Pollutants by Sector, $1997 \ldots \ldots \ldots \ldots \ldots \ldots \ldots$. . . . . 2

Total National Emissions of Carbon Monoxide, $1970-97 \ldots \ldots \ldots \ldots \ldots \ldots \ldots \ldots \ldots \ldots$. . . . . . . . .

Emissions of Carbon Monoxide from Highway Vehicles, $1970-97 \ldots \ldots \ldots \ldots \ldots \ldots \ldots \ldots$. . . . 4

Total National Emissions of Nitrogen Oxides, 1970-97 . . . . . . . . . . . . . . . . . . 4 4-5

Emissions of Nitrogen Oxides from Highway Vehicles, $1970-97 \ldots \ldots \ldots \ldots \ldots \ldots \ldots \ldots$. . . . . . .

Total National Emissions of Volatile Organic Compounds, 1970-97 . . . . . . . . . . . . . 4-7

Emissions of Volatile Organic Compounds from Highway Vehicles, 1970-97 . . . . . . . . . 4-8

Total National Emissions of Particulate Matter (PM-lo), 1970-97 . . . . . . . . . . . . . . . 4-9

Emissions of Particulate Matter (PM-1 0) from Highway Vehicles, 1970-97 ........... 4-1 0

Total National Emissions of Particulate Matter (PM-2.5) 1990-97 . . . . . . . . . . . . . . . 4-1 1

Emissions of Particulate Matter (PM-2.5) from Highway Vehicles, 1990-97 ........... 4-12

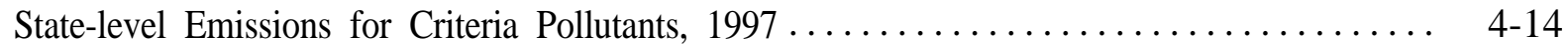

Changes in Per-Mile, Fuel-Cycle Energy Use and Criteria Pollutant Emissions

for Passenger Cars using Near-Term Technologies .................... 4-17

Changes in Per-Mile, Fuel-Cycle Energy Use and Criteria.Pollutant Emissions
for Passenger Cars using Long-Term Technologies $\ldots \ldots \ldots \ldots \ldots \ldots \ldots \ldots \ldots \ldots \ldots$

Employees

Employees of Motor Vehicle and Related Industries, 1990 and $1995 \ldots \ldots \ldots \ldots \ldots \ldots$ 5-1 8

Employment

Motor Vehicle Manufacturing Employment Statistics, $1972-97 \ldots \ldots \ldots \ldots \ldots \ldots \ldots \ldots$. . . 17

Employment in Transportation and Related Industries, 1960-97 ................ 5-19 
Energy

World Production of Primary Energy by Selected Country Groups, 1988-97 . . . . . . . . . . 2-2

World Consumption of Primary Energy by Selected Country Groups, 1988-97 . . . . . . . . 2-3

U. S. Consumption of Total Energy by End-Use Sector, 1970-98 . . . . . . . . . . . . . . . . 2-4

Distribution of Energy Consumption by Source, 1973, 1980, and $1998 \ldots \ldots \ldots \ldots \ldots \ldots$........ 2-5

Domestic Consumption of Transportation Energy by Mode and Fuel Type, 1997 . . . . . . . 2-6

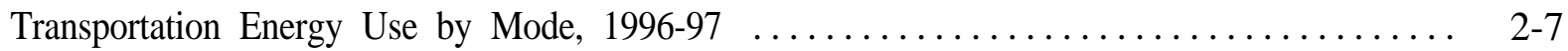

Transportation Energy Consumption by Mode, $1970-97 \ldots \ldots \ldots \ldots \ldots \ldots \ldots \ldots \ldots \ldots$

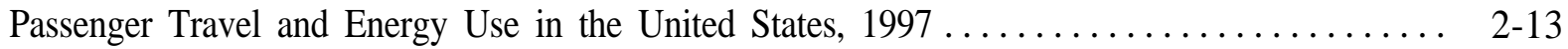

Energy Intensities of Passenger Modes, 1970-97 . . . . . . . . . . . . . . . . . . . 2-14

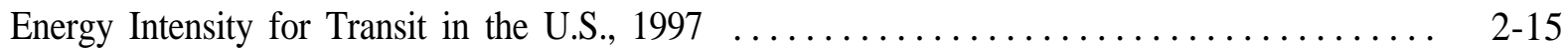

Intercity Freight Movement and Energy Use in the United States, $1997 \ldots \ldots \ldots \ldots \ldots \ldots$. 2-16

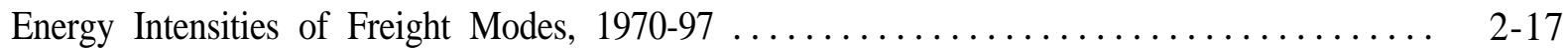

U.S. Carbon Dioxide Emissions from Fossil Energy Consumption by End-Use Sector, 1984-97 3-7

U.S. Carbon Dioxide Emissions from Energy Use in the Transportation Sector, 1980-97 . . . . 3-8

Changes in Per-Mile, Fuel-Cycle Energy Use and Greenhouse Gas Emissions

for Passenger Cars using Near-Term Technologies . . . . . . . . . . . . . . . . .

Changes in Per-Mile, Fuel-Cycle Energy Use and Greenhouse Gas Emissions

for Passenger Cars using Long-Term Technologies ..................... 3-13

Changes in Per-Mile, Fuel-Cycle Energy Use and Criteria Pollutant Emissions

for Passenger Cars using Near-Term Technologies ................... 4-1 7

Changes in Per-Mile, Fuel-Cycle Energy Use and Criteria Pollutant Emissions

for Passenger Cars using Long-Term Technologies ................... 4-19

Energy Policy Act Purchase Requirements of Light Alternative Fuel Vehicles.......... 10-8 Engine

Sales-Weighted Engine Size of New Domestic and Import Automobiles

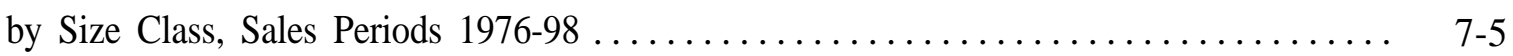

Engine Size, Curb Weight, and Interior Space of New Domestic and

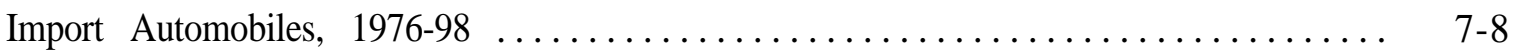

Sales-Weighted Engine Size of New Domestic and Import Light Trucks

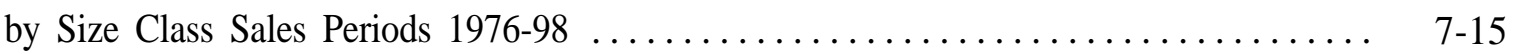

Estimated

Estimated U.S. Emissions of Greenhouse Gases, $1990-97 \ldots \ldots \ldots \ldots \ldots \ldots \ldots \ldots \ldots \ldots \ldots$

Estimates

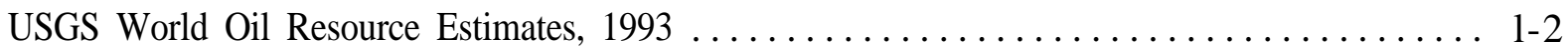

Numerical Estimates of Global Warming Potentials Compared With Carbon Dioxide ....... 3-9

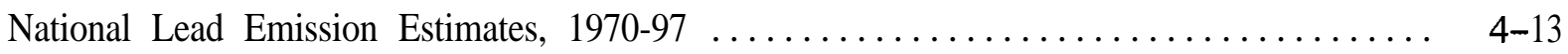

Corporate Average Fuel Economy (CAFE) Standards versus Sales-Weighted

Fuel Economy Estimates for Automobiles and Light Trucks, 1978-98. . . . . . . . . 7-17

Estimates of Alternative Fuel Vehicles in Use, 1992-99 . . . . . . . . . . . . . . . . . 9-3

Estimates of Light Alternative Fuel Vehicles, 1995, 1997, and $1999 \ldots \ldots \ldots \ldots \ldots \ldots \ldots$. . . . .

Estimates of Heavy Alternative Fuel Vehicles, 1995, 1997, and $1999 \ldots \ldots \ldots \ldots \ldots \ldots$........ 9-5 
Ethanol

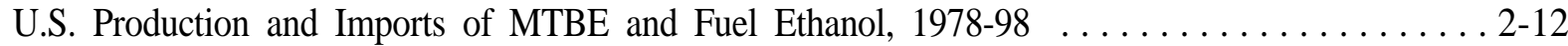

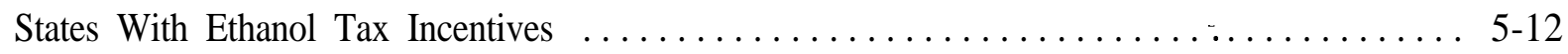

Excise

Federal Excise Taxes on Motor Fuels $\ldots \ldots \ldots \ldots \ldots \ldots \ldots \ldots \ldots \ldots \ldots \ldots \ldots \ldots \ldots \ldots \ldots \ldots$

Exemptions

State Tax Exemptions for Gasohol, November $1998 \ldots \ldots \ldots \ldots \ldots \ldots \ldots \ldots \ldots \ldots$. $\ldots \ldots \ldots$

Exhaust

Federal Exhaust Emission Certification Standards for Gasoline- and

Diesel-Powered Light Vehicles . . . . . . . . . . . . . . . . . . . . . . 4-28

Federal Exhaust Emission Certification Standards for Gasoline- and

Diesel-Powered Light Trucks (Category LDT1) . . . . . . . . . . . . . . . . . . . . . 4-29

Federal Exhaust Emission Certification Standards for Gasoline- and

Diesel-Powered Light Trucks (Category LDT2) . . . . . . . . . . . . . . . 4-30

Federal Exhaust Emission Certification Standards for Gasoline- and

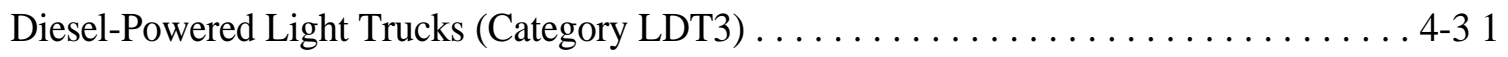

Federal Exhaust Emission Certification Standards for Gasoline- and

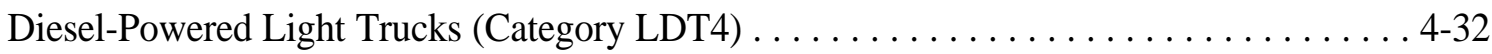

Federal Exhaust Emission Certification Standards for Gasoline- and

Diesel-Powered Light Heavy Trucks . . . . . . . . . . . . . . . . . . . 4-33

Federal Exhaust Emission Certification Standards for Gasoline- and

Diesel-Powered Heavy Heavy Trucks . . . . . . . . . . . . . . . . . . . 4-34

Expenditures

Summary of 1996 Military Expenditures for Defending Oil Supplies

from the Middle East . . . . . . . . . . . . . . . . . . . . . . . . . . . . . 1-11

Average Annual Expenditures of Households by Income, $1997 \ldots \ldots \ldots \ldots \ldots \ldots \ldots \ldots 11-3$

Facility

Percentage of Trucks by Fleet Size and Primary Refueling Facility, 1992 . . . . . . . . . . . 8 8-7

Percentage of Trucks by Major Use and Primary Refueling Facility, $1992 \ldots \ldots \ldots \ldots \ldots \ldots$. . . 8 8

Federal

Federal Exhaust Emission Certification Standards for Gasoline- and

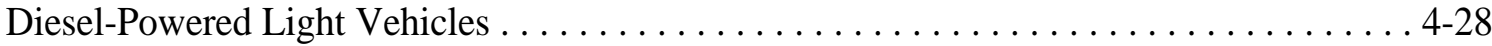

Federal Exhaust Emission Certification Standards for Gasoline- and

Diesel-Powered Light Trucks (Category LDT1) . . . . . . . . . . . . . . . . . . . 4-29

Federal Exhaust Emission Certification Standards for Gasoline- and

Diesel-Powered Light Trucks (Category LDT2) . . . . . . . . . . . . . . . . 4-30

Federal Exhaust Emission Certification Standards for Gasoline- and

Diesel-Powered Light Trucks (Category LDT3) . . . . . . . . . . . . . . . . . . 4-3 1

Federal Exhaust Emission Certification Standards for Gasoline- and

Diesel-Powered Light Trucks (Category LDT4) . . . . . . . . . . . . . . . . 4-32

Federal Exhaust Emission Certification Standards for Gasoline- and

Diesel-Powered Light Heavy Trucks . . . . . . . . . . . . . . . . . . 43 
Federal (continued)

Federal Exhaust Emission Certification Standards for Gasoline- and

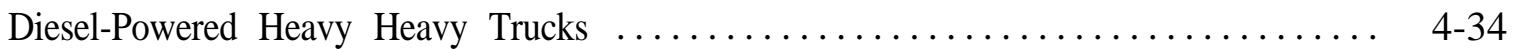

California Passenger Cars and Light Trucks Emission Certification Standards ........... 4-35

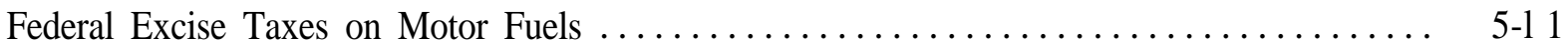

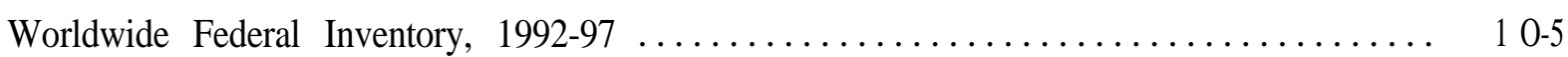

Average Miles per Federal Vehicle by Vehicle Type, $1997 \ldots \ldots \ldots \ldots \ldots \ldots \ldots \ldots \ldots . \ldots$

Federal Government Vehicles by Agency, Fiscal Year 1997 . . . . . . . . . . . . . . . . . 10-6

Federal Fleet Vehicle Acquisitions by Fuel Type, FY $1997 \ldots \ldots \ldots \ldots \ldots \ldots \ldots \ldots \ldots$ 10 . 7

Fuel Consumed by Federal Government Fleets, FY $1997 \ldots \ldots \ldots \ldots \ldots \ldots \ldots \ldots \ldots$ 10-7 Fines

Corporate Average Fuel Economy (CAFE) Fines Collected, 1983-97 . . ............ 7-18 Fiscal

Federal Government Vehicles by Agency, Fiscal Year $1997 \ldots \ldots \ldots \ldots \ldots \ldots \ldots \ldots \ldots$. . . . . . 6 Fixed

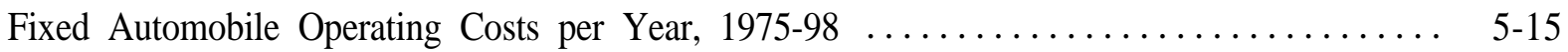

Fleet

Percentage of Trucks by Fleet Size and Primary Refueling Facility, 1992 . . . . . . . . 8-7

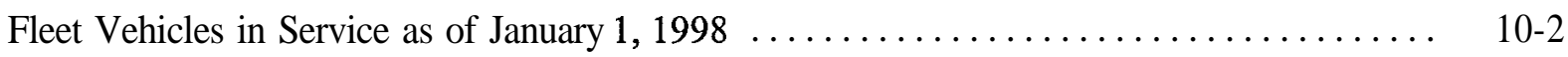

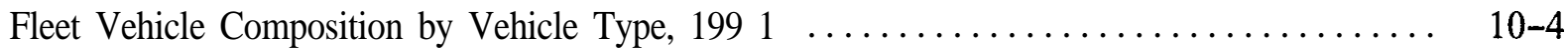

Average Length of Time Fleet Vehicles are Kept Before Sold to Others, $1991 \ldots \ldots \ldots \ldots$. . . 10-4

Average Annual and Daily Vehicle-Miles of Travel for Fleet Vehicles, $1991 \ldots \ldots \ldots \ldots . . .10-4$

Federal Fleet Vehicle Acquisitions by Fuel Type, FY $1997 \ldots \ldots \ldots \ldots \ldots \ldots \ldots \ldots \ldots \ldots$. . . . . . . . Fleets

Top Ten States with Fleets of Ten Vehicles or More, $1999 \ldots \ldots \ldots \ldots \ldots \ldots \ldots \ldots \ldots$ 10-3

Fuel Consumed by Federal Government Fleets, FY $1997 \ldots \ldots \ldots \ldots \ldots \ldots \ldots \ldots \ldots$ 10-7 Fossil

U.S. Carbon Dioxide Emissions from Fossil Energy Consumption by End-Use Sector, 1984-97 3-7 Freight

Intercity Freight Movement and Energy Use in the United States, $1997 \ldots \ldots \ldots \ldots \ldots \ldots$. 2-1 6

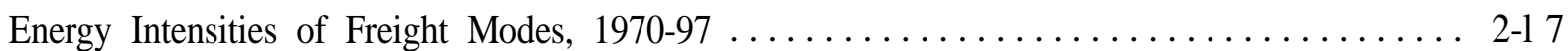

Class I Railroad Freight Systems in the United States Ranked by Revenue Ton-Miles, 1997 . . 12-7

Summary Statistics for Class I Freight Railroads, $1970-97 \ldots \ldots \ldots \ldots \ldots \ldots \ldots \ldots \ldots$. . . . . . . . . Fuel

Domestic Consumption of Transportation Energy by Mode and Fuel Type, 1997 . . . . . . . 2 2-6

Highway Usage of Gasoline and Special Fuels, $1973-97 \ldots \ldots \ldots \ldots \ldots \ldots \ldots \ldots \ldots \ldots$

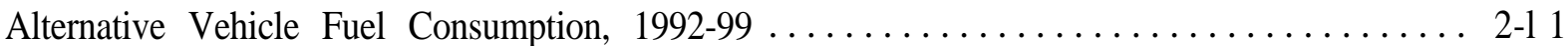

U.S. Production and Imports of MTBE and Fuel Ethanol, 1978-98 . . . . . . . . . . . 2-12

Changes in Per-Mile, Fuel-Cycle Energy Use and Greenhouse Gas Emissions

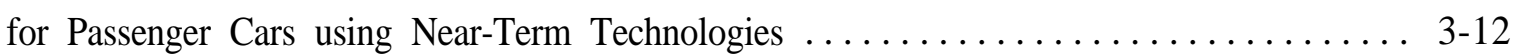

Changes in Per-Mile, Fuel-Cycle Energy Use and Greenhouse Gas Emissions for Passenger Cars using Long-Term Technologies .................. 3-13 
Fuel (continued)

Changes in Per-Mile, Fuel-Cycle Energy Use and Criteria Pollutant Emissions

for Passenger Cars using Near-Term Technologies .................... 4-1 7

Changes in Per-Mile, Fuel-Cycle Energy Use and Criteria Pollutant Emissions

for Passenger Cars using Long-Term Technologies ..................... 4-19

Diesel Fuel Prices for Selected Countries, $1978-98 \ldots \ldots \ldots \ldots \ldots \ldots \ldots \ldots \ldots \ldots \ldots$. $\ldots \ldots \ldots$

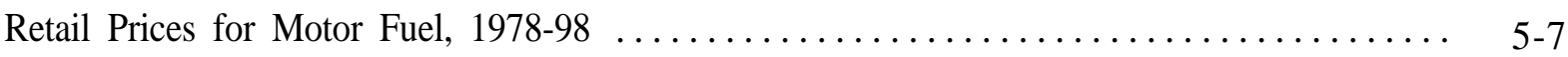

Period Sales, Market Shares, and Sales-Weighted Fuel Economies of New

Domestic and Import Automobiles, Selected Sales Periods 1976-98 . . . . . . . . . . . . 7-4

Period Sales, Market Shares, and Sales-Weighted Fuel Economies of New

Domestic and Import Light Trucks, Selected Sales Period 1976-98 . . . . . . . . . . . . 7-14

Corporate Average Fuel Economy (CAFE) Standards versus Sales-Weighted

Fuel Economy Estimates for Automobiles and Light Trucks, 1978-98 . . . . . . . . . . . 7-1 7

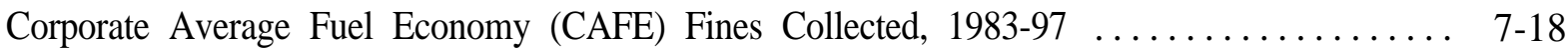

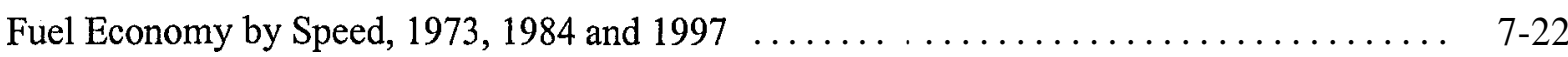

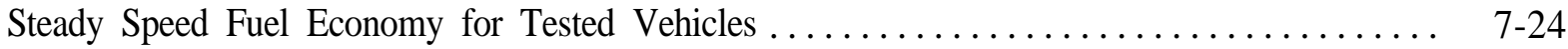

Truck Fuel Economy by Size Class, 1977, 1982, 1987, and 1992 . . . . . . . . . . . . . 8 8-7

Truck Fuel Economy by Fuel Type and Size Class, $1992 \ldots \ldots \ldots \ldots \ldots \ldots \ldots \ldots \ldots$. . . . . . . . .

Estimates of Alternative Fuel Vehicles in Use, $1992-99 \ldots \ldots \ldots \ldots \ldots \ldots \ldots \ldots \ldots . . \ldots 9.3$

Estimates of Light Alternative Fuel Vehicles, 1995, 1997, and 1999 ................. 9-4

Estimates of Heavy Alternative Fuel Vehicles, 1995, 1997, and 1999 . . . . . . . . . . 9-5

Alternative Fuel Vehicles Available by Manufacturer, Model Year 1999 . . . . . . . . . . . . 9-6

Number of Alternative Refuel Sites by State and Fuel Type, 1998 . . . . . . . . . . . . 9-7

Federal Fleet Vehicle Acquisitions by Fuel Type, FY $1997 \ldots \ldots \ldots \ldots \ldots \ldots \ldots \ldots \ldots \ldots$. . . . . . . . . . . . . . . . . . . . . .

Fuel Consumed by Federal Government Fleets, FY $1997 \ldots \ldots \ldots \ldots \ldots \ldots \ldots \ldots \ldots \ldots$ 10 .7

Energy Policy Act Purchase Requirements of Light Alternative Fuel Vehicles. ........ 10-8 Fuels

Highway Usage of Gasoline and Special Fuels, 1973-97 . . . . . . . . . . . . . . . . . . 2-9

Prices for Selected Transportation Fuels, $1978-98 \ldots \ldots \ldots \ldots \ldots \ldots \ldots \ldots \ldots \ldots \ldots \ldots$. . . . . . . . . . .

State Taxes on Motor Fuels, $1999 \ldots \ldots \ldots \ldots \ldots \ldots \ldots \ldots \ldots \ldots \ldots \ldots \ldots \ldots \ldots$

Federal Excise Taxes on Motor Fuels $\ldots \ldots \ldots \ldots \ldots \ldots \ldots \ldots \ldots \ldots \ldots \ldots \ldots \ldots \ldots \ldots \ldots$

Gallon

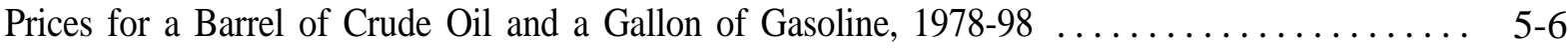
Gas

Changes. in Per-Mile, Fuel-Cycle Energy Use and Greenhouse Gas Emissions

for Passenger Cars using Near-Term Technologies . . . . . . . . . . . . . . . 3-12

Changes in Per-Mile, Fuel-Cycle Energy Use and Greenhouse Gas Emissions

for Passenger Cars using Long-Term Technologies . . . . . . . . . . . . . . . 3-1 3

Tax Receipts from the Sale of Gas Guzzlers, 1980-97 . . . . . . . . . . . . . . . . . 7-18

The Gas Guzzler Tax on New Cars .............................. 7-19 
Gases

International Man-Made Emissions of All Greenhouse Gases, 1990-96 . . . . . . . . . . . . 3-2

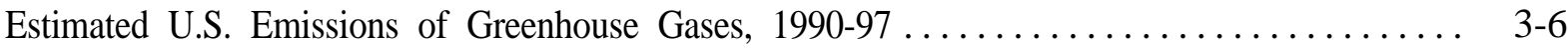

Gasohol

State Tax Exemptions for Gasohol, November $1998 \ldots \ldots \ldots \ldots \ldots \ldots \ldots \ldots \ldots \ldots \ldots$ 5-11

Gasoline

Highway Usage of Gasoline and Special Fuels, $1973-97 \ldots \ldots \ldots \ldots \ldots \ldots \ldots \ldots \ldots \ldots$

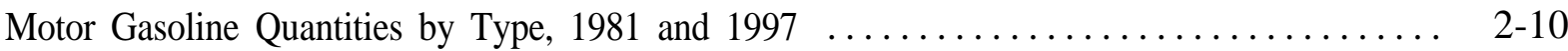

Federal Exhaust Emission Certification Standärds for Gasoline- and

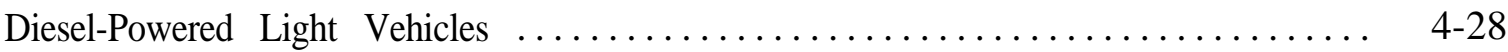

Federal Exhaust Emission Certification Standards for Gasoline- and

Diesel-Powered Light Trucks (Category LDT1) .................... 4-29

Federal Exhaust Emission Certification Standards for Gasoline- and

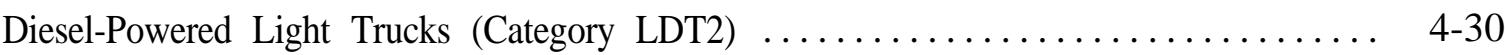

Federal Exhaust Emission Certification Standards for Gasoline- and

Diesel-Powered Light Trucks (Category LDT3) $\ldots \ldots \ldots \ldots \ldots \ldots \ldots \ldots \ldots \ldots \ldots \ldots \ldots \ldots \ldots \ldots$

Federal Exhaust Emission Certification Standards for Gasoline- and

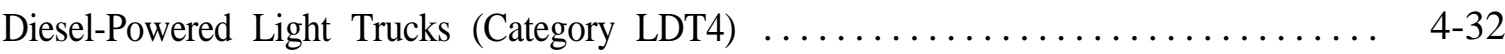

Federal Exhaust Emission Certification Standards for Gasoline- and

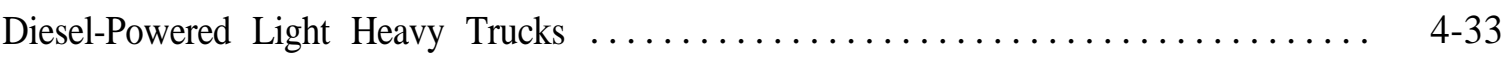

Federal Exhaust Emission Certification Standards for Gasoline- and

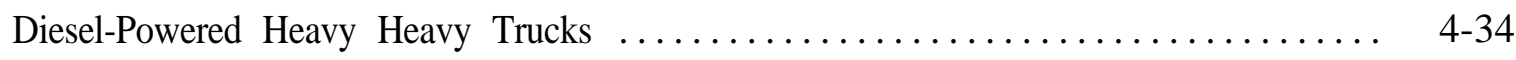

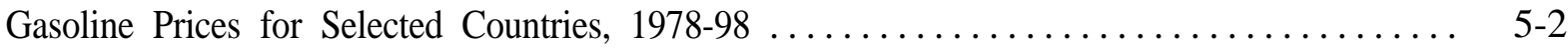

Prices for a Barrel of Crude Oil and a Gallon of Gasoline, 1978-98 . . . . . . . . . . . 5-6

Global

Numerical Estimates of Global Warming Potentials Compared With Carbon Dioxide . . . . . . . 3-9 Goals

U.S. Advanced Battery Consortium Goals for Electric Vehicle Batteries . . . . . . . . . . . 9-11 Government

Federal Government Vehicles by Agency, Fiscal Year $1997 \ldots \ldots \ldots \ldots \ldots \ldots \ldots \ldots \ldots \ldots$

Fuel Consumed by Federal Government Fleets, FY $1997 \ldots \ldots \ldots \ldots \ldots \ldots \ldots \ldots \ldots$. . . . . . . . .

Greenhouse

International Man-Made Emissions of All Greenhouse Gases, 1990-96 . . . . . . . . . . . . . . 3-2

Estimated U.S. Emissions of Greenhouse Gases, 1990-97 . . . . . . . . . . . . . . . . . . . . . . 3-6

Changes in Per-Mile, Fuel-Cycle Energy Use and Greenhouse Gas Emissions

for Passenger Cars using Near-Term Technologies . . . . . . . . . . . . . . . . . 3- 12

Changes in Per-Mile, Fuel-Cycle Energy Use and Greenhouse Gas Emissions

for Passenger Cars using Long-Term Technologies . . . . . . . . . . . . . . . . 3-13 
Gross

Refinery Gross Output by World Region, $1998 \ldots \ldots \ldots \ldots \ldots \ldots \ldots \ldots \ldots \ldots \ldots \ldots$. . . . . . . . . . .

New Retail Truck Sales by Gross Vehicle Weight, 1970-97 . . . . . . . . . . . . . . . . . . 8-3

New Retail Sales of Light Trucks by Gross Vehicle Weight, 1970-97 . . . . . . . . . . . . . 8-4

Truck Statistics by Gross Vehicle Weight Class, $1992 \ldots \ldots \ldots \ldots \ldots \ldots \ldots \ldots \ldots$. . . . . . . 8-6 Guzzler

The Gas Guzzler Tax on New Cars $\ldots \ldots \ldots \ldots \ldots \ldots \ldots \ldots \ldots \ldots \ldots \ldots \ldots \ldots \ldots \ldots$ Guzzlers

Tax Receipts from the Sale of Gas Guzzlers, $1980-97 \ldots \ldots \ldots \ldots \ldots \ldots \ldots \ldots \ldots \ldots$. . . . . . . . . . GVW

New Retail Sales of Trucks 10,000 pounds GVW and Less in the United States, 1970-97. . . . 7-13 Heavy

Federal Exhaust Emission Certification Standards for Gasoline- and

Diesel-Powered Light Heavy Trucks . . . . . . . . . . . . . . . . . . 4-33

Federal Exhaust Emission Certification Standards for Gasoline- and

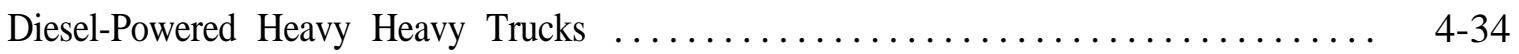

Estimates of Heavy Alternative Fuel Vehicles, 1995, 1997, and $1999 \ldots \ldots \ldots \ldots \ldots \ldots \ldots$........ 9-5 Highway

Highway Usage of Gasoline and Special Fuels, 1973-97 . . . . . . . . . . . . . . . . 2-9

Emissions of Carbon Monoxide from Highway Vehicles, $1970-97 \ldots \ldots \ldots \ldots \ldots \ldots \ldots \ldots$. . . . .

Emissions of Nitrogen Oxides from Highway Vehicles, 1970-97 . . . . . . . . . . . . . 4-6

Emissions of Volatile Organic Compounds from Highway Vehicles, 1970-97 . . . . . . . . 4 4-8

Emissions of Particulate Matter (PM-1 0) from Highway Vehicles, 1970-97 . . . . . . . . . 4-10

Emissions of Particulate Matter (PM-2.5) from Highway Vehicles, 1990-97 ........... 4-12

Highway Vehicle Miles Traveled by Vehicle Type, $1970-97 \ldots \ldots \ldots \ldots \ldots \ldots \ldots \ldots \ldots$. 6-7

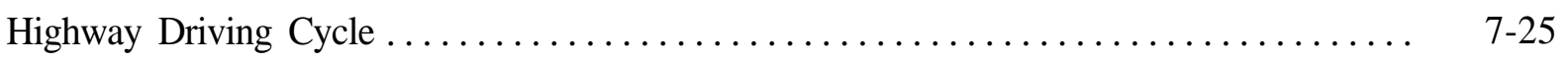

Household

Average Number of Vehicles and Vehicle Travel per Household, 1991 and 1994 RTECS .... 1 1-4

Household Vehicle Ownership, 1960-90 Census ....................... 11 .5

Average Annual Vehicle-Miles, Vehicle Trips and Trip Length per Household

$1969,1977,1983,1990$, and 1995 NPTS . . . . . . . . . . . . . . . . 11-7

Average Annual Person-Miles Traveled (PMT), Person Trips and Trip Length

per Household by Selected Trip Purposes, 1983, 1990, and 1995 NPTS . . . . . . . . . 1 1-8

Average Annual Miles per Vehicle by Household Vehicle Ownership, 1995 NPTS ....... 11-12

Average Annual Miles per Household Vehicle by Vehicle Age ................. 11-13

Long-Distance Household Trips by Mode and Trip Distance, $1995 \ldots \ldots \ldots \ldots \ldots \ldots \ldots$. . . . . . . . 19

Shares of Long-Distance Person Trips by Mode and Household Income, 1995 . . . . . . . . 1 1-20 Households

Average Annual Expenditures of Households by Income, $1997 \ldots \ldots \ldots \ldots \ldots \ldots \ldots \ldots \ldots$. . . . . . . . 
Import

Import Share of U.S. Petroleum Consumption, 1973-98 . . . . . . . . . . . . . . . . 1-17

Period Sales, Market Shares, and Sales-Weighted Fuel Economies of New

Domestic and Import Automobiles, Selected Sales Periods 1976-98 ............... 7-4

Sales-Weighted Engine Size of New Domestic and Import Automobiles

by Size Class, Sales Periods 1976-98. ....................... 7-5

Sales-Weighted Curb Weight of New Domestic and Import Automobiles

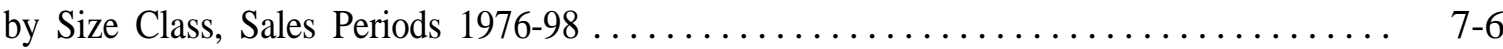

Sales-Weighted Interior Space of New Domestic and Import Automobiles

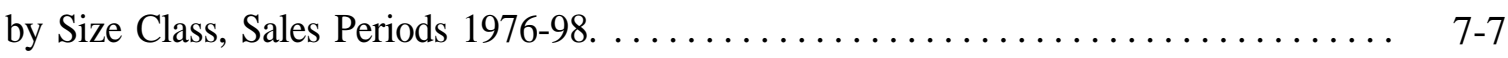

Engine Size, Curb Weight, and Interior Space of New Domestic and

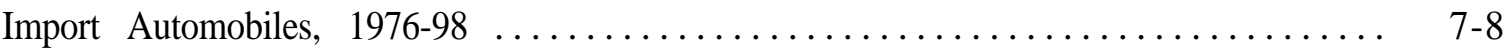

Period Sales, Market Shares, and Sales-Weighted Fuel Economies of New

Domestic and Import Light Trucks, Selected Sales Period 1976-98 . . . . . . . . . . . 7-14

Sales-Weighted Engine Size of New Domestic and Import Light Trucks

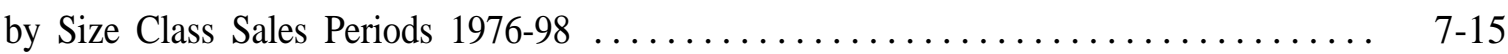

Imports

U.S. Petroleum Net Imports by World Region of Origin, $1960-97 \ldots \ldots \ldots \ldots \ldots \ldots \ldots \ldots$ 1-10

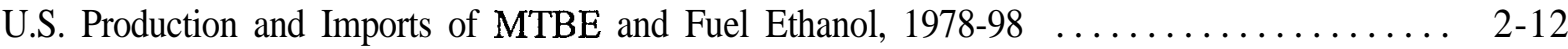
Incentives

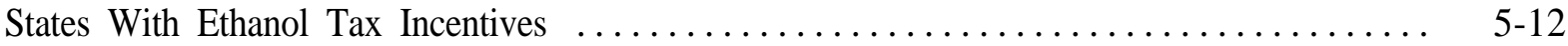
Income

Average Annual Expenditures of Households by Income, $1997 \ldots \ldots \ldots \ldots \ldots \ldots \ldots \ldots$. $11-3$

Shares of Long-Distance Person Trips by Mode and Household Income, 1995 . . . . . . . 1 1-20 Indicators

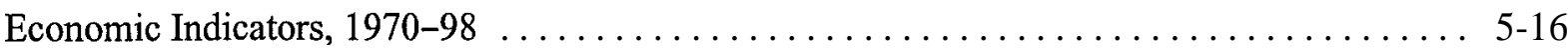
Indices

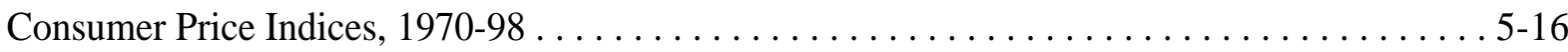
Industries

Employees of Motor Vehicle and Related Industries, 1990 and 1995 . . . . . . . . . . . . 5-18

Employment in Transportation and Related Industries, 1960-97 . . . . . . . . . . . . . . 5-19 Input

U.S. Refinery Input of Crude Oil and Petroleum Products, 1987-97 . . . . . . . . . . . . . 1-13 Intensities

Energy Intensities of Passenger Modes, 1970-97 . . . . . . . . . . . . . . . . . 2-14

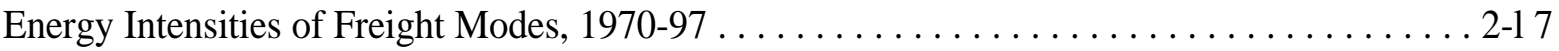
Intercity

Intercity Freight Movement and Energy Use in the United States, $1997 \ldots \ldots \ldots \ldots \ldots \ldots \ldots$ 
Interior

Sales-Weighted Interior Space of New Domestic and Import Automobiles

by Size Class, Sales Periods $1976-98 \ldots \ldots \ldots \ldots \ldots \ldots \ldots \ldots \ldots \ldots \ldots \ldots \ldots \ldots \ldots$

Engine Size, Curb Weight, and Interior Space of New Domestic and

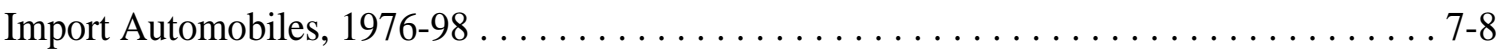

Intermodal

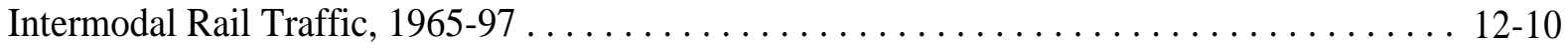

International

International Man-Made Emissions of All Greenhouse Gases, 1990-96 . . . . . . . . . . . . . . 3-2

International Man-Made Emissions of Carbon Dioxide, 1990-96 . . . . . . . . . . . . . . . . . 3-3

International Man-Made Emissions of Carbon Dioxide by Source Category, 1990 and 1996 . . . 3-4

International Man-Made Emissions of Nitrous Oxide, 1990-96 . . . . . . . . . . . . . . . . . . . 3-5

Summary Statistics for U.S. Domestic and International Certificated

Route Air Carriers (Combined Totals), 1970-97 . . . . . . . . . . . . . . . . . . . . . . . 12-2

Tonnage Statistics for Domestic and International Waterborne Commerce, 1970-97 . . . . . . . 12-4 Inventory

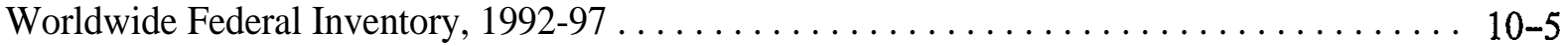
January

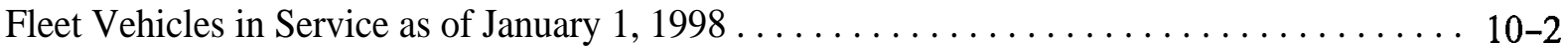
Journey

Journey-to-Work Statistics, 1983, 1990, and 1995 NPTS $\ldots \ldots \ldots \ldots \ldots \ldots \ldots \ldots \ldots \ldots$. . . . . . . . . . . .

National and Metropolitan Area Comparisons of Journey-to-Work Statistics, 1990 Census . . . 11-16 Lead

National Lead Emission Estimates, $1970-97 \ldots \ldots \ldots \ldots \ldots \ldots \ldots \ldots \ldots \ldots \ldots \ldots \ldots$ Length

Average Length of Time Fleet Vehicles are Kept Before Sold to Others, $1991 \ldots \ldots$. . . . . . . 10-4

Average Annual Vehicle-Miles, Vehicle Trips and Trip Length per Household

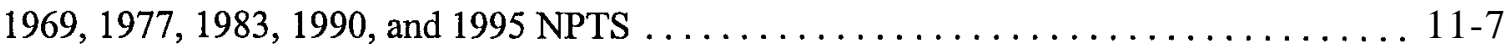

Average Annual Person-Miles Traveled (PMT), Person Trips and Trip Length

per Household by Selected Trip Purposes, 1983, 1990, and 1995 NPTS . . . . . . . . . . . . 11-8 Light

Federal Exhaust Emission Certification Standards for Gasoline- and

Diesel-Powered Light Vehicles . . . . . . . . . . . . . . . . . . . . . . . 4-28

Federal Exhaust Emission Certification Standards for Gasoline- and

Diesel-Powered Light Trucks (Category LDT1) . . . . . . . . . . . . . . . . . . . . 4-29

Federal Exhaust Emission Certification Standards for Gasoline- and

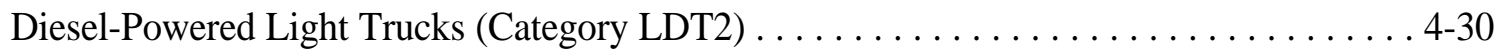

Federal Exhaust Emission Certification Standards for Gasoline- and

Diesel-Powered Light Trucks (Category LDT3) . . . . . . . . . . . . . . . 4-31

Federal Exhaust Emission Certification Standards for Gasoline- and

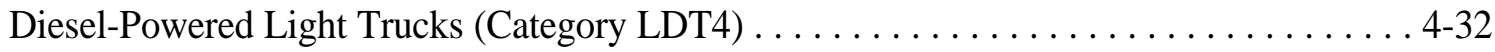


Light (continued)

Federal Exhaust Emission Certification Standards for Gasoline- and

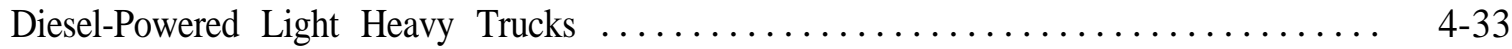

California Passenger Cars and Light Trucks Emission Certification Standards ......... 4-35

California Vehicle Emission Reduction for Passenger Cars and Light Trucks. ......... 4-36

Light Vehicle Market Shares by Size Class, Sales Period 1976-98 ............... 7-10

Sales-Weighted Wheelbase of New Automobiles and Light Trucks, Sales Period 1976-98 ... 7-1 1

Period Sales, Market Shares, and Sales-Weighted Fuel Economies of New

Domestic and Import Light Trucks, Selected Sales Period 1976-98 .............. 7-14

Sales-Weighted Engine Size of New Domestic and Import Light Trucks

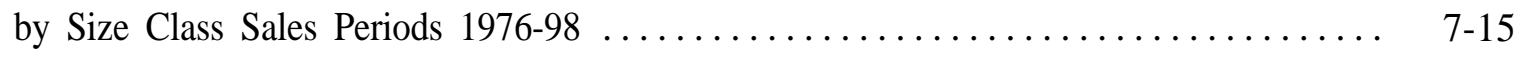

New Light Vehicle Dealerships and Sales, 1970-97 .................... 7-16

Corporate Average Fuel Economy (CAFE) Standards versus Sales-Weighted

Fuel Economy Estimates for Automobiles and Light Trucks, 1978-98. . . . . . . . . 7-17

New Retail Sales of Light Trucks by Gross Vehicle Weight, 1970-97 . . . . . . . . . . 8-4

Estimates of Light Alternative Fuel Vehicles, 1995, 1997, and $1999 \ldots \ldots \ldots \ldots \ldots \ldots \ldots$. . . . .

Energy Policy Act Purchase Requirements of Light Alternative Fuel Vehicles .......... 10-8 Long

Changes in Per-Mile, Fuel-Cycle Energy Use and Greenhouse Gas Emissions

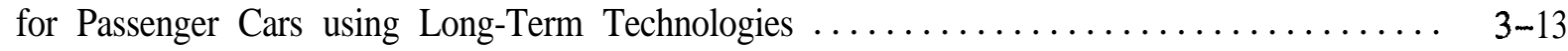

Changes in Per-Mile, Fuel-Cycle Energy Use and Criteria Pollutant Emissions

for Passenger Cars using Long-Term Technologies $\ldots \ldots \ldots \ldots \ldots \ldots \ldots \ldots \ldots \ldots$ 4-19

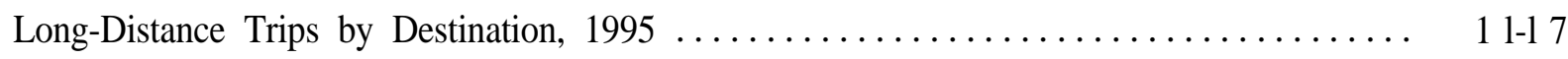

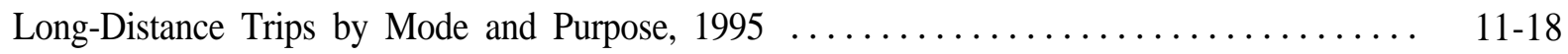

Long-Distance Household Trips by Mode and Trip Distance, $1995 \ldots \ldots \ldots \ldots \ldots \ldots \ldots \ldots$. . . . . 19

Man-Made

International Man-Made Emissions of All Greenhouse Gases, 1990-96 . . . . . . . . . . 3-2

International Man-Made Emissions of Carbon Dioxide, $1990-96 \ldots \ldots \ldots \ldots \ldots \ldots \ldots \ldots .3$

International Man-Made Emissions of Carbon Dioxide by Source Category, 1990 and 1996 ... 3-4

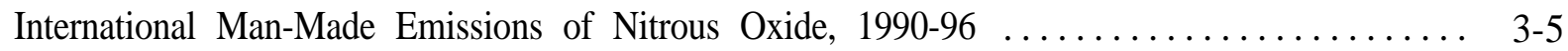

Manufacturer

Alternative Fuel Vehicles Available by Manufacturer, Model Year 1999 . . . . . . . . . . . 9-6

Manufacturing

Motor Vehicle Manufacturing Employment Statistics, 1972-97 ............... 5-17 Map

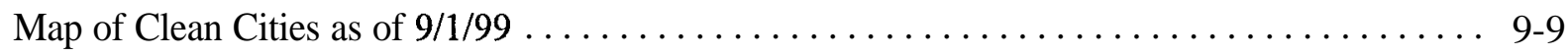
Marine

Breakdown of Domestic Marine Cargo by Commodity Class, $1997 \ldots \ldots \ldots \ldots \ldots \ldots \ldots \ldots$. . . . . . 6 
Market

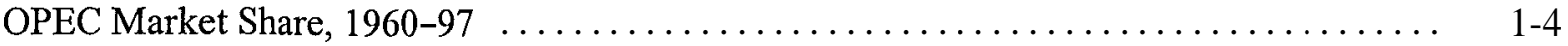

Period Sales, Market Shares, and Sales-Weighted Fuel Economies of New

Domestic and Import Automobiles, Selected Sales Periods 1976-98 ................ 7-4

Light Vehicle Market Shares by Size Class, Sales Period 1976-98 .................. 7-10

Period Sales, Market Shares, and Sales-Weighted Fuel Economies of New

Domestic and Import Light Trucks, Selected Sales Period 1976-98 ............. 7-14

Material

Average Material Consumption for a Domestic Automobile, 1978, 1985, and $1998 \ldots \ldots \ldots$ 7-9

Matter

Total National Emissions of Particulate Matter (PM- 10), 1970-97 . . . . . . . . . . . . . . . . 4-9

Emissions of Particulate Matter (PM-10) from Highway Vehicles, 1970-97 . . . . . . . . . 4-10

Total National Emissions of Particulate Matter (PM-2.5), 1990-97. . . . . . . . . . . . . . . . 4-1 1

Emissions of Particulate Matter (PM-2.5) from Highway Vehicles, 1990-97 . . . . . . . . . 4-12 Meeting

California Air Resources Board Requirements for Meeting Emission Standards ......... 4-37 Metropolitan

National and Metropolitan Area Comparisons of Journey-to-Work Statistics, 1990 Census . . 11-16 Mile

Changes in Per-Mile, Fuel-Cycle Energy Use and Greenhouse Gas Emissions

for Passenger Cars using Near-Term Technologies . . . . . . . . . . . . . . . . . . . . . 3-12

Changes in Per-Mile, Fuel-Cycle Energy Use and Greenhouse Gas Emissions

for Passenger Cars using Long-Term Technologies ......................... 3-1 3

Changes in Per-Mile, Fuel-Cycle Energy Use and Criteria Pollutant Emissions

for Passenger Cars using Near-Term Technologies $\ldots \ldots \ldots \ldots \ldots \ldots \ldots \ldots \ldots$ 4-1 7

Changes in Per-Mile, Fuel-Cycle Energy Use and Criteria Pollutant Emissions

for Passenger Cars using Long-Term Technologies ....................... 4-19

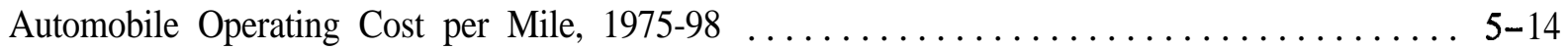

Miles

Highway Vehicle Miles Traveled by Vehicle Type, $1970-97 \ldots \ldots \ldots \ldots \ldots \ldots \ldots \ldots \ldots$. . . . . . . .

Value, Tons, and Ton-Miles of Commodity Shipments, $1993 \ldots \ldots \ldots \ldots \ldots \ldots \ldots \ldots \ldots$. . . . . . . . .

Average Annual and Daily Vehicle-Miles of Travel for Fleet Vehicles, $1991 \ldots \ldots \ldots \ldots$. . . $10-4$

Average Miles per Federal Vehicle by Vehicle Type, $1997 \ldots \ldots \ldots \ldots \ldots \ldots \ldots \ldots \ldots \ldots$. . . . . . . . .

Average Annual Vehicle-Miles, Vehicle Trips and Trip Length per Household

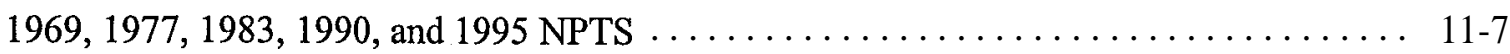

Average Annual Person-Miles Traveled (PMT), Person Trips and Trip Length

per Household by Selected Trip Purposes, 1983, 1990, and 1995 NPTS . . . . . . . . . . 11-8

Vehicle-Miles by Trip Purpose, 1995 NPTS . . . . . . . . . . . . . . . . . . . . . . . . $11-11$

Average Annual Miles per Vehicle by Household Vehicle Ownership, 1995 NPTS . . . . . . 11-12

Average Annual Miles per Household Vehicle by Vehicle Age .................... $11-13$

Class I Railroad Freight Systems in the United States Ranked by Revenue Ton-Miles, 1997 . . 12-7 
Mode

Transportation of Petroleum and Petroleum Products in the U.S. by Mode, 1975-97 . . . . . . 1-19

Domestic Consumption of Transportation Energy by Mode and Fuel Type, 1997 . . . . . . . 2-6

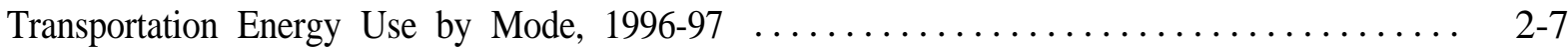

Transportation Energy Consumption by Mode, $1970-97 \ldots \ldots \ldots \ldots \ldots \ldots \ldots \ldots \ldots \ldots . \ldots \ldots$

1993 Commodity Flow Survey: Shipment Characteristics by Mode of Transportation ...... . 8-13

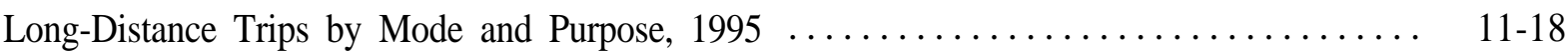

Long-Distance Household Trips by Mode and Trip Distance, $1995 \ldots \ldots \ldots \ldots \ldots \ldots \ldots \ldots$. $11-19$

Shares of Long-Distance Person Trips by Mode and Household Income, $1995 \ldots \ldots \ldots \ldots$. 11 1-20

Middle East

Summary of 1996 Military Expenditures for Defending Oil Supplies

from the Middle East . . . . . . . . . . . . . . . . . . . . . . . . . . . . . . . . . . .

Military

Summary of 1996 Military Expenditures for Defending Oil Supplies

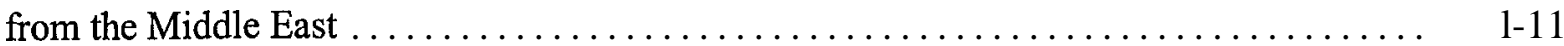

Model

Scrappage and Survival Rates for Automobiles 1970, 1980 and 1990 Model Years ........ 6-1 1

Alternative Fuel Vehicles Available by Manufacturer, Model Year 1999 . . . . . . . . . . . 9-6 Modes

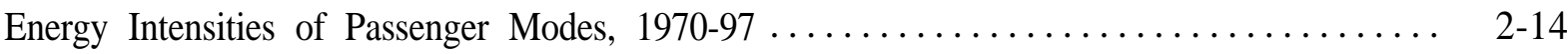

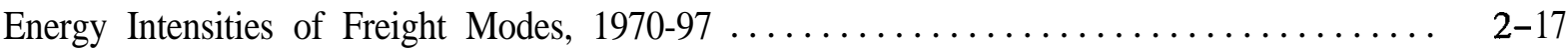

Monoxide

Total National Emissions of Carbon Monoxide, 1970-97 . . . . . . . . . . . . . . . 4-3

Emissions of Carbon Monoxide from Highway Vehicles, $1970-97 \ldots \ldots \ldots \ldots \ldots \ldots \ldots \ldots$. . . . . .

Motor

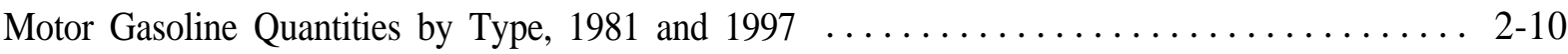

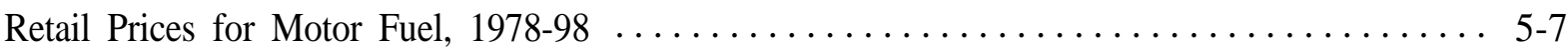

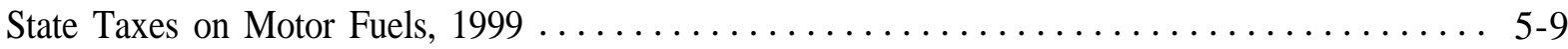

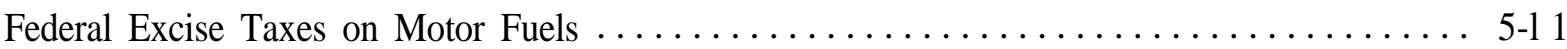

Motor Vehicle Manufacturing Employment Statistics, 1972-97 .................. 5-17

Employees of Motor Vehicle and Related Industries, 1990 and $1995 \ldots \ldots \ldots \ldots \ldots \ldots \ldots$ Movement

Intercity Freight Movement and Energy Use in the United States, $1997 \ldots \ldots \ldots \ldots \ldots \ldots$. . . . 16 MTBE

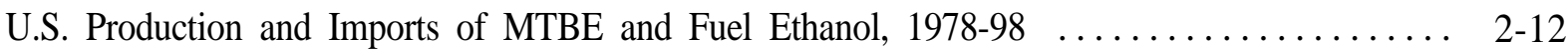
National

Total National Emissions of the Criteria Air Pollutants by Sector, $1997 \ldots \ldots \ldots \ldots \ldots \ldots$. . . . 2

Total National Emissions of Carbon Monoxide, 1970-97 ................... 4-3

Total National Emissions of Nitrogen Oxides, 1970-97 . . . . . . . . . . . . . . 4 4-5

Total National Emissions of Volatile Organic Compounds, $1970-97 \ldots \ldots \ldots \ldots \ldots \ldots \ldots$. . . . . .

Total National Emissions of Particulate Matter (PM-lo), 1970-97 . . . . . . . . . . . . . . . . 4-9 
National (continued)

Total National Emissions of Particulate Matter (PM-2.5), 1990-97. . . . . . . . . . . . . 4-1 1

National Lead Emission Estimates, $1970-97$. . . . . . . . . . . . . . . . . . . . . . . . 4-13

National and Metropolitan Area Comparisons of Journey-to-Work Statistics, 1990 Census . . 11-16

Summary Statistics for the National Railroad Passenger Corporation (Amtrak), 1971-97 . . . 12-11 Near

Changes in Per-Mile, Fuel-Cycle Energy Use and Greenhouse Gas Emissions

for Passenger Cars using Near-Term Technologies ........................ 3-12

Changes in Per-Mile, Fuel-Cycle Energy Use and Criteria Pollutant Emissions

for Passenger Cars using Near-Term Technologies ..................... 4-1 7

New York

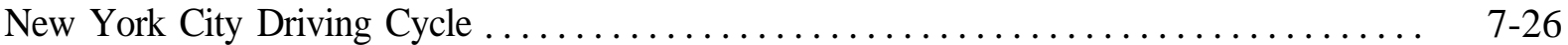

Nitrogen

Total National Emissions of Nitrogen Oxides, 1970-97. . . . . . . . . . . . . . . . . . . . 4-5

Emissions of Nitrogen Oxides from Highway Vehicles, $1970-97 \ldots \ldots \ldots \ldots \ldots \ldots \ldots \ldots .4$. . . . . . .

Nitrous

International Man-Made Emissions of Nitrous Oxide, $1990-96 \ldots \ldots \ldots \ldots \ldots \ldots \ldots \ldots . \ldots \ldots$

NPTS

Demographic Statistics, 1969, 1977, 1983, 1990, and 1995 NPTS $\ldots \ldots \ldots \ldots \ldots \ldots \ldots$

Average Annual Vehicle-Miles, Vehicle Trips and Trip Length per Household

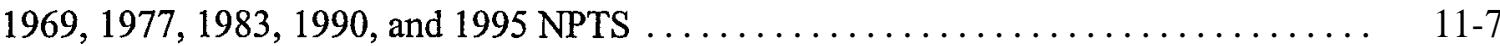

Average Annual Person-Miles Traveled (PMT), Person Trips and Trip Length

per Household by Selected Trip Purposes, 1983, 1990, and 1995 NPTS . . . . . . . . . . . . 1 1-8

Average Vehicle Occupancy by Vehicle Type, 1995 NPTS . . . . . . . . . . . . . . . . . . . 11 1-9

Average Vehicle Occupancy by Trip Purpose, 1977, 1983, 1990, and 1995 NPTS . . . . . . . 11 1-10

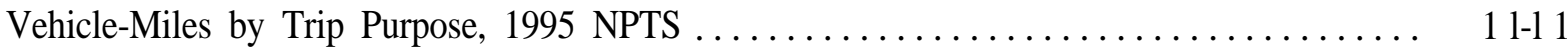

Average Annual Miles per Vehicle by Household Vehicle Ownership, 1995 NPTS . . . . . . 11-12

Average Age of Vehicles by Household Vehicle Ownership, 1995 NPTS . . . . . . . . . . . 11-12

Journey-to-Work Statistics, 1983, 1990, and 1995 NPTS . . . . . . . . . . . . . . . . . $11-14$

Numerical

Numerical Estimates of Global Warming Potentials Compared With Carbon Dioxide . . . . . . . 3-9

Occupancy

Average Vehicle Occupancy by Vehicle Type, 1995 NPTS . . . . . . . . . . . . . . . . . . . . . . . . 1 1-9

Average Vehicle Occupancy by Trip Purpose, 1977, 1983, 1990, and 1995 NPTS . . . . . . 11 1-10 OECD

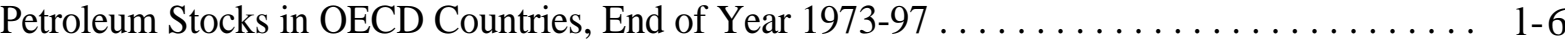

Share of OECD Petroleum Stocks to OECD Petroleum Consumption, 1973-96 . . . . . . . . . . . . 1-7 Oil

World Oil Consumption, $1960-96 \ldots \ldots \ldots \ldots \ldots \ldots \ldots \ldots \ldots \ldots \ldots \ldots \ldots \ldots \ldots$

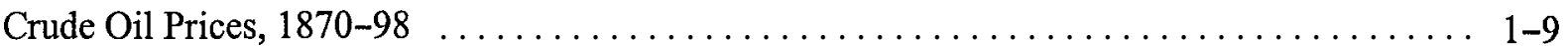

Summary of 1996 Military Expenditures for Defending Oil Supplies

from the Middle East . . . . . . . . . . . . . . . . . . . . . . . . . . 11 
Oil (continued)

U.S. Refinery Input of Crude Oil and Petroleum Products, 1987-97 . . . . . . . . . . . . 1-13

Refinery Yield of Petroleum Products from a Barrel of Crude Oil, 1978-97. . . . . . . . . . 1-14

Prices for a Barrel of Crude Oil and a Gallon of Gasoline, 1978-98 . . . . . . . . . . . . 5-6 OPEC

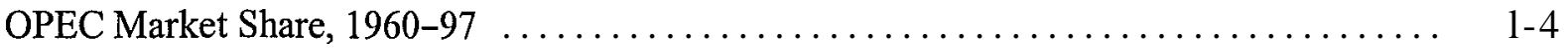

Operating

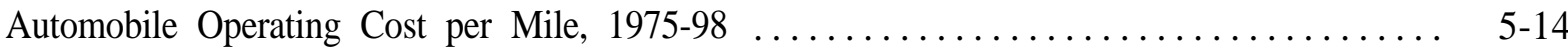

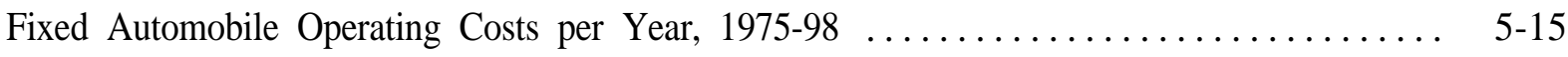
Operation

Automobiles in Operation and Vehicle Travel by Age, 1970 and 1997 .............. 6-8

Trucks in Operation and Vehicle Travel by Age, 1970 and $1997 \ldots \ldots \ldots \ldots \ldots \ldots \ldots$. . . . . . . 9 Operations

Summary Statistics for Rail Transit Operations, $1970-97 \ldots \ldots \ldots \ldots \ldots \ldots \ldots \ldots \ldots \ldots \ldots$. . . . . 12 Organic

Total National Emissions of Volatile Organic Compounds, $1970-97 \ldots \ldots \ldots \ldots \ldots \ldots$. . . . .

Emissions of Volatile Organic Compounds from Highway Vehicles, 1970-97 . . . . . . . . . 4-8 Origin

U.S. Petroleum Net Imports by World Region of Origin, $1960-97 \ldots \ldots \ldots \ldots \ldots \ldots \ldots \ldots$ 1-10 Others

Average Length of Time Fleet Vehicles are Kept Before Sold to Others, $1991 \ldots \ldots \ldots \ldots$. 1 0-4 Output

Refinery Gross Output by World Region, $1998 \ldots \ldots \ldots \ldots \ldots \ldots \ldots \ldots \ldots \ldots \ldots \ldots \ldots \ldots \ldots$ Ownership

Household Vehicle Ownership, $1960-90$ Census . . . . . . . . . . . . . . . . . . . . 11 1-5

Average Annual Miles per Vehicle by Household Vehicle Ownership, 1995 NPTS . . . . . . . 11-12 Oxide

International Man-Made Emissions of Nitrous Oxide, $1990-96 \ldots \ldots \ldots \ldots \ldots \ldots \ldots \ldots \ldots$ Oxides

Total National Emissions of Nitrogen Oxides, $1970-97 \ldots \ldots \ldots \ldots \ldots \ldots \ldots \ldots \ldots .4 . \ldots \ldots$

Emissions of Nitrogen Oxides from Highway Vehicles, 1970-97 . . . . . . . . . . . . . . . 4-6 Particulate

Total National Emissions of Particulate Matter (PM-1 0), 1970-97 . . . . . . . . . . . . . . . 4-9

Emissions of Particulate Matter (PM- 10) from Highway Vehicles, 1970-97 . . . . . . . . . . 4-10

Total National Emissions of Particulate Matter (PM-2.5), 1990-97 . . . . . . . . . . . . 4-1 1

Emissions of Particulate Matter (PM-2.5) from Highway Vehicles, 1990-97 . . . . . . . . . . . 4-1 2 Passenger

Passenger Travel and Energy Use in the United States, $1997 \ldots \ldots \ldots \ldots \ldots \ldots \ldots \ldots \ldots \ldots$. . . . . . . . . . .

Energy Intensities of Passenger Modes, 1970-97 . . . . . . . . . . . . . . . . . 2-14

Changes in Per-Mile, Fuel-Cycle Energy Use and Greenhouse Gas Emissions for Passenger Cars using Near-Term Technologies . . . . . . . . . . . . . . . . . . 3-12 
Passenger (continued)

Changes in Per-Mile, Fuel-Cycle Energy Use and Greenhouse Gas Emissions

for Passenger Cars using Long-Term Technologies ................... 3-13

Changes in Per-Mile, Fuel-Cycle Energy Use and Criteria Pollutant Emissions

for Passenger Cars using Near-Term Technologies .................. 4-17

Changes in Per-Mile, Fuel-Cycle Energy Use and Criteria Pollutant Emissions

for Passenger Cars using Long-Term Technologies ................... 4-19

California Passenger Cars and Light Trucks Emission Certification Standards .......... 4-35

California Vehicle Emission Reduction for Passenger Cars and Light Trucks.......... 4-36

Summary Statistics for Passenger Cars, 1970-97 . . . . . . . . . . . . . . . . . . . 7-2

Summary Statistics for the National Railroad Passenger Corporation (Amtrak), 1971-97 . . . 12-11

Percentage

Percentage of Trucks by Size Class, 1977, 1982, 1987, and $1992 \ldots \ldots \ldots \ldots \ldots \ldots \ldots \ldots$

Percentage of Trucks by Fleet Size and Primary Refueling Facility, 1992 . . . . . . . . . 8-7

Percentage of Trucks by Major Use and Primary Refueling Facility, $1992 \ldots \ldots \ldots \ldots \ldots \ldots$. . . 8-10

Percentage of Trucks by Size Ranked by Major Use, $1992 \ldots \ldots \ldots \ldots \ldots \ldots \ldots \ldots \ldots$. . . . 11

Periods

Period Sales, Market Shares, and Sales-Weighted Fuel Economies of New

Domestic and Import Automobiles, Selected Sales Periods 1976-98 . . . . . . . . . . . . 7-4

Sales-Weighted Engine Size of New Domestic and Import Automobiles

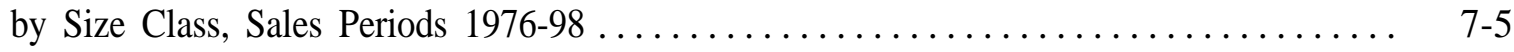

Sales-Weighted Curb Weight of New Domestic and Import Automobiles

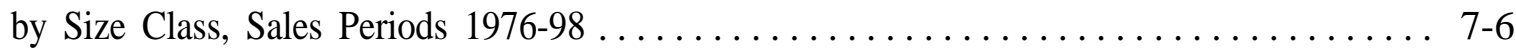

Sales-Weighted Interior Space of New Domestic and Import Automobiles

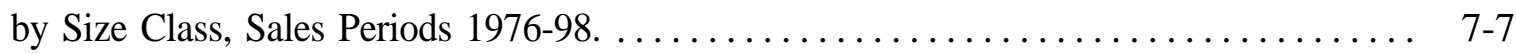

Sales-Weighted Engine Size of New Domestic and Import Light Trucks

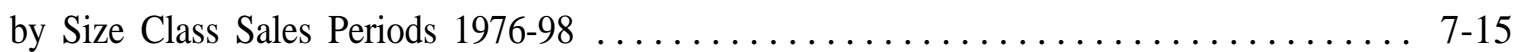

Person

Average Annual Person-Miles Traveled (PMT), Person Trips and Trip Length

per Household by Selected Trip Purposes, 1983, 1990, and 1995 NPTS . . . . . . . . . $11-8$

Shares of Long-Distance Person Trips by Mode and Household Income, 1995 . . . . . . . 11 1-20

Petroleum

Petroleum Stocks in OECD Countries, End of Year 1973-97 . . . . . . . . . . . . . . 1-6

Share of OECD Petroleum Stocks to OECD Petroleum Consumption, 1973-96 . . . . . . . . 1-7

Share of Petroleum Stocks to Petroleum Consumption by Country, $1996 \ldots \ldots \ldots \ldots \ldots \ldots$. . .

U.S. Petroleum Net Imports by World Region of Origin, $1960-97 \ldots \ldots \ldots \ldots \ldots \ldots \ldots \ldots$

U.S. Refinery Input of Crude Oil and Petroleum Products, 1987-97 . . . . . . . . . . 1-13

Refinery Yield of Petroleum Products from a Barrel of Crude Oil, 1978-97 . . . . . . . . . 1- 14

United States Petroleum Production and Consumption, $1973-98 \ldots \ldots \ldots \ldots \ldots \ldots \ldots \ldots$ 1-1 5

Import Share of U.S. Petroleum Consumption, $1973-98 \ldots \ldots \ldots \ldots \ldots \ldots \ldots \ldots \ldots \ldots \ldots \ldots$

Consumption by Petroleum by End-Use Sector, $1973-98 \ldots \ldots \ldots \ldots \ldots \ldots \ldots \ldots \ldots \ldots$

Transportation of Petroleum and Petroleum Products in the U.S. by Mode, 1975-97 ...... 1-19 
Phase

U.S. Advanced Battery Consortium Research Agreements, Phase II ................. 9-10 $\mathrm{PM}$

Total National Emissions of Particulate Matter (PM-1 0), 1970-97 . . . . . . . . . . . . . . 4-9

Emissions of Particulate Matter (PM- 10) from Highway Vehicles, 1970-97 . . . . . . . . . . 4-10

Total National Emissions of Particulate Matter (PM-2.5), 1990-97 . . . . . . . . . . . . . . . 4-1 1

Emissions of Particulate Matter (PM-2.5) from Highway Vehicles, 1990-97 . . . . . . . . 4 4-12 PMT

Average Annual Person-Miles Traveled (PMT), Person Trips and Trip Length

per Household by Selected Trip Purposes, 1983, 1990, and 1995 NPTS . . . . . . . . . $11-8$

Policy

Energy Policy Act Purchase Requirements of Light Alternative Fuel Vehicles ......... 10-8 Pollutants

Total National Emissions of the Criteria Air Pollutants by Sector, $1997 \ldots \ldots \ldots \ldots \ldots \ldots$. . . . . 2

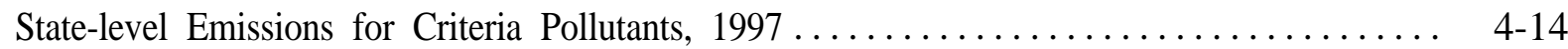

Population

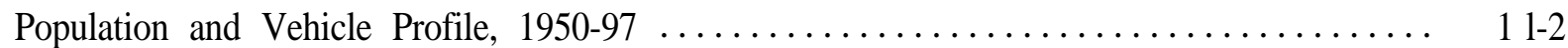

Potentials

Numerical Estimates of Global Warming Potentials Compared With Carbon Dioxide ....... 3-9 Pounds

New Retail Sales of Trucks 10,000 pounds GVW and Less in the United States, 1970-97. ... 7-13 Price

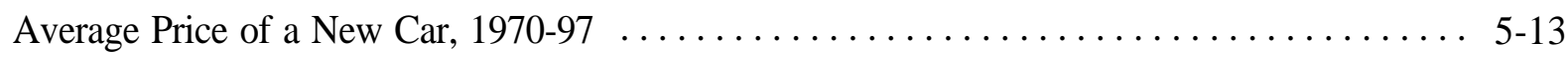

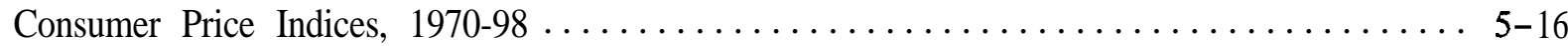

Prices

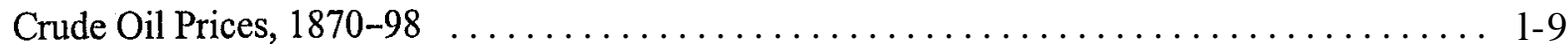

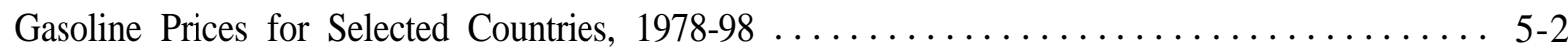

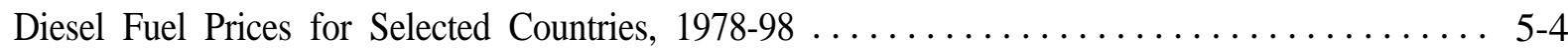

Prices for a Barrel of Crude Oil and a Gallon of Gasoline, 1978-98 . . . . . . . . . . . 5-6

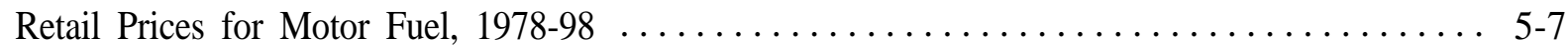

Prices for Selected Transportation Fuels, $1978-98 \ldots \ldots \ldots \ldots \ldots \ldots \ldots \ldots \ldots \ldots \ldots \ldots$

Primary

World Production of Primary Energy by Selected Country Groups, 1988-97 . . . . . . . . . 2-2

World Consumption of Primary Energy by Selected Country Groups, 1988-97 .......... 2-3

Percentage of Trucks by Fleet Size and Primary Refueling Facility, 1992. ........... 8-7

Percentage of Trucks by Major Use and Primary Refueling Facility, $1992 \ldots \ldots \ldots \ldots \ldots \ldots$ 8 Products

U.S. Refinery Input of Crude Oil and Petroleum Products, 1987-97 . . . . . . . . . . 1-13

Refinery Yield of Petroleum Products from a Barrel of Crude Oil, 1978-97 . ........... 1- 14

Transportation of Petroleum and Petroleum Products in the U.S. by Mode, 1975-97 . . . . . . 1-19 Profile

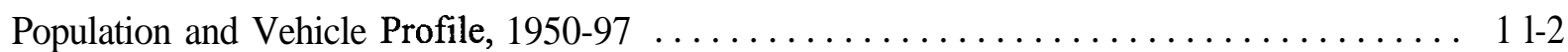


Purchase

Energy Policy Act Purchase Requirements of Light Alternative Fuel Vehicles.......... 10-8 Purpose

Average Vehicle Occupancy by Trip Purpose, 1977, 1983, 1990, and 1995 NPTS . . . . . . 1 1-10

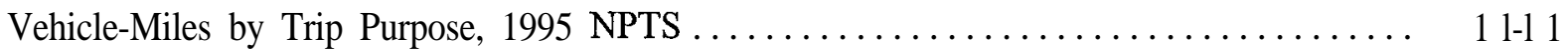

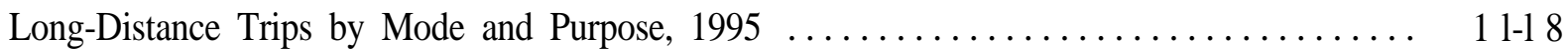

Purposes

Average Annual Person-Miles Traveled (PMT), Person Trips and Trip Length

per Household by Selected Trip Purposes, 1983, 1990, and 1995 NPTS . . . . . . . . . $11-8$ Rail

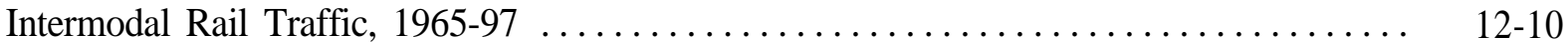

Summary Statistics for Rail Transit Operations, $1970-97 \ldots \ldots \ldots \ldots \ldots \ldots \ldots \ldots \ldots$ 12-12

Railroad

Class I Railroad Freight Systems in the United States Ranked by Revenue Ton-Miles, 1997 . . 12-7

Railroad Revenue Carloads by Commodity Group, 1974 and 1997 ................ 12-9

Summary Statistics for the National Railroad Passenger Corporation (Amtrak), 1971-97 ... 12-11

Railroads

Summary Statistics for Class I Freight Railroads, $1970-97 \ldots \ldots \ldots \ldots \ldots \ldots \ldots \ldots \ldots \ldots$

Ranked

Percentage of Trucks by Size Ranked by Major Use, $1992 \ldots \ldots \ldots \ldots \ldots \ldots \ldots \ldots \ldots$. $8-11$

Class I Railroad Freight Systems in the United States Ranked by Revenue Ton-Miles, 1997 . . 12-7 Rates

Scrappage and Survival Rates for Automobiles 1970, 1980 and 1990 Model Years ........ 6-1 1

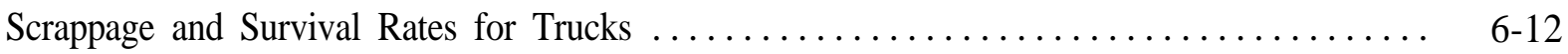

Receipts

Tax Receipts from the Sale of Gas Guzzlers, $1980-97 \ldots \ldots \ldots \ldots \ldots \ldots \ldots \ldots \ldots \ldots \ldots$

Reduction

California Vehicle Emission Reduction for Passenger Cars and Light Trucks. .......... 4-36

Refinery

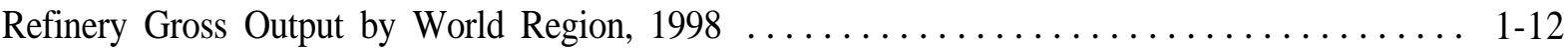

U.S. Refinery Input of Crude Oil and Petroleum Products, $1987-97 \ldots \ldots \ldots \ldots \ldots \ldots \ldots$. $\ldots \ldots$

Refinery Yield of Petroleum Products from a Barrel of Crude Oil, 1978-97. ........... 1-14

Refuel

Number of Alternative Refuel Sites by State and Fuel Type, $1998 \ldots \ldots \ldots \ldots \ldots \ldots \ldots \ldots$. $\ldots-7$

R e f u e 1 i n g

Percentage of Trucks by Fleet Size and Primary Refueling Facility, 1992 . . . . . . . . 8-7

Percentage of Trucks by Major Use and Primary Refueling Facility, $1992 \ldots \ldots \ldots \ldots \ldots$. 8-10 Region

U.S. Petroleum Net Imports by World Region of Origin, $1960-97 \ldots \ldots \ldots \ldots \ldots \ldots \ldots \ldots$ 1-10

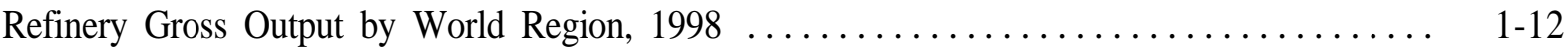


Registrations

Automobile Registrations for Selected Countries, $1950-96 \ldots \ldots \ldots \ldots \ldots \ldots \ldots \ldots$. . . . . . . . . . . .

Truck and Bus Registrations for Selected Countries, 1950-96. . . . . . . . . . . . . . . 6 6-3 Representative

Representative Number Five Driving Cycle $\ldots \ldots \ldots \ldots \ldots \ldots \ldots \ldots \ldots \ldots \ldots \ldots \ldots \ldots \ldots$ Research

U.S. Advanced Battery Consortium Research Agreements, Phase II . . . . . . . . . . . . 9 9-10 Resource

USGS World Oil Resource Estimates, $1993 \ldots \ldots \ldots \ldots \ldots \ldots \ldots \ldots \ldots \ldots \ldots \ldots \ldots \ldots$ Resources

California Air Resources Board Requirements for Meeting Emission Standards . . . . . . . . . 4-37 Retail

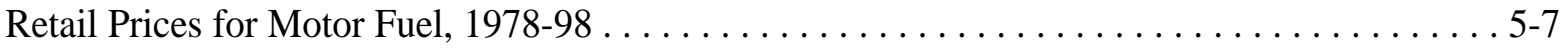

New Retail Automobile Sales in the United States, 1970-97 . . . . . . . . . . . . . . , . . . . . 7-3

New Retail Sales of Trucks 10,000 pounds GVW and Less in the United States, 1970-97. . . . 7-13

New Retail Truck Sales by Gross Vehicle Weight, 1970-97 . . . . . . . . . . . . . . . . . . . . 8-3

New Retail Sales of Light Trucks by Gross Vehicle Weight, 1970-97 . . . . . . . . . . . . . 8-4 Revenue

Class I Railroad Freight Systems in the United States Ranked by Revenue Ton-Miles, 1997 . . 12-7

Railroad Revenue Carloads by Commodity Group, 1974 and 1997 . . . . . . . . . . . . . . . 12-9 Route

Summary Statistics for U.S. Domestic and International Certificated

Route Air Carriers (Combined Totals), 1970-97 . . . . . . . . . . . . . . . . . . . . 12-2 RTECS

Average Number of Vehicles and Vehicle Travel per Household, $199 \mathbb{1}$ and 1994 RTECS . . . . 11-4 Sale

Tax Receipts from the Sale of Gas Guzzlers, $1980-97 \ldots \ldots \ldots \ldots \ldots \ldots \ldots \ldots \ldots \ldots$. . . $\ldots \ldots$ Sales

Vehicle Stock and New Sales in United States, 1997 Calendar Year . . . . . . . . . . . . . . . . 6-6

New Retail Automobile Sales in the United States, 1970-97 . . . . . . . . . . . . . . . 7-3

Period Sales, Market Shares, and Sales-Weighted Fuel Economies of New

Domestic and Import Automobiles, Selected Sales Periods 1976-98 . . . . . . . . . . . . . 7-4

Sales-Weighted Engine Size of New Domestic and Import Automobiles

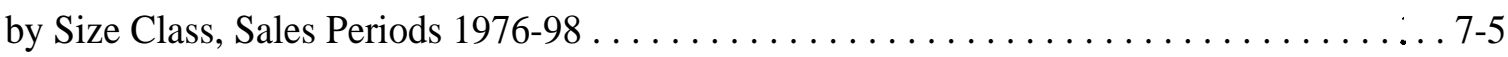

Sales-Weighted Curb Weight of New Domestic and Import Automobiles

by Size Class, Sales Periods $1976-98 \ldots \ldots \ldots \ldots \ldots \ldots \ldots \ldots \ldots \ldots \ldots \ldots \ldots \ldots$. . . . . . . . . . . . . . .

Sales-Weighted Interior Space of New Domestic and Import Automobiles

by Size Class, Sales Periods $1976-98 \ldots \ldots \ldots \ldots \ldots \ldots \ldots \ldots \ldots \ldots \ldots \ldots \ldots \ldots \ldots$

Light Vehicle Market Shares by Size Class, Sales Period 1976-98 . . . . . . . . . . . . . . 7-10

Sales-Weighted Wheelbase of New Automobiles and Light Trucks, Sales Period 1976-98 . . . 7-1 1

New Retail Sales of Trucks 10,000 pounds GVW and Less in the United States, 1970-97. . . . 7-13 
Sales (continued)

Period Sales, Market Shares, and Sales-Weighted Fuel Economies of New

Domestic and Import Light Trucks, Selected Sales Period 1976-98 . . . . . . . . . . . . 7-14

Sales-Weighted Engine Size of New Domestic and Import Light Trucks

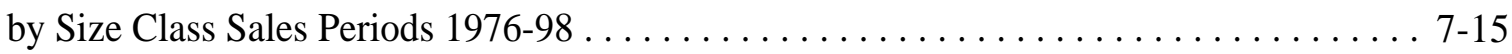

New Light Vehicle Dealerships and Sales, 1970-97 . . . . . . . . . . . . . . . . . 7-16

Corporate Average Fuel Economy (CAFE) Standards versus Sales-Weighted

Fuel Economy Estimates for Automobiles and Light Trucks, 1978-98 , . . . . . . . . . . . . 7-17

New Retail Truck Sales by Gross Vehicle Weight, 1970-97 . . . . . . . . . . . . . . . . . 8-3

New Retail Sales of Light Trucks by Gross Vehicle Weight, 1970-97 . . . . . . . . . . . . . . 8-4 Scrappage

Scrappage and Survival Rates for Automobiles 1970, 1980 and 1990 Model Years . . . . . . . 6-1 1

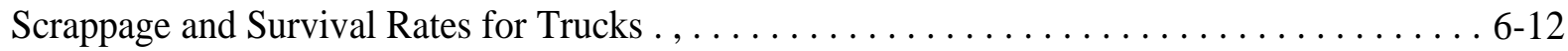
Sector

Consumption by Petroleum by End-Use Sector, $1973-98 \ldots \ldots \ldots \ldots \ldots \ldots \ldots \ldots \ldots$

U. S. Consumption of Total Energy by End-Use Sector, $1970-98 \ldots \ldots \ldots \ldots \ldots \ldots \ldots$. . . . . 2-4

U.S. Carbon Dioxide Emissions from Fossil Energy Consumption by End-Use Sector, 1984-97 3-7

U.S. Carbon Dioxide Emissions from Energy Use in the Transportation Sector, 1980-97 . . . . 3-8

Total National Emissions of the Criteria Air Pollutants by Sector, 1997 . . . . . . . . . . . . . 4 4-2

Selected

World Production of Primary Energy by Selected Country Groups, 1988-97 . . . . . . . . . 2-2

World Consumption of Primary Energy by Selected Country Groups, 1988-97 .......... 2-3

Gasoline Prices for Selected Countries, $1978-98 \ldots \ldots \ldots \ldots \ldots \ldots \ldots \ldots \ldots \ldots \ldots \ldots$. $5-2$

Diesel Fuel Prices for Selected Countries, 1978-98 ....................... . 5-4

Prices for Selected Transportation Fuels, $1978-98 \ldots \ldots \ldots \ldots \ldots \ldots \ldots \ldots \ldots \ldots \ldots \ldots .6 .8$

Automobile Registrations for Selected Countries, $1950-96 \ldots \ldots \ldots \ldots \ldots \ldots \ldots \ldots \ldots$. . . . . . .

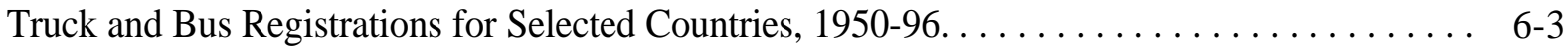

Period Sales, Market Shares, and Sales-Weighted Fuel Economies of New

Domestic and Import Automobiles, Selected Sales Periods 1976-98 . . . . . . . . . . . . . 7-4

Period Sales, Market Shares, and Sales-Weighted Fuel Economies of New

Domestic and Import Light Trucks, Selected Sales Period 1976-98 . . . . . . . . . . . . . 7-14

Average Annual Person-Miles Traveled (PMT), Person Trips and Trip Length

per Household by Selected Trip Purposes, 1983, 1990, and 1995 NPTS . . . . . . . . . . . 11 1-8 Service

Fleet Vehicles in Service as of January $1,1998 \ldots \ldots \ldots \ldots \ldots \ldots \ldots \ldots \ldots \ldots \ldots \ldots$ 10-2 Share

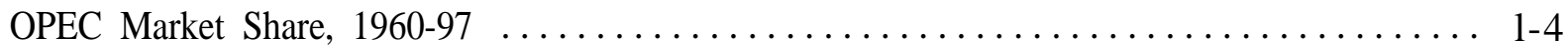

Share of OECD Petroleum Stocks to OECD Petroleum Consumption, 1973-96 . . . . . . . . 1-7

Share of Petroleum Stocks to Petroleum Consumption by Country, $1996 \ldots \ldots \ldots \ldots \ldots \ldots$ 1-8

Import Share of U.S. Petroleum Consumption, 1973-98 . . . . . . . . . . . . . . . . 1-17 
Shares

Period Sales, Market Shares, and Sales-Weighted Fuel Economies of New

Domestic and Import Automobiles, Selected Sales Periods 1976-98 . . . . . . . . . . . . . . 7-4

Light Vehicle Market Shares by Size Class, Sales Period 1976-98 . . . . . . . . . . . . . . . 7 7-10

Period Sales, Market Shares, and Sales-Weighted Fuel Economies of New

Domestic and Import Light Trucks, Selected Sales Period 1976-98 . . . . . . . . . . . . . . . 7-14

Period Sales, Market Shares, and Sales-Weighted Fuel Economies of New

Domestic and Import Light Trucks, Selected Sales Period 1976-98 . . . . . . . . . . . . . 7-14

Shipment

1993 Commodity Flow Survey: Shipment Characteristics by Mode of Transportation . . . . . . 8-13 Shipments

Value, Tons, and Ton-Miles of Commodity Shipments, $1993 \ldots \ldots \ldots \ldots \ldots \ldots \ldots \ldots$. . . . . . . . .

Single-Unit

Summary Statistics for Other Single-Unit and Combination Trucks, 1970-97 . . . . . . . . . 8-2 Sites

Number of Alternative Refuel Sites by State and Fuel Type, $1998 \ldots \ldots \ldots \ldots \ldots \ldots \ldots$. . . 9-7 Sold

Average Length of Time Fleet Vehicles are Kept Before Sold to Others, $1991 \ldots \ldots$. . . . . . . 10-4 Source

Distribution of Energy Consumption by Source, 1973, 1980, and $1998 \ldots \ldots \ldots \ldots$. . . . 2-5

International Man-Made Emissions of Carbon Dioxide by Source Category,. 1990 and 1996 . . . . 3-4 Space

Sales-Weighted Interior Space of New Domestic and Import Automobiles

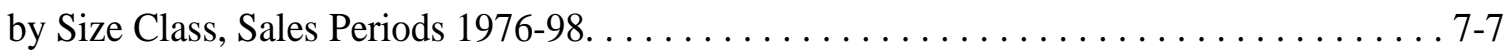

Engine Size, Curb Weight, and Interior Space of New Domestic and

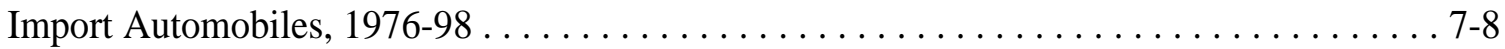

Specifications

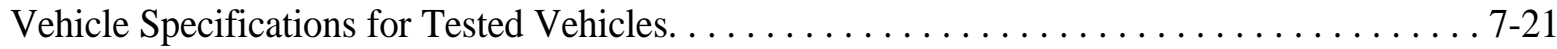

Speed

Fuel Economy by Speed, 1973,1984 and $1997 \ldots \ldots \ldots \ldots \ldots \ldots \ldots \ldots \ldots \ldots \ldots \ldots \ldots \ldots \ldots .22$

Fuel Economy by Speed, 1973, 1984 and $1997 \ldots \ldots \ldots \ldots \ldots \ldots \ldots \ldots \ldots$. . . . . . . . . . . . . . .

Steady Speed Fuel Economy for Tested Vehicles. . . . . . . . . . . . . . . . . . . 7-24

Standards

Federal Exhaust Emission Certification Standards for Gasoline- and

Diesel-Powered Light Vehicles . . . . . . . . . . . . . . . . . . . . . 4-28

Federal Exhaust Emission Certification Standards for Gasoline- and

Diesel-Powered Light Trucks (Category LDT1) . . . . . . . . . . . . . . . . . . . . 4-29

Federal Exhaust Emission Certification Standards for Gasoline- and

Diesel-Powered Light Trucks (Category LDT2) . . . . . . . . . . . . 4-30

Federal Exhaust Emission Certification Standards for Gasoline- and

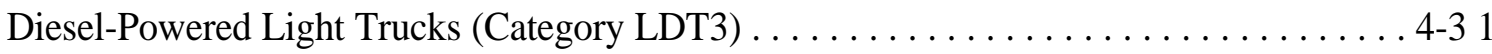


Standards (continued)

Federal Exhaust Emission Certification Standards for Gasoline- and

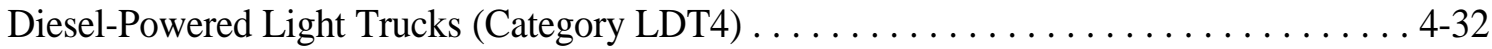

Federal Exhaust Emission Certification Standards for Gasoline- and

Diesel-Powered Light Heavy Trucks . . . . . . . . . . . . . . . . . . . . 4-33

Federal Exhaust Emission Certification Standards for Gasoline- and

Diesel-Powered Heavy Heavy Trucks . . . . . . . . . . . . . . . . . . . 4-34

California Passenger Cars and Light Trucks Emission Certification Standards . . . . . . . . . . 4-35

California Air Resources Board Requirements for Meeting Emission Standards . . . . . . . . . . 4-37

Corporate Average Fuel Economy (CAFE) Standards versus Sales-Weighted

Fuel Economy Estimates for Automobiles and Light Trucks, 1978-98 . . . . . . . . . . . . . 7-17

Statistics

Motor Vehicle Manufacturing Employment Statistics, 1972-97 . . . . . . . . . . . . . . . . 5 5-17

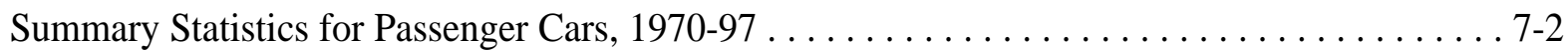

Summary Statistics for Two-Axle, Four-Tire Trucks, $1970-97 \ldots \ldots \ldots \ldots \ldots \ldots \ldots \ldots$. . . . . . . . .

Summary Statistics for Other Single-Unit and Combination Trucks, $1970-97 \ldots \ldots \ldots \ldots$. . . 2

Truck Statistics by Gross Vehicle Weight Class, $1992 \ldots \ldots \ldots \ldots \ldots \ldots \ldots \ldots \ldots \ldots \ldots$. . . . . . . . . . . .

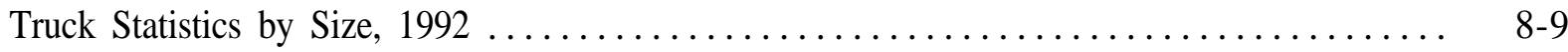

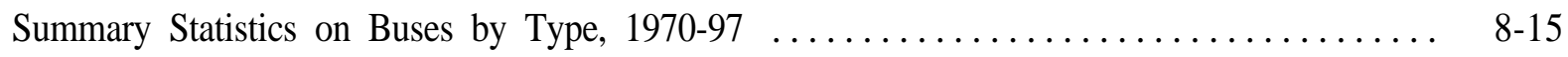

Demographic Statistics, 1969, 1977, 1983, 1990, and 1995 NPTS $\ldots \ldots \ldots \ldots \ldots \ldots \ldots$ 11-6

Journey-to-Work Statistics, 1983, 1990, and 1995 NPTS . . . . . . . . . . . . . 11-14

National and Metropolitan Area Comparisons of Journey-to-Work Statistics, 1990 Census . . 11 1-1 6

Summary Statistics for U.S. Domestic and International Certificated

Route Air Carriers (Combined Totals), 1970-97 . . . . . . . . . . . . . . . . . . 12-2

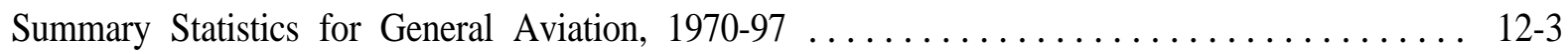

Tonnage Statistics for Domestic and International Waterborne Commerce, 1970-97 . . . . . . 12-4

Summary Statistics for Domestic Waterborne Commerce, $1970-97 \ldots \ldots \ldots \ldots \ldots \ldots \ldots$. . . . . . . 5

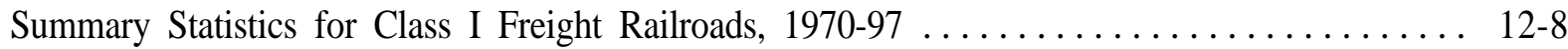

Summary Statistics for the National Railroad Passenger Corporation (Amtrak), 1971-97 . .. 12-11

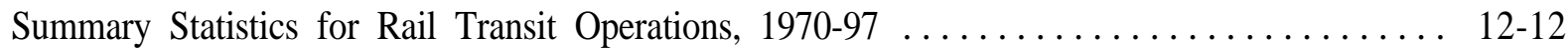
State

State-level Emissions for Criteria Pollutants, 1997. ......................... 4-14

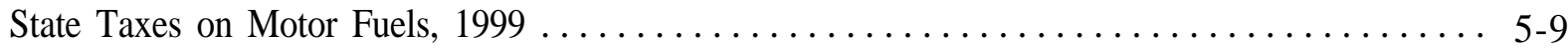

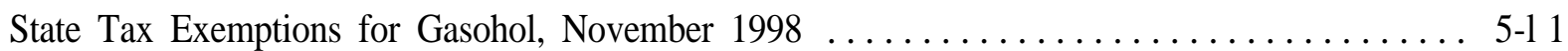

Number of Alternative Refuel Sites by State and Fuel Type, $1998 \ldots \ldots \ldots \ldots \ldots \ldots \ldots$. . . . . . . States

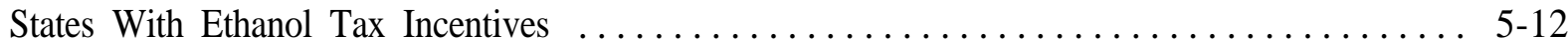

Top Ten States with Fleets of Ten Vehicles or More, $1999 \ldots \ldots \ldots \ldots \ldots \ldots \ldots \ldots \ldots$ 10-3 Steady

Steady Speed Fuel Economy for Tested Vehicles $\ldots \ldots \ldots \ldots \ldots \ldots \ldots \ldots \ldots \ldots \ldots$ 7-24 Stock

Vehicle Stock and New Sales in United States, 1997 Calendar Year . . . . . . . . . . . . 6-6 
Stocks

Petroleum Stocks in OECD Countries, End of Year 1973-97 . . . . . . . . . . . . . . . 1-6

Share of OECD Petroleum Stocks to OECD Petroleum Consumption, 1973-96 . . . . . . . . 1-7

Share of Petroleum Stocks to Petroleum Consumption by Country, $1996 \ldots \ldots \ldots \ldots \ldots \ldots$ 1-8

Summary

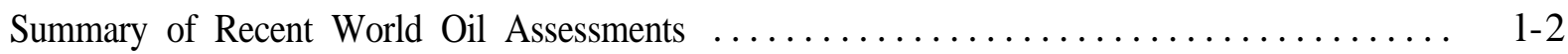

Summary of 1996 Military Expenditures for Defending Oil Supplies

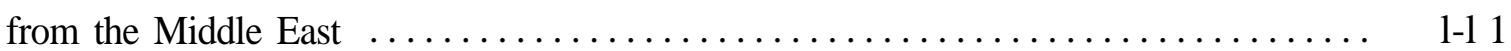

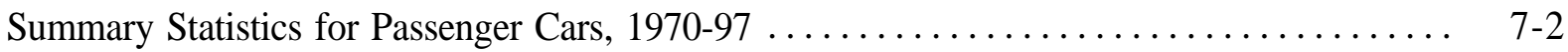

Summary Statistics for Two-Axle, Four-Tire Trucks, $1970-97 \ldots \ldots \ldots \ldots \ldots \ldots \ldots \ldots \ldots$ 7-12

Summary Statistics for Other Single-Unit and Combination Trucks, 1970-97 ......... 8-2

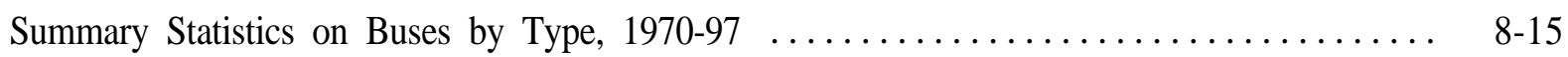

Summary Statistics for U.S. Domestic and International Certificated

Route Air Carriers (Combined Totals), 1970-97 ..................... 12-2

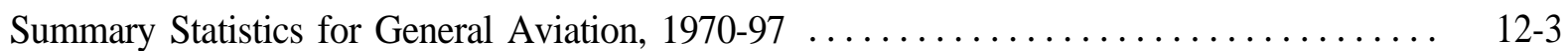

Summary Statistics for Domestic Waterborne Commerce, $1970-97 \ldots \ldots \ldots \ldots \ldots \ldots \ldots$. . . . . . . 5

Summary Statistics for Class I Freight Railroads, $1970-97 \ldots \ldots \ldots \ldots \ldots \ldots \ldots \ldots \ldots \ldots$. . . . . . . . .

Summary Statistics for the National Railroad Passenger Corporation (Amtrak), 1971-97 . .. 12-11

Summary Statistics for Rail Transit Operations, $1970-97 \ldots \ldots \ldots \ldots \ldots \ldots \ldots \ldots \ldots$ 12-12

Supplies

Summary of 1996 Military Expenditures for Defending Oil Supplies

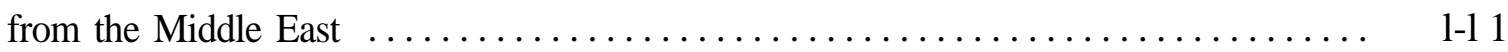

Survival

Scrappage and Survival Rates for Automobiles 1970, 1980 and 1990 Model Years . . . . . . . . 6-1 1

Scrappage and Survival Rates for Trucks . . . . . . . . . . . . . . . . 6 12

Systems

Class I Railroad Freight Systems in the United States Ranked by Revenue Ton-Miles, 1997 . . 12-7 $\operatorname{Tax}$

State Tax Exemptions for Gasohol, November $1998 \ldots \ldots \ldots \ldots \ldots \ldots \ldots \ldots \ldots \ldots \ldots \ldots \ldots$

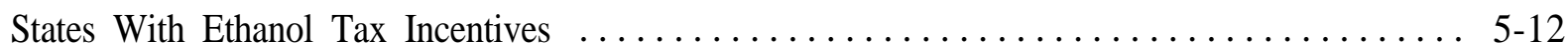

Tax Receipts from the Sale of Gas Guzzlers, $1980-97 \ldots \ldots \ldots \ldots \ldots \ldots \ldots \ldots \ldots \ldots$. . 8 . 8

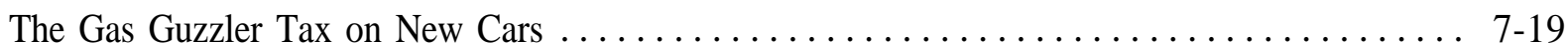
Taxes

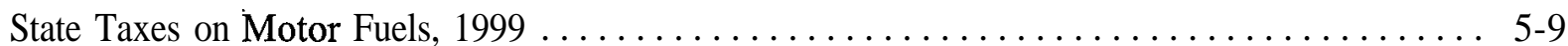

Federal Excise Taxes on Motor Fuels . . . . . . . . . . . . . . . . . . . 5-11

Technologies

Changes in Per-Mile, Fuel-Cycle Energy Use and Greenhouse Gas Emissions for Passenger Cars using Near-Term Technologies . . . . . . . . . . . . . . . . . 3-12

Changes in Per-Mile, Fuel-Cycle Energy Use and Greenhouse Gas Emissions for Passenger Cars using Long-Term Technologies . . . . . . . . . . . . . . . . . 3-13

Changes in Per-Mile, Fuel-Cycle Energy Use and Criteria Pollutant Emissions

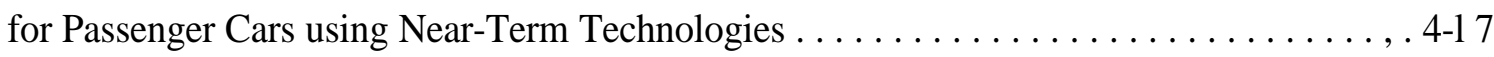


Technologies (continued)

Changes in Per-Mile, Fuel-Cycle Energy Use and Criteria Pollutant Emissions

for Passenger Cars using Long-Term Technologies . . . . . . . . . . . . . . . . . . . . 4-19

Tested

Vehicle Specifications for Tested Vehicles . . . . . . . . . . . . . . . . . 7-2 1

Steady Speed Fuel Economy for Tested Vehicles. . . . . . . . . . . . . . . . . 7-24

Time

Average Length of Time Fleet Vehicles are Kept Before Sold to Others, $1991 \ldots \ldots$. . . . . . 10-4 Tire

Summary Statistics for Two-Axle, Four-Tire Trucks, 1970-97 . . . . . . . . . . . . . . . . . . . 7-12 Ton

Value, Tons, and Ton-Miles of Commodity Shipments, $1993 \ldots \ldots \ldots \ldots \ldots \ldots \ldots \ldots$. . . . . 8-14

Class I Railroad Freight Systems in the United States Ranked by Revenue Ton-Miles, 1997 . 12-7 Tonnage

Tonnage Statistics for Domestic and International Waterborne Commerce, 1970-97 . . . . . . . 12-4 Totals

Summary Statistics for U.S. Domestic and International Certificated

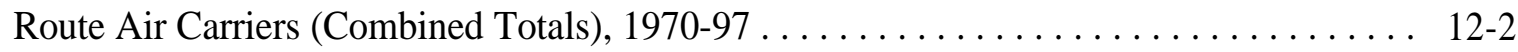

Traffic

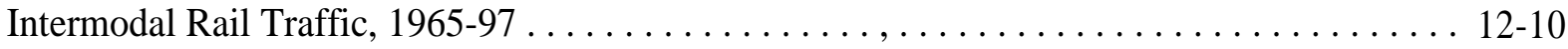

Transit

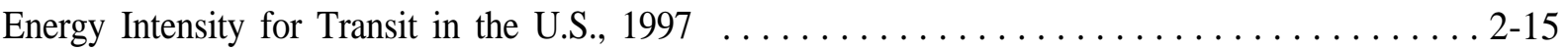

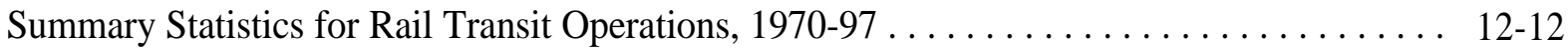
Transportation

Transportation of Petroleum and Petroleum Products in the U.S. by Mode, 1975-97 . . . . . . . 1-19

Domestic Consumption of Transportation Energy by Mode and Fuel Type, 1997 . . . . . . . . 2-6

Transportation Energy Use by Mode, $1996-97 \ldots \ldots \ldots \ldots \ldots \ldots \ldots \ldots \ldots \ldots . \ldots \ldots$

Transportation Energy Consumption by Mode, 1970-97 . . . . . . . . . . . . . . . . . . . . 2-8

U.S. Carbon Dioxide Emissions from Energy Use in the Transportation Sector, 1980-97 . . . . 3-8

Prices for Selected Transportation Fuels, $1978-98 \ldots \ldots \ldots \ldots \ldots \ldots \ldots \ldots \ldots \ldots \ldots \ldots \ldots \ldots$

Employment in Transportation and Related Industries, $1960-97 \ldots \ldots \ldots \ldots \ldots \ldots \ldots \ldots$. . . . . . . .

1993 Commodity Flow Survey: Shipment Characteristics by Mode of Transportation ... . . . 8-13

Means of Transportation to Work, 1980 and 1990 Census .................. 11-15 Travel

Passenger Travel and Energy Use in the United States, $1997 \ldots \ldots \ldots \ldots \ldots \ldots \ldots \ldots \ldots \ldots$. . . 13

Automobiles in Operation and Vehicle Travel by Age, 1970 and 1997 . . . . . . . . . . . . . . . , 6-8

Trucks in Operation and Vehicle Travel by Age, 1970 and $1997 \ldots \ldots \ldots \ldots \ldots \ldots \ldots \ldots$. . . . . . 6-9

Average Annual and Daily Vehicle-Miles of Travel for Fleet Vehicles, $1991 \ldots \ldots \ldots$. . . . . 10-4

Average Number of Vehicles and Vehicle Travel per Household, 1991 and 1994 RTECS . . . . 11 1-4 
Traveled

Highway Vehicle Miles Traveled by Vehicle Type, 1970-97 . . . . . . . . . . . . . . 6-7

Average Annual Person-Miles Traveled (PMT), Person Trips and Trip Length

per Household by Selected Trip Purposes, 1983, 1990, and 1995 NPTS . . . . . . . . . 11-8

Trip

Average Annual Vehicle-Miles, Vehicle Trips and Trip Length per Household

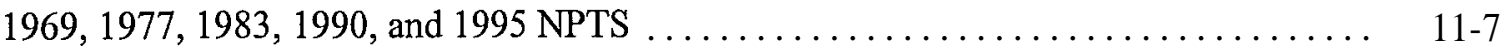

Average Annual Person-Miles Traveled (PMT), Person Trips and Trip Length

per Household by Selected Trip Purposes, 1983, 1990, and 1995 NPTS . . . . . . . . . . $11-8$

Average Vehicle Occupancy by Trip Purpose, 1977, 1983, 1990, and 1995 NPTS . . . . . . . 11 1-10

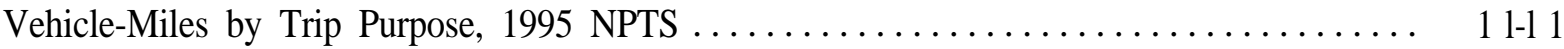

Long-Distance Household Trips by Mode and Trip Distance, 1995 . . . . . . . . . . . . . . 11-19

Trips

Average Annual Vehicle-Miles, Vehicle Trips and Trip Length per Household

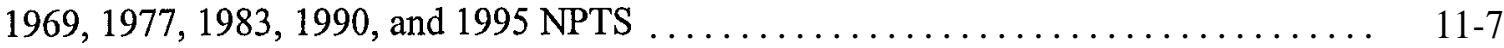

Average Annual Person-Miles Traveled (PMT), Person Trips and Trip Length

per Household by Selected Trip Purposes, 1983, 1990, and 1995 NPTS . . . . . . . . . $11-8$

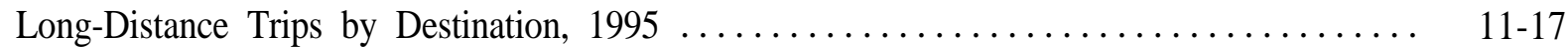

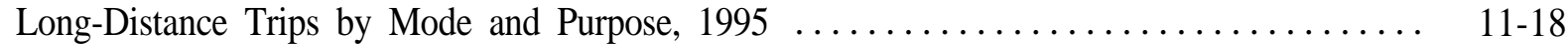

Long-Distance Household Trips by Mode and Trip Distance, $1995 \ldots \ldots \ldots \ldots \ldots \ldots \ldots \ldots$. 11 .1 9

Truck

Truck and Bus Registrations for Selected Countries, 1950-96. . . . . . . . . . . . . . . 6-3

New Retail Truck Sales by Gross Vehicle Weight, 1970-97 ....................... 8-3

Truck Statistics by Gross Vehicle Weight Class, $1992 \ldots \ldots \ldots \ldots \ldots \ldots \ldots \ldots \ldots \ldots$. 6 .6

Truck Fuel Economy by Size Class, 1977, 1982, 1987, and $1992 \ldots \ldots \ldots \ldots \ldots \ldots \ldots$.

Truck Fuel Economy by Fuel Type and Size Class, $1992 \ldots \ldots \ldots \ldots \ldots \ldots \ldots \ldots \ldots$. 8 . 8

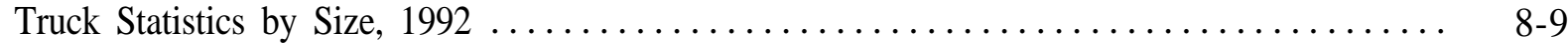

Trucks

Federal Exhaust Emission Certification Standards for Gasoline- and

Diesel-Powered Light Trucks (Category LDT1) ...................... 4-29

Federal Exhaust Emission Certification Standards for Gasoline- and

Diesel-Powered Light Trucks (Category LDT2) $\ldots \ldots \ldots \ldots \ldots \ldots \ldots \ldots \ldots \ldots \ldots . . \ldots \ldots \ldots$

Federal Exhaust Emission Certification Standards for Gasoline- and

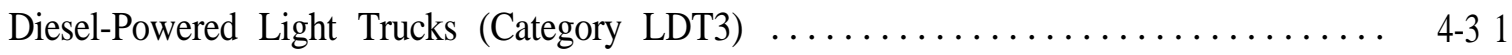

Federal Exhaust Emission Certification Standards for Gasoline- and

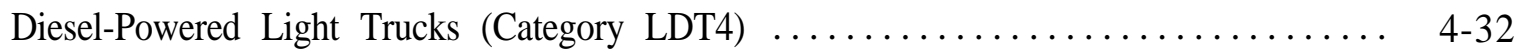

Federal Exhaust Emission Certification Standards for Gasoline- and

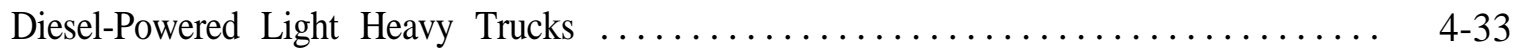

Federal Exhaust Emission Certification Standards for Gasoline- and

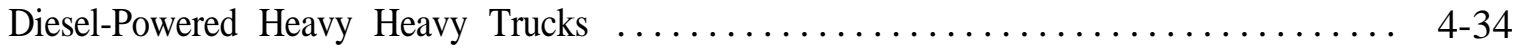

California Passenger Cars and Light Trucks Emission Certification Standards .......... 4-35

California Vehicle Emission Reduction for Passenger Cars and Light Trucks. .......... 4-36 
Trucks (continued)

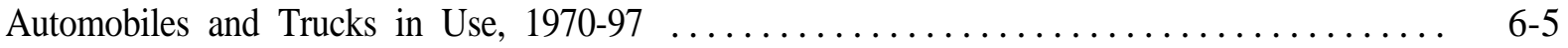

Trucks in Operation and Vehicle Travel by Age, 1970 and 1997 . . . . . . . . . . . . . . . 6-9

Average Age of Automobiles and Trucks in Use, $1970-97 \ldots \ldots \ldots \ldots \ldots$. . . . . . . . . 6-1 0

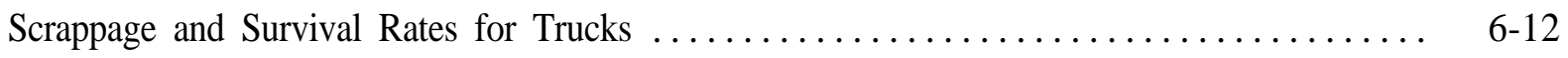

Sales-Weighted Wheelbase of New Automobiles and Light Trucks, Sales Period 1976-98 ... 7-1 1

Summary Statistics for Two-Axle, Four-Tire Trucks, 1970-97 ................... 7-12

New Retail Sales of Trucks 10,000 pounds GVW and Less in the United States, 1970-97. ... 7-13

Period Sales, Market Shares, and Sales-Weighted Fuel Economies of New

Domestic and Import Light Trucks, Selected Sales Period 1976-98 ................ 7-14

Sales-Weighted Engine Size of New Domestic and Import Light Trucks

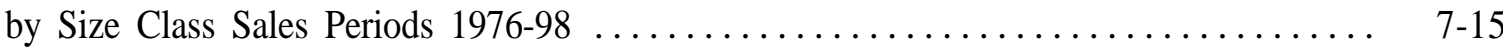

Corporate Average Fuel Economy (CAFE) Standards versus Sales-Weighted

Fuel Economy Estimates for Automobiles and Light Trucks, 1978-98 ............. 7-17

Summary Statistics for Other Single-Unit and Combination Trucks, $1970-97 \ldots \ldots \ldots \ldots$. . . . 2

New Retail Sales of Light Trucks by Gross Vehicle Weight, 1970-97 ............... 8-4

Percentage of Trucks by Size Class, 1977, 1982, 1987, and $1992 \ldots \ldots \ldots \ldots \ldots \ldots \ldots$. $8-6$

Percentage of Trucks by Fleet Size and Primary Refueling Facility, $1992 \ldots \ldots \ldots \ldots \ldots \ldots$. $8-7$

Percentage of Trucks by Major Use and Primary Refueling Facility, $1992 \ldots \ldots \ldots \ldots \ldots$. 8-10

Percentage of Trucks by Size Ranked by Major Use, $1992 \ldots \ldots \ldots \ldots \ldots \ldots \ldots \ldots . . . \ldots$. $8-11$

Period Sales, Market Shares, and Sales-Weighted Fuel Economies of New

Domestic and Import Light Trucks, Selected Sales Period 1976-98 ................ 7-14

United

United States Petroleum Production and Consumption, 1973-98 .................. 1-15

Passenger Travel and Energy Use in the United States, $1997 \ldots \ldots \ldots \ldots \ldots \ldots \ldots \ldots \ldots$ 2-13

New Retail Automobile Sales in the United States, $1970-97 \ldots \ldots \ldots \ldots \ldots \ldots \ldots \ldots$. $7-3$

New Retail Sales of Trucks 10,000 pounds GVW and Less in the United States, 1970-97. . . . 7-13

Class I Railroad Freight Systems in the United States Ranked by Revenue Ton-Miles, 1997 . 12-7 Urban

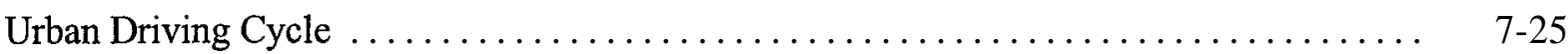
US06

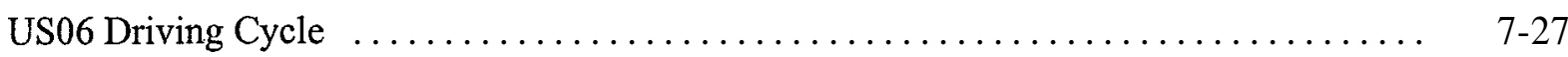
Value

Value, Tons, and Ton-Miles of Commodity Shipments, $1993 \ldots \ldots \ldots \ldots \ldots \ldots \ldots . \ldots$. $8-14$ Vehicle

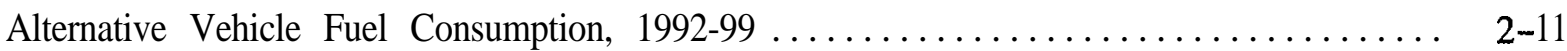

California Vehicle Emission Reduction for Passenger Cars and Light Trucks. .......... 4-36

Motor Vehicle Manufacturing Employment Statistics, 1972-97 ................. 5-1 7

Employees of Motor Vehicle and Related Industries, 1990 and 1995 ................. 5-1 8

Vehicle Stock and New Sales in United States, 1997 Calendar Year ................... 6-6

Highway Vehicle Miles Traveled by Vehicle Type, 1970-97 ...................... 6. 6-7

Automobiles in Operation and Vehicle Travel by Age, 1970 and 1997 ................. 6-8 
Vehicle (continued)

Trucks in Operation and Vehicle Travel by Age, 1970 and 1997 . . . . . . . . . . . . . . 6-9

Light Vehicle Market Shares by Size Class, Sales Period 1976-98 . . . . . . . . . . . . . . . 7-10

New Light Vehicle Dealerships and Sales, 1970-97 ...................... 7-16

Vehicle Specifications for Tested Vehicles ............................ $7-21$

New Retail Truck Sales by Gross Vehicle Weight, $1970-97 \ldots \ldots \ldots \ldots \ldots \ldots \ldots \ldots \ldots$. $8-3$

New Retail Sales of Light Trucks by Gross Vehicle Weight, $1970-97$. . . . . . . . . . . 8-4

Truck Statistics by Gross Vehicle Weight Class, $1992 \ldots \ldots \ldots \ldots \ldots \ldots \ldots \ldots \ldots \ldots \ldots$. . . . . . . . .

U.S. Advanced Battery Consortium Goals for Electric Vehicle Batteries ............ 9-11

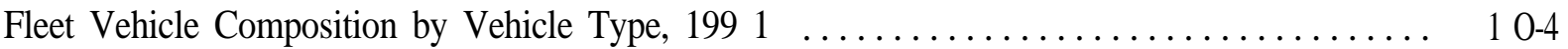

Average Annual and Daily Vehicle-Miles of Travel for Fleet Vehicles, $1991 \ldots \ldots \ldots \ldots \ldots$. . . . .

Average Miles per Federal Vehicle by Vehicle Type, $1997 \ldots \ldots \ldots \ldots \ldots \ldots \ldots \ldots \ldots$. . . . . . . . . . .

Federal Fleet Vehicle Acquisitions by Fuel Type, FY $1997 \ldots \ldots \ldots \ldots \ldots \ldots \ldots \ldots \ldots \ldots$. . . . . . . . . .

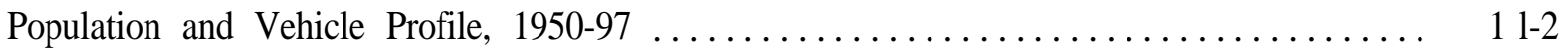

Average Number of Vehicles and Vehicle Travel per Household, 1991 and 1994 RTECS . . . . 1 1-4

Household Vehicle Ownership, $1960-90$ Census $\ldots . \ldots \ldots \ldots \ldots \ldots \ldots \ldots \ldots \ldots \ldots \ldots . \ldots 11-5$

Average Annual Vehicle-Miles, Vehicle Trips and Trip Length per Household

$1969,1977,1983,1990$, and 1995 NPTS . . . . . . . . . . . . . . . . . . . . . 11-7

Average Vehicle Occupancy by Vehicle Type, 1995 NPTS . . . . . . . . . . . . . . . . $11-9$

Average Vehicle Occupancy by Trip Purpose, 1977, 1983, 1990, and 1995 NPTS ....... 11-10

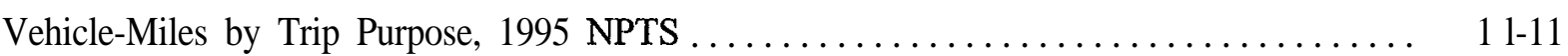

Average Annual Miles per Vehicle by Household Vehicle Ownership, 1995 NPTS ... . . . . 11-12

Average Annual Miles per Household Vehicle by Vehicle Age ................. 11 .1 3

Vehicles

Emissions of Carbon Monoxide from Highway Vehicles, 1970-97 . . . . . . . . . . . . . . 4-4

Emissions of Nitrogen Oxides from Highway Vehicles, $1970-97 \ldots \ldots \ldots \ldots \ldots \ldots \ldots \ldots . .4$. . . . . . . .

Emissions of Volatile Organic Compounds from Highway Vehicles, 1970-97 . . . . . . . . . . 4-8

Emissions of Particulate Matter (PM-10) from Highway Vehicles, 1970-97 . . . . . . . . . 4-10

Emissions of Particulate Matter (PM-2.5) from Highway Vehicles, 1990-97 . . . . . . . . . . 4-12

Federal Exhaust Emission Certification Standards for Gasoline- and

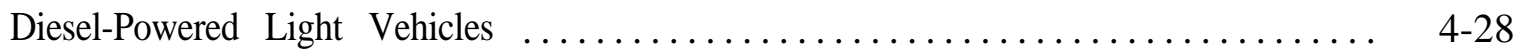

Vehicle Specifications for Tested Vehicles. ........................ 7-21

Steady Speed Fuel Economy for Tested Vehicles. . . . . . . . . . . . . . . . . . . . . 7-24

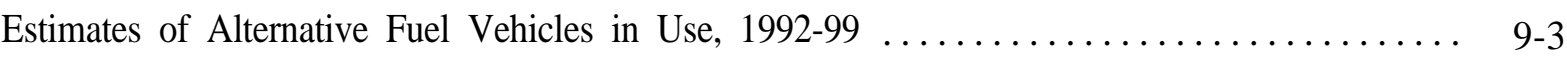

Estimates of Light Alternative Fuel Vehicles, 1995, 1997, and $1999 \ldots \ldots \ldots \ldots \ldots \ldots \ldots . . .49$

Estimates of Heavy Alternative Fuel Vehicles, 1995, 1997, and 1999 . . . . . . . . . . . . 9-5

Alternative Fuel Vehicles Available by Manufacturer, Model Year 1999 . . . . . . . . . . . . . . 9 9-6

Fleet Vehicles in Service as of January $1,1998 \ldots \ldots \ldots \ldots \ldots \ldots \ldots \ldots \ldots \ldots \ldots \ldots \ldots$ 10-2

Top Ten States with Fleets of Ten Vehicles or More, $1999 \ldots \ldots \ldots \ldots \ldots \ldots \ldots \ldots \ldots$. . . . . . . . . .

Average Length of Time Fleet Vehicles are Kept Before Sold to Others, 1991 . . . . . . . . . 10-4

Average Annual and Daily Vehicle-Miles of Travel for Fleet Vehicles, $1991 \ldots \ldots \ldots \ldots$. . . $10-4$

Federal Government Vehicles by Agency, Fiscal Year $1997 \ldots \ldots \ldots \ldots \ldots \ldots \ldots \ldots \ldots \ldots$. . . . . . . . . 6 
Vehicles (continued)

Energy Policy Act Purchase Requirements of Light Alternative Fuel Vehicles. ......... 10-8

Average Number of Vehicles and Vehicle Travel per Household, 1991 and 1994 RTECS . . . . 11 1-4

Average Age of Vehicles by Household Vehicle Ownership, 1995 NPTS . . . . . . . . . . 11-12

Volatile Total National Emissions of Volatile Organic Compounds, 1970-97 .......... 4-7

Emissions of Volatile Organic Compounds from Highway Vehicles, 1970-97 ........... 4-8

Warming

Numerical Estimates of Global Warming Potentials Compared With Carbon Dioxide ........ 3-9

Waterborne

Tonnage Statistics for Domestic and International Waterborne Commerce, 1970-97 . . . . . 12-4

Summary Statistics for Domestic Waterborne Commerce, $1970-97 \ldots \ldots \ldots \ldots \ldots \ldots \ldots$. . . . . . 5

Weight

Sales-Weighted Curb Weight of New Domestic and Import Automobiles

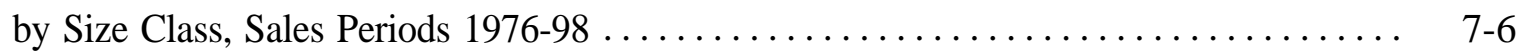

Engine Size, Curb Weight, and Interior Space of New Domestic and

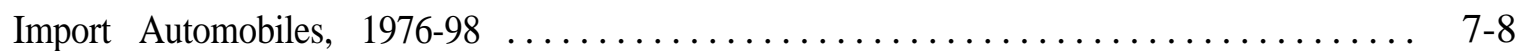

New Retail Truck Sales by Gross Vehicle Weight, $1970-97 \ldots \ldots \ldots \ldots \ldots \ldots \ldots \ldots \ldots$. . . . 3

New Retail Sales of Light Trucks by Gross Vehicle Weight, 1970-97 . . . . . . . . . . . 8-4

Truck Statistics by Gross Vehicle Weight Class, $1992 \ldots \ldots \ldots \ldots \ldots \ldots \ldots \ldots \ldots \ldots \ldots$. . . . . . . . . .

Weighted

Period Sales, Market Shares, and Sales-Weighted Fuel Economies of New

Domestic and Import Automobiles, Selected Sales Periods 1976-98 ............. 7-4

Sales-Weighted Engine Size of New Domestic and Import Automobiles

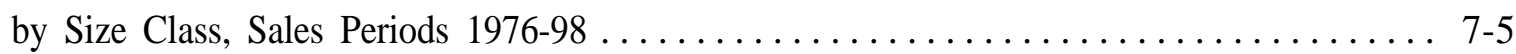

Sales-Weighted Curb Weight of New Domestic and Import Automobiles

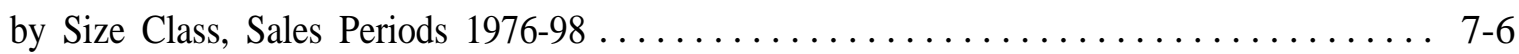

Sales-Weighted Interior Space of New Domestic and Import Automobiles

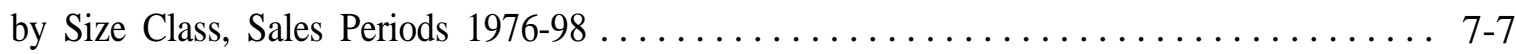

Sales-Weighted Wheelbase of New Automobiles and Light Trucks, Sales Period 1976-98 . . . 7-1 1

Period Sales, Market Shares, and Sales-Weighted Fuel Economies of New

Domestic and Import Light Trucks, Selected Sales Period 1976-98 .............. 7-14

Sales-Weighted Engine Size of New Domestic and Import Light Trucks

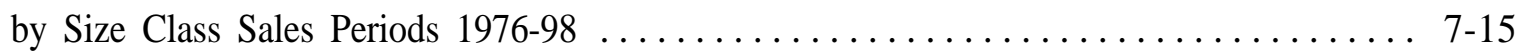

Corporate Average Fuel Economy (CAFE) Standards versus Sales-Weighted

Fuel Economy Estimates for Automobiles and Light Trucks, 1978-98 . . . . . . . . . 7-17

Period Sales, Market Shares, and Sales-Weighted Fuel Economies of New

Domestic and Import Light Trucks, Selected Sales Period 1976-98 .............. 7-14

Work

Journey-to-Work Statistics, 1983, 1990, and 1995 NPTS . . . . . . . . . . . . . . . . 11-14

Means of Transportation to Work, 1980 and 1990 Census . . . . . . . . . . . . . . . . 11-15

National and Metropolitan Area Comparisons of Journey-to-Work Statistics, 1990 Census . . 11-16 
World

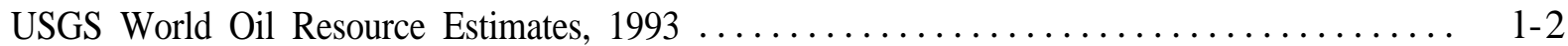

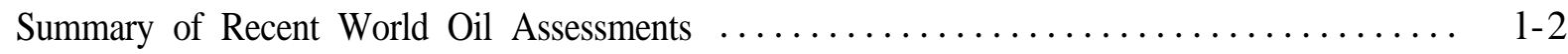

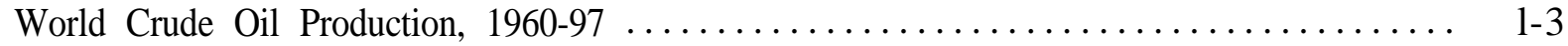

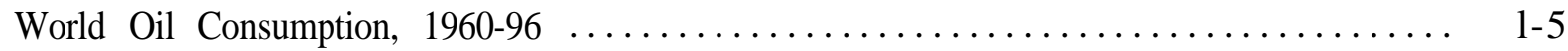

U.S. Petroleum Net Imports by World Region of Origin, $1960-97 \ldots \ldots \ldots \ldots \ldots \ldots \ldots \ldots$ 1-1 0

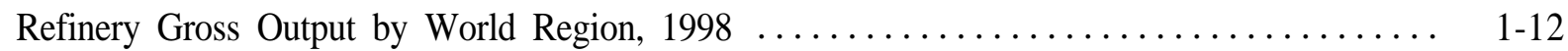

World Production of Primary Energy by Selected Country Groups, 1988-97 ............ 2-2

World Consumption of Primary Energy by Selected Country Groups, 1988-97 ........... 2-3 Worldwide

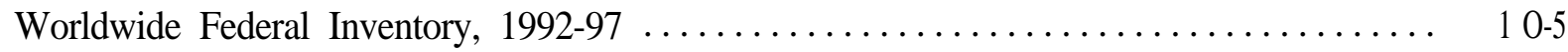
Years

Scrappage and Survival Rates for Automobiles 1970, 1980 and 1990 Model Years ........ 6-1 1 Yield

Refinery Yield of Petroleum Products from a Barrel of Crude Oil, 1978-97. .......... 1-14 


\section{INTERNAL DISTRIBUTION}

1. V. D. Baxter

2. T. J. Blasing

3. R. Boundy

4. J. E. Christian

5. G. E. Courville

6. T. R. Curlee

7. S. Das

8. S. C. Davis

9. D. L. Greene

10. L. D. Han

11. P. S. Hu

12. C. R. Hudson

13. P. J. Hughes

14. D. W. Jones

15. P. N. Leiby

16. G. Marland

17. L. N. McCold

18. W. N. Naegeli

19. R. D. Perlack
20. B. E. Peterson

21. C. G. Rizy

22. A. C. Schaffhauser

23. R. B. Shelton

24. S. Singh

25. J. E. Sorensen

26. F. Southworth

27. J. Terry

28. I. E. Treitler

29. L. F. Truett

30. J. W. Van Dyke

31. J. VanCoevering

32. ORNL Patent Office

33-34. Central Research Library

35. Document Reference Section

36-37. Laboratory Records

38. Laboratory Records-RC

\section{EXTERNAL DISTRIBUTION}

39. L. A. Abron, President, PEER Consultants, P.C., 1460 Gulf Blvd., $11^{\text {th }}$ Floor, Clearwater,, Florida 34630

40. S. L. Cutter, Director, Hazards Research Lab, Department of Geography, University of South Carolina, Columbia, South Carolina 29208

41. S. G. Hildebrand, Director, Environmental Sciences Division, Oak Ridge National Laboratory, Post Office Box 2008, Oak Ridge, Tennessee 3783 1-6037

42. P. R. Rittelmann, FAIA, Executive Vice President, Burt Hill Kosar Rittelmann Associates, 400 Morgan Center, Butler, Pennsylvania 1600 1-5977

43. S. F. Tiemey, The Economic Resource Group, Inc., One Mifflin Place, Cambridge, Massachusetts 02138

44. C. M. Walton, Ernest H. Cockrell Centennial Chair in Engineering and Chairman, Department of Civil Engineering, University of Texas at Austin,E Cockrell, Jr. Hall I, Suite 4210, Austin, Texas 78712-1075

45-996. Center for Transportation Analysis, Energy Division, Building 3 156, Room 102.

997-999. OSTI, U.S. Department of Energy, P.O. Box 62, Oak Ridge, Tennessee 37831

1000. Office of Assistant Manager for Energy, Research and Development, DOE-ORO, P.O. Box 2001, Oak Ridge, Tennessee 37831 